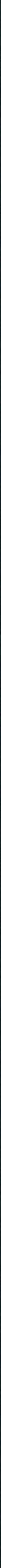




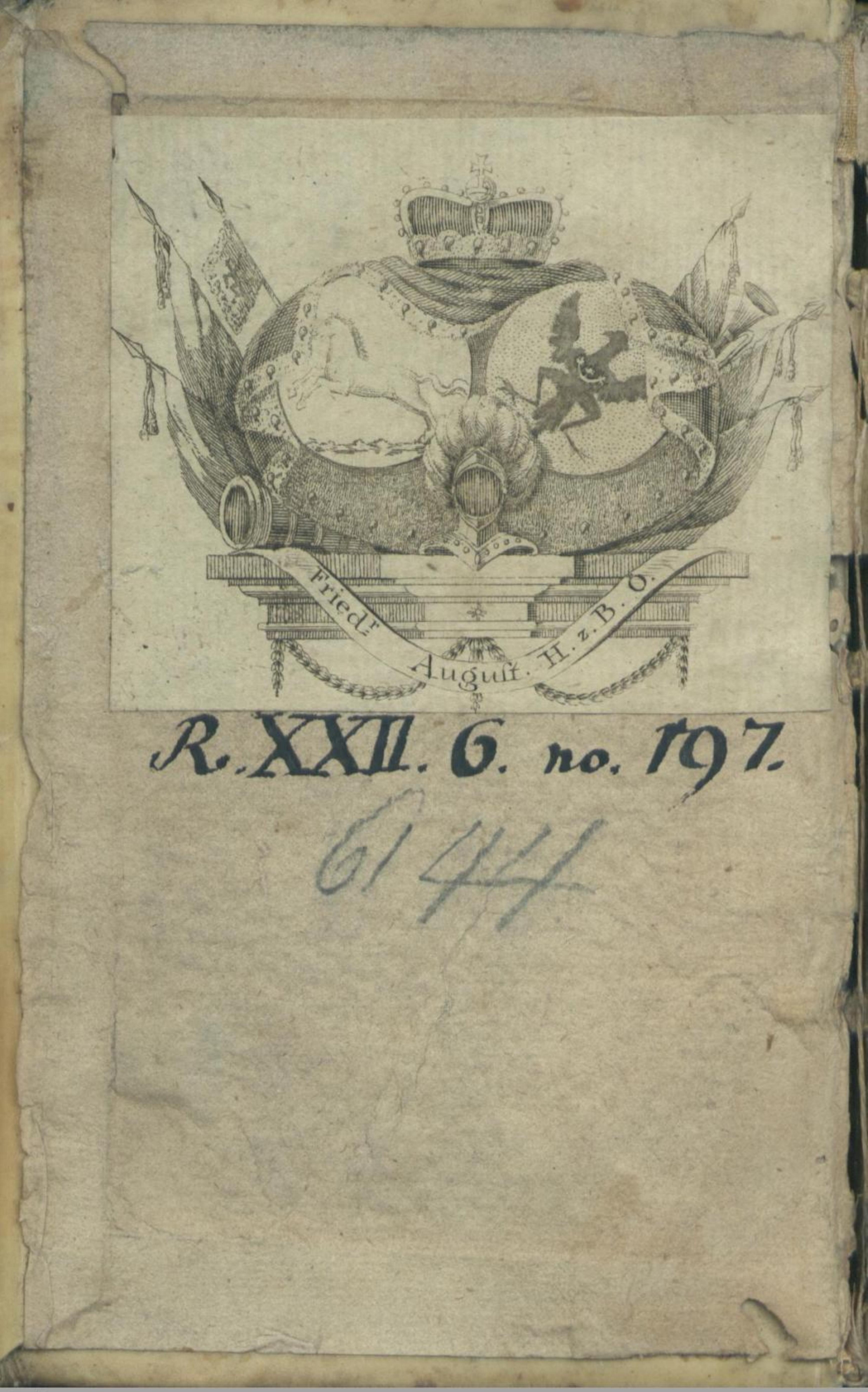






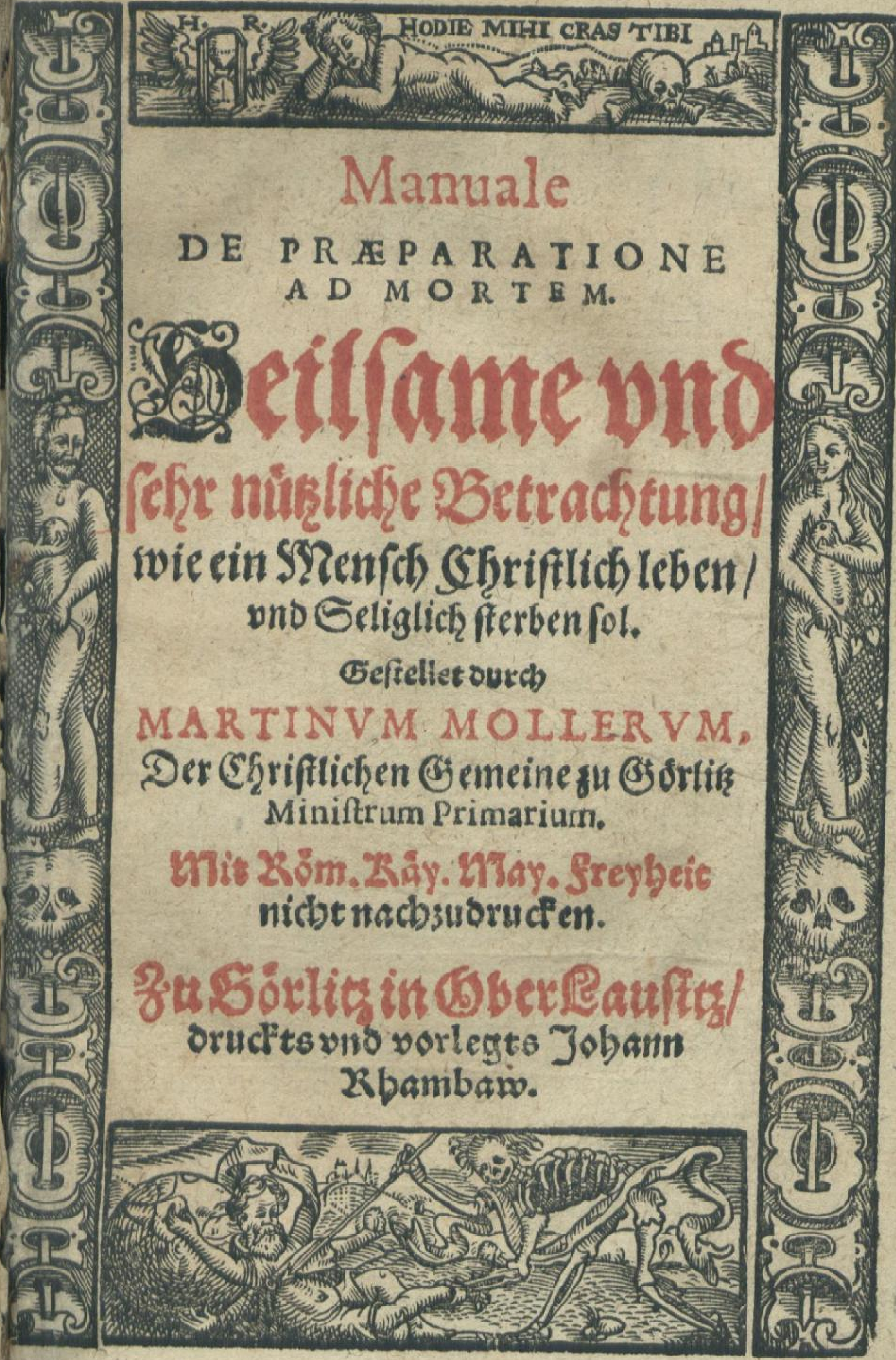



Gex Jolen/ biel Ebrentugenoretchen / Geftrengen frawen/ POL Y$\mathrm{X}$ E N A / gebornen Táchersin von Suchwato: Sep Eden/Beftrengen Ser.

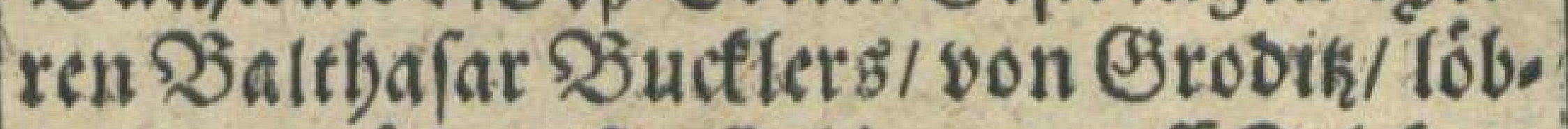
licher geoachentí / Erbberm auff Falce'en. berg/Sujaw vno Canterfoorff/zc. bin.

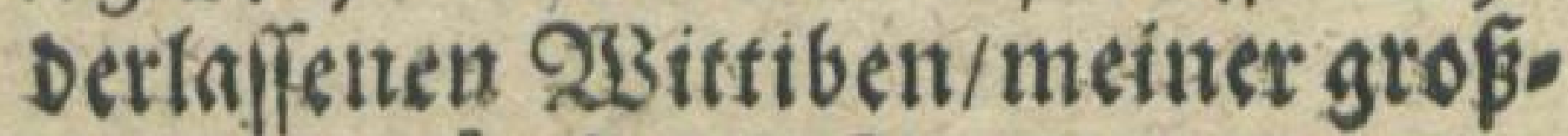
ginftigen Srawen.

Ginabevno friebe/ Durcb Jefint Chsiftum vnfern b隹rrn.

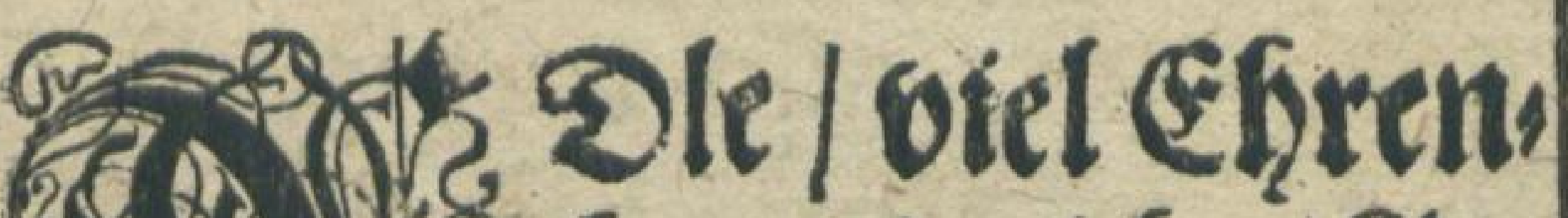
(5) thugenoreidbe/Ge3) am offenbar / oafialles/ was ein (Chriften SYenfith aus GD ttes 2Bort ftudiren/behalten vito thun fol / fiefertin ocm/ Dafer lerne:

$$
\text { A ij I.Shrift }=
$$




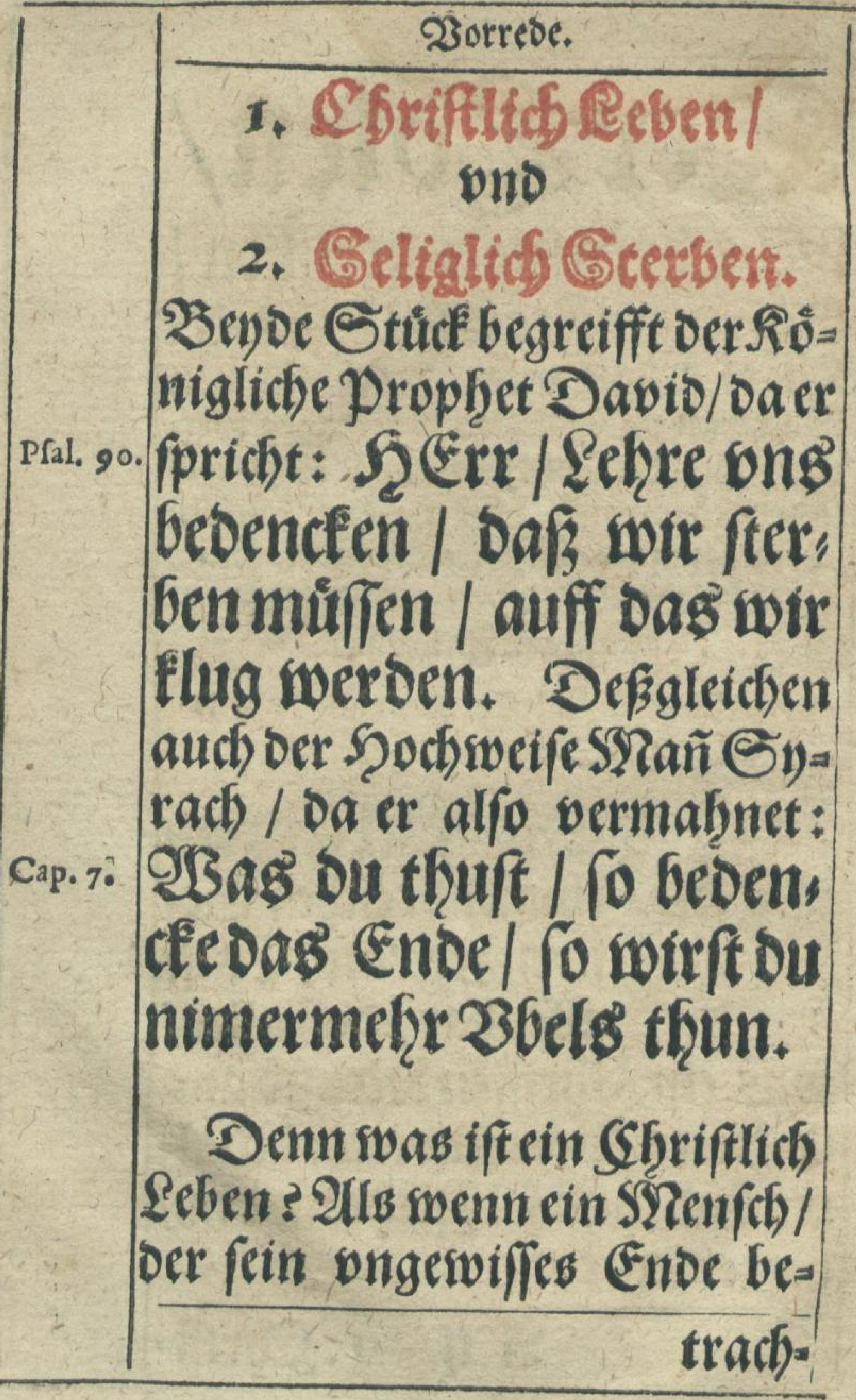


Worrede.

tract)tet/(ich) tåglich) $v$ bet in rect) $=$ tem Erfenonisßsottes/lebet in

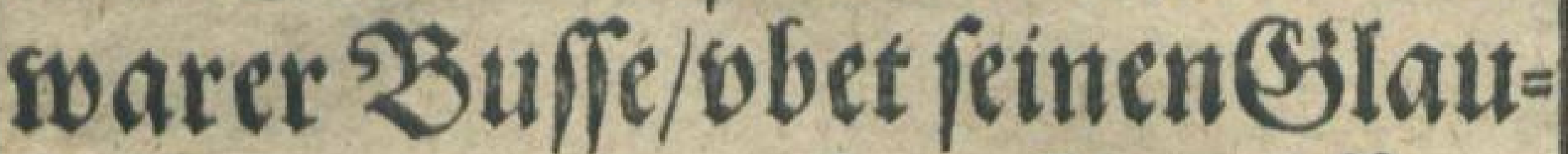
ben / berwabret fein Geswiffen/ Dajer (pricht Syrach) nimer vbels thute/Das ift / Er leffet fidh feinen Flugenbliat in etnem foldien Gtande fin Den / Darinnen er nicht ges trawet Selig zu werben. Zno was beiflet auch Selig= lich) fertbent ? Qlls weñ ein Şrtifens?enfth nicht allein tåglich bebendfet/ o a fer fterblich/ ono fur bem Eobenirgeno fietser ift fondern auch alle Stunden vin 2fugenblidf trachtet alfo ab $z$ t $=$ fobeioen/Daf er ein geswiffer $\mathfrak{E r}$. be bleibe oer erwigen Seligf eit.

$$
\mathfrak{A} \text { ii Daf }
$$




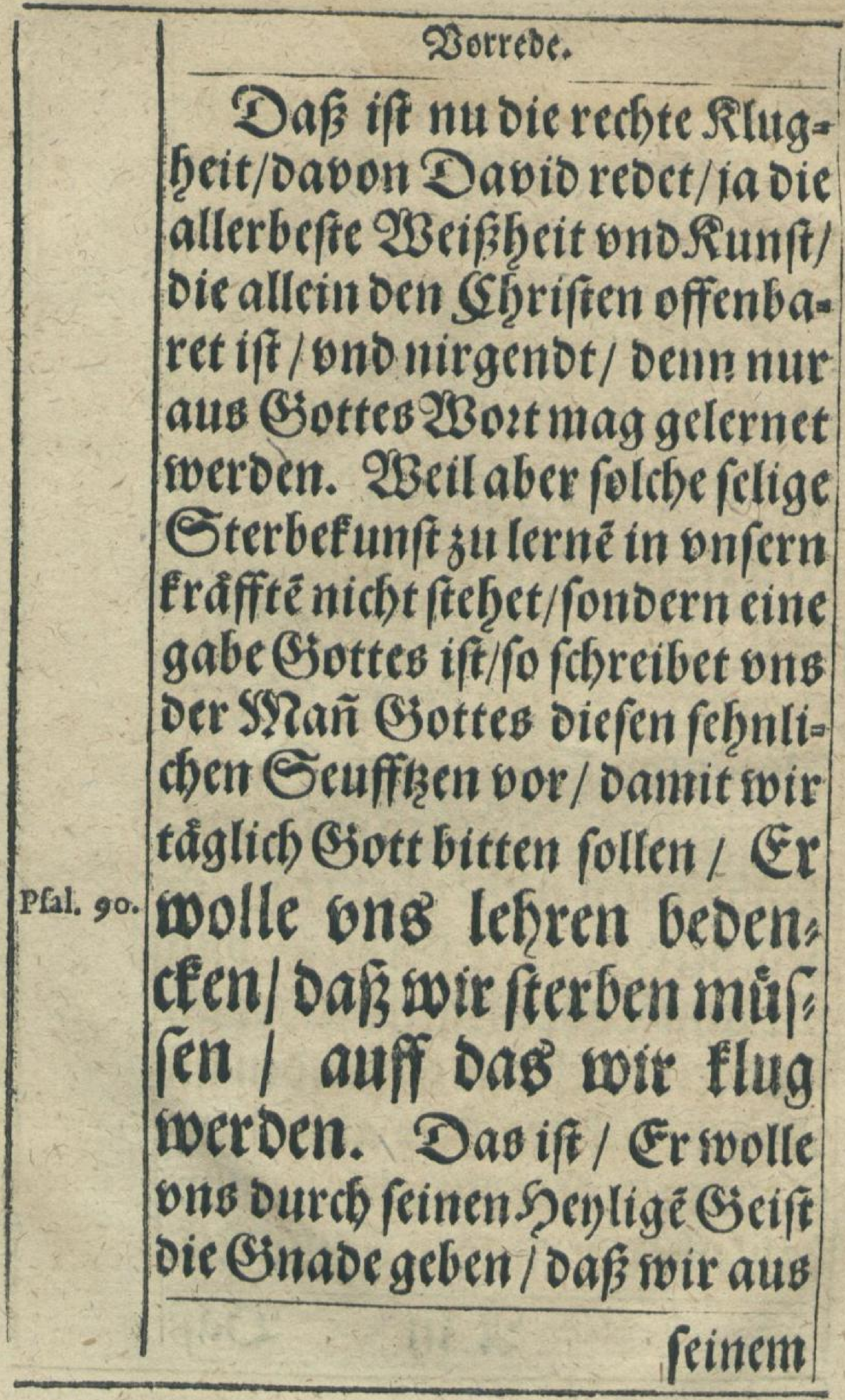


Dorrebe.

feinem feyligen \$3orte lernen onfer Seben alfo anfellen ono befchliefien / bamit wir auch borte eswiglich leben mògen.

Diefebeylige Sterbefunft haben vor Zeiten bie Seyben garnicht geswuft / if auch noch beutiges Eages verborgen alIen / Die Jefum SErriftum onfe= ren SDerrn nicht wollen zum Shenlande baben. Denn ob rod Cicero aub erfahrunge faget: Vita turpis ne morti quide bo- Orat. nefta locum relinquit. $\mathscr{W}$ ill $[a-$ puintio. gen:(S)leich/swie nach einem eht $t=$ licben Seben / gemeiniglich folget ein efrrlich Sterben / 2ulfo

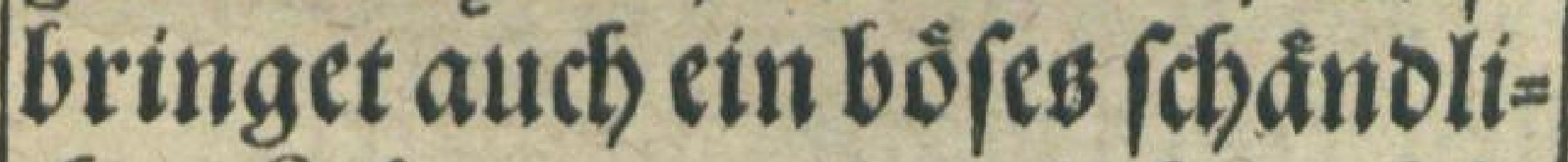
(b) $\mathrm{E}$ eben/gemeiniglich ein bo:= (és (ch)måbliches Sterben: Sp a iii i if 
Dorreos.

ift bod wber alle maffe ein grof: fer onterfocid intervitam boneftă, JointerChriftianam:intermortem boneftam, ¿o inter Chriftianam five Salutarem. Das ift / Bwifchen einem ebrrli= chen/auffrichtigen/euflerlichen IBandel / vno sfwifthen einem

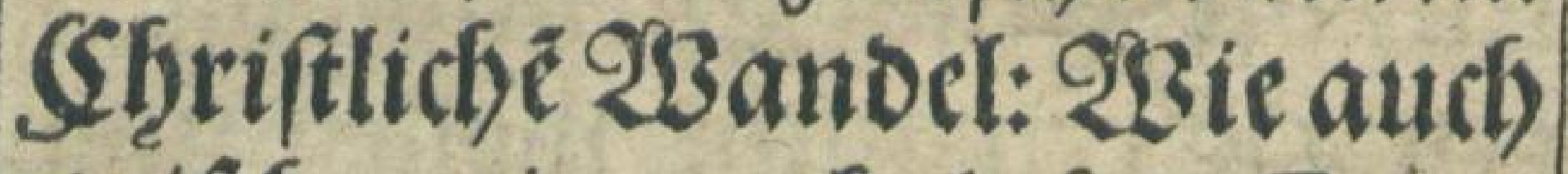
zivifben einem ebrlichen ter $=$ ben/vno zroifcben ellhem /eligen 2tofcheyde.

$\mathscr{B}$ enn Rânfer Auguftus Rom in tåglich swúnofchet $\mathrm{Eu}$. thanafian/ oas ift / Dafer fool fterben móge: 3 erfitehet er nich)= tes mebre / als das er bet) be we gen feines swandels bñfterbens citten efrrlicben Ramen finter fich laffenmoge. 98 ente mit 


\section{0 rrede.}

mit im oñ andern Şenden zuแ fterbe formet/fo ift oa fein sroft/ fein Soffinung / fonbern lauter

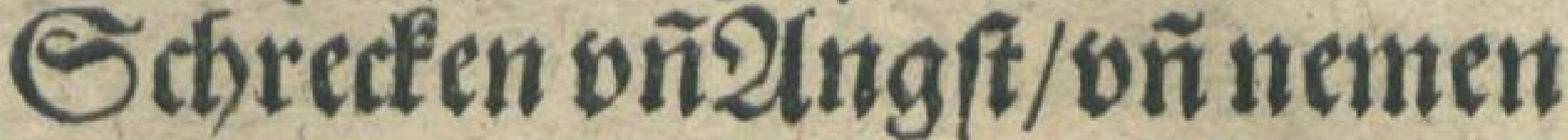
cin Enoe / swie Virgilius faget:

Vitaq́; cum gemitu fugit indignata Aeneid. fub vmbras':

Zitgernt liế er Das Seben fein /

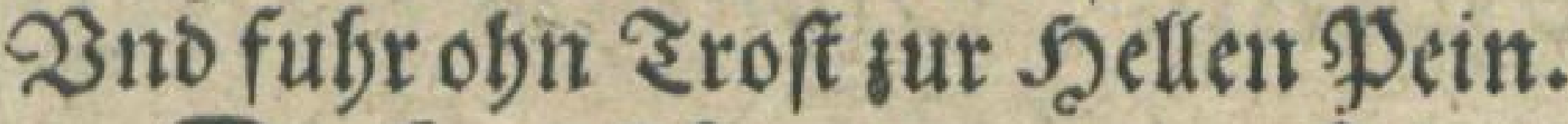

Daber fomet es/ oaf in oen (c) riffen oer23ngläubigen nur citel Rlagen wber SYenf(h)lich Eleno / ober oie furke 3eit but= fers $\mathfrak{E e b e n t / w n ̃ ~ b b e r ~ d e n ~ T o o ~ z u ~}$ finden fino/ Dagegé aber alles Trofts gefdrwiegen swirb. Totum, quod eft bomo, mi feria e $t$, fagt einer. Das ift $/ \mathbb{E}_{\mathfrak{s}}$ if nichts nebre ant Sienfeben / oenn nut lauter Trubjal ond Elenb. Rio: nig Xerxes weinete bitttcre

2f 0 ebr:




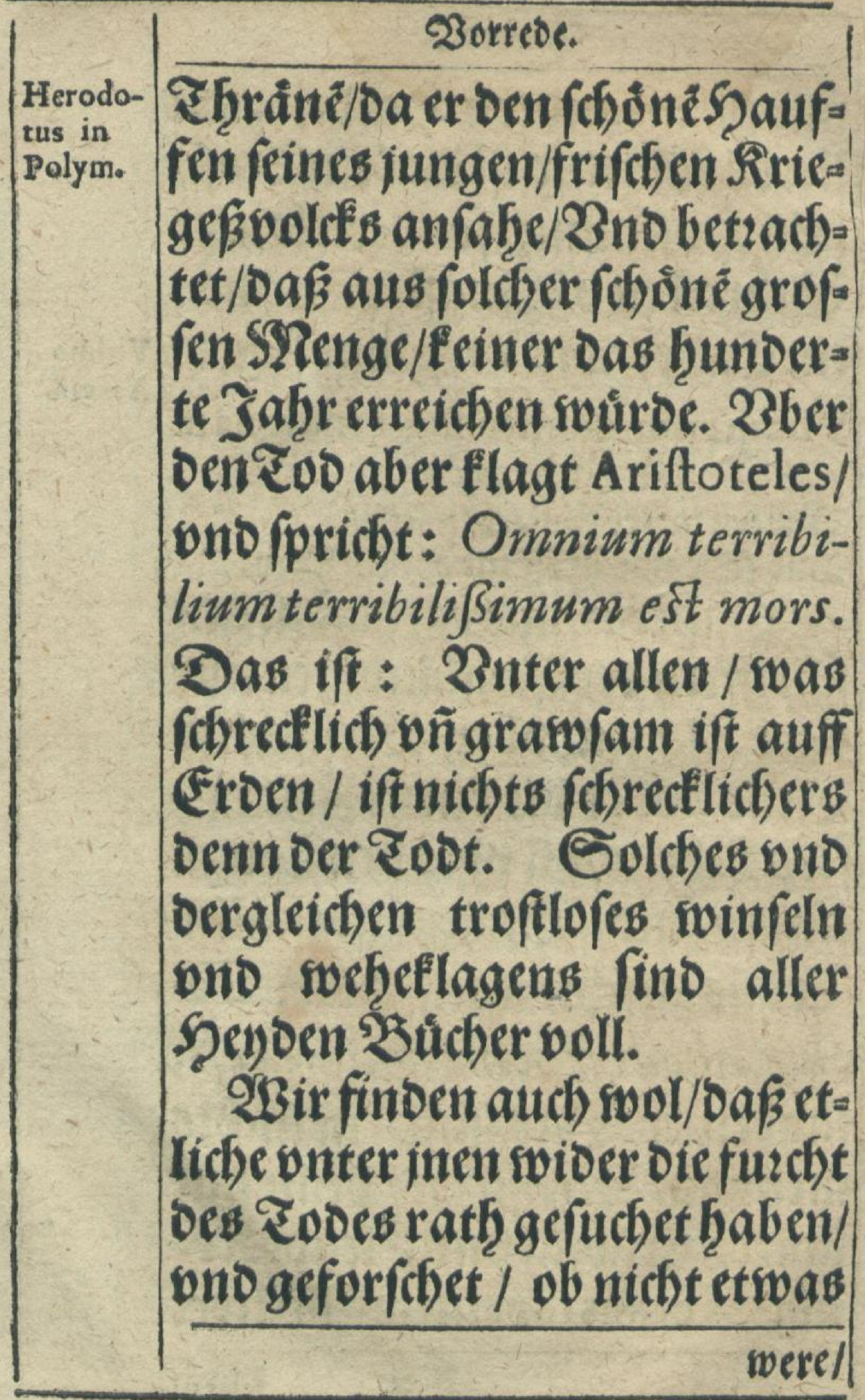


Worrcoe.

soere / Danit einer in folebem febreden Das Sperkeffillen/ond fich) sum fterben swilligergeben fonte. Olber fie find bojber nicht fomen/als woic Seneca faget: DenatuEffice mortem tibi cogitatione raxust. familiarẽ, vt, fi ita fors tulevit, poßisilliobviamire. Das if $/$ Du muft oir ben Tootfwol ein= bilden / vno ftets oaran gedent= dfen/bamit ou oich imt/weñ maldazu fömet/willig ergeton fanft. Daber lefen worr/oaj oic Egyptier cine gewonbeit gef̧a $=$ Herodobet / Daf fie in jren Pandieten Euter.

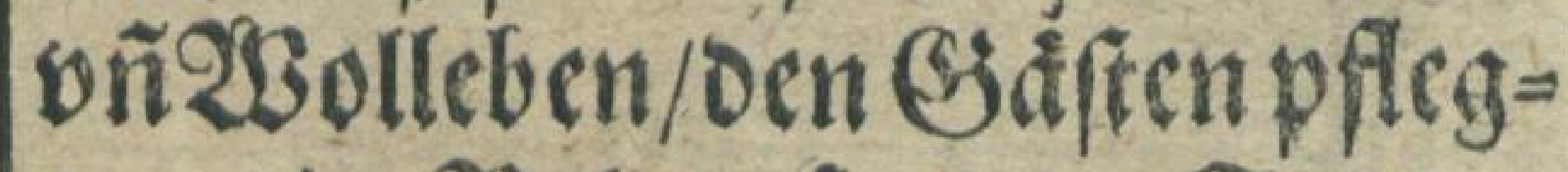
ten cin $\mathfrak{B}$ ilenif cincs Footen in einem Sarge fürtutragen vno zuseigen/ mit ang fiencle te Borten: In bunc intuens, bibe 
Dortede.

¿fis animo bilari, post mort $\vec{e}$ enimtaliseris. Das ift: $\mathcal{D}$ fchan an oif 23 ilo/ onto trinte/ vno madbe oids frolich/3 $3 \mathrm{coch}=$

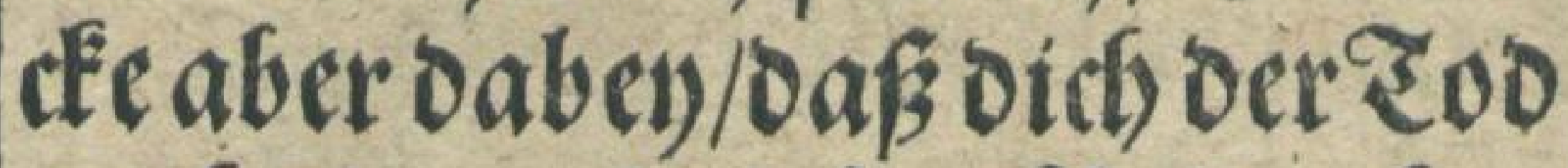
aucb cin mal Diefem 23 illocefrn licf machen wirb. 2no difí war ite praparatio vno vorbe $=$ reitung sum willigen ferben.

Soldber Runft gebraucbet fich Der (Sottlofe Amaleftififoe

r. Sam. Roontig Agag/Denn ba er fabe) 15.

Dafin ocrprophet Samudzu Stúden hamen wolte/gieng er fu ifim getroft / ono /prach: Xlio muF man Def To, Des bitterfett bertreiben. 2lls wolt er fagen/:Eg mus cin mal cin Sturk fein/orumb 
Sorrese.

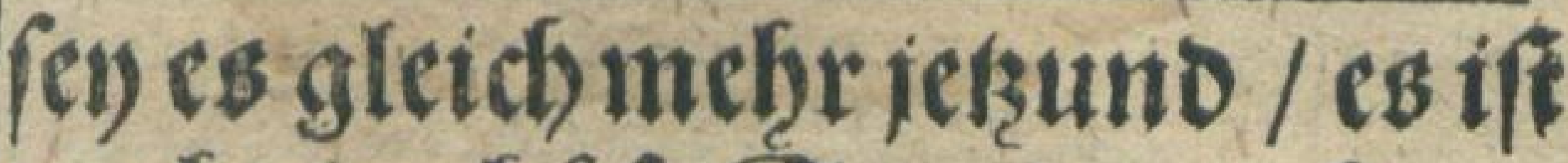
bumb eine bôfe Stunde zutthun. In Dés aber zitterten ire Soer $=$ ken/ hatten feinen Eroft / wno giengen mit grim ono murren

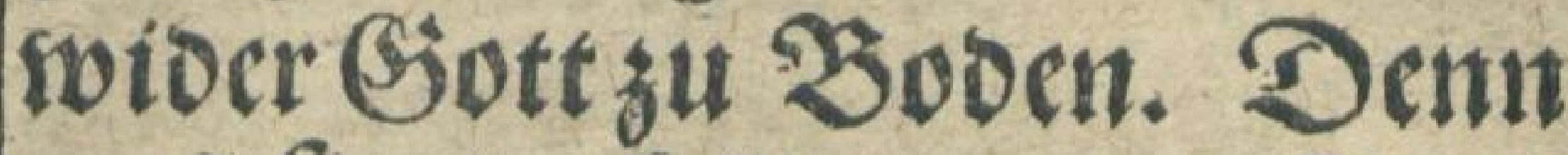
weñ fie gleich lange nact) Troft forftheten/ ond ire Soersenffil= Ien wolten / blieb es boch end t lich o abey) / woie Cicero faget: - Ego tentatis rebus omnibus, nibil invenio, in quo acquie cam. Das ift: Joh babe alle meine Runft herfür gefuebt/ die id jes mals ftudiret/ $\$$ b ich jirgeno $\mathrm{ei}=$ nen Iroft Daraus foboffien fơndec/ a Q 93enn fie aber gefehen faben oer Sterbenoé fleinmútigfeit/ ober ffer zagen fur Dem Tooc/

ppotte" 
Sorrede.

fpotteten fic iter nod o ofu/ond Senea fageten : O te dementem, $2-$ denatu-
ralibus oblitum fragilitatis twa, fitunc quxit. timesmortem, cum tonat. Das

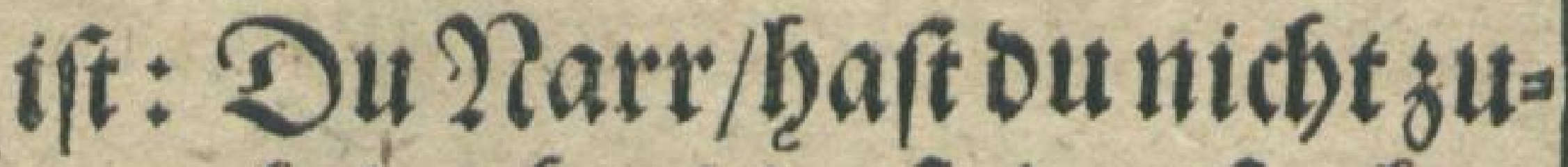
vor bebacht / baj ou ferben muft / Fill ou oen Tos nuerft fürchten / nu er fobon Dafer raufichet?

23as aber bas Evige 2 eben belanget / bemuiben fich Plato / Cicero/vno andere wottrefflich fehtr/zuersweifen/Dazbie Ser= len oer SWenfachen wnferblich finto (oenn was oie Leibe belanget / ift feinem niemals in Siñ fomen/ Daß fie wioer aufferfteben vno leben folten) vno oas nach Diefen Ieben bie Seclen oer fromen einen orth Der ? $\mathrm{lu}=$ 


\section{Wortebe.}

he haben wúroen. GaCicero swil ifm autb cinen Sguth ma* d)en/als fresvete er fich zur verfamlung oer Seelen fu fomen/ ono fpricft: O praclarum diconcilium cettumǵs proficiicar, ¿o cumm ex bac turba \&o colluvione difcedam . Das ift: 0 weldh ein ferrtictser Tag wird Das fein / Da ich aus diefem muthereligen Eeben abjecter)= Den/ ono zur Berfanlung oer Geelen fomen seroc. 98seil aber in inen fein rechter (5lau= be / feine soirffung be gen Sesiftes/vonfeine Soffinun= ge noch beftendiger Eroft war/ fo belt foldser erticfter Sziuth oen ftich) nicht/fondern ift oulbe: eture. 


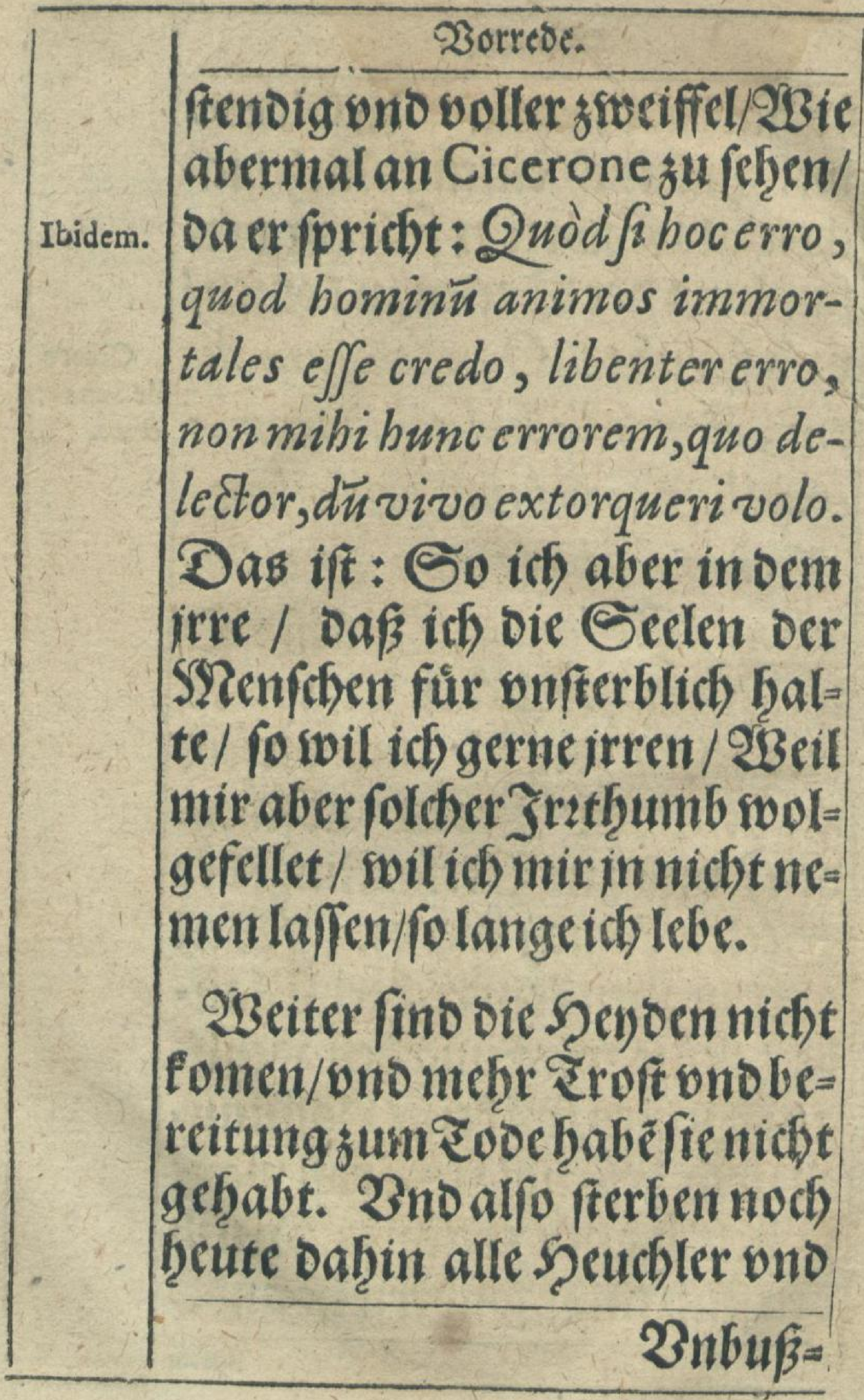


Borrebe.

Znbuffertige / bie nicft fint oes foaren Slaubens an Jefum Sthriftum vnfern ŞErrm.

Ja er follen auch biebey alle Şeนchler/2 $/ 2$ glåubige oñ $2 \mathfrak{n}=$ busfertige vinter ben SEriften genvis ono wolmerten / Daß it Zerbamnif an jenem Tage viel fehtwerer ono grofler fein woiro/als der Shendê/Eben barvmb / Dá̧ inen mefre als oen Senden/Dafiecht Def Euangeliigefebienen / Sic aber bod

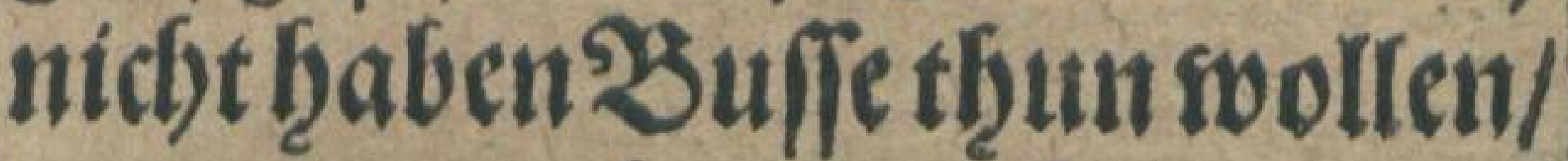
ono gum nolwersnt fieb) gebalten. Denn poppricht oer ŞErr felfer: OSBo elth jemanot Matt.zo nidf t annemen wiro/nodf erwer Pedeforen / Soge

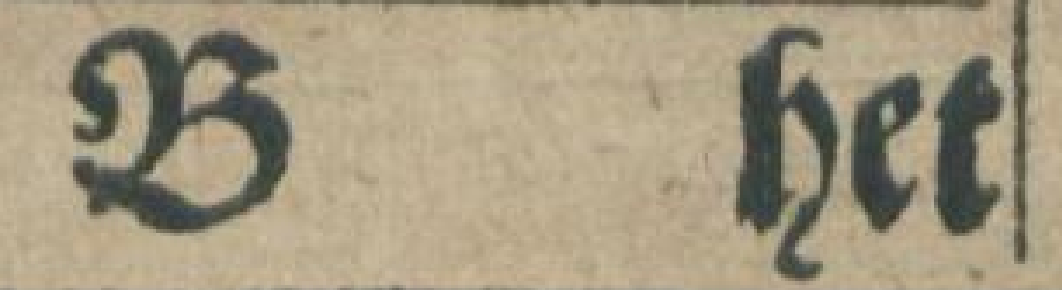




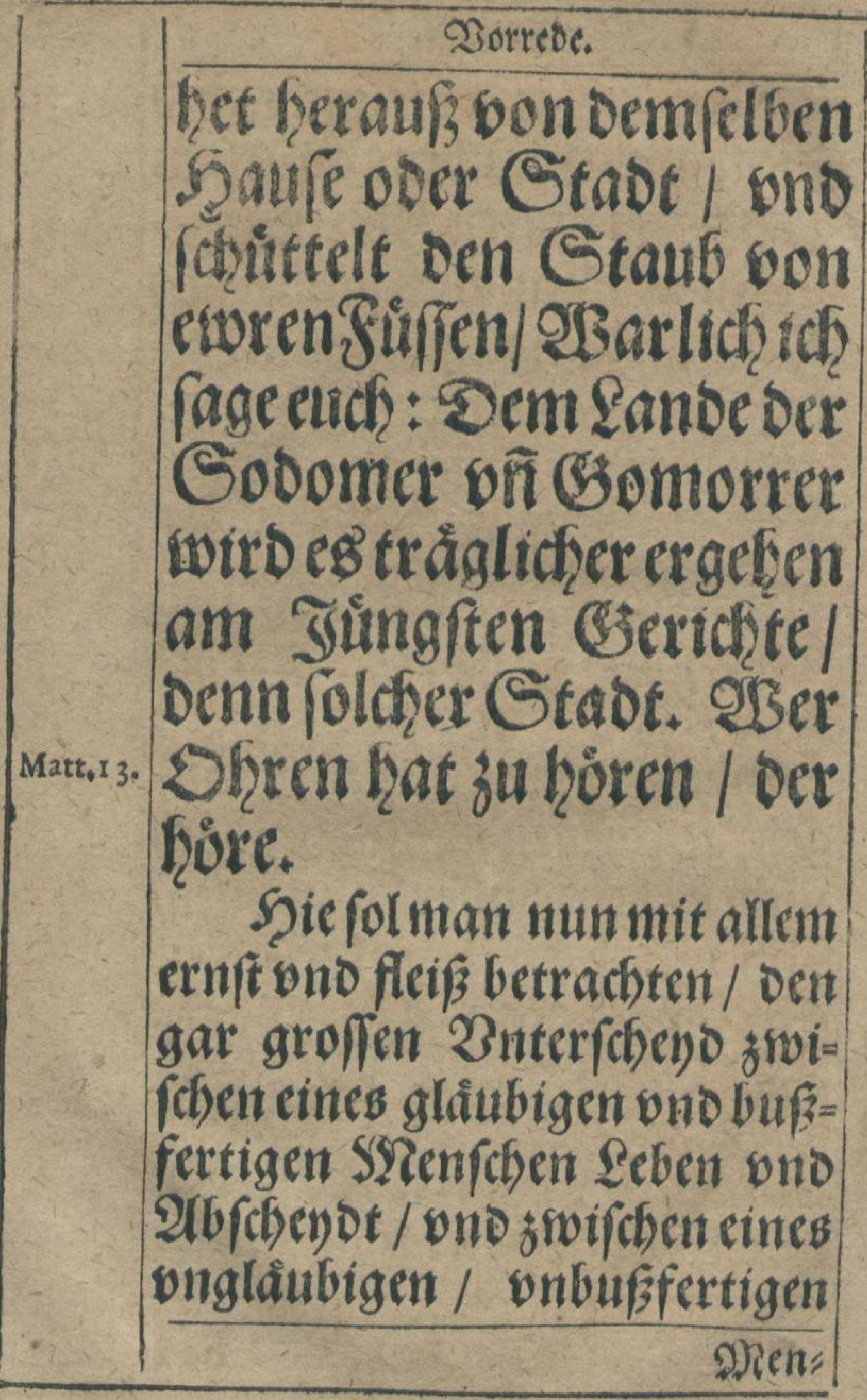




\section{Borreoe.}

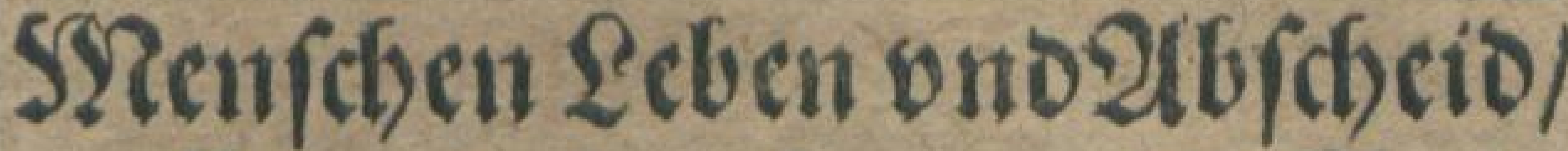
auffo oas wir erfennen oieSina. oc Siotteb / fo bus SEgriften ge geben ift / ono in von Setrizen růbemen ono prey fenlernen.

$$
\text { I. }
$$

Dettin was exfflich bas Lee

I. ben ber Menfchen anlanget/bat ber

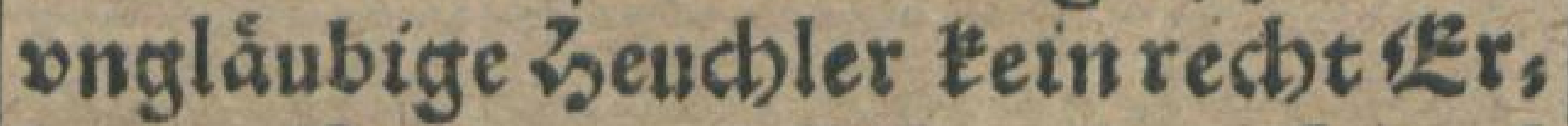
tenouif Gottes / Gibet ben bimel an / wie die Züub ein netw Gchenn Chor / 25raudbt ber eolen Gefboipfi Gottes / wie die Gave ber dEycbeln vntern 25ame/ DencFet nicbs ein mal/oafier audb foúloig fey Gott für fein Leben vin Doolfartb zu oans cten / Sraget nichts nach dem KE'wis gen Leben / Sonbern were feine zjerben luft / wenn er nur jumer bie leben/ und feintes fleifcbes lífte trei, ben folte. Dito iff gewif / dafjoas Diebe/ ja die Såwe cin beffer Leben fübren / als folche IMen/wen/Die

$$
25 \text { if Doch }
$$




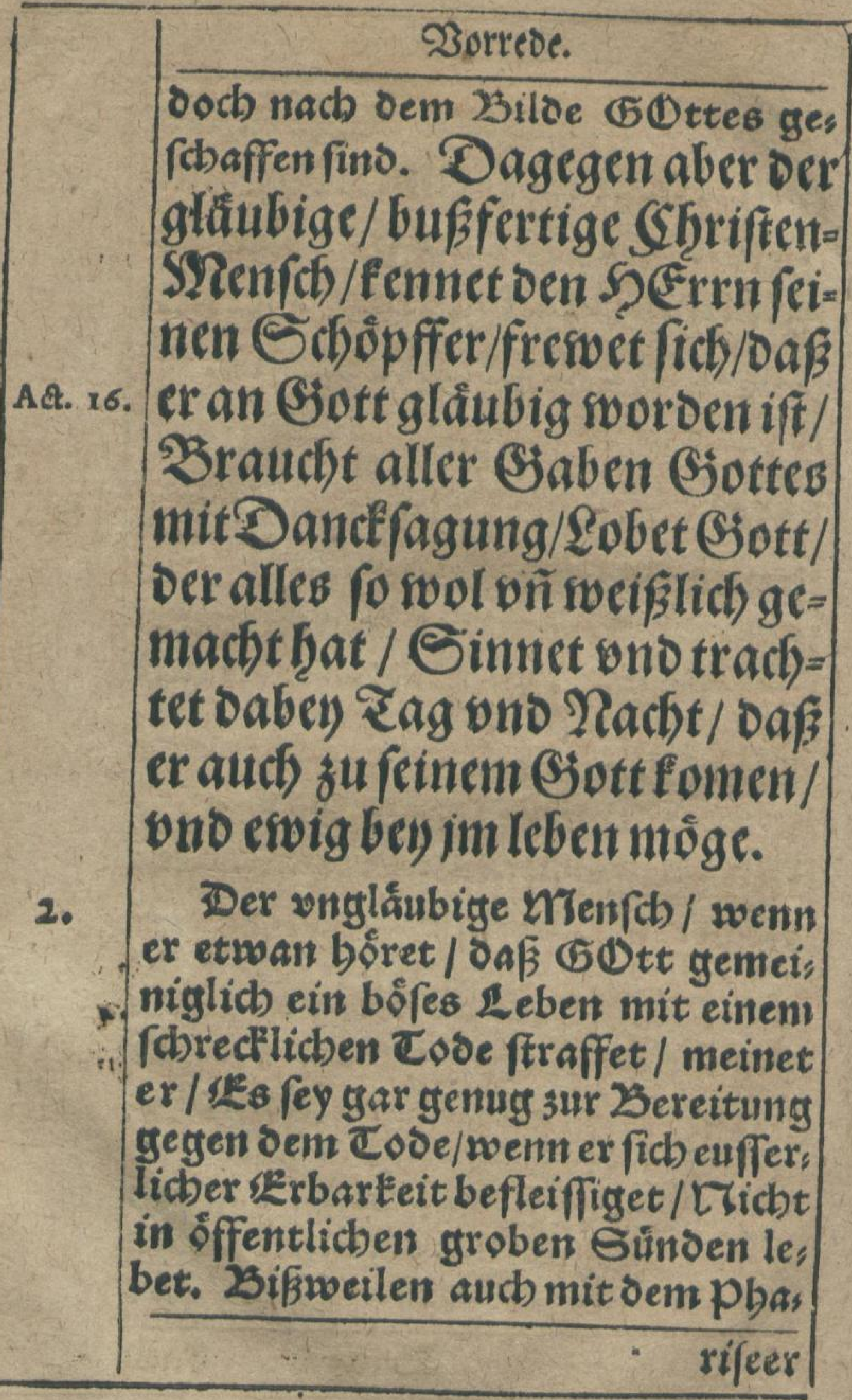




\section{Sorrebe.}

rifeer faytet: Jh) sancte sir (Gott/ oafich nicht bin/ wie anoer feus te/Xtáuber/Dngeredte/2̌bebre" cher/zc, vno foblieffeis balo oaranfi/

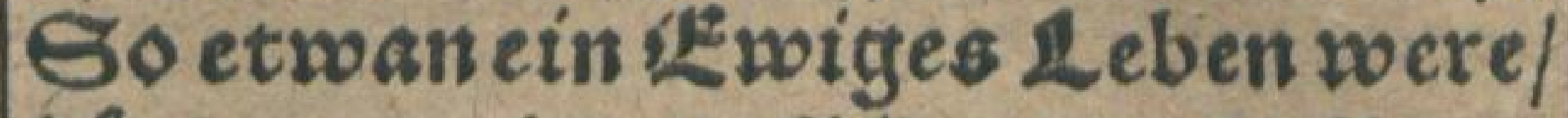
Énote es jun GOtt berbalben/ als erbarn Leuten / nicbt verfagen. Dagegé aberft ubiret oer glălu- Pfal. 90. bige ShriftenSzenf b oiered) bev)lfanc Sterbefunft/Erfen= Syr.7. net ono berefoet feine Sưnoe von Serken/Trófet fich feines Set)lantes beroienftes onto genugthutung/in frevoiger \&uber = fict) ono frolicber Soffinutge/ Siott foolle? oe annenten / ono lebet taiglich alfo/Dajer er beswaret Den Sjlau= ben bno gut Sierviflen bi Enoe.

Daf beucblifde Delttind/ weil es immolgebet/ifts ein guterCbrift/

t.Tim.1.

25 if tent 


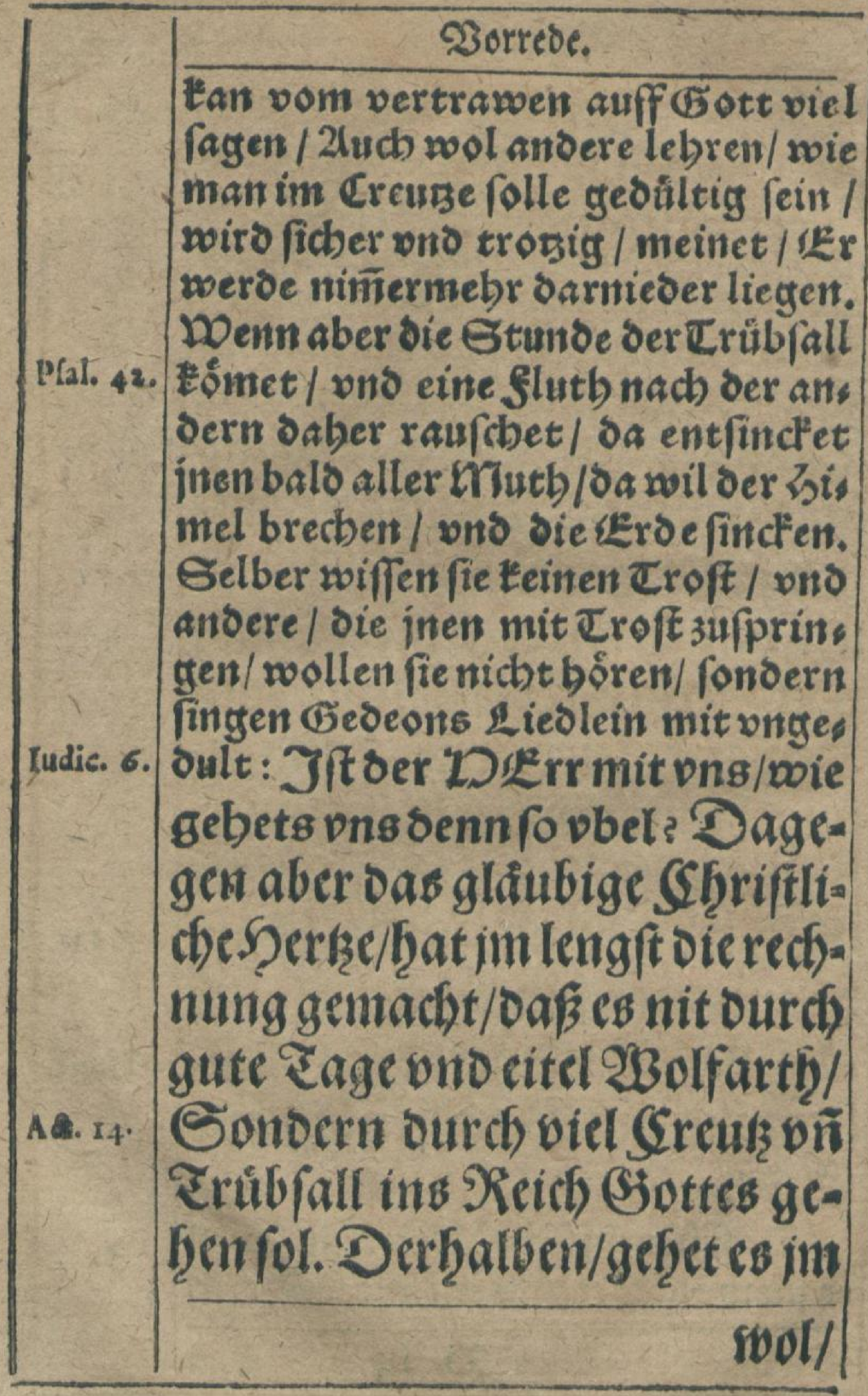




\section{Sortede.}

sol / fo bandéter Gott / Der in oef Suten im Lande genieffen Efaix x. lefiet / Sutet fich vor ficherbeit।

Dienet Giott Dem Soerrnmit Pralm.2. Furd)t/wno fresvet fich mit sit = tern. Sebets int wbel/ fo finget er mit bem lieben 306: Sgaben Hiob. 2. wotr Das Csute etmp fangen oon ber Sandot bef Sert ren / waromb folten wir Das 30 ofe nidfet aud ans netten? Sclt bas Sreutze an/ fo finget er mit bent lieben $D a=$ vio: O6es weret bifis in die pal.rzo 2acht/ ono wiber an oen seorgen / Doch fol metn Sorts an (Sottes Seachel oerguberfeln nidnt nock 25 uif Torgen. 


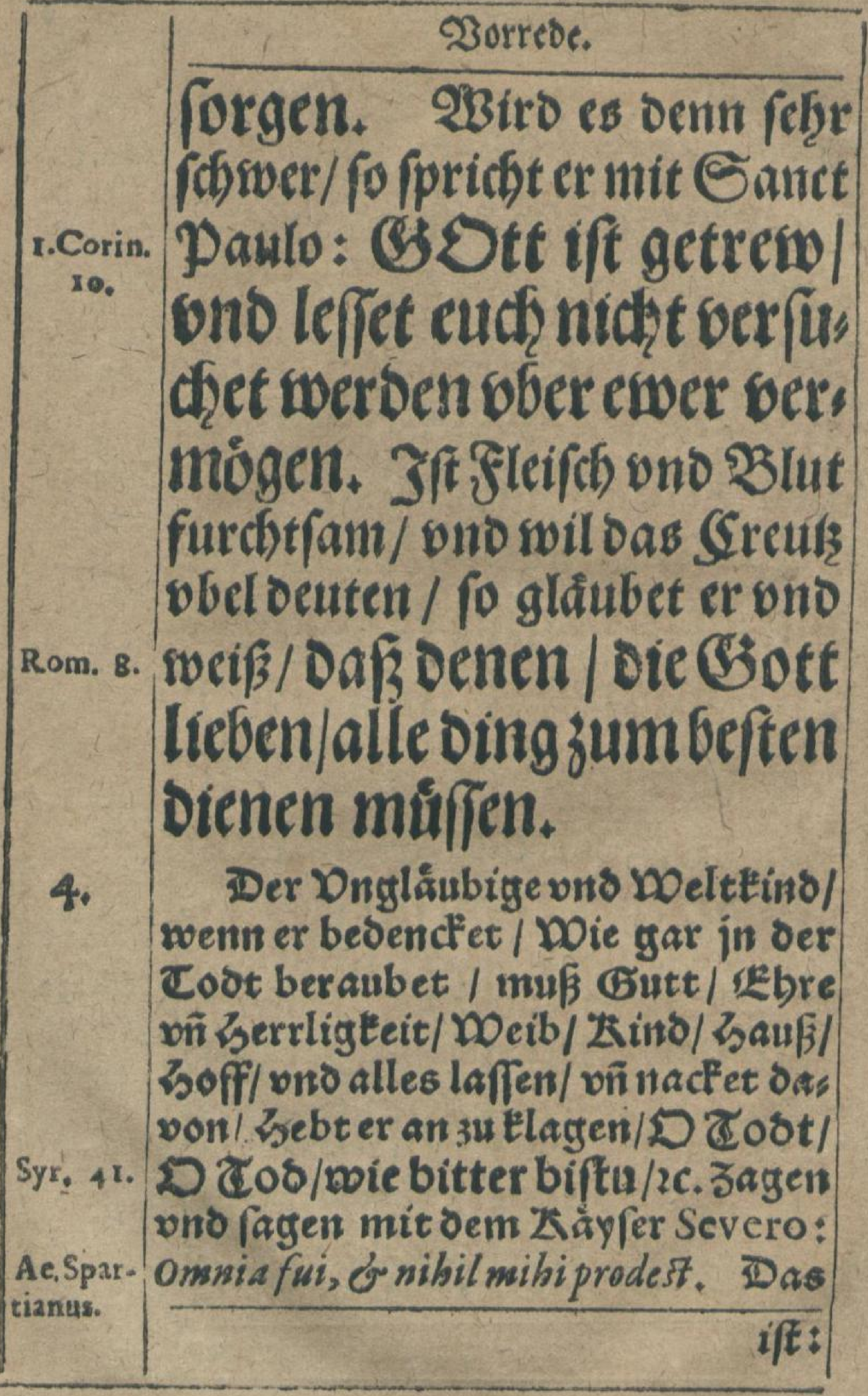




\section{Sorrebe.}

ift: Ydb bin tgrop/berrlidh/mádbtig/ vino alles gewefen/ Thu idh aber fier, ben fol/ ift nichts vnter allen / oaj midh retten Esnte. Dagegen aber bat der recbte Sforiftens?enfich Das rectite gute Efenleriveblet//Luc. zo. frewet fidb oefz oberforwengli(b) Ren Sefthumbs/Der esvigen Sutter@sottes/fan das zetrlitbe alle Qugenblidf wollaflen/ ono faget mit ben fornige Davio: Sgert / Duerfreweft mein Palm.4. Serk / of jene gleich biel SBetn ono erorn baben.

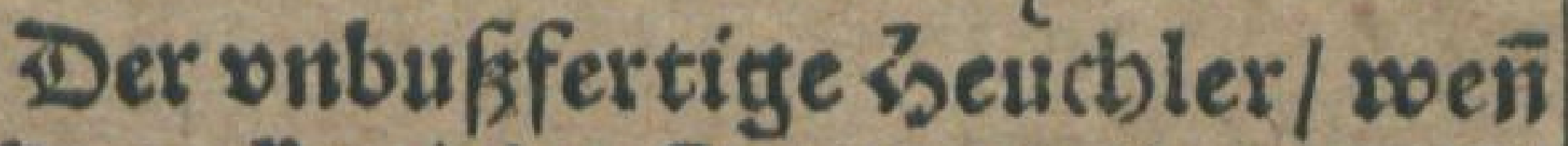
er trand" wird / finnet nur barauff / Do er die Zirand"beit betommen/ Doas er vutgefindes tgeffen / WO er eituen bo̊fen Crund" getban/ Doer fidberEáltet/erbitzet / verderbet ba: be / Ift zornig vno vrigeoriltig/ oa er in fold Dnglicte geratben ift.

$$
\text { S. Der }
$$


Dortede.

Der Gläubige SIenfeb aber

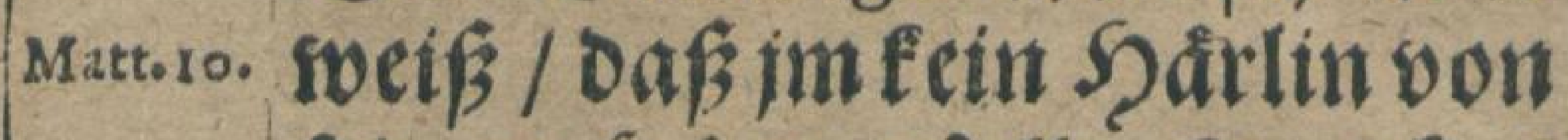
feitem Şâute fallen fan/ofine ferines Sottes willen/ge/chroeige oenit/ oafs er die edele Gabe oer (Siefundheit verlieren folte/ ofine befonoern willen Gottes. Derfialbengibter feinen Stut

terem.s. Den oie fobulot / Damit er bie Straffe der Rrandfleit verdie net/2 Bno erfennet/o a F in Bjott 1.Corin. fundfiget / onto alfo zur ßuffe

locfet / PBeif atich wol / woenn (Sott soas thun swil / ond cinen sqenfeben beimfucben / múffen fich alle SRittel bagu fotiden / ono 2 r facbe zur Rrandifeit ge= geben werden.

6. Der vnglínbige / fichere Noelts menfb/beoendit / eint Eeele nicht eber/bif er Erand wiro/ Da miler 


\section{Borrede.}

exft from nerben/ zuvor ifter fein lebenlanty ein prediger feind gewe: fen/ Thu aber mufs man anfpannen eilend vno gefdowinde/ond dent pres oigerbolen/zuvor bat er jn nimmer anders/als einen $P$ faffen/ia wollo, fen vnnuisen $p$ faffen gebeiffen/ T7u aber nennet er jbn einen Doiroigen Diener Gottes/vno /dreyet arffin 3u/O lieber/ frommer/ ebrwirdiger borr/belffet mir/gebt guten Kath/ oafich inicht werdampt werbe. WaAf beiffet beñ warlich an ftatt beß 3 Benedicamns/0 $/ 0$ Deus in adiutorium ange fangent. Gebet auds mit mans chem/wie wir fingen: J b fürcbt fürnoar sie (Gòttlich Gnas / Die er allzeit Derfpottet bat / wirb fbowerlicb ob im fabweben. Der glăubige/ buffertige Gfriften Srenfel) aber ift alle Stunoen ono Qlugenblicf fertig/ fikset in Pral. 90. tåglidber bercitf(bafit sum feli $=$ gen Sterben / Seflet fid atud) 


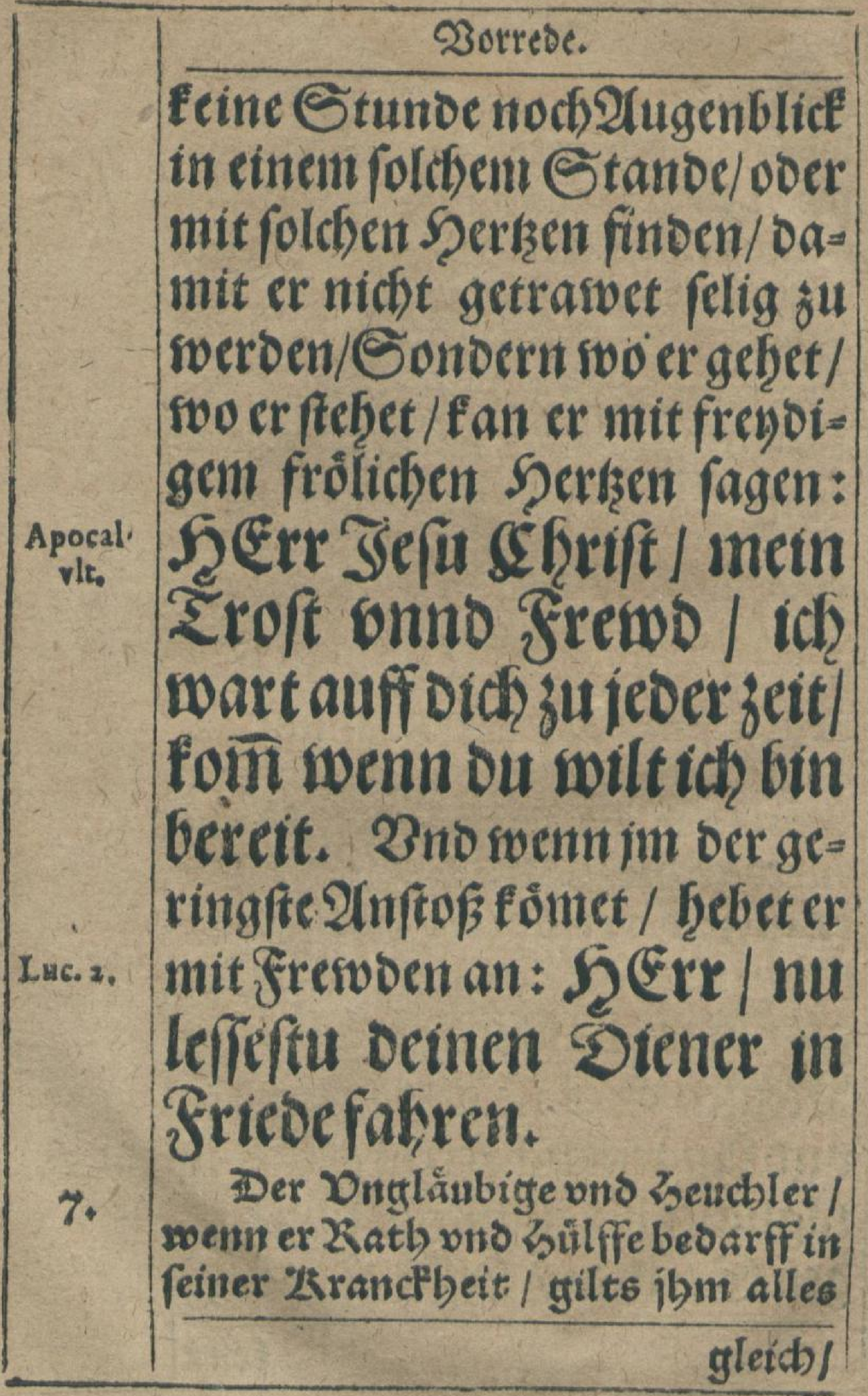




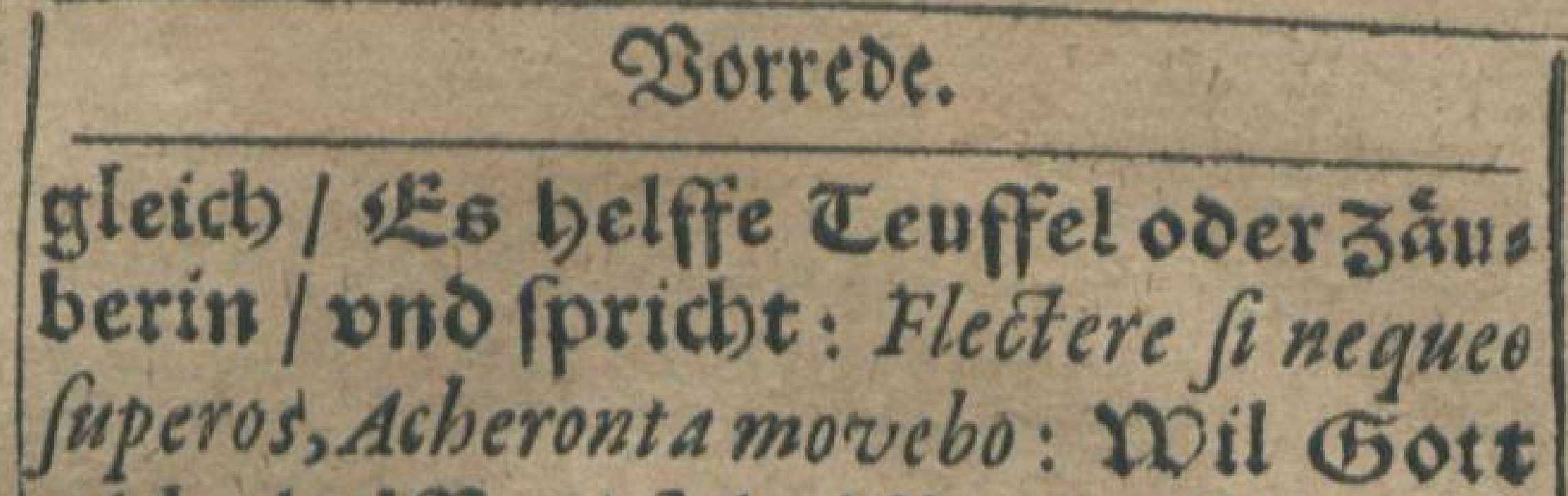
nicbcbelffen / fo belffe Teuffel / ono wer vor teomet/Da af mir nur gebolf fen wiro. 2lber Der Stăubige

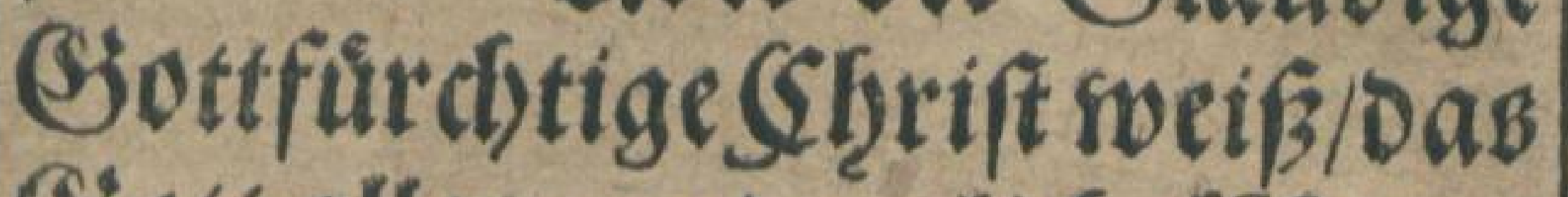
Sott alle onordentlicbess?itter Virgilius 7. Aene.

vno 2lberglauben ernftlich ver= boten bat/fentet auch oe 3 Sa $=$ tans Eift wol/o af er burch feine 3auberer vno Segenfprecher feinem SVenfoten bilffit zum be= 1. Sam. fen/fonbern nur Daromb/oaf Exod 20 or jer Seelen måcbtig werbe/ ono fie alfo von Giott abreiffe. za er glàubet bno soeíz/oaf vid Taufent mal beffer (el) in Sottes ?amen ferben/als ins Eeuffels शamen gefundt fein vno leben. Derbealben braucbet 


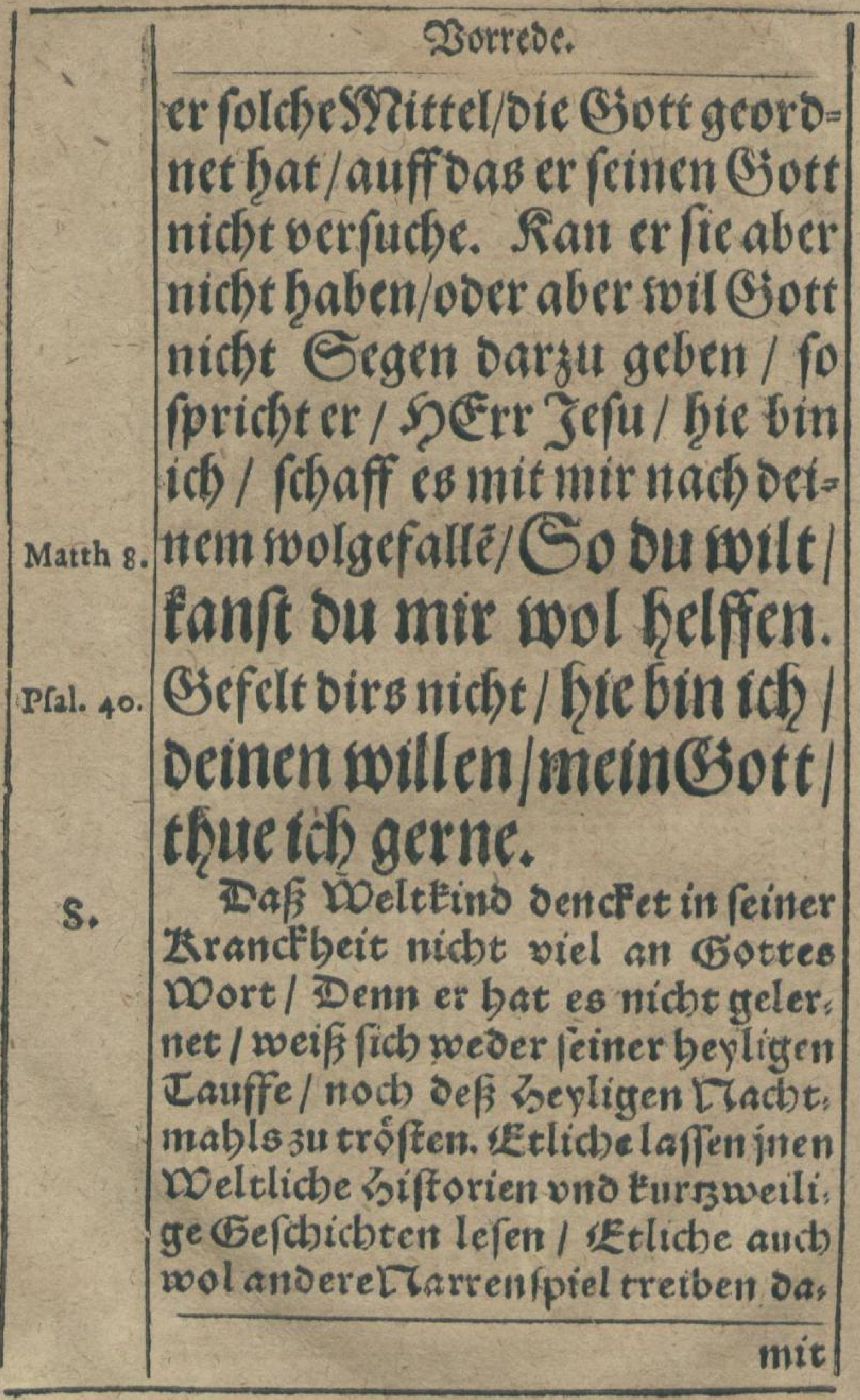




\section{Sortebe.}

mit fie def Codes Gedancien thin, Dern/vnto dafjocrse munter madsen wollen. 2tber Der Bjlâubige Shrift ift in Sottes 2 sort erfaren/2Beif oafferbe fein rect)t $z$ u they)len/Giefers vin (Euangeliumt 2.Tim.2. recht $z$ u brauchen 7 freswet fich oéß Ginabenßundes / ben er azar.s. mit feinem G5:tt in Shenliger Eauffe gefololofien / Dourftet nach oef3 S Errm 2lbenomabl/ Dabureb $\mathfrak{e b}$ rectst gefpeifer ono erquicfet foiro / ond von Shrifto newen Troft onofrewoecr= langet / 2lle Fbeltliche oinge fino im onmefir / 2leb)tet feiner Purbatil/Sondern redet ofin wnterlas von SErifto peinem Seligmadber / vom Ewigen Seberi / ont freivet fich auffo as relige Stunbelein. 
Qorrede.

Darbmb folche gereet)te Straffe oef Todes wber ons fómet/ond (prich)t mit Denutitigem Şerkzen: Iram Dominiportabo, quia Mich \%. peccavi ei: ฐch wil Den Born Def SुErrn gerne trägen| Denn ic babe wiber inges funbiget: Bnno abermal: Serr ou bift geredft / ond pas.no Dein Esort if recht. 2ilo gibet er Gott Dem S Ertn Dic E5. re / Daß er ons nicht burecht thue / ono bereitet fich tiglich zum feligen Stund lein.

Der vnglăubige beucbler / wenn

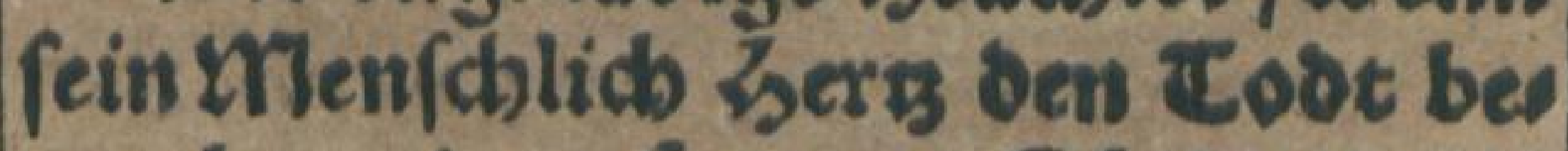
tracbtet / entferget ex fích / vno ers

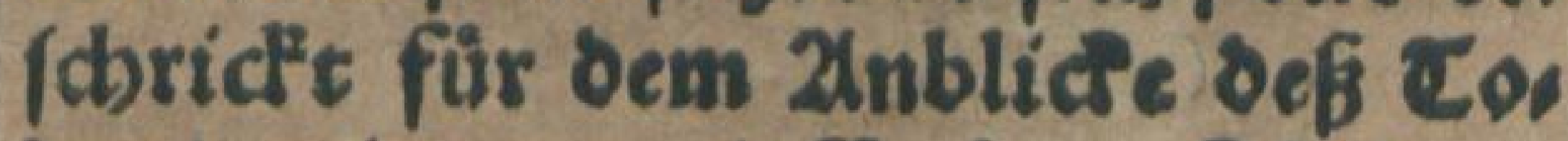
Des/ersittert vno fürdbet fich/semn Sleifb vno 28lut Eannidbt anders !

$c$

fintes 


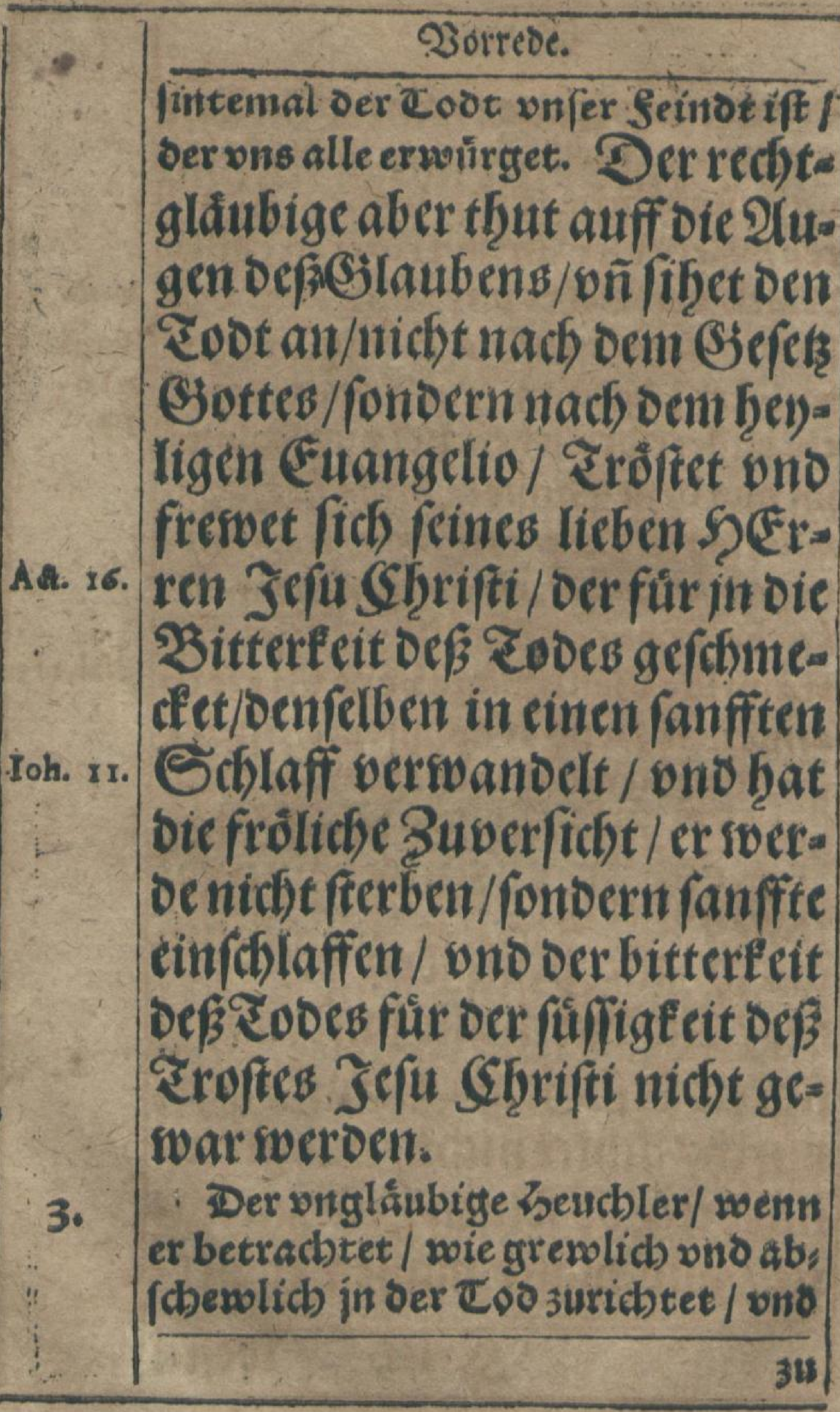




\section{Sorrebc.}

3u einer vnigeffalten kalten Leychent madbenwerbe / die man in bie talte viffennotlidbe derbe bientein forsars ret/Zjebet er ant 3 u eranoren/aller Inutb verfinctee jm / Eans aber befs fer wicht machen/als Zionnig Xerxes/ welcher / Ja man vom Coberedete / cin anders orein bracbt/ vno /dblugs Herod. in $\mathrm{P}$ \%. jim aus bem Sinne: Miffa bac faciamiss, inquit, neque triftium rerusm mentiosem faciamus, cùm iucunda in manibushabeamus. Dasift: Laffet das trawrige Gef throgise vont Sterben anfteben / vno was anoers für bie Zano nemen/dafimeb) Strewde brin, get. Dagegen aber bendet ber rectotgldubige plus vltra, fibet ourch alle befligfeit oeß oes/ ono burct) alle onfreunot $=$ ligeeit oef Sirabes frade bin = oureb/achtet nicht / wie greswe It to ber Too feinen \&elb zurichtet / ono roafer maffen er ber-

\section{$S$ ii wejer}




\begin{tabular}{|c|}
\hline 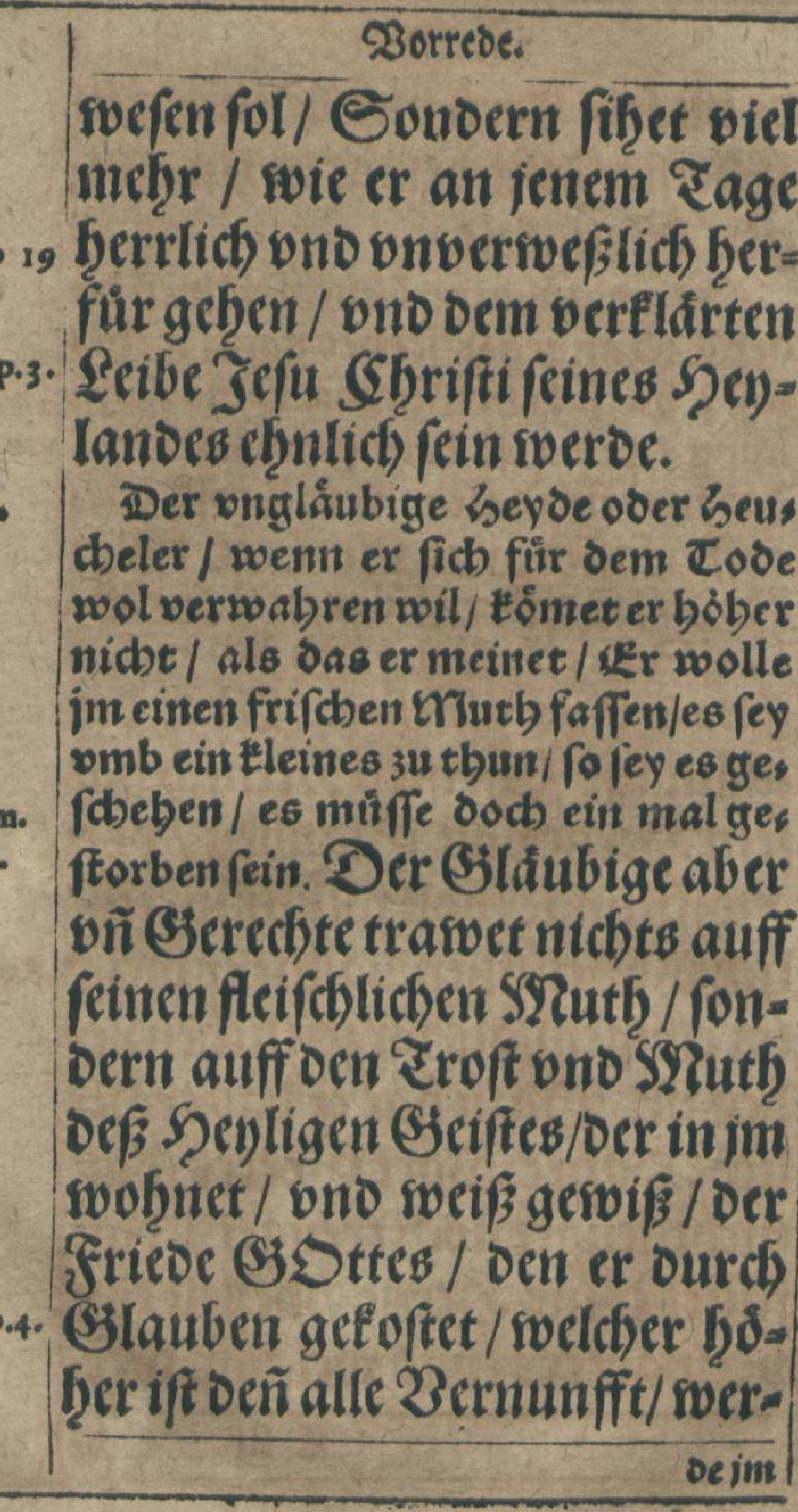 \\
\hline
\end{tabular}




\section{Borrebe.}

oejim fein Serks bno Sinnen in Sibrifto Jefu berwaren zur eswigen Eeligetit.

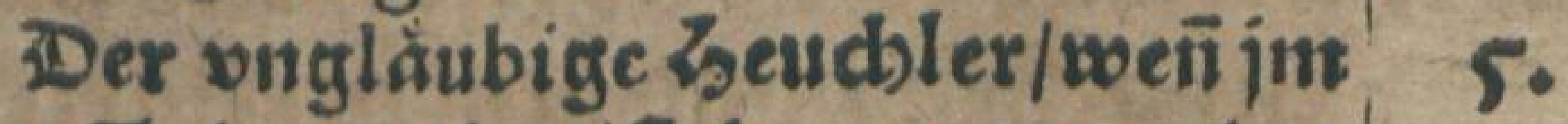
ber Cos angebet/ftebet ganz webrof

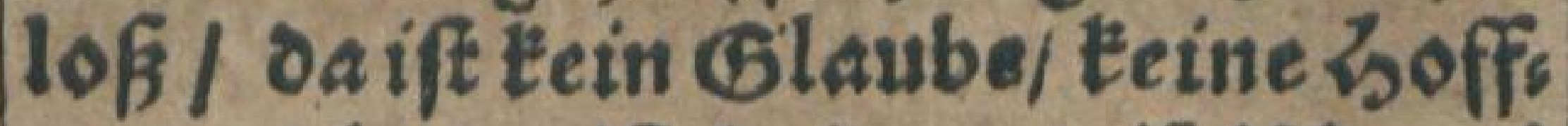
nung/ ja da ift Eein gewoif Doort/ Darant er fidb balten/Eein Eroft/oar, auffer furfen / ono fein bers frillens lónbte/ Gonbern fület eitel Surcht/ citel Gdbrecten/vno Ean fids nidtes guts 3 Gote verfehen. Afber ocr glåubige Stbriften S2Zenfeb bat fein Serkze voll Slauben / voll Soffinunge/ boll onausfiprech)= licher Seuffizen/Damit er mebre Rom s. im erwigem/als im zeitlichen \&eben ift. Dasu hat er Das trofft: liche wahre 2Bort Stottes / Daf nicht leuget noch) treuget / Da faffet er ins Sperkęe / behelit es / verwaret es/ono weifiz/imsocr.

E iij bel




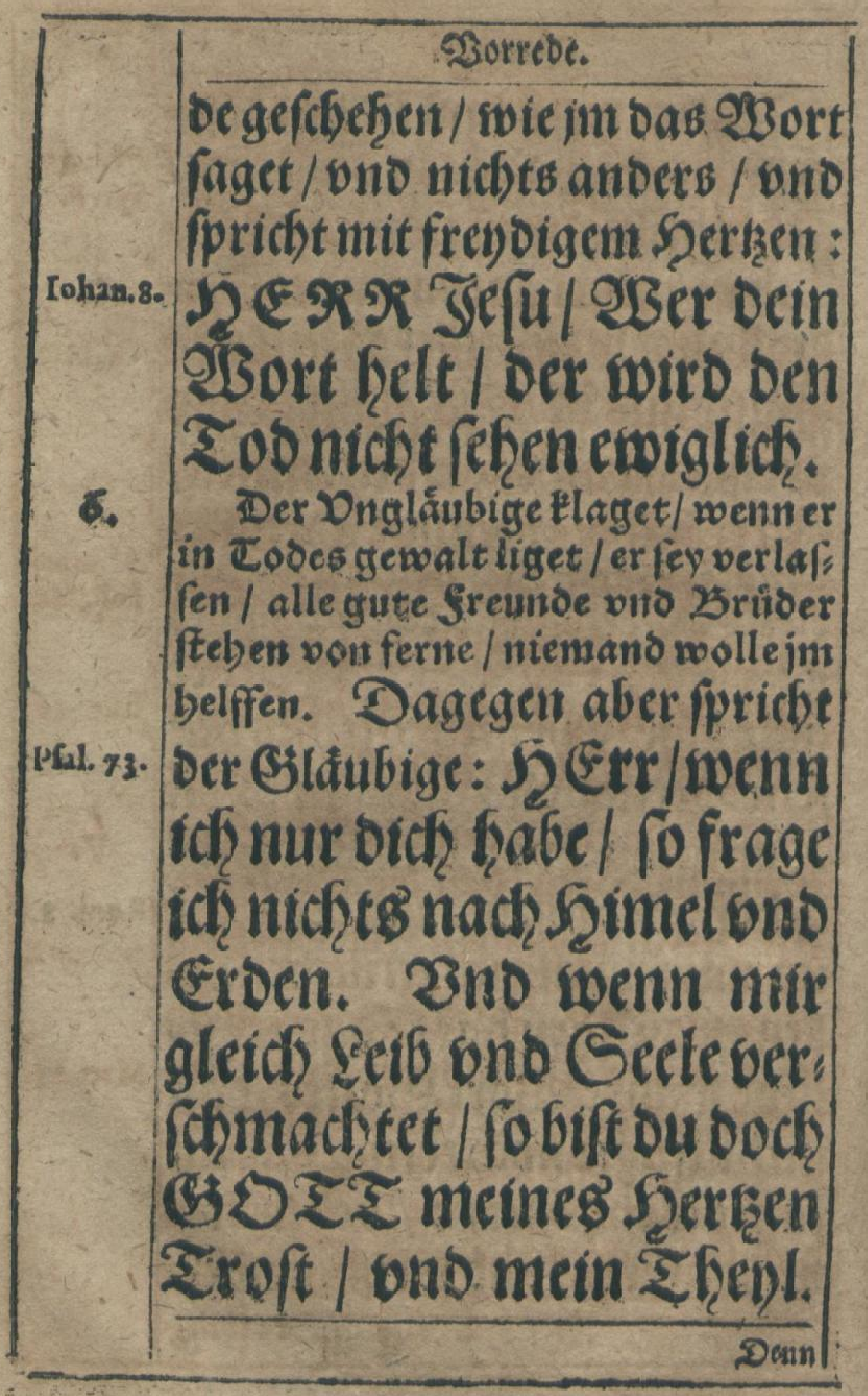


Sorrebs.

Denner socif wol/ Daf STen(ch)en nicht baltent in oer 2loth/ pal. Go.

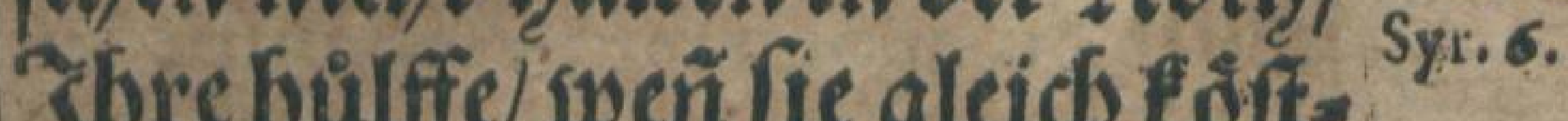

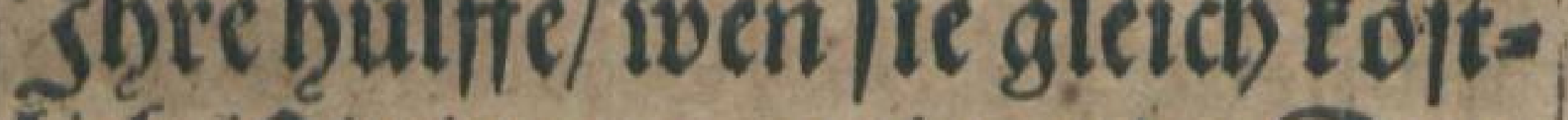
lich) ift/nimet gaz cint end. Oer. balben / FBenn aller SYenforen builffe aus ift / So glåubet ono socif er/oafs (Sott bey im belt/ Jaer bat die Senlige Drenfaltigfeit in feinem Sorkzen / ono toh, x4. vmb fein Sice) Bette heromb viel beylige Engelein/ Dic auff Lue. 16. feinte Secle warten.

Die Dngláubigen vñ Gூottlo[en/ wernm fie bören/Dafí dic Geele von fterblicb ift / vno nach Diefem Leben cin anber Leben fey / ba oie Geiredb; ten ewige Do anne/ oie Dargeredbten aber ervige Deroammiz vno pein baben weroen / KEx f chrecten fie vber alle maffe / vno winofchen / Des folches mint war were / wno wolten oaf fie nimermebr voun cobeauffo

$$
\text { C iií erftûn: }
$$




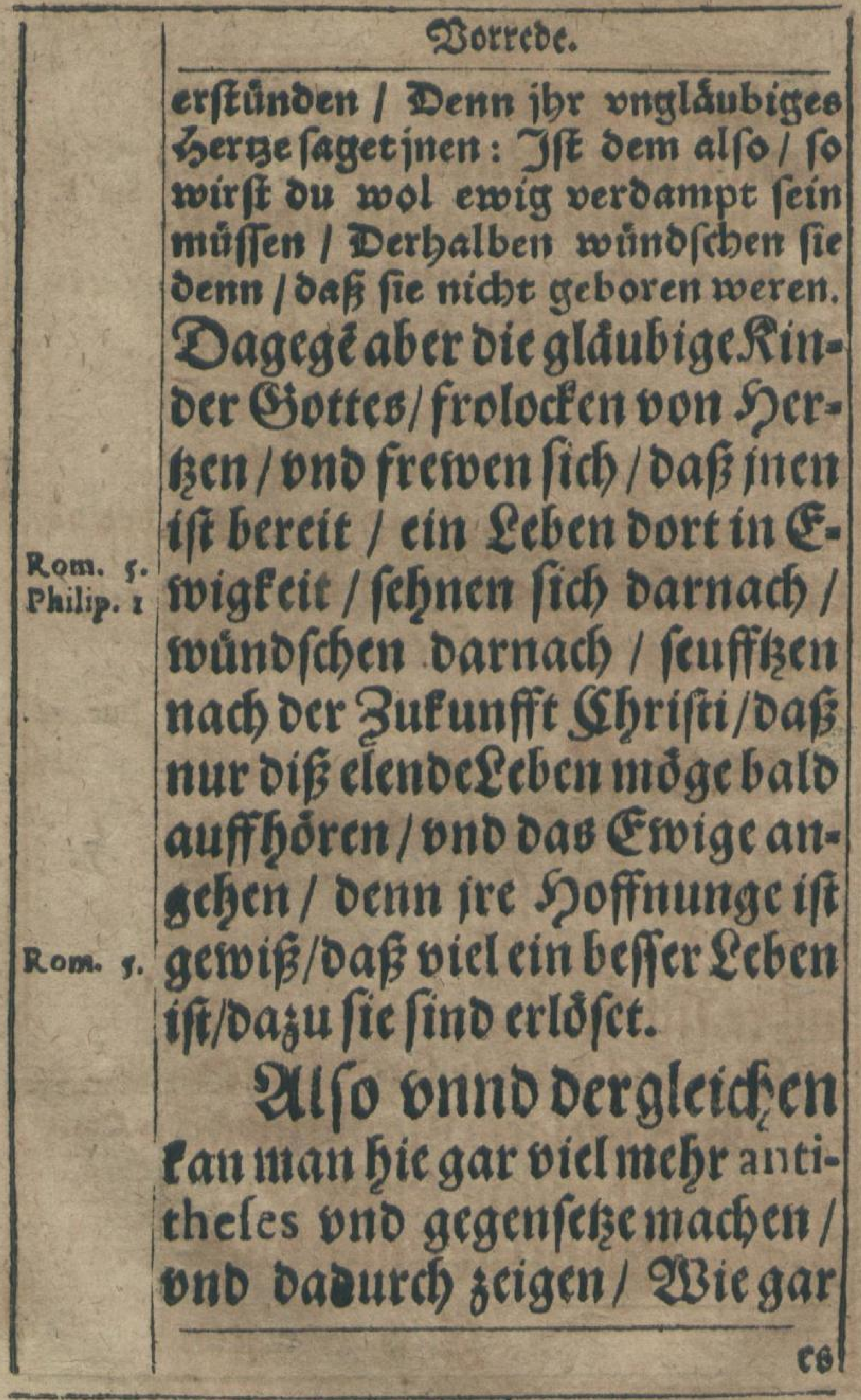




\section{Borrede.}

es ein anber oing if / mit bem frandel eines 3nglåubigé ober Soenden/ swenne es gleict) noct) po herrlich) f(d)einet / ono mit bem ¿Bantbel eines Rechtgtglâbigen ShriftenSZenfecten. 2no aber: mal/ soie gar oie Bjlåubigen ei= nen antern $26 f(5)$ en ot nemen Lue. 16. won biefer $2 B$ elt / alb Die wn= buffertigen Seudbler oñ $2 B$ eltRinber.

\section{Soldbes fol iajedermanmit} bुöch/tem ernft betract)ten/ tåg= liç) groflen fleiß̧ aniwentoen/wñ fies nicht eferer z $u$ frieden geben/ bifiser ficts sool gepruffet / ond

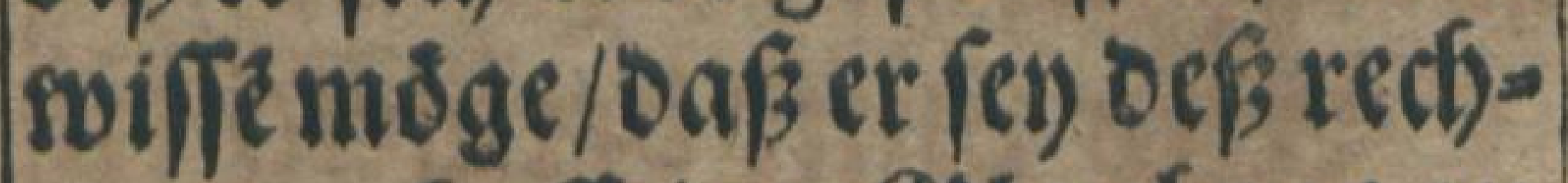
ten swarbaffitigenSlaubens an 2.Cor.3. Jefum Shrifum feinen Şey= land/oenfelben fúr fecinen bjớch=

\section{S $D$}

ften 


\section{Dortede.}

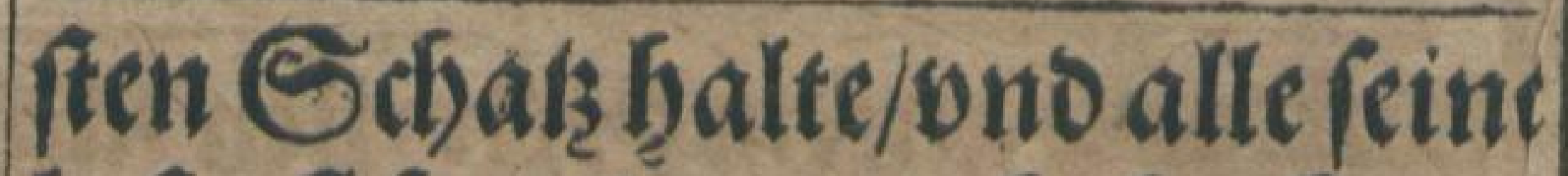
Iuft vin fresud an im babe. Ja er fol fein Şäupt nicht eher ianffi= te legen/ biß ers mit fleifigem Gebet / vno feter obung Dé 2.Tim. 4 Gilaubens / Dureh Rrafit Def́ Srenligen Geiftes Dab̨in brin= r.Toh,2. ge/ oafi er Das Eswige metbrlie= be/als dab scitliche/Den Sunt= x.Tim.6 ben ernftitict) feino (ci)/ who nur lufthabe an Der Gerechtigfeit/ Dafer das nichtige Eeno dic= fes fundichen Sebens recht er.

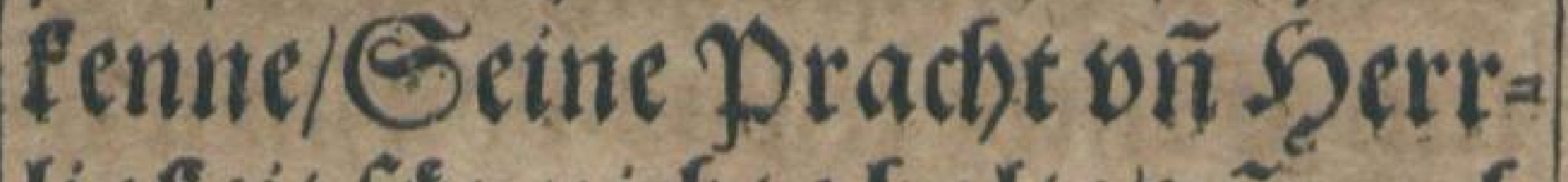
ligfeit für nichts balte/wñ nach. Den erwigen $2 B o n n e n$ verlanroh, 21. gen trage/ Daß er Jefum Sfritftum von Sperken lieb habe / oñ Apocal. nach feinter Zufunfit tåglich vlt.

$$
\frac{\text { wutnofebe / Daferfentes Se- }}{\text { ruffos }}
$$




\section{Sortebe.}

ruffe mit gutem 63 erviffen tag lich) wabrememe/vno alle Stun: oen berent (ey) auszupanten / vino einen feligen feyerabenot zumacben.

Denn das if die rectete Bereitung zum /eligen /ferbett/ oñ alfo follen wir vong abfondern alle rage bon ben onglaubigen Sely den / Surcfen/ Juben/ 1.Cor.6. Apoc. 18 23nbusfertigenScucblern bno 23 elte indern/ Derer etliche boin SSErrn Sfrifto gar nichts wif. fer. Etlicbe etwas von im swif. fen fơnten/swollen co aber nicht iviffen: (etriche ssuar bon im fioren bno biffen / in aber für iren Seylano nié)t erfennen: Mate.ss. Etlicbe aber nurmit becuss?un= De gute SEziften fein/aber nidst

mit 


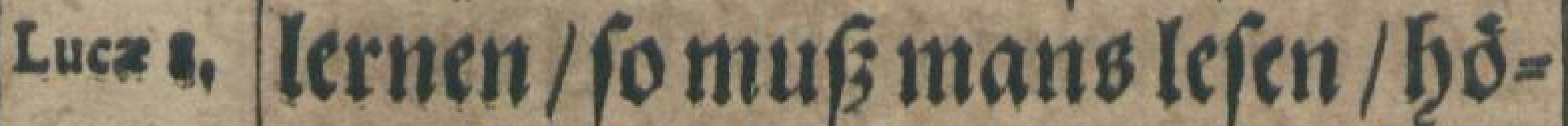
$\mathrm{ren} / \mathrm{beb}$ alten / ono auch vben. ?u ift ja/Gott lob/verbanoen oas liebe fechone Bucb Gottes/ Die beylige Bibel / oic einjeder fo nur sil/haben ono lefen fan. So bat man auds fonften viel

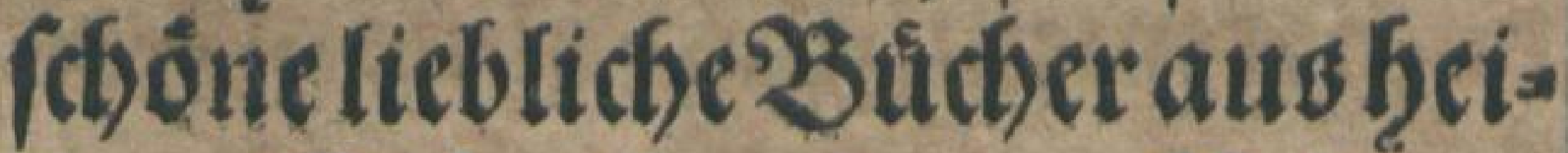
liger Schriffit vñ alten 3 atern sufamen gefdrieben/ Deutich ond \&ateinif(t)/Daraus man al-

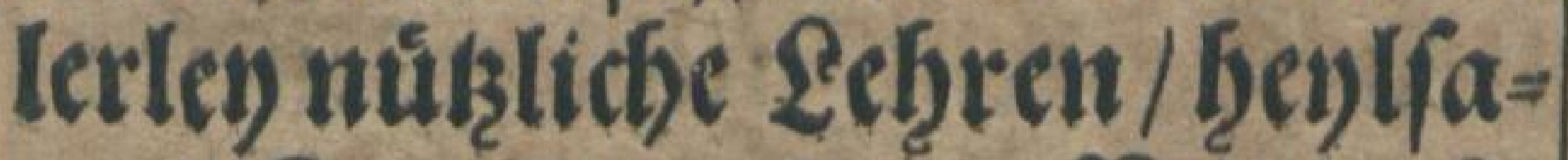
men Troft ono gute 3 ermahnungen fob ofpfien fan/Dte fum

Ebrifto.

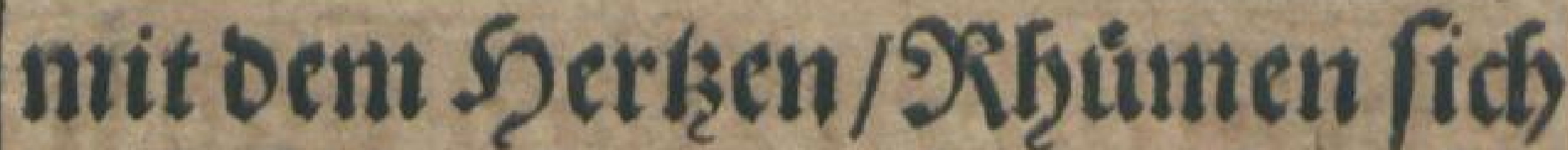
oeß Silaubens / berocifen ibn aber nicbt / Reden vom Eivi= gen Leben / lernen aber niedt / wie man bientin fomen folle. Sol matt aber folctere alles 
Sefriftlichen Eeben ono $\mathrm{e}_{3}$ ligen Sterben vonnotben fino.

Daßj mufich aber fagen/ich binnun ober Eit ono froankzig Jar im beyligen Predigaumpt/ vino febte oas folche? ticher/wie gemeloet/ \&war sum thenl / find gute\&ef̧rbuicher/bedorrfien aber fúr oie Einfältigen imer mefor erflarung / 3um the)l finos nukslicbe Tro/t3úchlein / aber Doch gemeiniglich ofine anley): tung/ woie fich Das Einfåltige Sherke drein foritfen folle/ Bum thenl aber fino etliche auch gar zu bodb / voller Difputationen

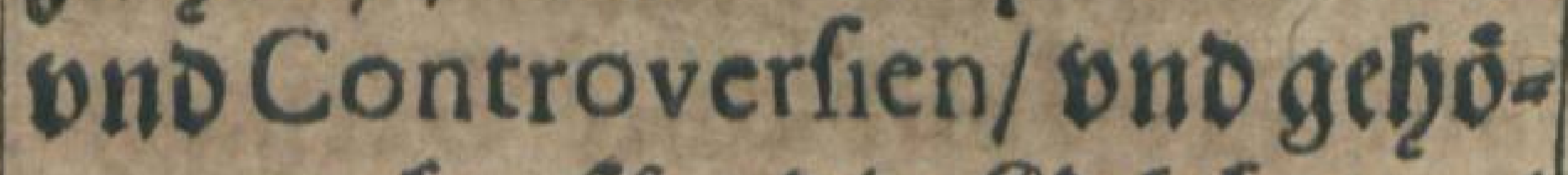
ren methr für dic Gielef̧rten/ Denn für oie Einfáltigen. 


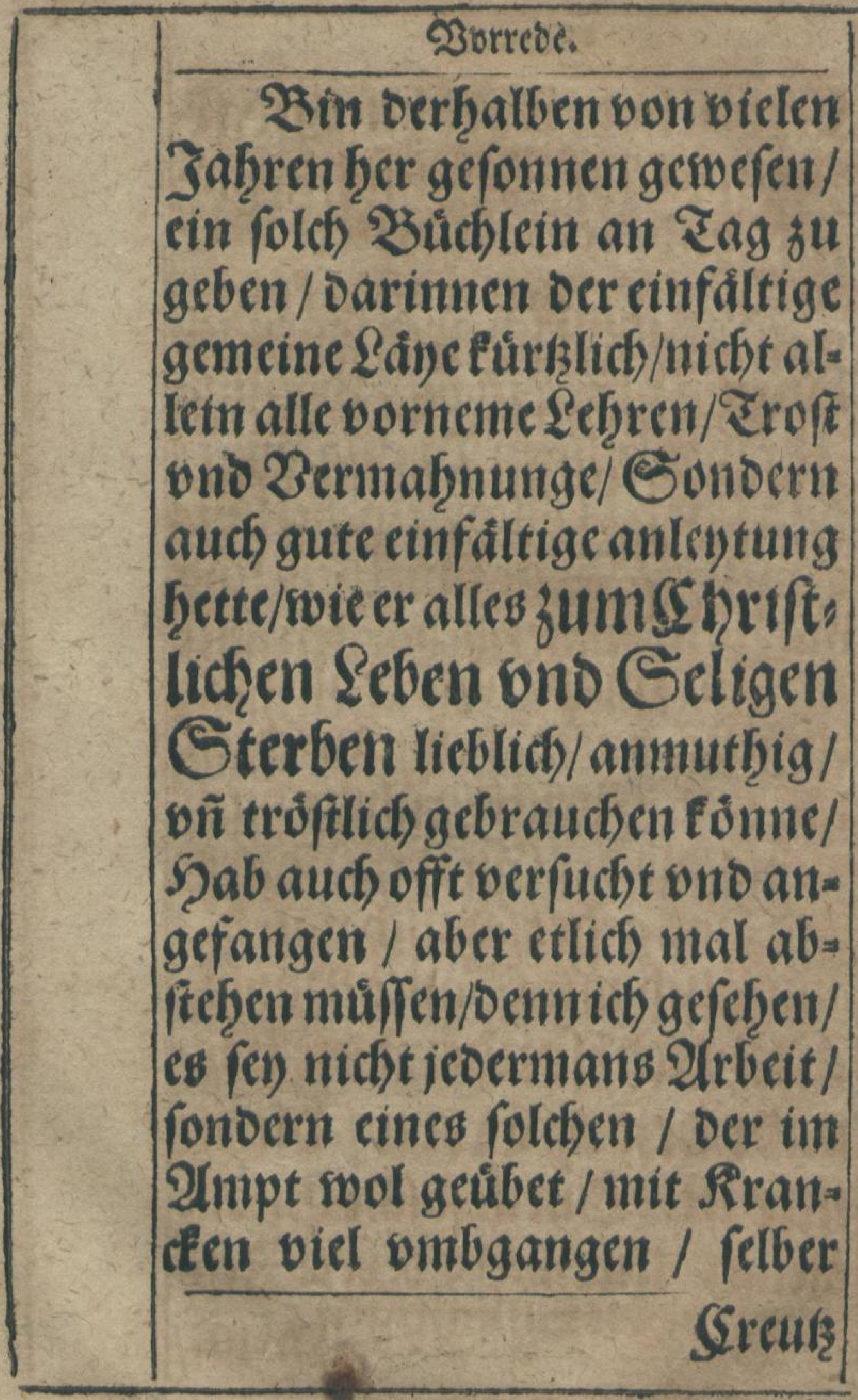


§orrebe.

Sreuk ont 2Anfect)tinge ge-

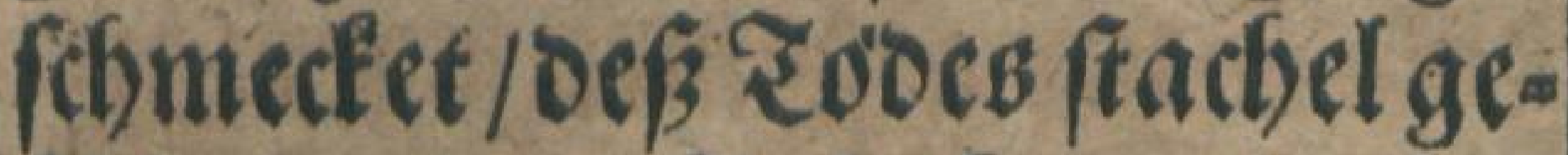
fullet/wno oieGabe bette/Dafer betrubte Serken troffen fonte. Ran audb freislict) wol fein/o a f ocr Satan an mir folch ?Berd gefbindert/ oñ micb Dahin britt= gen wollen/ Dafi ichs gankz wno gar nackliefle.

Jeb) zroar / als ber afler ge= ringefte vonter ben finechtent I E S V Şbrifti / ruffeme mich) nictsta/als oer SnadenStotteb/ ono fage gefor famlict) vito de. mutiglich mit Sant Pauld:

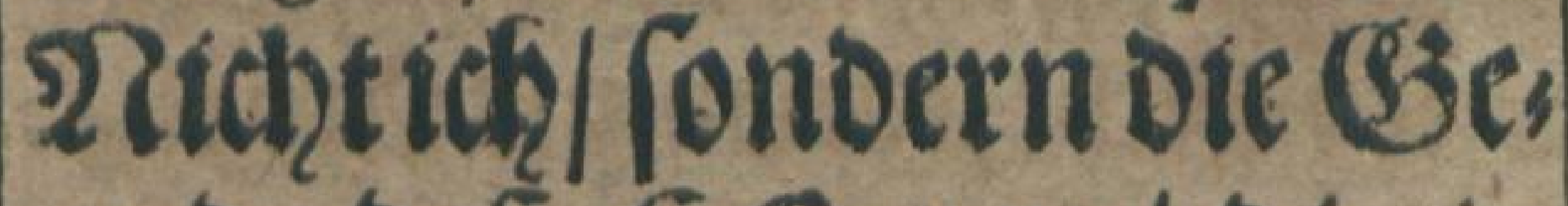
1.Corin. 15.

nade DeF SEerren / ote in mir ift. Durds foldse (Sinabe

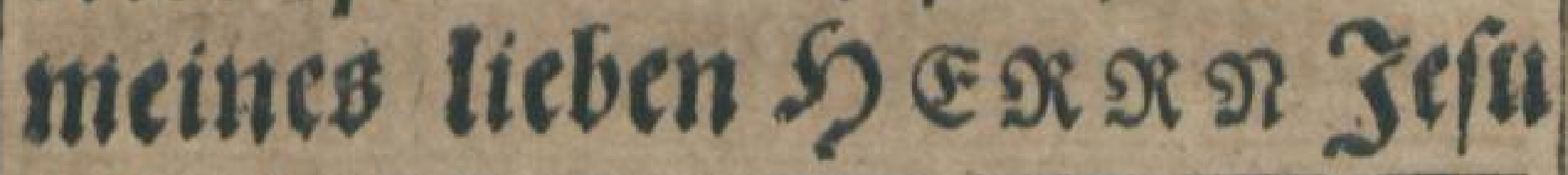




\section{Borrede.}

Shrifti/habe idfo burch Srafft vno 2 enfitanot de Gieifes endlich) verfertiget/ond laffe $\mathrm{es}$ ans Siect)t fommen in Sotteb?amen/in bemes aud angefangen ift. 2Berbofie es soeroecin folch) 2̧Bercf fein/Daf oa gelangẽ swiro meinem Giott ju \&ob ono Efbren / mir ond al-

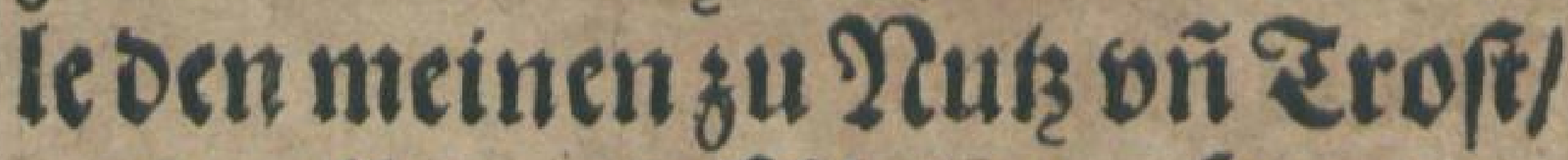
ond allen cinfältigen STerkzen jum chriftlichen Eeben ono jeltgen Sterben.

Die (jeleffrten bitte ids//ie wodlen mir foldbe Esinfalt / Der ich) micts in biefem brauche/zum beften ocuten/oeñ

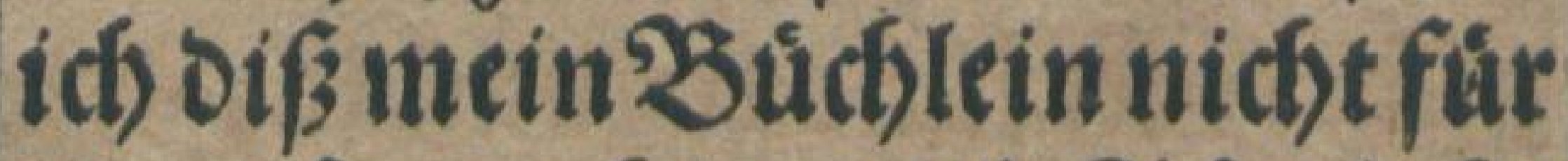
oie / fo vorbin viel Gjottlidbe

Gaben 
Sorrede.

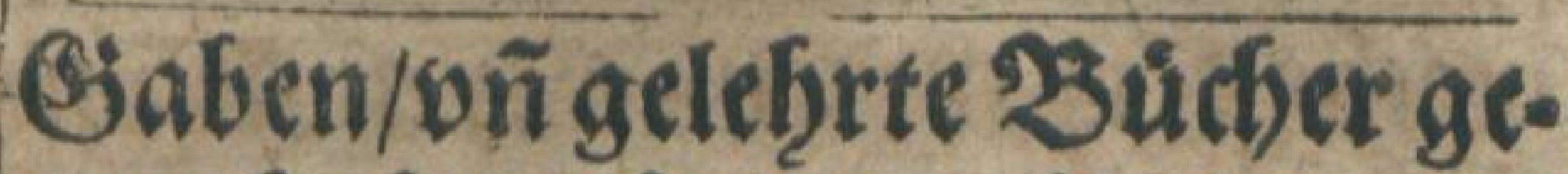
uug biaben/fonoern für Die einfăltige\&aiven zufamen gefçbrieben brabe. Die Einfältigen aber bitte ich/Sie wollen folche meine 2rbeit fleiffig gebraucben/ ono fich sool Darinnen oben / ich) weif gerwißz//fe werben beilfamen nus ono guten fromen oavon haben.

SBie offt treget fid $b$ su $/ \mathrm{baf}$ ciner fies) in fremboen Eanben mus finden laffen/Da er feinen reinen Prediger baben fan? 2Bie offte ift einer in Rriege $\beta=$ leufiten/ onv andern gefebrlig. feiten / o a er mandimalfeinen Preoiger erreichen fan ? 28 ie

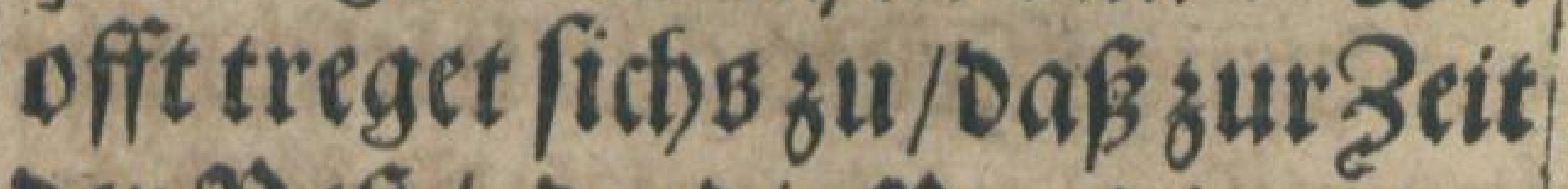
Der Peft/ Da Die Drxoicanten

$$
\text { D nicht }
$$


Borrede.

nicbt verb̧anden/ooer abgeftot: ben fino / mand Sorke nach Sefor / nach Troft ono Znterricht feuffizet? Da fon imeiner aus oiefen cinfâltigen $\mathfrak{Z}$ ích)= linfelber Sroft zu/precben/ooer ourch anoere/die bmb infino/ veno lefen fơnnen / sujprecben lafien/nach allen Stúcten/oie fein Serch begeret / Damit er in feinem Sauff nicft twande/fonoern befendig fortfabre / ono Dureb die Snade Sottes einen guten 2 fofcheio oñfeyerabento mach)en mòge.

\section{Soldie meine Proett}

vno Büchlein/habenun/Eole Seftrenge frawo / E. Geftr. ich ocoiciren/ ono onter oerfelben

lóbli= 
Ioblicben / 2lorlicben Namen / ausgeben lafien wollen / Der trofflicben zuver/idst/(E. Steftr. werben bieran feinen ongefalIen tragen/oarbmb ocnn Esoer Gieftr. ich sum aller bienftlichfen soil gebeten baben. 2lutb soolle E. (Seftr. Fidb foldbes meineB boznemens nicbt soundern/ Detun nach Dent ich) vor $2(d)$ kze ben Jabren / als ein Fremboer vno Alußlanoer/nacb Dem wilk Ien Sottes alfier zur Sprottal fun Sevligen Predigampt be ruffen/ono nicb) twenig anfto $\beta$ । fonderlicb) fum anfange batte/ waren bie beyoe alten Serrm

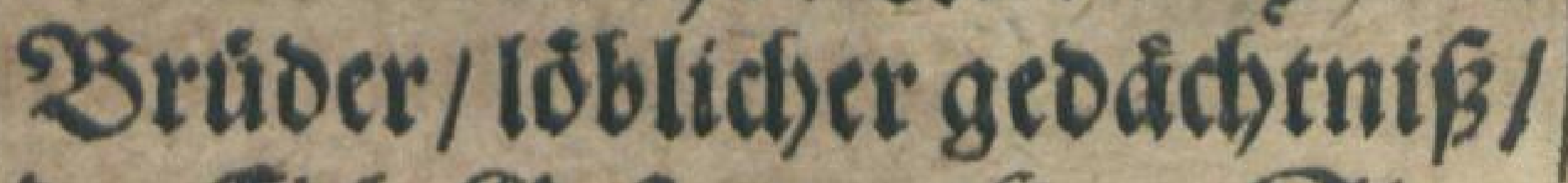
ber Ede Bieftrenges Sert Sieg. fried von Nachern/auff Run-

$$
\text { D ii k̨en. }
$$




\section{Bortede.}

kenoorfi ono Rortnik/2c. So wol ber auth (EDle Sieftrenge Şerr Srabes von Naicbern /

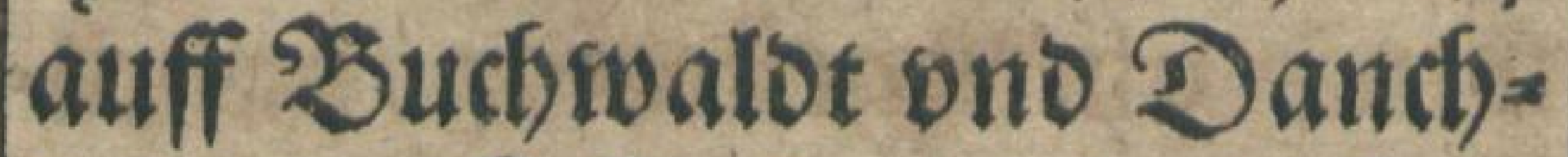
wik/ze. E. Geftr. viel geliebter Sperr Zater/nebenmeinen lie $=$ ben Sprottern/Dicerften onter DenSzen/chenf inoern/bie nicht allein meine/als eines geringen SZannes/funofchaffit begerten/ vno mich su fich lutoen/ fonoern mir auct) alle trew vno for to $=$ rungerseigten. Shabe fie auds bei) oe/ folange fie 35 tr leben lafien/nichts anders/ als meine grofsgunftige Şerrn ono grof fe freunde vno forderer erfent net.

Feil ich benn nach beralten Şerrn Eode/Derer geoåchtnif 
Worrebe.

im Segen bleibe/ ire bielgeliebte hinterlaffene Rinter ond Er = ben / als won benen mir nicht senig gutes gefecteben / billich auch liebe bno chre / fie auds fámptlich in meinem tâgliçen Gebet befialte / ono nunbejon= oers Ew. Seftr. gleich fan zum andern mal verwaifer / 20no gleith/ woie vor swenig Jabren/ jreb geliebten Serrn 23atern/ alfo auch nun ires froct)gelieb= ten Şerrn ono Şerizene/ ourch) oen zeitlicben Foot verluftig roorben/ ond in ben betrúbten tratorigen $23 i$ iofenftanot ge= fest fino / 2lls act)te ich oafur / Dif Butchlein gebure E. Sieftr. gankz billich / Damit es nicht al= lein ein Dendfmab̨l $/$ en meines

$$
\text { D iif Danct. }
$$




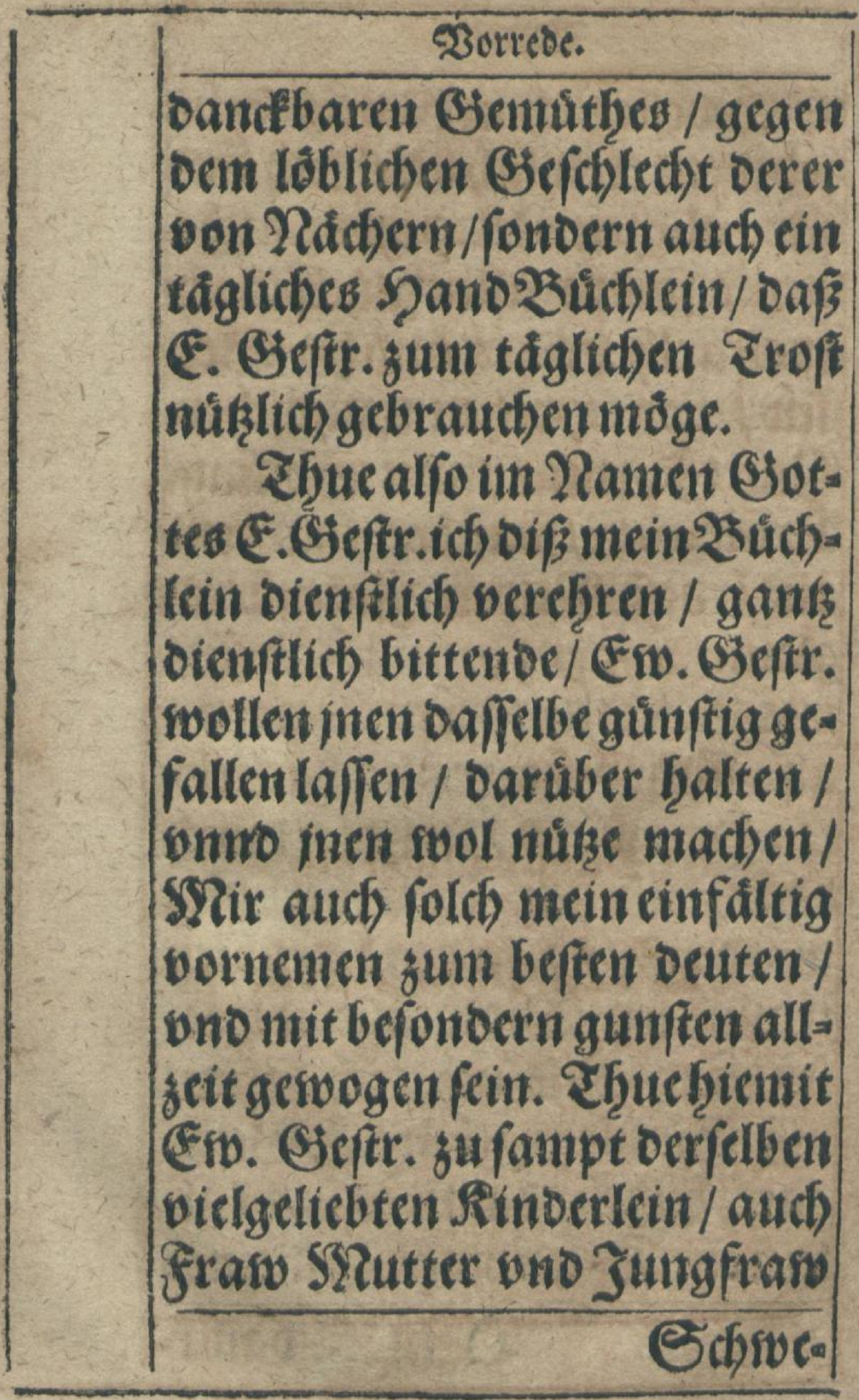


Sorrebe.

Edfroefter/ith in Gottes fof uts jedergeit treswlict) befeblen/ bno

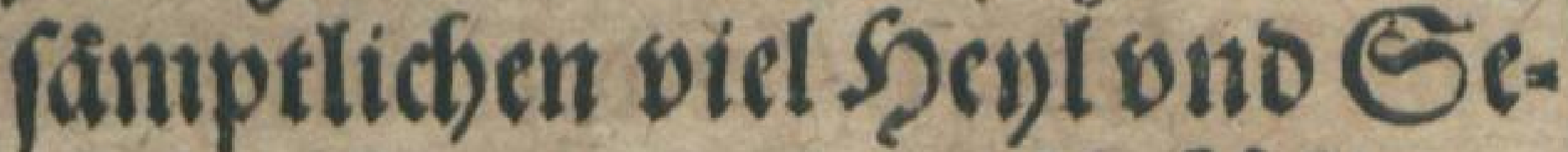
gen / fampt aller glúdéfeligen soolfarth leibes ond oer Ser= len / von Soerken sountofhen. 3ur Eprotta / am Eontage Palmarum/war ber 11 . Aprilis / Def Retwen Salenders / Anno 1593.

REw. Geftr.

Dienf fwilliger alls.

- Martinus Mollerus. 


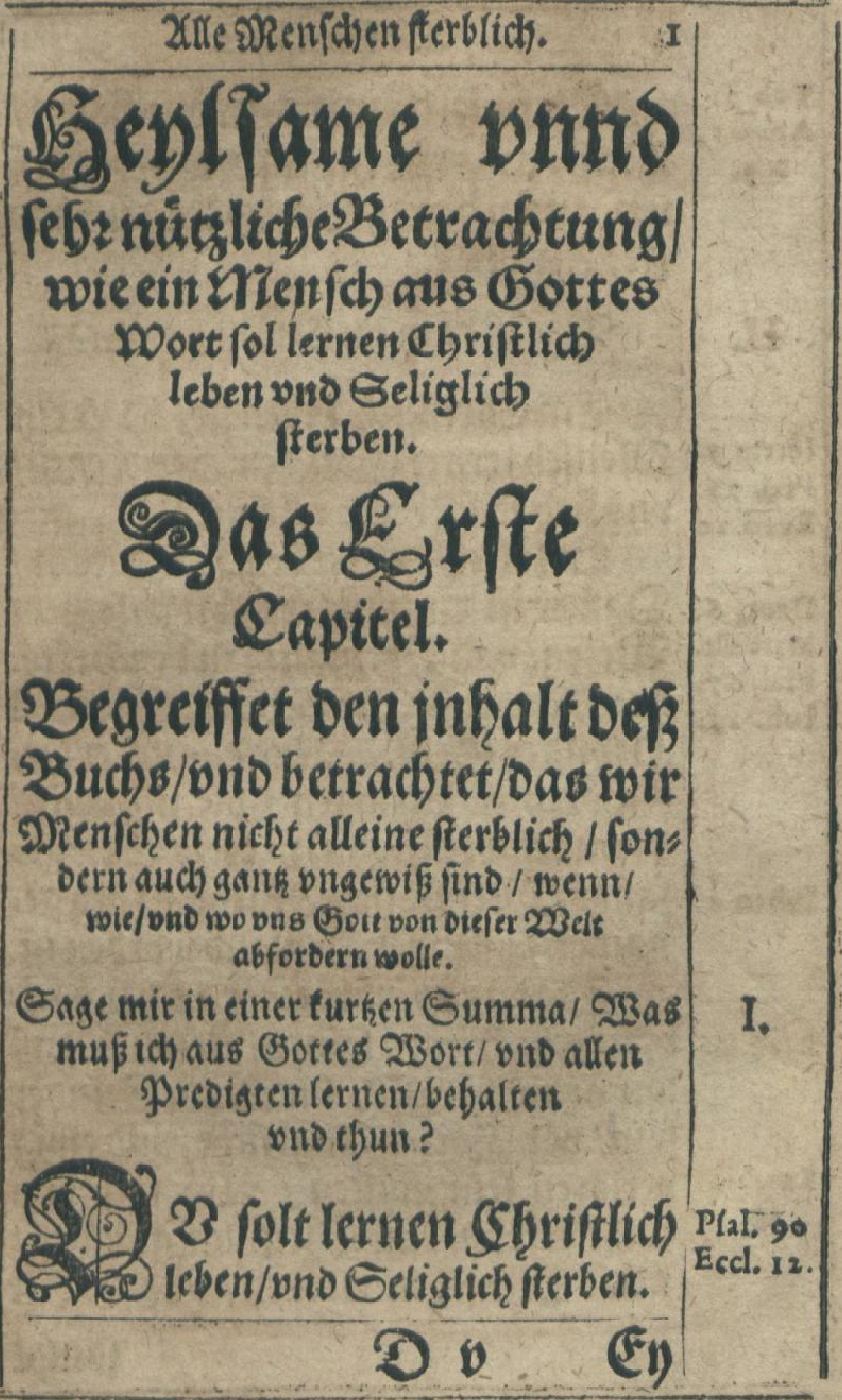




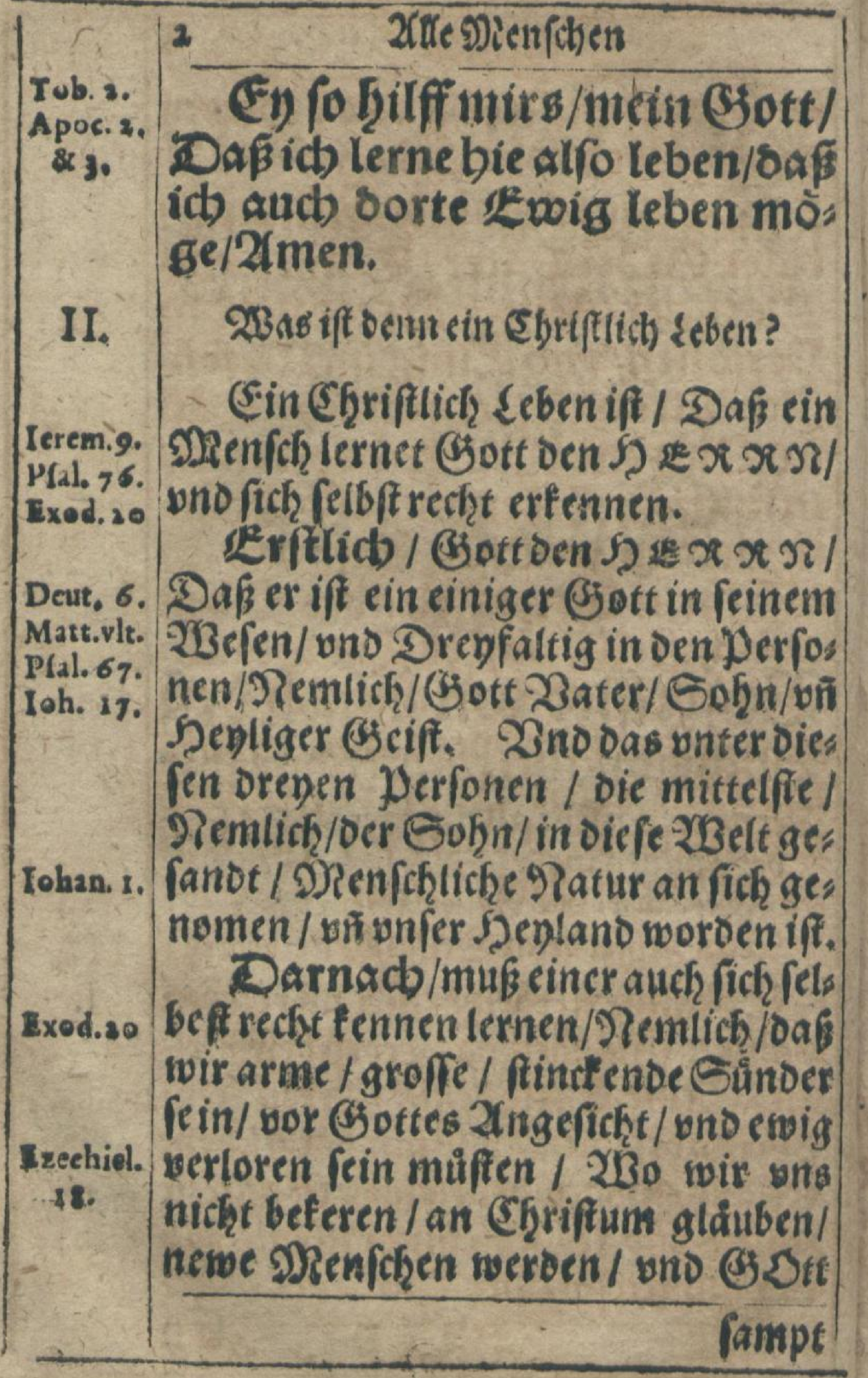




\section{fterbility.}

fampt vnferm Nefieften von Serfen Dienen/bigan vnfer Ende.

Deiniger/eswiger/2Illutåch) tiger (BD)tt Dater / Sobn / vns Deyliger Geift / Dreyfaltig in Perfonen/pno LEinig im DDe en/ Jdb bitte oich / lebre mich / Dâs icb Dich ono IDich recbt ertenne/

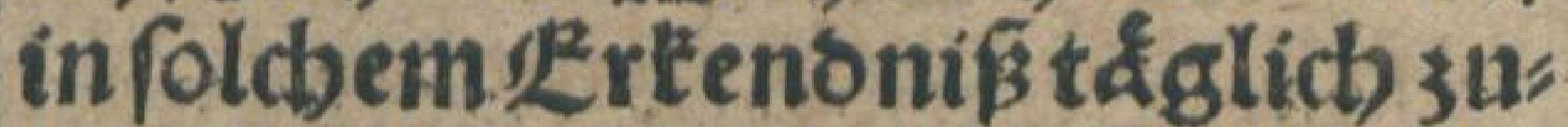
neme ons wach $/$ / Zuff oas ich oî́ mein turtzes elendes \&eben alfo anftelle/ oaj es Dir löblich/ meinem hebeften nutylid / ons Dir felber nicht perbamlid)/ fons. bern ein recht 23 uffertiges ons Cbriftlicbes deben rey fit oeis nem 2ingefichte / 21men.

2Bas beilfet dentr Seliglich ferben?
Belighioh ferben heiffer im recheen wabren Glauben das Seben befebliefs Luez 2. fen / Seine Beele dem Sherren Yeflu philip. x.

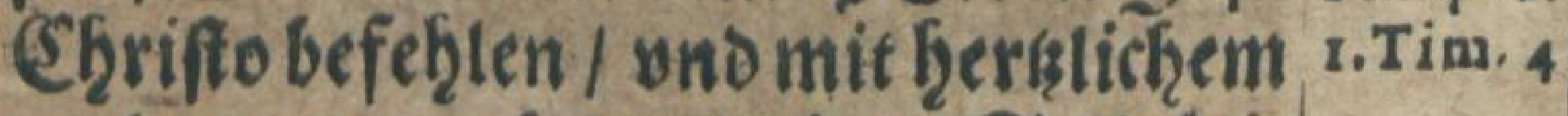
verlangen -nach ber ewigen Seligteit fanffite uno frólich einfchlaffen / vno von hinnen faberen.

atere 


\begin{tabular}{|c|c|}
\hline & dule Renfeden \\
\hline & SุEx ร̌fu Shrifte / Du \\
\hline & allein weifreft mein Stunblein / \\
\hline & Jcb bitte sidb/befcbere mir aud) \\
\hline & ein feliges SEno/ons nim alsbeñ \\
\hline Actor.7. & meine $\Theta$ eele inn beine Dånbe / \\
\hline & 2umen. \\
\hline IIII. & NRiffen bent sbir WRenfichen \\
\hline & alle Sterben? \\
\hline & Ja. Denn fo fpricht die Schrrifft: \\
\hline Ebre. 9. & DendDenfbenift geretst ein mal \\
\hline & 3u fterben / Darnach aber oas \\
\hline & Gerichte. Sore Doch/meine Eeele \\
\hline & fehen gefest ein mal 3 u fterben / Eo \\
\hline & wird es gewiß auch ein mat an Dich \\
\hline & fommen. Denn fo fpricht der weife \\
\hline 8. & Dann Syrach : Geftern wars an \\
\hline & mir/Deute ifts an oir. Ta/ lies \\
\hline & be Geele / mercte gar wol / gaß die \\
\hline Ebre.9. & Gatrint pricht : Lin mal zulter? \\
\hline & Dienffhent gefest were/ swey mal su \\
\hline & fterben / fo fonde ciner / went er je \\
\hline & bas erfte mal nief̧t wol geftorben wes \\
\hline & re/ oaj anber mal beffer fiesben. \\
\hline & $2(6, t$ \\
\hline
\end{tabular}




\section{fterblict).}

Abernein / \& in mal Dbel fterben/ bringet ewoiges Derberben.

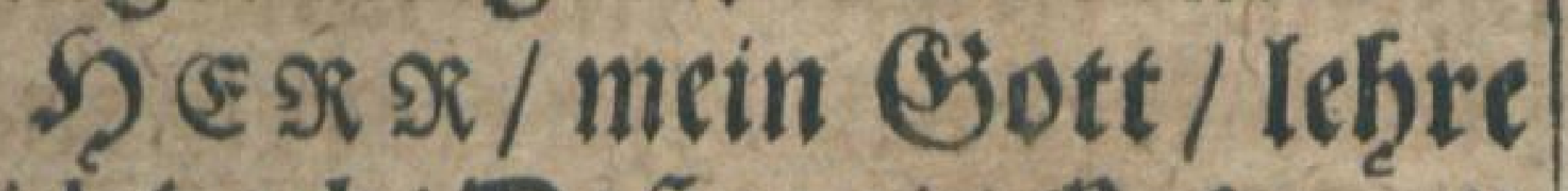
mich soch/ Dafis es ein Ënoe mit Pfal, 39. mir baben mus/ono mein feben ein Zielbat / pno icb oavon muŝ. Sibe / meine aage fins einer Danot breit bep oir / Dno m ein Lebè ift woie nichts für oir. DDein Gott/lebremich besenclen/ $\mathrm{ba \beta}$ ich ftezben muß/auffoss ich tlug Pral. go. werbe / ons sie rechte beylfame Sterbekunft lerne/21men.

2̧Genn vns boch Gott ber Sc Err bie Stun De vnfers Sodes offenbaret bette/ Daß wir vns su rechter seit oajufchicfen sno beteiten tondoten.

Ta / meine Eeele / nicht allein bie Gtunde if dir verborgen / fondern noch vielmefir. Die Alten fagten: Quatuor circa mortem funt incerta, nimirum, tempus, locus, ftatus, modus. Das ift: (Es iftung Dien: feflen nicht allein die Gtunde vno Beit vnfers godes berborgen / fondern wir wifien 


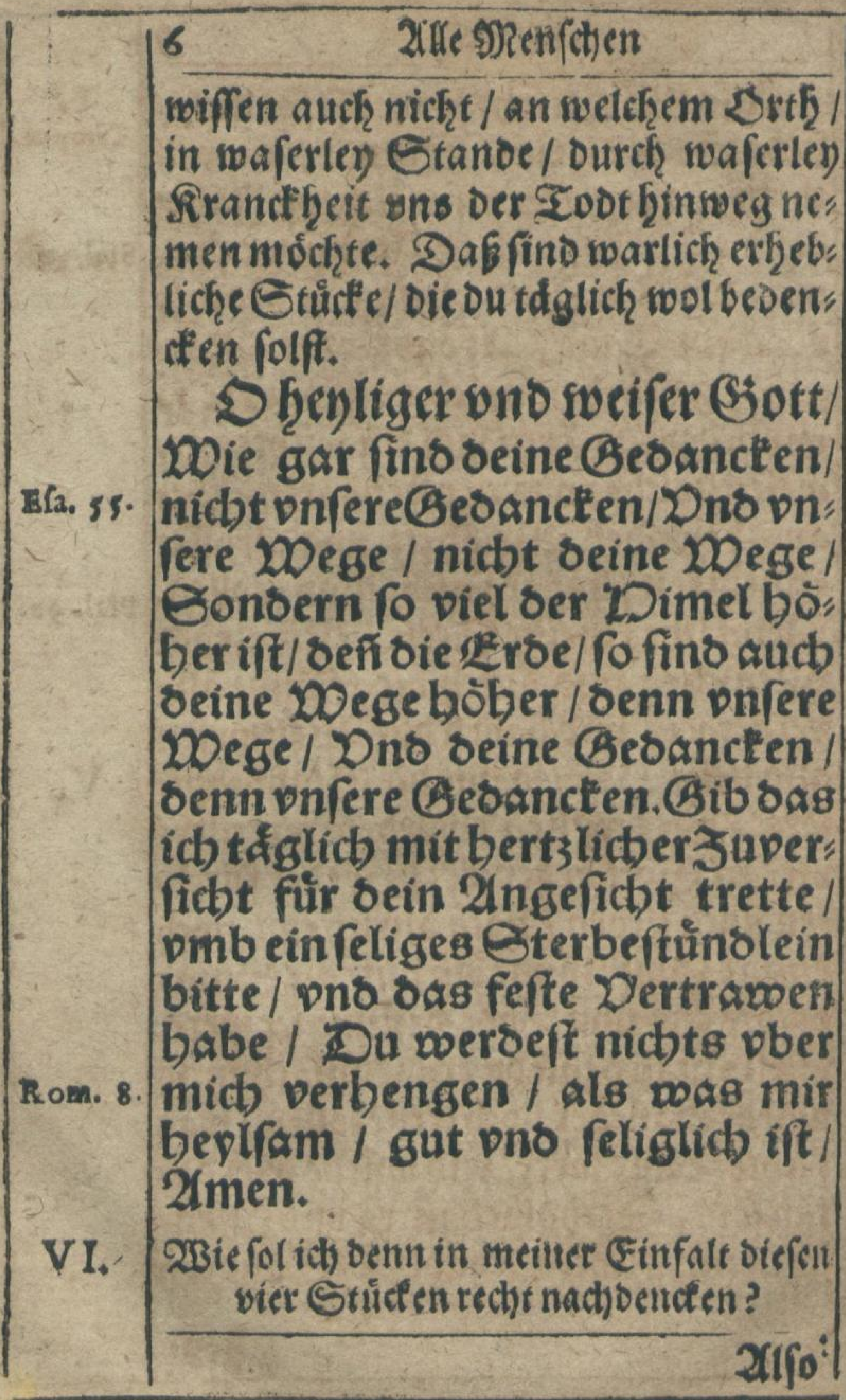




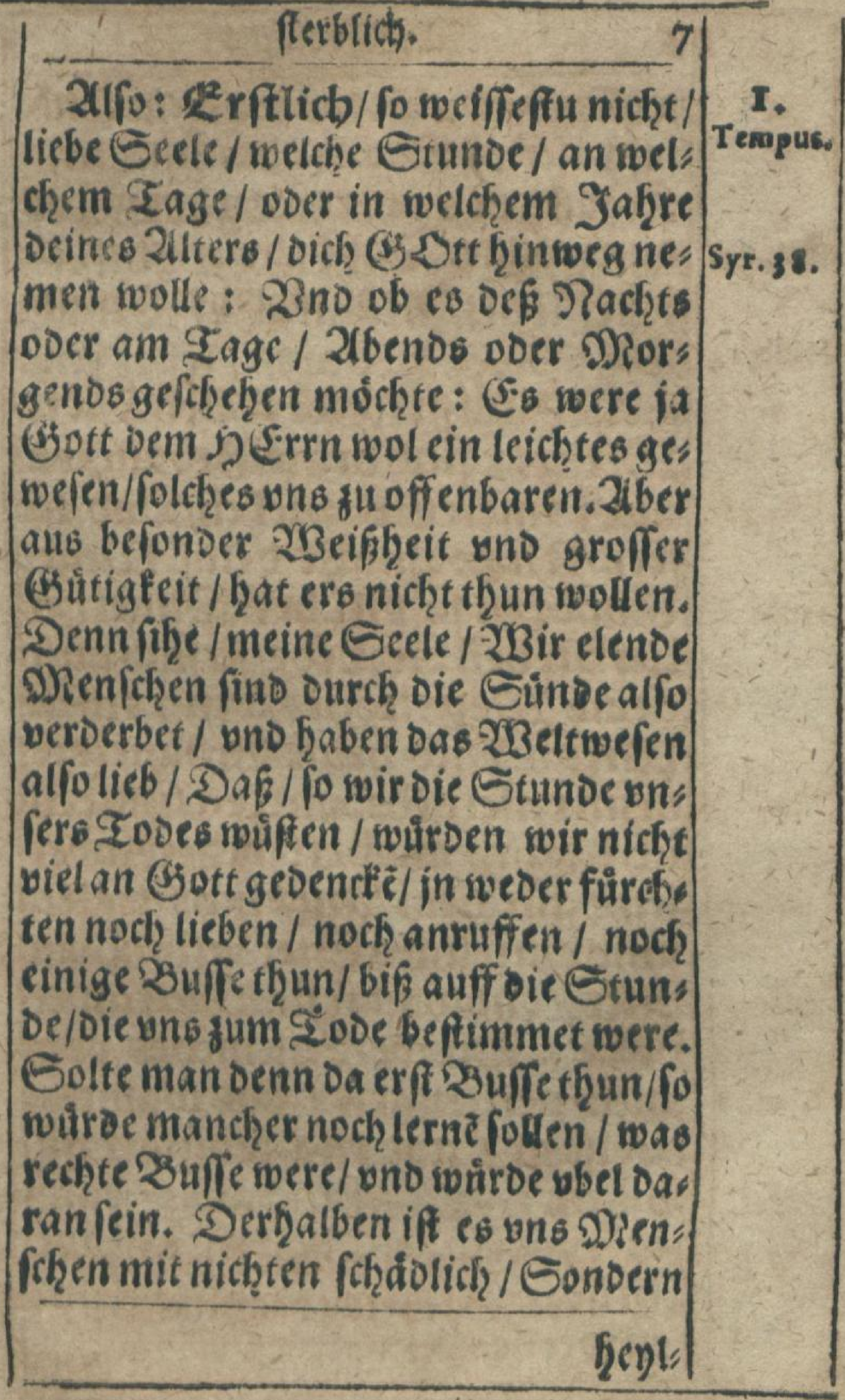




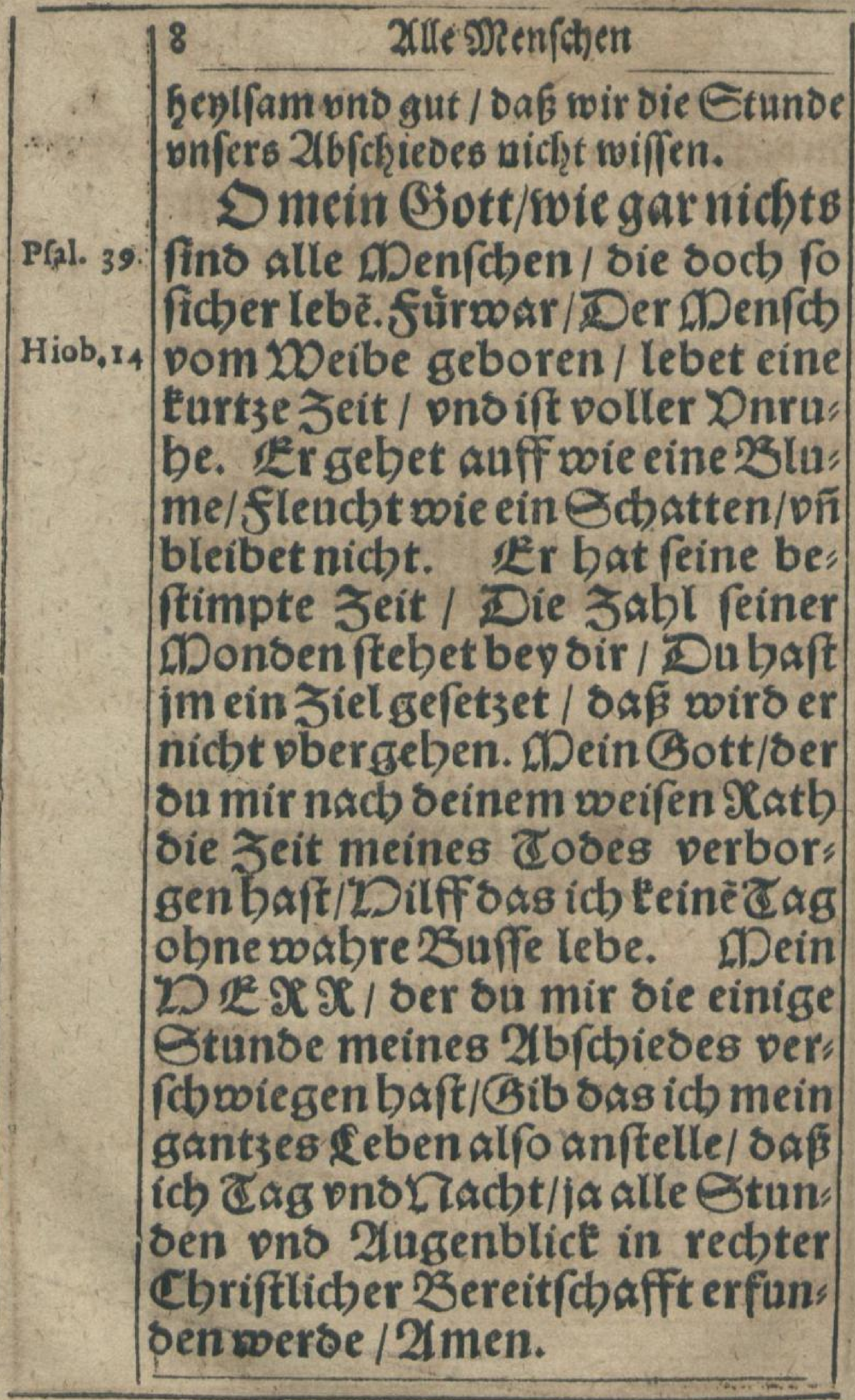



an welchembrth oir Dein Ende moecht Locus. befeheret fein / sob ou Dabeimen / odex in oer Sremboe / Auff Deinem Sette / ober auff Dem gelde abfcheiden folft. Ta / meine Eecle / es fiefiet gerwib Dee Zెenger onfers Lebens nimmer fitlle/ ond fein Dien fech weiß / wenn er auss gelauffen ift. Eo ift denn ber Foot binter wns her / uno feket uns auff ber Serfen nach /nimet bns hin/ wo er vns findet / $5 r$ wartet bein an allen Sr: then. Bift Du nu ein fluger Sinech $t /$ io warte auch feiner allenthalben mit bereitembugfertigen Serken.

Dulein Gott/gib mir guertennen/bẩ idb an allen Drtben ein elenser fterblicher [Denfdb / vno fur sem cobe nirgeno ficber bin. Weil er benn auff midb belt pno lauret allentbalben/fo bilff/

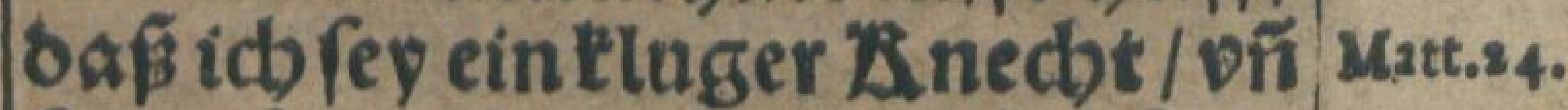
reiner binwiber an allen Drtben mit buffertigem 'Dertzen wars te/2lmen.

$\mathrm{Matt}_{0} \mathbf{2}_{4}$ 


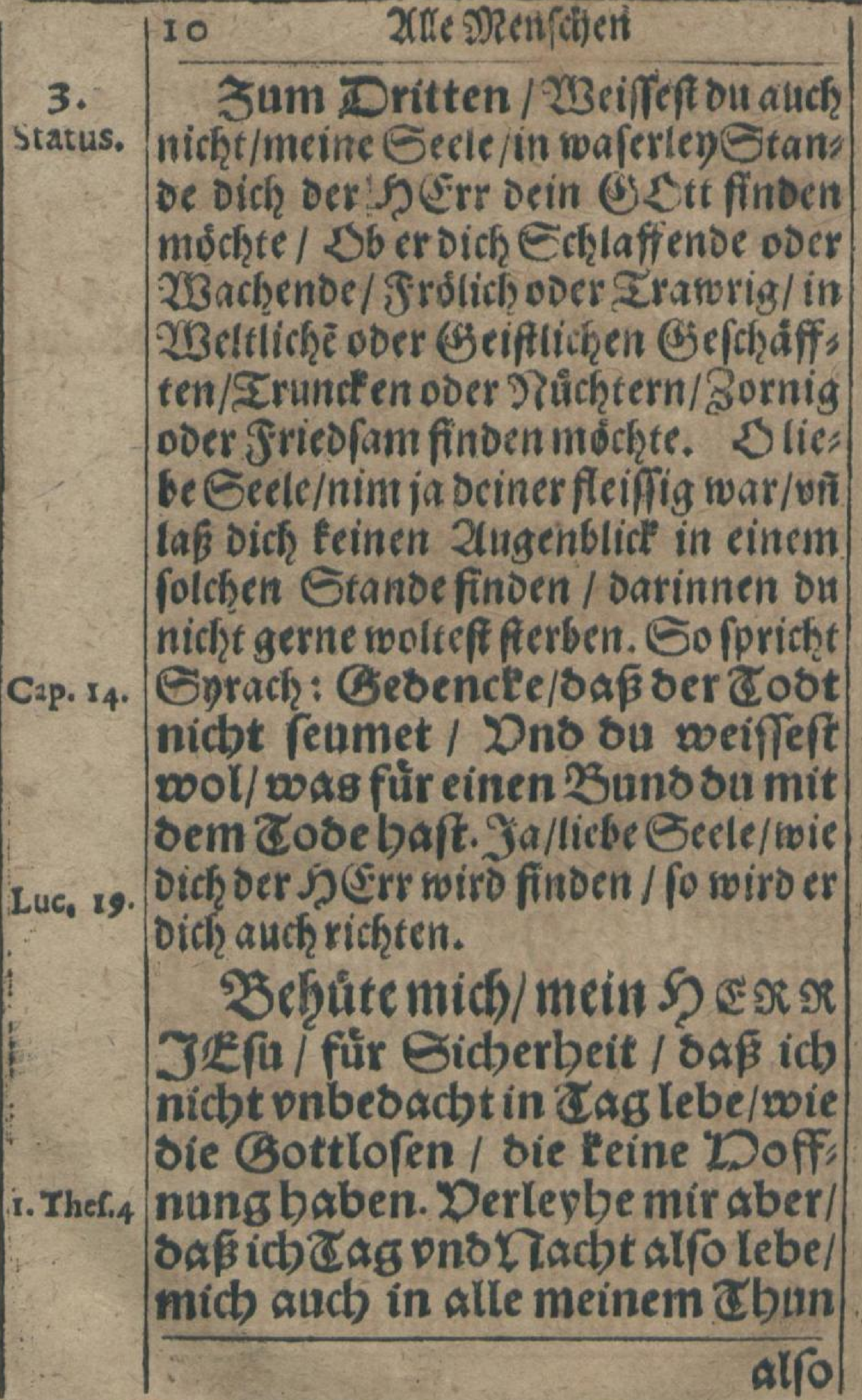




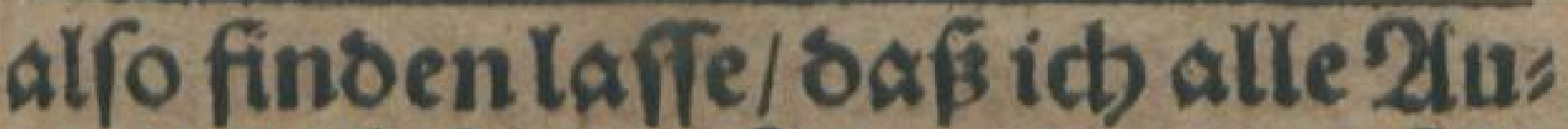
genblick fertig fev/onb einen relis gen 2abfoieot nemen tơnne। 2imen.

Zum Dierben/2Weiffeft ou auch nicht / liebe Eecle / Durch waferley Modus

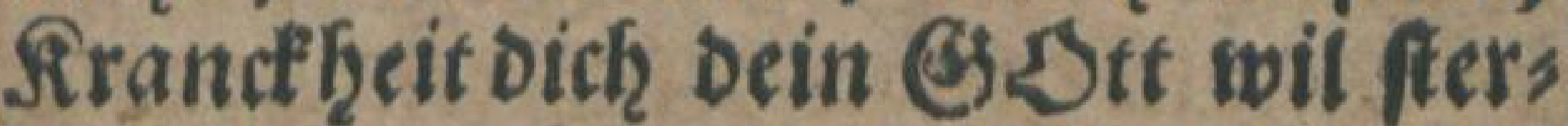
ben laffen / Sboureh Deftilens/ober

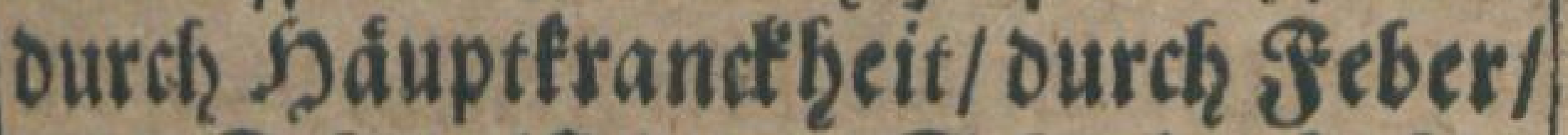
oderEdimulf / oder Echnoind fucht/ SOb Du etwan in 2Baferesnoth odee getwersnoth bleiben / Dureh gistoer

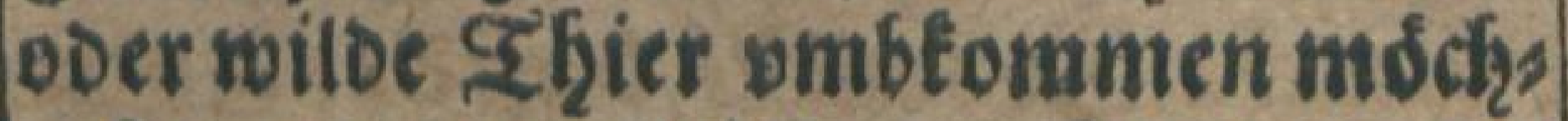
tef / oder bergleichen. Ia / meine Seele/ es [pricht wolmanther/2Benn ich werbe trandt werben/wil ich 2 buffe than / vno mide berenten. Er weib aber nicht / obes im auch fo gut wers Den mochte / Daf in Ëottlieffe frande werben. Saben wir nicht tiogliche Exempel: YBie gar mancher rother Dento if verbliechen bey Deinem ges bend"en/ burch den Eschlag ober ander re Etraffen/ehe ert trandt worben if : Decin BS Stt / WBie unmeife fint boch wir elenbe vnachtfame Diens 


\begin{tabular}{|c|}
\hline 2lle s)enfchen \\
\hline 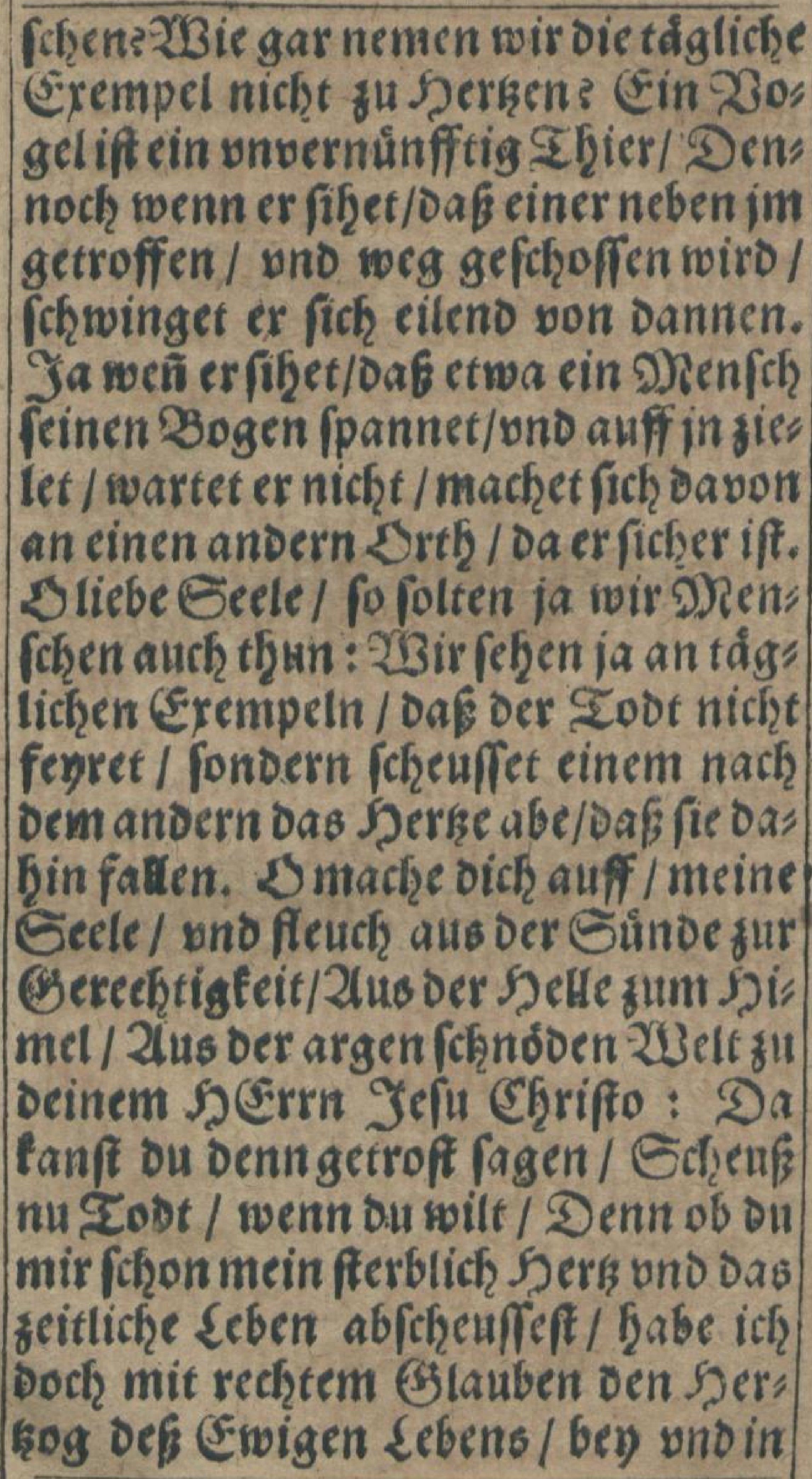 \\
\hline
\end{tabular}


mir / Der gibet mir mehr / als Dumir nemen lanf.

Darvmb / liebe Ecele / Fare beis Syr. 18. neß3 uffe nicht/ soeil bu nodb fün: oigen tartif/20rzeuch nicht from 3uwerben/ ons barre nidht mit Defferung beines febens bifs in ben root.

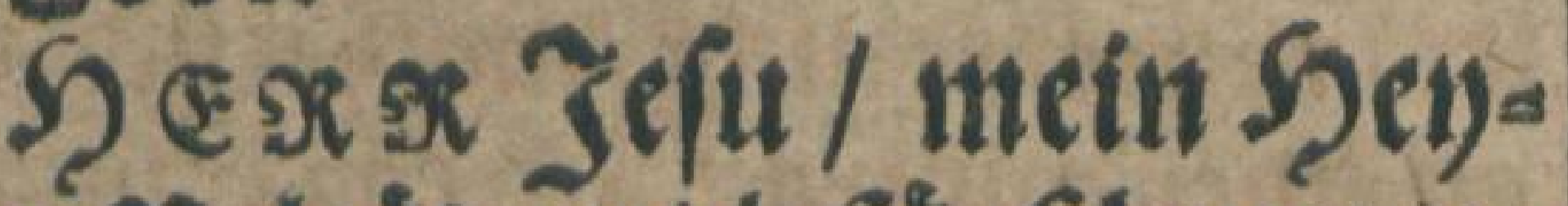

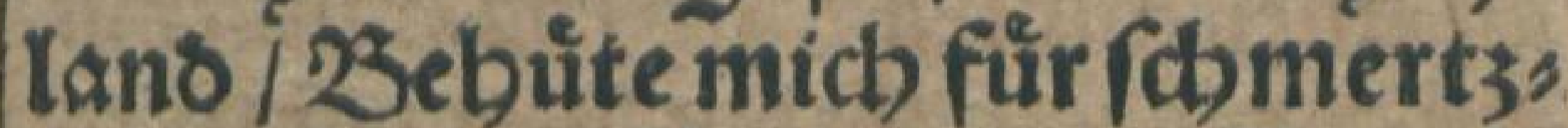
lichen $\mathrm{A}$ ranctbeiten vns forecle: líbem rose. Dberfatle mid nicht in meinen Stingen/vns lap mich nicht obne Buffe fterben. Derlepbe mir aber ein ftitles fanfftesienoe/obne groffe2lngit pinestbmertzen/auff oafid bey guter Dernunffit/ mit gutem 23es badbt / ein gutes dénoe madoen / Deinen Ramen bíz ala meine Dinnefartb betennen / ons meis ne Seele in beine Denoe an: Dácbtig vno Dertłlicb

$$
\begin{aligned}
& \text { befeblen mögel } \\
& \text { 2imen. }
\end{aligned}
$$

Eiij Das




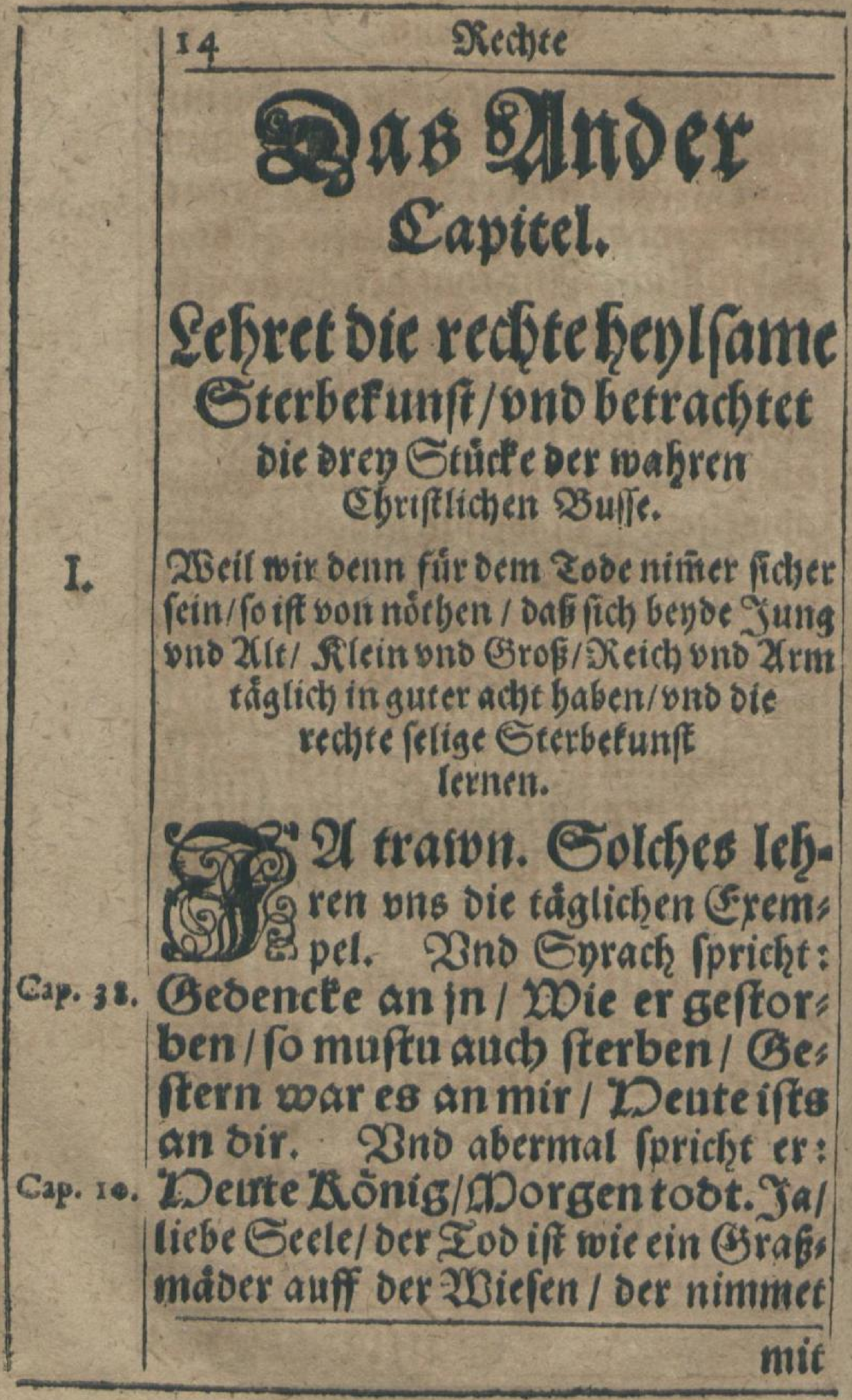


mit ber Sainfen alles meg / sno achtet nichts / es fer) Gradslein oder Sllims tein/ Irdutlein oder Siofకlein / Sraget auch nicht barnach / wie alt/ wic fch onn/ wie foftich: 2alo thut ber $\mathfrak{Z}_{00}$ aud / Es ift im feiner fu Stard / feiner fu Tung/5o if im tein Echlof su foch/ feine Burg zufefte. Boleffet erfich auth mit feinem Gelde ooer Ssolde abweifen. Ya wenns mugglich were/ dak ou ifrm aller -2Belt Giter geben fondeft/[o vermóchteftu im nichst cine Stunbe abs̆tåufẽ / Dte er vertogge/ vi Dir beines gefallens fugebe. Da b fol ja jebermantáglich bedendfen/ wol zu Sorzen nemten / fein Săupt nicht elje fanffite legen / bif ev oie felige Sterbes funft rechit uno wolgeternet

2(t) mein Siott/2Bie ift Doch ber [Denfch gleid) woie nicbts a pal. 344 20 ie febret reine 3 eit/ons arle reis ne DDerrtigleit oabin / wie ein Ecbatten: Dno weiff nicbt/ ob er Jung oser2Alt fterben fol/zian ficb aucb weber mit (Bewalt nodb

$$
\text { (2) iiti }
$$

mit 


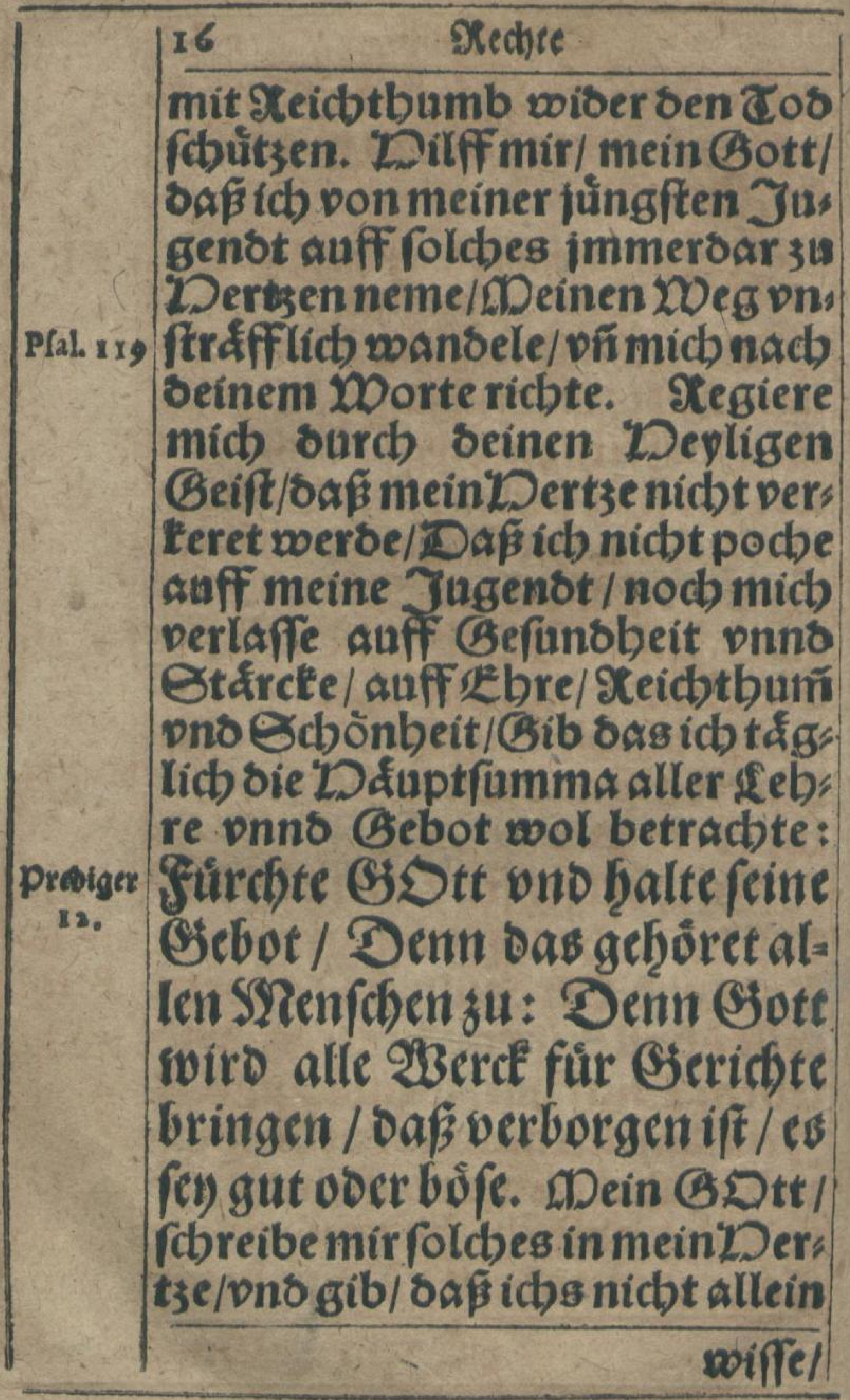




\section{Sterbefunft.}

wiffe/fonsern auch tbue/vfi micb oarnacb ricbte / biß on mein LEn' be/21men.

XBie for ich mich benn sumt feligen Sterben bereiten?

Die rechte 3 ereitung su einent fe" ligen Ende / ift cin täglichę / Ehriftit: ches/ Busfertiges Leben. 23itt ou nu wol uno felig ferben / liebe Eeele / fo thue sechte wahre 20uffe/vno verfigarre oarillenbis ans Ende. Deftes fpricht Die Echrifft: GDtt will2zuffe für sap. 12. bie Eưnoe annemen. 23 n aber mal pricht dic Echriffit: 200er bes

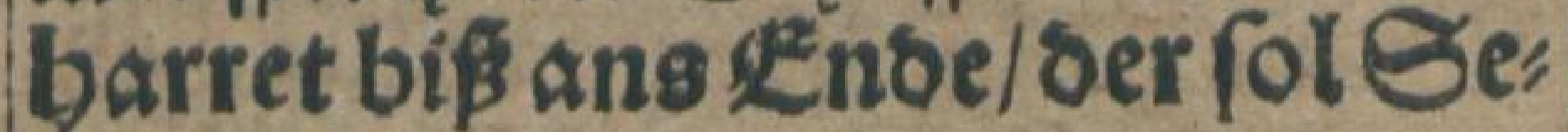
Must. 24 lig werben.

Selobet fenftu / mecin Siott / sas ou níbt luft baft an vnferm Cost pns Derbamnißß/Gonsern wilt / saji woir pns beteren/ ono leben. Dilff mir/mein'D R X $x$ Jefa/bapi ich nicht t verziebe/mich 3u sir 3 ubeteren/Dn' meine 23 trs syr. s. fe nicbt aufficbiebe von einem a age sum anbern / Denn beine)

Eze. 18. 8* 33 . 位

Gnw: 
Ginabebaft bu mir jasugefaget/ 2 ber ben morgenben ₹agbaftu mirnicht verbeifchen. Ey fo ver: Pfal, 95. Lepbe mir / Oafs icb beute 23 uffe tbue / vno mich betere / 2uff oas id) nicht 2 Dorgen in meinë Sùn oen ergriffen vno bingeraffet werbe/21men.

III.

$$
\begin{aligned}
& \text { 23as ift Denu rechte wabre Ebrifto } \\
& \text { liche Soufe? }
\end{aligned}
$$

Buffe if niclits anders / denn das cingienfth von Eumben abfertict/vnd fich von Sorken zu (Sott beferet. Etes het aber in oiefen oresen Sturten:

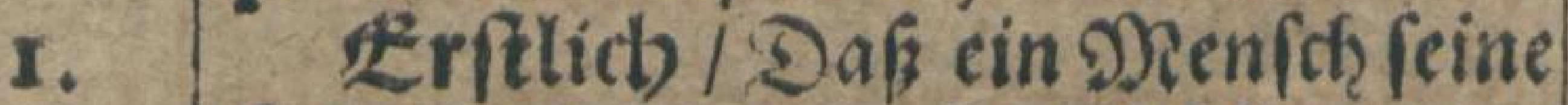
Eimbe erfenne und herglich berewe.

2. Jum andern / Da a crin feinen Günden nicht verzage / Eonoern an Gefium Efriftum gläube/Der für vafes re Sinde begaflet bat / vnd fich feiner von Serken frewe vno trófte.

3. Jum britten/Dak er auch feinen Bilauben berbeife mit tåglictrem ncw: en Geflorfam / bende gegen Gott und Dienfichen. 
SEerr/mein (Sott/ict) weif/ Saßj idb nicht aus eigener Dep" nunfft noch Atrafft / zu wo abrer 2Buffe tomen / noch an meinen Dérrn Jefum Cbriftum gldu: ben tan : Idb bitte sidh/ rübre mir mein Dertze ourdb beinen Deyligen Geift / Dno gib / oâf meine 23 eterungenicbt Deucbe: Syz. x. ley/ Dif mein Glaube nidbt falf (b)/ Bonbern recbtifbaffen vfibert lich fey / Dno an feinen rechten früdbten allezeit möge gefpuiret Math. 3. ons ertanot werben/21mers.

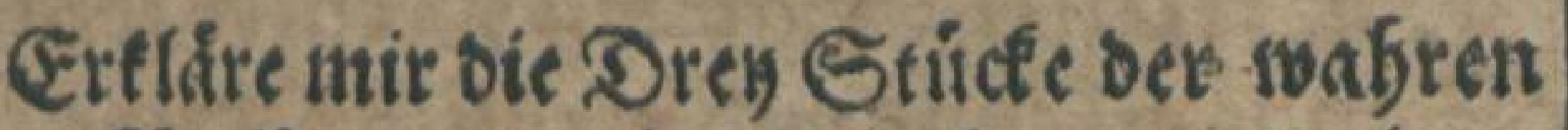
S3uffe/ viro gib mir antertung/ woie. ich mich barinner etseigen/. ond taglicl) wben for.

Bar gerne. Daf erfie Städe ifte rechte wafire Reewe uno Seno uber bie Sinde. Denn of ne Erfenonif ber IIII. Etinden / ift ber Bs laube nur Sheuches Iey/und nichtsechtifthaffen. Derthals ben lerne beine Sinde erfennen / den Sorn CSEtteb wiber dit Sünde bes. 


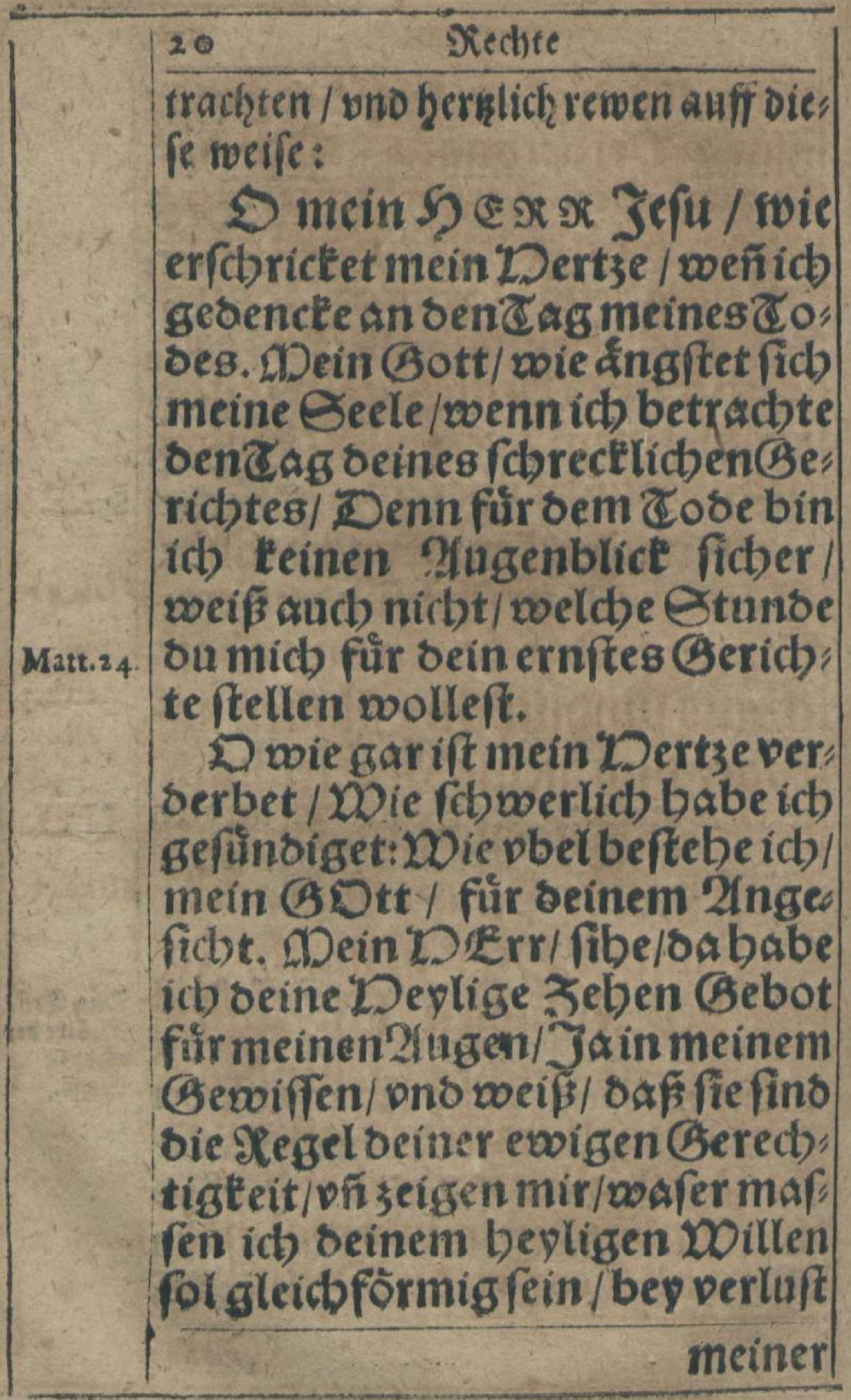


meiner Seligl"eit. 24db ber graws famen Derberbunge. 2(d) ber ficutzlichen Zerruittunge aller

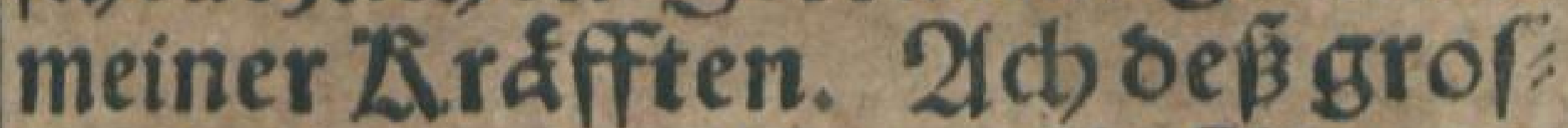
ren Echabens / ro burch 2loams fall gefcheben/vnb auffons alle geerbet ift. DDein Gott/ woie gar ftimmet mein 'Dertze mit beinen Geboten nicbt pberein.

freylicb ertenne ono befinse id) / bẩ ich in KRrbfünoen em/ Prab si. p fangen onø geboren bin/Daj ich) aus fundlicbem Samengefeuget /ono meine SYutter mich in Stunden empfangen bat. Daßz ganke Shâupt iff frand? / Elaix 1. Daf gantse Sortz if math/ Za von oer fusfolen bif aufits Şaupt iff nichts gefundes / nicbts recbtf(b)affenes an mir / Eonbern alles verberbet burch bie Eúnbe/ bie in mir woobnet. Rom. 7. Pfup sich Teuffel/bu "Dellifther 


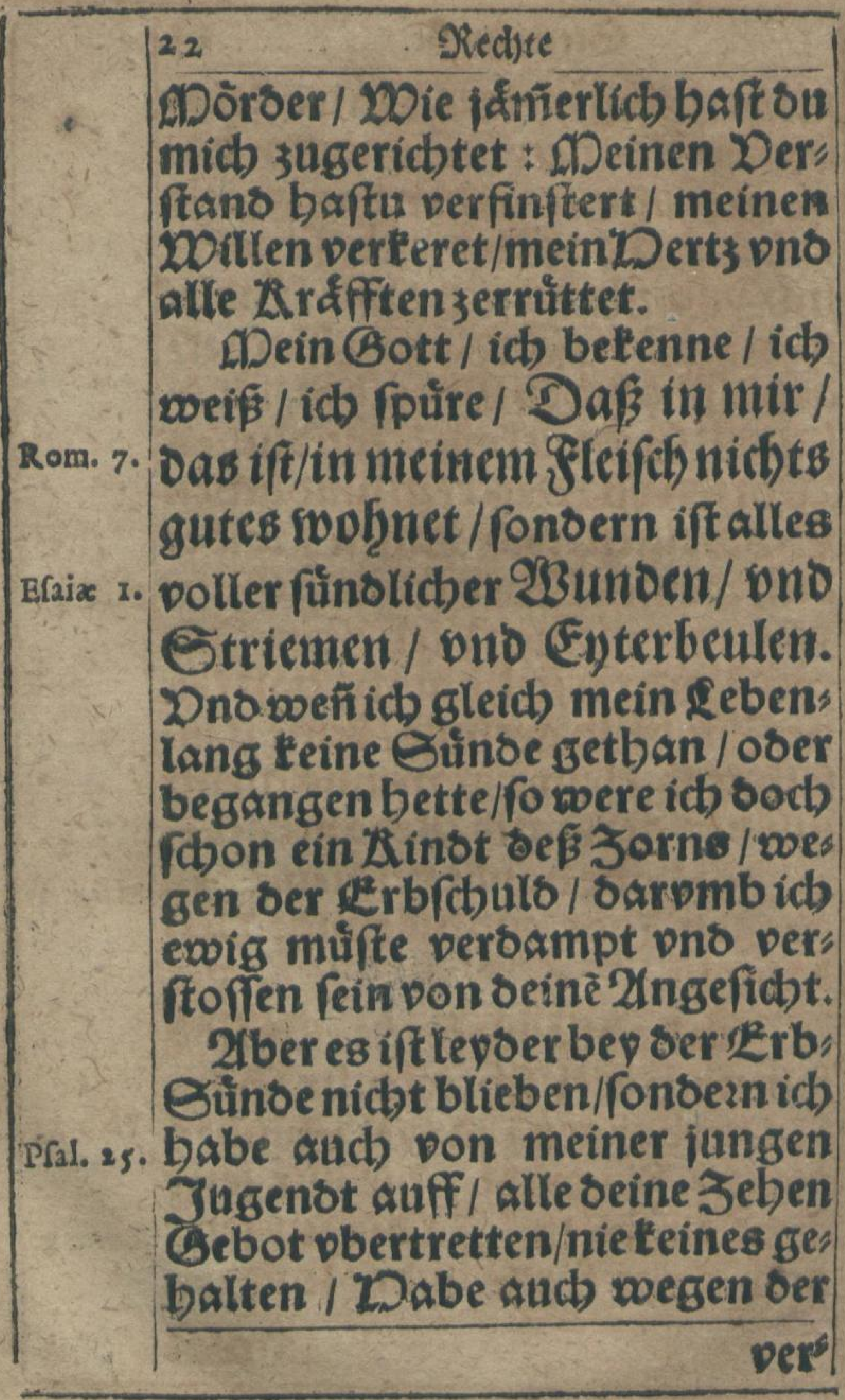




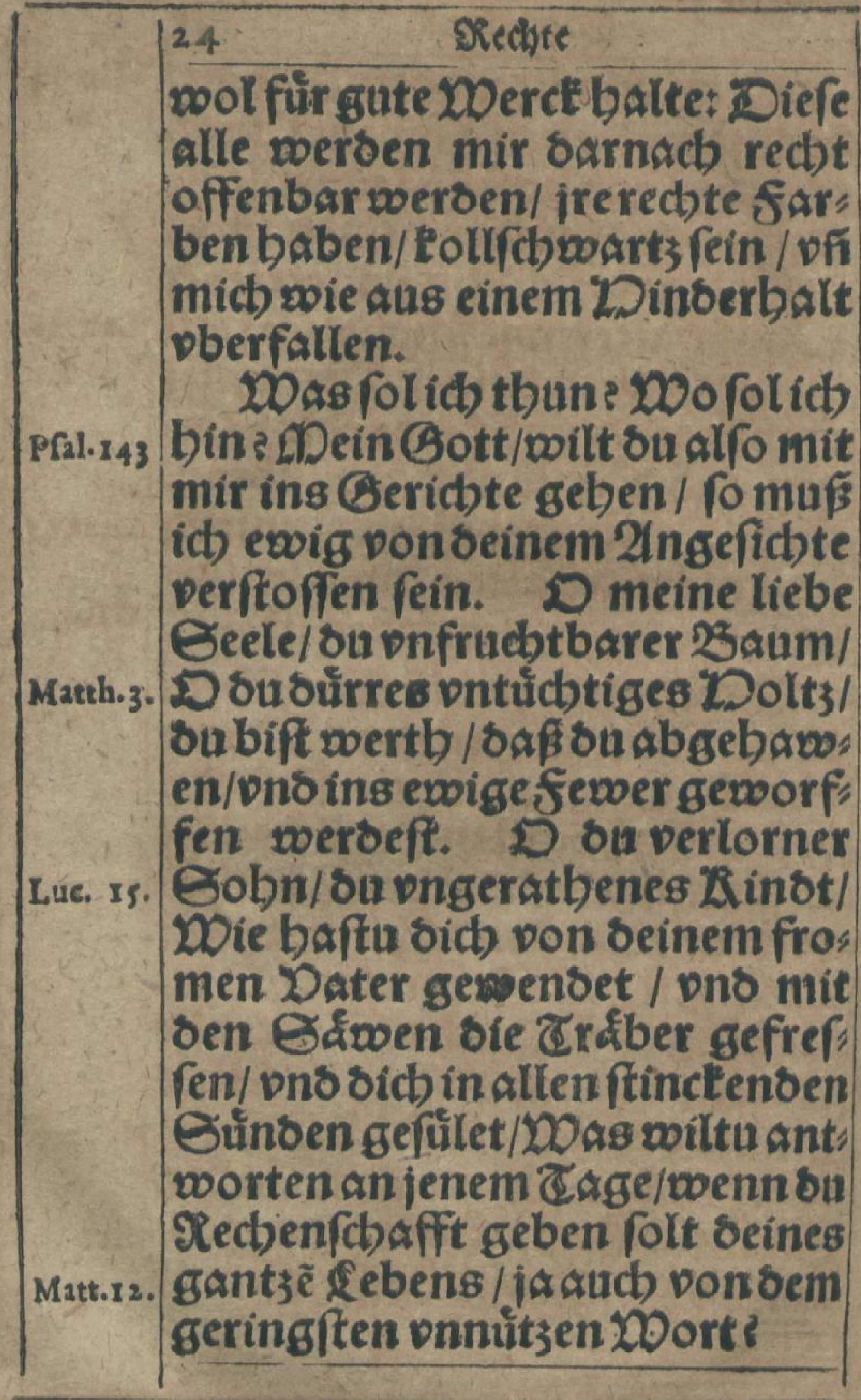




Sterbetunf. 25

D ou vnnuitzer bōfer Ânecht/ Mak. 18. oubift wolseben Taufent Pfuno fchultoig/vnobaft viel onseblicbe taulens mal woiber seinen Gott gefünoiget. D ou vngetrewer Dauph balter/wie fbenolid) baft Lue, s6. ou bie Gaben seiner Dernunfft ombbracht / ons seine eole $\Theta$ in: nen ons Derftanot gemifbrau: det. Derflucbt bift ou / Senn ou Deut,27. baft nicht gebalten / was im Ge: fetse Gottes gercbrieben ift.

D webe mir elensẽ \&Denfcben/ 2Das babe ich getban? D wie vbel babe icb getban? $D$ Jorn beß 2ullerböchiten / falle nicht auff mich/D Grin se 10 atlerbo:s beften / DDer tan bich ertragen : $D$ 2Angft / $D$ Ecbrecken / D ou onflátige / fünsliche / ftinckense Eeele / bin id mir soch felber gram / oafí ich ein folch Grevoel worben bin fur meinem GDtt. Ja/mein Gott/idb bin nit wertb/

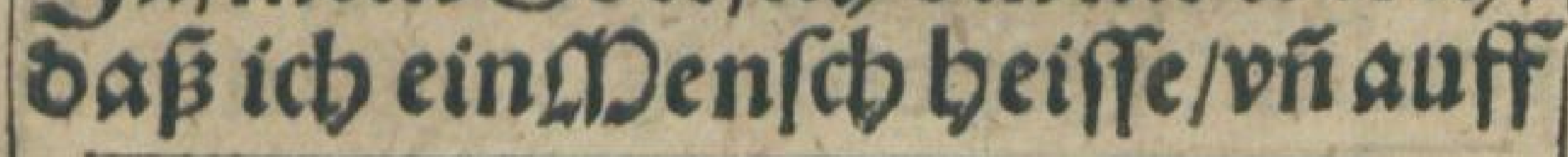

$$
\text { f beis }
$$


26

Pectite

seinen Grunot ons 2 Sosen tret, ten fol. KE were nicbt wunber/ o a fi fich bie \& ro aufftbete / pno mich verfchlùnge / o a pa ich leben: sig in sie Delle fibbre.

$D$ revoe ons traworesuelense Oeele/D beule ons weine ou bo:" fer aDenfa/ Ja Slut folt ou wei: nen/fo es miglích were/pber beis ne Sünsen/ons aus allen beinen Lirafften traworen. D webe bir/ subartes Dert3e/ tan bich bodb tein $8 d b r e c t^{2}$ en erweichen. WDe: be oir / oufchlaffenoes Dert3e I exn oich soch leind Devoen ervoe: cien. D ou töslicber Scblaff/ tan sich sodb tein 2 lit; noch Donner befi ernften Gefetzes Gotteg vertreiben. 2uff/liebe

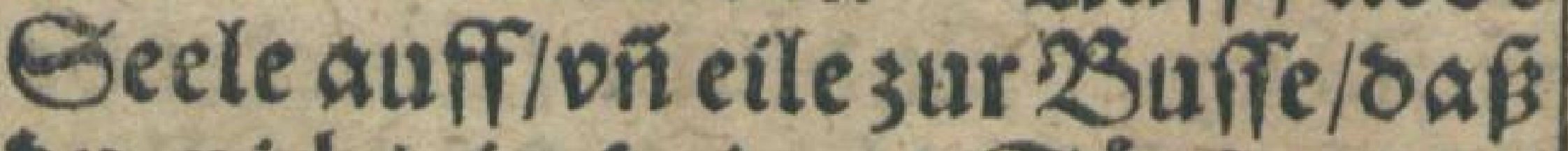
ou nicbt in seinen Günoen er" grieffen werbeft. Sibe/ber Jag

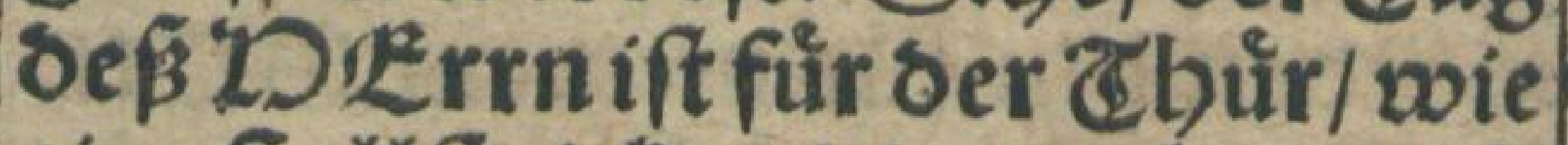
Lue, 2x. ein fallftrict wirb er vber sich Pomen. Eibe/ber zost greiffet nads 


\begin{tabular}{|c|c|}
\hline \multicolumn{2}{|l|}{$\begin{array}{ll}\text { Sterbetunft. } & 27\end{array}$} \\
\hline \multicolumn{2}{|l|}{ nacb bir / vns in einem 24ugens } \\
\hline blict wirs er bid erwifchen. & \\
\hline Darvmbrebeich/ons (a)wei: & \\
\hline ge nicht / Jch beid)te pno belens & \\
\hline ne für meinem (3) tt / alles wods & \\
\hline ich in meinem Destzenbabe 21d & \\
\hline bas ich 20affer genug bette in & m. \\
\hline meinem Dápte/Dfi meine 24 : & \\
\hline abranequellen weren/ ठaß̧ & \\
\hline $\begin{array}{l}\text { id Cag pfin ladbt weinent } \\
\text { te pbermeine Ouinde. } 5 \mathrm{~m}\end{array}$ & \\
\hline liebe Seele / laß̉ flieffen seine & \\
\hline abránen สag vns macbt / wie & 1 \\
\hline cine Bacb / ons bòre nicht auff/ & \\
\hline Dns sein 2lug2lpffel laffe nicht & \\
\hline 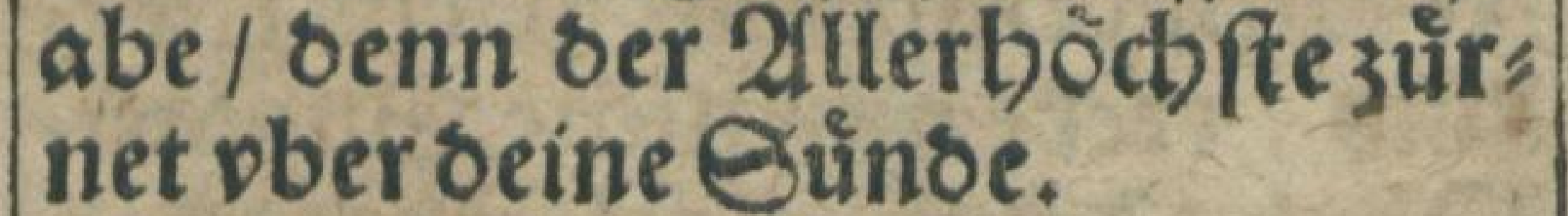 & \\
\hline $\begin{array}{l}\text { nfib in folctiem ?rane } \\
\text { wnd in Sunden }\end{array}$ & ? \\
\hline 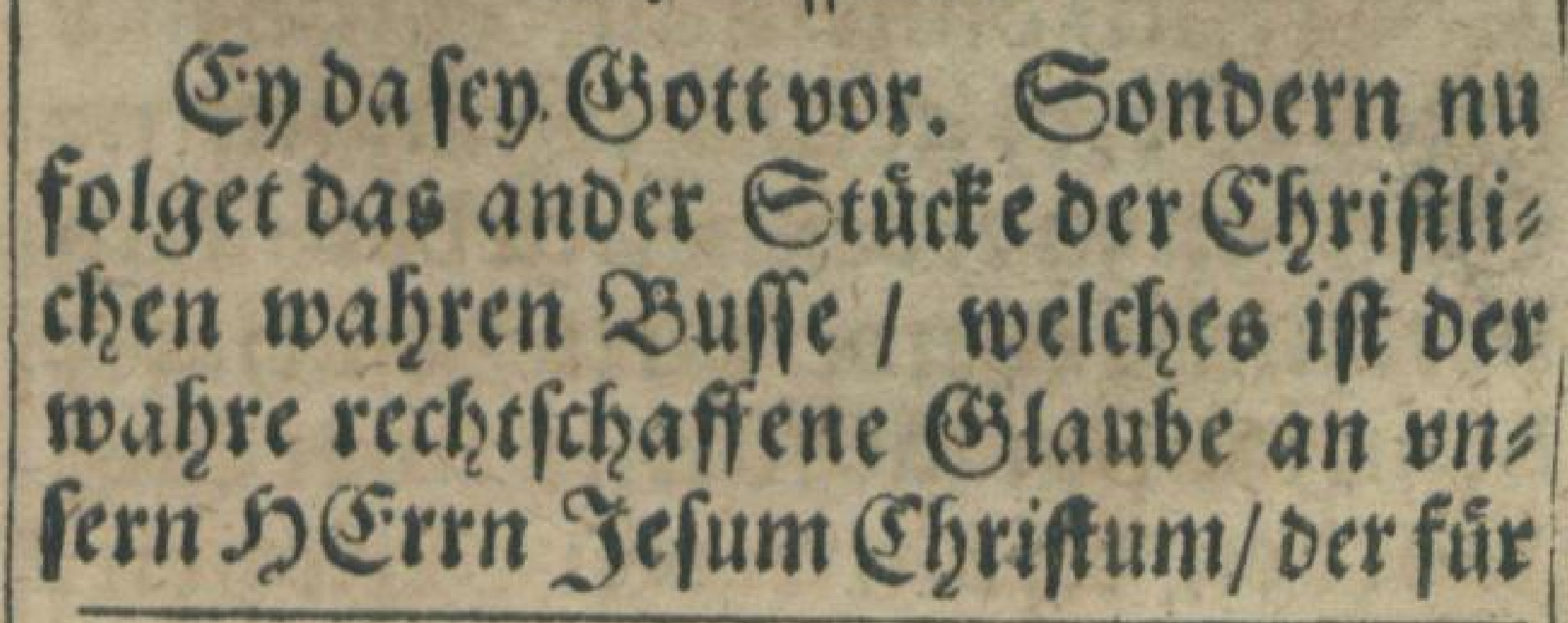 & $\begin{array}{l}\text { Das an } \\
\text { Siade } \\
\text { wabein } \\
\text { Douffe. }\end{array}$ \\
\hline if ij & \\
\hline
\end{tabular}




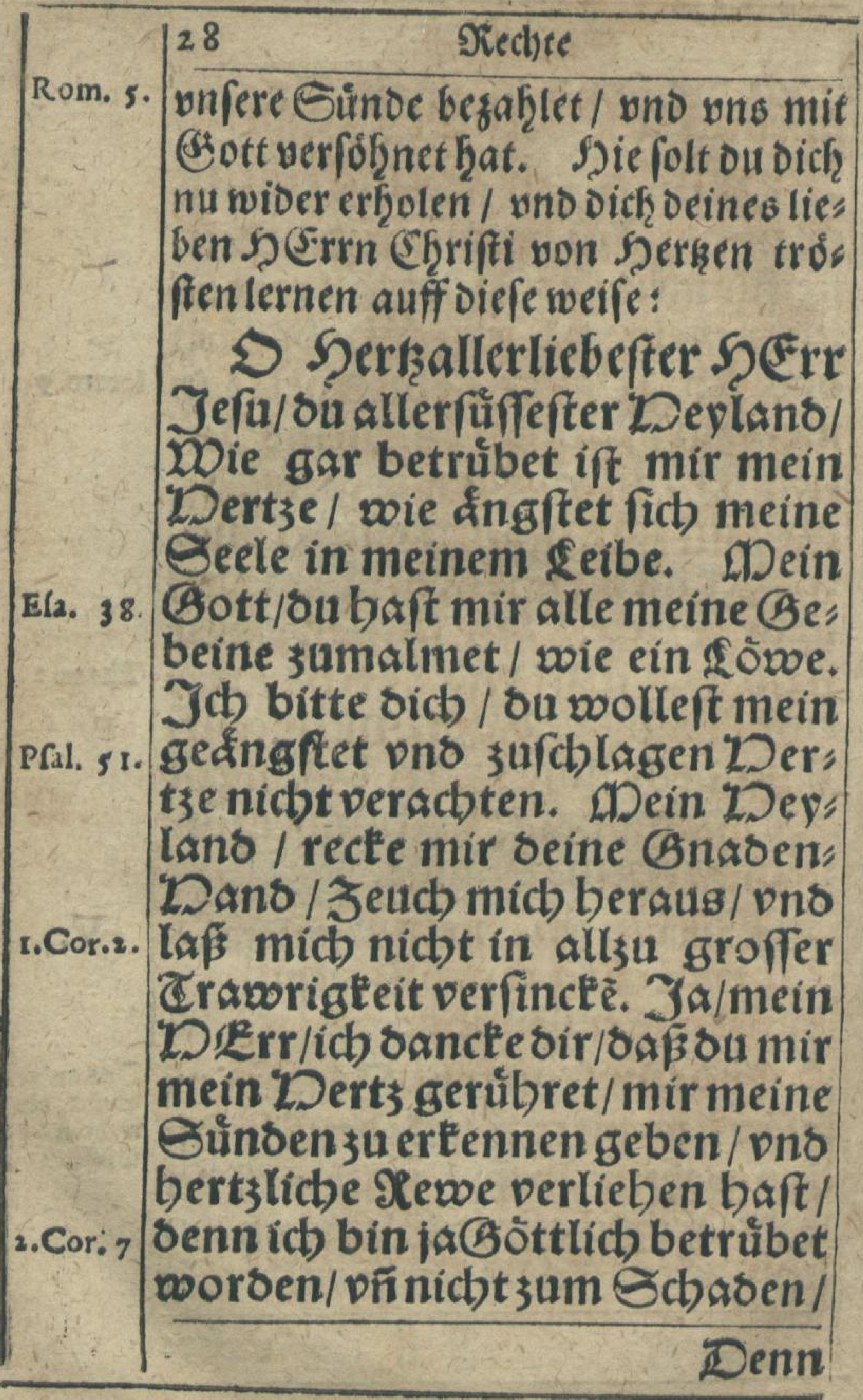


$\frac{29}{\text { Denn Dic Storbettliche Traforig }}$ feit woircfet zur Seligfeit cine Rerwe/ Die niemanden gererwet. 2Do fol icb aber nu croft fia: dhen für meine betríbte Eeele? Do o rol $i$ ch friese vno xube fin ben für mein 3 ufdalagenes D Der" tze ? $\mathcal{E}^{2} y$ bey oir mein allertiebes fter/allerfúffefter Deylano allevi: ne/ Denn su bift ja in biefe Dolett tomen / bie armen Eunber felig r.Tin.r. zu madben/ beiffeft audb Sarbmb Jefus/oasift/ein'Delffer ligmacher/oajs ou bein Dolct res: lig macben wilt von allen ibren Lucke 1. Euñen.

Die bin id) / mein Deylanot/ Db wol ein gar groffer/ oods ein busfertiger Eunber. Eibe/Jd) babe beine troftreid)e Stime ge: boret/basu ruffeft: Romet her Math. r. ğt mir alle / oic je múlefelig oñ beladen fevo/icb roil euct) erquicfert. Eibeda/mein DERer/icb $f$ iij tome 


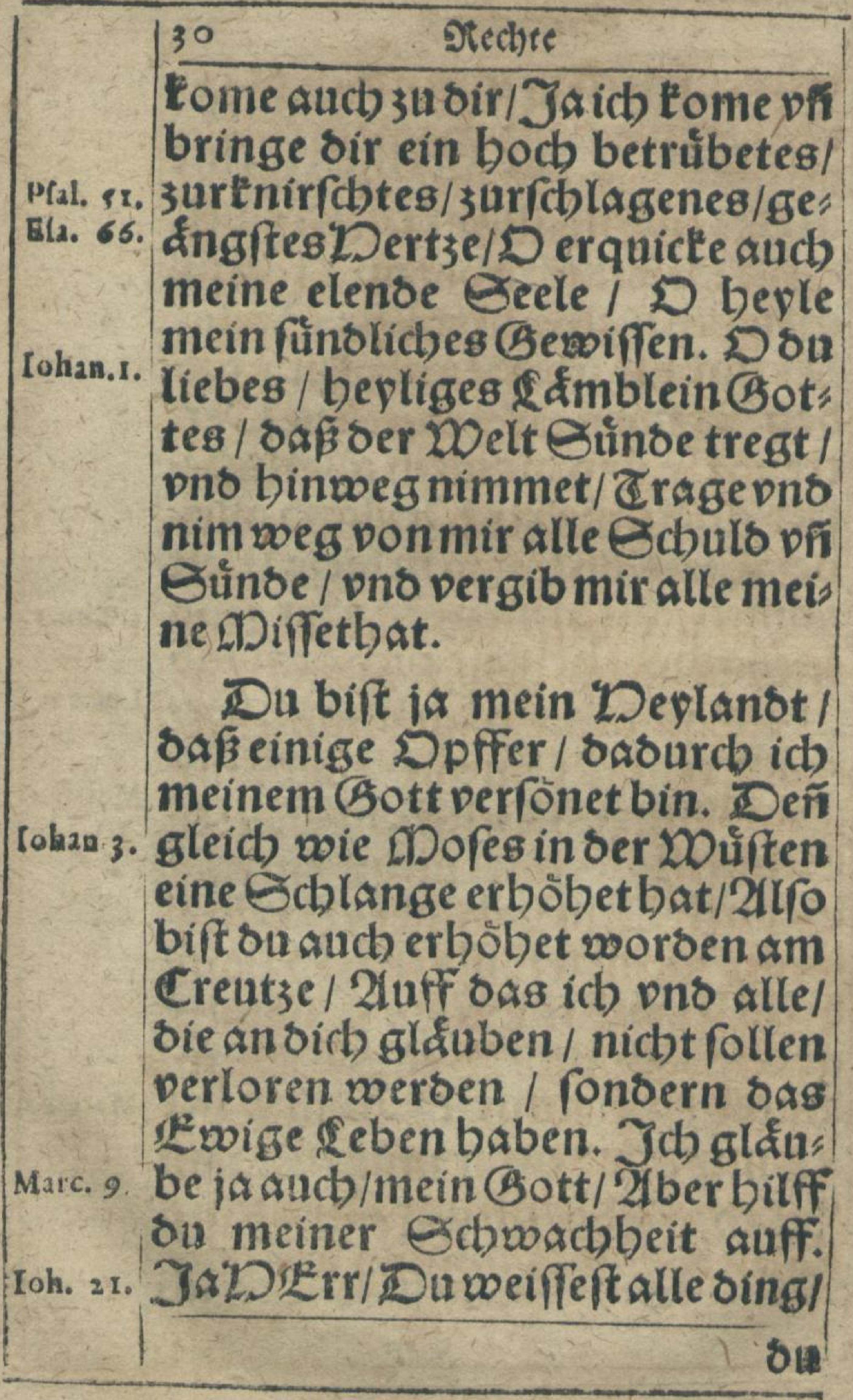




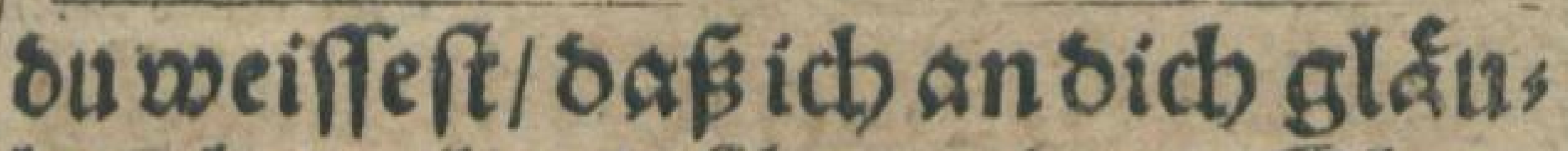
be/ob wool mit fobrachem (Slaus ben/Deñoch mit oiefem meinem geringen (Glauben / fetze ich alle mein Dertrawen vns Zuverficbt auffoich. Ich glåube ons 3 weif: fele nicht / Job gláube vf trawe। oafis alle meine foboere ons ftins ckense Eundon/burch bein 2 lut ons a 0 gevoiflich gebuffet ons bezablet fins.

Ja id) weî́p ons glåube / idb

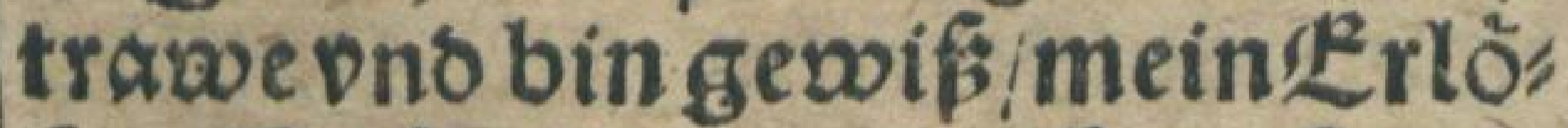
$\mathrm{fer} /$ Daj beine reine 'Empfeng" nif / reiniget meine funsliche 12mpfengniß/Deine onbeflectte Geburtb/beyliget meine onreine Geburt/Dein bevliger 20 anoel/ buiffet meinen fünolichen 20 ans oel/Deine derniorigung/ ift mei ne Rrböbung / Deine Dellens Zngft I if mein Dimelaroft Deine $2 S a n b e$ / fins meine SE rló, funge / Deine Sd)mad/ meine

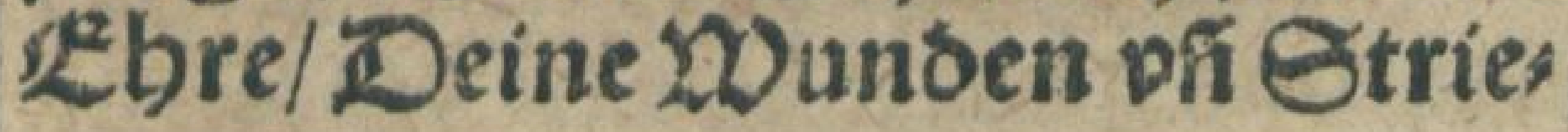

$$
\text { f iiù men/ }
$$




\begin{tabular}{|c|c|}
\hline & stedse. \\
\hline & men/meine Zier/Deine 21uß̧üb; \\
\hline & 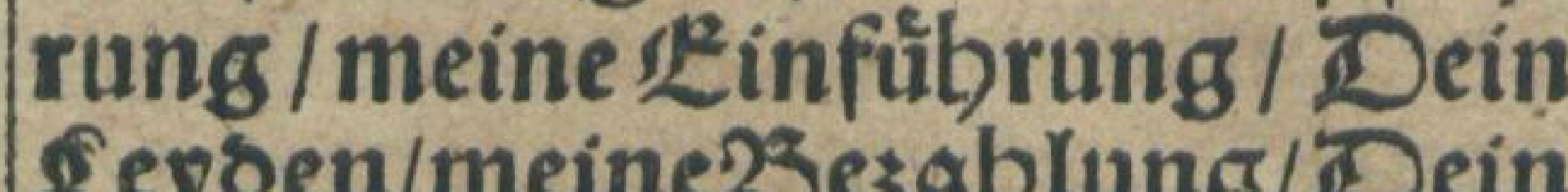 \\
\hline & $\begin{array}{l}\text { Levoen/meine } 2 \text { Sezablung/Dein } \\
\text { Deroien/t/ mein \&öregelo/ Dein }\end{array}$ \\
\hline & 23lutruinftiger aost iftsas recb: \\
\hline & 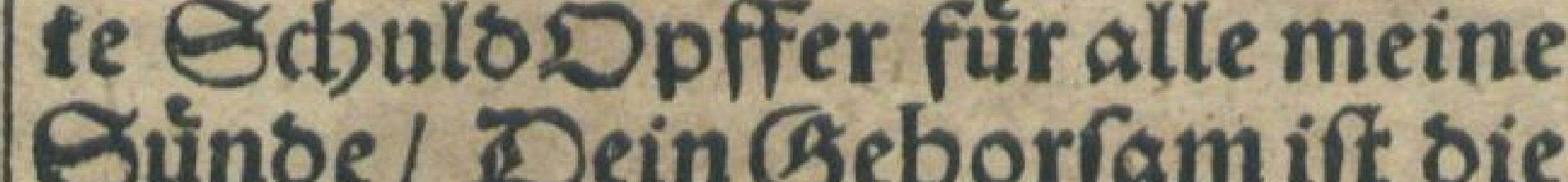 \\
\hline & recbte (Genugtbuung für alle \\
\hline & meine Diffetbat / Deine Delle \\
\hline & $\begin{array}{l}\text { fartbift mir eine Kzrettunge aus } \\
\text { oer Gewalt ber Dellen pns sef }\end{array}$ \\
\hline & aeuffels / Deine 2ufferftebung \\
\hline & ift meine Gerechtigleit/Dnobei \\
\hline & ne Dimelfartb meine Derficbe: \\
\hline & 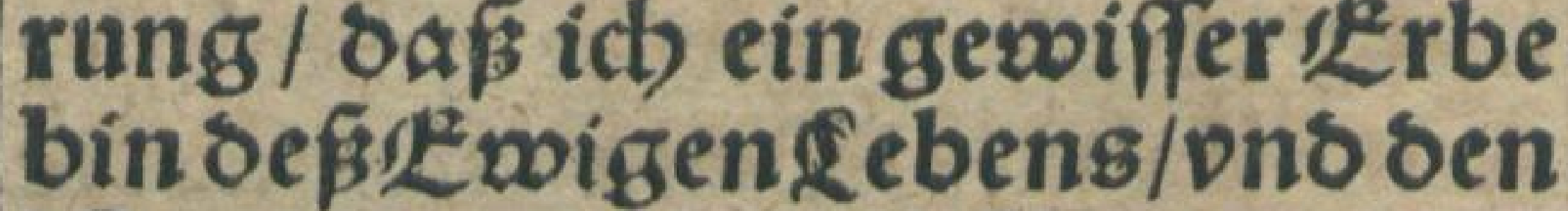 \\
\hline & $\begin{array}{l}\text { Dimel surcb sicb fobon innen } \\
\text { babe. }\end{array}$ \\
\hline & Ja/mein DiErr Jefu/ Saf́al \\
\hline & les baft ou mir getban / mir ge" \\
\hline \multirow{4}{*}{ Philip.3. } & en/ \\
\hline & netmir es $3 u$ / fo \\
\hline & $\begin{array}{l}\text { roienet bette I } \\
\text { erces oer Kerlo: }\end{array}$ \\
\hline & \\
\hline
\end{tabular}




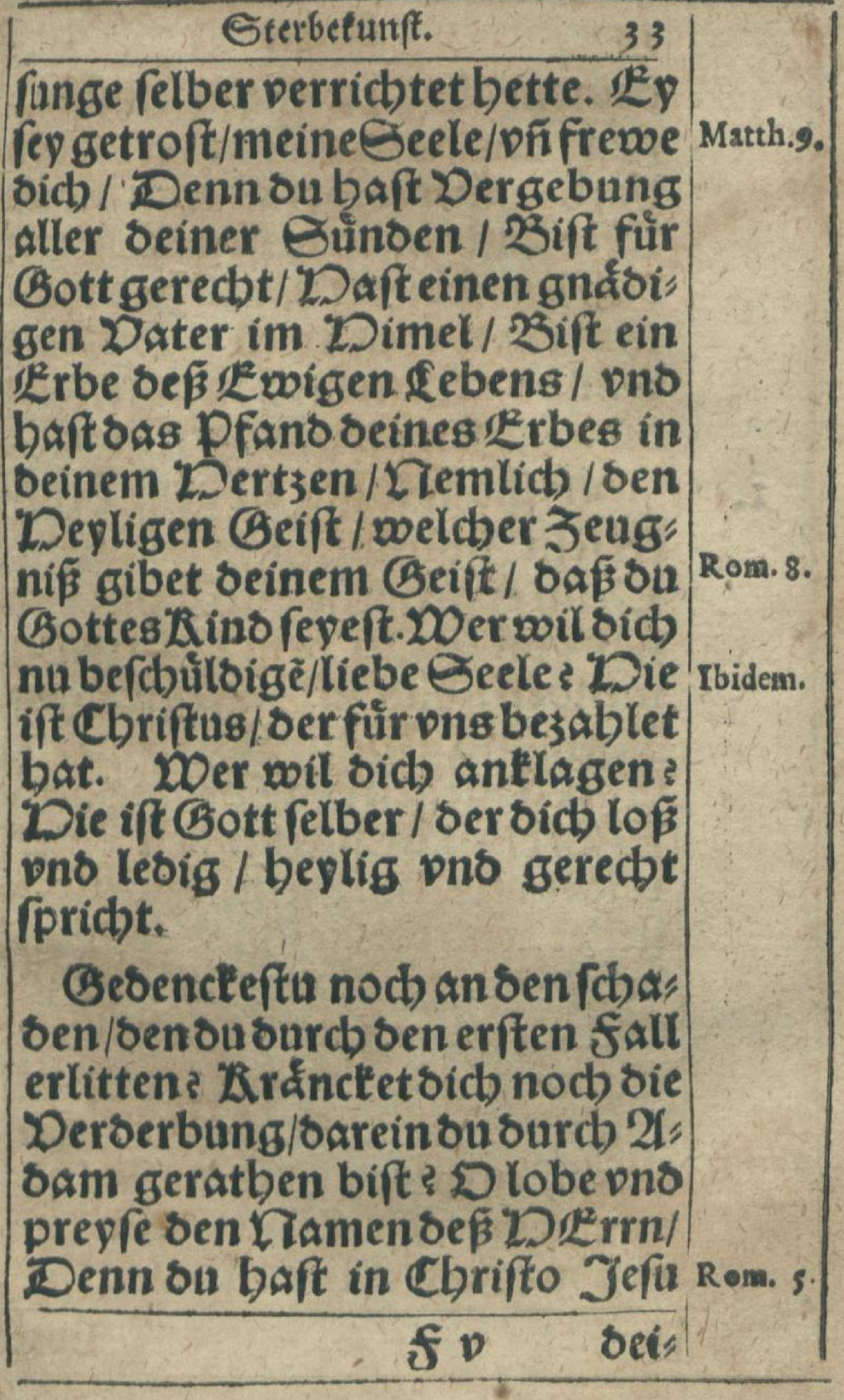




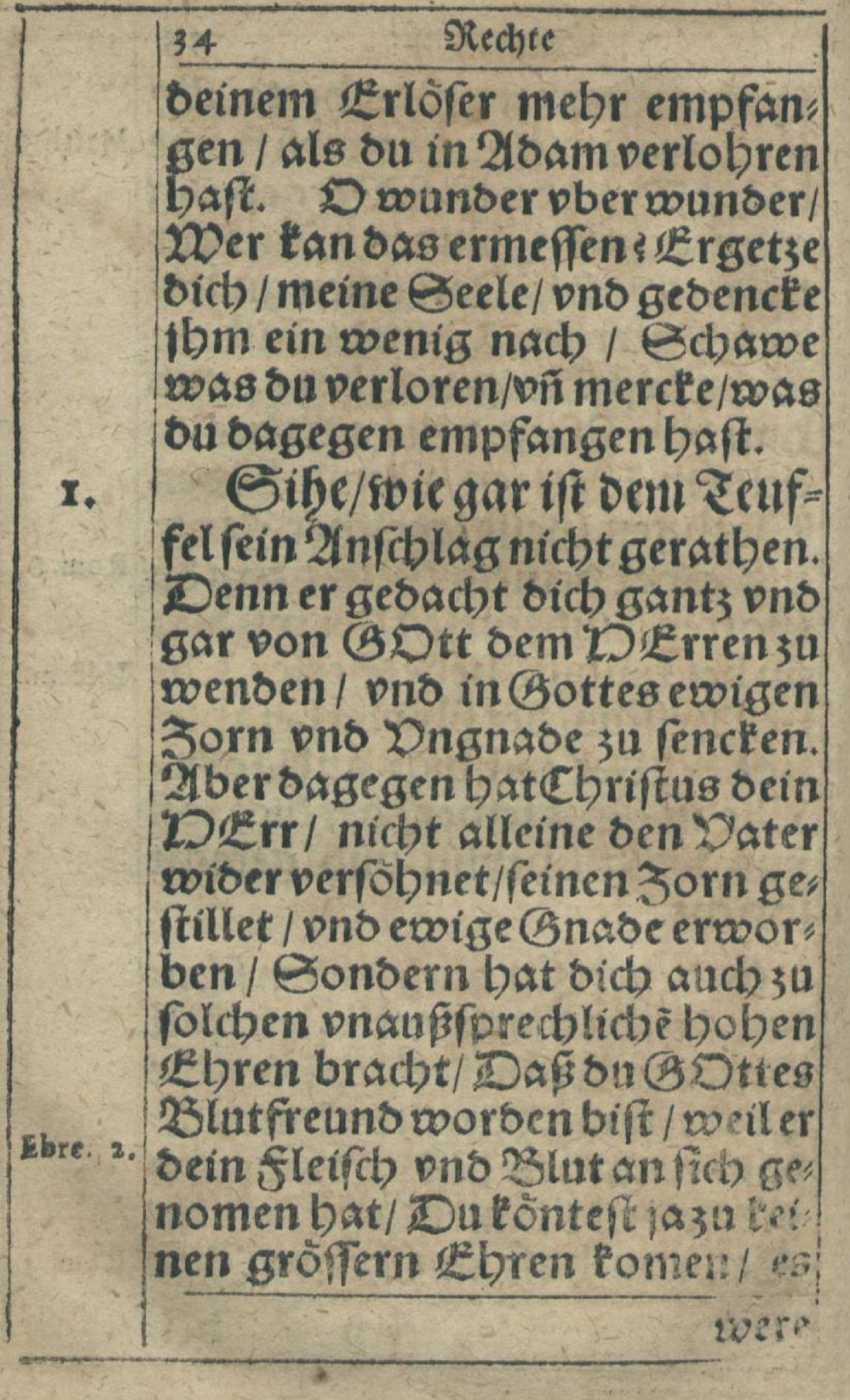




\section{Sterbefunft.}

weresenn / Saß̧ ou felber GDtt woorben woereft.

Der Satan battevor / Er 2. wolte bich aus oem fuftDaure be ber Rngel Gefellifthafft berau: ben. 2iber oagegen fübret oich Cbriftus beint Deylano/nicht at: leine in bas Dimliffhe Parabeis ono K'wige feben/Sonbern bat oich audb vber alle renget erba: ben/ vno berrlicb gemacht/ Def̂ er bat nicht KEngelifdoe/fonoern Ebre. 2. DDenf(bliche Itatur an fieb ges nomen.

Der Satan gebachte obich 3. pmb sie Epeife se位 \&ebens za: bringen / Es gerietb $^{2} \mathrm{~m}$ auch: Denn Gott der DDErr verftiés Gener, 3 . oie LDenfchen Dmb jrer Eunoe willen / oấ fie vom 23aum be Sebens im parabei nichteffen folten. Dagegenaberift Chri: ftus felber beine Speife wooroen/ Iohan.6. vno ift felber ber 23aum befís fe:

bens/ 


\begin{tabular}{|c|c|}
\hline & 36 \\
\hline & bens/ber viel beffer ift/beñ jener/ \\
\hline & Dns breitet feine $\mathbb{L}^{2}$ te pns 2 rme \\
\hline & aus / baß̄ woir pns pnter jm neis \\
\hline & gen/pntertried)en/ Dom im effen \\
\hline & ond fatt woerben/ Dñ pnter feinem \\
\hline & $\begin{array}{l}\text { Schattendube ons ewigenfrie: } \\
\text { se baben follen. }\end{array}$ \\
\hline 7 & Der Satan fooltedicboon \\
\hline & aller seiner Gerecbtigkeit brin: \\
\hline & 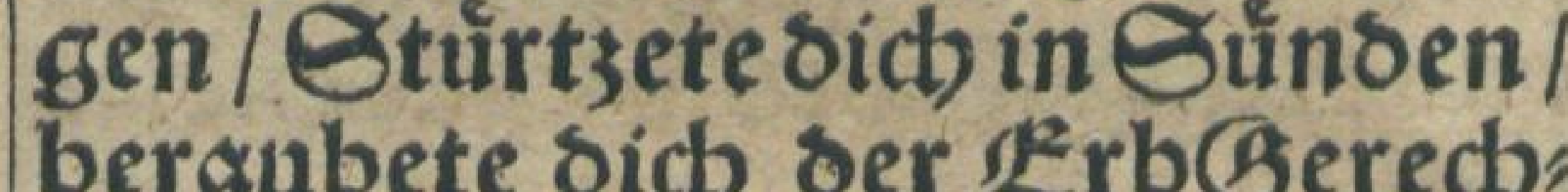 \\
\hline & tigleit/sarinnen su rein/ons ob \\
\hline & $\begin{array}{l}\text { ne Eunde gefbaffen / pns ein } \\
\text { Ejilse (Gottes wareft. Sber sa. }\end{array}$ \\
\hline & gegen fobenctet sir ser DSErr \\
\hline & $\begin{array}{l}\text { viel cine beffere Gerechtigleit } \\
\text { nemlich / feinen Derbienff / feine }\end{array}$ \\
\hline & Genugtbuung/weldbes sir voll \\
\hline Philip.3 & lomen burch ben (blauben zuge: \\
\hline & redbnet woird / Dno ift seine Ge; \\
\hline & $\begin{array}{l}\text { rechtigteit / Sie alleine vor (BDDtt } \\
\text { gilt. }\end{array}$ \\
\hline 5 & Der Satan bat oich outed \\
\hline & $\begin{array}{l}\text { 2loams falloeß } 2 \text { Zilseg Gotteg } \\
\text { beraubet/an allen Âraffen vers }\end{array}$ \\
\hline & muntost I \\
\hline
\end{tabular}


$\left|\frac{\text { Sterbetunft. }}{\text { wounbet/3erruttet/ pns balb tost }}\right|_{\text {Lucx }}$ liegen laffen/bas in oir tein eini: ge Ärafft / nocb einiges Glieb: mấ vbrig war/sapí Gottes wils len tbun vns er fưllen tònote / Dín betten woir alfo ewig fterben ons veroerben muifen. 2Aber oage" gen bat dich Cbriftus ber 'DSErr nicht allein wiser belleiset mit feiner eigen (Gerecbtigteit / Dei: ne DOunden gebeilet mit feinem E\{a. $\$ 3$. eigenen 2 lute ono arofte / Dich ourd) reinen'Deiligen Geift new geboren / ons a as Bilbe Gottes wiber angerichtet : Eonbern er wirb auch am Jüngften rage beinen nicbtigen feib verťlấren/ Philip. 3 ons ebnlich mactien reinem ver, tikrten feibe.

Suma/ber Satan gebach). te bich) subringen pmb alle beine Derrligteit/ ons mit fich in evois Ge Deroamnifßzufturrtzen. Da gegen aber fpringet Cbuftus oir 3u / Timet nicht allein an fict) 


\begin{tabular}{|c|c|}
\hline & Sect) \\
\hline & 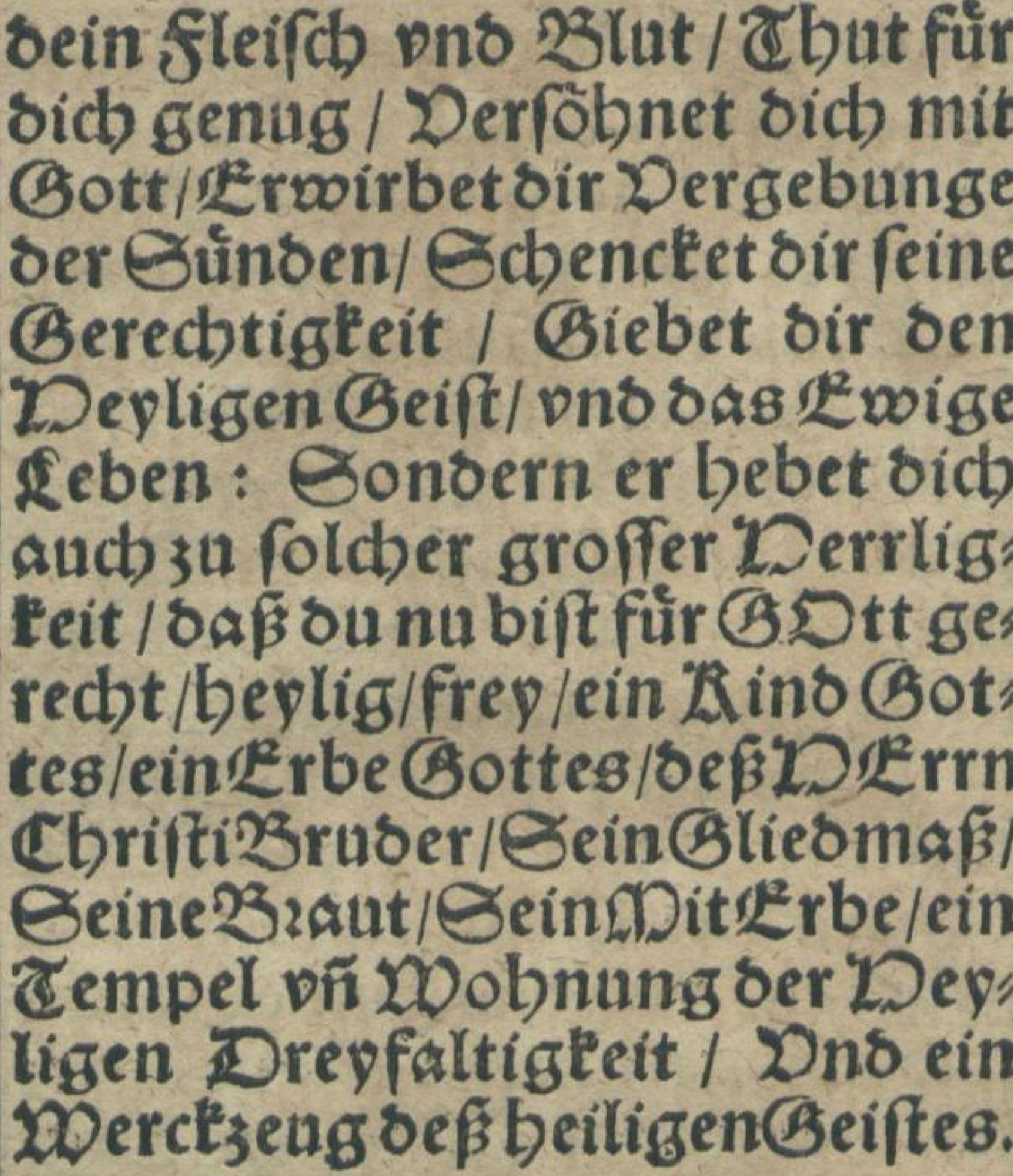 \\
\hline 7 & 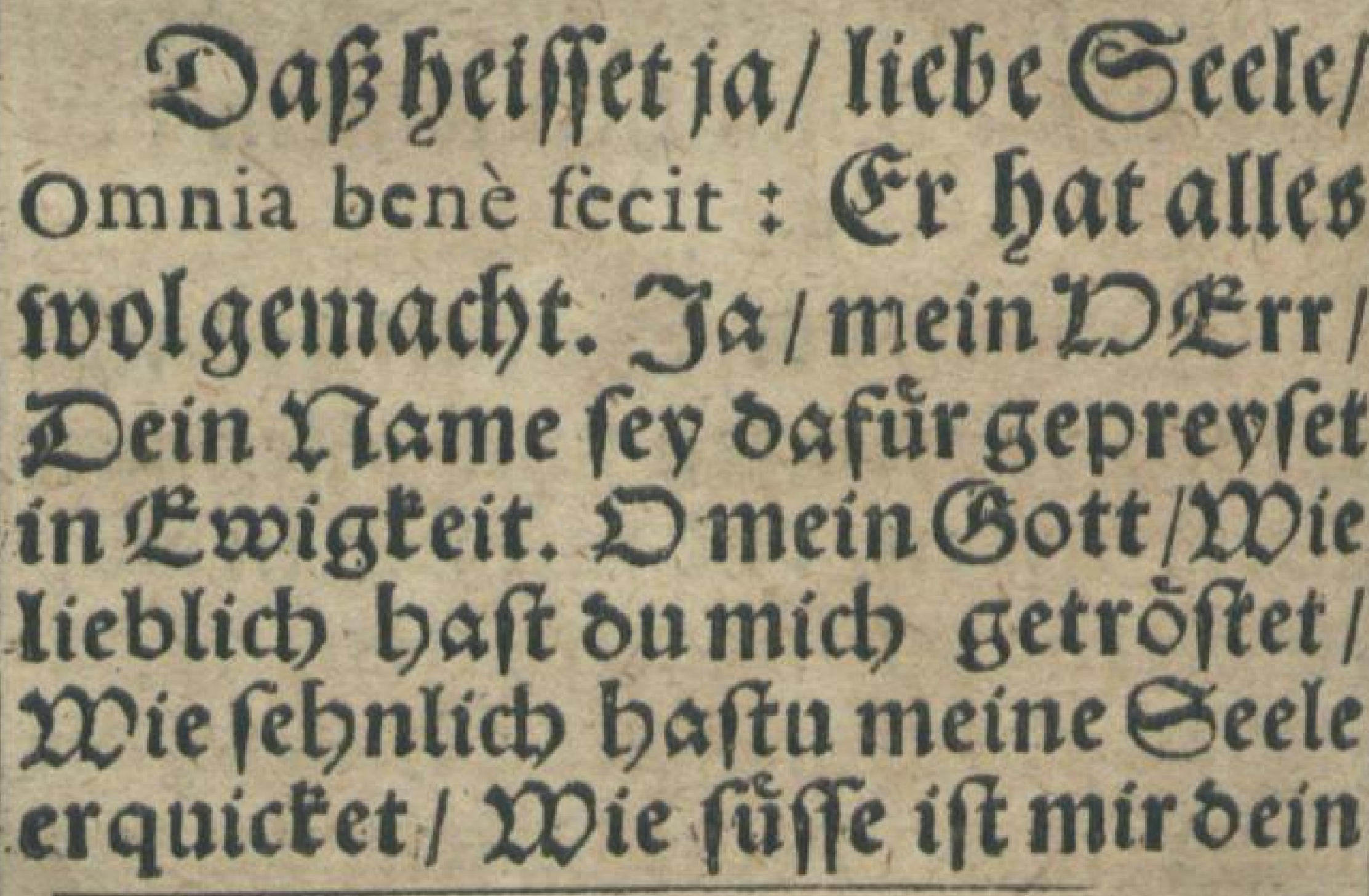 \\
\hline & ento \\
\hline
\end{tabular}


$\frac{\text { Eterbefunft. }}{\text { Troft / Wie fein gibt fich mein }}$ Dertzezufriesen.

Du bift ja bie redbte gúloene Gnaben Pforte 3 um SEvigenfe: ben/Dubift Der $23 e g /$ ie'sar: feit/onb bas Eebert. Ricmans toh. 14. fonct fum 2 ater / Denn ourch Dich.

Dubiffoer Q3eg. Dennes ift in feinem anderm S马en / iff Ator.4. aucb) fein anoer Rame ben STenfchen gegeben / Darinnen wir follen felig weroen.

Dubiftoie 2 Barbeit. Denn wer obertritt/ ono bleibet niche s. Toban. in ociner Leffre / Der hat feinen

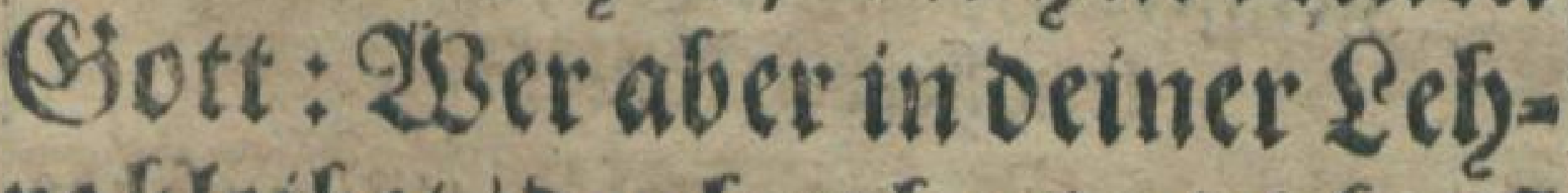
re bleifet / oer fat beyoe dich bin Deinen 2 ater.

Du bift auch Das Seben. Denn iver an bich) gldiubet/ oer rohan.s. bat 


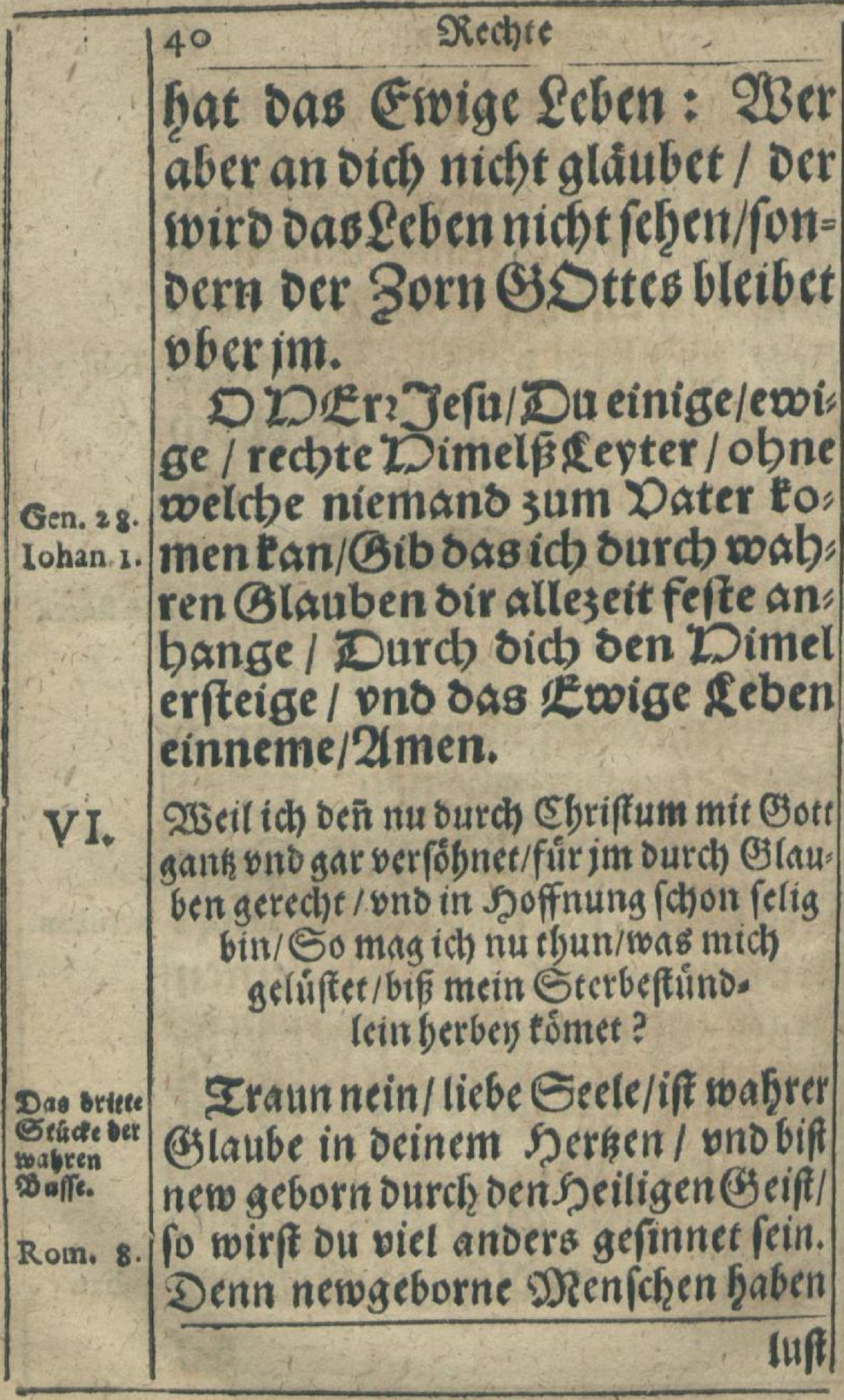


Secrbetunft. 4I

liff sum newen seben.23no ocr (slaus be hitet fich allewege fur muthwillis gen Gunden / wno ift allem Bottlofen wefen gram vno feind / 3 no ob ein gldibiger Mienfch vom తatan vbers cilet/vno aus angeborner Eshrwacheit Prov.24. einen Seiltritt thete/raffet ex fich Doch bald wider auff / berewet feinen Jall tdiglich / uno troffet fich feines S S Errn Sefu / Derfialben ift auch nichts vers Damliches an $j \mathrm{fm}$ / weil er burch ben Rom. s. @lauben feinem S de $x$ x $x$ Efrifto Jefu anfianget.

Dns oas ift nun oas oritte Etuide/zur waren Eghriftlichen Suffe gefjorenoe/Pemlich/oernewe G3ehor fam / nath Den Behen (S) eboten \&ots tes/Darinnen fich Der @laube täglich beweifet / wno in allen guten 213 erdten gegen @ott ono Denfehen Dand"bar ¿no liebreich ergeiget. Sie folt Du Dich nu táglicḩ al po vben:

Jds Dande Dir / mein Gsott / Dimlifcher Dater/ bấ ou mich 3 ubeinem 2Bilde erfcbaffen/Dno Genef. $x_{\text {. }}$ (B) 8s 


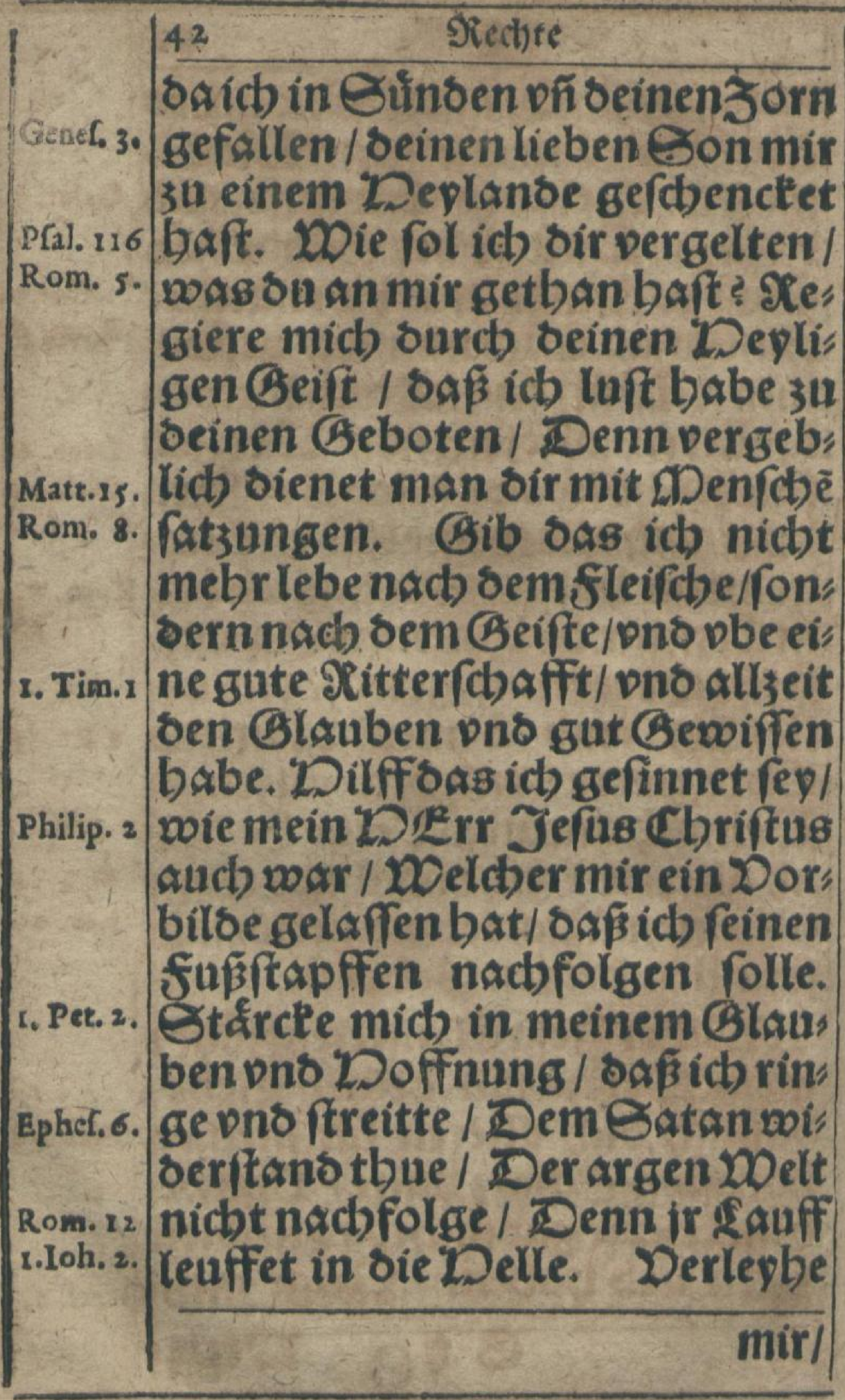




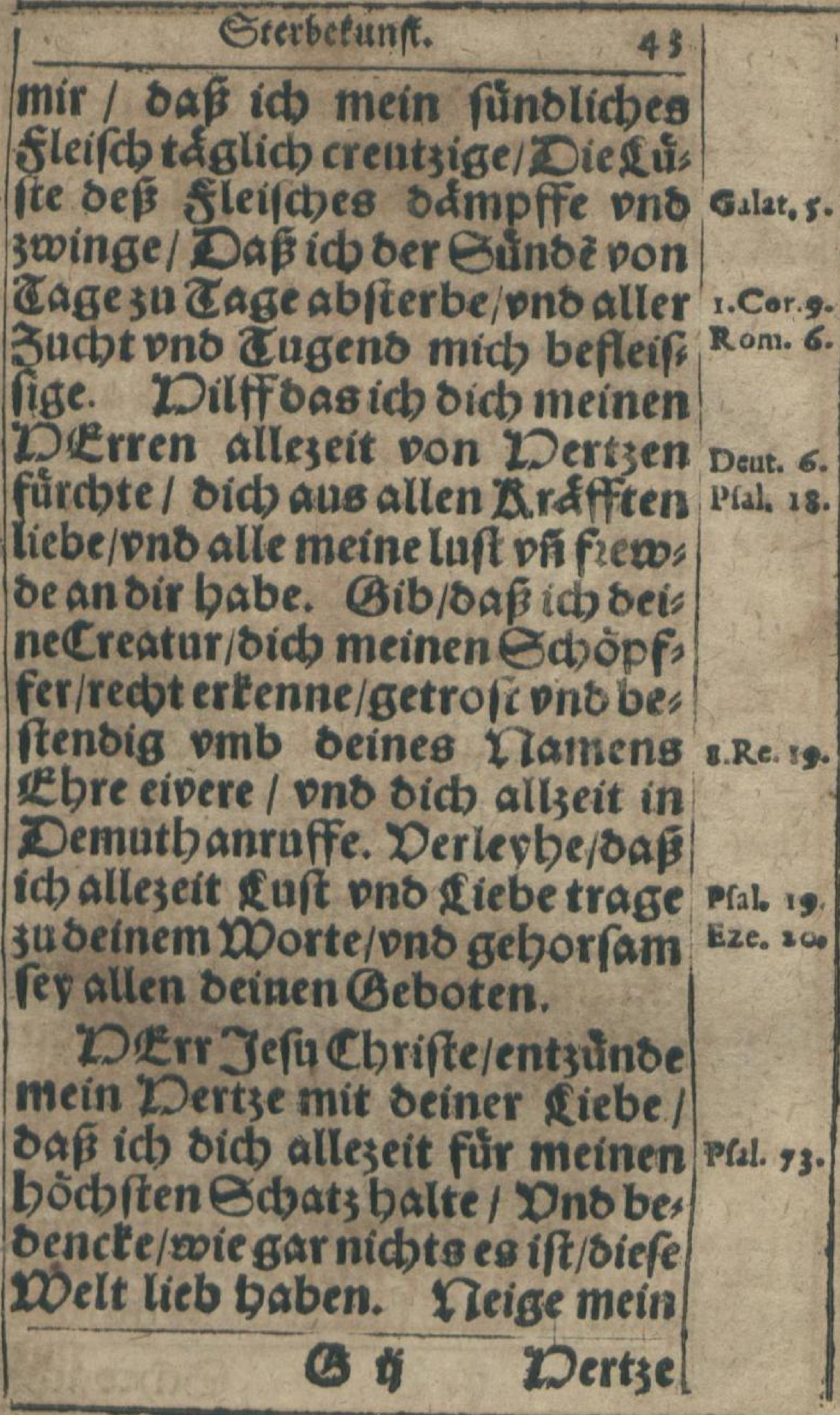


Matth.6.

44 Diechite

Dertse su sir / mein Deylandt oaji ich bie $\Theta$ chátze biefer 20 elt fir nichts adbte / Sonoern nadb oen exoigen Guitern perlangen trage. Bebuite mich für ber elen: ben 2 saudbrorge/ D no bilff/ oaf ich teinDertrawen auff oxsDer: gángliche fet3e.

Dein Deyland/ Derleybe mir wabre Geoult in allerlev Creats ons arub fall / ons beftensigen Dutb ons (rof in allen2infecb: tungen/Dilff bas ich in Dngluct

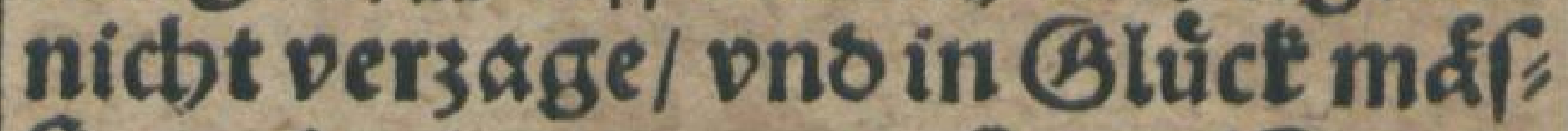
fig uno eingesogen fey. neyge mein Dert3e zur Gottreligeeit / ono gib/baf́ ich allezeit tbue ons bebencte / wagg ou wilt / Dns oir biene mit reinem / nuicbtern / ans odicbtigen / Eeurcben / recbt fabafs fenem Dertzen / pno willigem Bemútbe.

Dein DDRr/lebre mich alles 3eit Surch seinen Geift / pno lap micb nicbt fallen inn meiner

E(b) wach) 
Sterbetunft.

45

छd)wachbeit. Seite mich mit beiner Xecbten 'Dand/Dns fúbre mich/o afs ich alle mein bun ons Gebancten rid)te nadb beinem wolgefallen.

Dein Gott / bilff ods mein Dert3 nicbt Doffertig / $\mathrm{Fy} /$ LDein Gemitb nicht bon oir wancte I Daßs mein Duns nichts arges rebe / Lod beinen Ifamen mifs" braucbe: Dafi ich nuidbtern bns

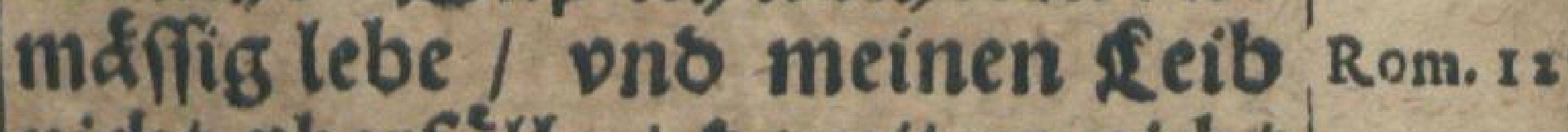
nidbt bberfútlle / bamit er nid) $t$ geyl werbe.

Derleybe mir / mein 'DERr/ oafi ich meinen riechiten nicbt fobmatbe/nicht verleumboe/nicbt aus meinẽ 3 eruff fobreyte/ nicht mit Dnrecbt nacb Teidbtbumb tradbte I Ifidbt nach eitel Ebre ftrebe/ hicht ein'Deud)ler/nidbt ein Ecbmeicbler fey : Gíb mir/ mein (Bott/ bafí id) bie (Seringen nicbt veracbte/Dem Duirfftigen gerne Dorreicbe I Dem 24rmen gers 


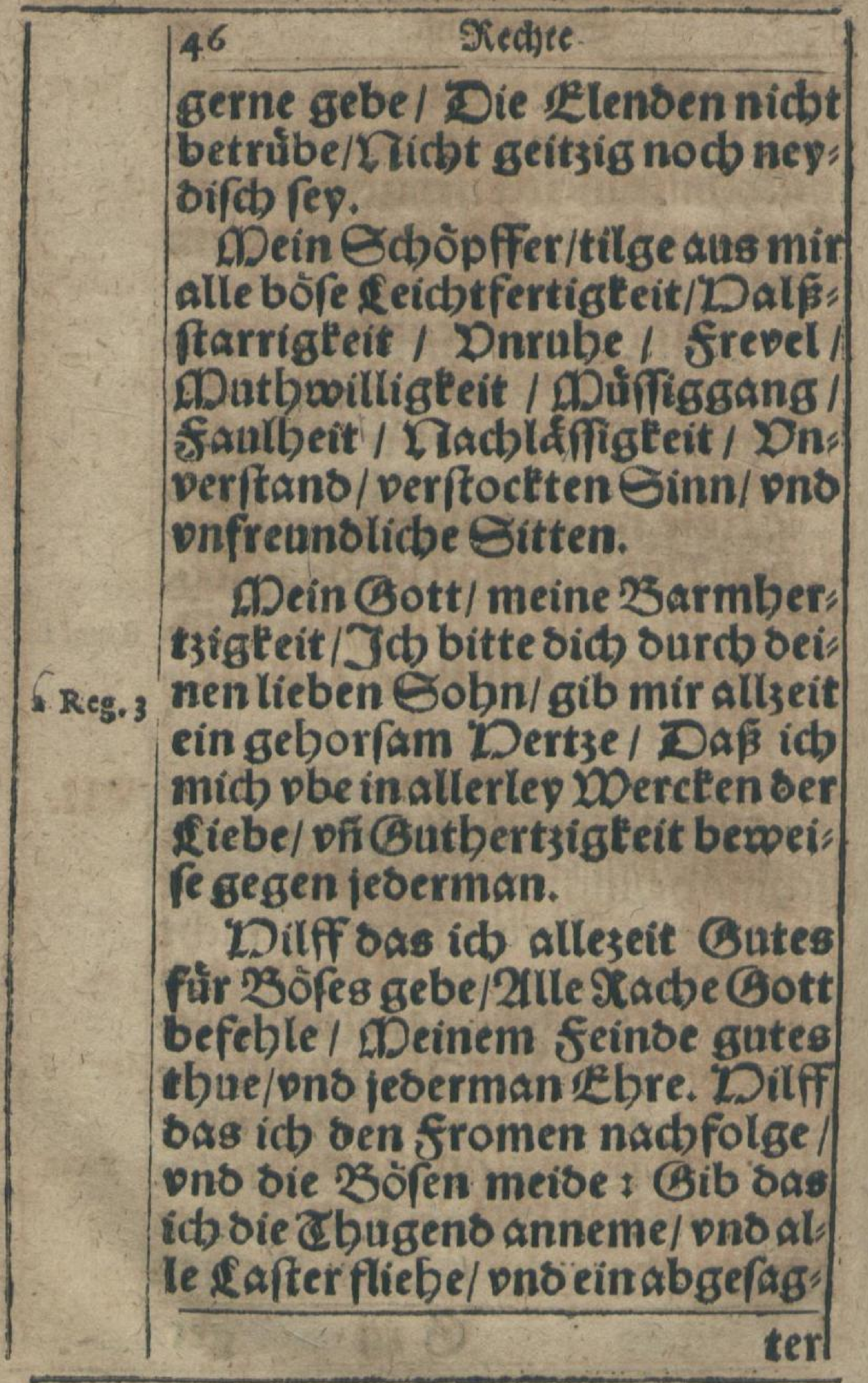


ter feinot aller Ginben pnno Echanben fterben mòge.

Erbalte mein Dertze bey sem rial. 86. Rinigen/baß̄ tb beinen namen fürcbte / Damit icb mich alfo fel, ber prúfe / ono ertennen moge/ oas icb auff rechtem Do bege wown bele/pno meinen (Blauben leud): ten laffe.

Errôfe micb enoliç von allem Dbel/2Dno gib/ bafs ich oas Jrr" bifche veracbte / vno nacb oem Dimlifben ernftlicb tracbte/ Zmen.

Ifts benn nu genug/ went citt \$Renfch al fo ein mal rectite So tife tbut/Doer

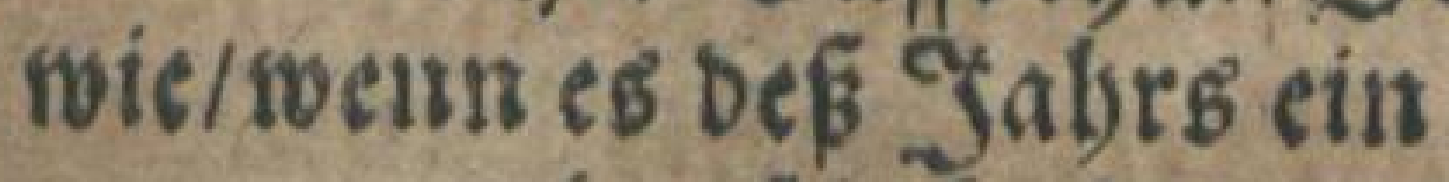
mal sefchche?

Eraun nein. Denn Egriffus ber

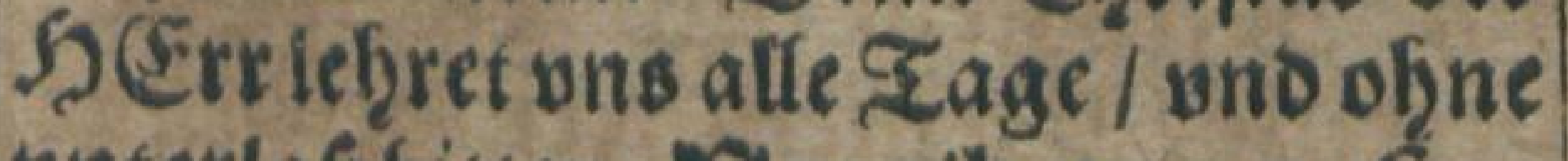
vinterlafi bitten: Dergib ons vnfere Masth.6. Ectulls. Who E. Detrus fpricht: 20 acbfet in ser Gnabe vnb SER: 2.Pet.2. Eenotnif Dnfers DDÉrren JE fii Cbrifti/ze. 


\begin{tabular}{|c|c|}
\hline \multirow{5}{*}{ Ephef. $x$. } & Steclite \\
\hline & Ta aucli S. Danlus bittet GS Stt ben \\
\hline & S) $x x x$ für bic (Epfefer / Daßfie \\
\hline & ftarct werben mtogen ourch den Spey. \\
\hline & ligen ङeift/andem inwendige \\
\hline \multirow{10}{*}{ Pfalo 32.} & fchen. 23no Davio pricht: Dafü \\
\hline & $\begin{array}{l}\text { wero } \\
\text { ten } 31\end{array}$ \\
\hline & i ou da / liebe Geele / Da B on \\
\hline & nich̨t aur ein mal / auch nicht nur bef \\
\hline & Sab̧rs ein malosufe thun / sno dar \\
\hline & $\begin{array}{l}\text { nach barauff wioer in mutfwilligen } \\
\text { Sundenleben folleft / Sondern bein }\end{array}$ \\
\hline & gankes seben fol ono muz eine ftet \\
\hline & $\begin{array}{l}\text { nerende } 23 \text { ufe fein. Denn fols fein } \\
\text { ein Éfriftlich Seben / fo muß es auch }\end{array}$ \\
\hline & 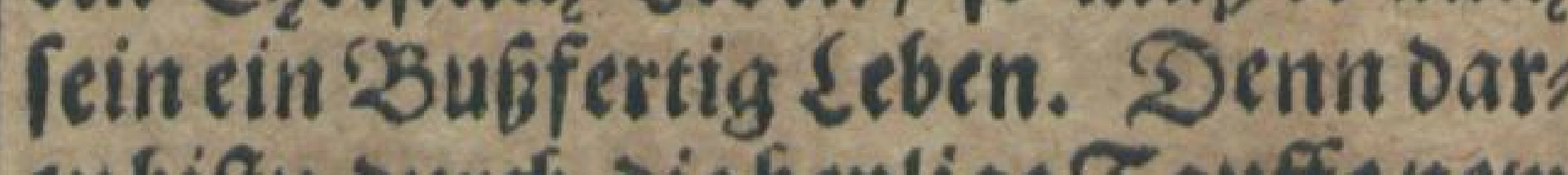 \\
\hline & $\begin{array}{l}\text { Jubifu durch oie benlige gallte new } \\
\text { aeboren? vno cin Rind Gottes wors }\end{array}$ \\
\hline \multirow[t]{7}{*}{ Rom. 6.} & ben / Daf oer alte $\mathcal{A}$ Dam in oir ourch \\
\hline & tågliche jiewe vn's Duffe fol erfeuffet \\
\hline & werben / no fterben mit allen Stins \\
\hline & $\begin{array}{l}\text { Den yno bofen suften/23nb widerumb } \\
\text { tảglich ferfür gef?en/vno aufferftehen }\end{array}$ \\
\hline & Dentch / Der in Sedigletit \\
\hline & \\
\hline & lebe.] \\
\hline
\end{tabular}


Sebe. 3 Sterbeturter. $4 \%$ mon : Der Gerechten Pfáb glén: Pròr. 4. tzet wie ein Riecht 1 baß $\delta$ a fort gebet / pno leuchtet bis auffoen Dollen a ag.

S SEtt vito Bater aller Gnaoen vns Barmbert3igteit / ber ou mich in Cbrifto Jefu bes s. Pei.s. ruffen baft zu beiner ewigẽ berr: ligkeit / Dno verlieben / $\partial \alpha \beta$ ic ourd) rechten wabren (Glauben/ die Euiffigteit beiner Bnaben pî

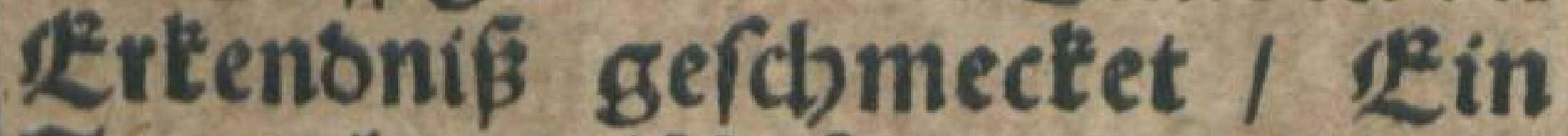
Tempel vno 20 obnunge seines Devligen Geiftes/ vno ein UÉrbe Rom.8. beß Dimelreichs worben bin I Regiere mich allezeit burdb ben, felben seinen Devligen Beift / o\&ß id in woabrer 23 uffe alleseit fort fabre/ woiber alle Eunbe ob: ne vnterlaß ftreite/in beinemEer, renoní̄ zuneme / pnno als cin newogeborner LDenfd in aller Gottfeligteit woad) fe / vno je lán: ger fe ftárcter im Blauben woer: (5) be. 


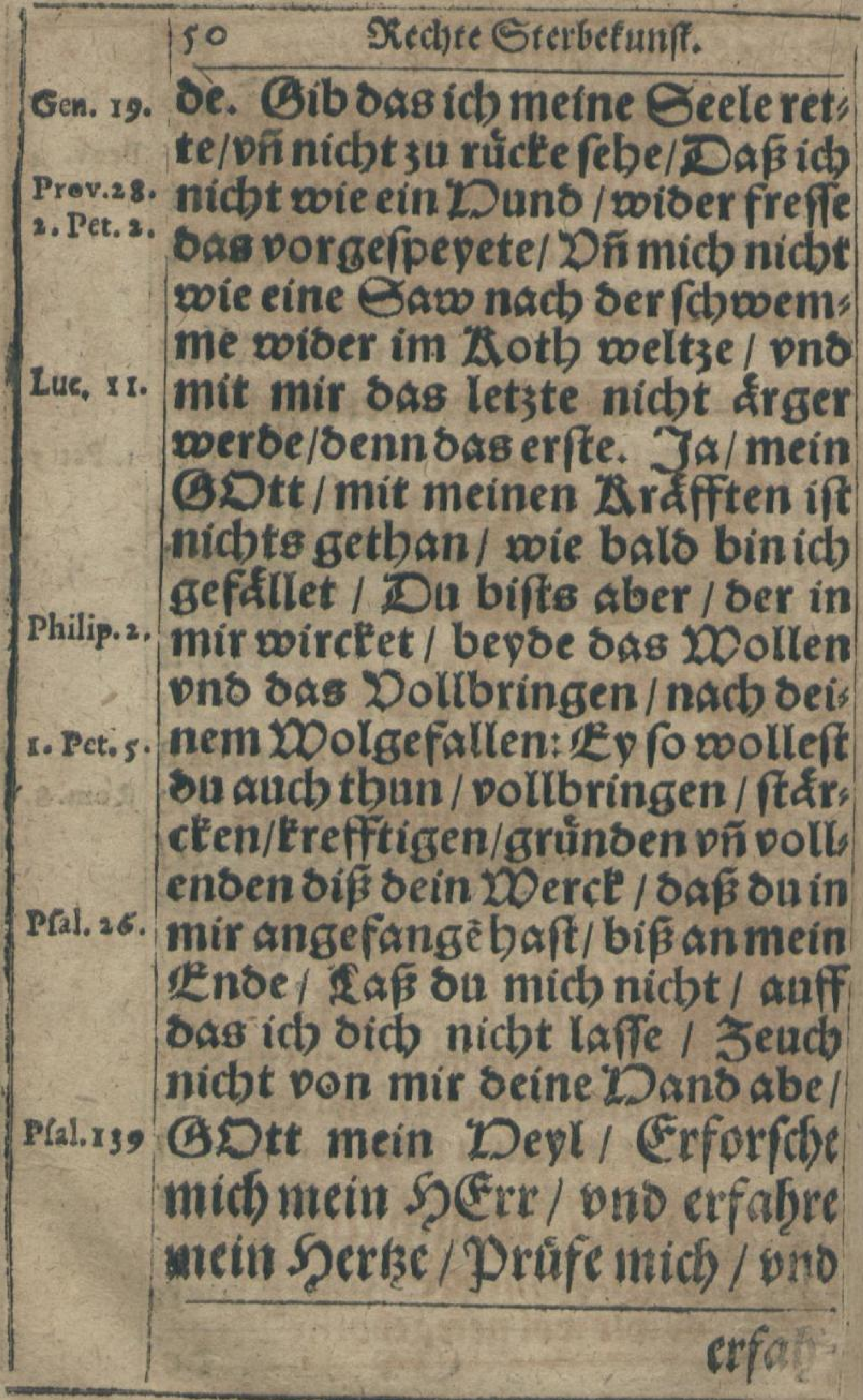


Sechs Stuicfe cinen Ebrift. \{ebens. II erfabre/soie id s meine/onto fis be/ ob ich auff bofem 2Bege bin/ vno lente mich allgeit auffersigem recf)ten $23 \mathrm{egc} / 2 \mathrm{Lmen}$.

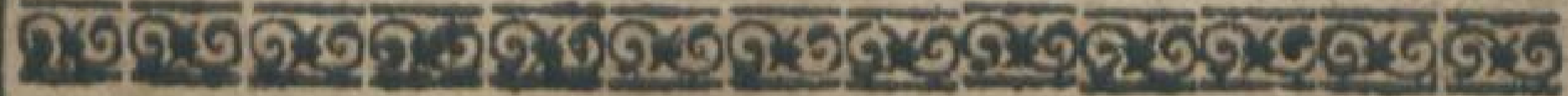

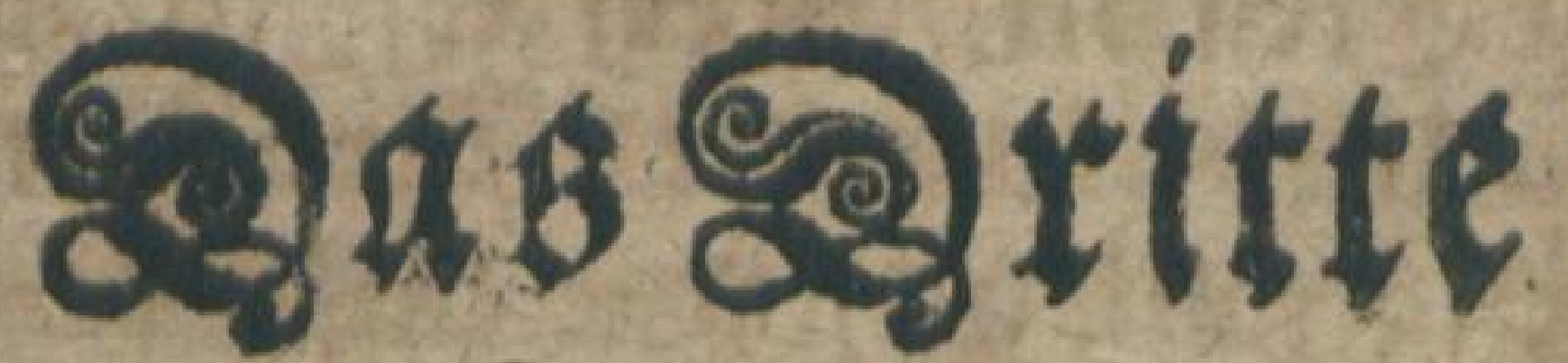

\section{Eapitel.}

DBegreiffet fechs borneme Sturte/ Die zut einem Shriftli-

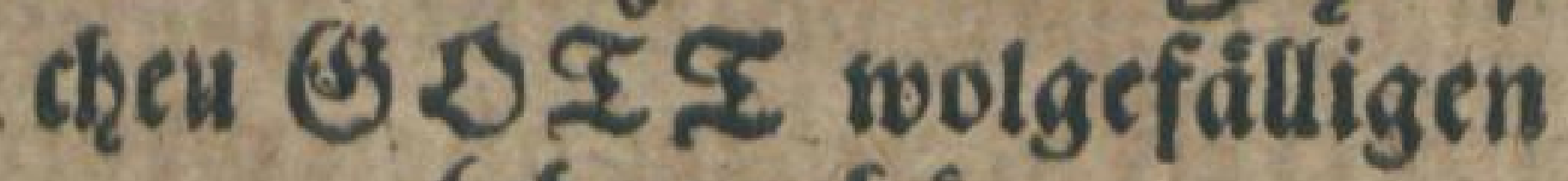
seben gefioren.

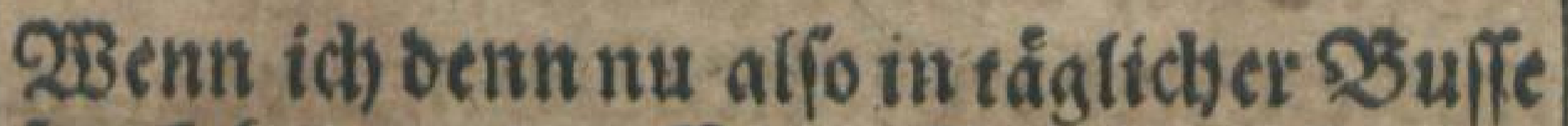
fort fabre I und auff bem fotbmalen Steige viel zbioerwertigfeit baben mochte / Sbie [ol tich mich) benn verbalten/Damit mein

Blaube nicht aufffodre 1 ond ich nicht jere geben móge?

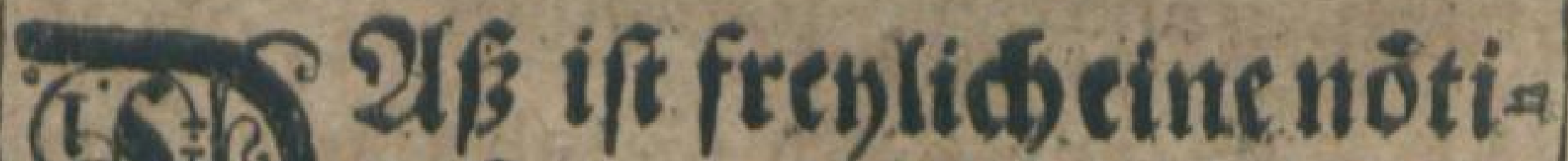
2) ge Sragelliebe Eeele. Denn ob wir wol newgeborne Dien 


\begin{tabular}{|c|c|}
\hline & Sechs Stridectincs \\
\hline & [chen/ vnd Dureh o \\
\hline & $\begin{array}{l}\text { Eottes worden find / Eotrage } \\
\text { Doch folche edle Eclodise in irroil }\end{array}$ \\
\hline & (B) fálfen/ uno fchrwachen Seiben/ bno \\
\hline & ift vns dexieluffel gram/vno verfuchet \\
\hline \multirow{6}{*}{ t.Ioh.2. } & शुelt mit jrem fęornen sauff gibt viel \\
\hline & 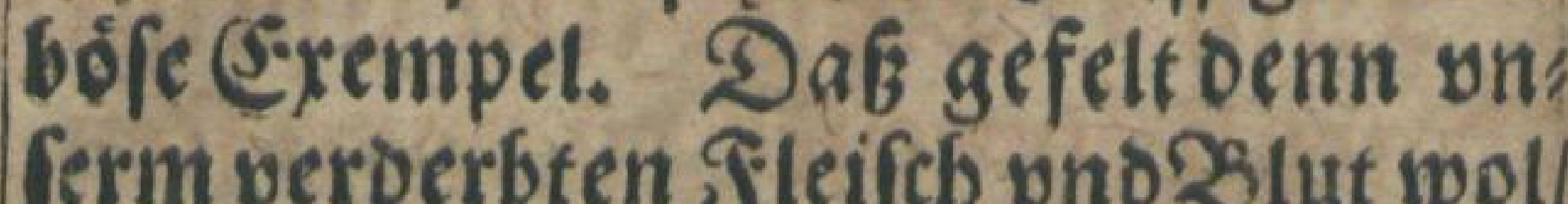 \\
\hline & Sprin \\
\hline & DaÉ polches fey oic brente Dabn / Die \\
\hline & in abgrumb oer sellen führet. Dermes \\
\hline & $\begin{array}{l}\text { gen/liebe Seele/ wilt Du beinen sauff } \\
\text { Sgriftich bollenden / vno Das End }\end{array}$ \\
\hline \multirow[t]{7}{*}{ 8. Pe.t 8. } & DeB @laubens / 9 emich / der Ecelen \\
\hline & e táglich nor 2lls \\
\hline & Daß Du Di \\
\hline & Diefi \\
\hline & 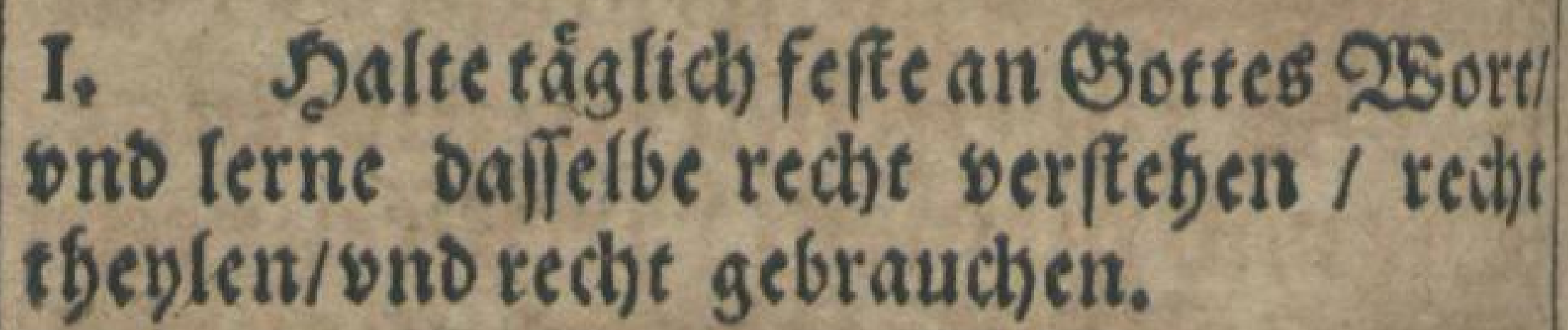 \\
\hline & $\begin{array}{l}\text { IT. Erinnete dich triglich Deiner Şeylio } \\
\text { gen Znuffe. }\end{array}$ \\
\hline & 11. Salt \\
\hline
\end{tabular}




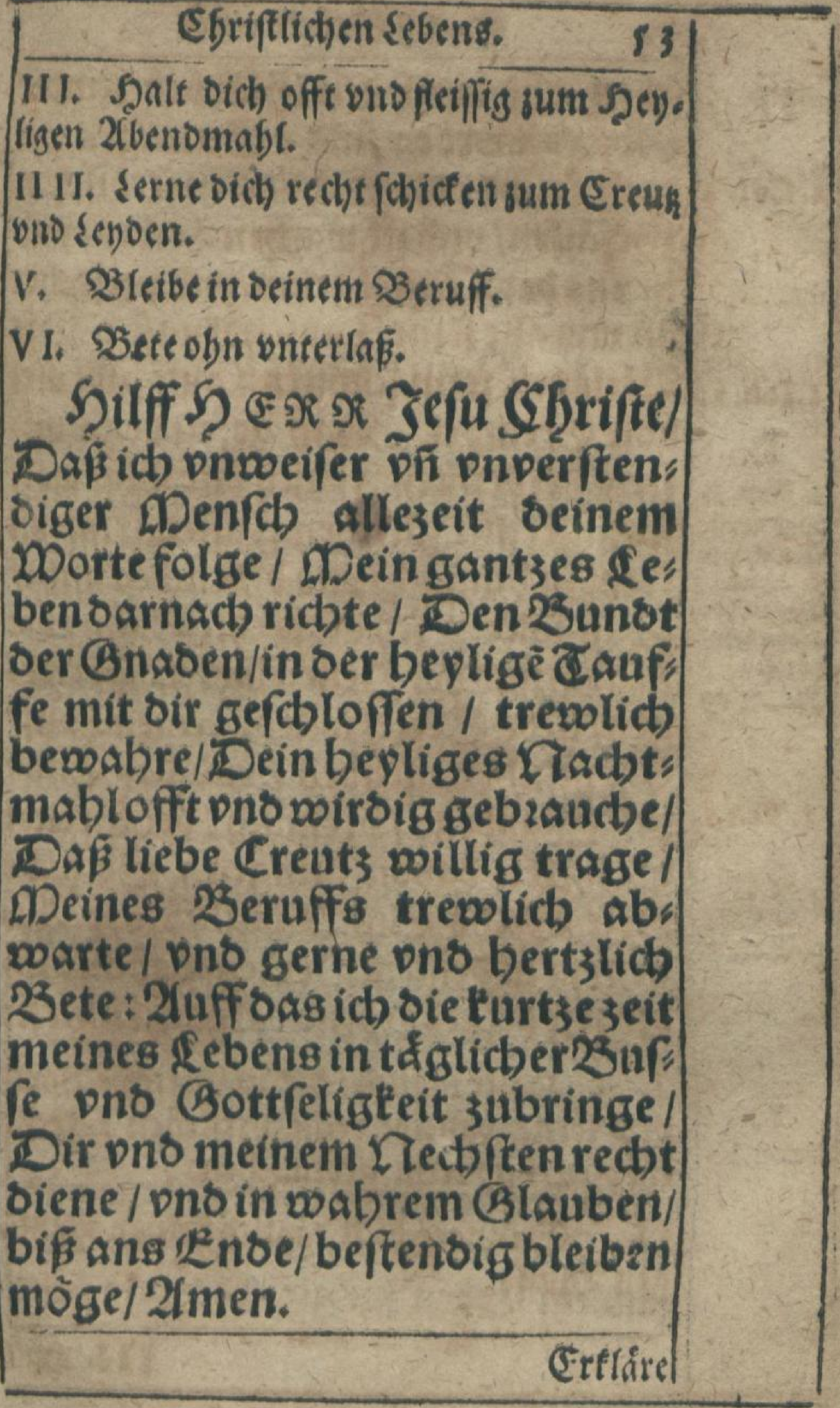




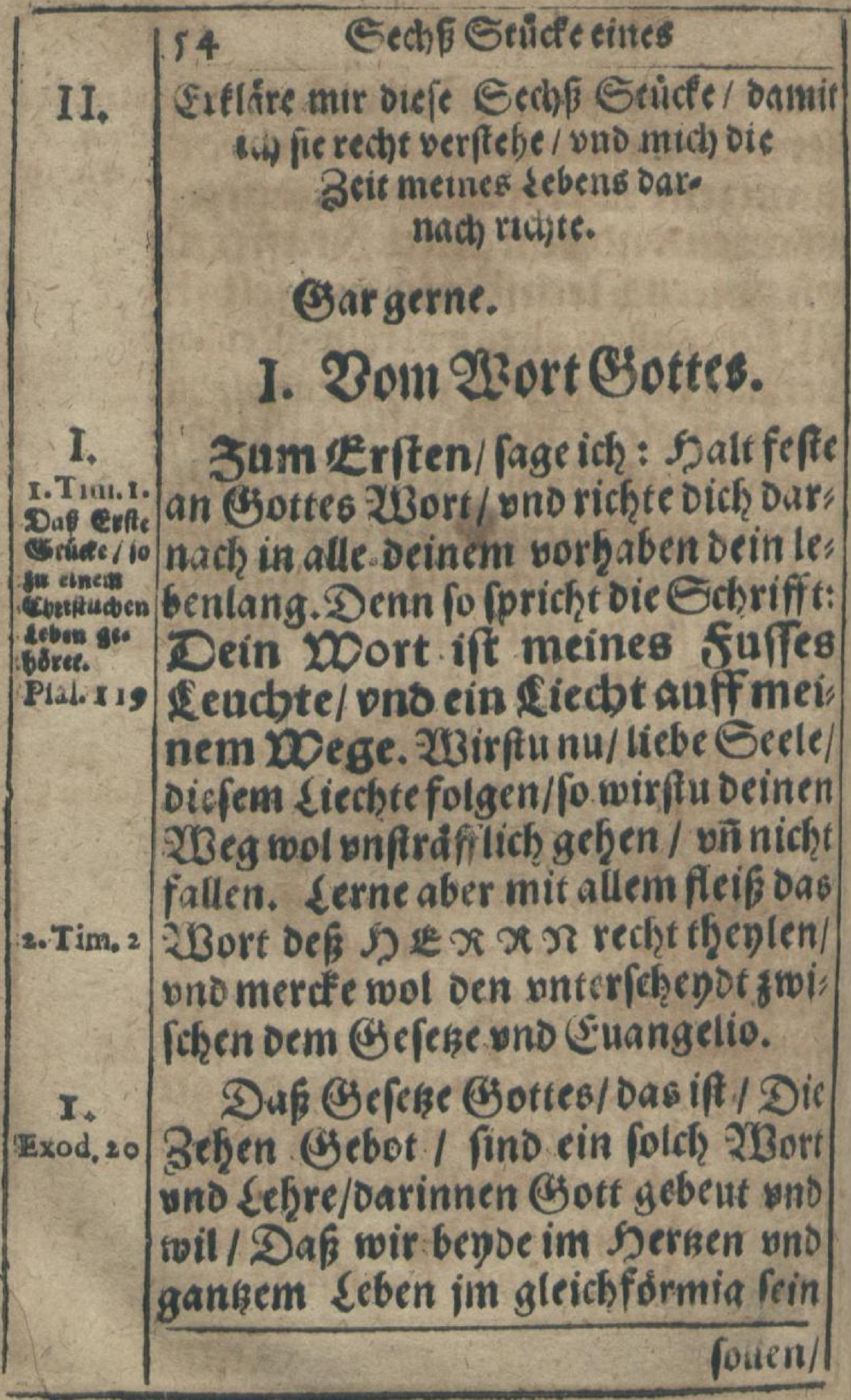




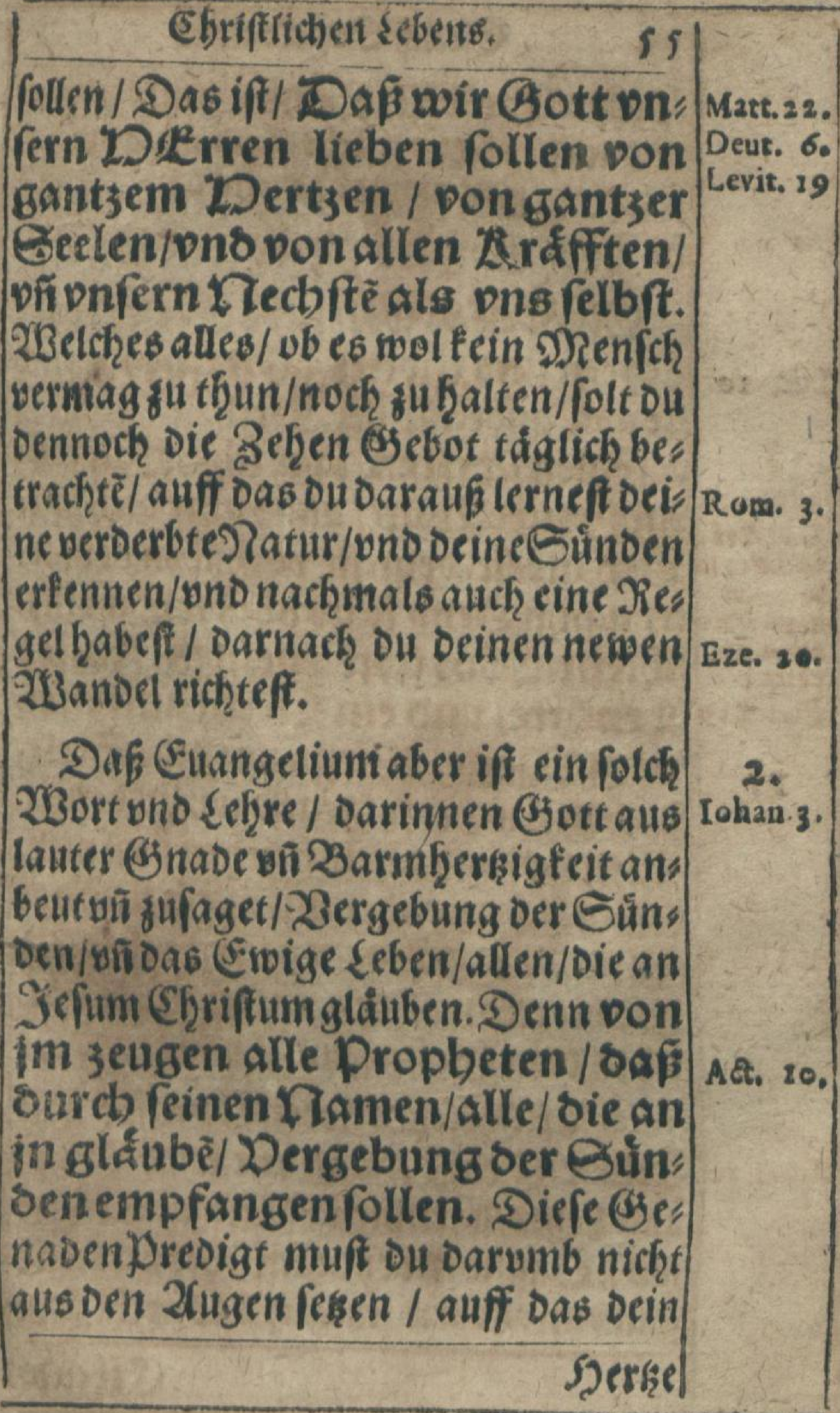




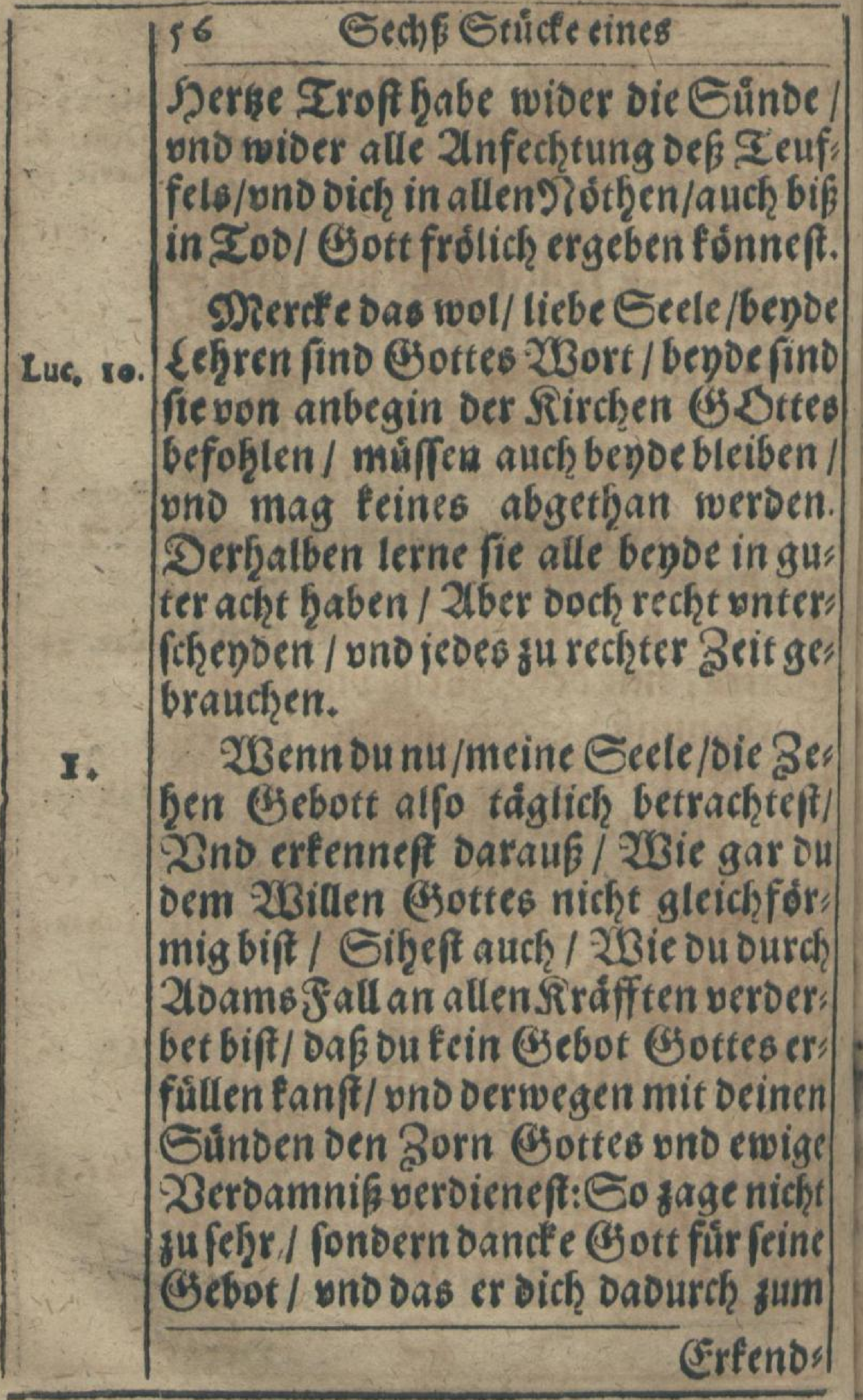




\begin{tabular}{|c|c|}
\hline Ebrifflicten sebens. & \\
\hline 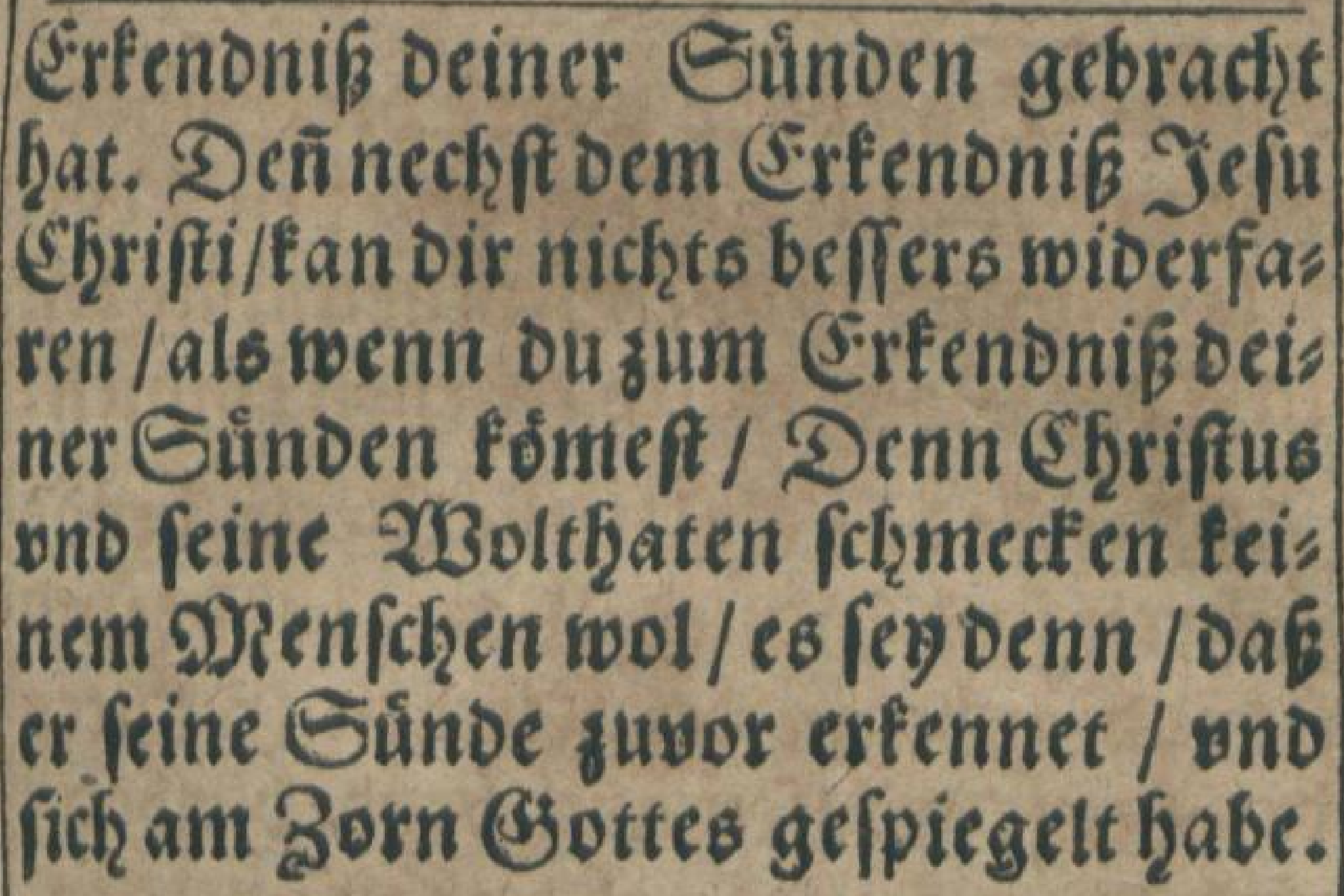 & \\
\hline 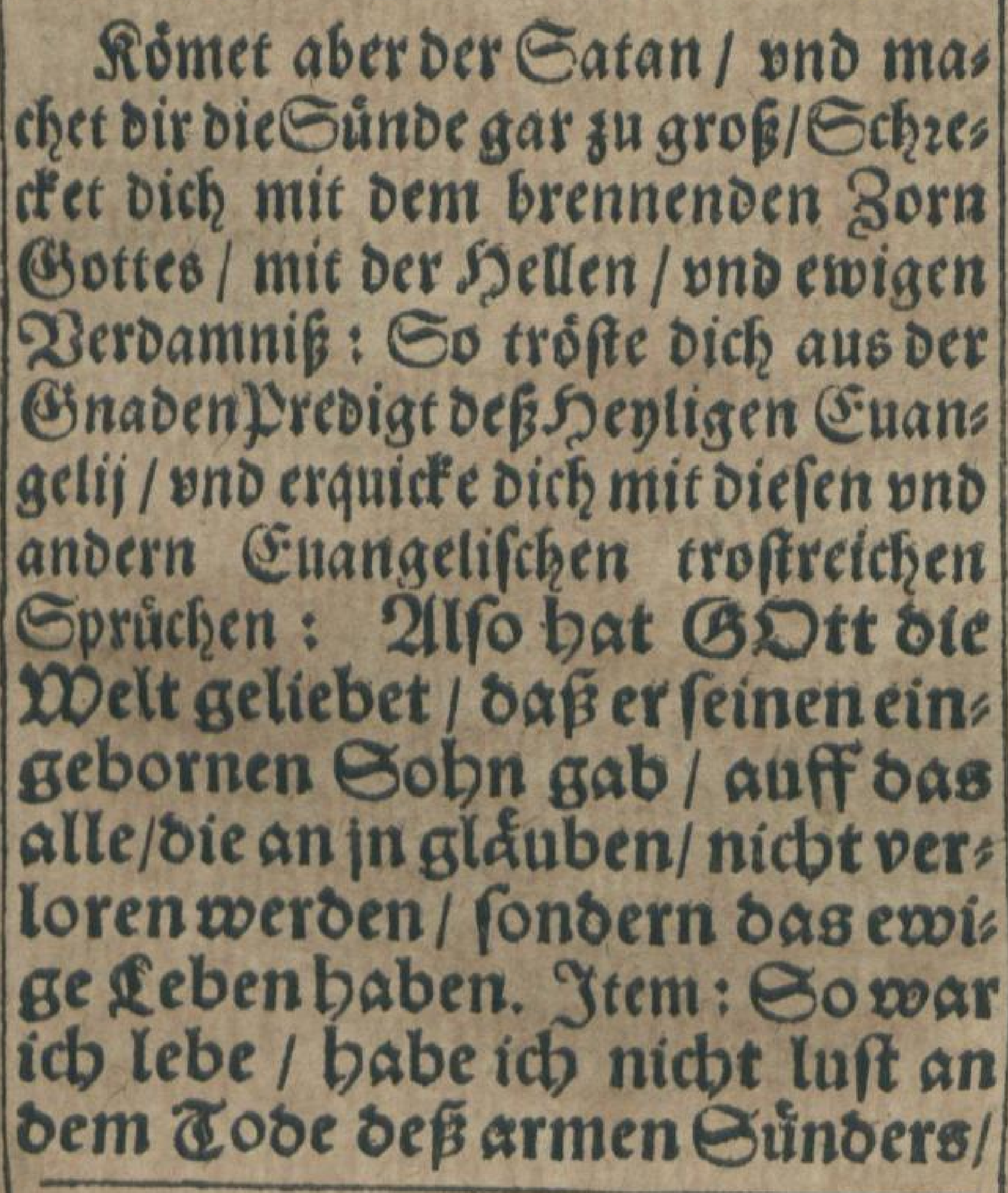 & tohano 3 \\
\hline & \\
\hline
\end{tabular}


fonsern sas er fícb betere / ono

lebe. Ytem : Job woil fie erlören ausber Dellen / ons bom Tose erretten. Ytem: 2xo sie Sừnbe maichtig wooroen ift/ ox ift sod) bie Bnabe viel mádbtiger wor" oen. Gtem: Datifif je gewifflich poar / ons ein tbewreg werbes Dort / Dấ Jefus Cbriftus in die WDelt Eomen ift / bie Sunser felig su macben. 2ulo wiberftebe Luc, 21. fefter/Denn Simel vno Eroen. Denn Ioh. 17. rein 200rt ift bie 200 arbeit.

3. Simet aber ber Gatan auff ber andern Geiten/verftellet fich in cinen 2:Corin. Sngel dek Siechtes / Dachet Dir bie 11. Sundeflein/ ond den Sorn (3) Sttes gering / Raumet oir cin / Stinde fen) leicht gebúffet / fen boch Gs nade genug verhanden: Go hitute dich / liebe Gee: le / gefe jm nicht an. 23no gebende

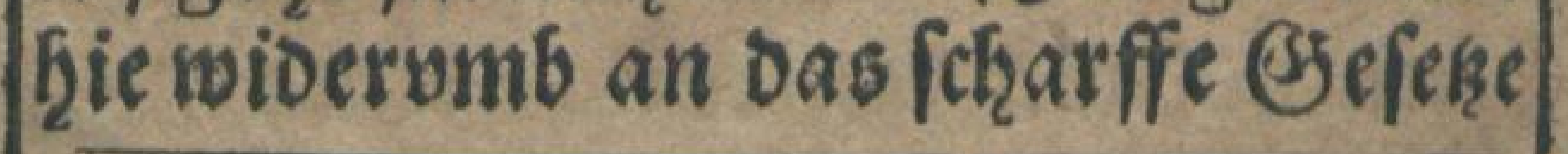

Crot: 
Ebrifteticlien sebens.

USUStes / Darinnen alle Gúnoen fo fchredt'lich angetlaget werben / Dars vmb fönnen fie nicht gering noch flein fein. Gebente Dargu an bie 2 Ingft Deines lieben ŞErren Jefu Şrifil ono fprich in oeinem Serken: Dfuy dich Eatan/ou signer / Sch febe mie fchwer uno faner es meinem Ş $x$ : rex $n$ Yefu worden ift / Der ein mal vnter DemgItuch Def@Sefeses/für meis ne Sunde gebuffet/ond feines 2 aters Sorn für mich getragen f̧at: Darvmb wil ich alten wno jeden Sunden gram fein mein Lebenlang/ vno mish Dafür butten / als fürm Zeuffel felbeft. Sch Dandfe meinem Sott / Daß̧er mich ein

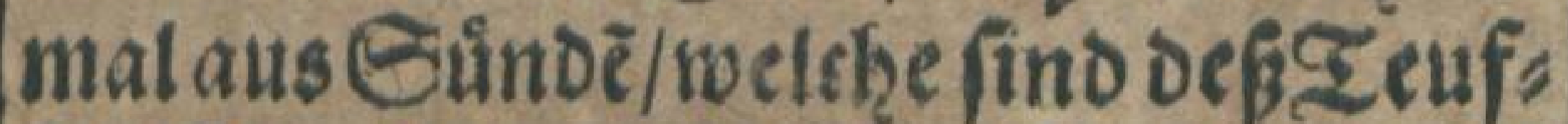
fels Sande/ erlofet hat / Eolt ich mich denn wider alufio newe in feine Stris d'e flechten: Die Etricte fins ent: 3wey/Dno ich bin frey/oep DKr" Plal. 124 ren Llame ftebe mir bey/bepbot: tes Dimels ons ber $t^{2}$ roen.

3eiget bir aber ber Gatan oen Fehoinen Sauff oer 2Belt / uno reiget 万ु $i j$ Dich 


\begin{tabular}{|c|c|}
\hline & Sedh Sticte eines \\
\hline om. 12 & 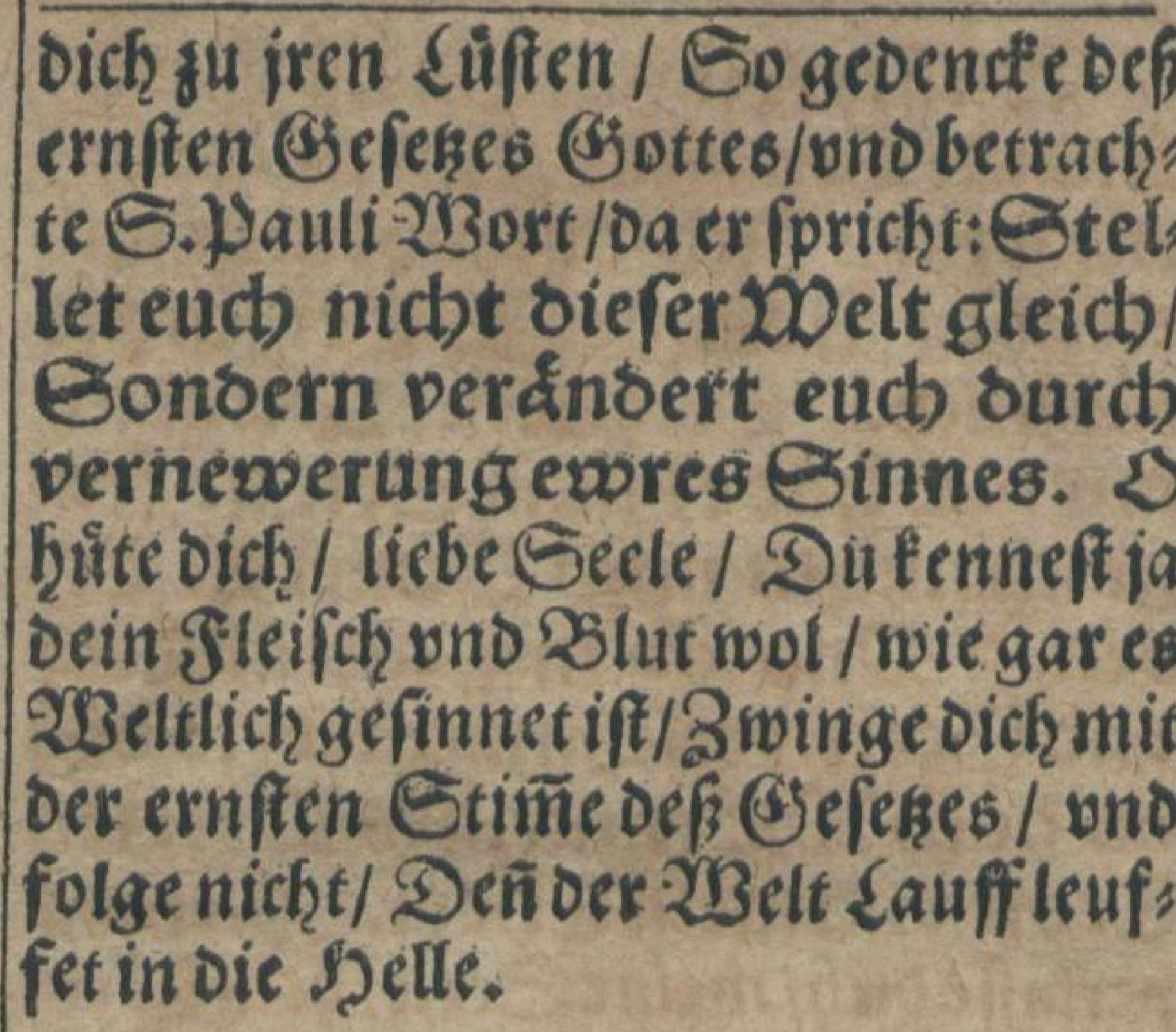 \\
\hline 5. & 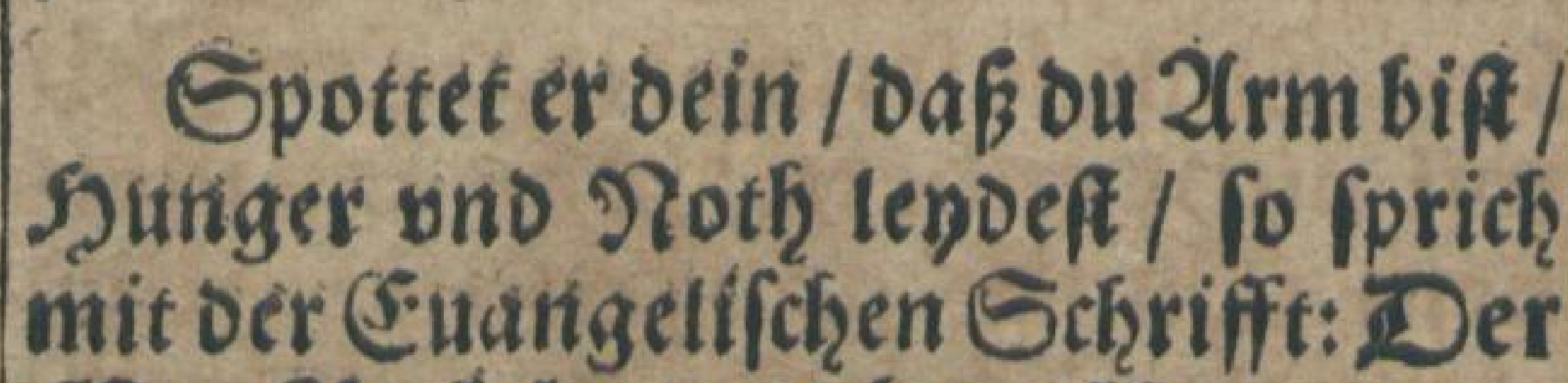 \\
\hline tth.4. & 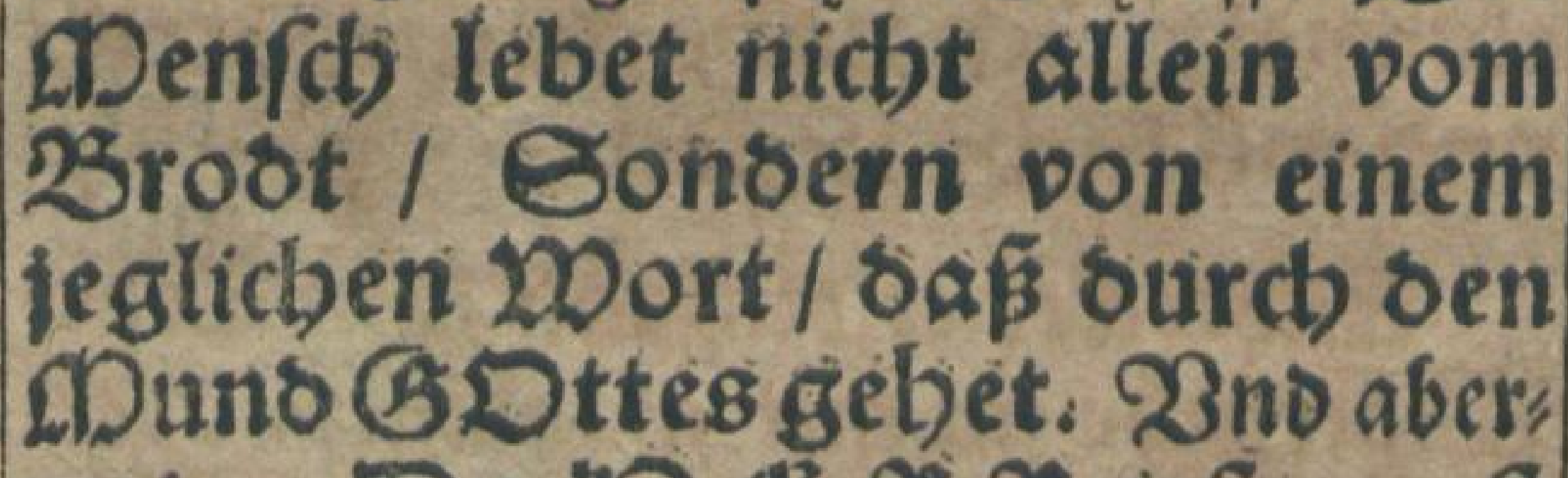 \\
\hline 1 & 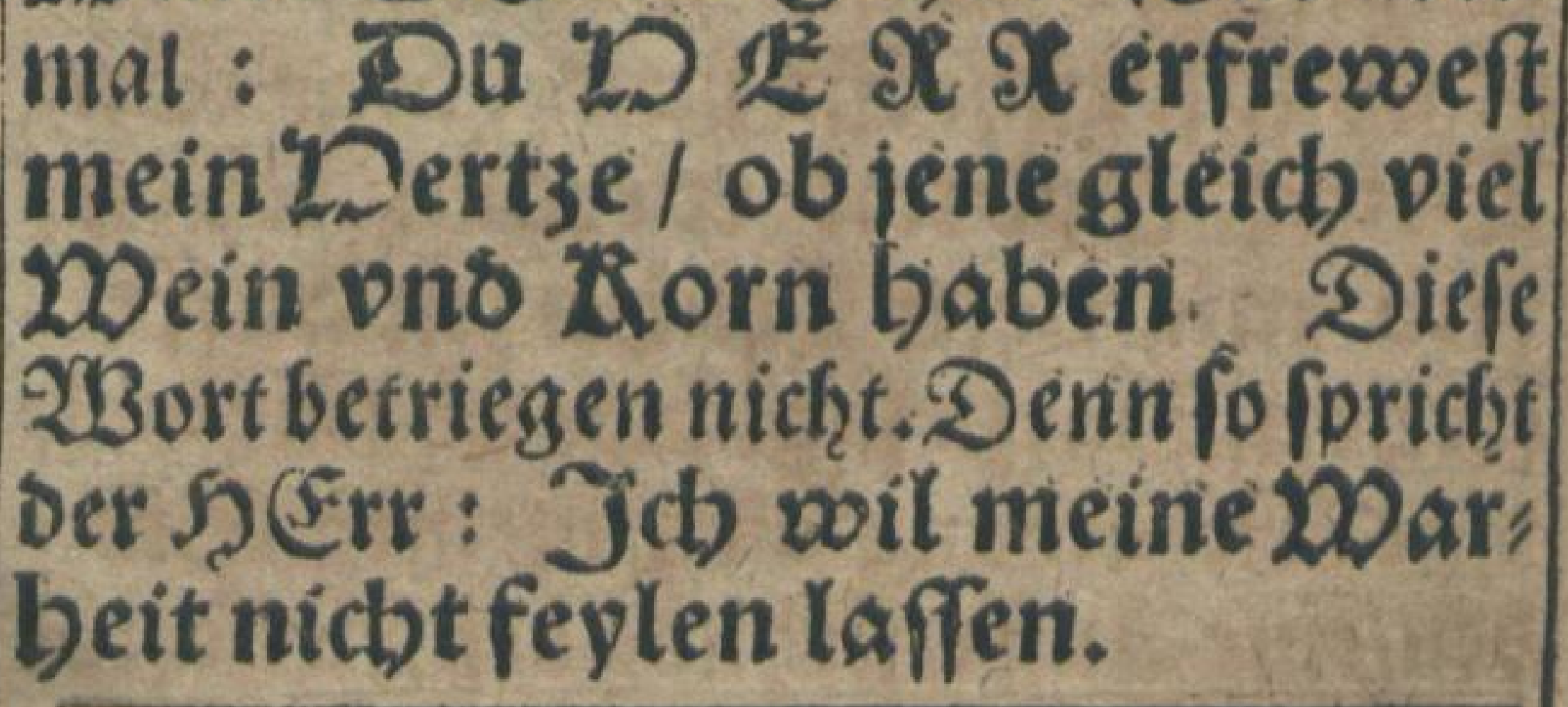 \\
\hline & \\
\hline
\end{tabular}


Ebriftichen sebents. 61

WBiffit er bir vor/ Dubift Elenoel Rrandk/23erlafien: WBioerftehe im/ vno prich : Jach bin vool arm ond Pfal. 10. elenot / 21ber ber 'DSErr forget fiir mich. Derḩalben / Denn idb $\mathrm{P}_{21}, 73$. nur bich babe / mein ङsott / fo fra: ge ich nichts nad) Dimel pí Érs ben. Dno ob mir gleich Reib uns Seele verfamadtet / fo bift ou boch DER meines 'Dertsen aroft ons mein สbeyl. Shierauff verlaffe dich/meine Geele/Dein leben: lang. Denn fo pricht ber S Srr: Jdb wil nicht ensern / wass aus meis płal. 89. nem Dunbe gangen ift. 20no abermal: Jcb babe gefdyworen / ons wil nicht liegen.

Gchrectet en bith mit bem Fobel rBioerftefe im mit glaubigem Sers Ben/wno pprich: Eo fpricht dee f) Err: Deine zooten werben tében/ I/2. 26 . pns mit bem feicbnam auffer: fteben. Dargu fo gldube ich eine gnds Dige 2 ergebung aller meiner Sun: pen/eine 2lufferftefyung oef Sleifenges/

$$
\text { S) } \text { ii und }
$$




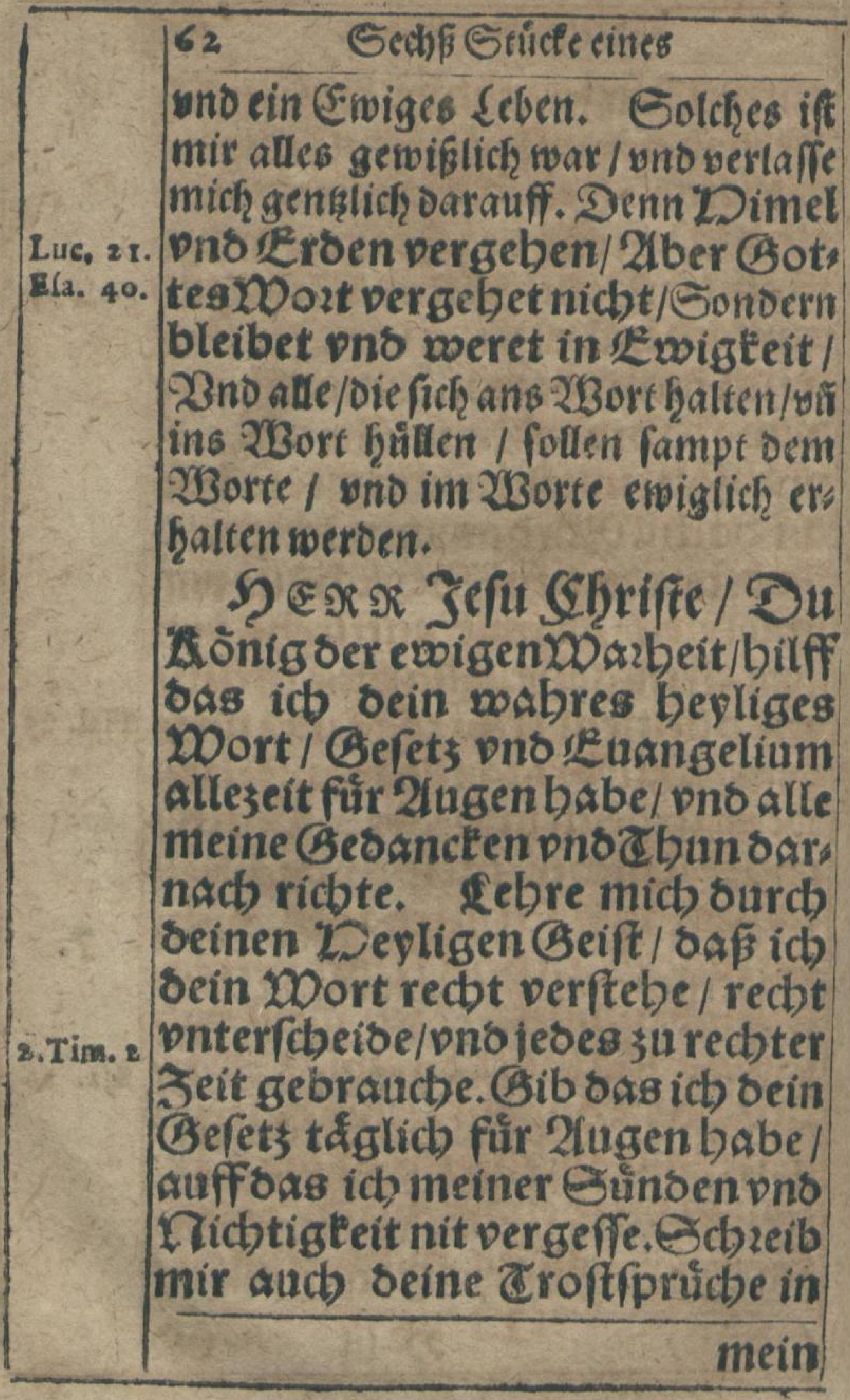


1) Ebriftlitiben sebent. 63

mein Dert3e / ons erinnere mich oerfelben in all meinem 2Inligen/ oas fie allezeit fein meine DDebre pno 200affen/ $30 \mathrm{fb}$ lagen meine feinde/Dein Sichirm bfi Edbils prov.3०. wiber benđenffel/mein Etecten P\{al. 23. vno Stab / Saran id) micb balte ons ftewore / Deine Epeife ons eabrall in allen 2 ngften ons Tötben. (Gib oag id mich gants vno gar in bein 20ort butlle pns wickele / Damit auffitebe ons fchlaffen gebe. Ja sas icblebe nacb beinem 20 ort/Eterbe nacb scinem Wort 1 Wiser aufferftes benacb beinem 20 ort. Dns ob mein Dertse in Erbwadbeit wolte 3 agē / fo bilffoas ich nicht Derzage/ Defídein 2 Dort betreus: get micb tricht. Job weißs ons gláube 15 Gott Su ewige Dors beit / oafs oubein Wort belteft / Darvinb werbe ids ta aucb er: balten werben. Zुb weís vns slaknbe / dafi ou nidbt leugeft /

$$
\text { Do iif Dars }
$$




\begin{tabular}{|c|c|}
\hline & Sect) B Stuicte tintes \\
\hline 1.Ioh. 3 & 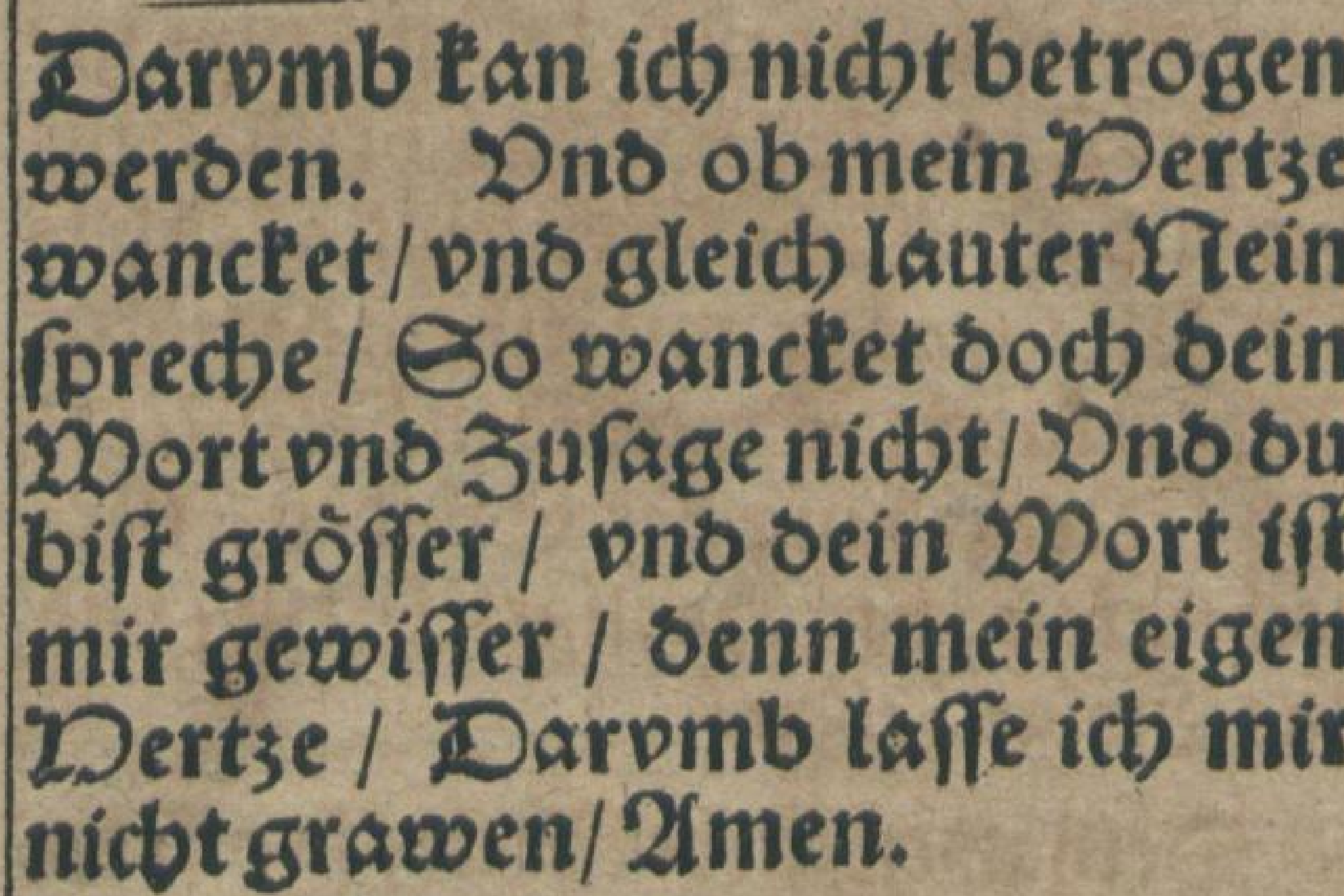 \\
\hline & IT. 23onber Sauffe. \\
\hline IT. & Şumanbern/lic \\
\hline & 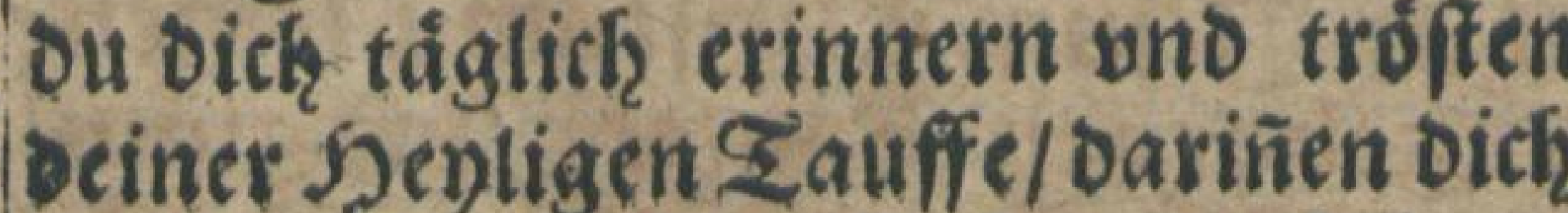 \\
\hline $\begin{array}{l}\text { Uddenteber } \\
\text { sebspet. }\end{array}$ & 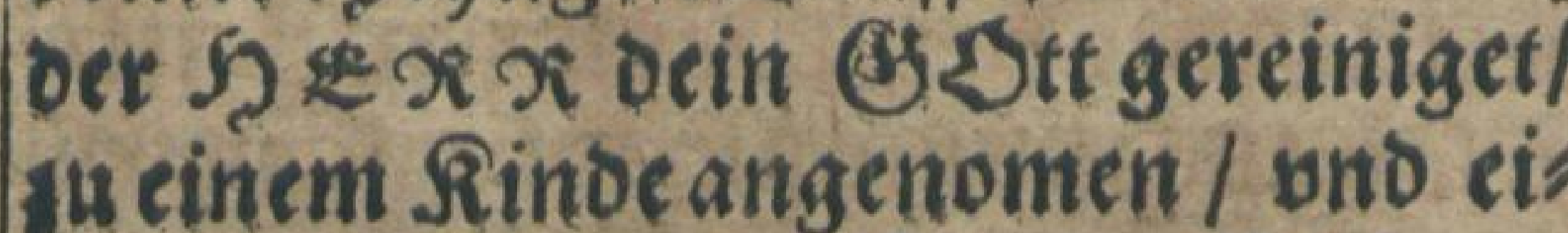 \\
\hline I, Pet, 3 & nen ewigen @snaben2sund mit dir ges \\
\hline & 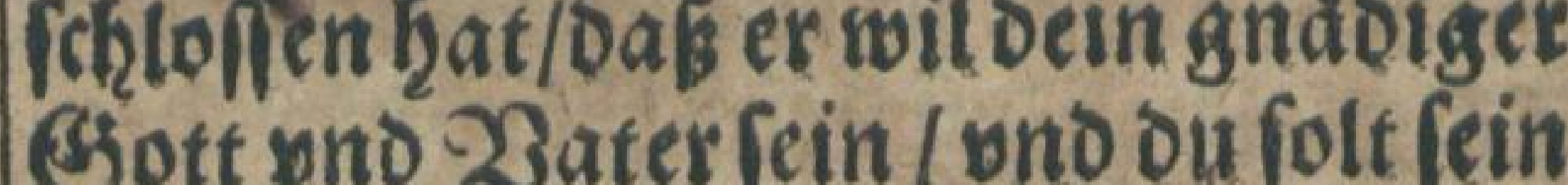 \\
\hline & $\begin{array}{l}\text { liebes Sind und Srbe fein. Ja/mei } \\
\text { ne Seele/ in ber Şenligen Iauffe hat }\end{array}$ \\
\hline & Iffen bey \\
\hline ES & Detinem) \\
\hline & ben / ono bich nach feinem ?amen eis \\
\hline & nen \\
\hline
\end{tabular}




\section{Ebriftitchen \{ebens. \\ 65 \\ nen Egriften nennen laffen / sum ges wiffen Beugnif / Daf ou Ëfriftizoru}

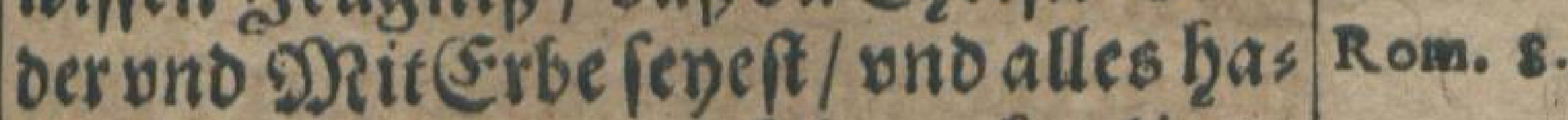
ben folt / was er mit feinem fienligen Blut erfauff oñ erworben frat $/ 2 \mathrm{em}$ lich / Dergebung oer Einden / Bie" rechitigleit / Den Serdligen Geift / ond Das swoige Seben.

Derb̧alben/liebe Seele / fo offit ou tdiglich in beinem @ebete Deine Glaw: bens 2lrtict el prich ff: Ich glaube an Gott ben Dater/2c. Jab glku: be an Jefum Cbriftum / reinen eingebornen $\Xi$ on/2c. J̧) glaku: be an Gott ben 'Deyligen Geift/

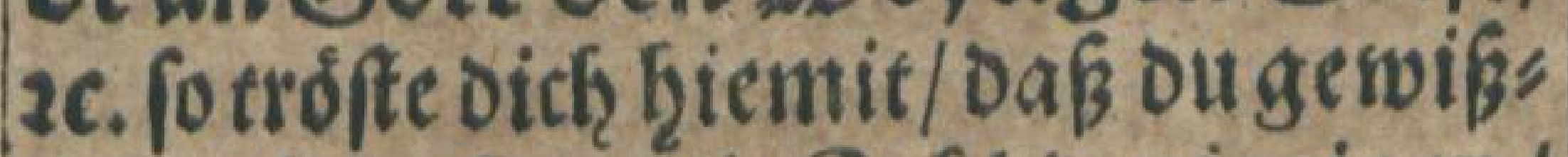

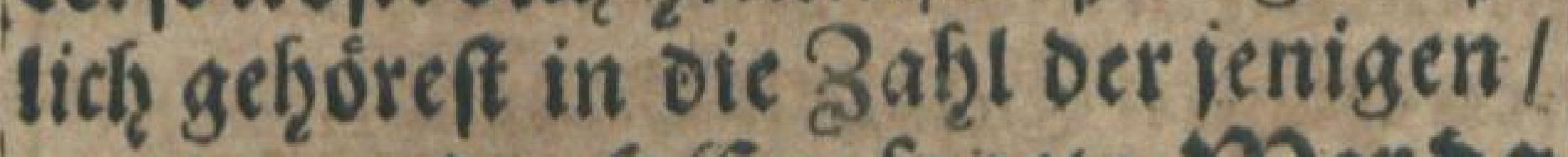

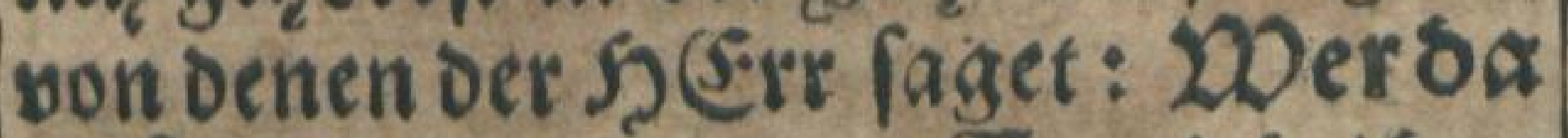
glánbet / ono getsufft wiro / oer wirb felig. Dagegen aber fonderft

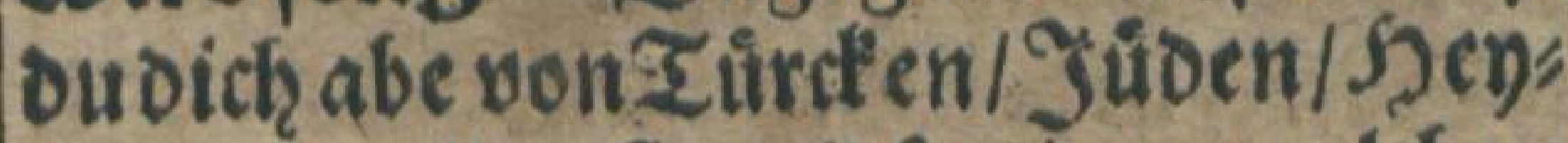
ben/snd allen Gottlofen/won welchen Der Sh Sirr faget: Woer nicht glew: bet/oer wir's veroampt. Ya foleh bein Betenoniberinnert dich/oaß ou S马 $y$ wegen

Marc. 16 


\begin{tabular}{|c|c|}
\hline \multirow{5}{*}{ Rom. 8.} & Sed) Strictectites \\
\hline & wegen ber Zauffe vnd beffelben 3 un \\
\hline & Des verpflichtet bift/nichet nach ocinem \\
\hline & fleifoblichen 2 Bitlen vno saften sule \\
\hline & ben / Gondern gu dienen dem lebendi \\
\hline \multirow[t]{8}{*}{ Iucx 1.} & gen @ott / in Seyligfeit vno Berech \\
\hline & tigfeit \\
\hline & Têีu \\
\hline & ausfprecblicbe sooltbat / Daf \\
\hline & Su mich nicbtallein lebenoigaul \\
\hline & eibe gezogen / Gonber \\
\hline & auch oxichin Sur \\
\hline & Homenlap \\
\hline \multirow[t]{6}{*}{ s. Pet. 3.} & benbuns gefchloffen/Durch sei \\
\hline & t new geboren/2us sef \\
\hline & (ceuffels Xacben geriffen/Dno 36 \\
\hline & 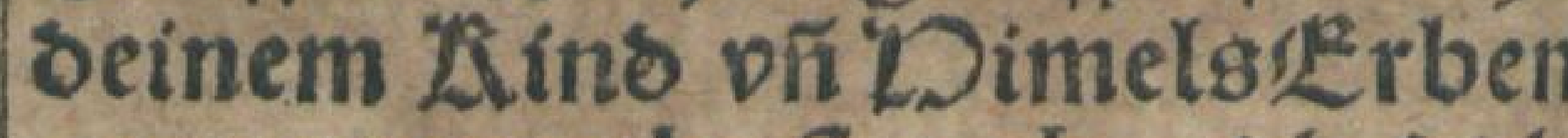 \\
\hline & angenomen baft I ebe ich sich \\
\hline & Eanote. Dein Deplano/ DDie fo \\
\hline \multirow{3}{*}{ Pfal. 126} & icb oir pergelten / was su an mir \\
\hline & getbai \\
\hline & 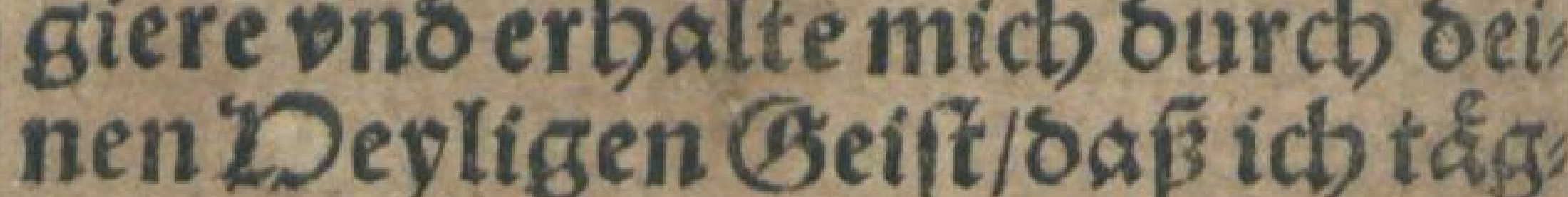 \\
\hline \multirow[t]{2}{*}{ 19et. 3.} & licb in seinem Gnsoenbunse fe \\
\hline & \\
\hline
\end{tabular}


(Esriffict)en Eebent. $\quad 67$ licb trớlte / EDeinen Blauben be: wabre/ Dir meinem Gott allein anbange / 2Alter 216 gòtterey/ 2 / berglauben vno Teuffelizroefen gram rey / Dno allezeit bruinftig Epher. r. im (Beift/ 1 î frölicb in 'Doffinung beinem namensiene.

Ja/mein (Bott/weil idb) burcb bie ben/pno ein nevoer LDenfCh voor" ben bin / fo gib ons bilff auch / bajich binfortb in einem newoen Seben wanbele/Damit meinfe? ben ein Zeugní̈ gebe / beybe für Gott vis aDenfhen/oapich oie Deylige zauffe nuttslicb ono fes liglidb empfangen babe. Ceyte vin futbre midb pollens surch oip muibefelige'Janĩertbal pno Laffe mich nimmermebr / bis ou mich bringen woirft ins Xeich ber ewi: gen Eeligteit / Dno mir baretbeft geworen woirf afle (Bnabenf a as t3e uns Gutter/ oie Su mir in mei/

Ibidem.

ner 


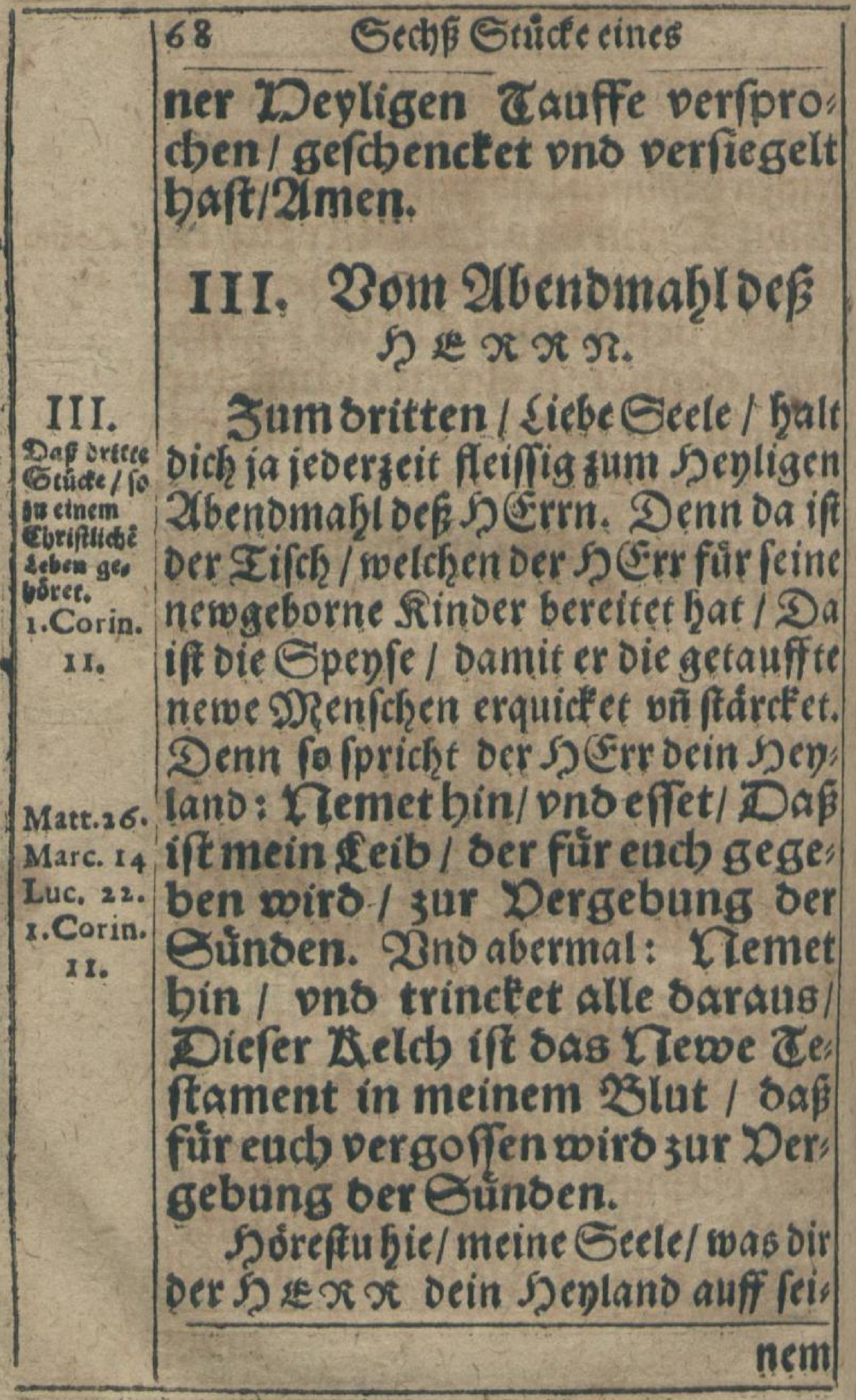




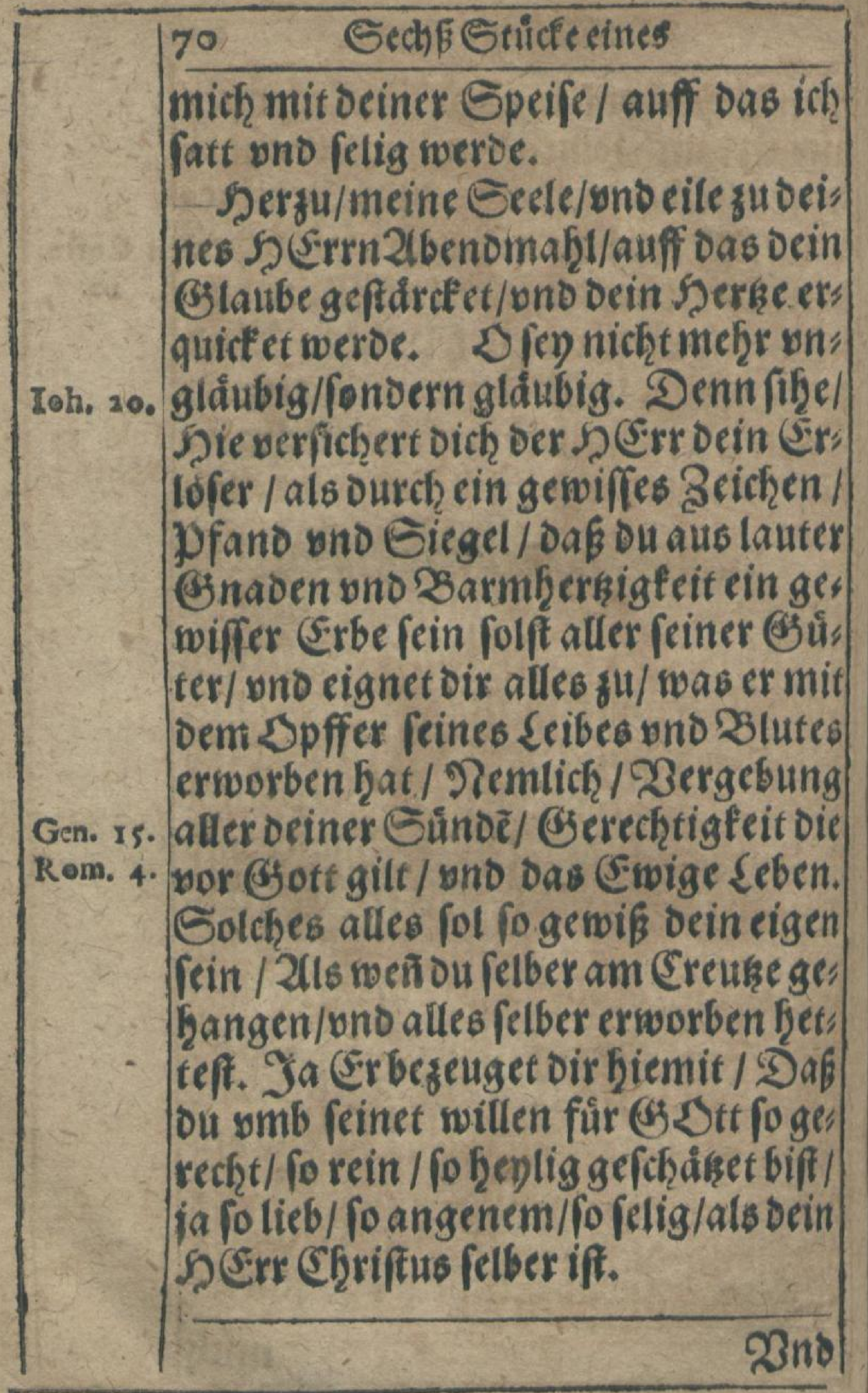


Wno vber das alles/ fentetet er fich Giemit Durch feinen Syenligen Eseif in

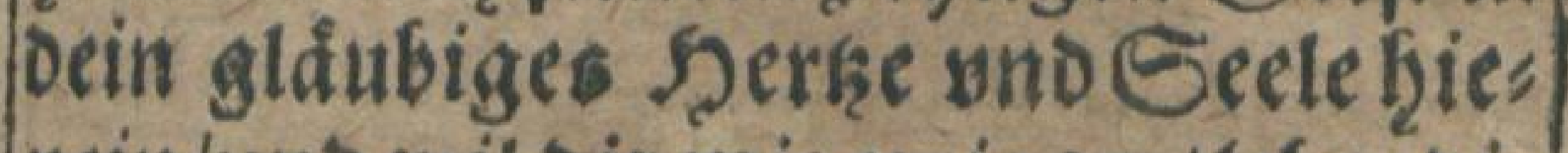
nein/wno wil bir mit ewigem/ lebendis gen Irofte beywohnen/ nimmermetis von dir laffen / vno new seben / siecht ynd Grerwde in bir wircten. Ja/mein ŞËr Iefu / alfo bin und bleibe ich in Dir/wno ou in mir. Joth fange dir meto nemErilofer fo fefte an/ZBie ein leben: biges@stiedmáz an feinem lebendigen Seribe/2rsic cine 20 raut an irem 20 reus tigam/2Ond bin alfo Fleifth won Dele nem fleifth / 23no Bein von beinem Bein. Ta ich bin in Dir/vnd hange oir an / wie cin 2B3eintebe an Feinẽ 23cin: frefle / und wic ein 3weng an feiner 23utkel/ 2 nd erlange alfo von bir/ snd alls dir/ alleseit newen Eaffi und Rraff $t$ newengroft vnd Frewoe/new seben wnd Sabfall/und bin mit bir an seib vno Seele bermaffen vereiniget ourch wafiren Ğlauben / vno werbun: Den Durch den Szentigen Eseif / Daß mich ond oich erviglich feine Roth 1 .Cor.7. noch Iodt fchenden for. 
72

Sechas Stricte eines

Gelobet feneftu SErr mein Deylanot / baß ou fo Dáterlich für micb geforget / pnosif (Bna: oenmabl mir binterlaffen baft/ oabey ich Spepfe pno fabfatt fuir mein bungeriges Dert3e/ $D$ ñ Eroft ons KRquickunge für mei: ne surftige Eeele bolen möge। oamiticb im Glauben ftarck ono fefte fortfabren / oir meinem \& lófer getroft nacb folgen/fefte an oir bleiben / pno bíp ans 促noe beftenoig verbarzẻ mòge/2Amen. Du fraftauch gefaget/mein STErr Yefiu: Soldbes thut 34 meinem Geodedtniß̧. Micin (jott/2Bic fan Das cin lieblich ङ̈ eddechtnis/ein/ WBie ref̧nlich fan es meine Geele trơften. Ta/mein STErr Jefiu/wenn ich an Deinem Tifche effe ober trintle/ fo balt x. ich Da bende Das Troft(Geoácbt:

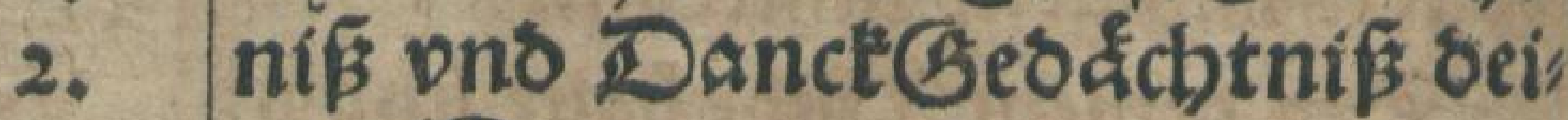
nes namens.

I. Erftlich / halte ich das Troftge o\&dchtnif / vato begef̧e in meinem Sher 
herken alle herrliche hiolie Seftegage des ganken Jafhrs / betrachte alle beis me 2Bolthaten / eigne mir fie gu/ sno troufte mich derfelben.

Bey beinem Iifche halte ich fros? liche werbenacbten. Denn bein Senliges 2lbenomaft bezeliget mir I Dak ou warfafftig mein fileifch yno Blut an Dich genomen fiaft / vno Dich mit mir befreundet/viel náḩer/als mit Den fenligen Engeln. Denn Du haft nichte Engelifche / fondern Deenichtlis che Satur an oich genomen.

Bendeinem Iifthe fralte ich auch bie liebe Darterwo be. Denn dein 2lbenomafl beseuget mir/ Daf Du das rechte Echuld Spffer worden bift fâr meine Sünde. Ta allfie effecich alfo Das rechte Sfter Samb / Remlich/ dich felbeft/sesrr Jefiu/ber ou für mich ges opffert und gefchllachtet biff.

23en ocinem Iifche fralte ich auth fróliche Ditern. Denn weil ich oeis nes Leibes und Blutes thenlhaffitig/ uno dein Bstieomaß bin / Eogldube

1.Cor.s.

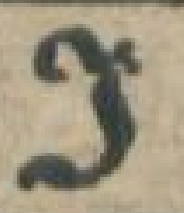

ynd

Ebre, 2. 2.

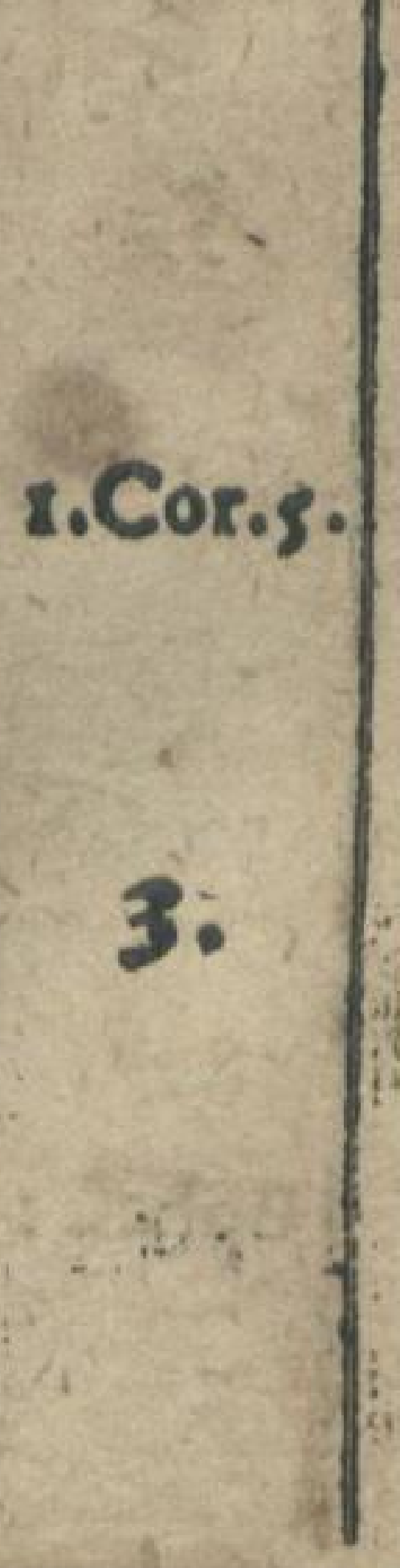




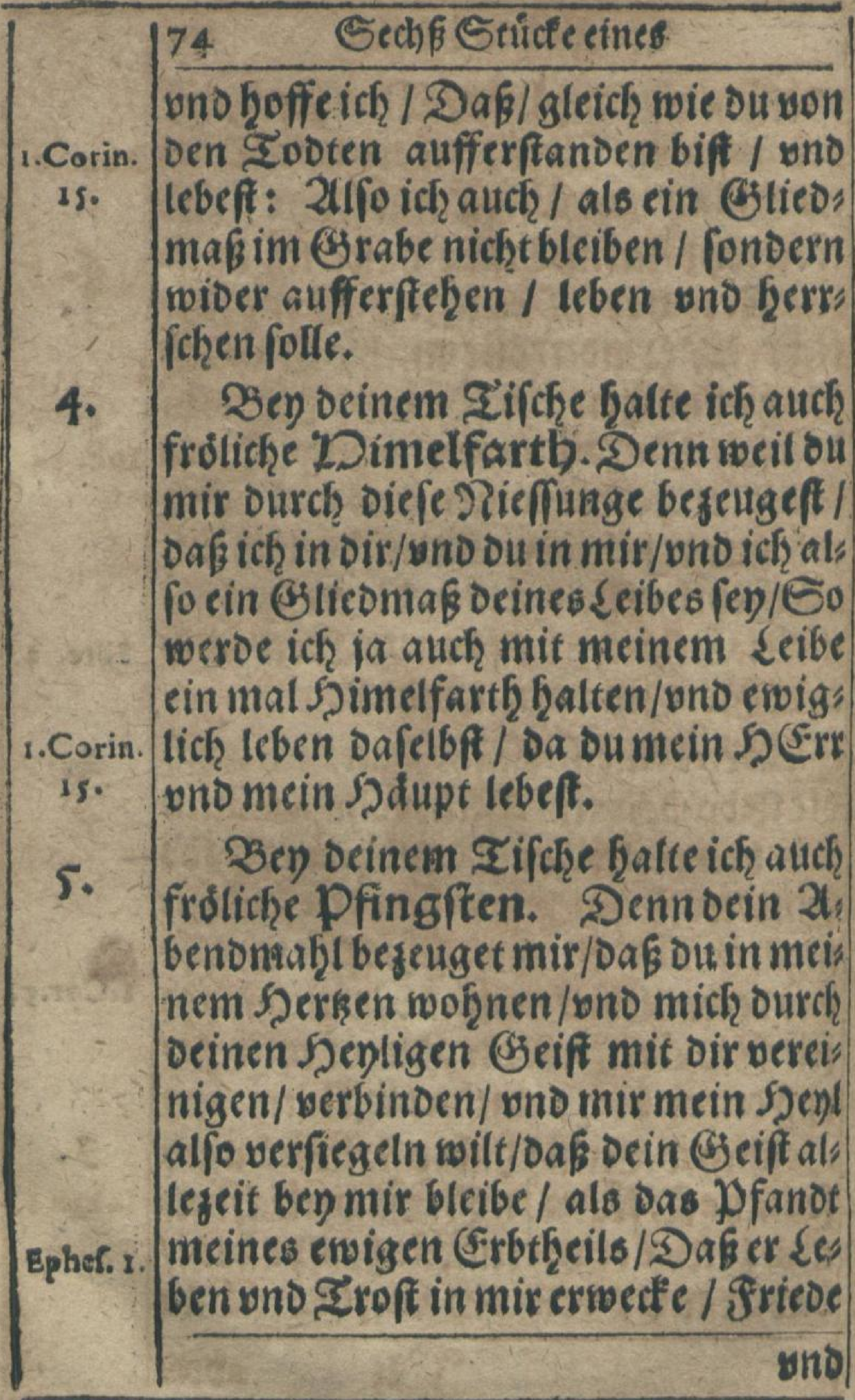


bisd Grenobe anflinde / 23no gougni gebe meinem @eift/ Dafich Dein Dorus zom.s. oer vno ewiger Mhit (5rbe [ey.

Bey deinem Iifthe halte ich atteh bas feft ber Deyligen Dreyfal: tigleit / 2no trofte mich / Dab ich forthin allezeit cin $\mathscr{Z}$ empel 32 ttes fein fol/23ndoie gankes seglige Drens Ioh. 84. faltigfeit fumir tomen/bno wobznung ben. mir machen wolle. Ya das ich auch nach bieferm seben bie ewige 5 es ligfeit befiken / bnd Den Ş Errm meis nen (B)ott foharen werbe won 2unges ficht fu Angefichte.

Dey beinem Iifche ha!te ich auth Das rechte 2aller Depligen feft I 2no trofte mich oer folt f ligen Eis nigfeit uno Bsemeinichaffi / weld he wir beine liebe Senligen / ourch wahs ren Blauben/mit birS马 ex $x$ Jefu/ yno ourch bich / mit bem 23ater vns Syenligem Bjeifte haben I VBie bu ges paget haft : Woer mein fleifd iffet/ Iohnu.6. ons trinctet mein 3 lut / oer bleis bet in mir/ ono ich in jm. 2Ho

$$
\text { Y if abers }
$$




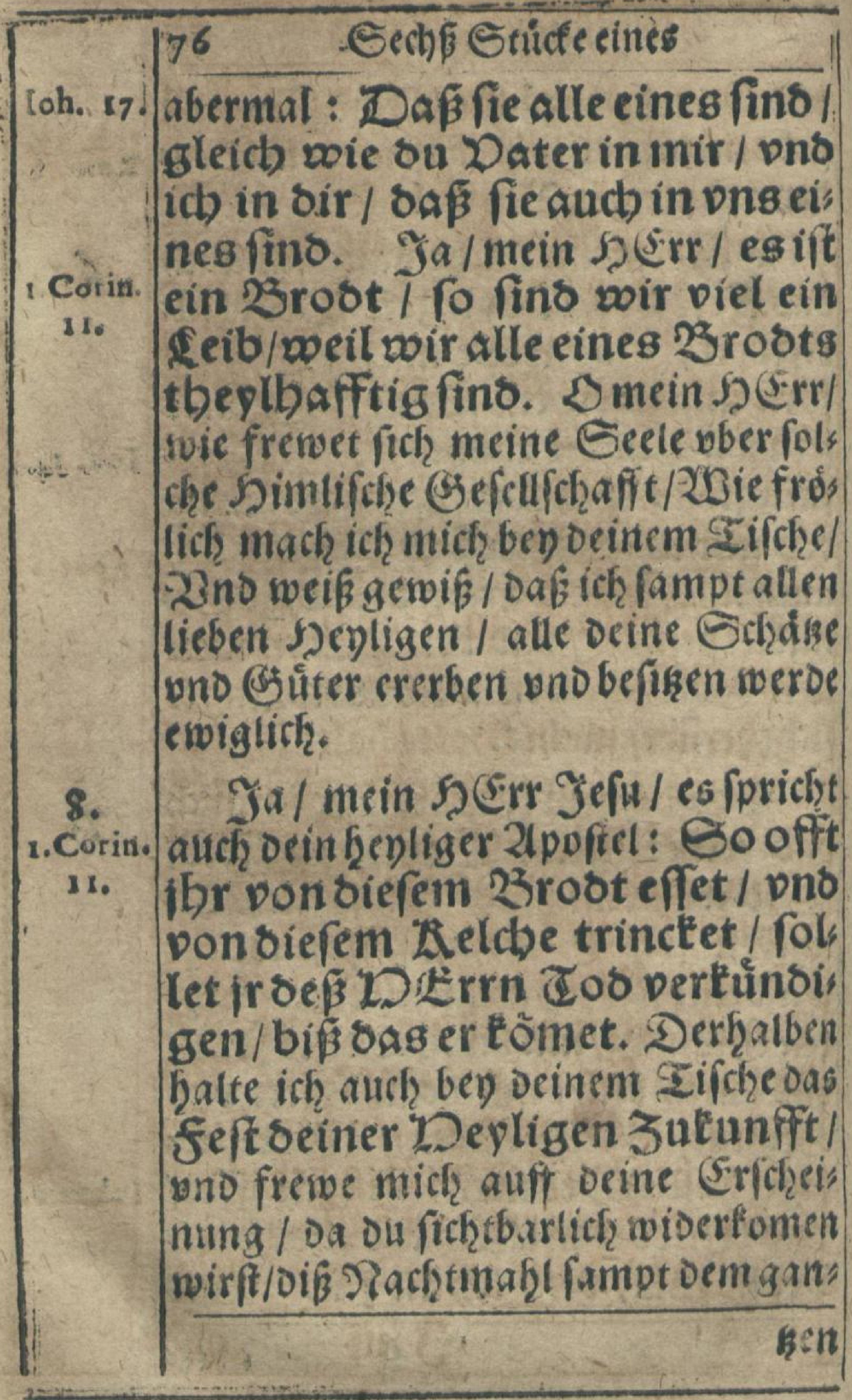


$\frac{\text { Ebrifflichen Sebelts. }}{\text { sen Dredig } 2 \text { mpt auffheben / mich zu }}$ oir nemen/Dafs icfifen wo ou bitit/oa ou Loh. 37. mir ein ander Enviges S. imeimafil bes reitet ḩaft in Deines 23 aters Xecich/vin ich mit 2braham/ Ifaac/ Jacob/und Matth.s.

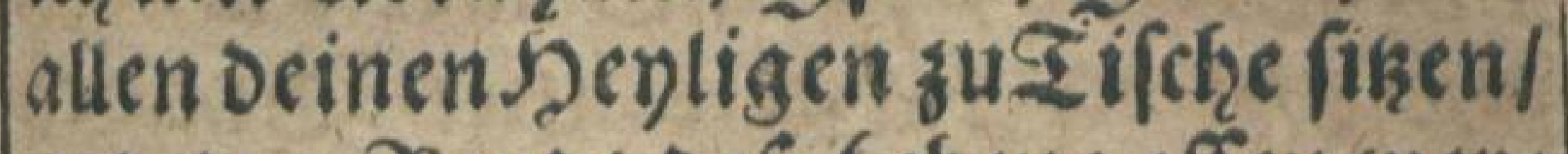
und Das Z Brodt Dés Lebens effen wers De enviglich, ऽ wiefefinlich betractrte ich folches ben Deinem Eifthe/vin feuffs" Be von grundt meines Serkens: DERr Jefia/tom/ fa tom balbe/ Apoc.22 mein'Deplano/pno fey nidbt lan: ge/zimen.

Serner/mein Sott/Latte ith auch II. ben beinē Sifche / Daf rechte Dance"s Gebácbtniß oeines naxmens. Denn weil ou mich da fo reichlich vino herrlich pes) feft / fo erbrennet ja bils lich mein Serke widerumb in rechter Dandt'barfeit gegen bir. 2 no dandte Dir Dabey fúr alle vno jebe beine 21306 thaten / Die ou mir armen fünolichen Dienfchen/Die Zeit meines sebens/ant Seib und Eecle er⿳亠口冋eiget fraft.

Ja ich falte hुie widerymb beine $I$ iij borie 


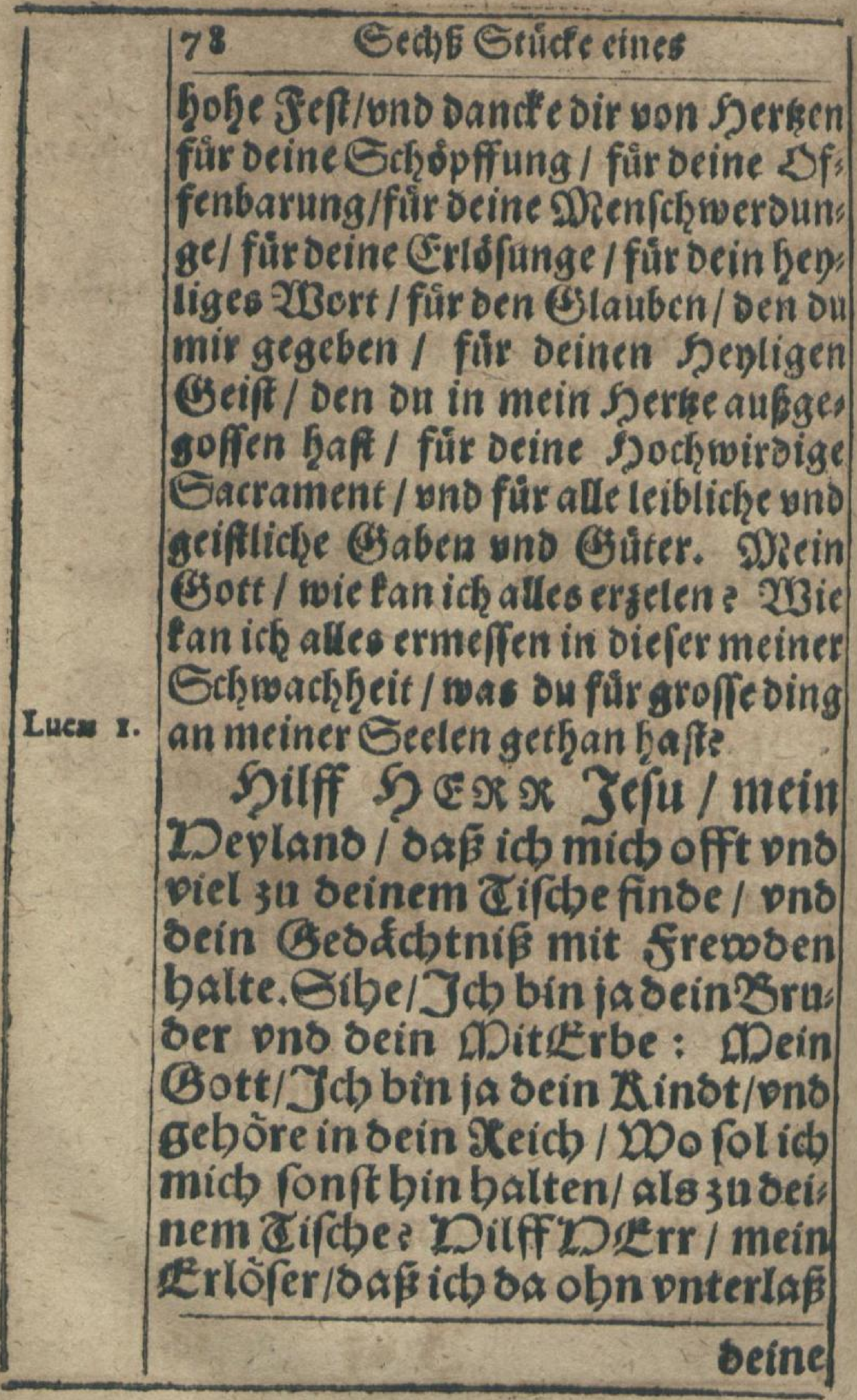


Ebrifftedentsebens.

beine bevlige / bobe fefteage andécbtig balte/mein'Dertse vir Blauben ftátie'e/aile meine fuift pno bochfte frevobe an oir babel Dir fir alle beine grofle $200 \mathrm{ol}$ thaten bert 3 lich oancke/Dir Don Lertzen biene biß an mein $\mathbb{Z}^{2} n$. be/ Dno nacb biefem Jammer: thal oas 23 rod oeß allen $2 \mathrm{Au}$ serwoblten in beinem Xeiche efien möge/2Imen.

Eeslich / liebe Secle / wenn ou von

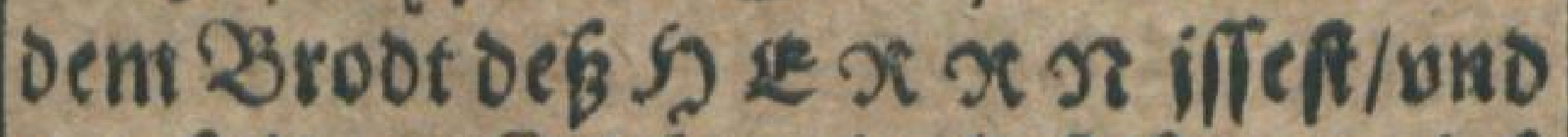
von feinem Jelche trintteft/fo vergié, auth de ines Prechffen nicht / Der neben dir auch mit fffet.

Ja/meit Şerr Jefu/idb betefie/baf er aucb bein (bliebmaßs/ oein Bruber / ein tAins (Gottes / pno bein DDit'Erbeift / gleich ro wol als ict/ Derbalben babe idt in bert 3 licb lieb omb beinet woil len/ Dns bin imbereft mit aller gutbertsigteit / als mir felber.

Dns wenn ou mein 2 ruintein

I iity $\mathrm{rel}$ 


\begin{tabular}{|c|c|}
\hline & Sedis Stricte tnes \\
\hline Mate. & 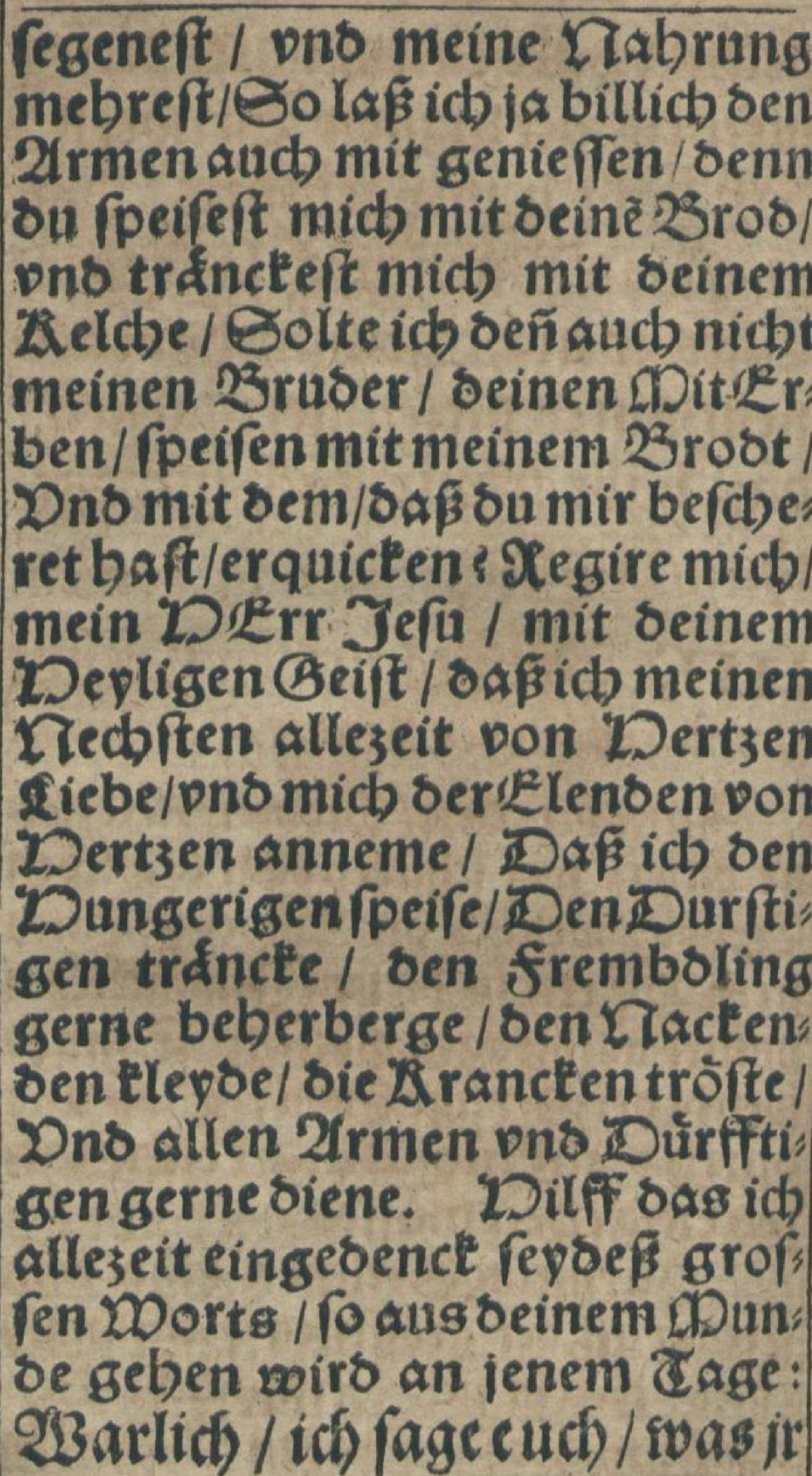 \\
\hline & \\
\hline
\end{tabular}




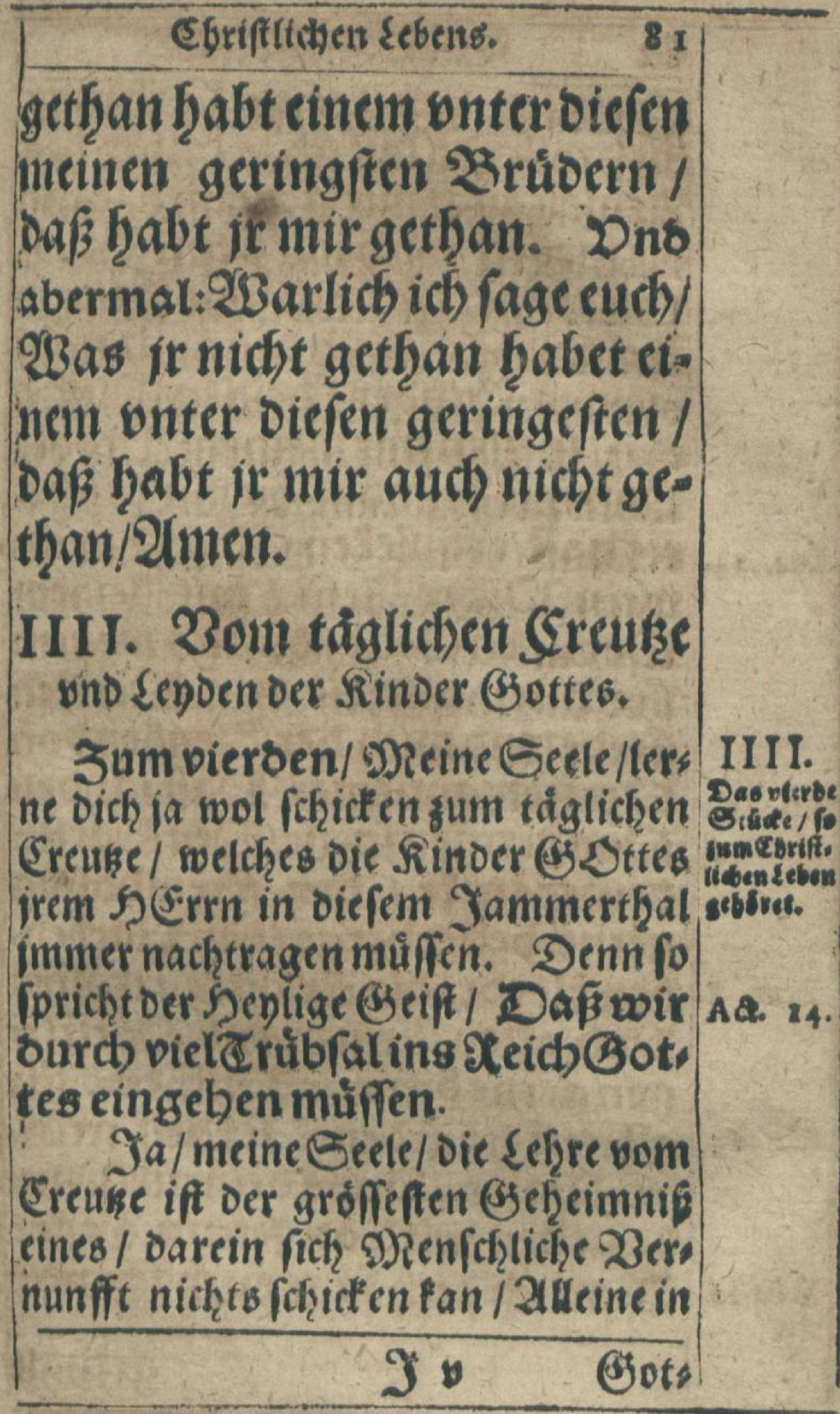




\begin{tabular}{|c|c|}
\hline & Sedt) Stictectines \\
\hline & (3)ottes 23ort ifts offenbaret / wie al \\
\hline & Dere SSeheimnif / Z̈Zno Daraus mus \\
\hline & mans lernen. Dahertomet es nun/ \\
\hline & liebe Seele / daß uns Das Creuse po \\
\hline & 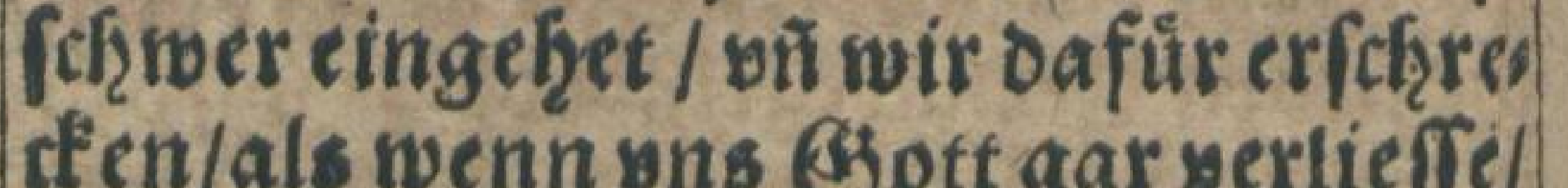 \\
\hline & $\begin{array}{l}\text { ifen/als wenn sns bott } 8 \\
\text { ober vnfer argefter fieind }\end{array}$ \\
\hline & ches if fehr fein vorgebiloet in den \\
\hline & lieben zwepen Jüngern / oie von Ses \\
\hline $10,24$. & sufalem gen Emahus giengen im \\
\hline & 1 ond fende/ weleffen ber. \\
\hline & ifre 2ugen worben gefialten / Dafifi \\
\hline & junicht lanoten. Eife/meine Gee \\
\hline & $\begin{array}{l}\text { Sreukes/Dat } \\
\text { offecalfo ver }\end{array}$ \\
\hline & flendet bnd verbirget / da \\
\hline & beften Sindern fremboe nor \\
\hline & İber alfo fqat er feine luff mi \\
\hline & tete \\
\hline & f feinem Srotboden. I \\
\hline & bart ge \\
\hline & Siebe vito SSute vergeffen \\
\hline & 2 aber su feiner 3eit giebt er fich wide \\
\hline & \\
\hline
\end{tabular}


Juerfennen / Daf ifgre Serken bollex Srewoe werden. Darumb lerne dich/ liebe Seele / in ois OSehreimnif recht fchicten/uno mercte biefe nach folgens de fieben Creukregeln wol / po wiro oir Gieben beine Erubfall nicht balb lo ftcher werden.

Crens Kegein.

\section{Die T. Sirturerget.}

2Alle Gld́ubigen fino von Gott oaju georonet / baß̧ fie Cbrifti Creutżtráger fein follen. Denn Fo fpricht 6 . Daulus \pm DDelche Gott Rom. 8 . 3uvor verfeben bat / bie bat dEr aucb Deroronet / bafi fie gleids fein follen sem ${ }^{Z}$ benbiloe feines Sobnes / auff oas serfelbe ber. Erftgeborne fev vnter viele23rú: bern. Sodreftuhie/meine Seele/ ba B bu fum Ereuke vnd sepoen serorenet biff / Richt alleine in Dutter seibel fondern autb effe die 2 Beft gegnindet worden iff / auff das ou deinem s) Exs ren Jefu gleichformig onns einnlich werben follefts Ja/meine Seele/ Dil 
84. Sieben Ereukregeltt.

bift Fleifh von feinem Jleifh / vno Wein von feinem Dein: 2Boltefo ou Denn nicht mit jm leyden s Sr ift Der S) $x x$ / vno ou fein Junger/ Er ift Lnex 6. Der Breutigam / vno ou feine SBraut / Iphei, 2 . Er ift Das S)áupt / vnd ou fein (5)lied: mas / 2 Saromb wolteftu cs beffer has ben / alser? Gehawe anden Sy Srrn ! als den Serisog Def Cretizes / vno fis Matt.r. be / 2Wie alle liebe Syenligen ifgm in Erubfall gefolget haben, Der S Srr gef̧et forn an mit Dem aller gróffeften Creuke / Dic ß゙láubigen folgen nach/ vno traget ein jeder fein Joch/ in fro: licher Sjoffnung/weil fie dem Sorrn

Rom. 8. Jefu fice cfinlich fein in Irvibfall / fo weroen fie im auch gleith formig wers 8. Coria. Den in Ewiger Serrtigfeit. 15.

A(b) ŞErr / mein Sjott/oer on mich beinem Gobne sum Crent3tråger veroronet pno be reitet baft / Gib mir ein verften: oig Derts / bafích foldben woun berbazenXatb.erkefie/bascreut; mit frewoen anneme/ meinem

Deys 
Sieben Ereuķregein. 85

Devlandenadb folge/pno im bie 3eitlich vno sort evoiglich gleidt: formig werbe/21men.

\section{Die II. Sreuzzregel.}

Esiftsef DERrn Cbriftibe: 2.

febl/ baj im feine Glkubigen fein Crentze nadbtragen follen. Denn fo fpricht er: Ilemet ouff Matt.xt. eacb mein Jocb. Wind abermal: 20 er mir folgen wil/ber verletigs Luce 9. nefich felbit/ono neme feinereas tze auff fich takglich / pno folge mirnach. Ia/meine Eeele/ Diefer Befeth! gefget nicht oie 23 ingláubigen an / Eondern feine Shenligen und GHláubigen. Denn das Sreukc beß

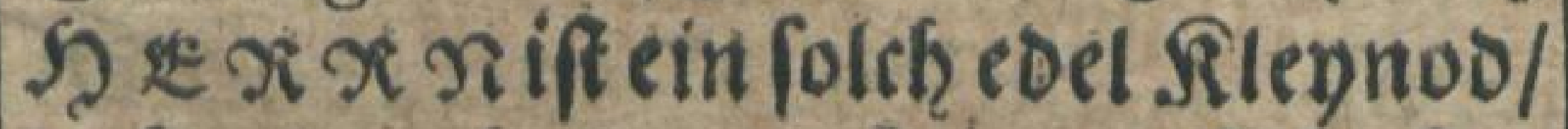

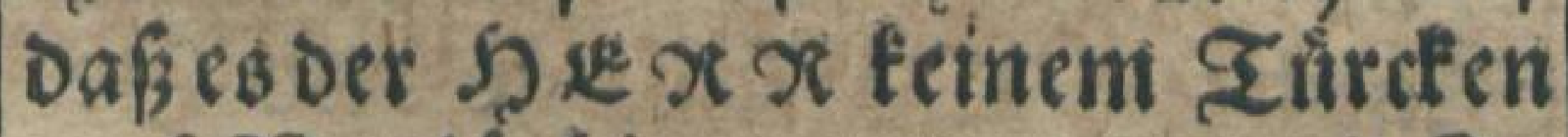
nocr. 23 ngláubigen vertrawen wil. Ia es iff eine folche 3ier/Daßzer es feinem Sottlofen gônnen wil. Scliane an Den Gimton yon Gyrent I welch cin Lues is

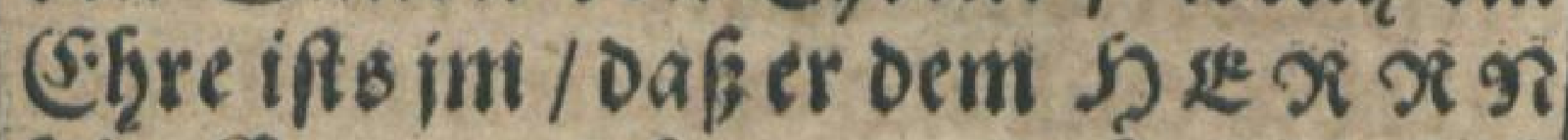
fein Ereuk nacligetragen bat / welch 


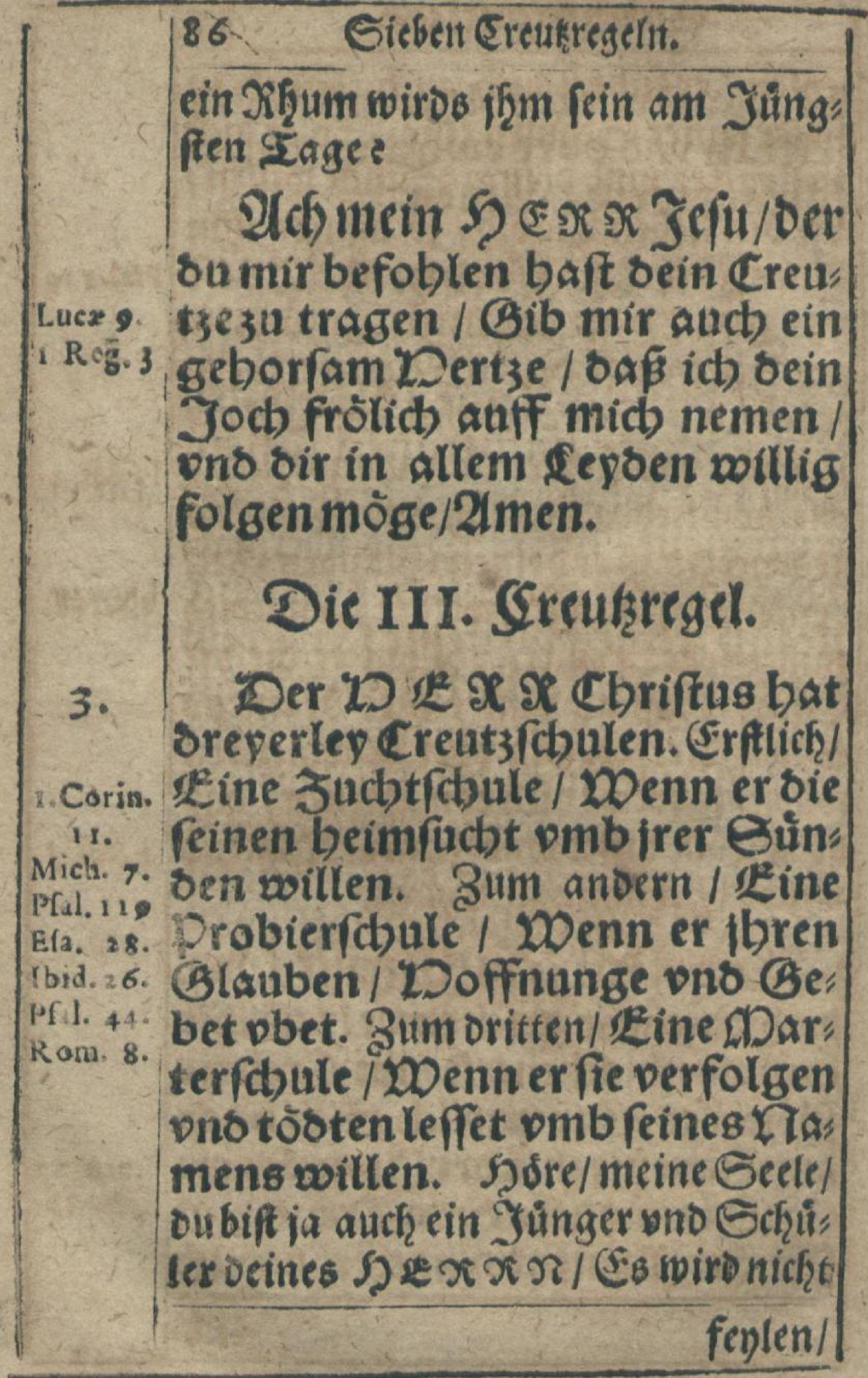


Eieben Ereuhreactn. 87

fenlen / er füfret Dich auch tdiglich in deefer feiner Estz̧ulen eine. Go er dich nufitaffet vmb deiner Eunde roillen/ mie Den lieben David / fo Dandte $15 \mathrm{~m}$

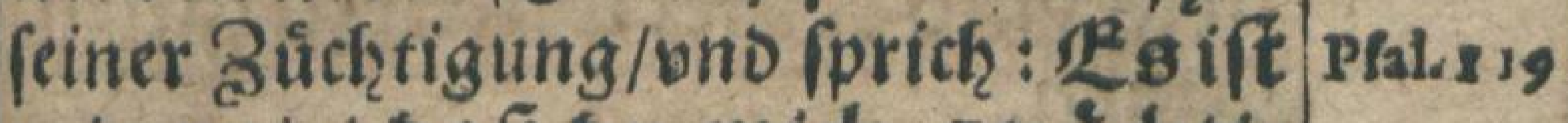
mir gut / oấ ou micb gezuัchti:

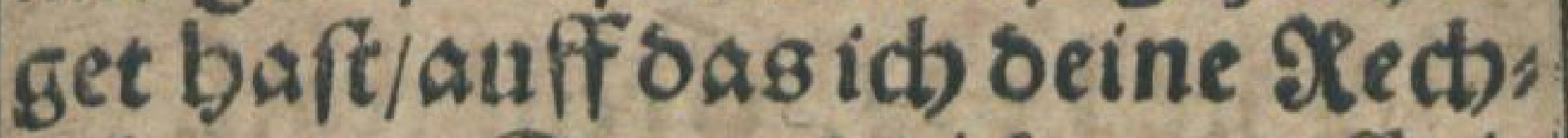
telerne. Eeket er dich nu auff die Jrobe / who finfyret bich wunoerlich / wie den lieben Jofeph und Siob / po gedendfe/Daf er beinen ङ) lauben vbet/ oeine Soffnung erwerlet / wno bich Ela, 26. gum (3)ebet reizet. Thqut er oir die Efire/ Dafs ou umb feines Jamens millen 2 erfolgung ober Zobt leyden folleft/ fo frewe dich von Szersen / Daf ou wirbig bift / umb feines Ramens willen Ectzmact? zu lenoen.

S๐ $\Re \Re$ Jefu SErrifte/ Der su bicb nicht gefdbemet baft mit bobn vno fdmmadb am Creutse 3u bangè / (Gib oas ich micb aucb ricbt fobeme mit ber Jungfraws en EDaria pnter bem Creutze 34 A Etor.s. Pfalm. 4. fieben: Za anft su mich wunbers 
88

Stebch Ereukregeln.

lich fübren / ro bilff / oaf́cich oir wunserlich folge/ ons bereit $\mid \mathrm{ey} /$ aucb mein seben/ ro oirs alfo ge; fiele/pmb seinet willen 3 ulaffen/ atmen.

\section{Die IIII. Sreukzregel.}

4. Daß Crentze ser Glḱubigen/ ift ein Jeichen oer fiebe botteg/ vno nicht oef́ Zorns. Denn fo

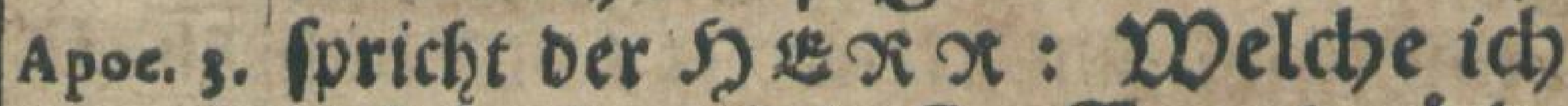
lieb babe I die ftraffe bno zuid), Ioh. 16. tige ich. 23no abermal : Die LDelt woirb fich freween / J br aber wer, bet traworig fein. Désgleichen:

1. Per.4. Daij Gerichte fenget an vom Daufe Gottes. Ond widerumb: Lere. 3s. In ber $\Theta$ tasot / bie nad) meinem riamen genennet ift / fabe ich an 3 uplagen. Shóreft dubas/meine Eeeles Ta / liebe Seele/2Ber pein Rindet lieb hat / Der fhelt es unter der Ebre.s2. Zuthen. Denn wo iftein 2 ater/ Der peinen Solinn nicht zuchtiget ? Siff ou aber ofgne 3úchtigung / welche alle

Sins 
Simber \&sottes gefohmedit haben / fo biftu ein 2 aftart / ond nicht ein Rino. Echawe beinen fु $x x x$ Yefum an/Daß cinige ßind (jottes / wie fefte

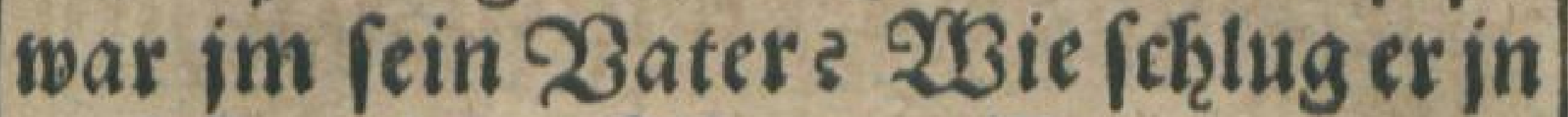
vmb fremboer Sunde willen ?

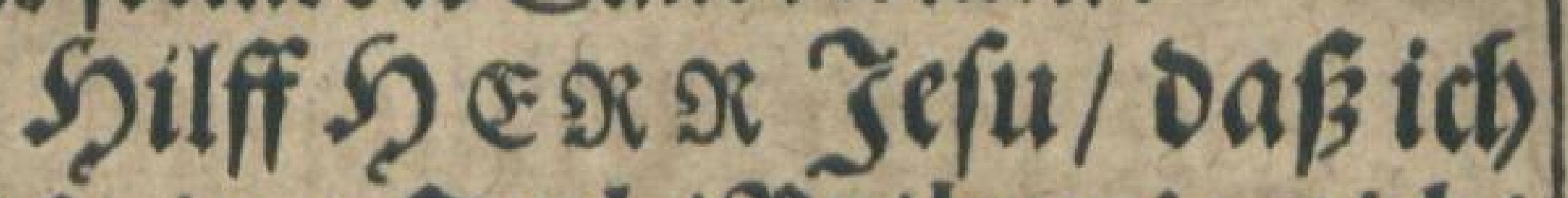
fúr beiner ZucbtXutben ja nicbt erfbrecke / fonbern als ein from:

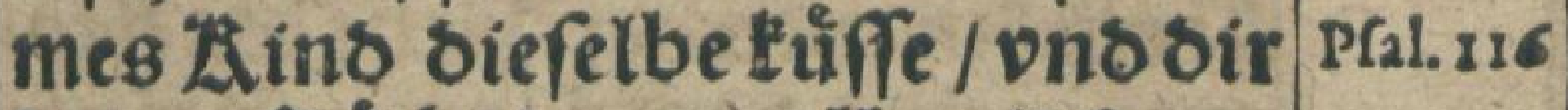
beiner З̌ib)tigung allezeit oan: cle/2Amen.

\section{Die V. Sreutzretgel.}

Je grôffer notb / ie náber Gott. Denn fo pricht oer Şerr:

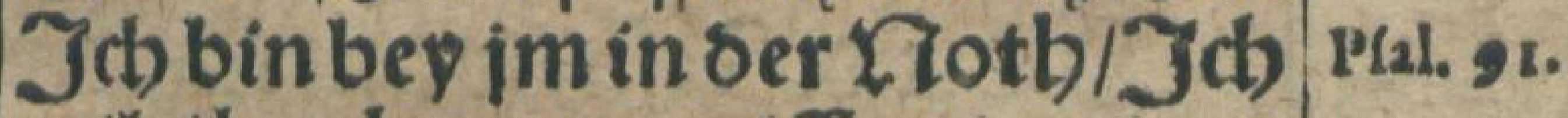
wil ibn beraus reiffen / pno 3 a Zubren macben. Emeine Eeclel wie gur fan fleifch yno blut folches nicḩt gläuben. 2ูBir meinen immer/ wenn Irúbfall oa ift / @S Stt habe vn: fervergeffen / Bott habe dem Ieuffel

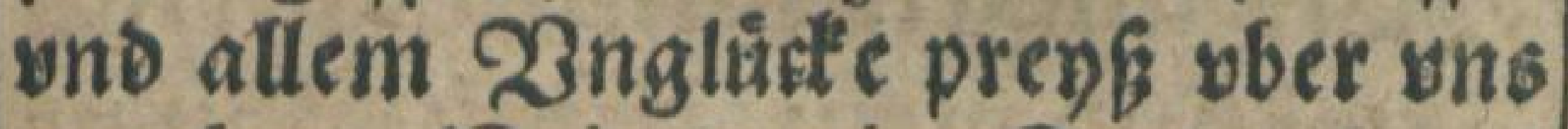
gegeben. Fein/meine Eecle/ ob wol

$\Omega$ oer 


\begin{tabular}{|c|c|}
\hline & Steben Ereutregelit. \\
\hline & oer Eatan ocin árgfer Seino ift / vni \\
\hline & Lag und Racht a \\
\hline att, 10 & bmbgefget/fan er di \\
\hline & vil \\
\hline Ela. 49 . & 1en. Ja/liebe Eeetel \\
\hline & oie 2 Barbeit felber ift ? 2 no ob e \\
\hline & fich einen fleinen 2lugenblict verbir \\
\hline & $\begin{array}{l}\text { I mitgrofer } \text { Sarm } \\
\text { famlen. Den fro }\end{array}$ \\
\hline & men gebet bas fiecbt auff in \\
\hline & \\
\hline m. 8 . & (3) Stt mit mir / wer wil wider mis \\
\hline & 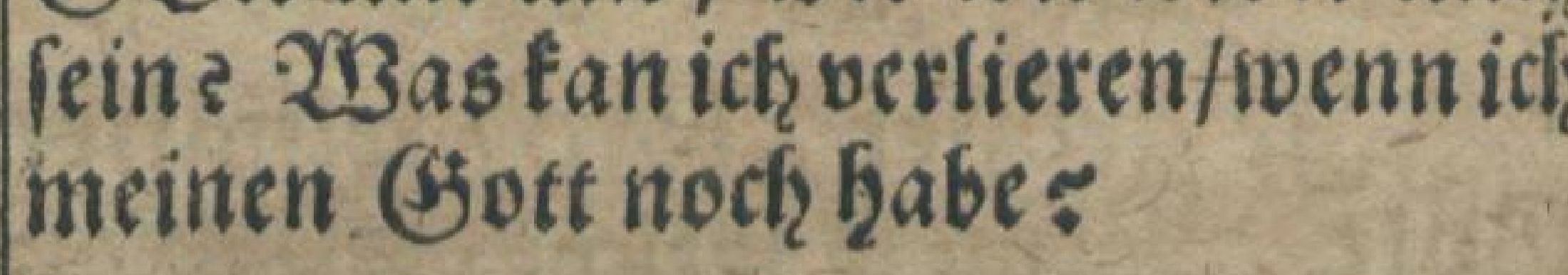 \\
\hline & tit! \\
\hline & bidf babe / fo frage idh nichts \\
\hline & nach Spimel bno Erden / కa \\
\hline & focñ mir gleich Seib ond Secle \\
\hline & berf(b)machtet / fo bift ou ooch \\
\hline & Serr \\
\hline & Eroft ond mein \\
\hline & \\
\hline
\end{tabular}


Steben Ereuktegelit. 93

Die VI. Sreukregel.

Daf Crentze ber Asinber (Bot: 6 . tis / oienet ibnen allezeit sum be: ften / ono nimmer zum árgften. Denn fo fpriçt 5 . Daullus : 20 ir Rom. 8. wiffen/Sás benen / fo GDtt lie, ben/alle sing sum beften sienen. 2 no abermal: Die Ochlekge be Siebbabers meinens recht gut. $\mathrm{Sa} /$ meine Eecle/ es dienet das Sreus Ëevielfáltig sum beften.

Dennes tefiret uns die Sünbeers fennen/2Wie gefthrieben ftethet: Jch Tere. 30. wil bicb júcbtigen mit maffe। auffoas ou sich nicbt pnf(b) utloig balteft.

Eis treibet uns auch sum 2 ชorte. Denn fo feffiet gefchrieben : 2lltein E[a. 28. oie 2infechtung lebret auffo wort mercten.

SB erwedtet vnfern Btallben/23ie geffrrieben fiefet: Eoldbe Truibfal woiderfubr pns oarumb/ bấp woir onfer Dertratwen nicbt auff ons I.Cor.I. a if relbeft 


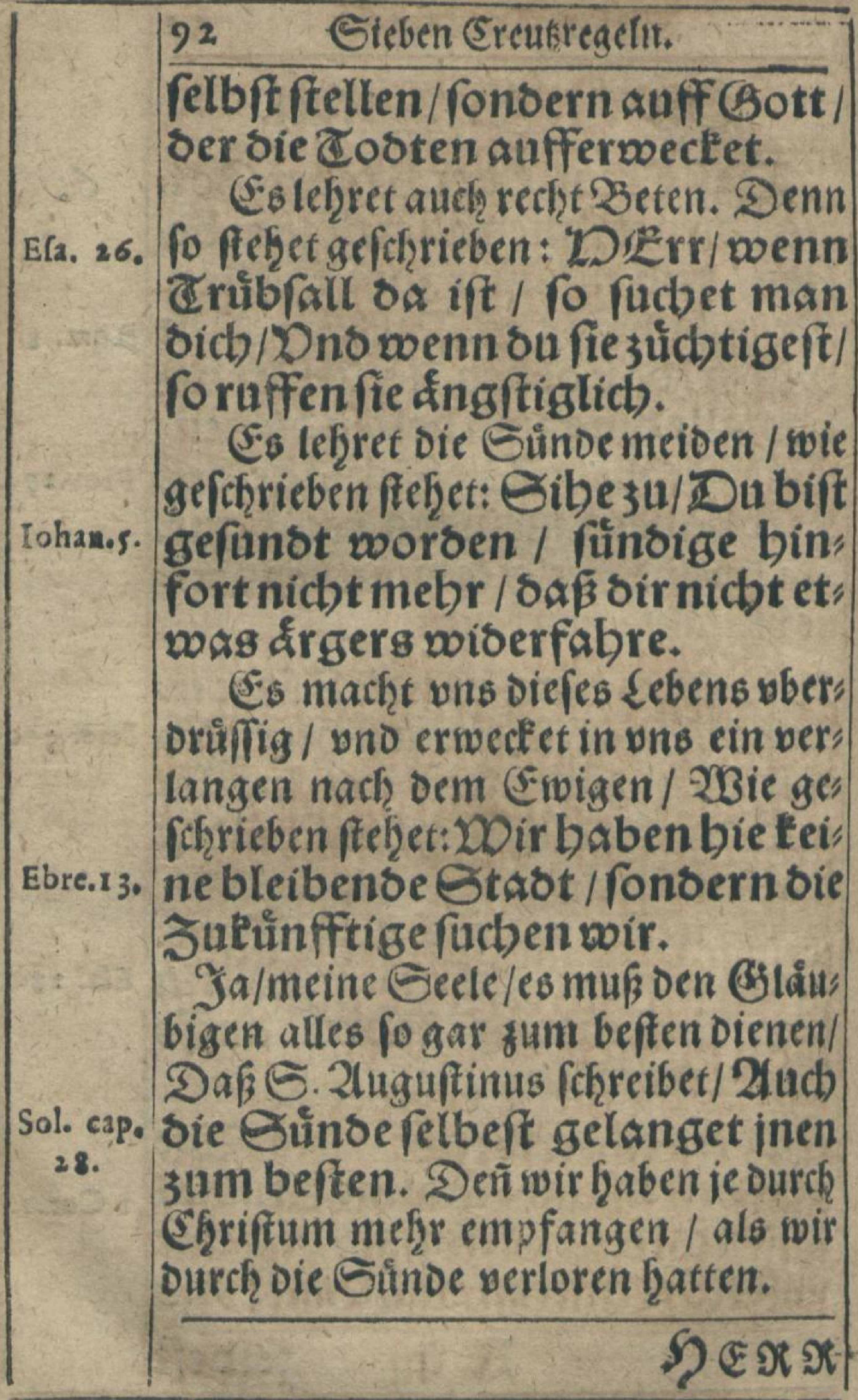


SูExx Jefu Shrifte/foreib mir siefen lieblicben aroft in mein Dert3e / vno bebuite mich / oấ ich ja in teiner T túbfall vers 3age / Eonbern micballezeit bís in oen Toot beß allerbeften $3 \mathfrak{u}$ bir verfebe / Denn wonserbars licb ift sein WDort/ono fúbreft al: Ela. 28 . les berrliab) bienauß $/ 2$ men.

\section{Die VII. Srcukzregel.}

Gesult/Gebet ons Doffnun: ge laffen teein Crent3 34 fchwoer werben. Deñfo fpricht der Drophet Ieremias: $\mathcal{R}^{2}$ s ift ein toôftlich) oing Thren, 3 . geoultigfein / Dno auff bie Gùte

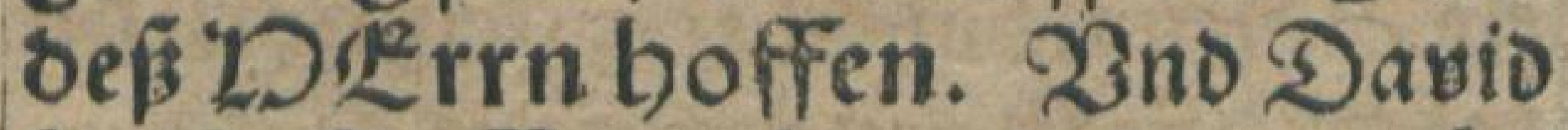
(pricht: Seuffe micb an in sernotb/ pral. so. fo woil ich oidb erretten/ fo folt ou mich preyfen. 2ino Eyrach faget: Eebet an bie Exempel ber 2Al, Cap. 2. ten / vno merctet fie / woer ift je: mals za fobanben worben / ber auff Gott geboffet bat? $\mathrm{Ya}_{\mathrm{a}} \mathrm{met}$; ne Secle/2Beñ einer eine Burroc oder $\sqrt{i}$ iij Safit 


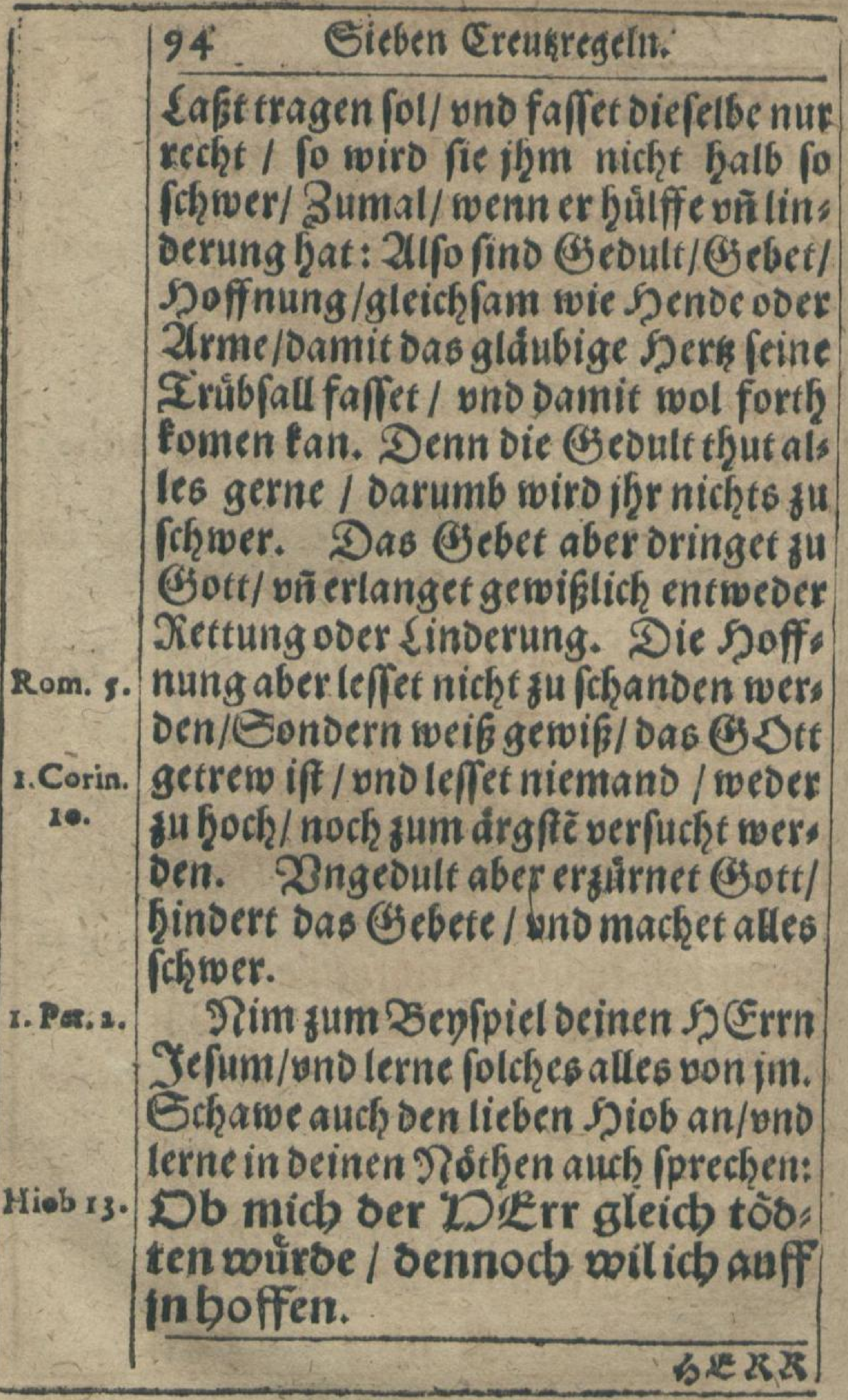




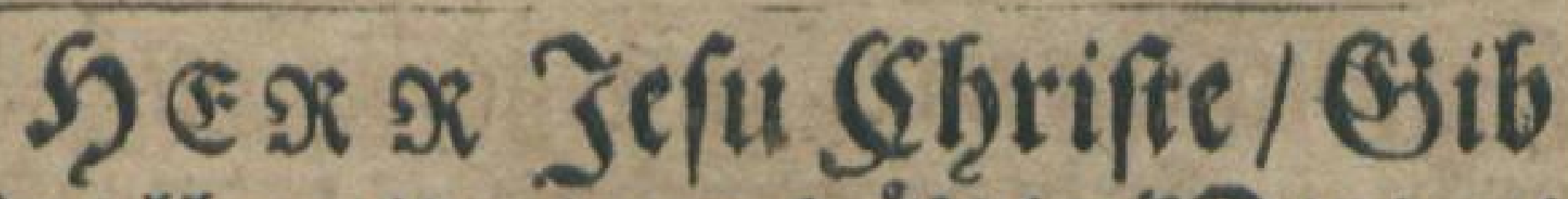
mir allezeit ein geoultig tDertze/ baßj wool warten ono beten tan/ bamit ich in meinem Creut3 nicht ongebuiltig fey/fonbern getroft/ vir mit aller Zuberfict)t mein 21n; liegen auffoich werffen/vns alle 2ugenblict be boffen möge/ 2imen.

\section{v. Bomenfferlichen Beruff} citres (Eqrifien פienfstzen.

Sum fünfften / liebe Geele | bleit e in Deinem Beruff / Darinne du von (ङ) Stt geferat bift / vnd warte bef: felben mit allent fleis vno trewen/ $\mathrm{Dak}$ oualles deines Ihuns fin @ Stt veno Dienfchen fönent Xectzenfichaffit ges ben. Denn (H) Dtt Der hy $x x$ wil nicht / Dafsoie Denfichen auff Eroen Eccle.6. mífig pein follen / Gondern hat ei, nem ieglichen Dienforen Zlrbeit auff; geleget / inach feiner maffe. Gif ooch

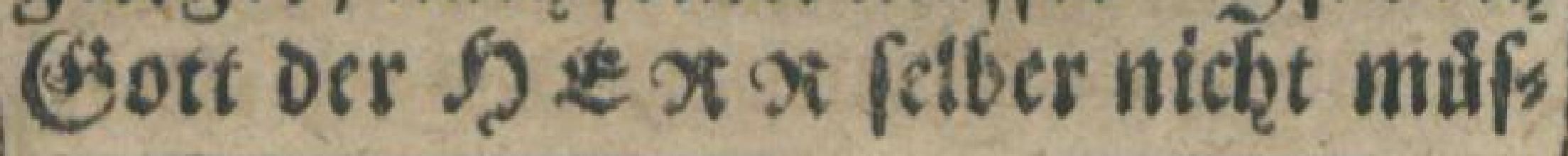

$$
\Omega \text { iiii }
$$
te Rude / fo su einems Cluiftióthen seben 800 botet.

Matt.12. Eccled.6. 


\begin{tabular}{|c|c|}
\hline & Sedip Sticte etnes \\
\hline & [ig/ [ondern mirffet/ regieret / tráget / \\
\hline & 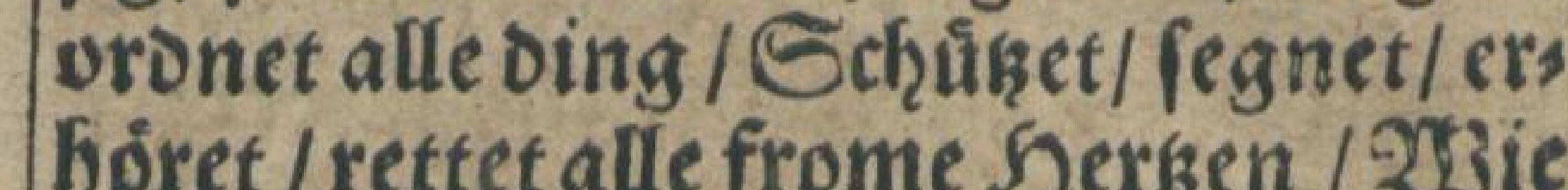 \\
\hline & Ęriftus felber fpricht: ¿Dein Dater \\
\hline & woircket bip̈ber / pno ich woircte \\
\hline & audb. Ya/meine Seele/folte@ott oer \\
\hline & Ş5re nur einen 2lugenblitt mutfig \\
\hline & Degaleichen auch oie begligen (s) \\
\hline \multirow{3}{*}{ Ebrea $x^{\prime}$} & getfing allsumal dienftbare Geifter/ \\
\hline & aurgefano sum Dienft berfelben/wels \\
\hline & $\begin{array}{l}\text { che oie Geligfeit ererben follen. } \\
\text { Ge auch Die liebe Gonne an/Den Dons }\end{array}$ \\
\hline \multirow{10}{*}{$\begin{array}{l}\text { Pal. } 19 . \\
\text { Pfalm. } 8 .\end{array}$} & Den und alle Eternen / wie cigentlich \\
\hline & $\begin{array}{l}\text { fie jren Sauff balten / und jren } 2 \text { eruff } \\
\text { werrichten. }\end{array}$ \\
\hline & xlfo/liebe Geele/ folauth ein jeocr \\
\hline & 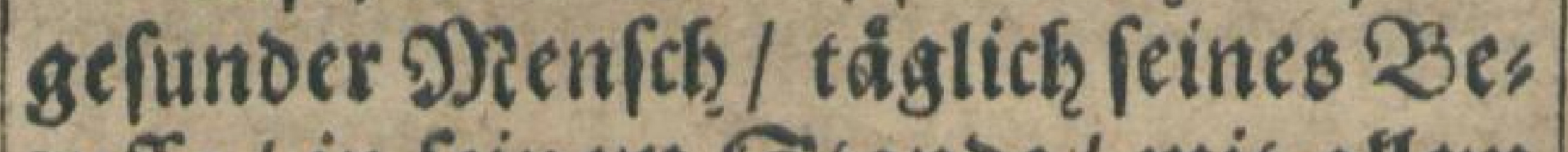 \\
\hline & em Gtanbe / mit allent \\
\hline & $\begin{array}{l}\text { leis yo crnft abwarten. Solauch } \\
\text { lefren / preoigen / regieren / fraffen / }\end{array}$ \\
\hline & foalten/banen/beffern/ \\
\hline & fur die feinen forgen / als folte er ewig \\
\hline & 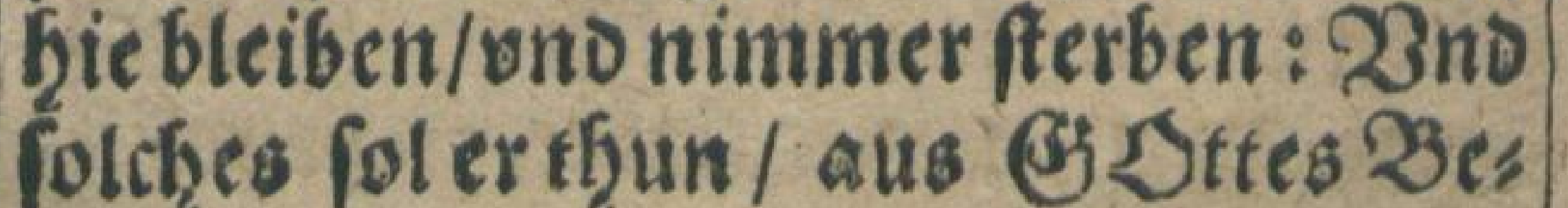 \\
\hline & \\
\hline
\end{tabular}




\section{(Sbrifftict)en \{ebeens. $\quad 97$}

febl/ wnd aus siebe gegen ben Rachy fómlingen. Dabey aber fol er táglich alfo leben / Glauben uno gut ESerwif, r. Tim.1 fen berwafiren / Daf er alle 2ungenblict" zur feligen Szeimfarth gefthictet fen/ yno fagen fónne mit 5 . Paulo: Die 2. Tim.4 Jeit meines $2 \mathfrak{b}$ f theioens ift vers banben/ Jcb babe einen gatten Aampff getémpffet / İd babe ben fauff pollenset / Jab babe Glauben gebalten/Dinfortb ift mir beygeleget die Ä ron ber Ge,

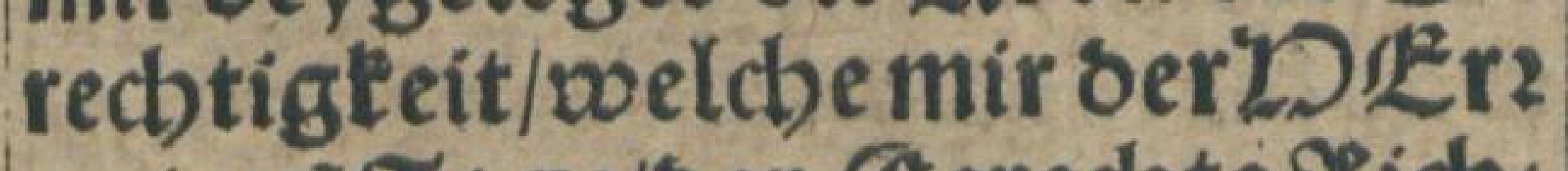
an jené đage/ber Berechte Xich" ter / geben woirs / ficht mir aber alleine / fonbern audb allen / bie feine $\mathbb{R}^{2} r f(b) e i n a n g l i e b$ baben.

Derfialben / tiebe Eeele/fitzeffeifs fig auff oeinen Beruff / Darinnen Dich, Ephes. 4. Der ŞErr georonet hat / Daßs Du dars innen wandelft / wie fiche gebuifret / mit aller (s) Coult und Eanff tmuth.

Eifze aber su/bleib gerne im nioris gen Stande / Dafifif beffer denn alles/

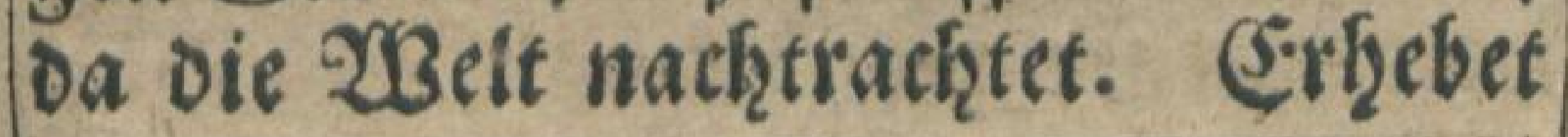

$$
\text { 尺ै Dich }
$$




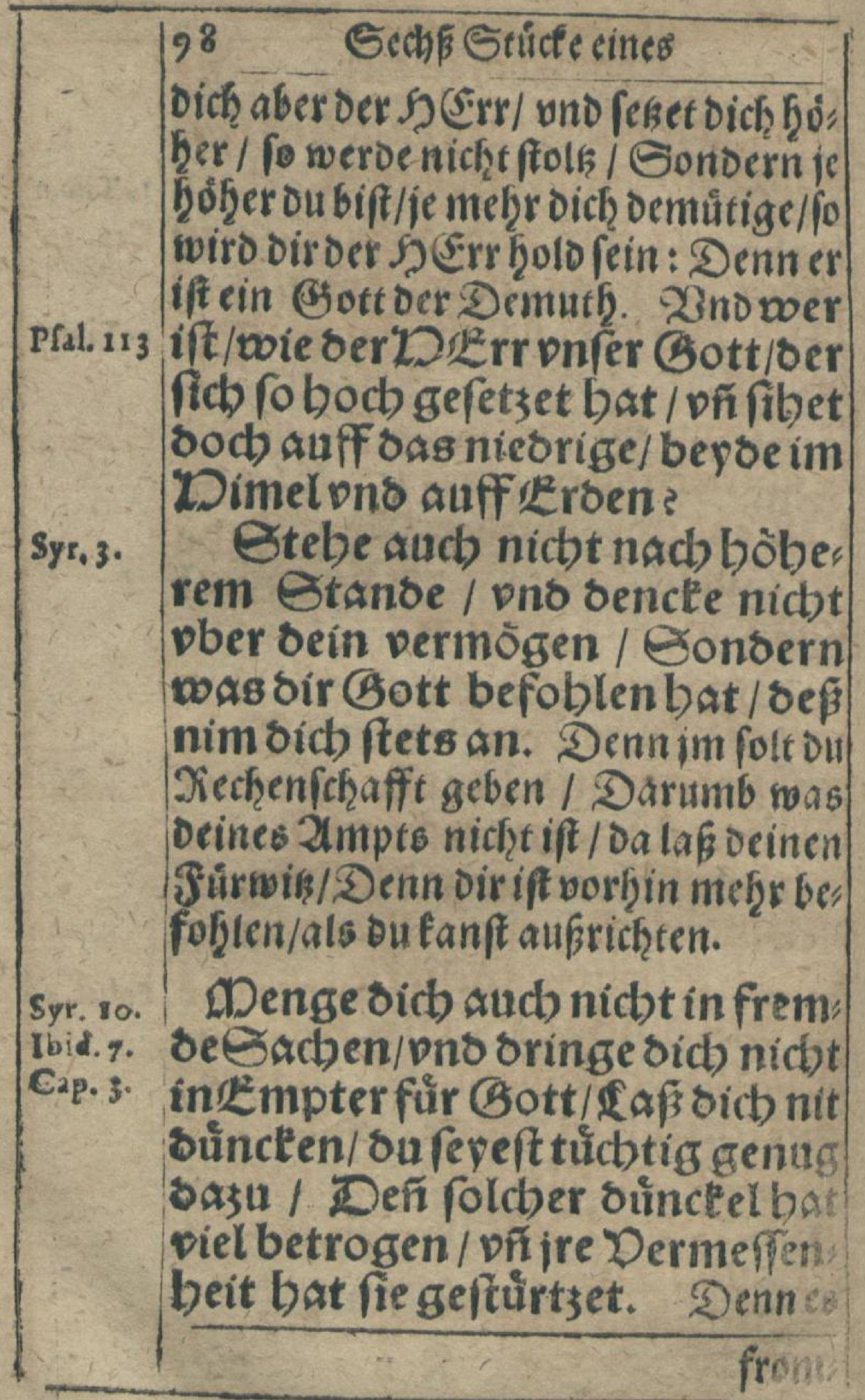




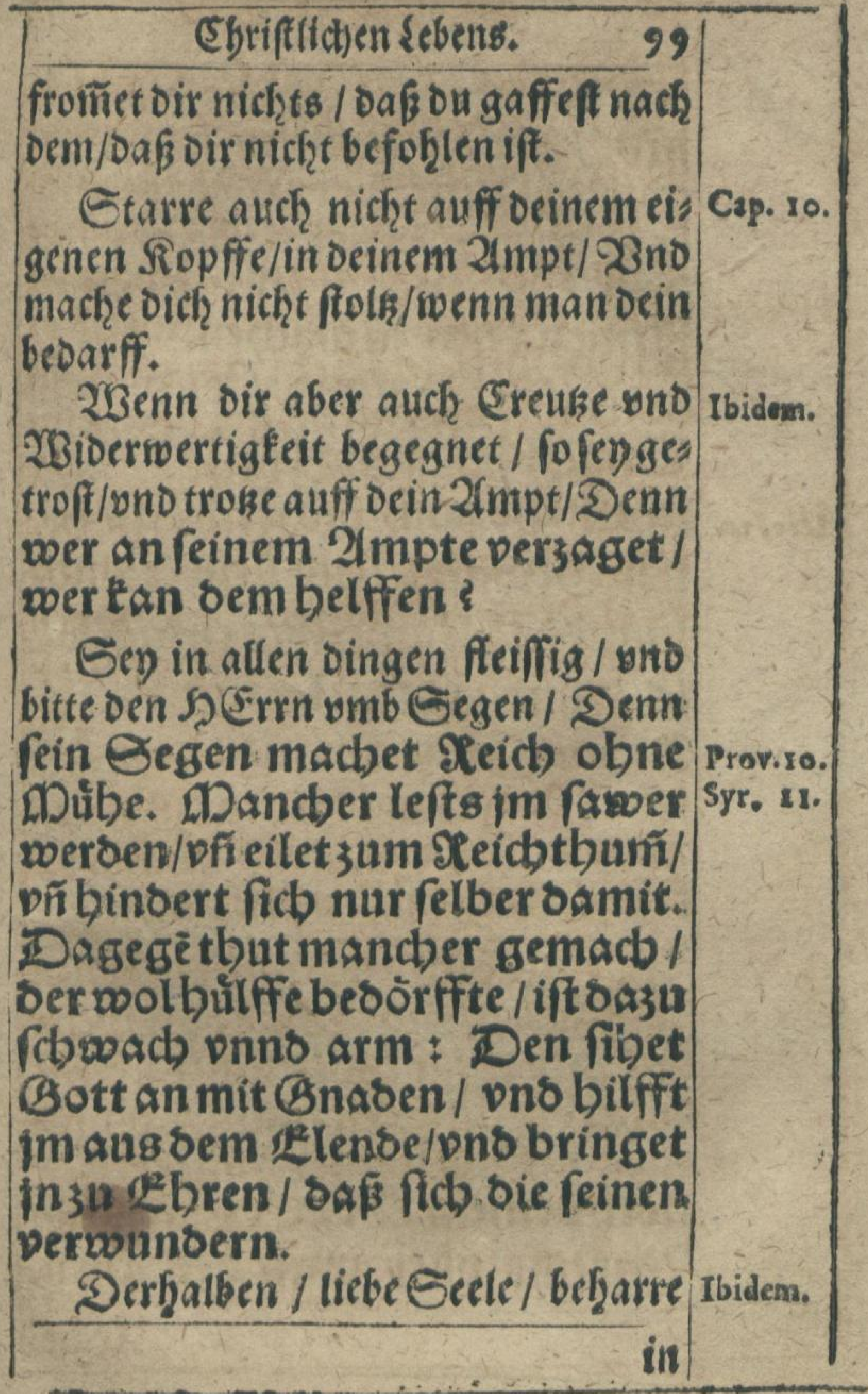




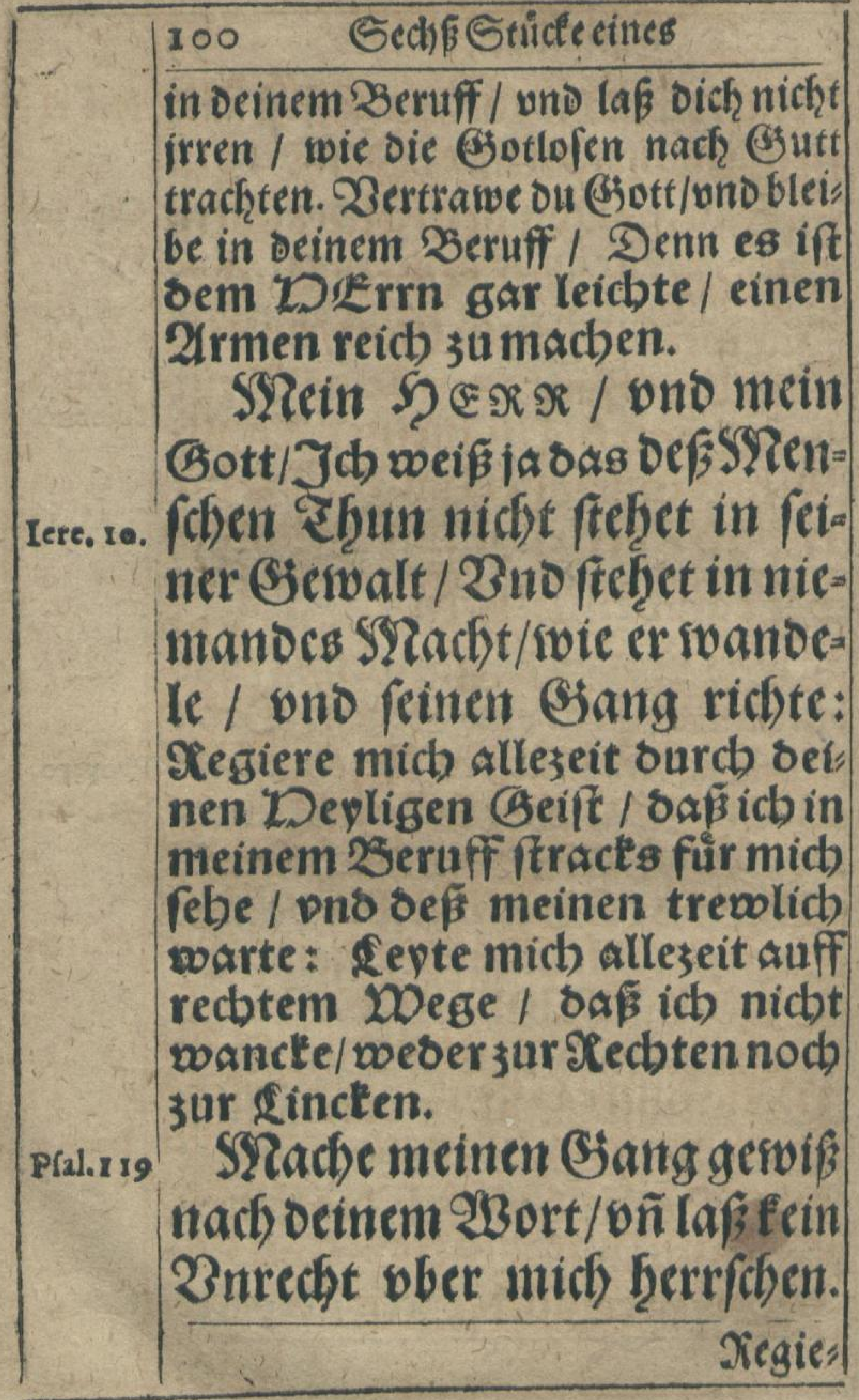


Ebrifflichen Sebens. - IOI

Regiere mich allegeit nach bei $=$ P(al. ${ }_{43}$ nem wolgefallen / Deñ ou bift meinGott/Bñ ocin guter fúf̧re mich auff rechter $\mathfrak{B a l h n}$. Jcb wei auch beruffen bin in beinẽ 20 ein: Matt,20. berg/ pns oir in meiner đauffe 2irbeit 3ugefaget babe. Jab bits te sicb / verleybe mir gefunben

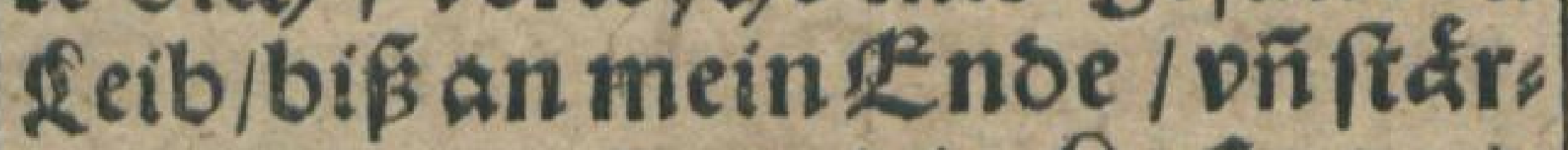
ckemich/ bafisich Die Lafist vito Sitze meincs $\mathfrak{B}$ eruffí willig trage/ pnosir meinem DSErren allezeit trewo vio gewebrfey.

DDeil mir sber sie Etunde meines feverabenos verborgen/

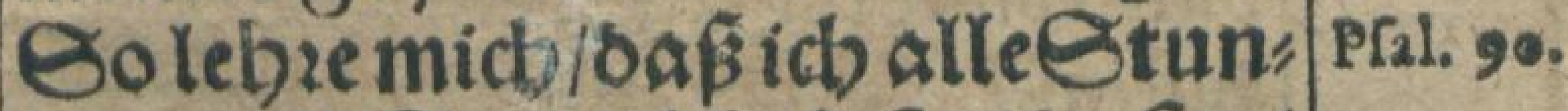
ben vis 2lugenblict fertig rev/ ein feligen 2lbtritt madben / oie woelt woilliglaften/mit frieb ori freboben einf(h)laffen / pno mit Luex s. bir pno allen 2lupierboeblten ben evigen Jube vno feyertag bal ten möge/2Imen.

VI. $25 \mathrm{~m}$ 


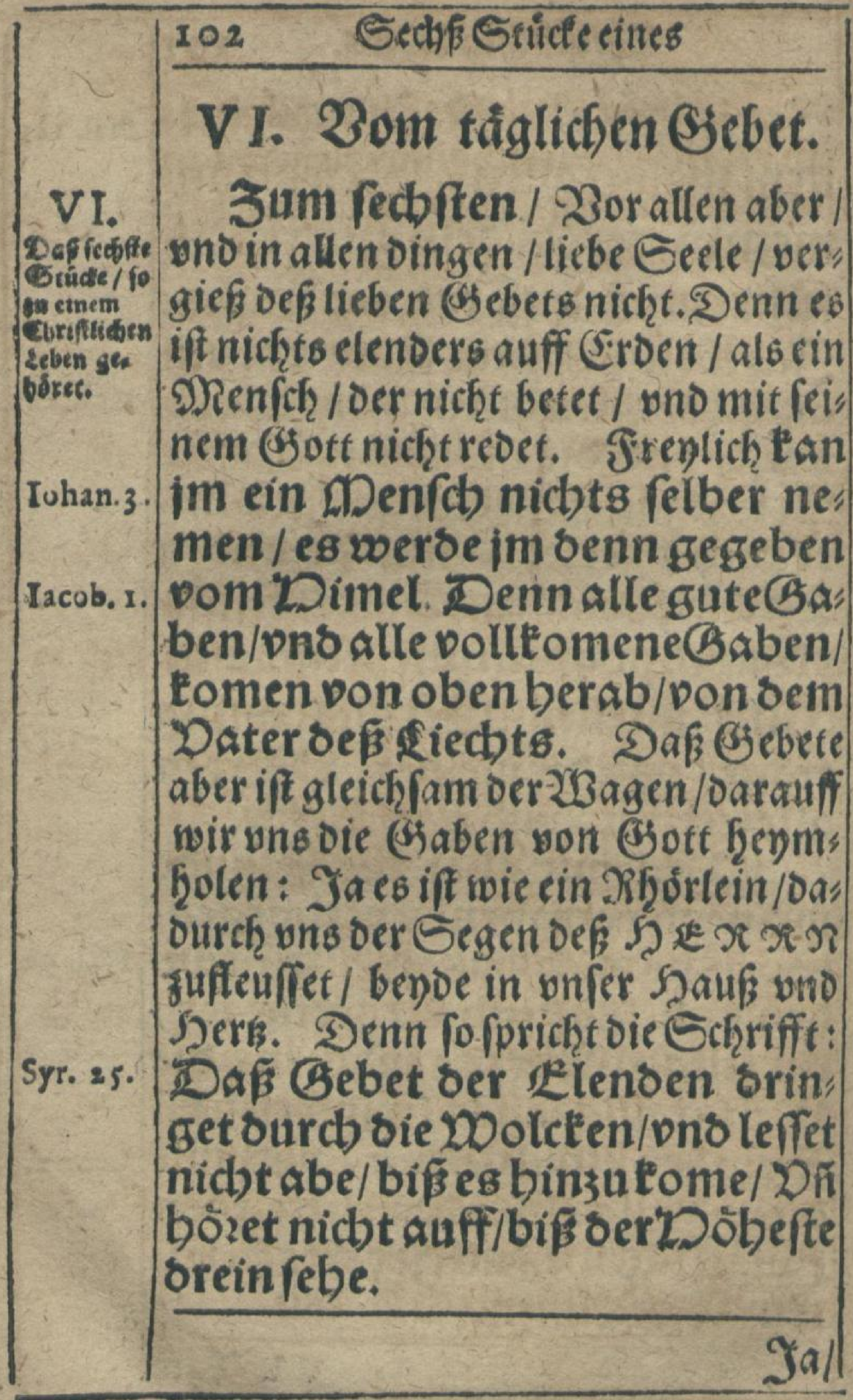




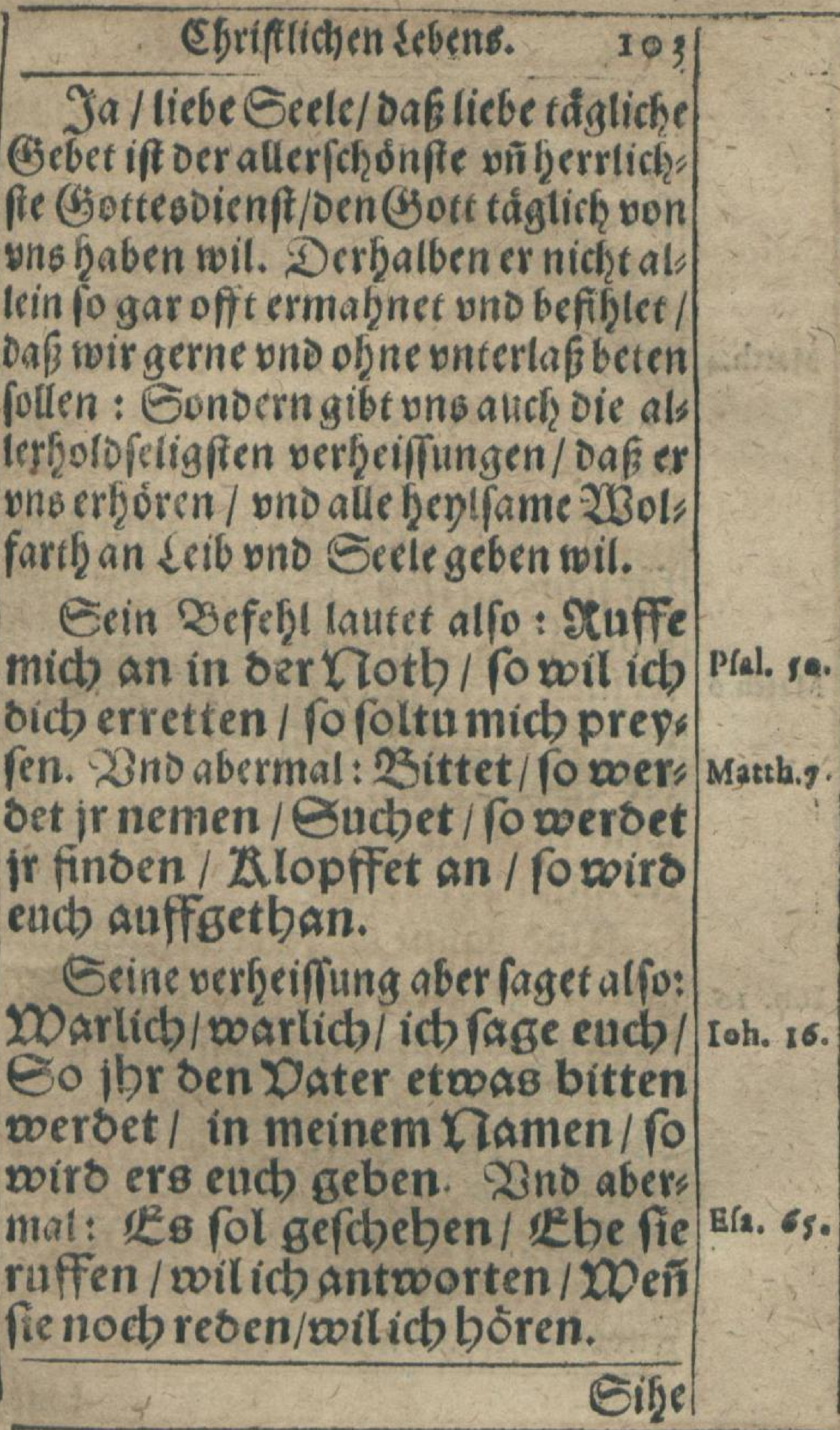




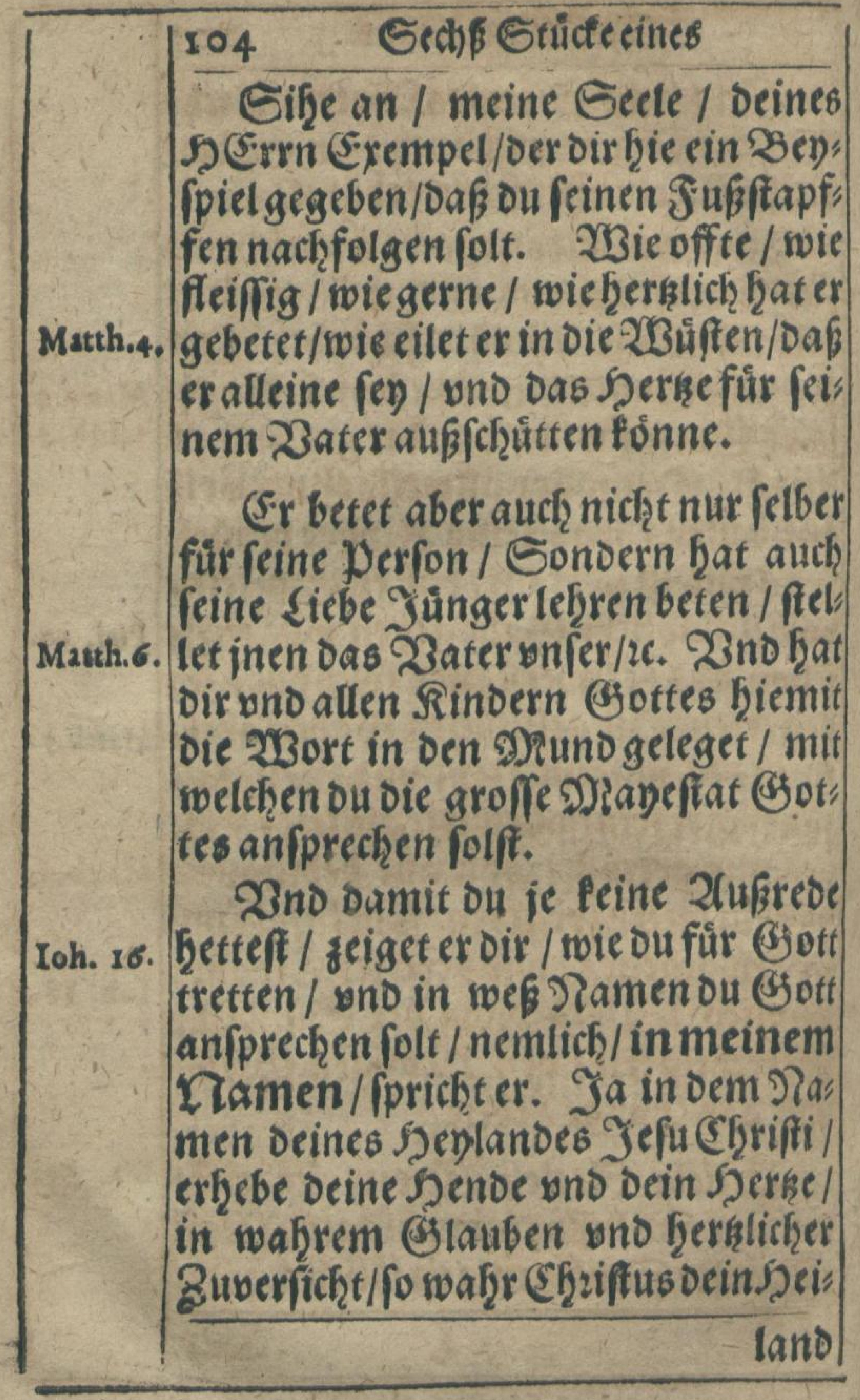


(s)rifticlien sebents.

108

land ift / vno su feines 23aters Xiechs ten fizet/[o gewisift auch oein Ssebet/ und alle bein Eeuffuen erhृoret.

2ber bas alles/[o weiffu auch/ was

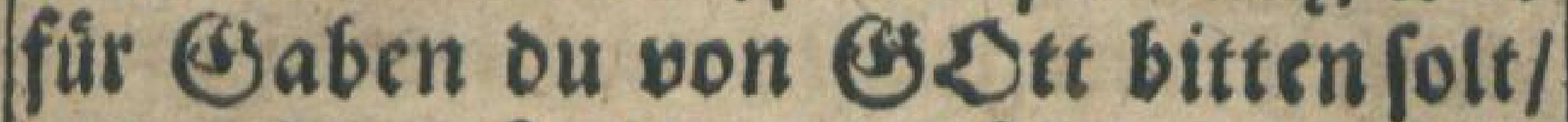
bno mit wajerley onterfchendt. Die yeitliche@saben bitte mit bem bedinge/ Mank.8. foes (S)ttes wille/ ond bir gut und fes s.Joh.s. lig ift. Denn ber allweife Sott weif am beften / was feine sinder beourfs fen / uno ift fo from / Dafer inen feine bofe/fchádlicḩe (3)abe geben will/ ob fie Lue. z3. fchon aus 2 neerftanot folche von im begerten. Die geiftlichen ङ3aben aber/

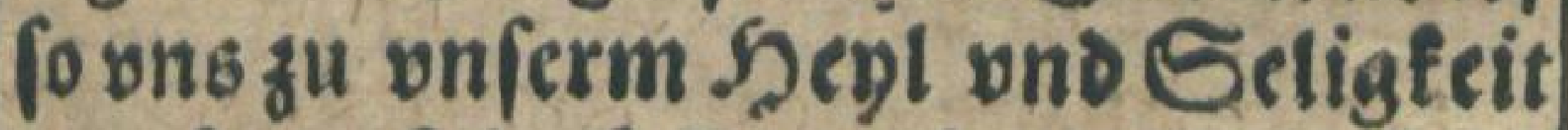
fuge faget fein/ follen wir alo tiebe Rins oer nur getroft / of̧n alle bebingung Mattb.6. von im bitten/begeren/ fodern/als vns fer erworbenes / vnd bereites (Exbgut / nemtich / Dergebung Der Eünen / (3)erechtigfeit / oen Segligen bुeif / yno das Swige Seben.

$\checkmark$ liebe Seele / WBer wolte nicht munter wno luftig fein gu Deten / weil alle onfer Eeufiken gewiflich erhoget

wers 


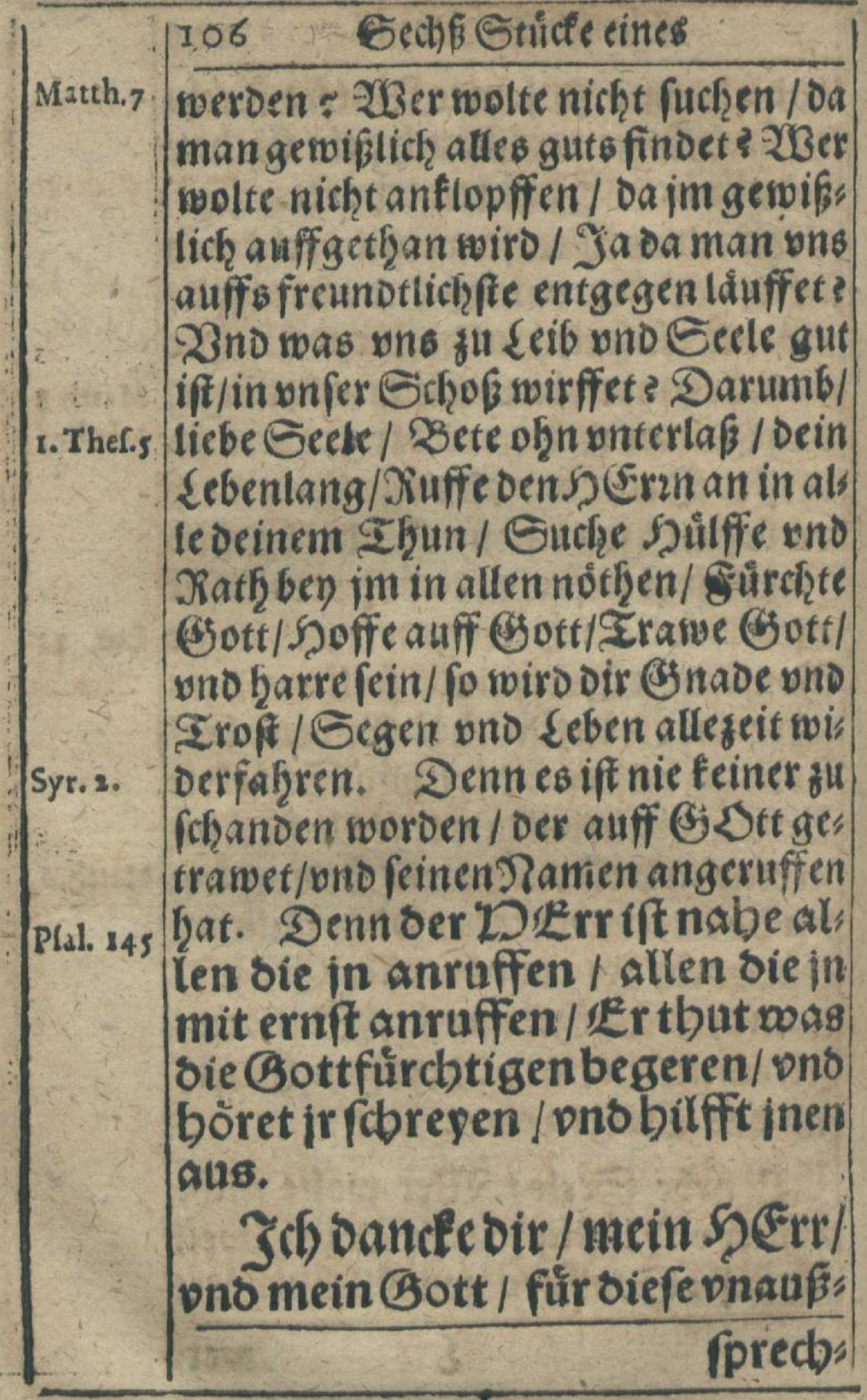


Ebriffichen sebens. 107

fprecblidbe Woltbat/8afís ou vns Ioh. so. nicht allein befoblē baft / beinen

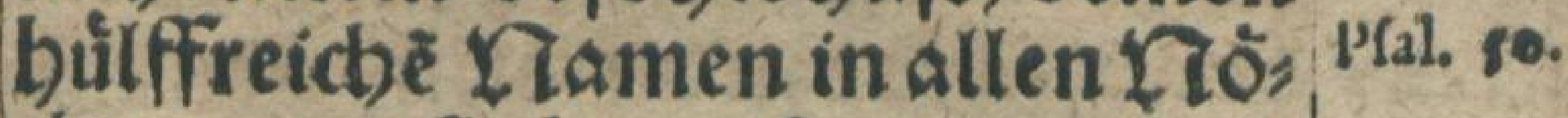
tben anzuflieben / fonsern auch bein Daterliches / liebreicbes Dert3 / fo garbolofeligerkléret/ oẩ ou vns gewif́licb erbören / pno allzeit geben wolleft 3 u rech; Pfal.s45 ter Jeit/alles / was pns berlfam ono gut ift / an seib ono ecele. Jich bitte oich / mein (bott / geus aus vber micb seinen Depligen

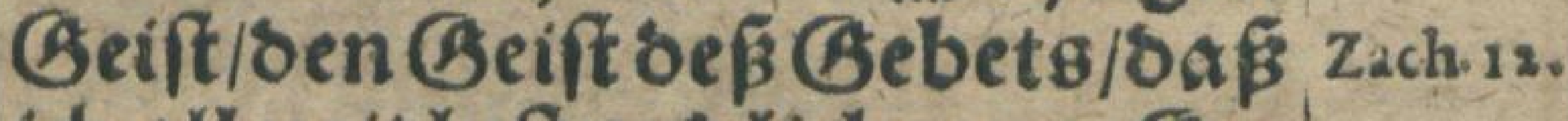
idb allezeit luft ono liebe sum Ge: bete babe / Dno t \&glid) beuge die Anve meines Dertzen/ für bir meinem allerliebften Dater/ber ouber rect) te Dater bift/ Dber al: les / wa as Linser beiffet / im Dis mel ono aufferoen. Dilff oas ich tagglicb binzu trette / getrofit ons mit aller zuderfidbt / im Lla: men meines DERrn Jefu Cbris ftisich bitte / anruffe / anfiprecbe in allen Rlòtben / als ein liebes

Ioh. 14 Id. 16 .

\section{Dhe 48}

Epher. 3.

I 1 ข

atins 


\begin{tabular}{|c|c|}
\hline & 108 Sech ffftict eines Efriftl. Sebents. \\
\hline & zins feinenlieben Dater. Gib \\
\hline & auch/baß̧ich allzeit beilige Den \\
\hline 1.Tim, 8 & se auffbebe 3 uir/obn 3 orn vns \\
\hline & Sweiffel / pno feftiglich trapoe / \\
\hline & oas alle meingebet/ja alle feutf" \\
\hline & t3en/ fo aus meinem Dert3en ge: \\
\hline & Derlevbe mir audb/oaf́ icb / weñ \\
\hline Haba. 2. & sie Duilffe Derseucht / gesult tra \\
\hline & ge/dir nicbt Jeit noch \\
\hline & te auff bein Stứlein / Denn \\
\hline Pfal. $x_{47}$ & ou baft wolgefallen an benen / \\
\hline & Die Dich furchten / ono auff bei- \\
\hline & ne (Situte watted. Xegiere mich) \\
\hline & auch / mein Gott / Dno errinnere \\
\hline & Geift / oẩ id t\&glich ons offte \\
\hline & an mein Sterbfuñ blein geben \\
\hline & cle / mich alle Stunben in guter \\
\hline & Sereitfdafft balte/ons oid) alle \\
\hline & 2ugenblick pmb einen relis \\
\hline & gen 21bphies pon Der: \\
\hline & \\
\hline & as \\
\hline
\end{tabular}




\section{8 fu thun (e) in sirand beitent. 109}

\section{RuB ơterde capitel.}

Bertchtet / wie eit (S6rt4 fen Srenich fich erzetgen foll wenn in B̈ott mit Srancthęt beimfucligt.

23ie fol fich ein Ebriften Menfols erjeto gen/senner eranct wirb?

a) Esele / ob oich (3) Dtt erante Dieuiden erand wato werden laffen / oder plogiclich / ben. ofine Seibes Echrwacḩheit / ab fodern wit. Derhalben ḩabe ich oich po offe ers innert/du folleft deine Suffe nicht fpas Syr. 28. ren/ bis ou frand werbeft / fonbern tåglich ben gefundem seibe/in Staus ben ond guter Berentichafft fehen I Zno bich feinen 2ugenblict in einem folchen Gtande finden laffen/Darinne ou nicht getrawef felig su weroen. Bebendte an ben nárrifchen / trundtes

r. Sam. 25. nen/ 


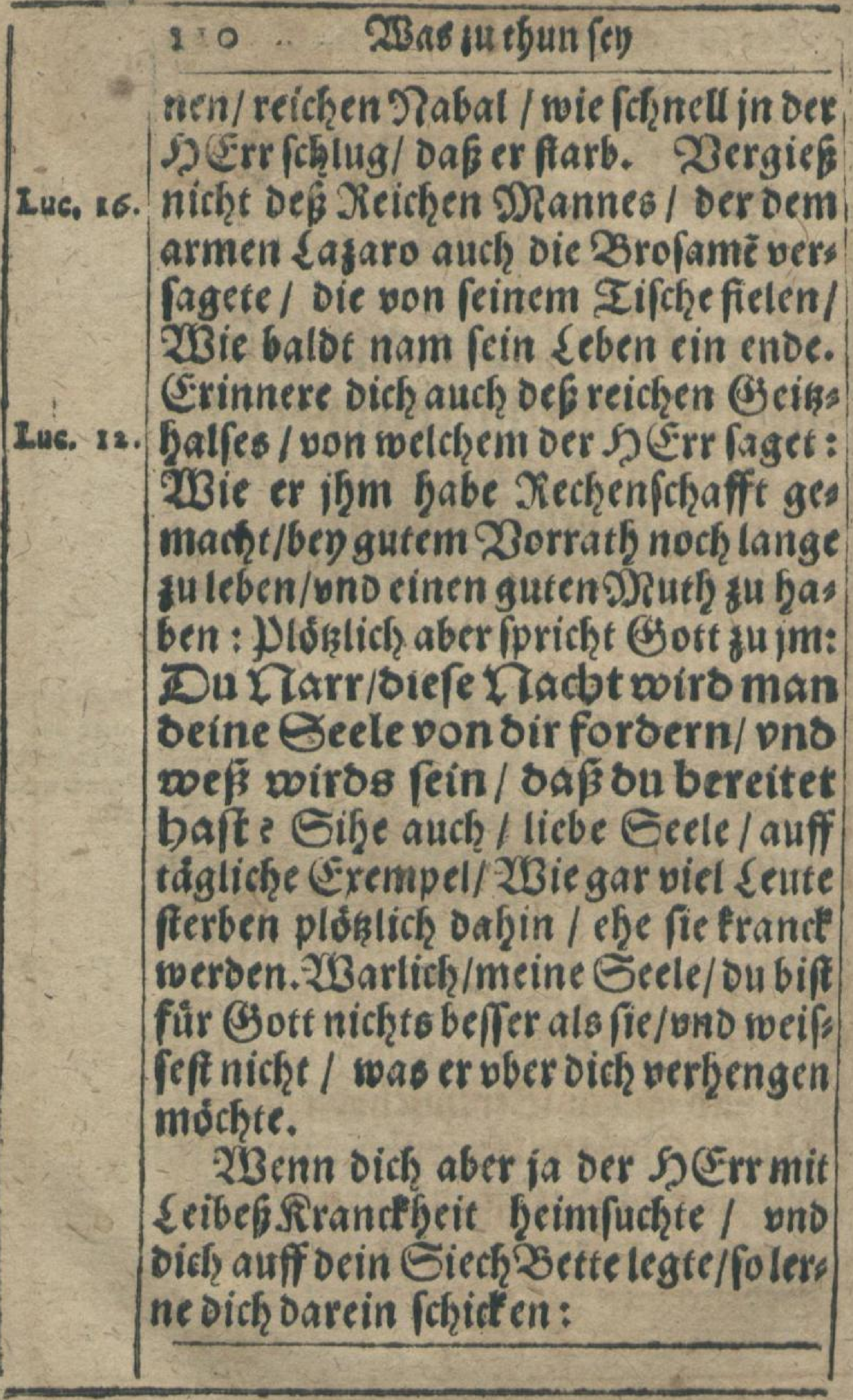




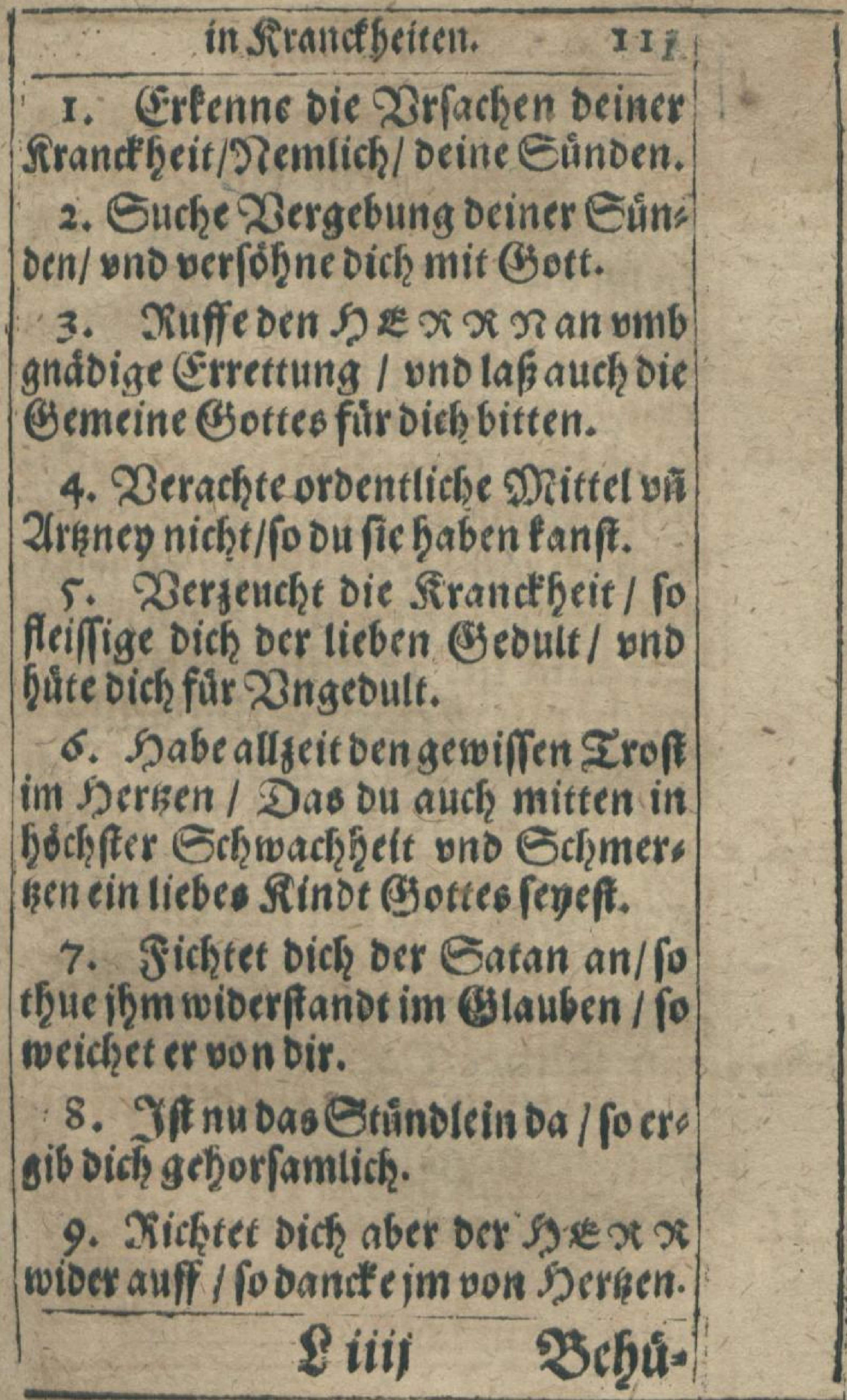




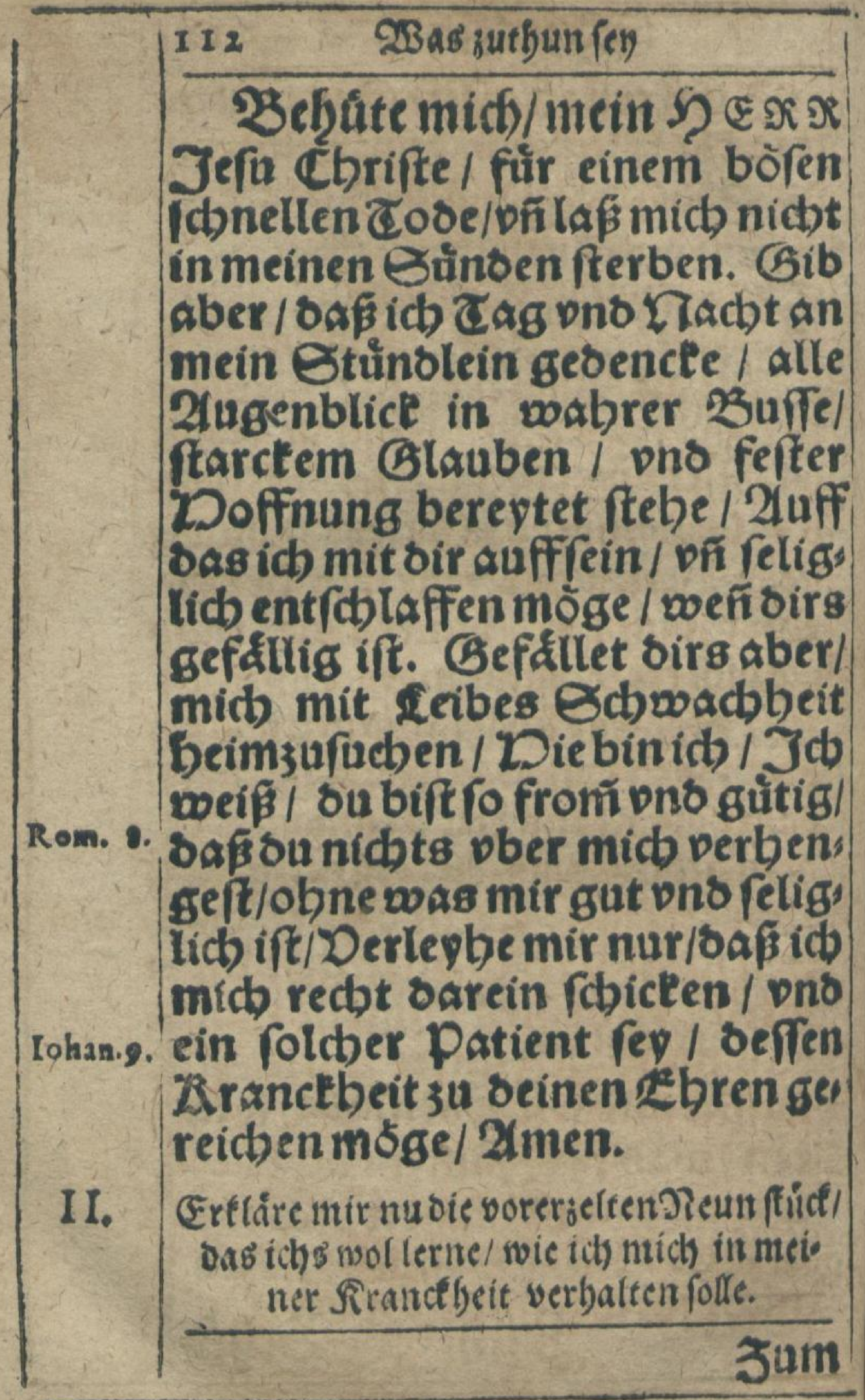


in Strand beiten. II3 it e wol / Sileich wie oer I Dot felber ift Der Sunden Bold/2lifo fint autch alle Siranttheiten Sottes ftraffen/Damit er vos heimfuchet vmb onfer DRiffes that willen. Th ḩue nicht/meine Sees

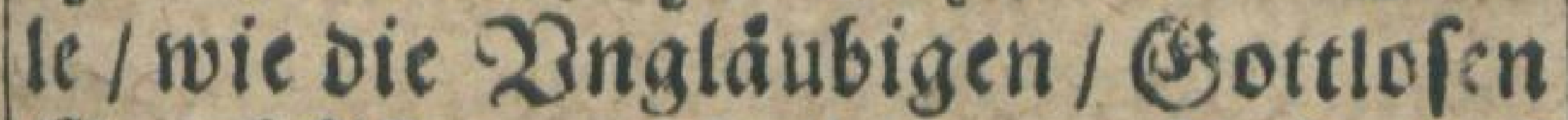

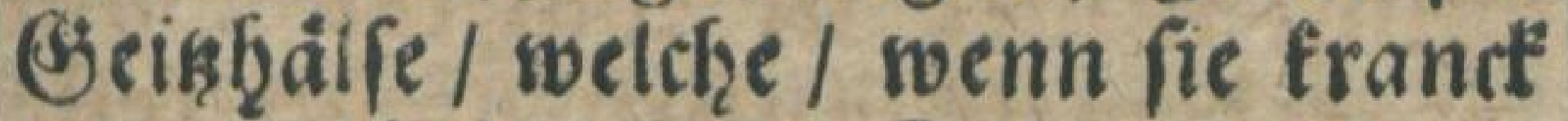
weroen / hecben fie an Beter und D)ior: Dio/ [orgen nur / Das nu iffre ?iafirung liege / vno nichts erwerben tónnen: Soer finnen yno dende en nur/ wo fie irgenot einen bngefunben Biffen gef: fen / ober was foliddliefres getruncten/ oder ifnen fonft was widerfafzren [en]/ Daffer die Rrantefgeit fomme: Son" bern gedend" zum aller erftew/ was ber

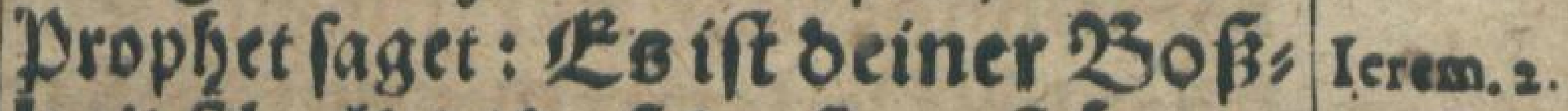
beit fonulot / oaj bu fo geftécupet wirft / Dí beines Dngehorfams/ oaßs bu ro geftraffet woirft/ 2llfo muftu jnnen woeroen/ vno erfab; ren/ wo as für Jamier pno DDert3" leyb es bringet/ben DD ocinen GDtt Derlaffen / ons in nicbt fürdoten/fpricbt ber "DERr a v . buter Rrandibeis ten fino (B) $2 \geq 3,8$ ฬiucben. Kow. 8. Kom. 8. . 3 


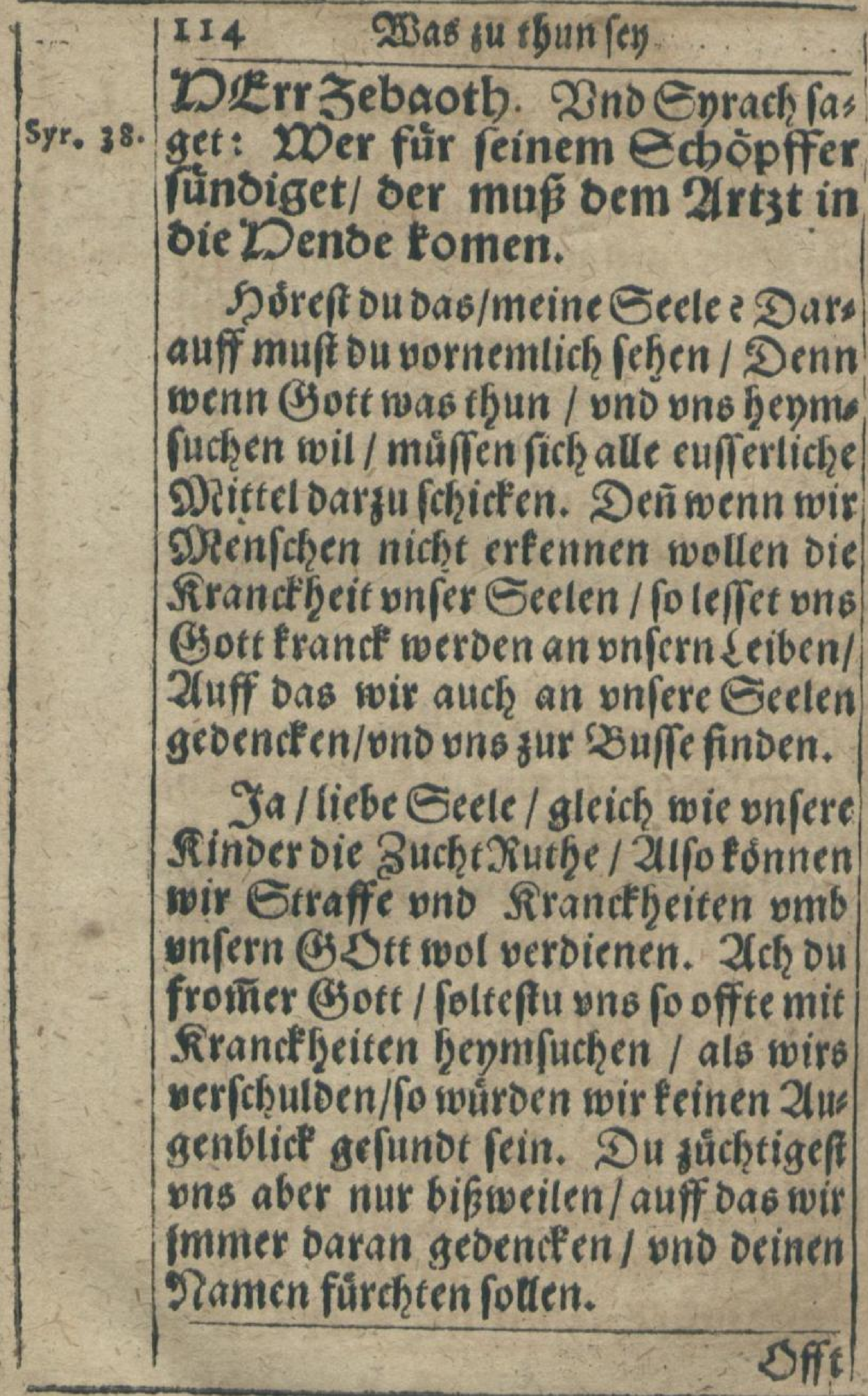




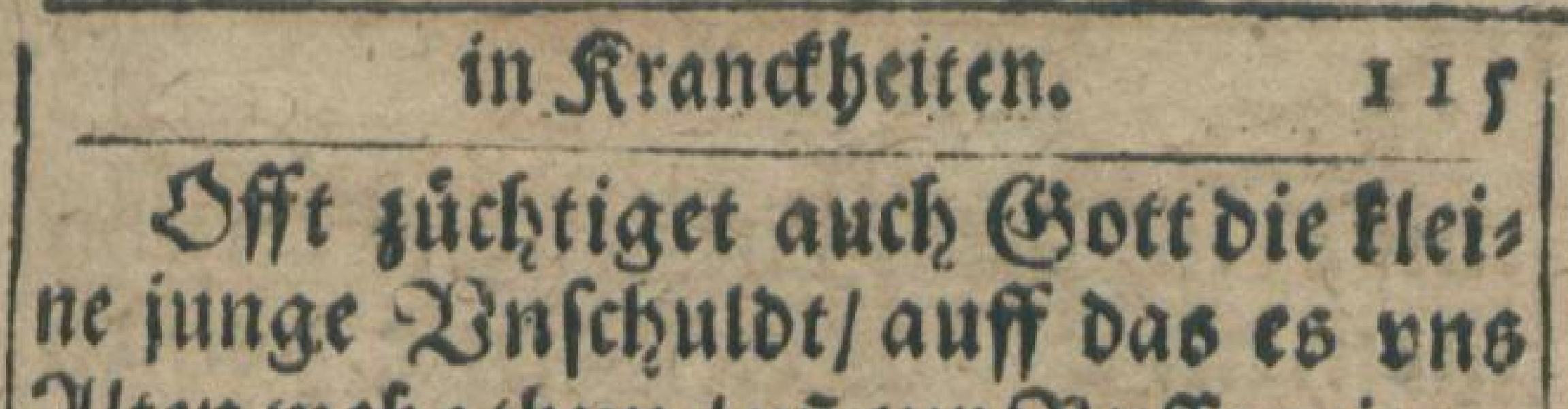

Zlten wef̧e thun / vĩ zur Buffe reizen fol. Eife/meine Geele/alfo find die 2. Sam. Rirandflgeiten gleich wic Bothen won Gott gefand/Die uns heiffen vonEan: ben laffen / vno Buffe thun / Daß wir nicht in vnferen Eunden verderben.

Dierdie nur/liebe Eeele/wie ficher die Menfehen in Tag leben / oie fein Ptal. 39. Creuke/feine Sirandtheit haben/ Sie achten weber Sottes 2Borts noch fei: ner Sacrament/23ergeffen def(Stau= bens an Sfrriftunt/ ono oeślieben Bes bets. Zno alfo ftefen oenn ire Eeelen in frochfter gefahr. Denn je weniger ein Water juchtiget / Je mef̧r ber Rinder 23 ngefforfam vberbano nim: met: 2lifo iemefre CBefundot und 2rBols farth oie Dienfehen haben/ ieficherey fie dabey werden.

ЭЕ $x$ Jefu Şrifte / Sie liege idb in meiner A ranckbeit/ ono ertenne / oaj foldbes oein Déterlisber Doille ift. Denn fo 


\begin{tabular}{|c|c|}
\hline & Trab gutbuntey \\
\hline \multirow{7}{*}{$\begin{array}{l}\text { Luex } 2 \text { I } \\
\text { Matt. } 10 .\end{array}$} & mir lein'Daar entfallen fan von \\
\hline & meinem Dofupte / obne seinen \\
\hline & Willen / Wie folte ich benn bie \\
\hline & $\begin{array}{l}\text { ede } \\
\text { Derlieren/wenns nicht bein Dil }\end{array}$ \\
\hline & le were ? Eibe/ mein GDtt / Du \\
\hline & idbtet \\
\hline & wie ou in beir \\
\hline \multirow[t]{2}{*}{ Psal. 22 . } & einDDurm/ono nicbt cindDen/cb \\
\hline & $\begin{array}{l}\text { alfo baft st } \\
\text { ebnlich aem }\end{array}$ \\
\hline \multirow[t]{4}{*}{ PSal. 39.} & wenn ou einen Srenct)en zuct \\
\hline & tigeft / vuib fei \\
\hline & len/ fo soirol \\
\hline & zefret/swien \\
\hline \multirow[t]{3}{*}{ Pfab 119} & er Juidbtigung \\
\hline & $\begin{array}{l}\text { vif / ठxil } \\
\text { Wein }\end{array}$ \\
\hline & \&iebbaber \\
\hline \multirow[t]{2}{*}{ Prar. 37} & berDDenfben/Die Sd)lage aber \\
\hline & oe \\
\hline \multirow{2}{*}{$\begin{array}{l}\text { Rom. } 8 . \\
\text { Apoc.5. }\end{array}$} & $g_{0}$ \\
\hline & \\
\hline
\end{tabular}


in Siranceffetiten. $\quad$ II 7

beit sum beften sienen werbe. Prov. 3.

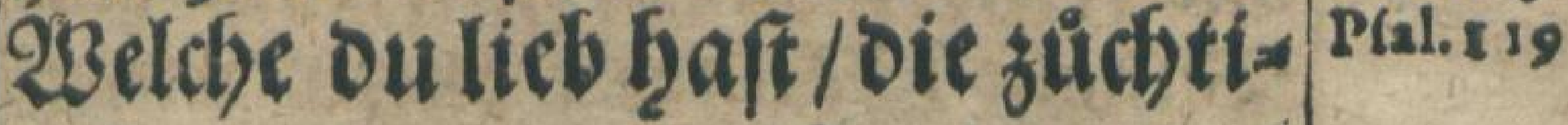
geft ou. Darumb ift mirs gut / Daßs ou michs zuchtigeft ono be= mutigeft / auf Das id ocine Rectyte lerne. Doilf/ mein Gott/ baf ich mein febenlang an fol:

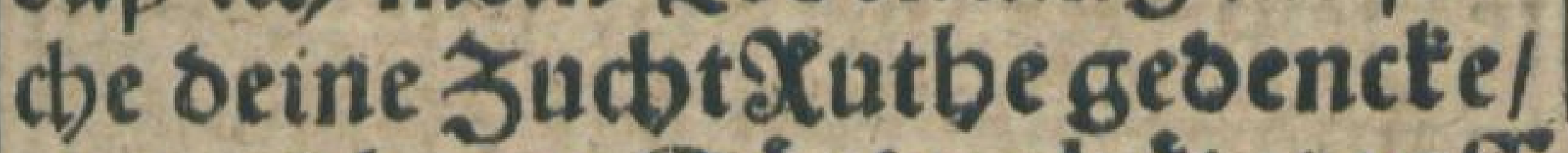
vno mich vor Sưnoen buite/ auff Iohas.s. oas mir nicbts árgers wiber fabs: re/ Amen.

Jum 2/nbern/2Beil bu nu/ liebe Eecte/ an Deiner eufferlicten Srand"; Theit ppireft / Daß beine Eecle in $2 \mathfrak{n}$ : buffertigeteit frantd lieget / So thue für allen Dingen Darzu/Dáz deine Eees le gefund weroe. Die Ëefundheit aber der Seelen/ift wahre Buffe/rechs ter (ङlaube an Jefum Efrriftum/burch welchen bu erlangeft 2 ergebung beis ner Sưnden / vin einen gnádigen 203 ter imt Shimel. Dern ourch den BSlaus:

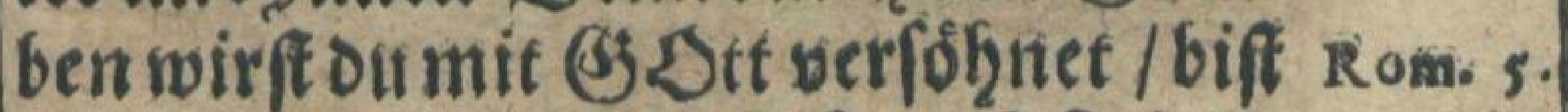
furr im gerecht/ und befindeff Sriede

II.

Doufie th ber Seetio gefinabbeles 


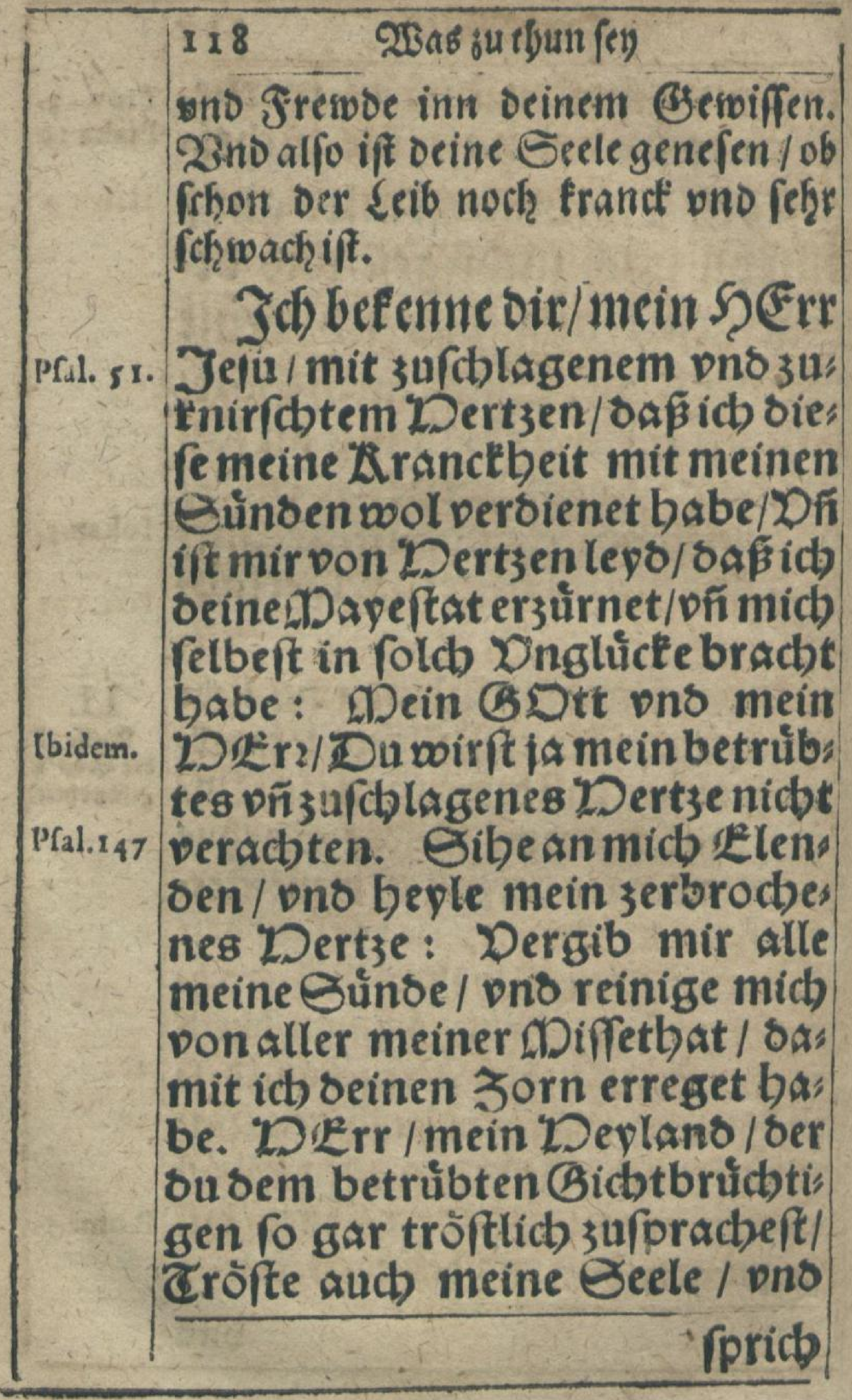


forich mir in mein Dertse bies nein sifí freesoenweiche wort: Sev) getroft / mein Rintot / Dir Math.9 fino alle bcine Sunde vergeb $D$ wie lieblich / wie trōfitid ift bas 2Dort meinem 'Dert3en/wie wol erquicket es f cib ons Seele/ LDarce uno 2 zeine. Ja DERr Jefu / wenn beinbeyliges Dort ono Gnabenverbeiffung mein Ẽrofi nicbt were/ fo muift ich per: Pfal. ix, geben in meinem selense. Id)

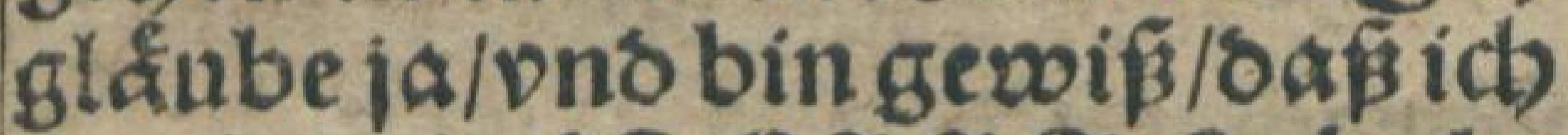
ourcb bich / D DE $x$ Jefu/ babe Dergebung aller meinereưnoèl Sin mit meinem (6) Dtt wols ran / pno ein Kzrbe ber epoigen Eeligteit/2Imen.

Jum Dritten/ Ruffe auch Den S) $x x x$ bcinen (jott bmb rettung an/snd flefge ifn/Das er dich wider ges funb inache. Denn fo fpricht Enrach: Drin Zinst / 20enn ou tranct Syr. 38. biff / fo verachte oif nicbt / Sons bern bitte sen DDRerrn / fo wiro

III. BDote for man ynib retcung btes terio. 


\begin{tabular}{|c|c|}
\hline & \\
\hline & \multirow{14}{*}{ 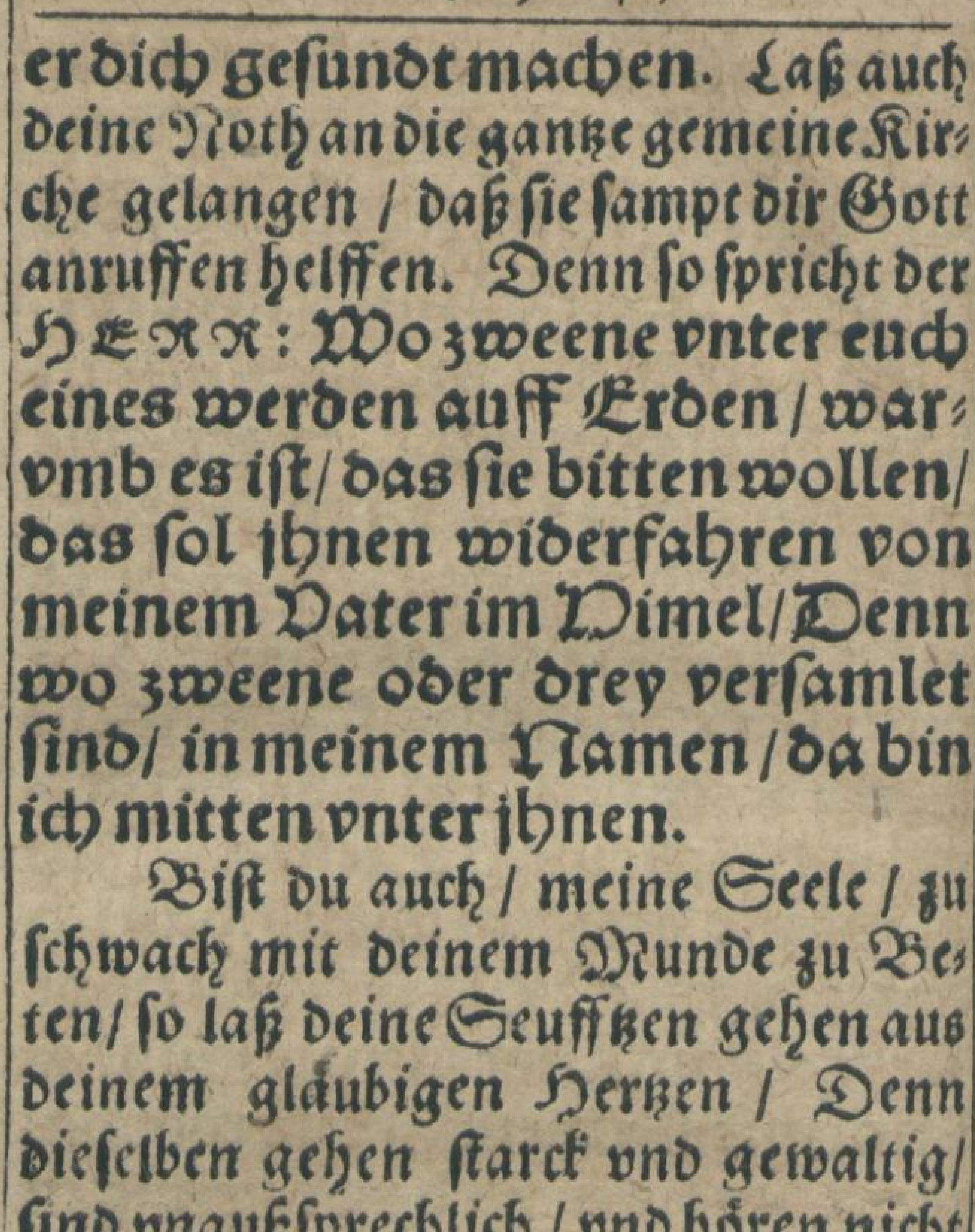 } \\
\hline & \\
\hline & \\
\hline & \\
\hline & \\
\hline \multirow{8}{*}{18.} & \\
\hline & \\
\hline & \\
\hline & \\
\hline & \\
\hline & \\
\hline & \\
\hline & \\
\hline \multirow{6}{*}{$\begin{array}{l}\text { Rom. } 8 . \\
\text { Syr. } 35 .\end{array}$} & \\
\hline & 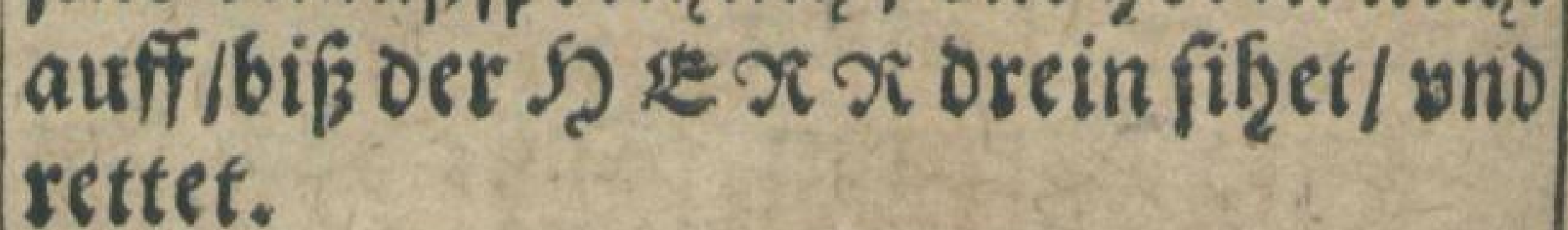 \\
\hline & u (Strifte/Du \\
\hline & $\begin{array}{l}\text { baft beinen } 230 \text { then } 3 \text { u mir ge: } \\
\text { faclt I Wemlich I siefe meine }\end{array}$ \\
\hline & $\begin{array}{l}\text { Aranctbeit / pno mid sur } 3 \text { uffe } \\
\text { ermabnen taffen. Eibe / mein }\end{array}$ \\
\hline & \\
\hline
\end{tabular}


DERrr / Jchertenne beinen gus ten 20 illen / bin geborfam / ino betere micb 34 sir. DDein (BDDtt Icb renbe sir woiber einen 230 ; tben / fremlid, / mein armeg (bes bet/Dñ meinEeufftsen/D DIErr nim mein fleben an/vîl hấ mein Beufftzen für bicb tomen/Befets let oirs / mein Lerlōper / pns ift mirs gut vno felig / bapicicb leben fol/Re fo ricbte mich auff / pno bilff / baf es ein newoes / gefuns oes / Cbriftlicbes ₹ Reben fey / biß an mein $\mathcal{E}^{2}$ be: Gefeltet oirs nicbt / baj icb lebe/ fonbern fol rein oas iznoe/LEy fo fey es ein fes liges En noe/Bo ḱom DDErr Jefu bebenoe / ono nim meine Oecle inoeine 'Denoe/2Imen.

Zum Dieroten / Siabe Gecle / iff bir erteubet oroentliche 2(rinney vno D) ittel zubrauctzen. Denn fo fagt ber weife Miann ferner: Darnadb Lấ ben 2Art3t zusir/bennoer DSRr bat ibn gefdbaffen. Dnolấ ibn

IIII. Drbentile. de Sortter nitoe ju: verabern. Syr. 38.

$$
\text { DD nicbt }
$$




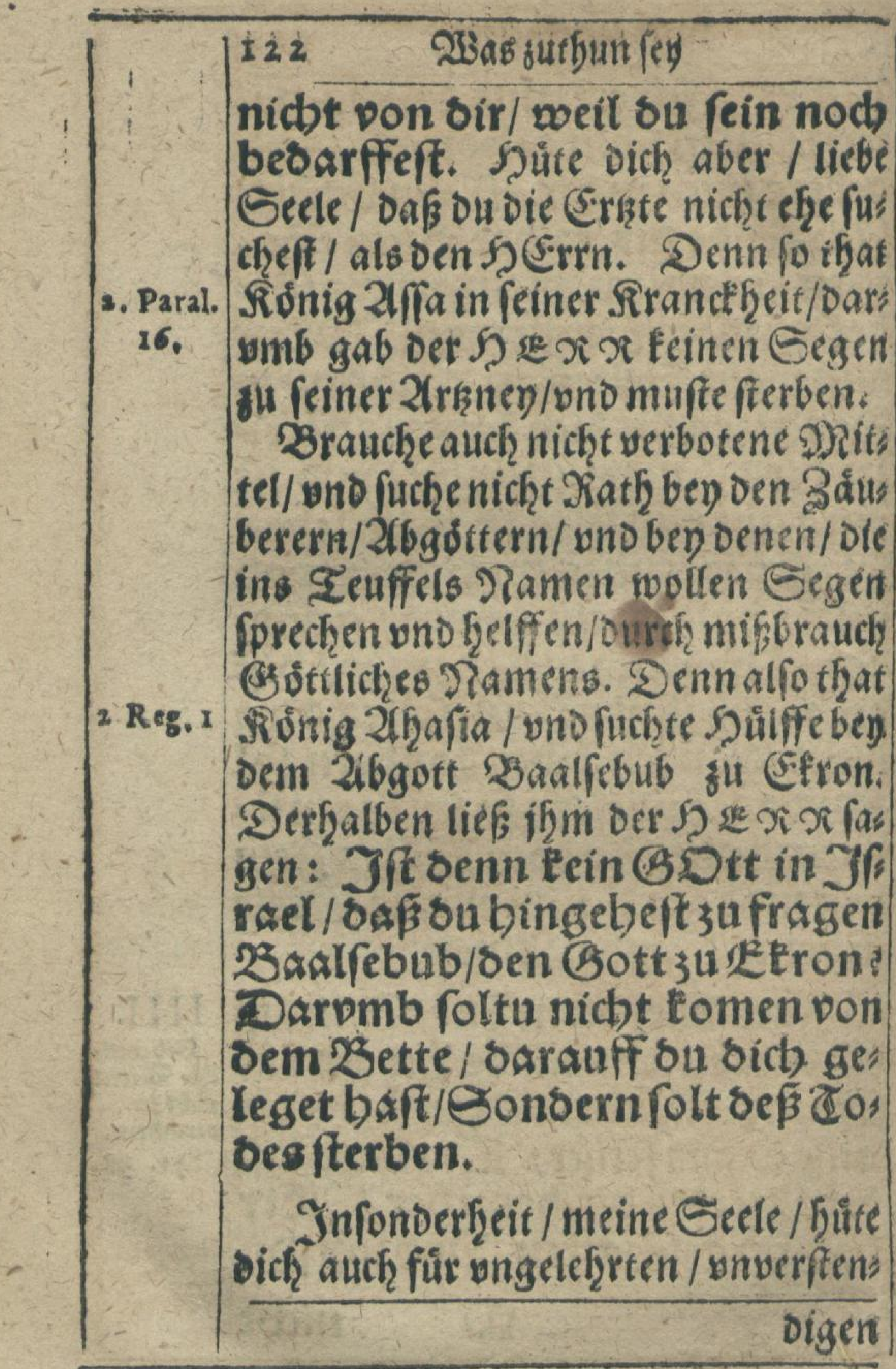


bigen Srrzten / Die Peine Runft geles net hiaben. Denn der Seib dé פ Mens chien ift eine ede Sreatur (S) ottes/vno folin s:frren gebzalten/ono nicht bers warlofet werden.

Idb Dande oir / mein Şerr JE fu Chrifte / ou trewoer 2Art3t Exod, is meiner Eeelen / oaf́p ou meiner trancken Eeelen gebolffen/ von Eùnben gereiniget / pno mein

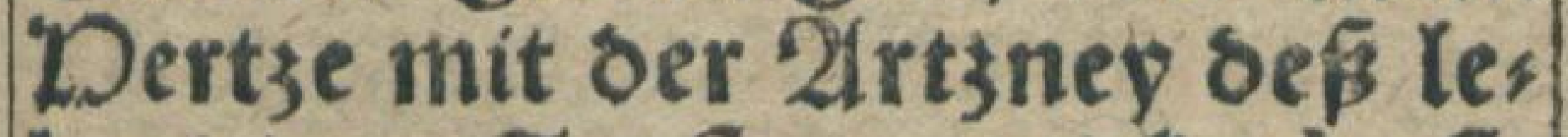
benoigen Trofts erquicket baft/ उ(b) bitte oid) / D su Ecböpffer aller binge / regene aucb nu biefe eufferlicbe 2frt3ney pno orbentlis: de LDittel/bie su geî́chaffé baft: DErr / So ou wilt / Eónnen fie Matth.8. mir bevlfam fein/ ono zumeiner Gefunobeit bienen: Oo suaber nicht woilt / toònnen fie mir nicbts fromen: Derr/bie bin icb/f(b)afs fe es mit mir/poie oire löblich/ pñ mir gut pro feliglicb ift/2tmen.

కum fưnfften / siebe Eeele / fleiffige bich ber lieben Bsebult / uno

y. Bebute an edet strane in Strands \\ Ni is bittelbotio}




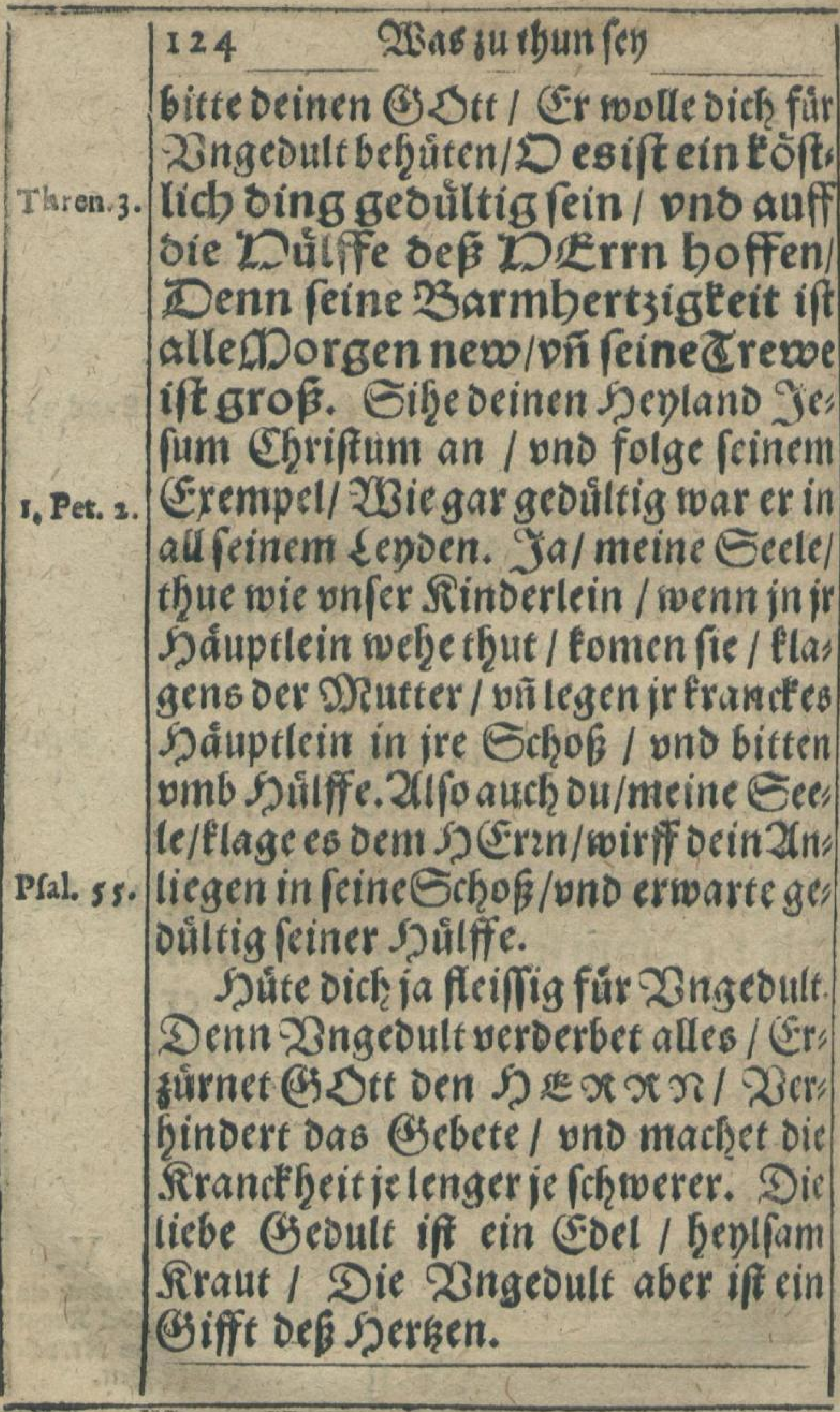


Nim weg von mir S马 Ex Jefu/ bñ tilge alle Dngeoult aus meinem Dertzen / Szebuite mich allezeit / baj fie nidbt vberbanot neme in mir / 2uff oas ich mir mein Creutz nicht gröffer macbe. Pflantze mir aber oie liebe chos ne Geoult in mein Dertze / oar ibb beinem Sexempel folge / ftille pno geouiltigfey / pno auff oeine Dúlffe voarte. Xùttele beinen Zeyger/fẩ tomen beine Stun: be / Denn beine Žit ift sie rechte Zeit / ond seine Etunde bie rech: te Etunde. (Gib oas ich ftets bof" feond ftarclefey / in gewoiffer J verficht / Du bift getrewe / vns leffeft teinen aDenfdben verfucbet 1.Corin. 10. weroen $p$ ber fein vermògen : $\mathbb{Z}^{2}$ fo rchaffeaucb/mein'D E rr Jefia/ oấ alle mein Creut; bî X ranct. beit fo ein enoe gewoinne / baj id) s tönne ertragen / Z b weip / ou wirfts nicht laffen/zimen.

DD iif รับ 


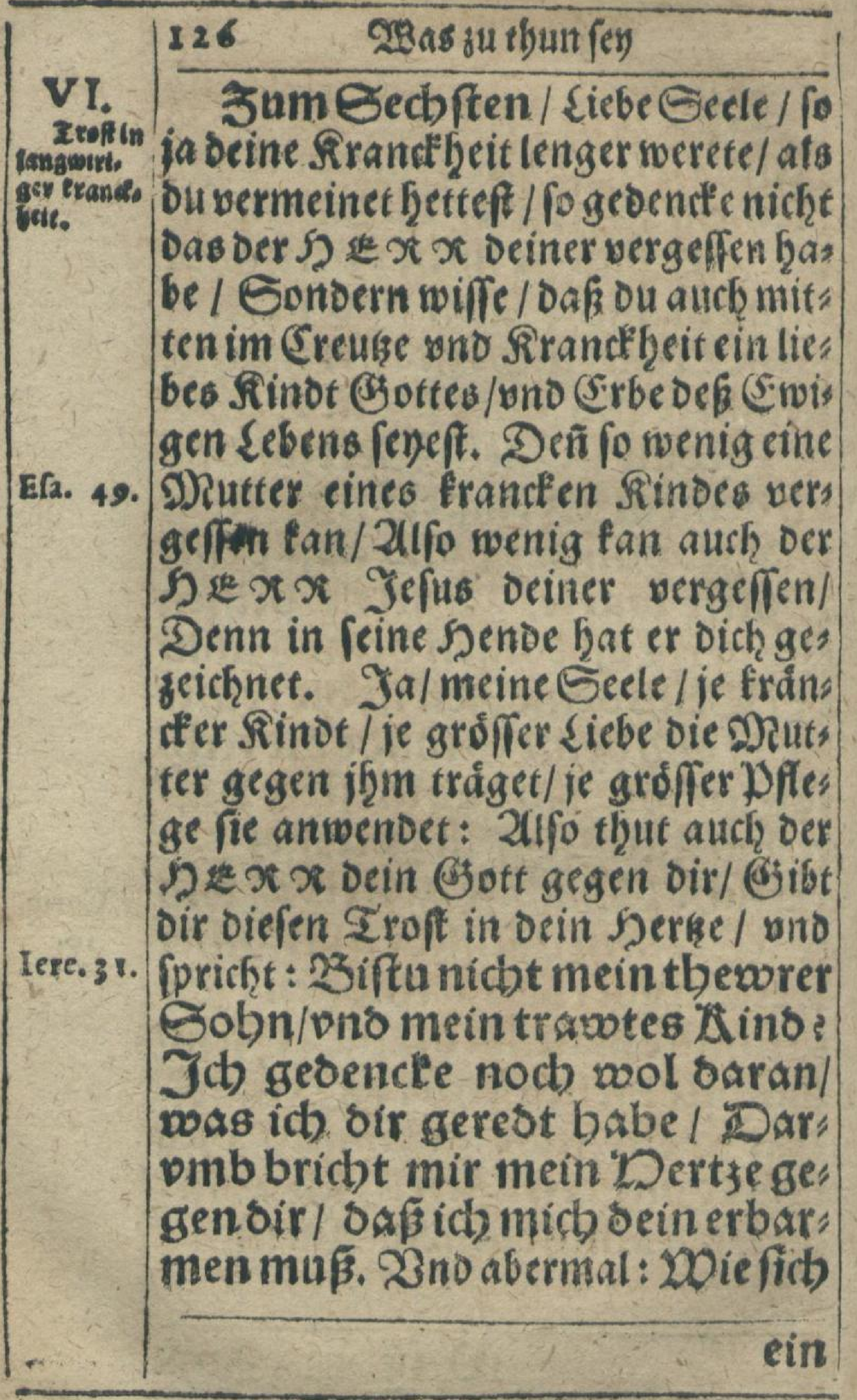




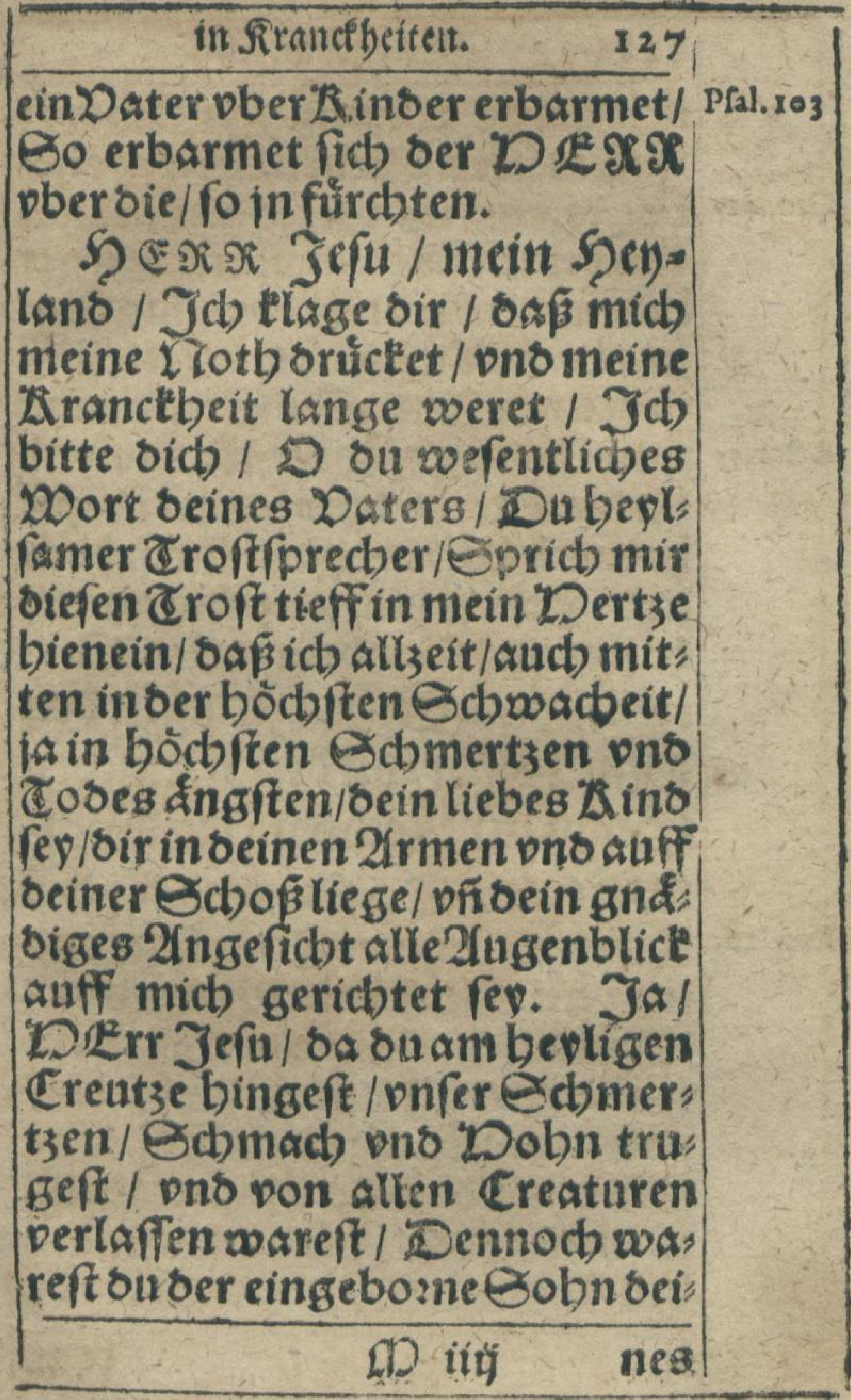




\begin{tabular}{|c|c|}
\hline & Sgsas fu tbun fen \\
\hline & $\begin{array}{l}\text { nes lieben Dimlifben Daters } \\
\text { Bift es aud blieben. Daberlies }\end{array}$ \\
\hline \multirow[t]{5}{*}{ Gen. 4I. } & be Jofeph im Gefengniés lage/ \\
\hline & Dno fein bruncelin Denfblicber \\
\hline & bulffe abe/wareftusennocbmit \\
\hline & im/ batteft in lieb / Dno retteit in \\
\hline & us \\
\hline \multirow{7}{*}{ Attor.7. } & e gerubre \\
\hline & berumb floben / batteftu in bocb \\
\hline & lieb / gabeft sich im su fawwen / \\
\hline & oxpermit frewbenreichen Der \\
\hline & tjen fagte: Sibe / Jablebedn \\
\hline & Deintel offen / on def Syen/eden \\
\hline & Solngur Kechtenpanoesot \\
\hline \multirow{9}{*}{ Iuc, 16.} & fenen. \\
\hline & Reicben MDannes o bur Lag / Dnd \\
\hline & Dorensennod seine $\mathcal{E}^{2}$ naelein \\
\hline & pmb in ber/Lamen feine Šeele / \\
\hline & ons fubreten fie in 2 brabams \\
\hline & Scboß̂/in oas E wige Paraøei \\
\hline & Sott/? \\
\hline & \\
\hline & \\
\hline
\end{tabular}


in Sirancffeiten.

fe an beine abuir mit meinen Eeufftzen: Die noth ift wool gar gro $/$ mein DDKXXK / oer Ecbmertzen fins viel / pno sie 2lngit wil mich Dberwáttigen:

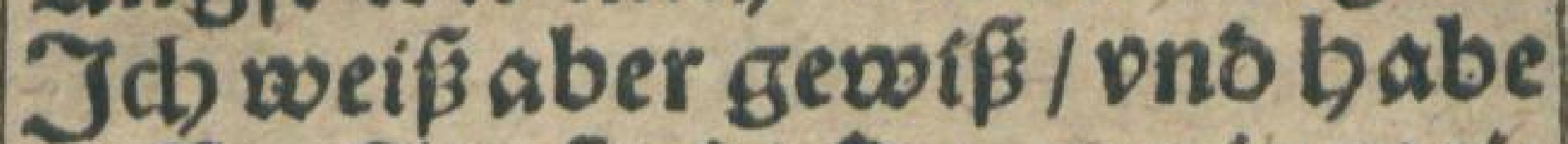
oefien caufenot Zeugen in mei: nemDertzen/Ourcb beinen Dey: ligen Geift / ba B ou micb nicbt wirft verlaffen/Deine2Augenfte: ben Tag vno nacbt offen vber mir / I d bin bein liebes LAinot / ons ein IErbe bef Kzooigen fe: bens / Derbalben bift ou ia mit mir in siefer notb / Du woirft Plal. on mich aucb berauß reiffen/ pns 3 u Ebren madben/3u diefem oder jes nem \&eben/ woie sirs gefátlig ift/ Daßjift gevoißłticb war/2men.

Jum Siebenoen/ siebe Seetel wiro Der Gatan fchwerlich auffen bleiben / wiro dir sufereen mit feiner Ephef. 6. Taufendtfunft/ yno dein Şerke mit feinen fewrigen Dfeglen berwunden. Shie if gar nicht gu rathen / Dak ou

SDe Difí

VII. Infects sung. 


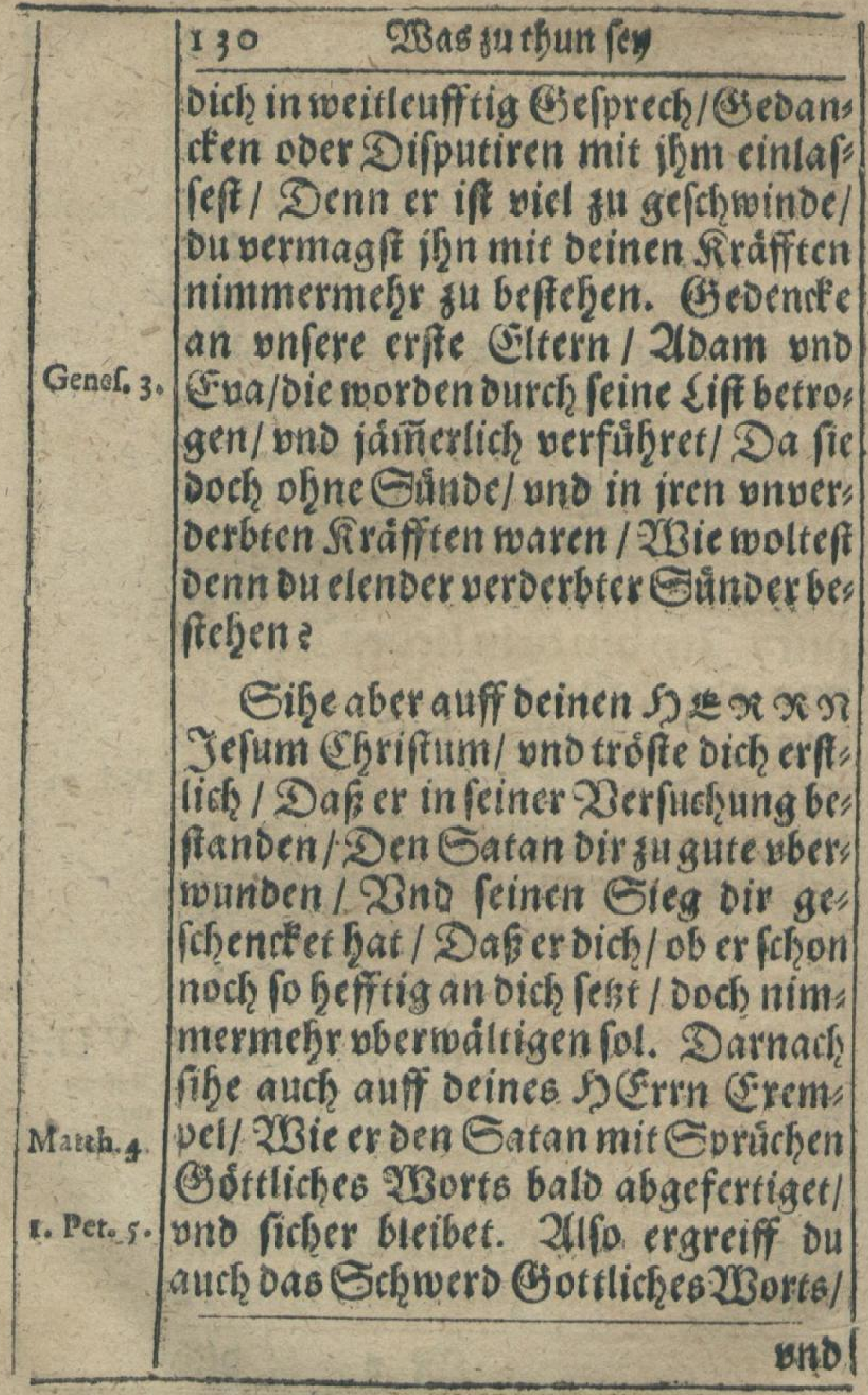


in Sirandbeiten. I3 I

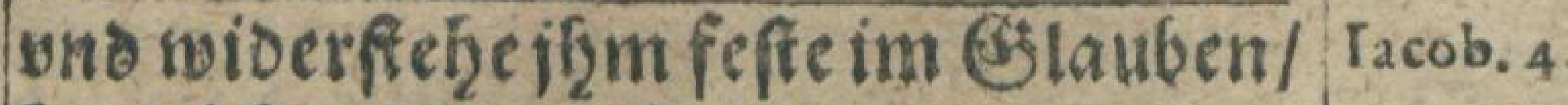
fo weichet er onon bir.

Eprichter / Deine Sunden find groffer / Denn das fie dir mogen verge" ben werben: So fehlag ifn mit Dem wafzren 2Bort Bottes / Erfreye und erfigle ofin wenterlaf: Duleugeft $S_{a}$ tan/Denn fo feflet gefchrieben: DO bie Eunde makbtig worben ift/ Rom.s. ox iftsoch bie (Bnabe viel måc): tiger worben.

Eprithter/Ëhriftiogerbienft getzet bief nichts an/. Du gefioreft nicht in oic 3afitoer 2ufserwelten / Go fchrey ofin onterlak: Duleugeft Gatam/ Denn fo fpricte mein fase $x x$ Jefus: Aommet ber 313 mir alle/ bie ibr muberelig ons belasen revo/Z̈h wil eucb erquicen. 2Nno abermal: Doie fie in 2 som alle fterben/21: fo werben fie in Cbrifto alle le: benoig gemad, t wersen.

Macher er Dir Den Jorn (e) Ertes gros 1 Eprich ofin unterlaf: Ziro lohan 3 bat GDett oie 2Delt geliebet/2C.

Matt, II.

1.Corin 


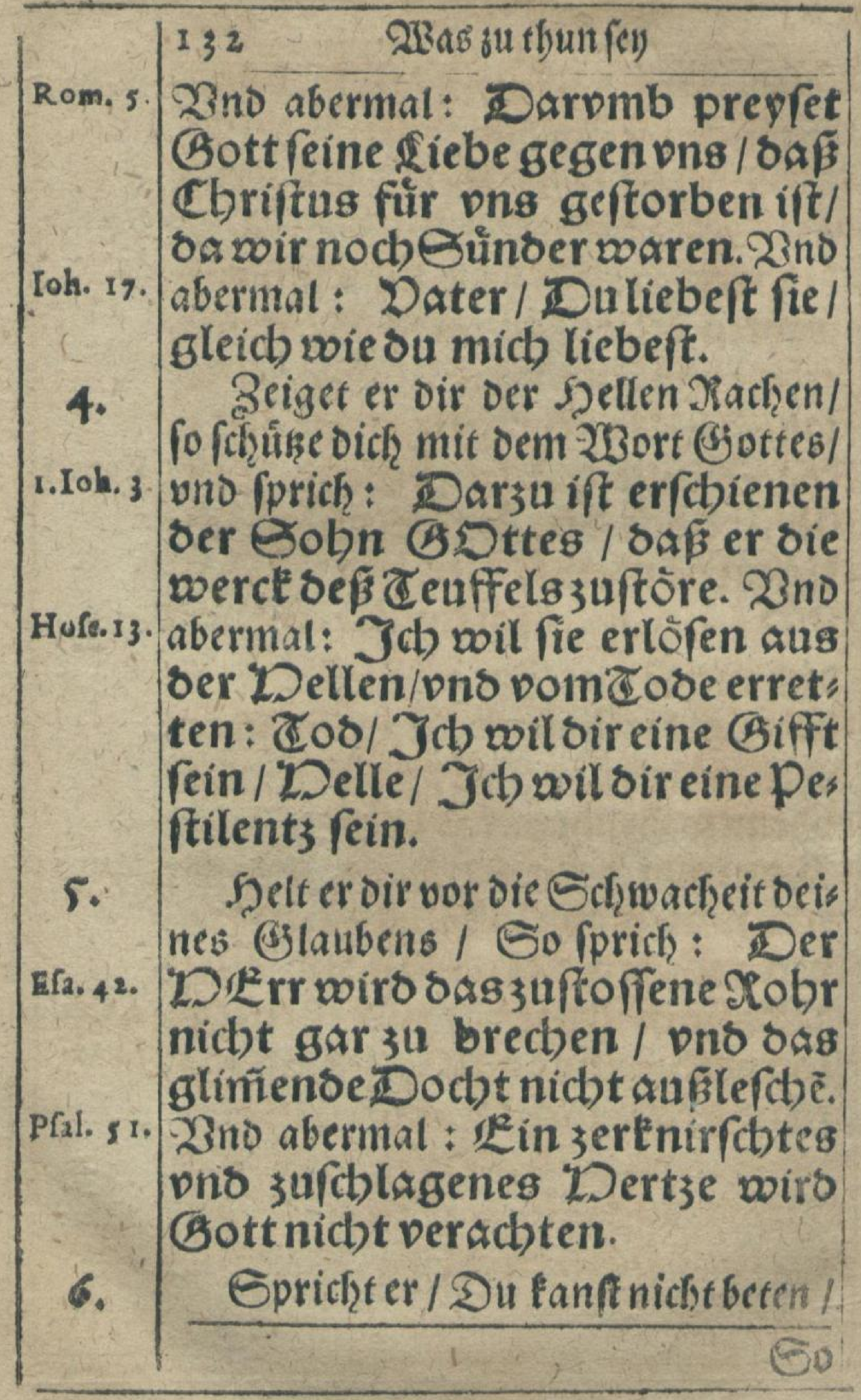


Eo antworte: (5o fo fan ich feuffizen] ono weis gewís / Daf folche meine Rom. s. Geuffigen vnauffprechlich fein / ond alle 2 Bolde en ourdizoringen.

Sprichter/Deine Srandfezeit und Srents weret lange/(5ott bat bein ver" geffen / Sprich: Za an aud eine Efa.49. WDutter ires Linses vergefien / oafi fie fich nicht erbarme vber ben Sobn jres Reibeg: Dno ob fie seffelben vergeffe/So woil ich sodb bein nicht vergeffen/Eibe/ In meine Denoe babe ich oich gezeidbnet/fpricht ber 'DSErr.

Gaget er/ Du muft verfermachten in beinem 2trmuth/ vno bift von jeoers man verlaffen / Eo fprich : Dater ond butter verlaffen mich/aber 7. oer DiErr nimet mich auff. Zino abermal : 2Denn ich nur meinen psal. 23 . Derrn Jefum Cbriftumbabe! fo frage ich nicbts nach Dimel

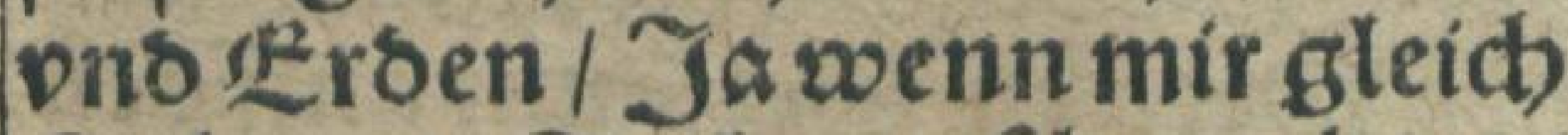
Seib vno Seele ver(d)machtet / ro bift su sodb Gott allezeit mei: 


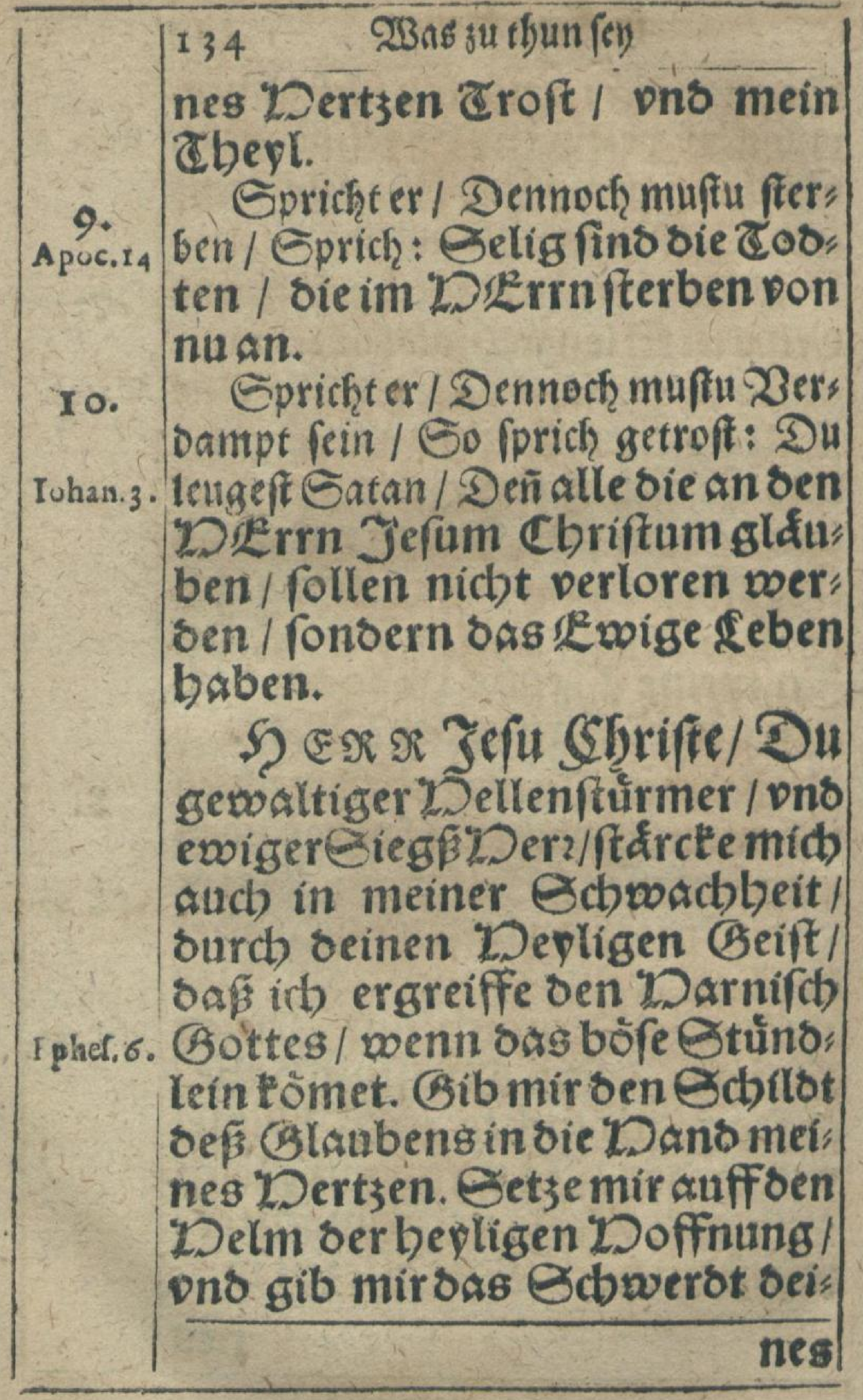


in Sirancefbeiten. -135

nes 20 orts in mein Dert3e pno Duno/Zuffoas ich recten 20i: berfans tbue/Die fenrigepfey, le bef̂́ 2 söfewichtes auflerche। भilles wo ol aufrichte / Daf felot ons sen Sieg bebalte/2Imen.

Zum 21dten vno Reunsten/ siebe Seele / fo fife auff Das enoc dei ner Sirandefeit. Sifeftu/Daf Deines sebens nicht mefir fein fol / Socrgib

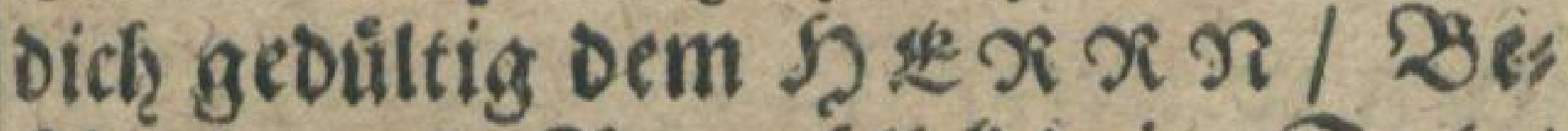
fight ithm alle Alugenbritt beine Eeele/ Zno prich mit aller Buverficht / ja peuffic mit allen Sráfften: "DEE SR Jefu/nim meinen Geift auff / Eo bif ou gewiflich, onverloren / onno twig felig.

Xichtet er bich aber wiber auff/ po (en) ifm dand bar bein lebenlang / mit Serken/23orten vno 2Wercten: 2 no

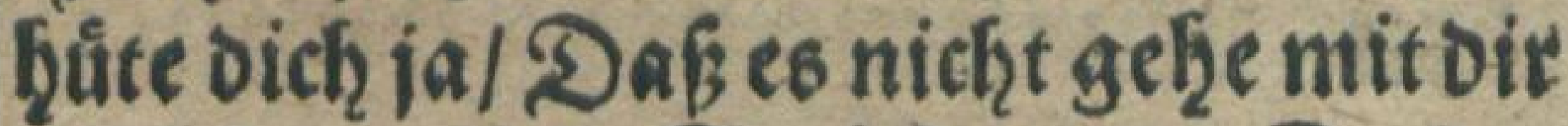
nach oem alten Eprithwort: Da Der Rrand'e genaf / warb cr årger / Denn er vor was. Sonbern gebencte ant oas Wort bef Sy $x x x /$ Da erfpricht:

- Sibe

VIII. IX. zobe oove scbun.

A\&tor. $\%$ 


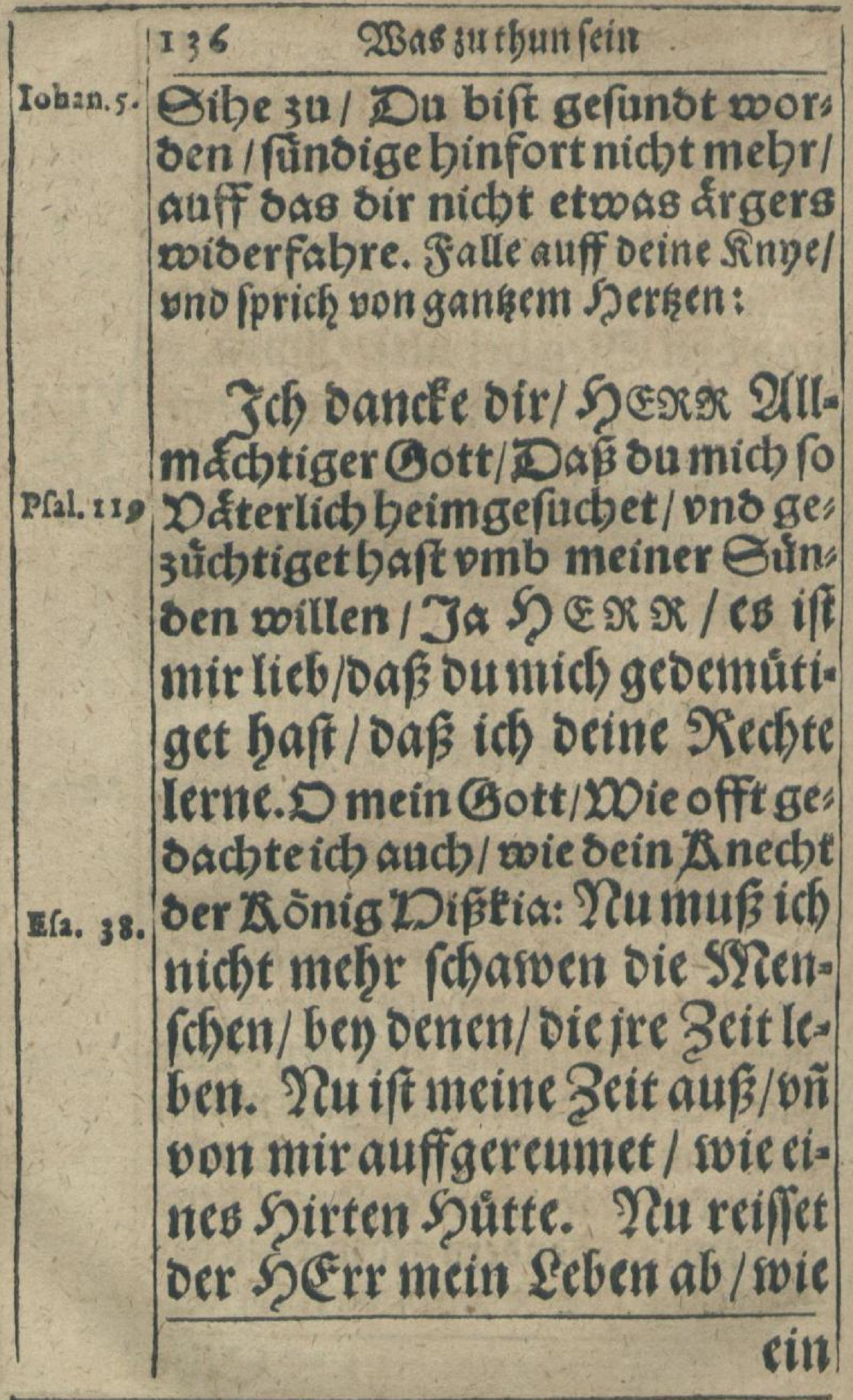


ein $2 B$ eber/ ono macjet es mit mir aus/oen Iag vor 2lbenos.

Jd) rebe aber/mein Gott / baj meine Â ranckbeit nicbt sum đos Ioh. 2x. be gewofenift/ fonbern 3 ur KE bre tohan.9. Gottes / baß ou mein D U $x$ Jefu (brifte oaburd) geprepfet werbeft. Denn ou baft bich mein erbarmet/ ono oich mei= Esa. 3 s. ner Eeclen berklitich angenommen. Du baft alle meine Sünoen hinter ocinen Plúden ge. worffen / pns mir mein seben gefriftet. Doiewol baft su meine a ranclibeit gewenoet / pno mir fo gnábiglich gebolffen.

Jcb bancke oir/mein (Bott/ ia id) oancke oir bon gruno meines Dertsen / Dấ ou mich wioer auffigericb)tet bir geftárctet baft/ Daji id) bas (bottes "Daús wi: ber befictben/ meine DDege ons Eteige geben / vno mein đbun

$$
\text { in vers }
$$




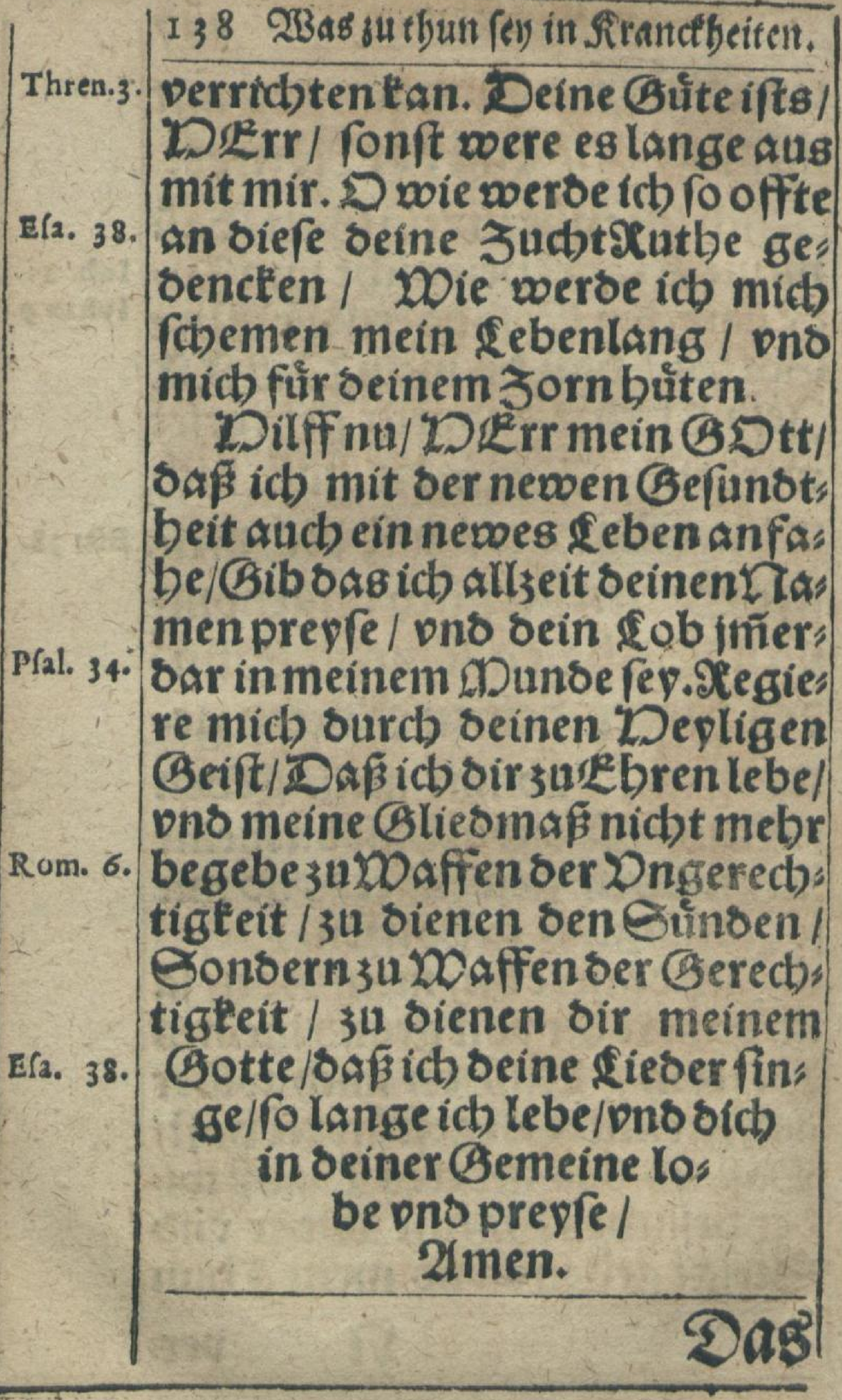


Seligane Biedan. Dek Yobes balben. I 39

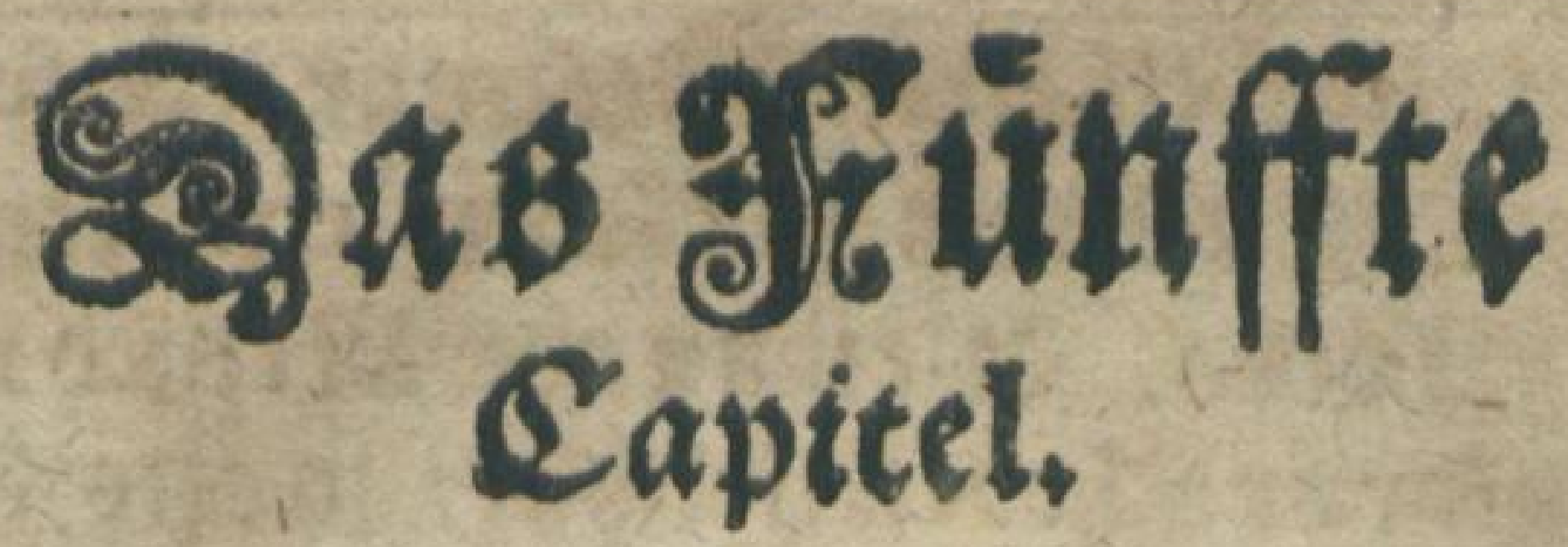

รobut beridft bon allerley) sueltlicten vind fleiffslictsen Ge. Dandten/ bie cinem einfomen/offite auch febr betimmern/ wenu er täglich in Der Sterbetunft ftubieret.

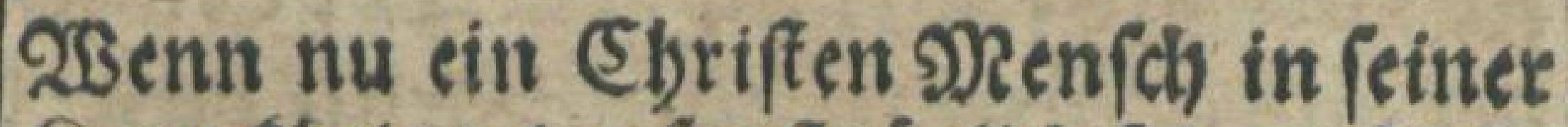
Siranctbeit / ooer fonft tigglich feintes fterbe. Stindeins gedenctet 1 Defindet fichs

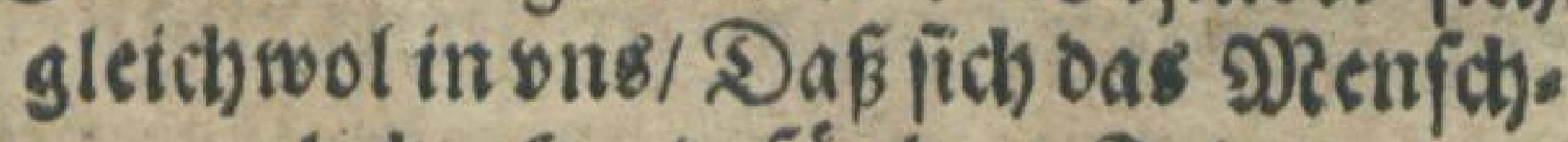

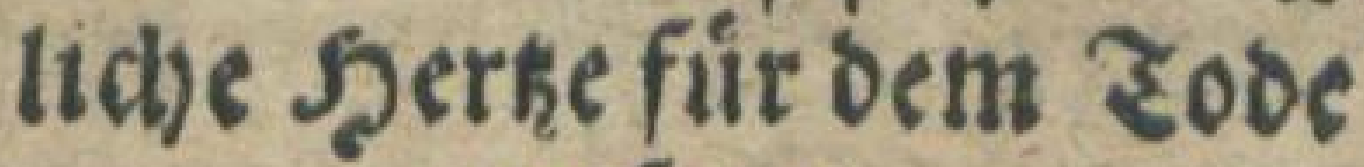
ent eहhet.

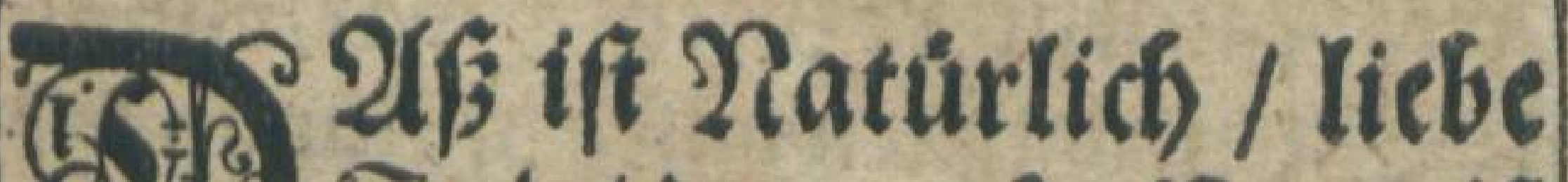
- Eeele / Denn vnfer Natur if 2. A anfänglich nicht tum İobel fondern zum Eeben erchitafien. Det Ioot aber ift vmb oer Sinden willen Rom. s. in bie 2k3elt fomen / und ift ber Euns 6 . Den Sold ond Etraffe. Ja/liebe Eees le / gleich wie ber Sob ift cine ierfids

$\Re$ ij rung




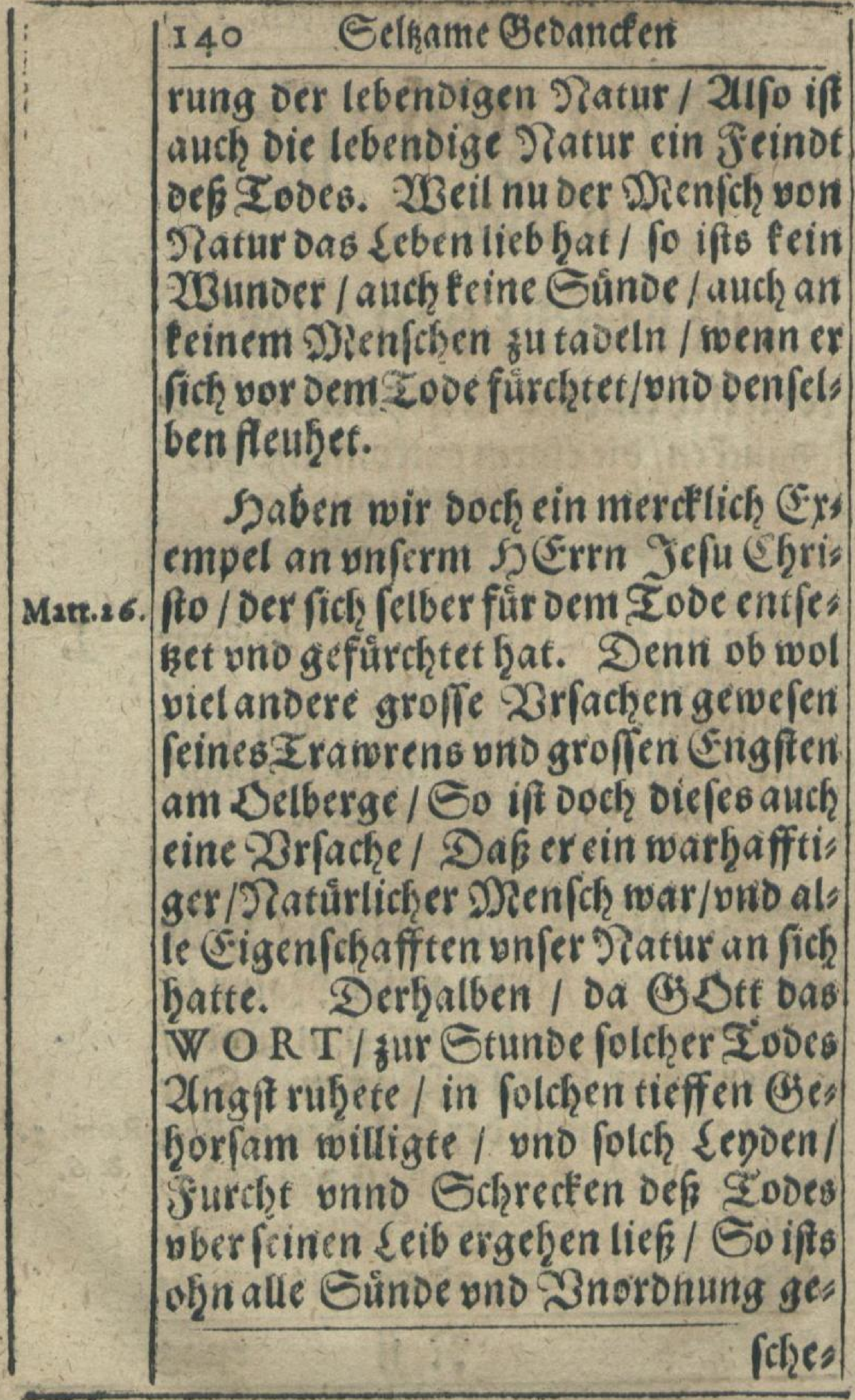




\section{bef robesbalben. $\quad 4_{4} I$}

fchefien/o afs fein Sleifch niche luft hats te sufterben. Ta er fpricht auch an eis nem andern Srthe: Job mus mich Lues 12 3ubor tåfufen laffen mit einer Tauffe / Dno wie ift mir fo ban: ge/bifífie vollendet werbe.

J゙C Dandfe oir SD Ex $x$ Jef Cbrifte/Daßsou midb nicht zum đode/ fonbern zum \{ebẽ erfchaf̈: fen/Dnosie fiebe zum feben in mein Dert3e gebilloet baft / J d) bitte oich pon grunst meines Dertzen/[febre mich bebencken/

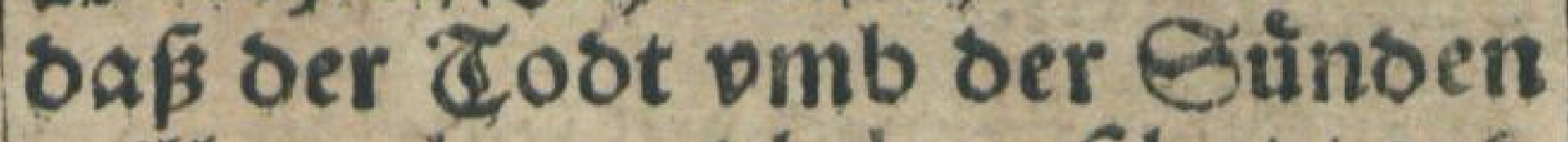
willen vber mich berrfdbet / vns mícb enslicb erwoirget. (Bib mir ourch oeinen Devligen Geift I Dấ / fo offte mich ber Costers

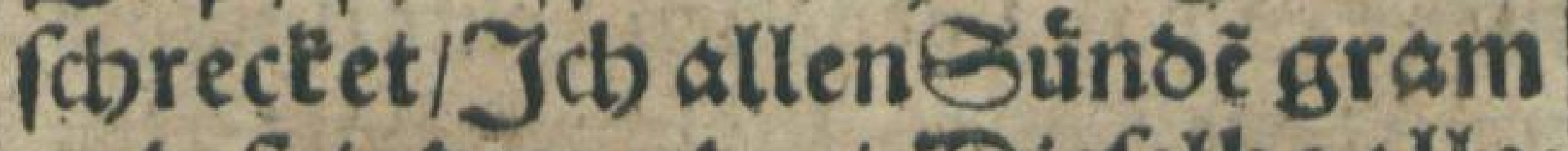
ono feino werbe / Diefelbe alles 3eit mevoe/ als ben (cos relbeft/ ber pmb ber Gtinbe voillen vber micb tomen ift. Dilffaber/Daf icb oir meinem DD E Rrn / oer oas Keben felberifít / ourd) wabrein Ioh. s1 II iี้ Glau: 


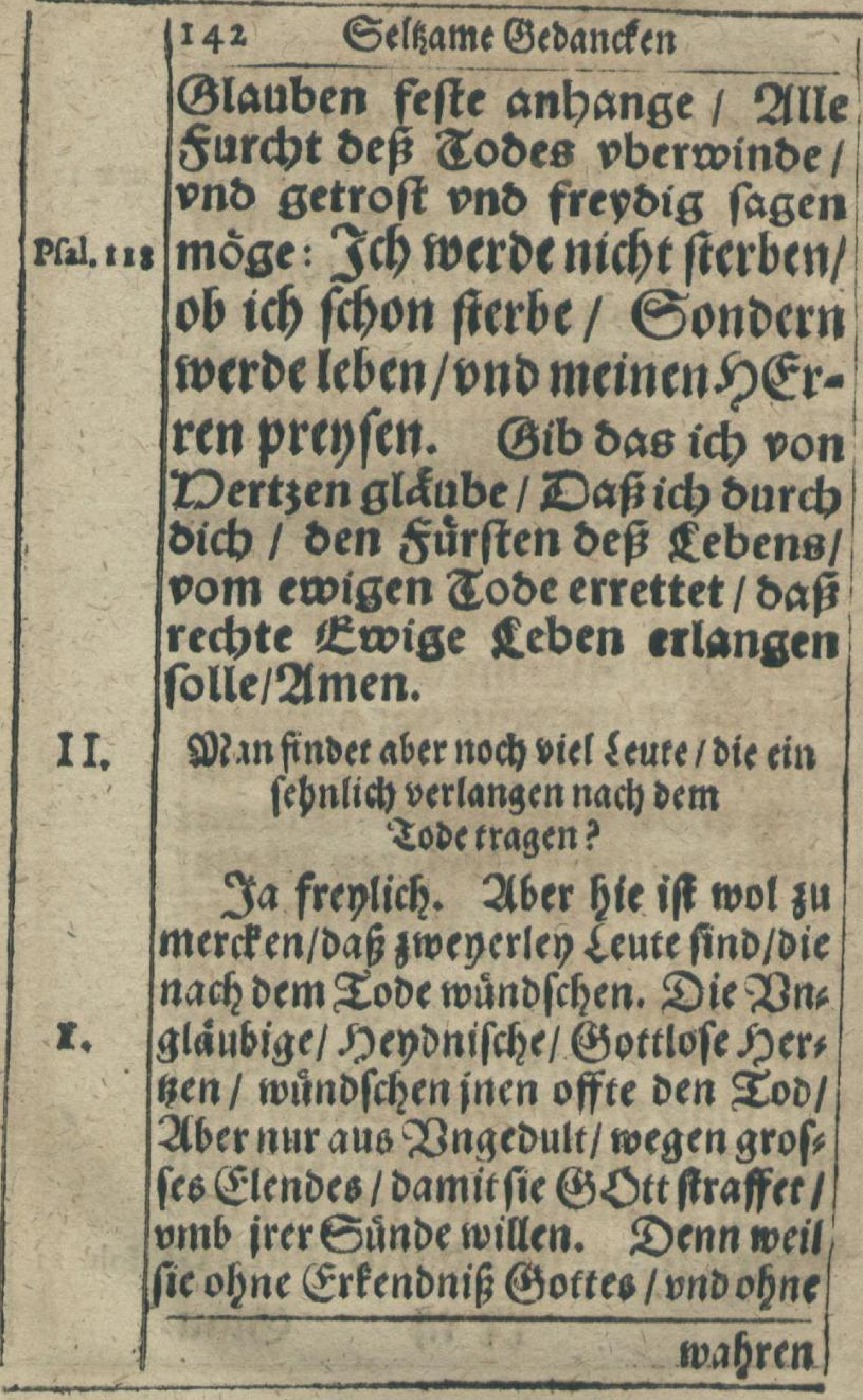




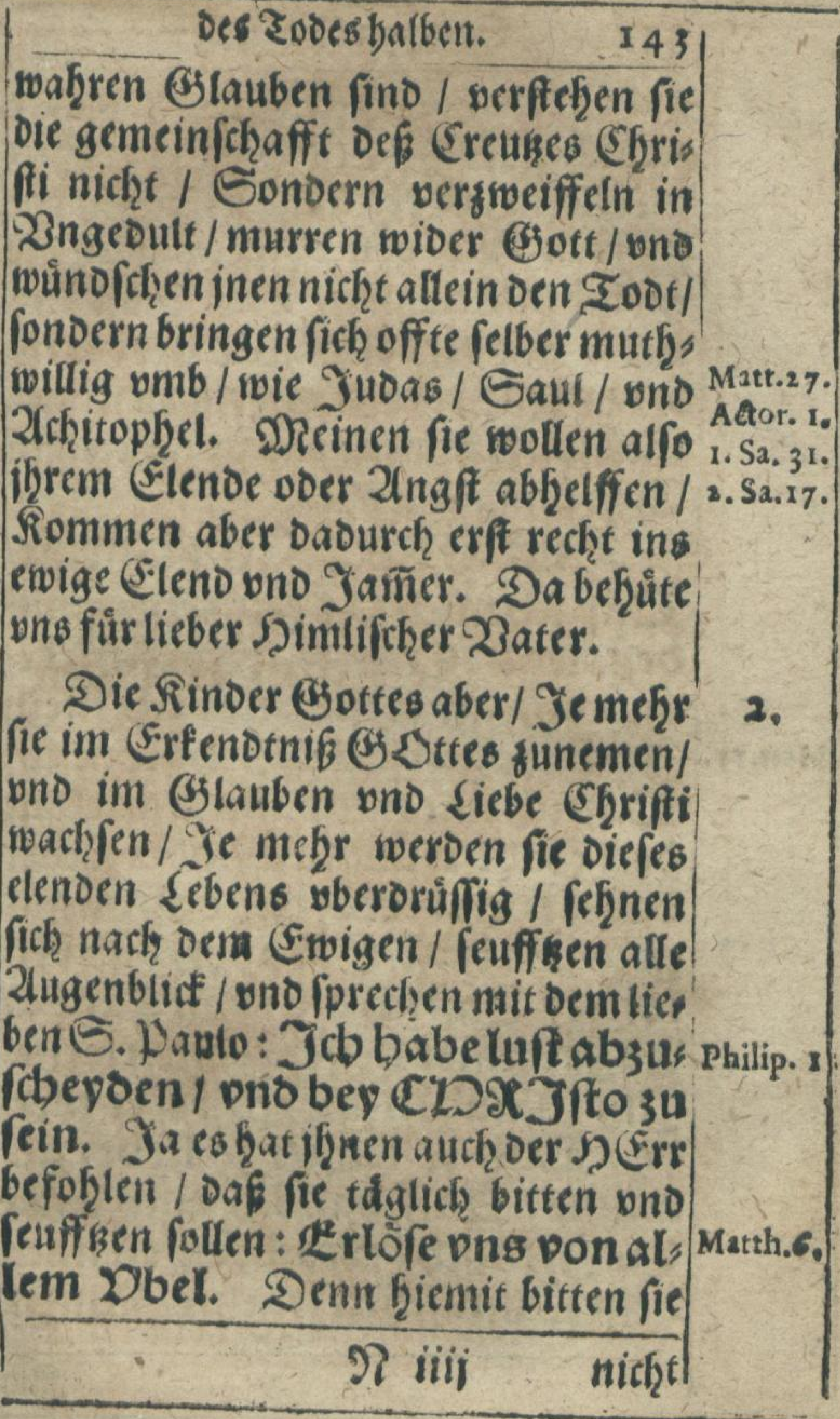




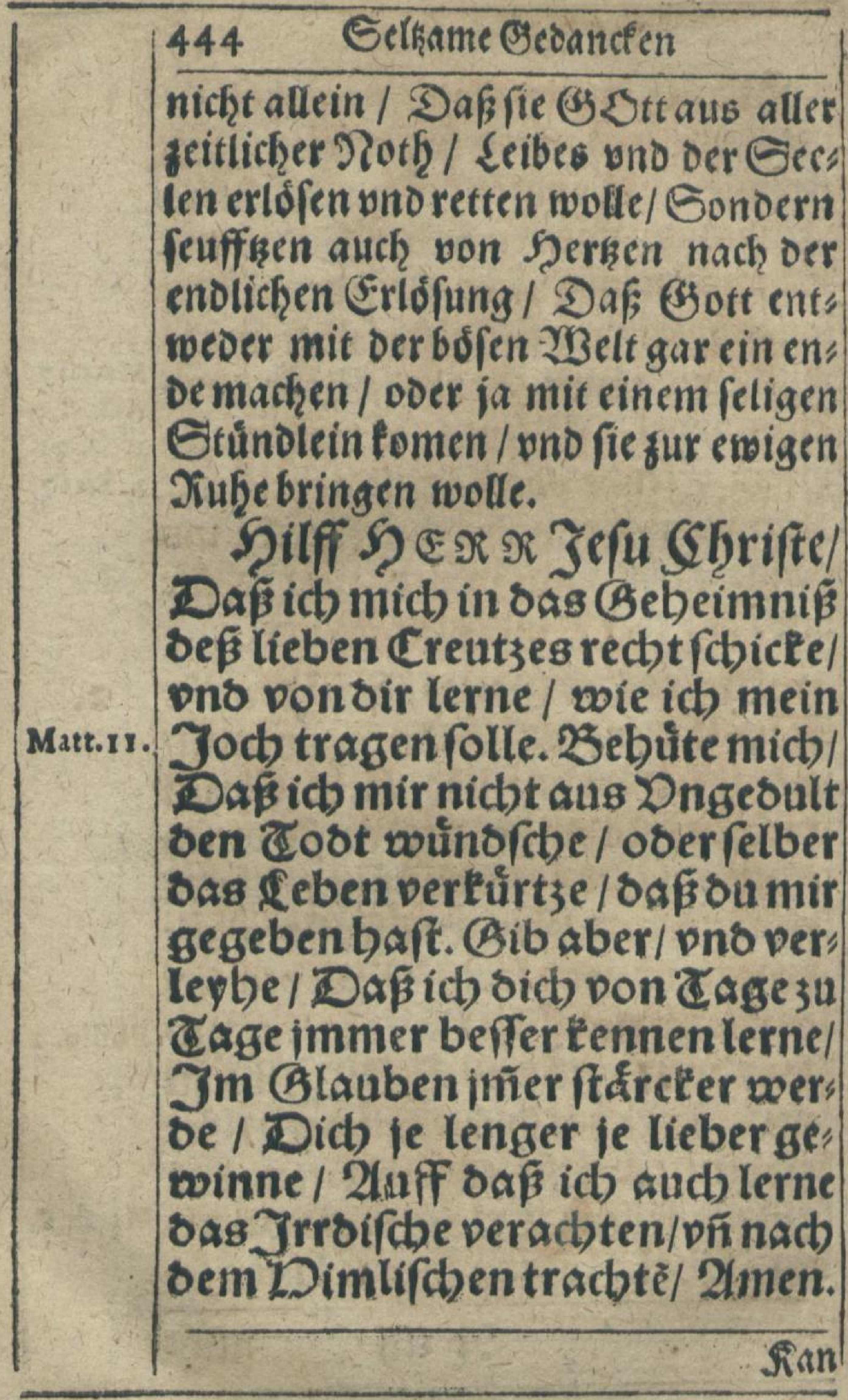




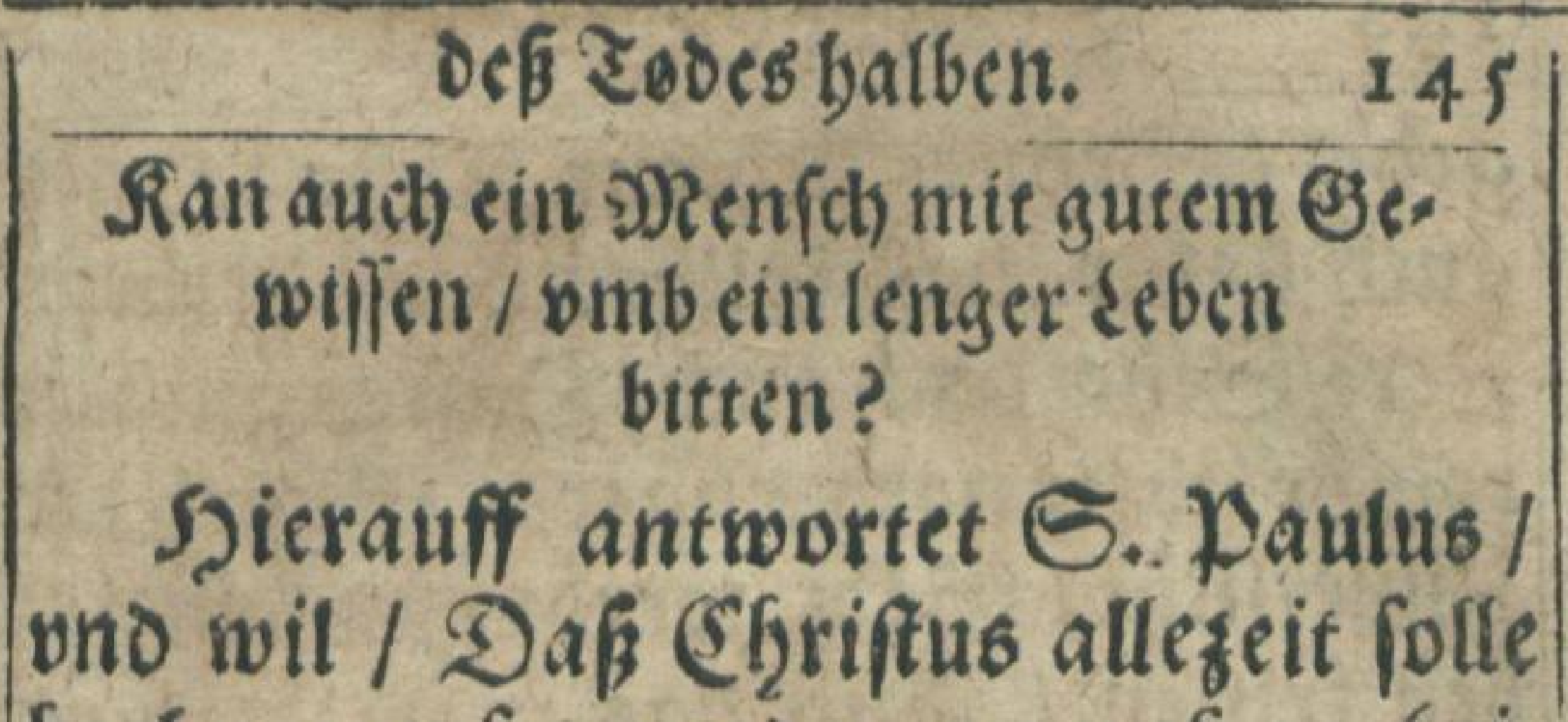
\begin{tabular}{ll|l} 
Des కobes balben. & 45 \\
ein Menfch mit gutem (5).
\end{tabular} III. fochgeprenfet werien an unfern Eeis Philip. I ben / Ei feg Durch Leben oder Durch Ioot. Denn (fpricht er) Cbriftus ift mein feben/ pno Eterben ift mein Geroinn. Eintemal aber im fleifch leben / sienet mebr frucbt zu fathaffen / So woeifich nicht / weldbes idb erwobleb fol/ Denn es lieget mir beyoes bart an: Idb babe luft abzurcheioen/ vno bey Cbrífto zu fein / welches audb Diel beffer were / 2 iber es ift nòtiger im fleif ch bleiben $/ \mathrm{vmb}$ evoren willen / D no in guter 3 w: verficbt weis ich / Jaßs ich blei" ben / pno bey euch fein werbe/ each zur föroerung / pnno zur frewoe beßs glaubens.

hie froreft du/liebe Eecle / dafs. Daulus mit beiderley gedandten umb; $i v$ gefeel 


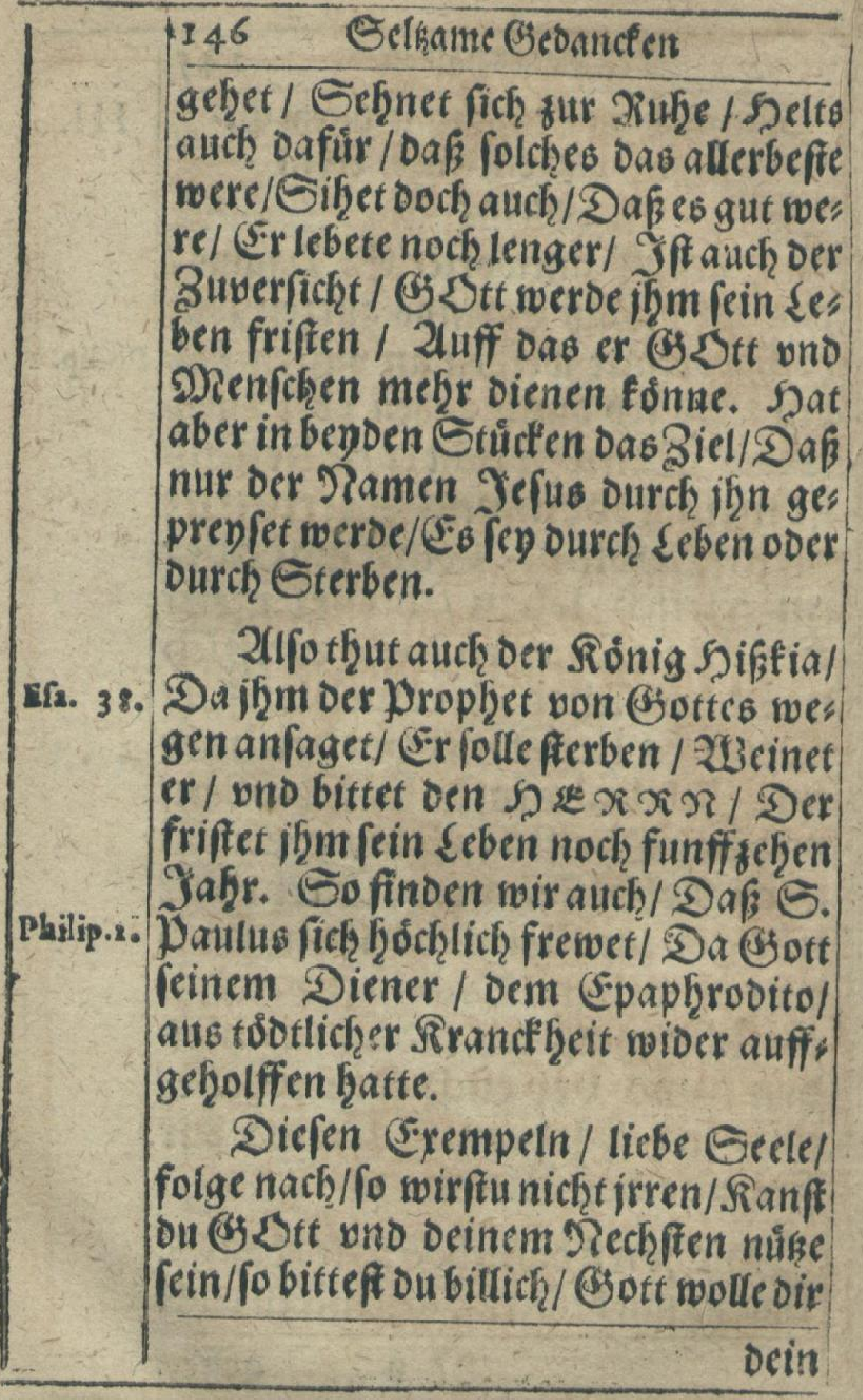


Defíp rodes balben. $\quad 147$

Dein Seben friften / Gs efunoen scib bes fcheren/Dich lenten wi regieren/Daf

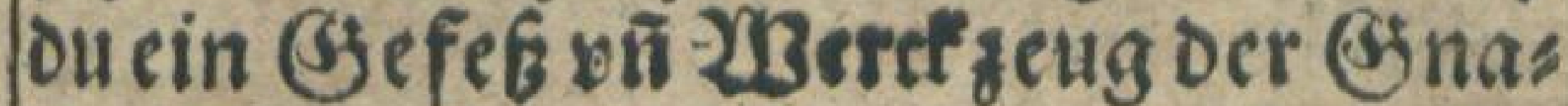
oen fein mógeft. Ergib oich aber alles seit in feinen VBillen / ono fey táglich berent aufiufpannen/Denn Das Birt ift dir verborgen.

Emiger/2Illmåchtiger Sott) Du bift meín feben / pno meine Zeit ftebet in beinen Denben. Denn in Dir leben / freben GHO Aa. $7 \%$ fino swir. Ich bancte sir von gruno meines L Dert3en / Dap bu mich biésaber erbalten/ Befun, ben \{eib/vno ricbtigenD Derftans gegeben / LDidb audb in einen fols ben 23 eruff ons 8 tans gefatzt / Daicb oir meinem DÉrrn vns meinem riebeften babe sienen toonnen. LDein Bott/ Du fibeft ja/ons fenneft mein Dertze/oaf id) willig vns bereit bin/ in meis nem 2 ieruff getrewolicb fortb 3 a: fabren/vns keinen böfen Dorfats 


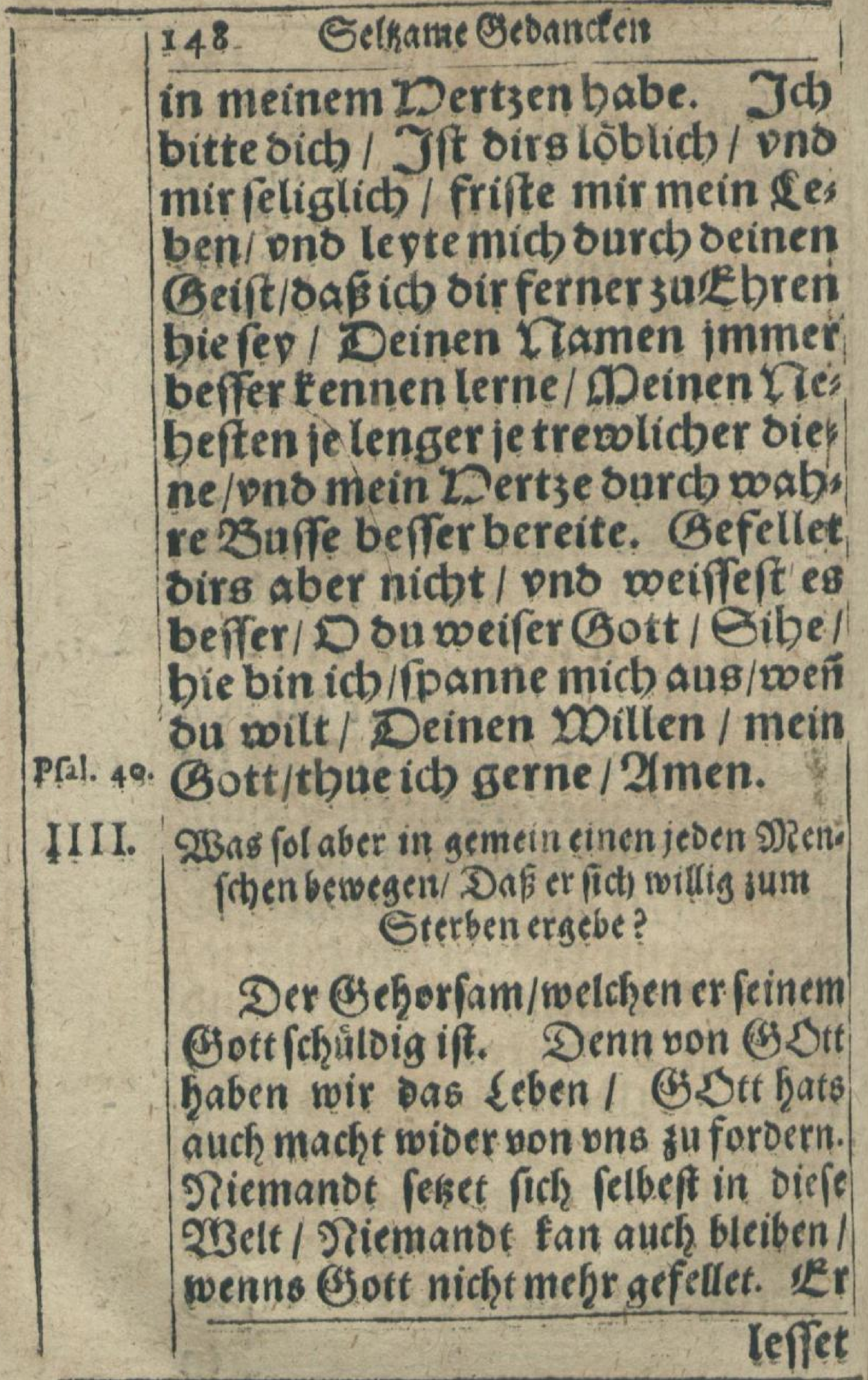


bef rodes balbert.

149

leffet bie DDen/fben fterben / pno Pral. go. fpridbt: DAomet wioer DDenf(ben Alinber.

Jưte Dich aber/lieber Geele/ wenn bein Etundein da ift/ Dak ou nicht ongerne ftirbeft / VBider deinen Bott nich̨t murreft/Sprich nicht: Ich mus mol ferben / Teh wil ooer wil nicht. Eo thun die Singláubigen / dié fecine Jofinung haber/onid nur auff oif $2 e$ s ben bawen/Denen gehet es oarnach / wie oer sou $x x$ fpricht: Wer fein tok. iz. Leben lieb bat/ser woiros perlie; ren.

Dit aber/meine Geele/gewere beis nem $\mathfrak{S}$ S.rrn ein gehorfam Soerke fu leben/ fo lantge er will Bufterben/weñ or wil : Sa B oich nicht ts jrren / nichto auffialtẽ snter alleri/was in oer $2 B e l t$ iff / Zelrte aiteh bein seben fur nichts / gegen oer vberfchrwenglichen Serrligs feit / Die an Dir fol offenbaret werben / Rेom: 8 So wiro dir widerfabren / wasbein Sogrrfaget: Wer fein Rebenauff rok. iz. biefer Delt baffet/ber wiro es ers baltenzum sewigen Keben.

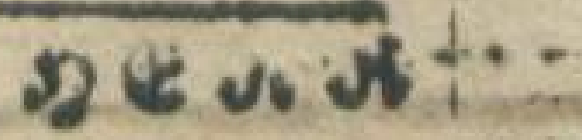


S E $\mathfrak{A}$ Jefu SErrifte/Der su fo willig pns berept wareft 3 u Philip. x. Deinem feysen/Dfi beinemDater geborfam vooroeft biß̄ zum खo: be/fa biszum tode oep Creatzes/ Matt.27. pno/pracheft/2 ater / nicht wie ich swil/ fondern foic ou swilt : J(b) bitte oicb / mein "Deylano / Gib mir alleseit ein geborfam Dertze/oapicich/woeri mein Etuins belein ba ift / mich willig ergebe/ ono gerne fterbe. Du kenneft DERr meine 23lōoigtét / pno weiffeft oas fleifch ons 23 lut folt d)es nicbt permag/LDerr lencke mich nacb seinem woillen / feyte midb in beinen füftapffen/Gib oas ich mich oir mit feib pno fes

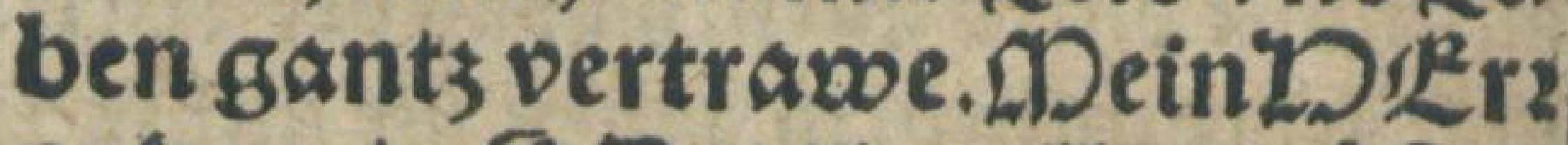
ono mein GDtt / bu aller trṓftli; Der Depland/Du bift gut / ons alles wass ou tbuft ift gut / Dno baft es niemals böfe gemeinet/ niemals böfe gemacht/Du bafts

ni 


\section{Deßs?odes balbent. Is $\mathrm{t}$}

nie teinem verberbet / ber auff oid) geboffet bat. Die bin id) DUR rr in seiner Gnabenbandt / sebeich/folebe ich oir / Sterbe Rom. 34 (id) / fo frerbe ich oir / Jeb lebe oder ferbe / fo bin icf Dein / ons werbe ewoiglich onverloren fein / Dafs ift gewoiflich war I $2 \mathrm{men}$.

Es iff aber gleichwol fein in bersselt/ ono ir saufft ift fchone / Wer wolte nicht lieber bie bleiben?

2llle 2 Belt Rinder fingen dif sieds leit. Du aber / liebe Eeele/fitlle dich nicht gleich Dem 2 Befen Diefer WBelt: Rom.12 Eprich nicht I Es ift allo Der 2 Belt Saufft / Gonbern gedentte / Dafs Der WBelt Saufft in die Solle leufft. Denn wir fins A Ainser ber 'Deyligen / Tob. 2. víi warten auff ein anber \&eben/ welchs Bott geben wirb/oenen/ fo im Glauben ftarce ons fefte bleiben bey im. Sore aber/was fagt bie Echrifft won Dem Laufft oer2Welt: 


\begin{tabular}{|c|c|}
\hline \multirow{3}{*}{ t.Ioh. 5 . } & Selkame Sebancfen \\
\hline & Die gant3e Welt (fpricht 6 . Jo \\
\hline & Lunnes) lieget im 21rgen. 21 \\
\hline $.10 \mathrm{n} .2$ & Welt / nocb was in ber Welt ift \\
\hline & So jemano oie Doelt lieb bat / in \\
\hline & sem ift nicht die fiebe be 3 D \\
\hline & ift / nemlich / be $\beta$ fleifches fuft \\
\hline & pno 2ugen Ruft/pnoboffertiges \\
\hline & $\begin{array}{l}\text { Leben/bafifit nicht vom Dater } \\
\text { Eonbern Donser Delt / pns bi }\end{array}$ \\
\hline & 20elt vergebet mit ibrer fuft. \\
\hline & ben Willen GDttes \\
\hline \multirow{10}{*}{ Mattb.7. } & igrelt. Dini \\
\hline & ge Pforte/ Denn sie Pforte if \\
\hline & weit / pnober Wegift breyt / oet \\
\hline & sum Deroamni \\
\hline & anff wandeln. \\
\hline & ftenge / pno ber \\
\hline & $\begin{array}{l}\text { Deg ift fchmal / ber sum leben } \\
\text { fúbret / Dno wenig ift ir / bie in }\end{array}$ \\
\hline & \\
\hline & $\begin{array}{l}\text { Soore boch / liebe Geele / was ber } \\
\text { 2mert Saufft ift / Sibe ooch / woer hin }\end{array}$ \\
\hline & leufft / \\
\hline
\end{tabular}




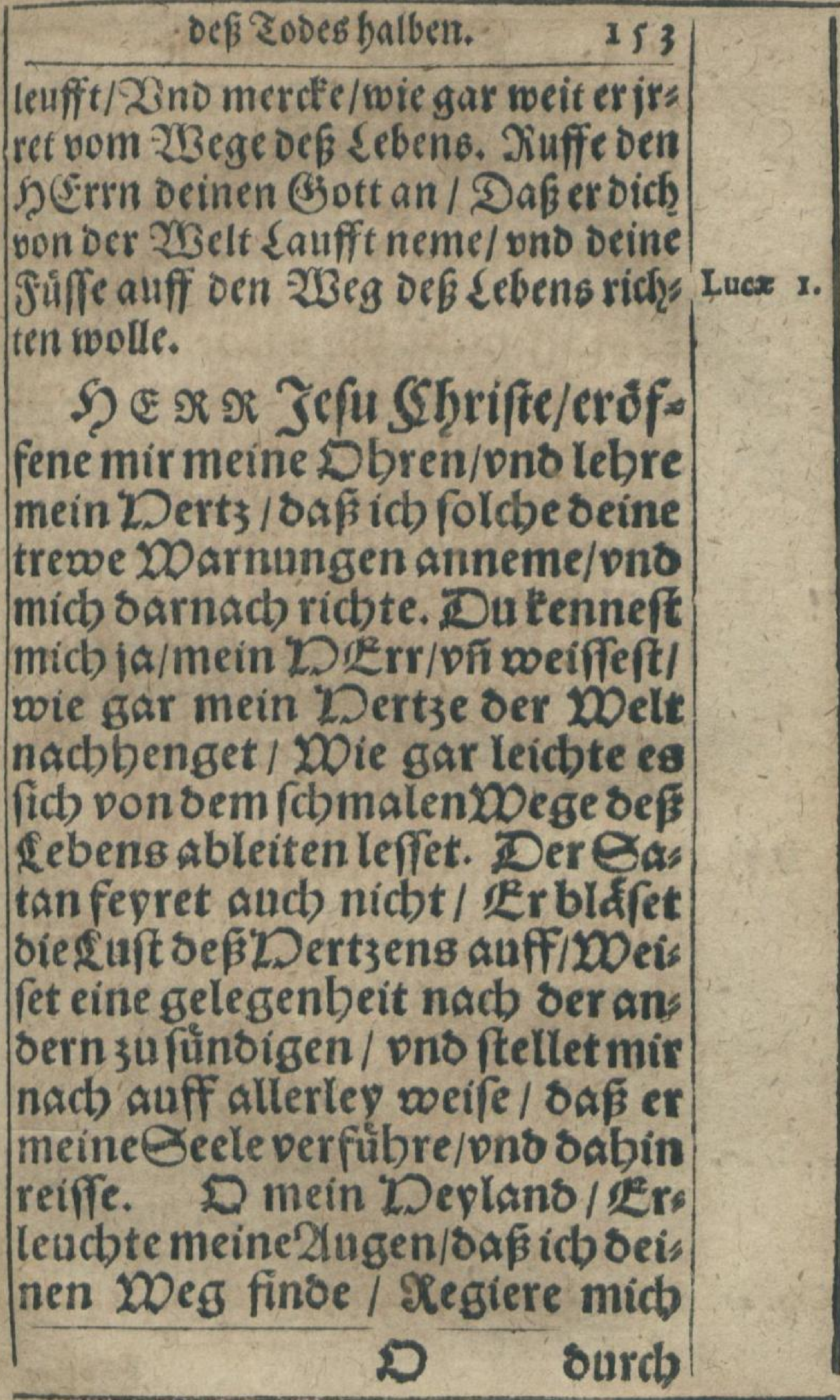




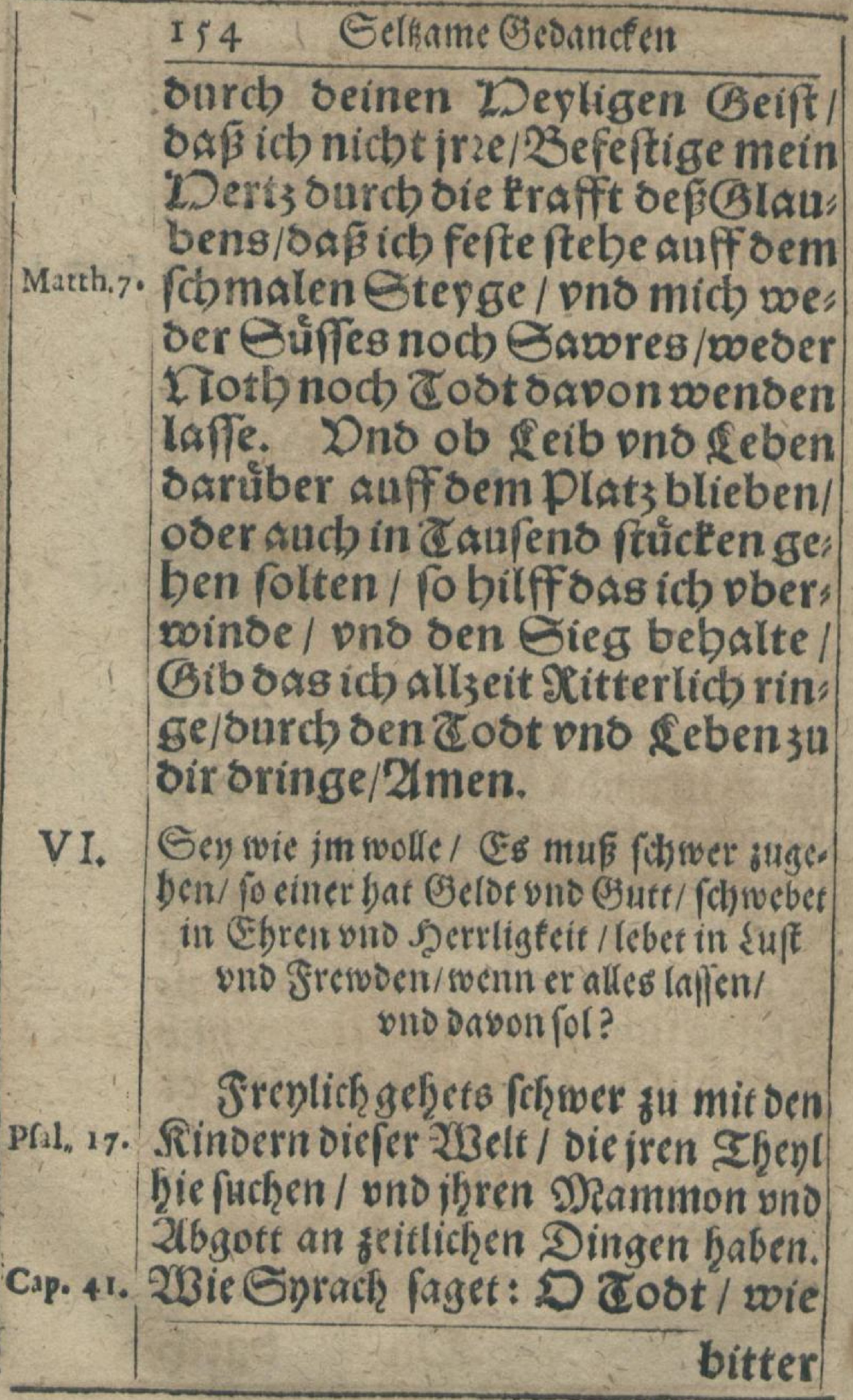


befs robesbalbent. Is

bitter biftu/wenn an sich geben clet ein DDenfch / ber gute a age / ons genug bat / vno obne forgen lebet / pno sem es wol gebet in allen singen/vno nob) wol effen mag.

Aber mit ben Sinbern Botteg ges hets mit nichten foflwer zu. Denn ob fiefchion von \&5 Stt gefegnet / G5eldt vno @utt biaben/ In hiohen Emptern

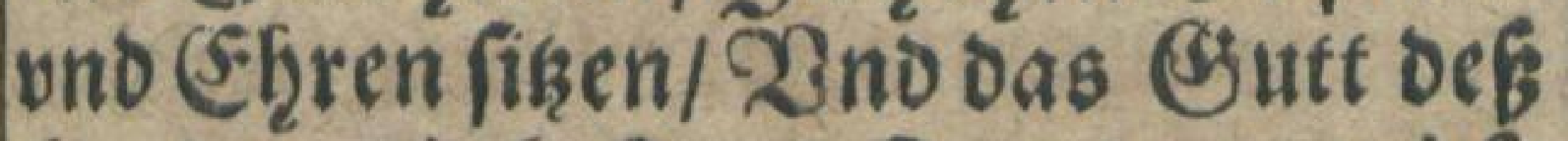
Sandes mit \{uft bno Srewoen geniefs Elaix 1. fen / Go machen fie doch feinen $2 \mathrm{ab}$ : gott Daraus/Şengen Das Şerkse nichte P\{al. 62. Daran / Gondern Dand"en Gott / wno brauchens recht / 20no wiffen / Daßes find Stiter biefer 2Belt / die nur f̧ie gelten / vno fiur @ott niehts. 2ino betrachten obze onterlaß Das 230 rt ock S⿹ Sirrn : 20as builffe es einen Mare. 8. Denfcben / Wonn er bie gantze welt gewinne/ ono liese 3 cbas ben an feiner Eeelen ?

2lifo thue ou auth/liebe Eeele/fels let oir Xieichthumb ju / fo henge das Pa.1. 62 .

$$
5 \text { ij Syerze }
$$




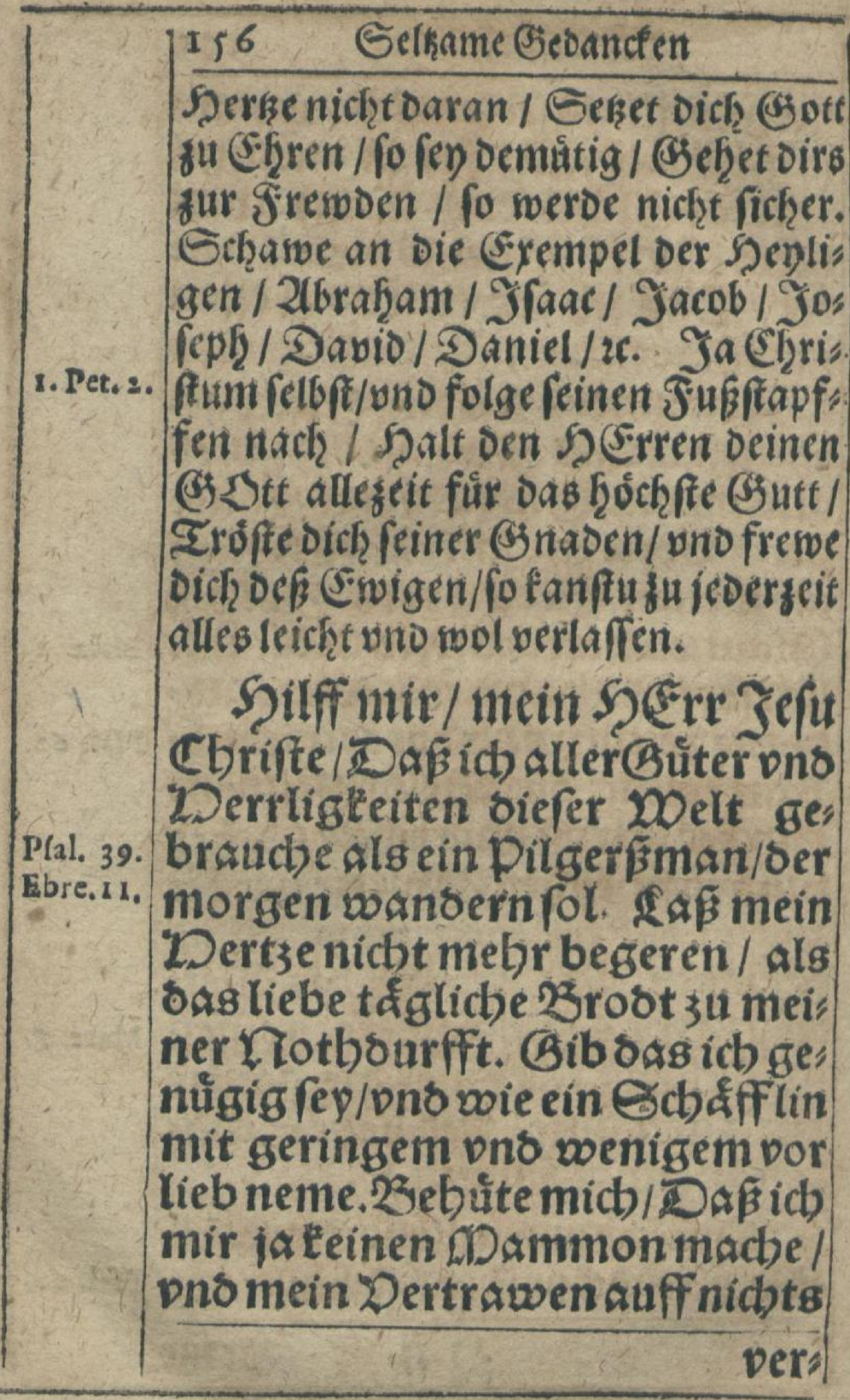


bef Tobes balbert. I5 7 vergånglichs fetzelauffoas mirs nicbt binberlich rey in meinem Eterbe Stundein. Dilff aber/ mein 'DKRr/Dríneige mein Der" tze / Daś id erweble oas rechte gute beyl/nemlidb/oich meinen Lue, so. DRErrn/meinen $\mathcal{R}^{2}$ rlö $\mathrm{er} / \mathrm{meinen}$ Eeligmacber / ono an sir allein alle meine suft ons frewo bas be. Derleybe mir/Dafích nur an meiner Seelen reich / pno im (G) (auben berrlicb/ons in sir fro", lich /ey/ So babe ich genug/ bey" be bie seitlich ons sort ewiglich/ A, an willig oie Welt gefegnen / ons frōlich טñ vnverbinsert von binnen fabeyon/21men.

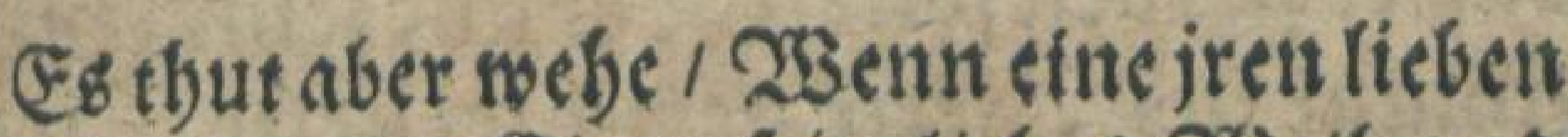
VII. Ebemann / Einer fein tiebeg $2 B_{\text {eib }}$ yno Simber berlaffent fol/ Die offt in 2(rmuth!

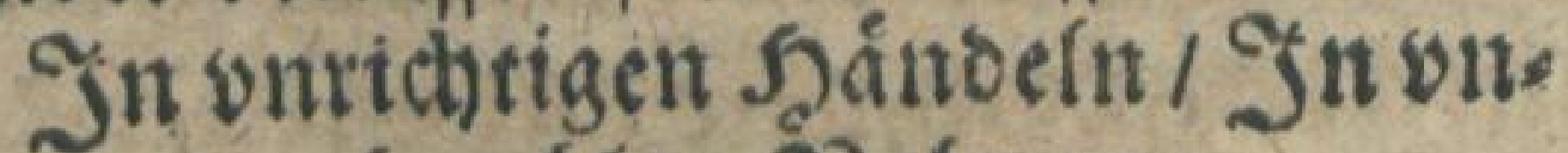
bejablter Piabrung bleiben.

Dis find nur fieifchliche mistrews liche Esedandten / Damit Doch Der Gas (5) iij tan 


\section{Seligame Bebantefen}

tan die Syerigen der Nenichen offte fehre onruhig ono jrre machet/ Daf 3 eg mit manchem Deiffe sno 2lrbeit hat / ehe fich s ergiebt / vno bott walten lef, fet. Dan erfehrets auch / Daf manche Serken fich gar Sobonifich ergeigen / wider (S)ott murren / als thet er inen onrecht / Daf erfie eben jerzt fterben lefs fet / Da fie noch viel richtig su machen / vno ben jbrigen ant beften folten vor: fetenen. 2lifo that fener cole Xiomer Caius Marius / ob er wol fum fieben: oen mal Conful / uno nu mef̧r ein Mann won Siebenkig Jafiren war/ murrete er ooch wioer feine Fortunam/bas ift/wioer feinen Gott/wñ bes fohweret fich / S s gefehebe im unrecht $/$ Daser fterben folte/ehe er alle feine 2 In: fchláge ins $2 \mathrm{Werdf}$ gerichtet Fette.

Zber die gláubigen Rinder Stottes machens nicht alfo / Sie wiffen/ Daf fie nicht oer jrigen Gott fino / noch fels ber fie serforgen fonnen / Gonoern Luex 16 nur Shaughalter/ imen von (S) St füs, geftellet/fo lange esjum gefellet.

2nol 
Def robeebalbet. $\quad$ I59

Dno weil fie ir Dertrawentág: lich fekzen auff oen Evwigen/2ullmách" tigen / Zarmferkigen / 2ullweblen

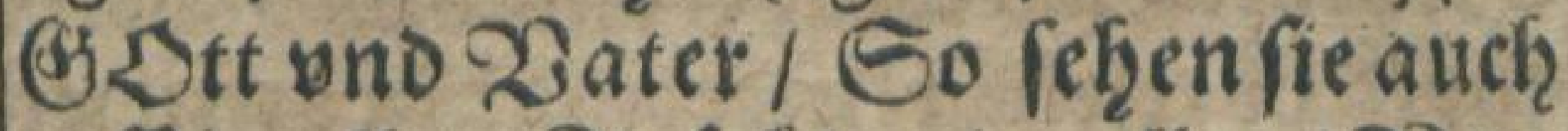
auff in alles/ Befeflen im alles / 2les" trawen im alles/ In trofflicher auver" ficht / 2Beil er juen bas Saughalters Luc, 16. 2umpt auff faget/vno fie ab fordert/6o werbe Er die Sinterlaffenen beffer wiffen guberforgen: Ta er werde felber wollen 23 ater wno pfifeger fein / 2 Beil er fich in Senliger Selzrifit fo offe oer WSiowen Water/uno 23aifenStelfer Pralo 68. riffemet. Ta weil benor dic Schriffit vno tågliche erfafrrungzenget / Dak Y3iowen offec viel beffer nach forer D) iantiner tove/Dianner offe viel bef: fer nach ibrer YBeiber tode / Rinoer ofite viel beffer nach irer eiltern tos beverforget vno verfefien werden / als menn ifgnen bie Ygrrigen noch gelebet bettent. Dern (Sott treuget vno lew

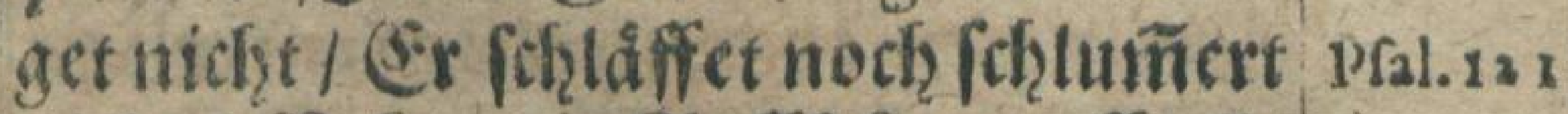
nicht / Erbat ein Zluffefien auff afle unb jede/ oie feinen ?amen anruffen/

$$
5 \text { iii } 23 n
$$




\begin{tabular}{|c|c|}
\hline & Selaname Siebancfen \\
\hline & Qno wiro im nimmermef̧r nach fa \\
\hline & gen laffen/ Dafarer ciniges Dienfebe \\
\hline & Segl verfeumet/oderjemandes zBol \\
\hline & farth vberfefen fette / Denner fibe \\
\hline \multirow[t]{18}{*}{ Pfal.113 } & auffoss fiorige / beyse im Di \\
\hline & mel uns aufferben. Ia/liebeEee \\
\hline & Ie / eben darumb leffet Er auth eleno \\
\hline & 2Bibwen und 2Waifen werden / Daf \\
\hline & or anjen leine Sute groz mache/vni \\
\hline & les ift aller Silenden gewiffer Girun \\
\hline & ono Zuverficht/Daferfie alfo verfor \\
\hline & gen/alfo berathen/befguten/lenten vnt \\
\hline & fufbren wil / fo fie jn nur su einem $2 \mathfrak{Z a}$ \\
\hline & $\begin{array}{l}\text { ter haben noflen/Daf feines wer in an } \\
\text { ienem Iage wiro Slage fübren/Daf }\end{array}$ \\
\hline & ift gewiflich war. \\
\hline & 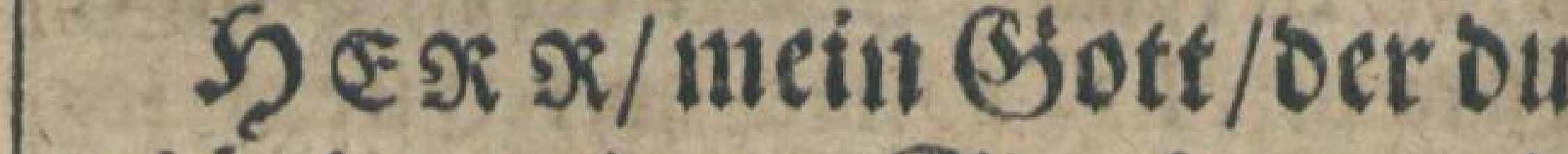 \\
\hline & $\begin{array}{l}\text { mich in meinem Etanbe su ci, } \\
\text { nem Dauphalter (ober Dous: }\end{array}$ \\
\hline & balterinne) beftellet/ons mirsen \\
\hline & $\begin{array}{l}\text { Deinigen trewolicb vorsulteben } \\
\text { befoblen baft । Du pociffeft ia }\end{array}$ \\
\hline & Dasicb fold es surdb seine Be; \\
\hline & ol in Sbr \\
\hline & \\
\hline
\end{tabular}




\section{DeE rodes Galben.}

$16 \mathrm{I}$

bod) mit allem fleif perrichtet babe / Dafí ich meín (Bevoiffen perpoabret/Dich für Dntrew ges buitet / Dns allen / sie su mir be: foblen baft/ beyse in seiblichen uns (Beiftlicben singen/alfo vor: gefinnet babe / als mir felbeft / Jbnen alfo geratben / als ich wünortbe/ oa símir felbeft fol ge" ratben werben. 2beil idb beñ nu foldbe Eorgen nicht mebr füb; rentan/oasu trancé bin/ons bie in beiner gevoalt liege / fo willich oir nu oas $2 \mathrm{mpt}$ wioer einreus men/ vielleicbt woilt ou esourch ein anberperfon beffer beftellen.

DDein Gott / foles ferner mein feben rein / fo rey es ein Cbrift: lich Seben / Xichte mich voiser auff/ Bib newe Gefunbbeit vns guten Derftanot / Dafí ich noch mit mebrem fleí̧/als zubor/ben aDeinen vorfteben mòge. Soles

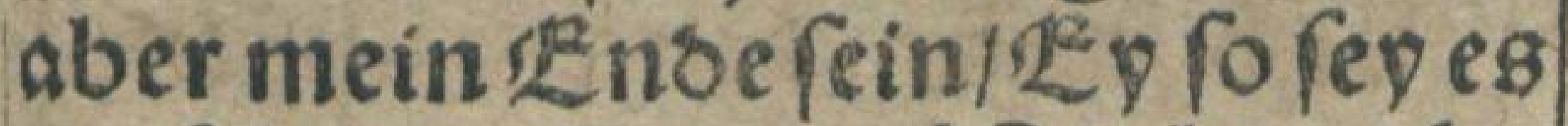
einfeliges iznoe. Die bin id)

$$
\text { D. } \ \vec{c}
$$




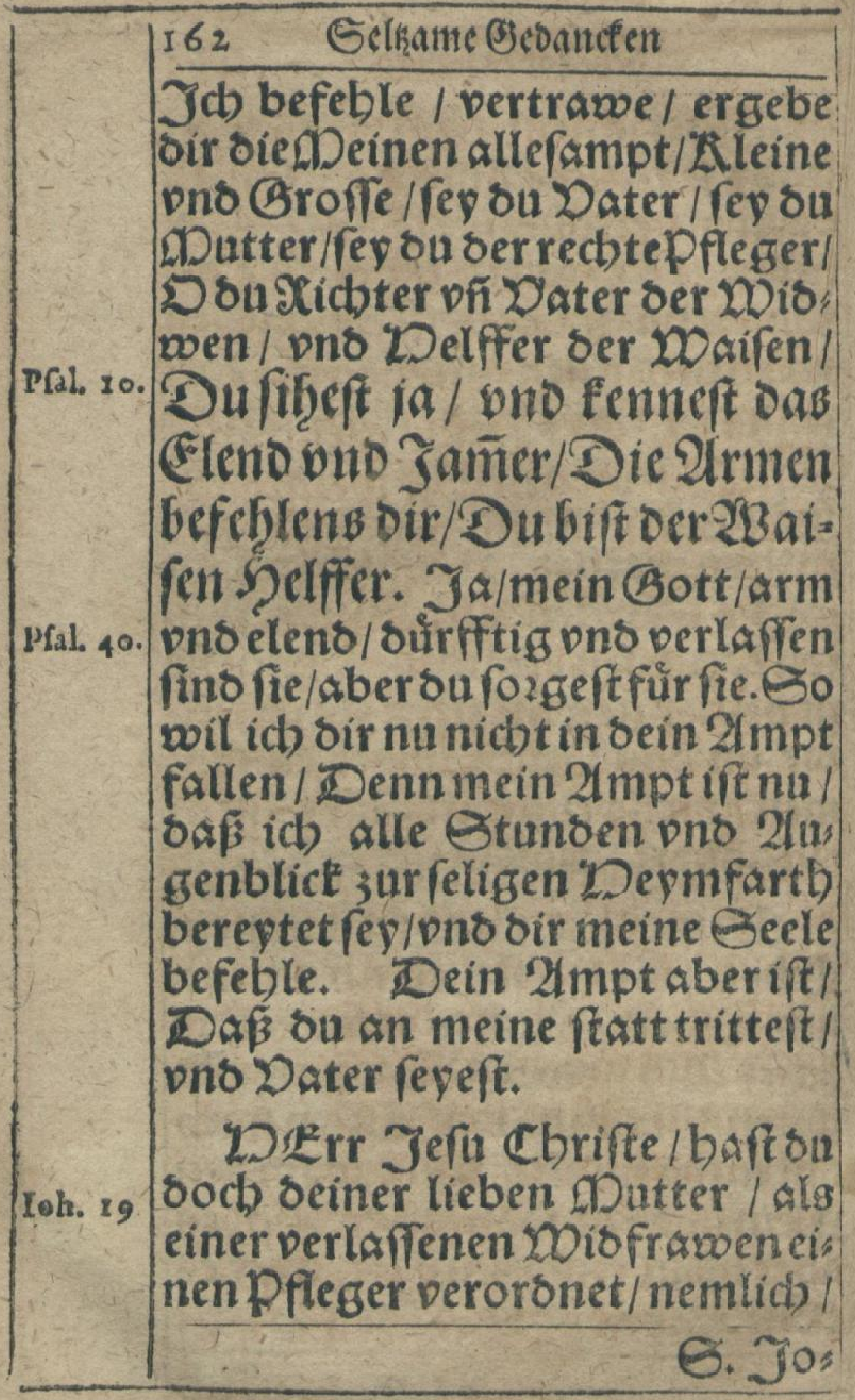


DeE Fodes balben.

163

ఠ. Jobannem ben teuangeli:

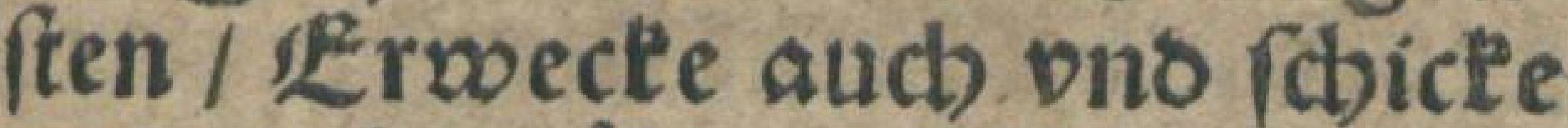
meiner betrúbten Wois wen guts bertsige feute $3 \mathrm{u} /$ bie fich irer an: nemen/Dñ 3 uoenen fie fich a rewe ono föroerung verreben mòge.

Ja / DRErr Jeru / gleid wie seineWutter senwo bowenftans/ alro baftu aucb ben Daifen: ftand Derfudbet / Dennoein Pfle; geDater Joreph bat sir nicht lange gelebet/So woeiftu ja/was Wiowen ons Daten fins/Ia noas ift oir perborgê / D ou Der,

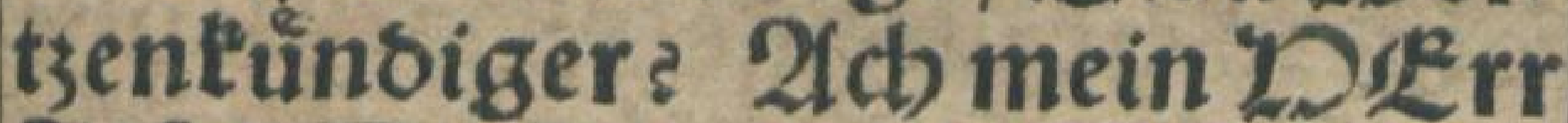
Jefu / Der bu ein waife worben bift / vmb aller elenben 20 aifen willen / Dir befeble idb meine Waifen / Da baft ou fie I Du wirfts wolmachen/Dasi ich ons fie oir am Jùngften Tage fob vno Danck fagen werben.

Rerfille pno regne ire Dertsen allezeit mit recbter warbafftiger Gottreligleit/ Jbre feibe mit

beften: 


\begin{tabular}{|c|c|}
\hline & Selkame Bedanten \\
\hline Pfal. 91. & 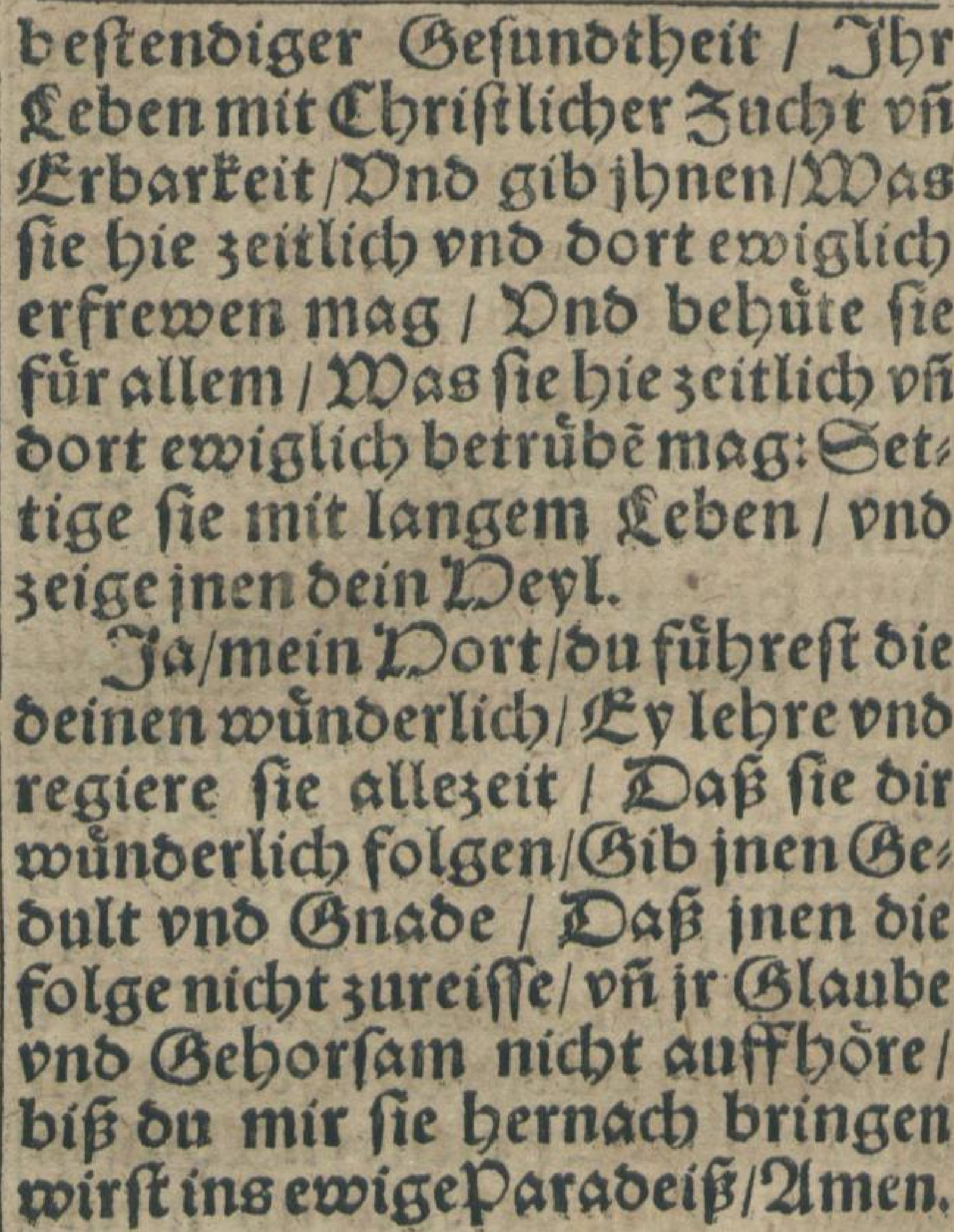 \\
\hline VIII. & 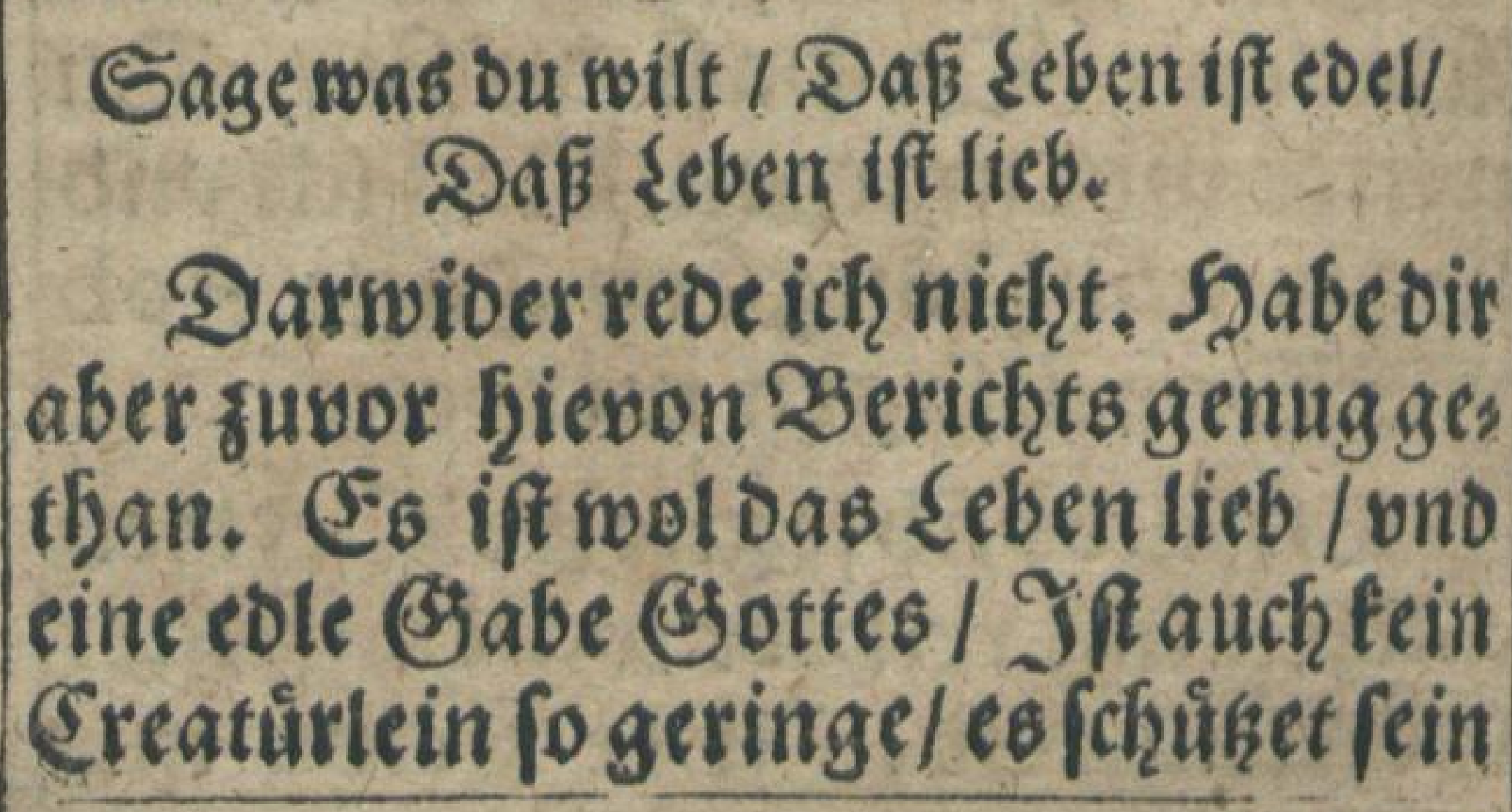 \\
\hline & \\
\hline
\end{tabular}


seben / ond fpreuffet fith wider den Foot. Du muft aber/liebe Seele/ Daf seben nicht lieber haben I als ben Gchopffer / Die \&abe nicht fröher achten/als den Syesrrn/Der dirs geges ben hat. Diuft autch nichterergeffen/ Dak er dir viel cin beffers erworben/ vno oir zum beften ourch feine Ssimel: farth fchon eingenomen fat / welches if verborgen mit Shrifto in (S) Ett. Color, 3 . 2Benn aber Gerriftus dein seben ficts offenbaren wiro / Denn wirfiu auch offenbar werden mit jm in Der Şers: ligfeit.

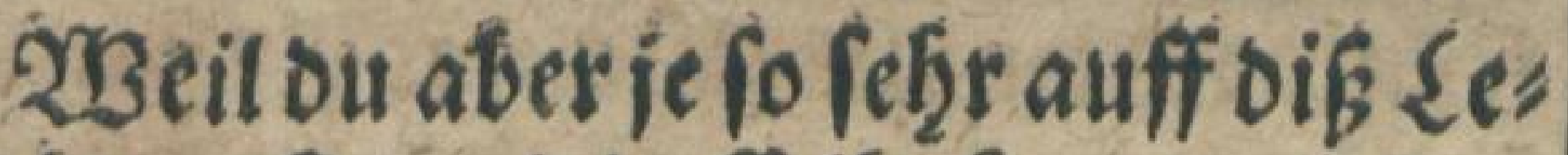
ben baweft / vno daffelbe fo gar viel zu lieb fraft/ MuE ich Dits cin wenig abs mafilen / ynd jeigen / was di seben fen / एo out bermal eines beffelben vberbrififig werben / who nact bem Eiwigen oich felfnen moidhteff.

Sore/was fagt der Zlteater Jacob Davon: Die Jeit meiner 20all, Geni, 47. fartb (fprichter) ift Dunsert pno orevfitis Jabr / weenig vno böre 


\begin{tabular}{|c|c|}
\hline & Selkame Bedantefen \\
\hline & eines cebens / on \\
\hline & langet nicbt an sie Zeit meine \\
\hline & Dáter in irer 20 allfartb. \\
\hline iob & 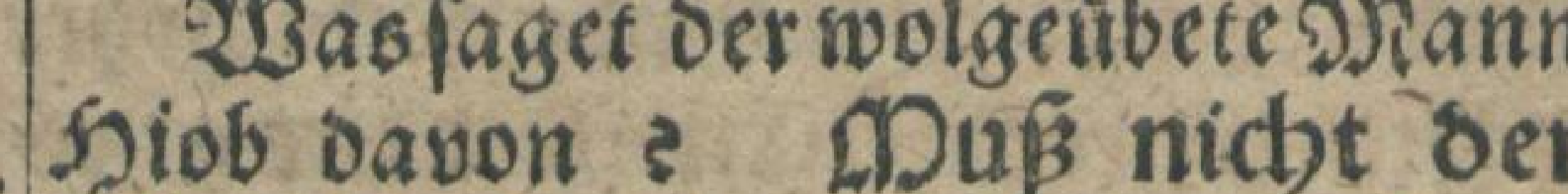 \\
\hline & Den/ch ( prichter) jiñer im ftreit \\
\hline & einaufferben / D \\
\hline & fin \\
\hline & Ecbatten / D D's ein Caglŏbner \\
\hline & oas feine 2 rbeit aus rey : 2 llo \\
\hline & $\begin{array}{l}\text { babe id, wol gant3e Donben } \\
\text { Dergeblich gearbeitet / pns elen }\end{array}$ \\
\hline & ber Iacht find mir viel worben. \\
\hline Ibidem. & abermal: Deine Cage fino \\
\hline & $\begin{array}{l}\text { leichter oabin gefloben/oenn ein } \\
\text { Deberionel / ons fins Deraans }\end{array}$ \\
\hline & gen / saj tein auffbalten oa ge, \\
\hline $\mathrm{Ca}_{\mathrm{a}}$ & poefenift. 23ns wioerumb : Der \\
\hline & eboren/le: \\
\hline & rube : Gebet auff wie eine 2 lu: \\
\hline & ofellet abe. fleucht wie \\
\hline & ein \\
\hline & \\
\hline & \\
\hline
\end{tabular}


Dep zober balbent. 167

tes im Dfalm Davone Dnfer Leben Ptal. 90.

( (pricht er) webret Eiebent3ig Jabr / woenns toch) tờmmet / fo finss 2fcht 3 ig Jabr / pno woenns Ròftlícb gewe en ift / ro ifts múbe pns arbeit gevoefen/ benn es feb; ret fonelloabin / als flöben woir oavon.

YKas fagt Der Sionnigliche l) rophet David davon? ZTt boch) (fpricht er) Ptal. 144 ber Denf(b) gleich) woie nicbts / reine Zeit febret oabin / wie ein Echatten.

2Bas prediget Ealomon Davon? Le ift (prrichter) alles tbun fo viel Hccle.r. mübe / oấ niemanot aupreben tan. Ono abermal: Solche pn: Ibidem. felige aD ibe bat Gottoen LDen: foben Âinoern gegeben / bap fie ficb oarinnen múffen quelen.

2Bas ḩalten bie lieben alten Rir: chentefirer davon 3 So ppricht S. Auguftinus: DDein feben ifte ein elen: des [eben/ einsuirfftiges \&eben/ je álter es woird / ie mebr es abni:

met: 


\section{Selthame Bebanten}

met. Je ferner es fidb erftreclet/ je náber es $\mathrm{sem}$ cose fömmet: zin betrieglich ons vergenglid) ₹ebẽ/Doller Etricte oe

23no abermal: 24d DER wie vberoruiffig bin ich biefes fe" bens / pnno siefer múbefétigen Wallfartb / Denn es ift ein elen oes seben / cin pnbeftendig fe: ben/ cin vngewoîs Leben/ ein mu' befeliges $/ \mathrm{eben} / \mathrm{ein}$ vnreines $f \mathrm{e}$ ben / ein feben barinnen bie '230", ren berrfben/ ons bie Dofferti, gen vberbanst nemen/es ift bols ler đrub/all ons Jrrtbumb/ ono folte nicbt oas Eeben / fonbern oer aost beiffen / Sarinnen woir in mandberley A.ranct beit ono Gebrecben/auff mancberley weirealle 2lugenblict fterben.

Dercte ooct fic / liebe Geele / was die Syenligen vñ Rinder @ottes ieder geit von Diefem elenden seben gefjal, ten/onto wie gar zeitlich fie oeffelben vberoruffig worben fint. 23 eil ou es aber 
Def Todes balben. 169 aber noch fo garlieb haft / uno po viel Davon fielteft/ fo gibftu ja Juverftefien/ Daks Di noch gar jrzoifch gefinnet bift। Die fündichen Süften noch in bir Kerz: fchen / Pricht viel Srfenonis Bottes / 2no wenig Buffe ono S3laubens in deinem Serken fein mus. \$S bedens de bich befler / liebe Geele / fen nicht To fieifehlich gefinnet / I raclite nach Dem/ DaE oroben ift / vnd nicht nach Colof, 3. $\mathrm{Dem} / \mathrm{was}$ auff Sroen ift. Dift ou mit Shrifto aufferftanden / fo fuche was broben if / Da SGriftus ift / fizeno fur Bechten Eottes.

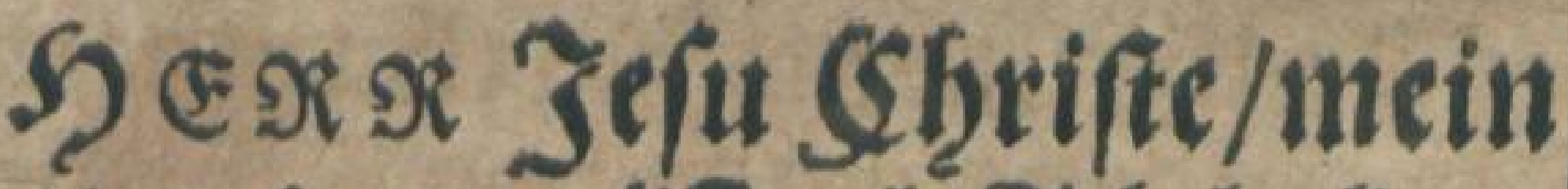
Gott vno mein'Deul/Iab beten: ne bir $j a /$ ons $\mathrm{flage} \mathrm{bir} \nu$ ber mein verberbetes fleifch onno 2 lut / oafí ich fo gar irroifd gefinnet bin/pno sas pergenglicbe DDepen biefes febens viel za lieb babe I Jit) bitte sich / mein (BD tt / er" leudte mir meine2lugen/sas ich

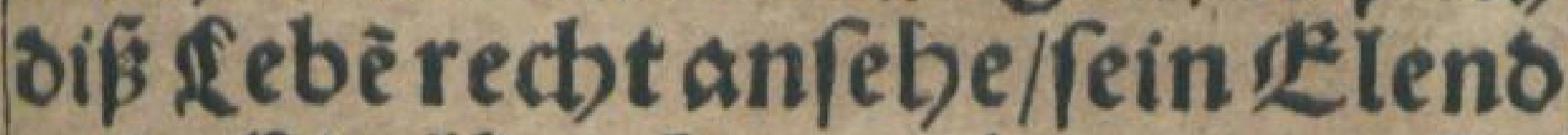
ons Eiteleit betrachte / ons rei:

p

nel 


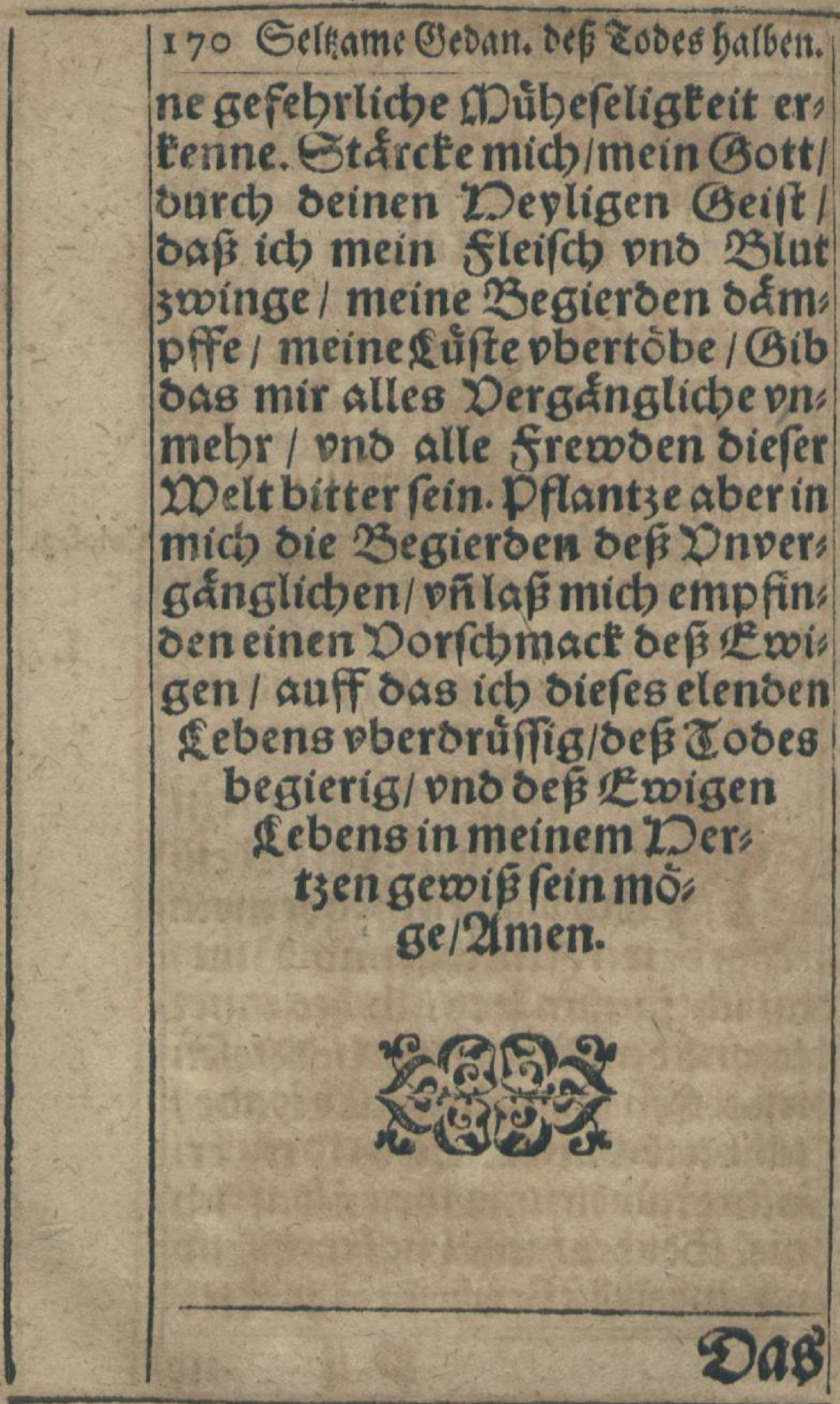


28ie man fretobis ferben tonne. I7 I

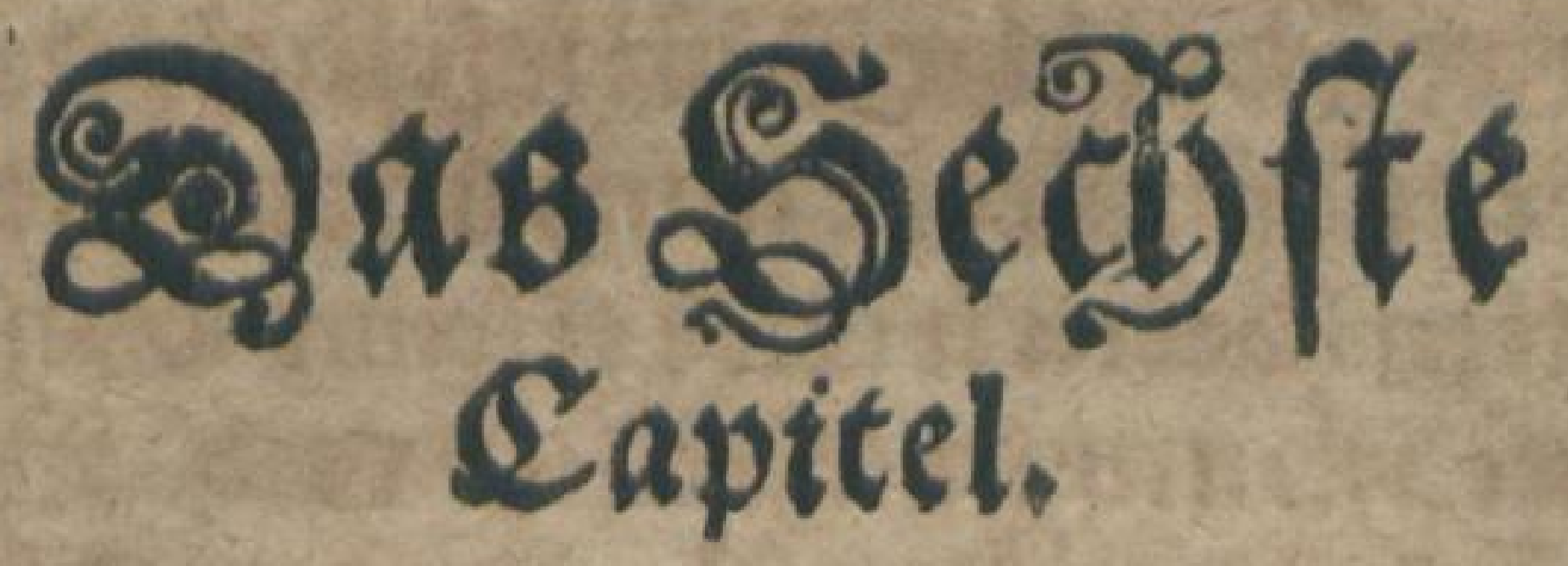

Berichtet / wie ein sefrit

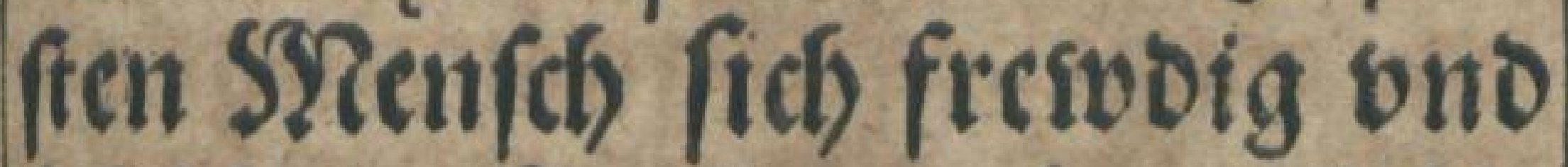
frólich zum Gterbeh ergeben / alle bit: terteit de B Soocs vberninoen/ vno in

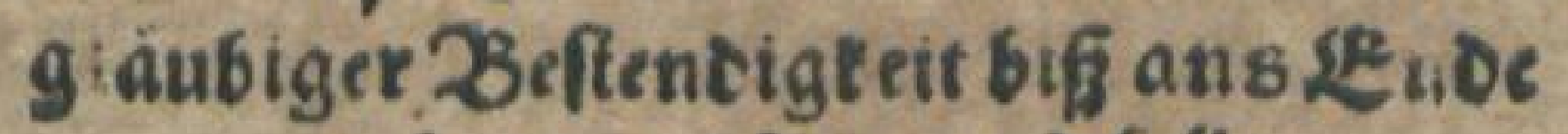
verbarren fonne vnd folle.

So fage mir nu/ $250 \mathrm{mit}$ for vnd fan id) I. bie Surcht deß ?odes ftillen/ Dan ich mich metnem GDtt gant frewdig wnb fròlich ergebe?

Q Orjeiten lefhreten bie

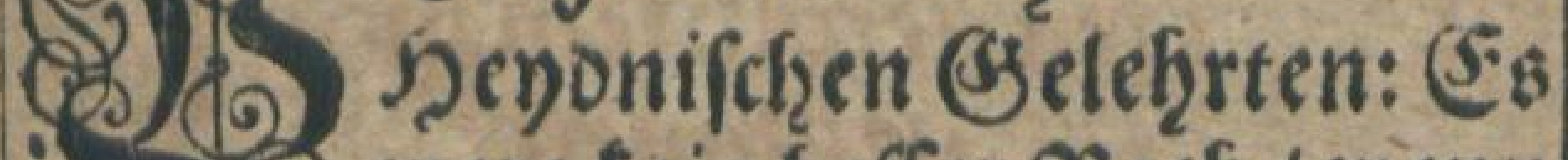
- 2 were fein beffer \atb / wern es sum Gterben feme/ als das man ei, nen friferzen Dath faffete / oen Ioot verachte / vno geodichte / (s s fornne nu nicht anders fein / 5 were vomb ein bojes Stúndein su thun / fo were es aus. Golcḩes hielten fie fúr cine grof:

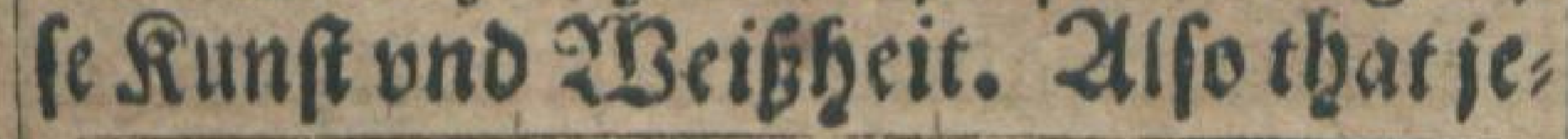

$p$ ii

ner 


\begin{tabular}{|c|c|}
\hline \multirow{3}{*}{$\begin{array}{l}1.5 a m, \\
150^{\circ}\end{array}$} & OSBic man frewoig \\
\hline & 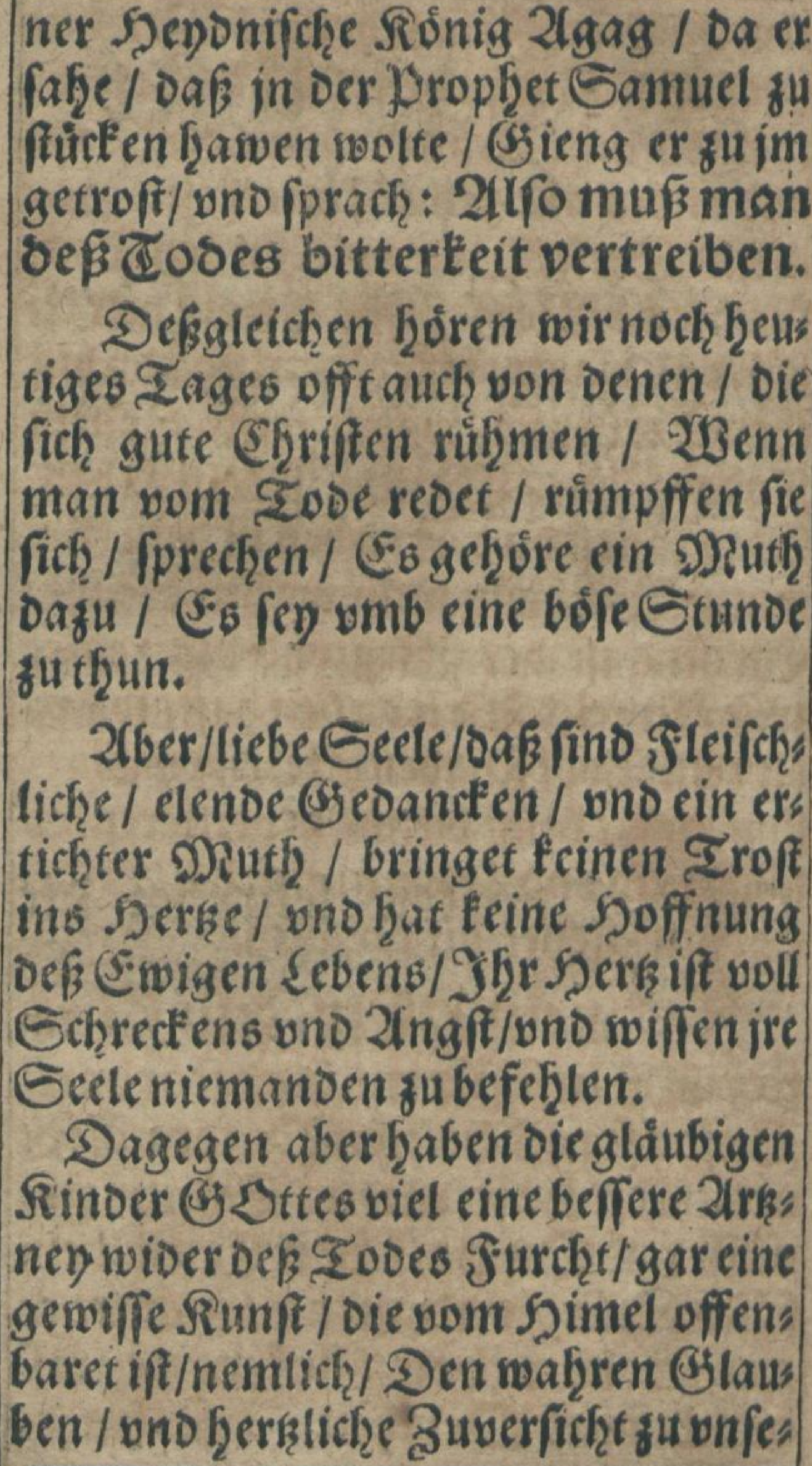 \\
\hline & \\
\hline
\end{tabular}




\section{ferben fónte.}

173

rem Sorren Jefu SGrifo/2Xer den frat / vin fich an in belt / Der fiat Dierech: te eigentliche 2(rkney wider Den IEOD/ ono wider alle feine Ditterfeit. Ia/ meine Seele/2Bas wolteftu den Foot fúreften ? VBeifleftu ooch wol / wer ou bift/ono was ou bift. Marlieh/ou bift thewer erfauffit / was sitterftu fir bent Zooe? Gife / Shriftus ift bein Leben. $25 a s$ zageft ous Sterben ift bein (b)e; winn: WBeiffeftu nicht no hin : Gifel Shriftus ift Der $2 \mathbb{B e g}$ uno die Senter Jum Simel. 23eil Duden ha af/ fo fan Dirfein Fodt fchaden / 2Beil ou EStris ftum baft / fanft ounichts verlieren/ob firon \{eib vns seben auffgefiet/Denn Chriftus fiefer dir fir alles.

Ja/mein SPerr Zefu Shri: fte $/$ Du bift für mich geftorben/ Du baft mir beinen Dater berfo" net / Du baft meine Eunbe ge, buffet / Du baft ben aost Ders fblungen / vno mir bas L'woige Seben erworben. Soldbes glån, be icb von Dertsen / ons balte $p$ iñ micb

Philip. $x$.

Ioh. 14. 
mich an bich feftiglid)/ Darumb babich in meinem 'Dertsen fries oe pno frewse/ Troft ono Don, ne/ pno adbte be cobes nicbts. Ja/DËrr Yefu/ourdb oidb babe idb Dergebung aller meiner $s$ un, ben / 2 in für (6D)tt gerecht pno rein / Dabe an imein gnáoigen lieb ẽ Dater/23in fein liebes A ins ons Krbe feines Dimelreicbs. Wein DERr Jefu idb bin bein Rigentbumb / Du baftmicb er, eaufft mit beinem 2 lute/ pno ers Iöfet aus ber Dellen / Ja ou bift mein 23 ruber $/$ ons ich bein DDit, Zerbe / Denn alles wassu baft / oafs ift audb mein/ Jaicb bin beis ne Dertsliebe 23 raut / oeine fuft vnd frewse/bein $\Theta$ atats/ben ou gar thewer erworben baft. Dar; 34 bin icb auch ein Tempel pns 20obnunge seines Depligen Geiftes/burdb welcben ich perfies gelt ons verficbert bin im Glau, ben. Ja serfelbe bat mich mit bir 


\section{fterben fointe $\quad$ 175}

meinem DDErrn alfo verbunsẽ/ Dereiniget/vno fo fefte vertnuipf" fet / Dafich bin Bein Don bei: nem 2 ein/Dñfleifd) Don beinem fleifch / Dîn muiten ebe alle Creas turen 3 grunbe geben / ebe mid) etwas von beiner fiebe fbeyoen Rom.8. folte. Was folte mir benn ber cos tbun: Do a:lidb nidhts meb?/ benn bas er midb náber 34 oir meinem DDErren Jefu bringe. Derbalben adbte idb sef cobes gleich wie nidbts/ Dabe oas \&e" ben im Dertzen / vns rese Don nichts / als vom feben: Denn Shriftub ift mein $\operatorname{Ieben} /$ bno Philip. s Sterben ift Inciii Ssentun. $j$ ath finge ons fage mit $\delta \mathrm{em}$ lieben Diob: Jih weif̧ Das Hiob x. inein erlófer lebet/vito ey foiro intid Gernads aus Der Erben auffersoeden / ond swerbe barund) mit biefer meiner Saut

$$
\mathcal{P} \text { iiij } \quad \text { omb }=
$$




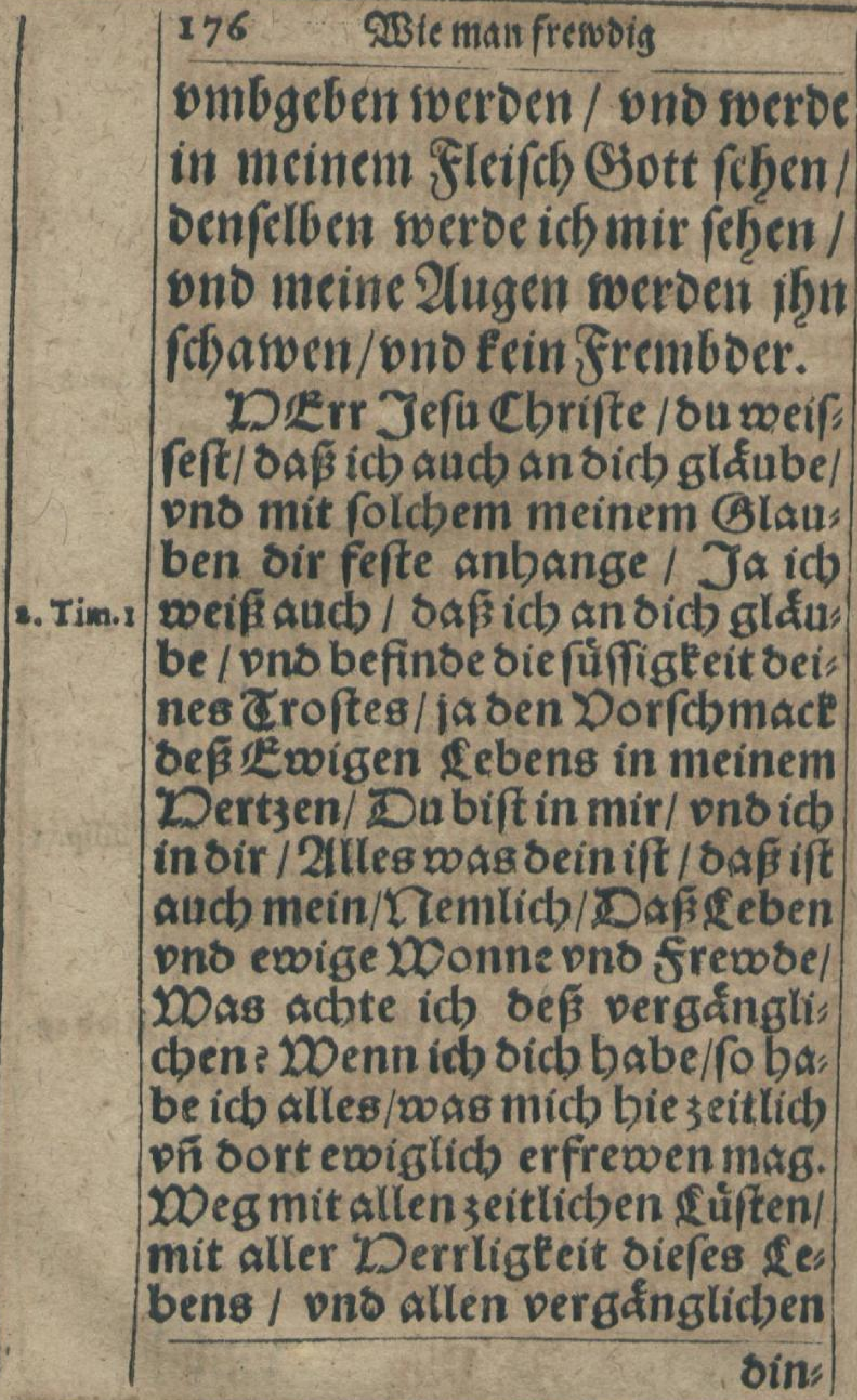




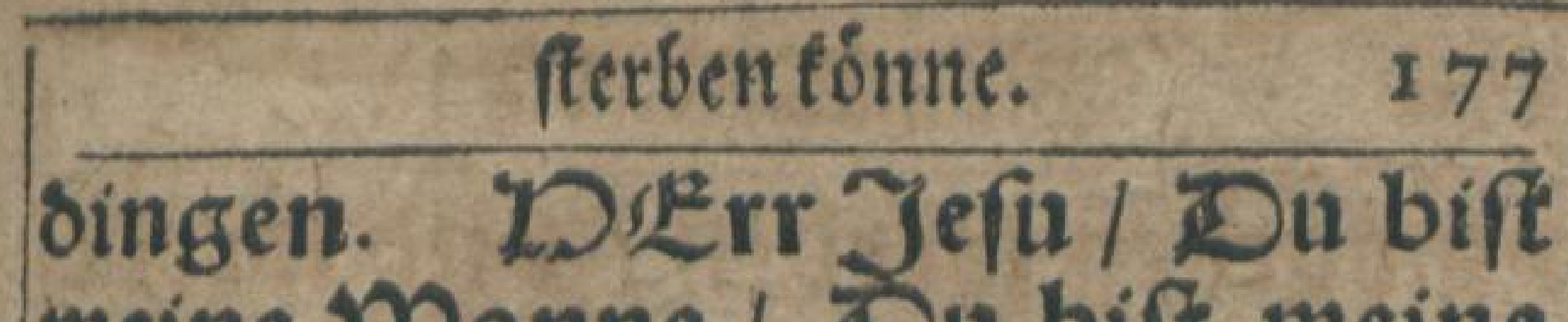
meine LDonne I Du bift meine frenobe I Du bift mein Gelot / mein (Gutt/Dubift meine K bre/ mein Xbum/meine "Derrligteit/ meine fuft ons evoiges Xeich: thumb. Sersilich lieb babe icf)

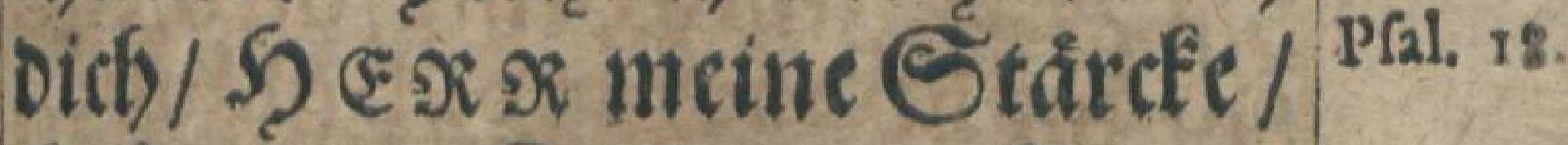

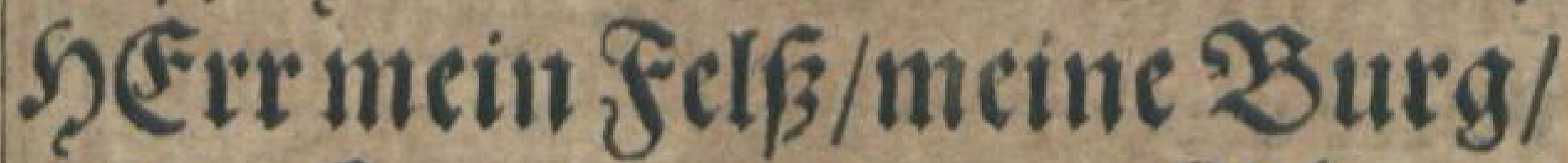
mein Ertetter / mein (SiStt / mein Sort/ auff Den ich trasbe/ mein Schildot ono Sport met nes Spenls ono mein Sctuts. 2Benn ich nut oid babe/fo fra = Pal. 73. ge idf nichts nach Simel ono Eroen/23no soenn ntir gleids Seib vñ Ged veridimaditet/ /o biftu boch Sbott allezeit meines Serken Eroft ono mein Ebe)l. Eoldber (Gtaube / foldbe fiebe / foldbe $23 r u n f t$ gegen bir / mein Derr Jefu / bat mein Dertse

$$
\text { P a alfo }
$$




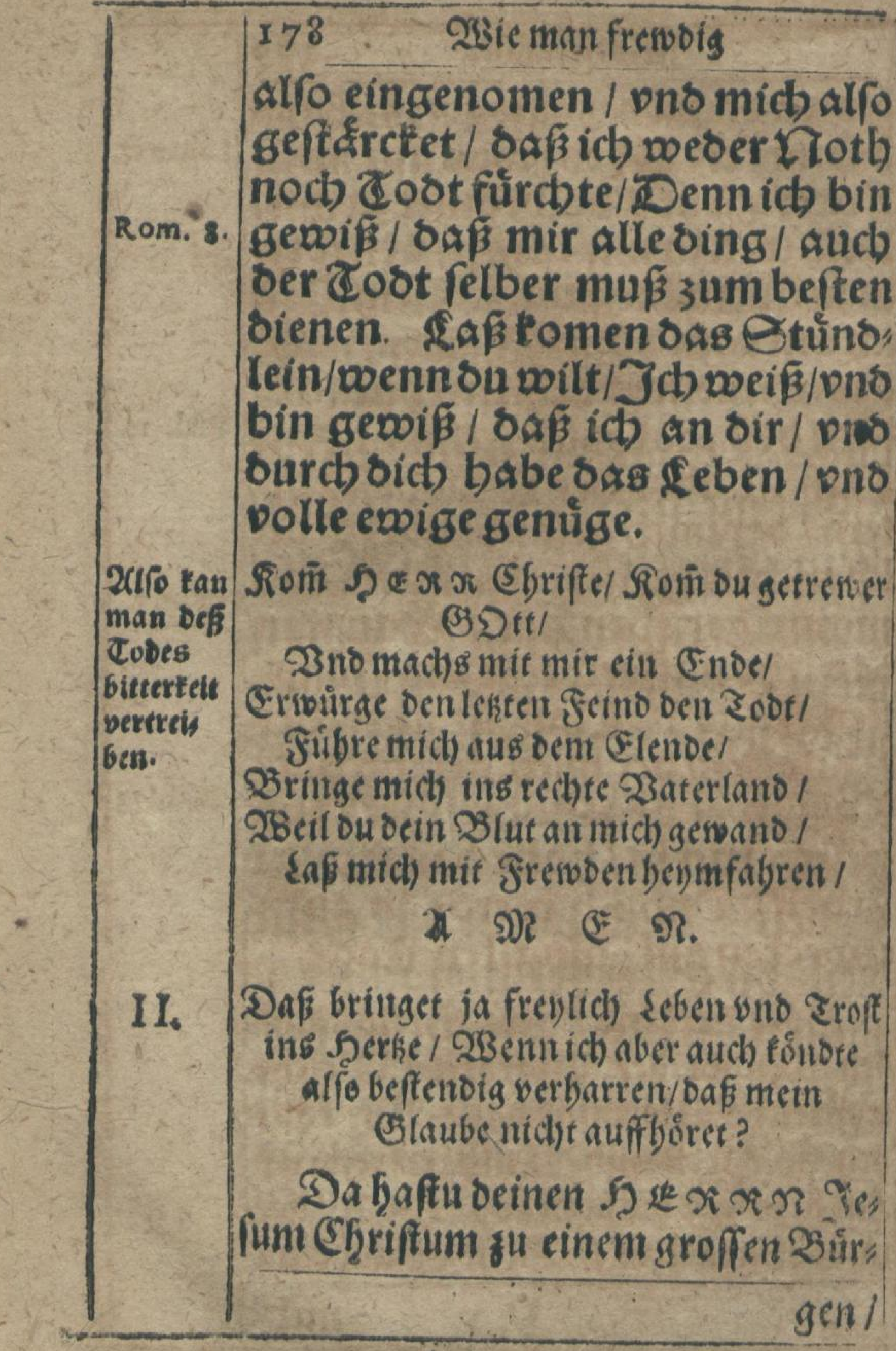




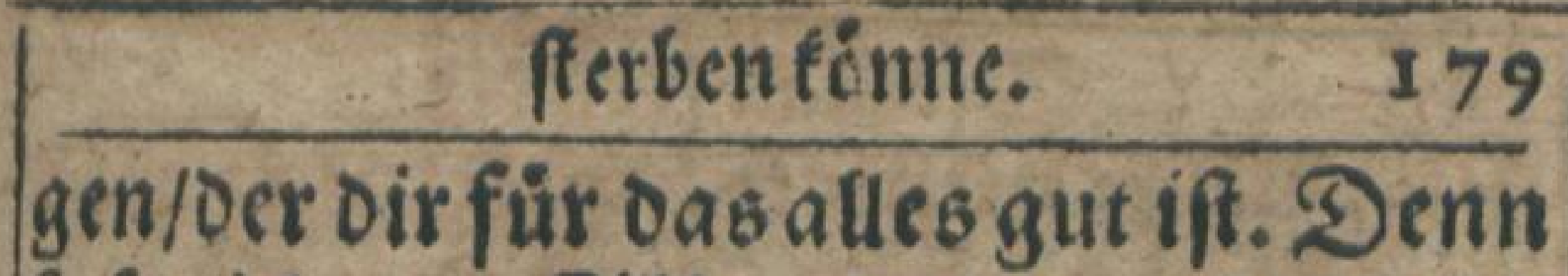
fo fpricht er: 2flles / woas mir mein Iohane. Dater giebt / baß̂ Pòmet 3 u mir/ Dno wer su mir loòmet/ oen wer, be ich nicht bienauŝ́ forfen/Deñ id) bin Dom Dimelt tomen/nicbt oasicb meinen Willen tbue/fon: bern be $/$ / ber mich gefandt bat. Daji ift aber ber Dollte be $\overline{3}$ Da: ters / Der micb gefanst bat / Daf icb nicbts verliere on allem oas er mir gegeben bat / Eonbern. oas icbsaufierwecte am Juing: ften rage : Das ift aber ber Dille be / / ber mid gefano bat / Daßj / wer ben Eobn fibet / pno gláubet an in / babe sas L'wige Leben/ Dns ich werbe in auffs erwecten am Jüngiten rage.

Shore doch f̧ie / liebe Grele / auff den Diunot Der 2Barfzeit / Welchie troftreiche 2Bort find Dass 2 Bie fons nen fie Seris und Druth erfrewen?

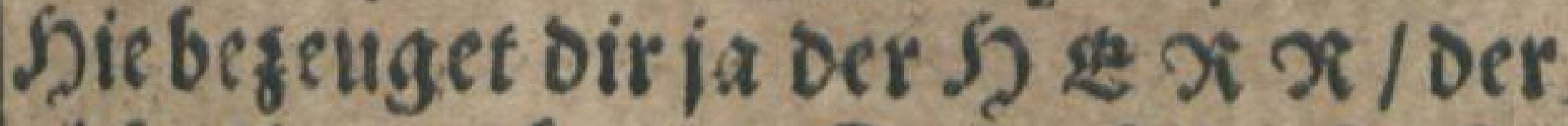
nicht liegen fan I Drin Blaube fot nichit 


\begin{tabular}{|c|c|}
\hline & 23ie man frentobig \\
\hline & nicht aufffioren / Du folft nicht ver \\
\hline & loren / Gondern beftendig erb̧alten \\
\hline & werben zumjsigen seben. \\
\hline Luc, 22 & 2lbermal pricht Der ŞEr / Jd \\
\hline & Gabe fir sich gebeten / oap sein \\
\hline & Glaube nid)t auff \\
\hline Loh, 10. & Dno abermal: Deine Schaffe \\
\hline & Goren meine stine / ons ich fen \\
\hline & ich gebe inen sas S wige \&eben \\
\hline & Dñ fie werben nimermebr vmb \\
\hline & $\begin{array}{l}\text { Eomen/Dñ niemand } \\
\text { meiner panst reiffe }\end{array}$ \\
\hline & ter / ber fie mir gegeben bat / ift \\
\hline & g:öfer benn alles/D no niemano \\
\hline & $\begin{array}{l}\text { wirs fie aus meines Daters } \\
\text { Dans reifen/Ith pns ser Dater }\end{array}$ \\
\hline & fins eines. SefBgleichen f pricht er \\
\hline Lис, 1 & auch : DDaria bat bas gute tbeyl \\
\hline & ofis folnicbt Don ir ge: \\
\hline & \\
\hline & onte dich der fy a $x x$ fchóner ber \\
\hline & no fu[agt I \\
\hline & urch die trafte leines @eifts \\
\hline & \\
\hline
\end{tabular}


ferben fónte. 181

gut Dafür fein / Dein (3) laube fol auch nichtauffiboren / Riemand fol dich. aus feiner bno feines 23aters Sandt reifen/23nd oas gute thenl/ Daf ou in Deinem fyerken haft 1 fol nicht von bir genomen werden Eobifunu gewis/ meine Seele / bende deß Swigen Le" bens / vno auch Der beftendigfeit fum Ewigen seben/Daf ou dureh feine (S) nade beftendig verharren werdeft/ big ans ento / Er roolle bich nicht lafien/ Ondou werdeft in nicht laflen.

Derbzalben fen getroft und frewe bich / vno fprich mit Ganct Daulo: Jch weifs an welcben ich gleube/ i. Tim.I. vno bin gevoî́ / baj er mir meis ne bevlage bewabren $\mathrm{e}$ an bifiz an ienen ag. Who abermal: Ich bin Rom. gewif́s / Daß weser rost nod Leben / weber is ingel nod) furr" ftentbumb/nocb Gewalt/ weber Begenwertiges noch Jutuinff: tiges / weber "Dobes noch Cief" fes/ nocb teine ansere Creatur/ mag midb fobyoen von ber Rie, 


\begin{tabular}{|c|c|}
\hline 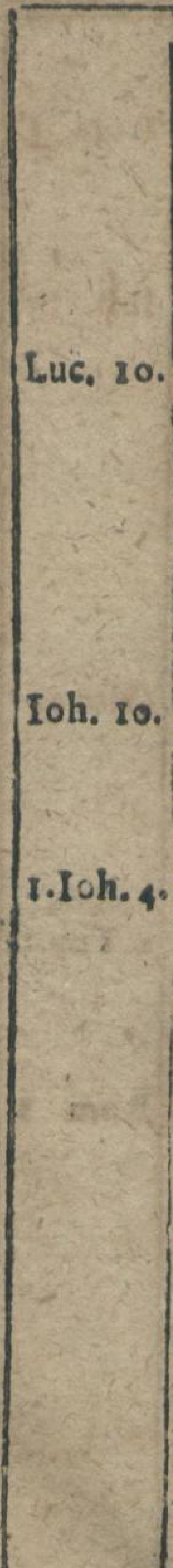 & 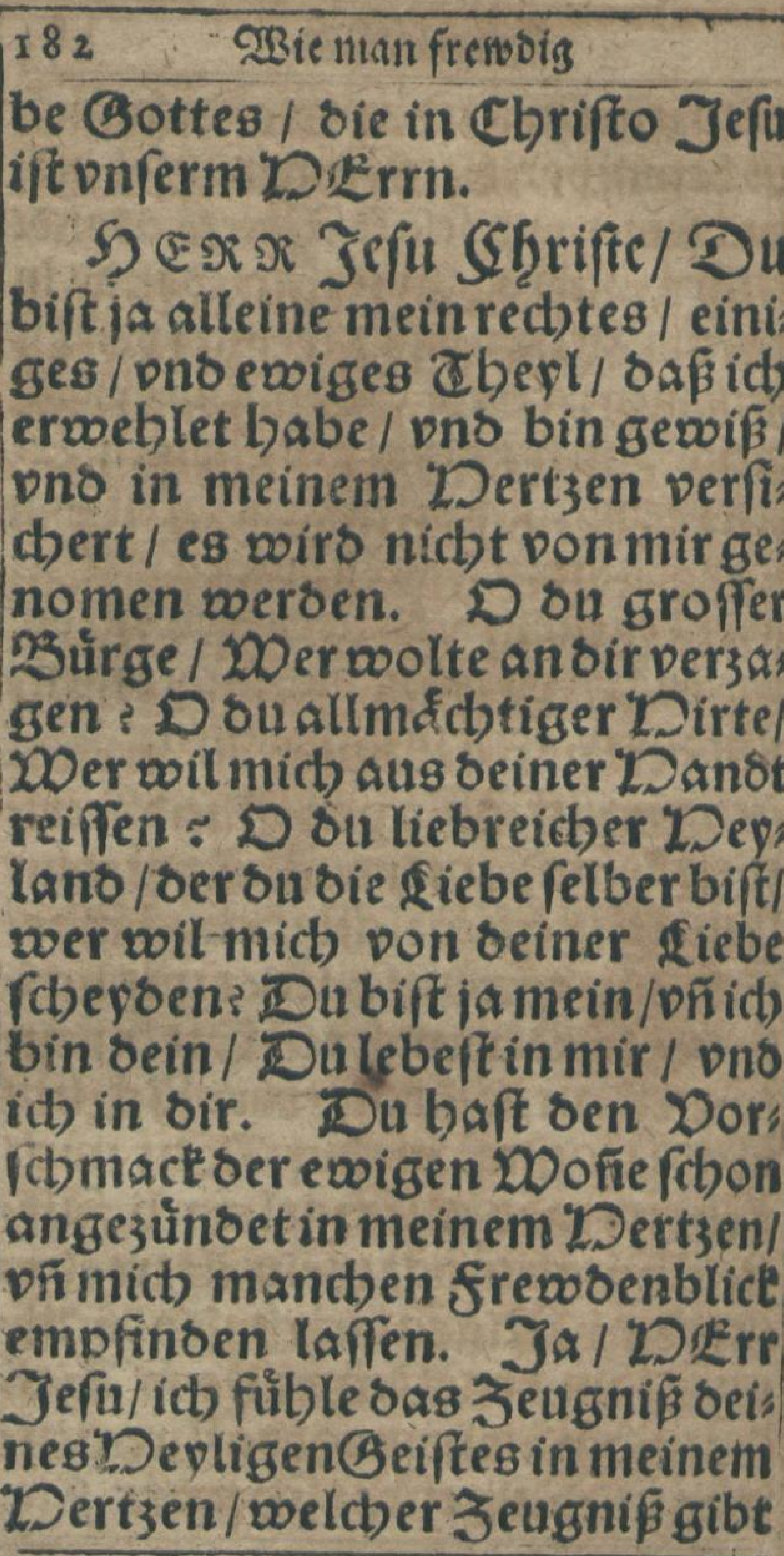 \\
\hline & \\
\hline
\end{tabular}


ferben fontre. $\quad 183$

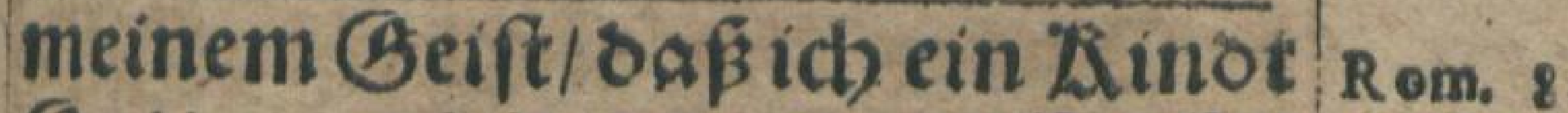
Gotteg/vns bein ewiger DDit: $\mathrm{R}$ : be bin.

Du bift ser 200einftock / Dno Ioh. 19. ich ein debe an bir / Du biftser Saum bef s Lebens / Dno ich bir fefte eingepfropffet ourch meis nen tebenoigen (slauben. Du bift oer 23 reutigam/pns icb bein Dertrawotes Dertze/ DDit bir Ders bunben/ LDit bir Dereiniget / LDit Ofe. 2. oir Derlenuipffet Surch einen ewo: gen vnauffiōslicben 3 uno/ wels c)en teine Totb nod) Zoot/tein reuffel/tein 21nglt / tein Ecbre: d'en sutrennen follen ewoiglich. LDein "DiRrr / mein GDtt / mein Dort / mein aroft / meine fuft / Du Donne meines Dertsen/ Du frewbe aller meiner "Ä raff" ten/Die brennet mein Dert; ges gen oir / Wie fol icb midb an bir gnugfam ergetzen: Donn wers be ich) Sabin fomen/Safí ich oein Pral. 42. tröftlich Zngeficbt fobawe. 


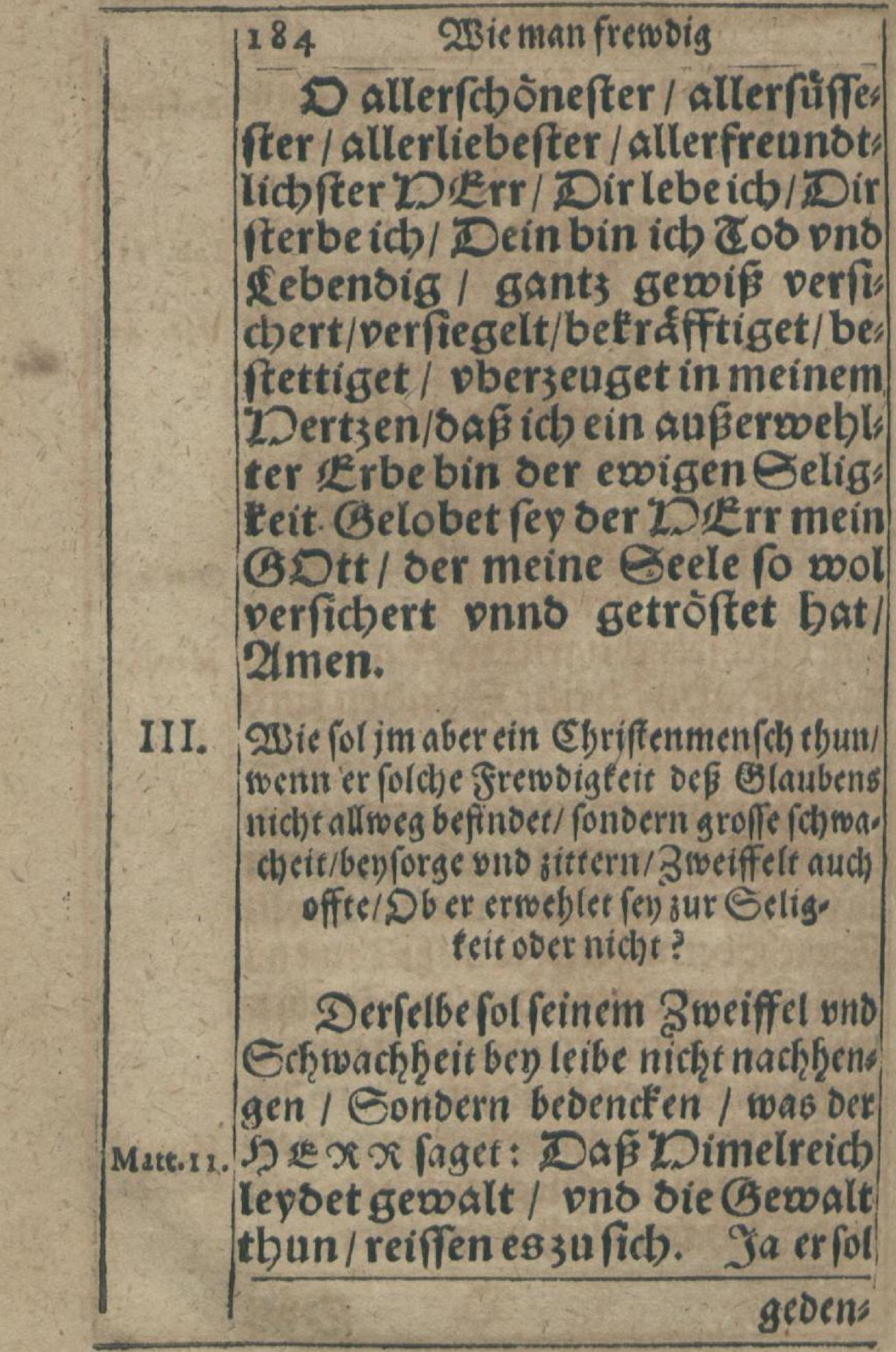


geterben fonne. $18 \mathrm{~s}$

li: Jcherinnere sich/Dais ou er" 2. Tim. 1 weckeft die Babe Bottes / bie in oir ift. Ex troffe fich auth Der hebl fa" men Bufage / Da der Şerr fpricht: Dấ zuftoffene Xobr wirb er Era. 42 nicbt subrecben / Dns bas glim: menoe (cocbt woirb er nicbt auks" lefhen. Er labe feine Eeele mit oem

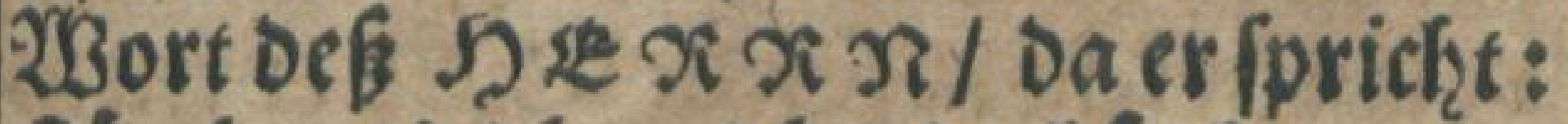
fuird)te sich nicbt / gléube nur. 2 no abermal pricht ber $\mathcal{S}$ se $x$; oem Esichtbrichtigen: Bey getroft/ Math.s. mein Sobn/beine Einoen fino oir vergeben.

Denn/liebe Seele/es gibtsbietågs liche Erffafrung/ Je mefhr fich einer

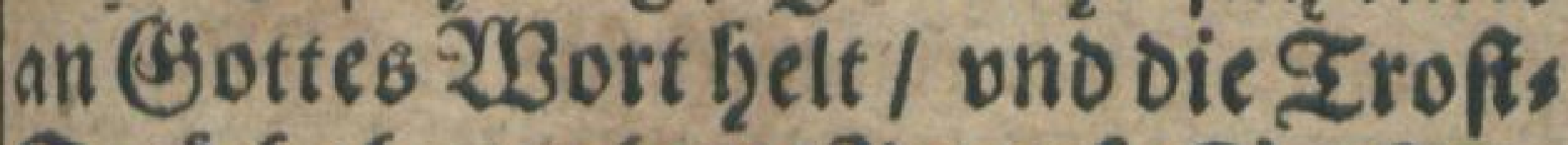
Epruiche betrachtet/ Je mef̧rąroft im auff gethet in feinem Serken. Sonders lich aber / betractite er ben Senligen

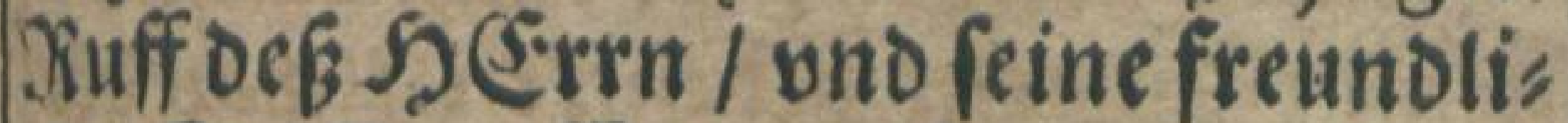
che Etime : No Omet ber su mir al: Matt. 1r. le / bie ir múbefelig vno belaben (epo/:J ch) wil eudb erquicken.

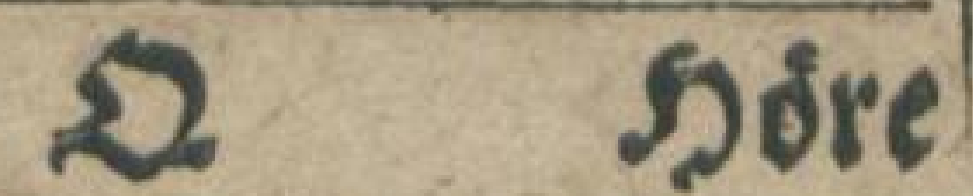

Mare. 5. 


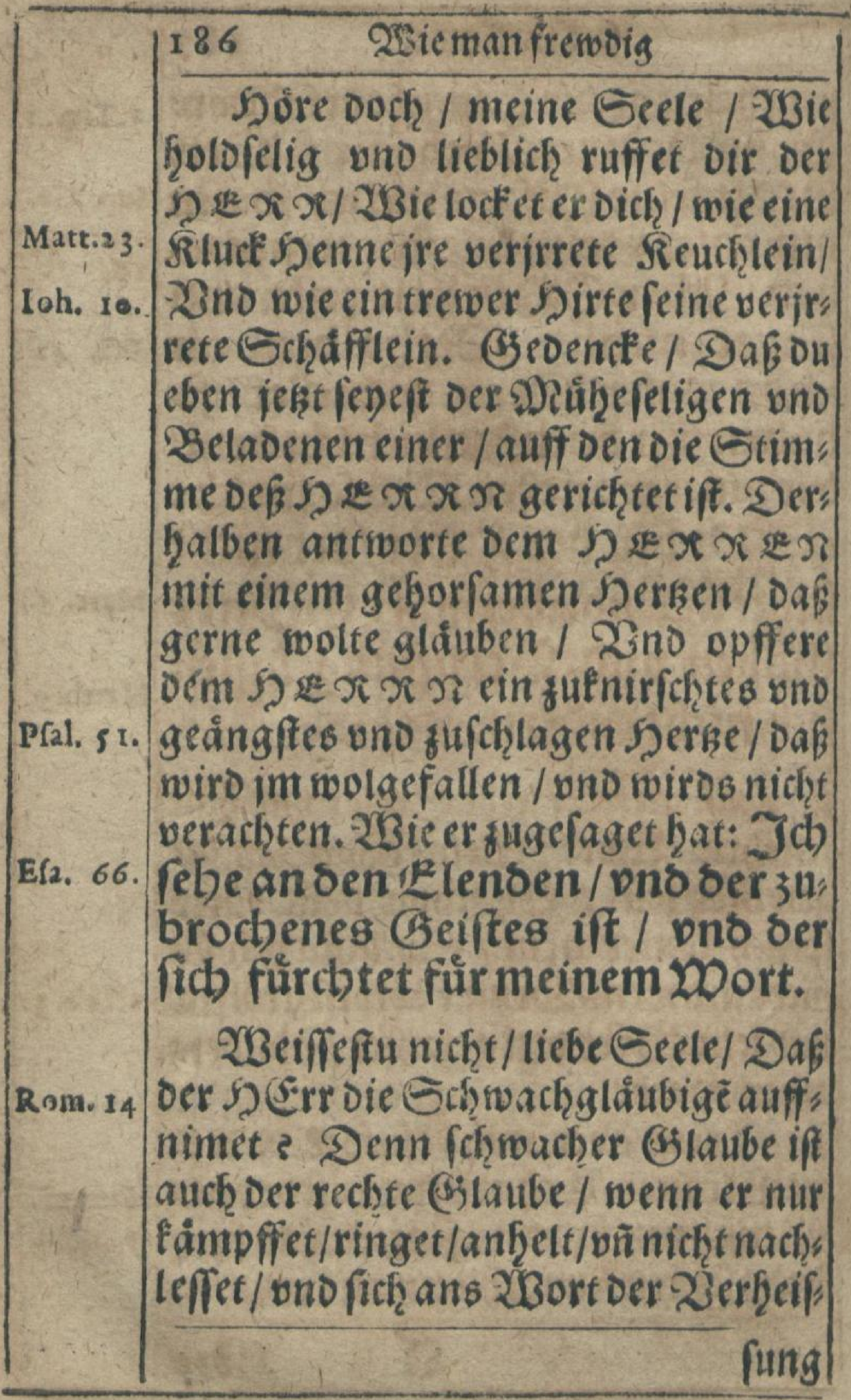


fineg belt. Denn fo fpricht ber themere Mann D. Mart. Lutherus : Delt GD tt feine Jufage nicht / fo ifts sup. Gemit pnfer $\Theta$ eligteit fobon gerebes ben: 2aber (OSDtt belt fefte ons ner. cap. 26. leuget nicht. Derbalben ob fbon onfer Derts wanciet / fo baben wir bod) Juflucbt 3 u bem/ ber nidbt wancket. Deñ fo fpricht er: Jidh bin Der S (Err/Dernicht letiget. Dno abermat: SSOttes Gaben ond Berufiung lafien fidb nicht endern. Hac ille.

Daf mert"e / liebe Seele / wno halt fefte am rBort: Sein 23 ort la bir

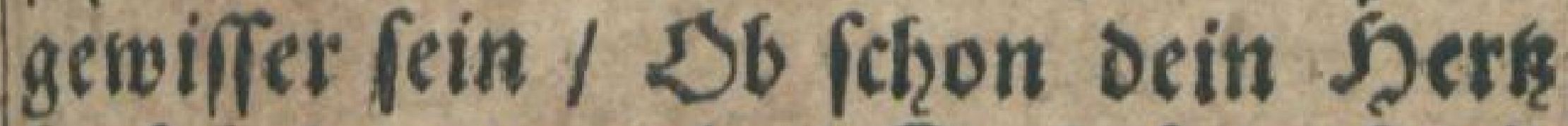
ipraiel lauter nein / So lak Doch Dir nicly grassen. Denn (Sott if grốfer/ ynd fein ZBort vielgewiffer/als dein eigen berke.

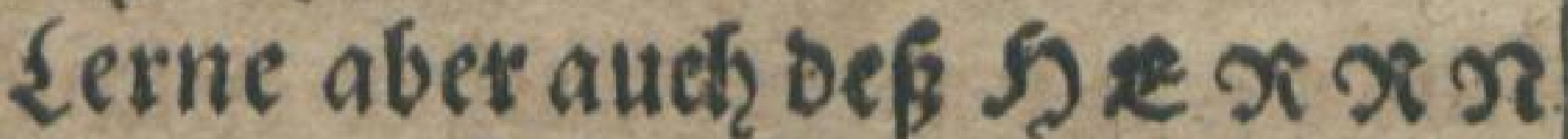
weife fennen/Er frat feine suft bey Den Dienfchen Rindern / Bärtelt vno fpies provo s. Ift mit jnen / wie Siltern mit jremties 


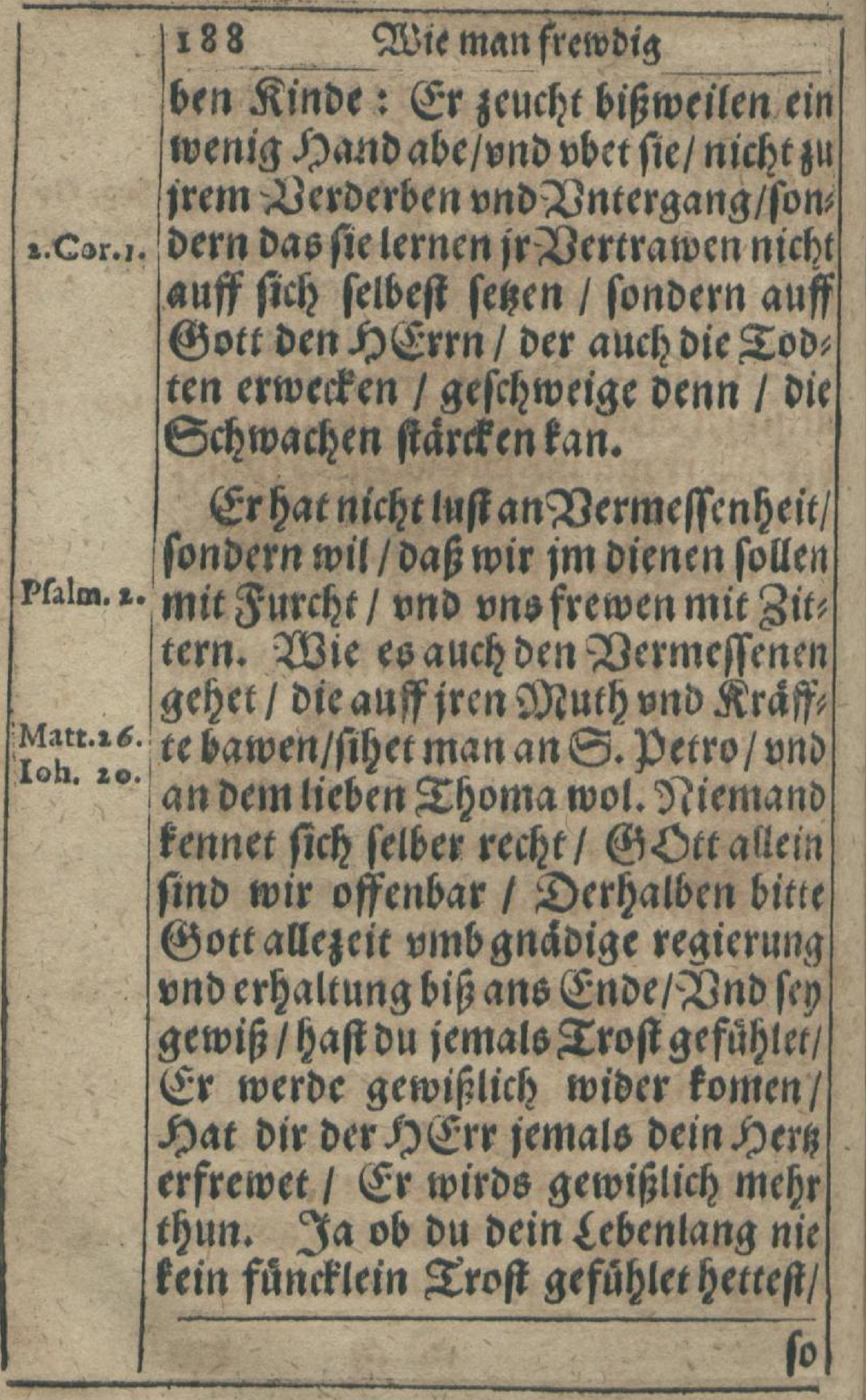


ferben fonnte.

189

fo verzage boch nicht / Becte / gláube/ hoffe / habe Geoult / oer Iroft Dé herren wiro gewiflich fomen / ono Abac, 2. nicht aufen bleiben/ fonderlich an beis nemlekzten Ender.

Bebende Das Exempel def Cana" neifchen 2WBeiblins/Eifie/ wie gărttelt Matt.1s. Der Sherr mitje / wie weifet er fie abe wolorey mals Daferfie mal ferzwei, get er gar ftille / bno antwortet ir fein 2Bort. Dafianoer mal leffet er fich Gơren / (se fey nicht ir gu gut fomen. fonbern Den Rindern Ifrael. Dafa oritte mal heiffet er fie sine Suindin/ die feines Eroftes nichte werth len. 23asthut fies Sumerfen / Oeketfie feinem ftill for weigen entgegen ein ges oiltig Jerke/Dak wol hoffen uno biar: renfan. Bum anoern/ Sezet fie oem S) Erren entgegen ein feft gláubige STerke/ Daf fich aluch ins zBort fiulllet] nno theyl an oer Zerbeifing haben wil. Zum oritten/Eecetfie int entges gett ein bemultfges 5 erase/vno wil ger:

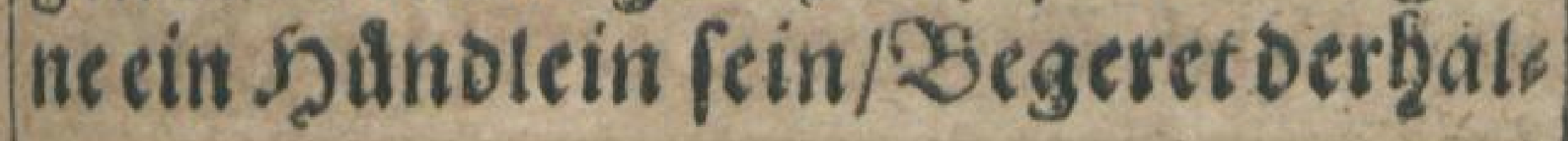
2 iii bent 
ben nut das Sgunderecht / nentich/oie Bródtein/oie vom S्Fifhe fallen/ Daß if / Pur eingar fleine Sulffe/2ur cin flein Sundtlein Irofts / Daran nuiroe fie fampt jrer Sochter gar genug has ben. Da fan fich enolich oer J) $x x$ nicht mehr halten / fehuitet ir das ga feiner Salffe gar umb / sno fpricbt:

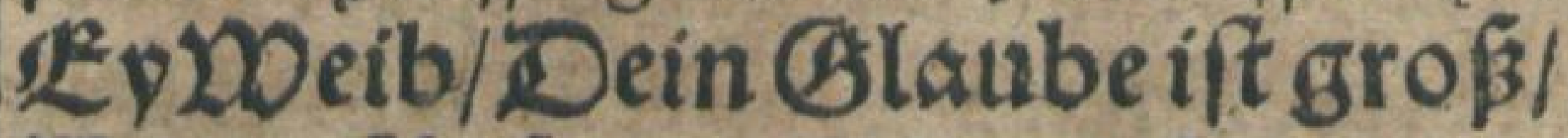
Dir gefbebe / wie ou wilt.

Sprichft ou aber/ Sa fie war ftart

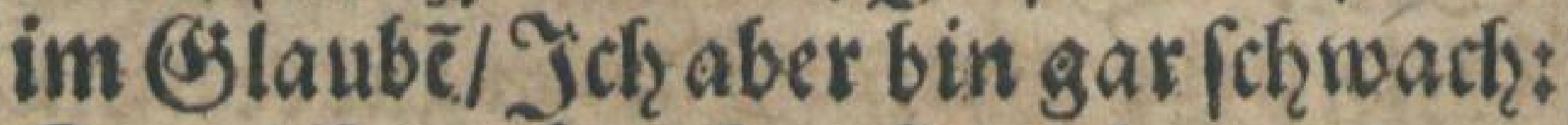
So wiffe/dak onfers@laubenseigen: ferbafft ift / wie cines Xingers. Sin Ringer beforget jifierdar / Ser móchte verlieren / ono feinem 2Biberpartb fu februach fein/2ufo unfer @laube/ weri cr ringet uno fámpffet / meinen wir / Sr werbe viel fu fochwach fein / onb im Gtrent bnterliegen. 2lbernein/Denn oer Gieg geburet dem @lauben f So fome noie es wolle/ po geburet der Sieg Dem Slauben/ Ja auch Dem. fichwache

1.Pet. 3. (Slauben / oer fol Dus Jelot befalten/ vno Dursh Sottes פRacht fur Seligs ete bewabret werden. 
Biebencte auch an oen Rampfi beß 2litvaters Yacob/2nicer mit \&5tt Gen. 32. ringet ono anţelt/nicht nachleffet/ono fpricht : Idb laß̧ sich nidbt / Du regneft mich benn. 20no al fo ges reriabees auch.

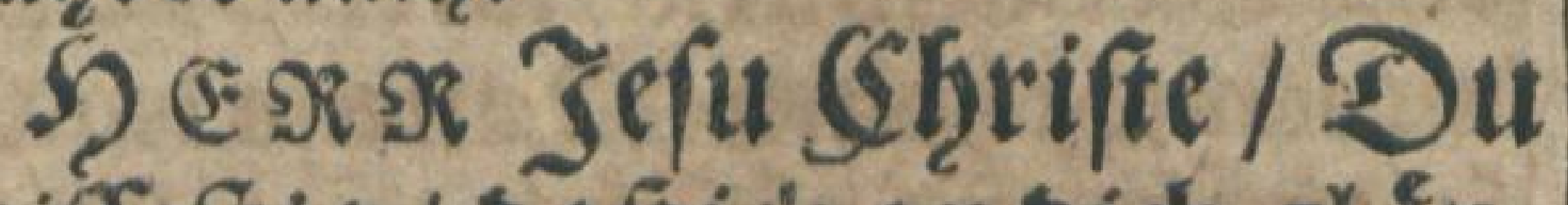
weiffeft †a / baßich an bich glåu be/ ob wolmit ft)watbem Glat: ben / Jerodo mit oiefem meinem f(t) wachẽ Glauben / fetzeich auff oich alle meine Suberfict) : :Tes cke mir beine trṓftlidie 'Danot! pno zeudb mich) 34 oir. LDein DDErr / ich bin ia faboach / $D$ mebre mir meinen Glauben / $D$ ftárcle mir meine Suberficht. Luca 7. Eey ou mir nur nicht fobrecetich/ mein Dort / Denn ou bift meine einige Suverficht / 200 fol ich fonft bin? Slafe auff oas teine finctlin in meinem Dertsen/ ourch beinen Deyligen Beift/ Eibe / woillen babe id) wool / D Rom. 7. gib ou bas Dollbringen. Jal

D iiư DËr




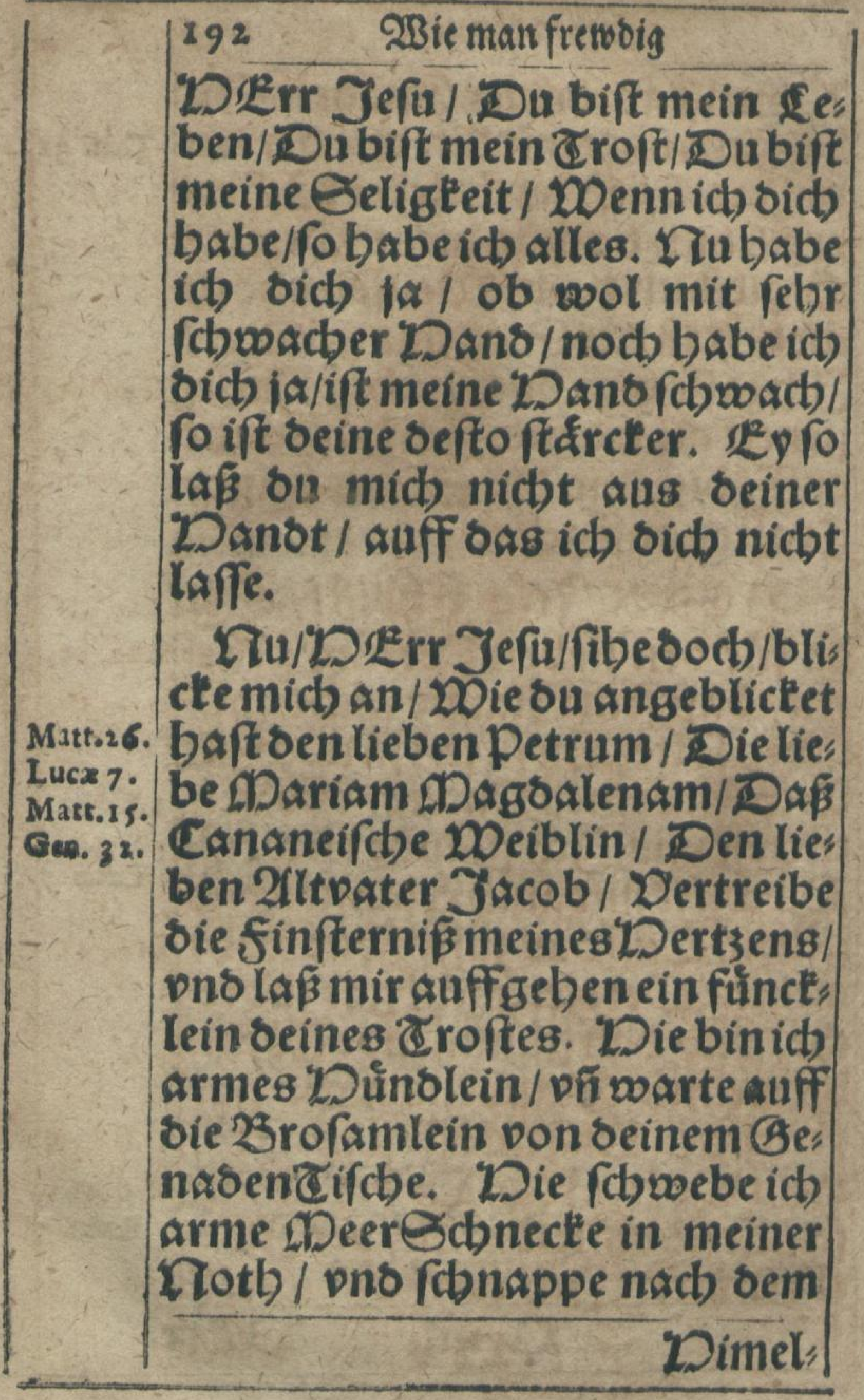




\section{ferben tơnne.}

193

Limel Tawo seiner Gnaben. $\theta_{i:}$ be/ Jch ringe mit oir/ wie ber lie: be Jacob/Douftarcter DERr ergib oich soch in mein Dertze I R'rgib sich soch/20eiffeftu sod wol/ baj ber Eieg mir geburret I Jab) laffe bich) so(b) nicht / Du fe: Gen. 32. geneft mich oenn/Icb börenicht auff / biß mir bie ઉonne beines roftes auffgebe.

HE $\mathfrak{A}$ Jefu SEgrifte/Der ou für sen lieben Petrum gebe: Luc, 22. ten / oaß fein (Glaube nicht auff" bơzen folte/Du fitsef $j$ a nodb sur Xecbten beines Daters/ vns Ders/,r.roh. 2.

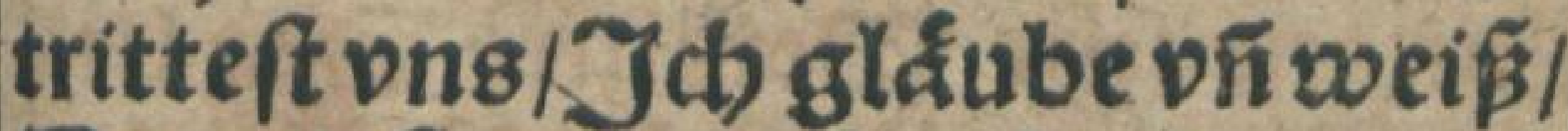
Du boirft mir audb erlangen ons

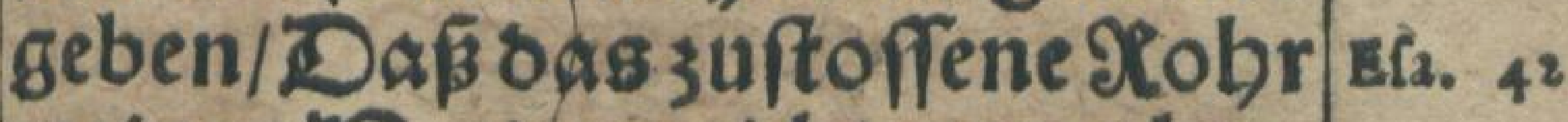
meines Dert;en nicht gar 3 ubre: dbe / pns oas glimmense co obt) lin meines Glaubens nicht gar Derlefobe. Du fableffert ia nicht/ mein Dort/Lein/Deñ er fchlaf//psal.121

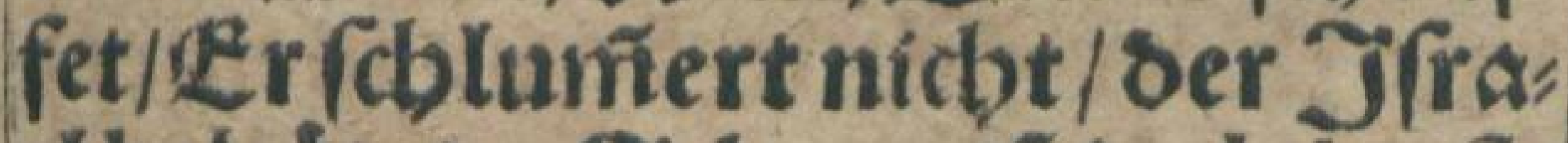
elbebuitet. Jdb weipósubŏreft/

$$
20 \text { Stes }
$$




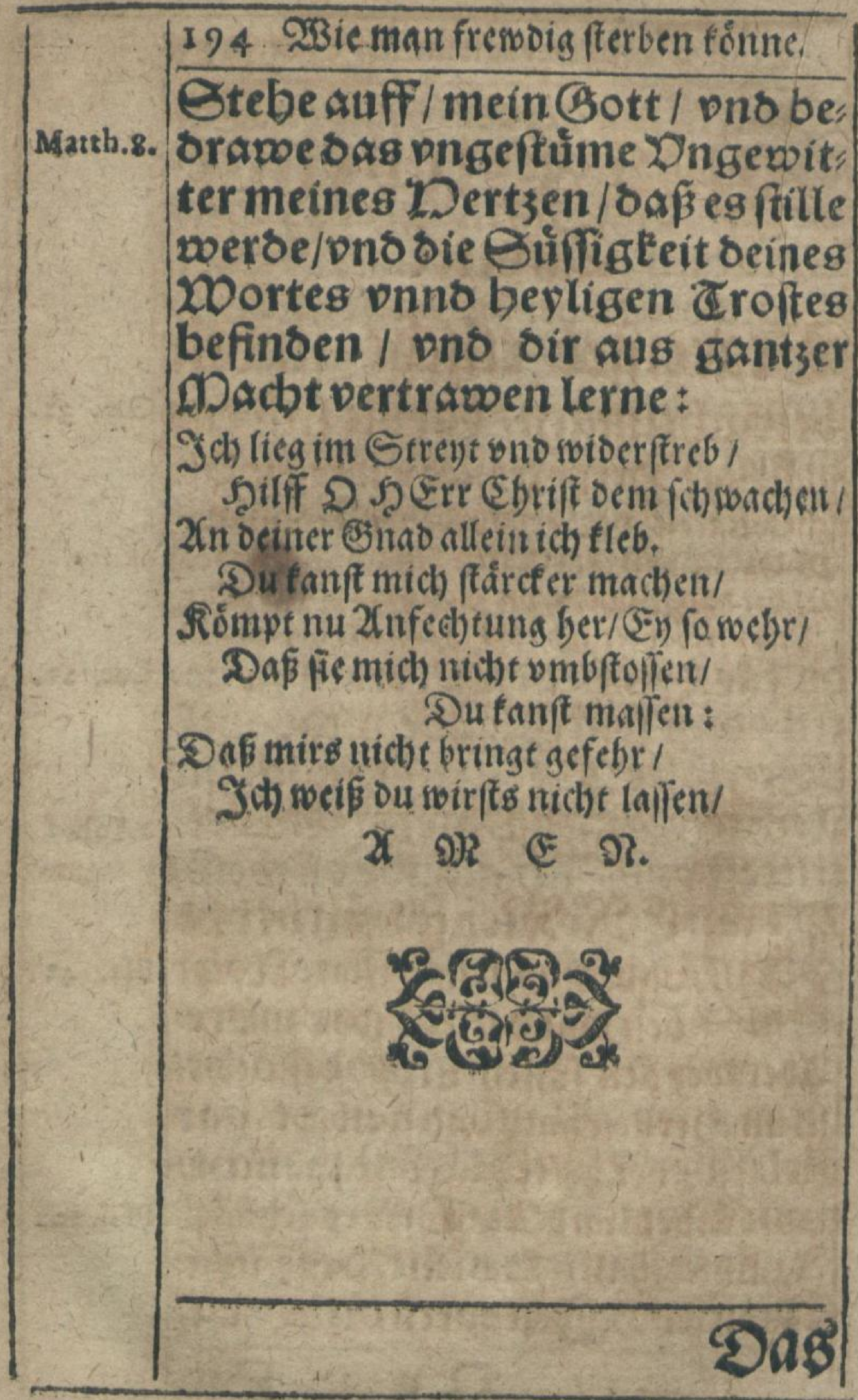




\begin{tabular}{|c|c|}
\hline & Sroft ivibet bett \\
\hline & ne Senm farth ins ewige 2 aterlant \\
\hline & Denn Shrifus / oer in inen wohnet \\
\hline & Lat fur fie den Iodt gefehmedfet / in \\
\hline & feine Bewalt genomen / feinen Sta \\
\hline & ubrochen/Daf \\
\hline & gen nichts mef̨renden fan / oenn nu \\
\hline & Seib und Geele fiche \\
\hline & aber / 2Bic jnen fo \\
\hline \multirow[t]{5}{*}{ Rom. 8 . } & fien bienen milfen / 2llfo oienet jner \\
\hline & auch der Todt Dargu/Daßfie von allen \\
\hline & $\begin{array}{l}\text { Wbel erlofet weroen / vnd fu Nufy } \\
\text { fomen. }\end{array}$ \\
\hline & Solenges ge \\
\hline & maltig \\
\hline Sap. 2. & $\begin{array}{l}\text { ocr V Beifheit: Die Eeelen ber Be } \\
\text { reditenfins in (SDttes Want }\end{array}$ \\
\hline \multirow[t]{8}{*}{ Ioh. 14.} & Q2no Ériftus fpricht felber: Ict \\
\hline & wil wiber fomen / ons eudb \\
\hline & /mirnemen / 2fuffoas ir fevo/ wo \\
\hline & $\begin{array}{l}\text { ich bin. Hoire fie / vnolerne / lieb } \\
\text { Seele / was dein Sterben ift / Richt }\end{array}$ \\
\hline & mehr als cin 2abfhenden / eine Spin \\
\hline & \\
\hline & \\
\hline & J) \\
\hline
\end{tabular}




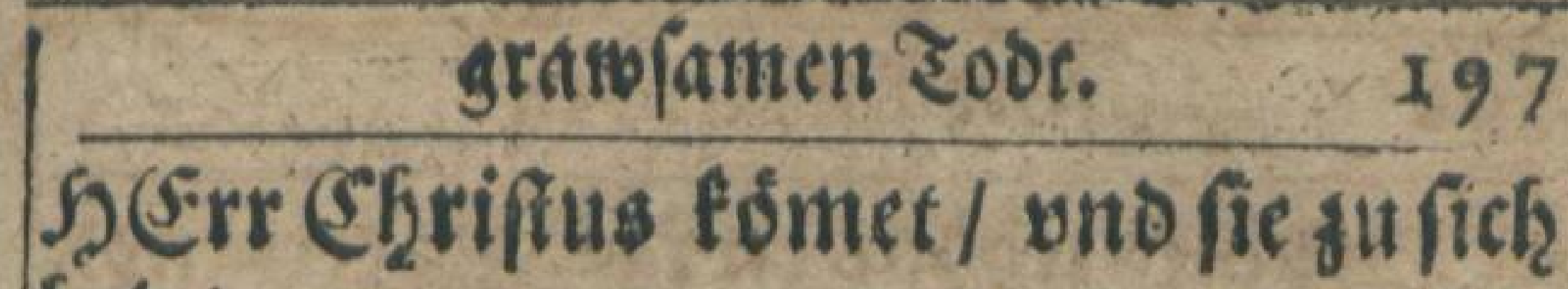
holet.

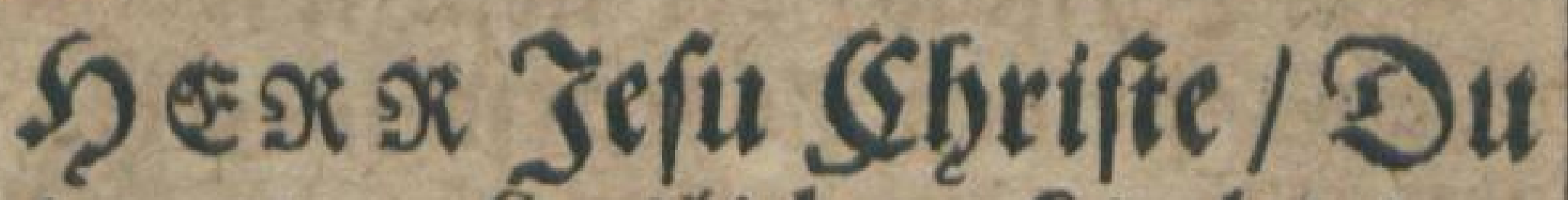
eroiges / wo entlicbes fiecbt / er: leudbte mir mein 'Dertze/oaks ich als ein newogeborner LDenf(b) । ben Tost mit newen 2lugen ans rebe / vno in nicht balte für mein Deroerben / Sonsern nur für beinen 30 ten/ ourd) weldben on micb ab foberftaus biefem Jam: mertbal/ ons zu Rubebringeft.

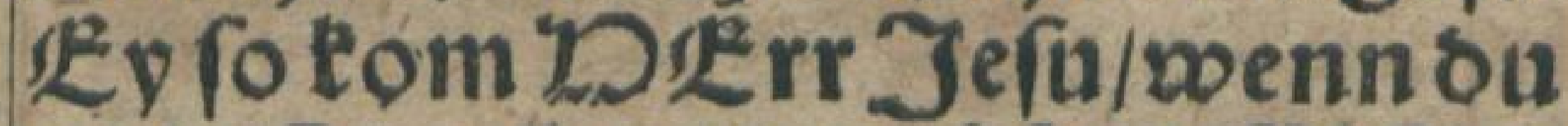

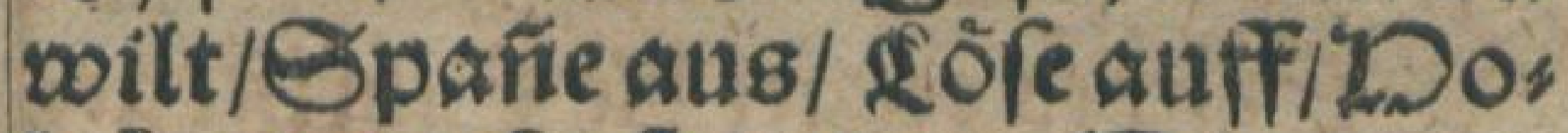
le heym / faßj beinen Diener in Lucx 2. friebe fabren/ ber ficb nacb bei: ner Xube rebnet/2Imen.

Es macket vits aber ber ?obt gar gremlich II. vino ablcherolich / falt und vingeftalt /. millen erfarten / ond im Strabe berwefen.

Dafift auth cinte Straffe/die Esott vmb ber Gunden willen 1 , vber Den seib ergelien leffet. Aber die gláubio gen 


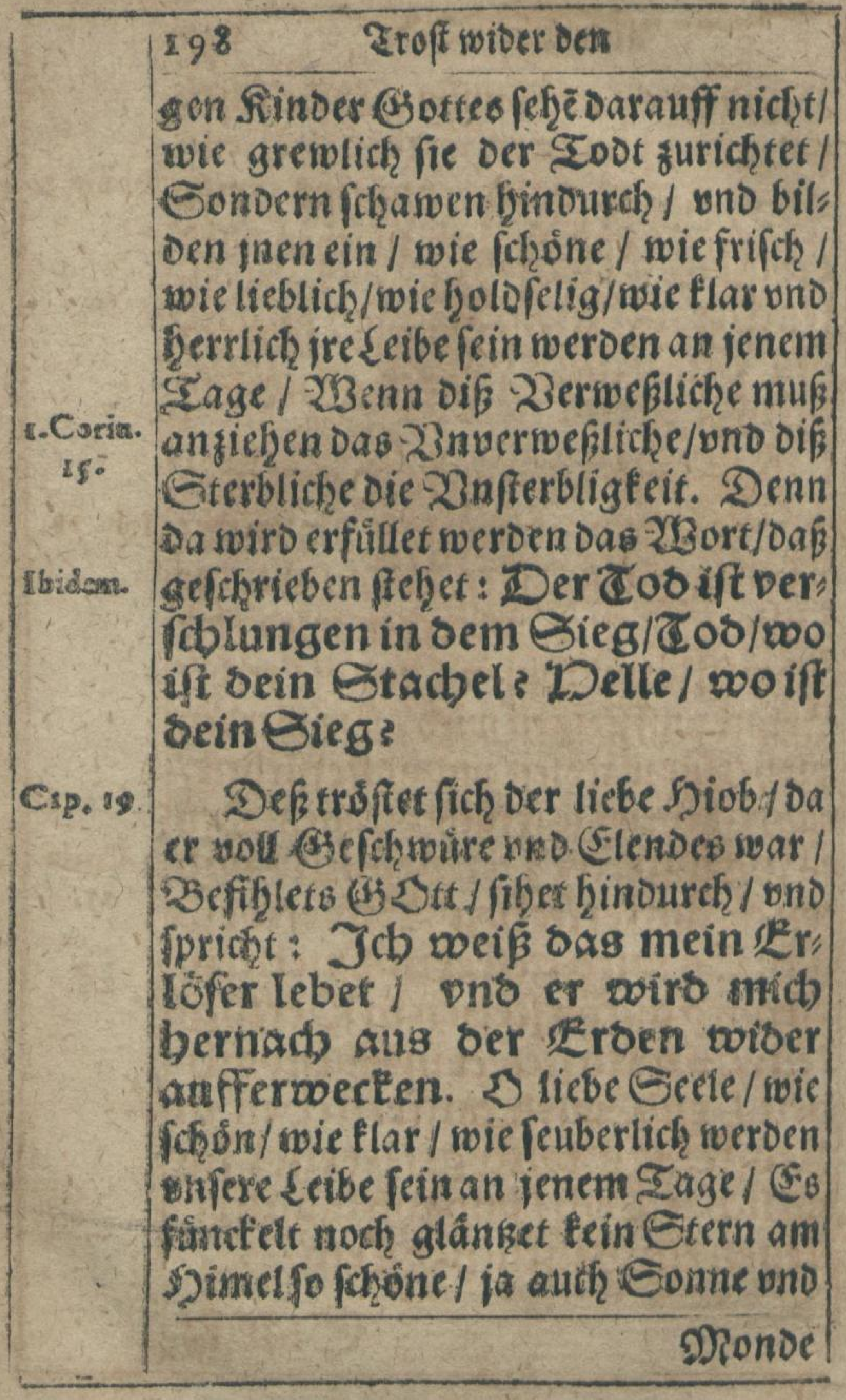


grabfamen ₹obr. 199

Dionbe nicht / als unfere seibe feheis nen vno fehinmern werden / VBent

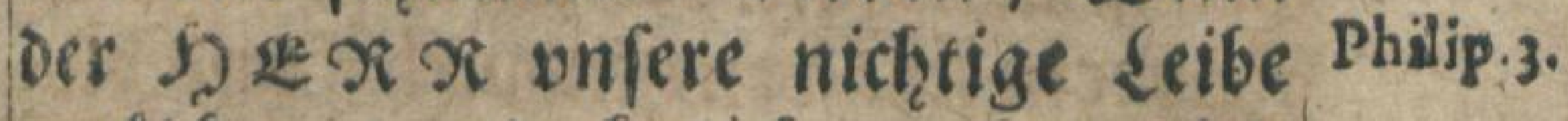
verfláren / vno ehntich machen wiro feinem verflárten seibe / Ia wenn wir nicht allein Den Engeln / fondern auch $0 \mathrm{~cm}$ So Serren felber gleich fein weroen.

Jekt finto wir unfers Şex $x$ Gottes 2Beizenfornlein / Die er feet Ioho 16. in feinen Botts Zlder/23enn aber ber Iriting Gottes anbrechen wiro/oie rethe 233 årme fomen / vno die rechte Eonne auffifeigen wird / Da werden wir herfir wachfen vond aufferftefen / uno rechte ewige fruchte tragen. Deñ es woirs gefeet Derweflich / ons wirs aufferfteben Dnverwe $\$$ " I.Corin. 15. lich: K 8 wirs gefeet in Dnebren/ pí woird aufferfteben in Derzlig: teit: $\mathcal{E} 9$ woirs gefeet in $\Theta$ cbwacb: beit I pno wirs aufferfiteben in Araffit: IEs woiro gefeet ein ha: tiirlicber feib/ pns woirs auffer, fteben ein Geiftlidber ceib. 


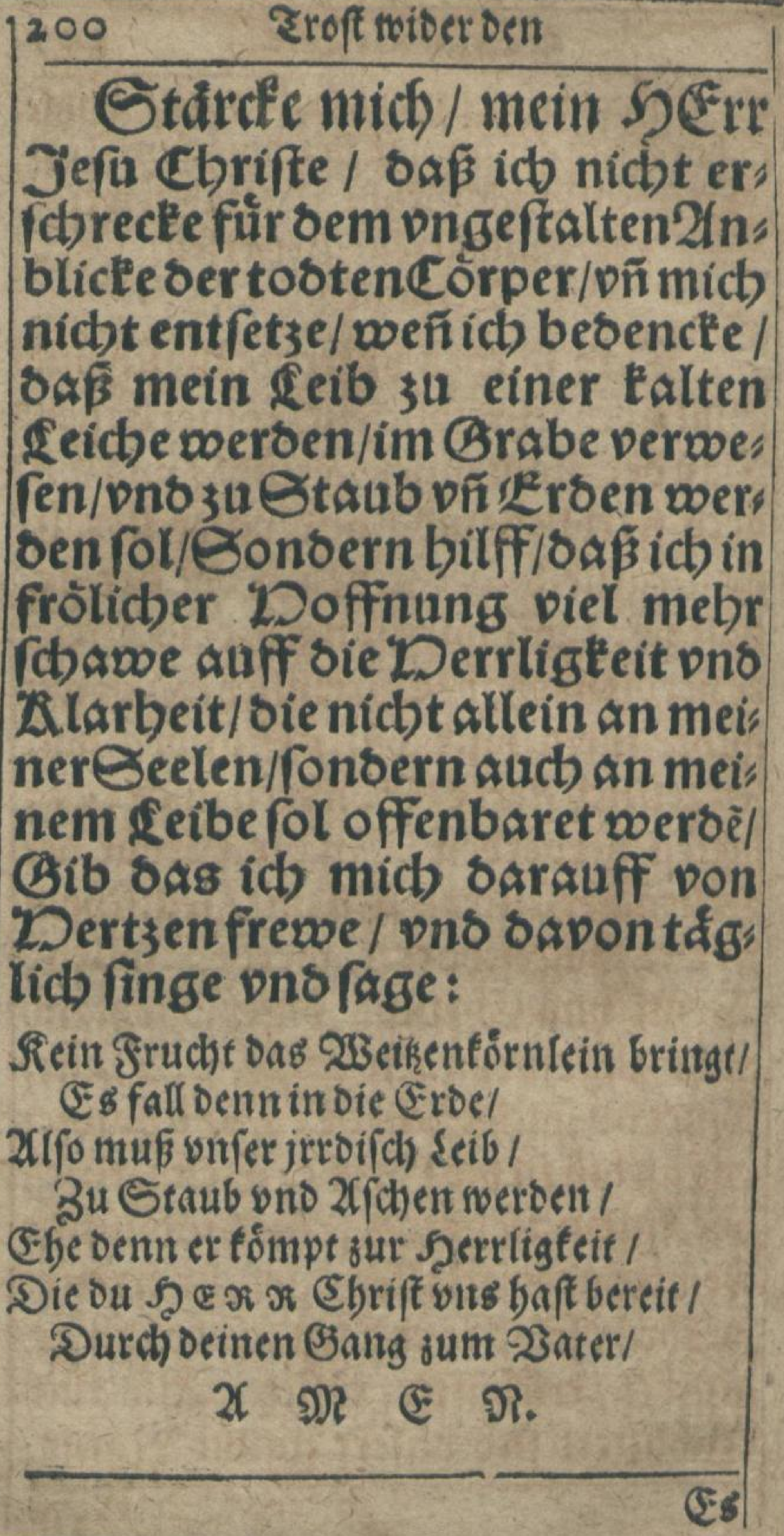


grawfafmen zooc. 201

Eg find aber gleichwol fiblectliche oinge iu borren/ich gefdhroeige su fchmecten/ ₹od/

Sterben/Grab/Grufft/3egraben wer.

Den/Zufcharren/Zerwefem/ Zerfaulen.

Daf foldzes onferm Fleifth wno B fut noch forfrectlich ift / fomet Daher/ Daf̧oer Zoot onfer Jecind / ono wir noch ferblich fino / ono unfer ferblis che Siatur bildet ir ben Ioot ber ge" ffalt vor / wie wir in nach Dem ESefes, Ge veroienet haben/2iemlich/D a nicht allein vmb onfer Günoen wits Ien zeitlich feterbẽ/ Gondern auch ewoig hetten veroerben / In Die Brrube Der

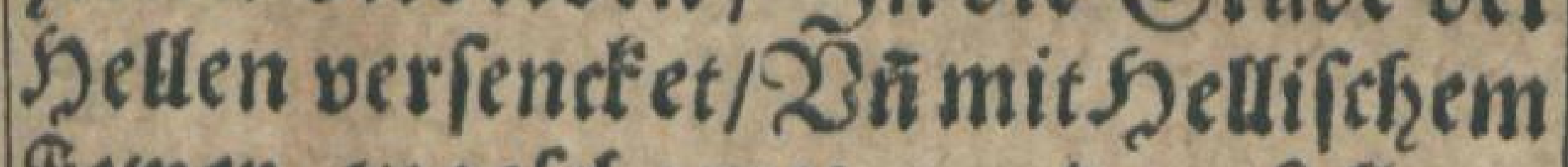
Fewer fugefcharret werben follen /

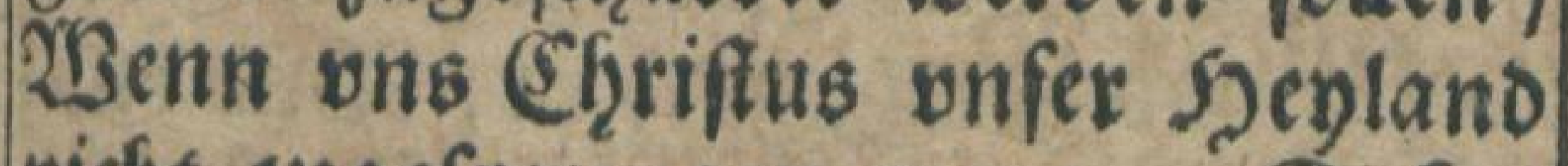
nicht zugefprungen were. Daber fómet nufolch Eefreetten/wno ḩanget vns an bifs in die Grube. Denn gleich wie einem ift Der aus einer tobtlichen Sewers oder 2Waffers gefafir errettet morben / fo offte er barnach oaran ges bendtet / erfeftuttert er fich: : Al fo auch erfohuttert fich vnfere ganise Natur

$x$ vno




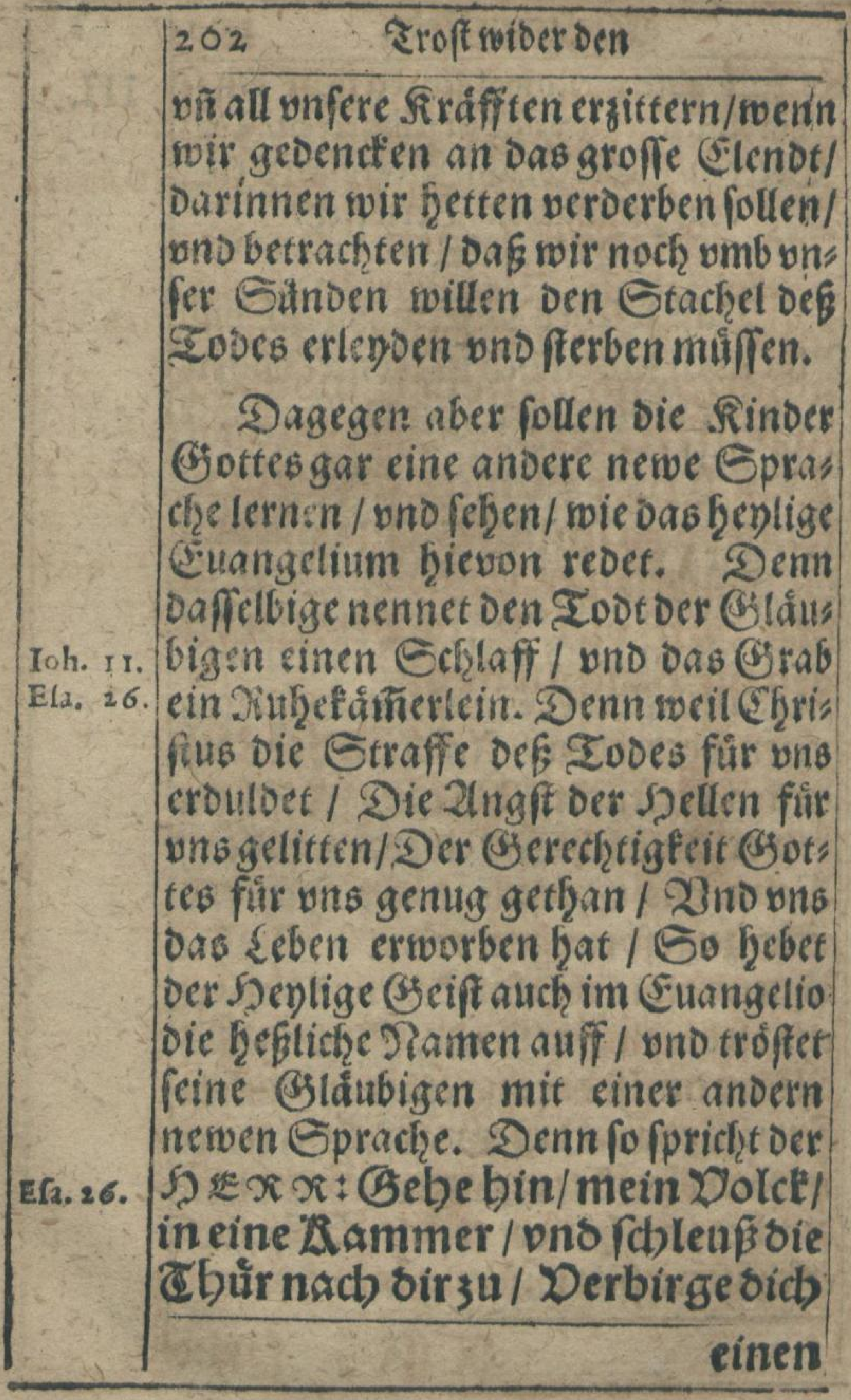


\begin{tabular}{|c|}
\hline gramfamen zoot. 203 \\
\hline einen Eleinen 2lugenblice / bif
\end{tabular} ber Jorn füruber gebe. 2ino abcrmal: Diel fo pnter ser $\mathbb{R}^{\mathbb{E}} \mathrm{r}$, Dan. 12. ben fcblaffen liegen / werben aufforacben. 2no Ef rrifus fpricht felber: fajarus onfer freunot [oh.ss.

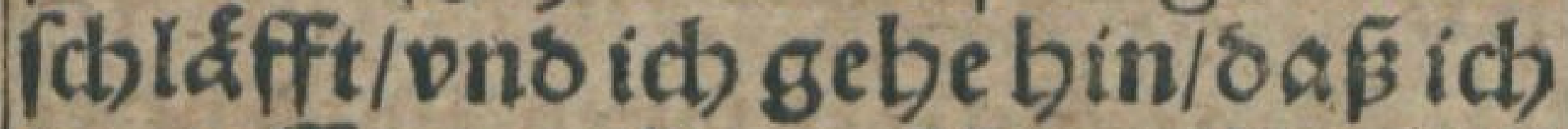
in aufferwecte. 23 nis abermal: Daßs LAinot ift nicbt geftorben / Marc: 8 . fonbern es (d)lafft 2lifo fagt auth oie Siftoria oer Daffion: $\mathbb{Z}^{\mathbf{S}}$ fun: Matt.2\%. ben auff viel Leibe ber "Deyli: gen bie bafdhlieffen.

Go lerne nun / liebe Geele / biefe nerwe Euangelifthe Gprache / mit newer Andacht falfen / Eilze / Der Şe $x$ i hat burch feinen zodt Dein Gterben in ein fanfites sinfchlaffen vermandelt / vno dir bcin (Strab guei: nem feuberlichen Jubbetlin gemachit. 2lienn ou ftirbeft/f̨ciffet es evinfohlaf fen: 2Benn ou ins \&rab gelegt wirft/ beiffet es zuDette getzen: -V1Benn man gufcharret / heiffet es die I buir nach? oir fufchlieffen: 23enn dein Seib ver:

$$
x \text { if faulet } /
$$


faulet / Geiffet es rufien / vno oich bers bergen/bif oer Born furnber ift.

Serr Giott Senliger Sicift nim bu mein Dertze ein / pnoers fütlle es mit sen trớftichen (Bna oen meines DD Ezren Jefu Cbri: fti/Rebre mich bie abermal(befe, t3e vno KEnangelium wol pnter, fabeioen / Darmpffe in mir os Ecbrecten vin Kentretzen für bem đode/ pno lebre midb seine beyl, fame pns troftreiche Kuangelis fhe Spracbe/Gib oas ichs nicht alleine mit oen $\Xi$ bren böre / pno mit meinemed unse narbripzechel Oonoern bilffand) / bapich Don Dertzen gláube ons empfinbe/ Dấ icb/ poenn mein Stừnolein oxift/nicbt fterbe/fonbern fanff" te einfdhlaffe : Ticht ing (Grab/

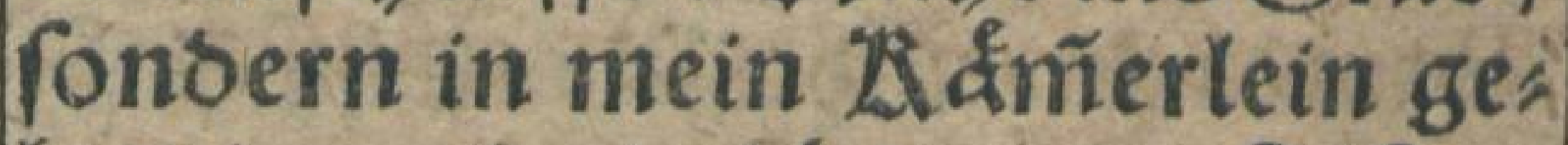
leget woerbe/ nicht verwoefe/fon: bern rube pno rafte/bifs ou mich woiber aufferwoecten woirft. So lege idb micb na/ DDErr Jefu / in

seine 
grampamen 20br. 205

seine 2(rme / woie cin Z inst in bie 2 Irme feiner Dutter/23efeble sir meine Seele / Ed)laffe ein / ono rube gar fein :

Ditemand én mich) erroecten/

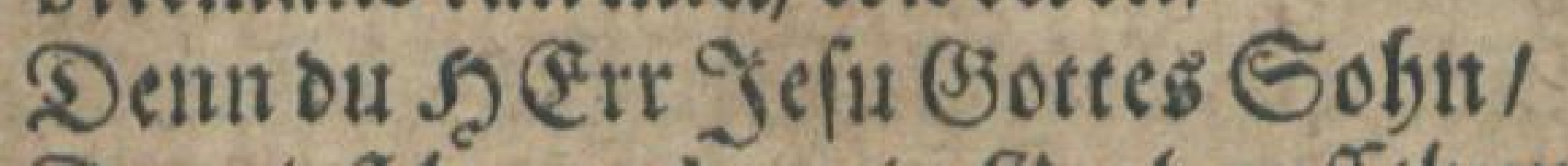
Du soitft bernach mein (3rab auffethut/ D)

Du fageft nol was / 2luer ich) febe gleich. wot an ben ferbenden we en/chen/ Daßj jnen mus seche gefecteben/Denn etlicte erfectuto tern fich / zucten/ fchlucten / verfeb. ren bie 2ugen / baben einen 2fingftich weißs.

Ta wol / Soldhesfifiet man nicht allein an ben Rinoern oie fer 23 elt/oie olgne Suffe fterben / Sondern auch offitmals an Den Estáubigen felbeft l Denn alfo mus Derfundtiche ferbli: che Seib / Den Etachel deß 3 Sodes fith: len / vno den Soldt Der Gunden em: pfangen. 2aber bie Rinder Sottes/ob 1.Corin. 15. Rom. 6. man wol an inen auch Sobesangften und Schmerken fif etet /werden fie Doch

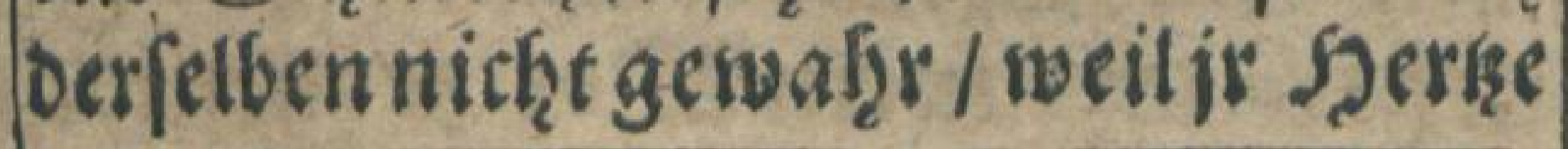
$x$ iij ver 


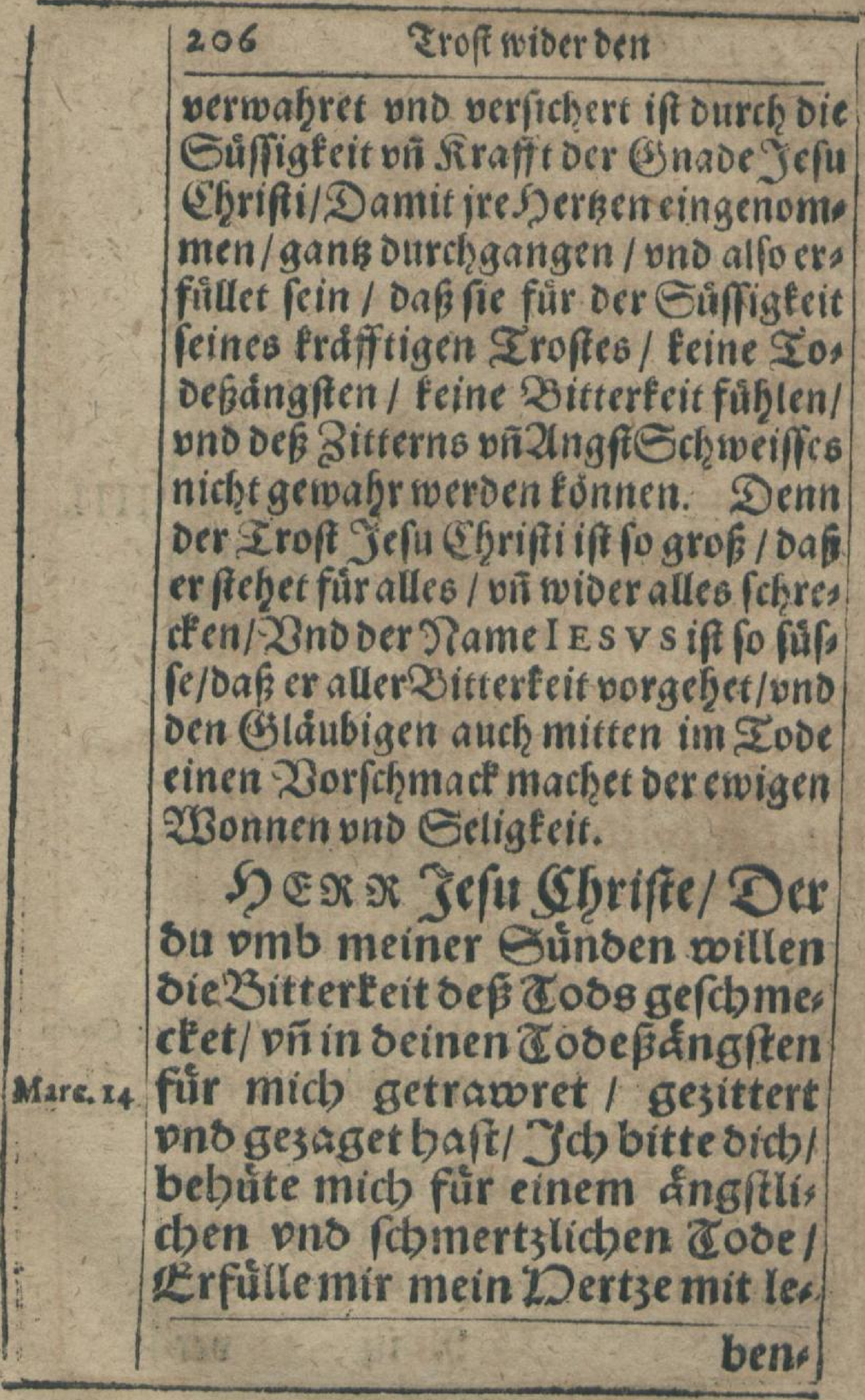


gratofamen ?obr. 207

benoigem Glauben ons reichem aroft / oa febr fuirchte/ ons mich fur reinem Otacbel nicht 3 a rebrentretze: 0 IE S V / berfüfre I lame oein/ Im cose erquicte bie Eeele mein/ 2 Imen.

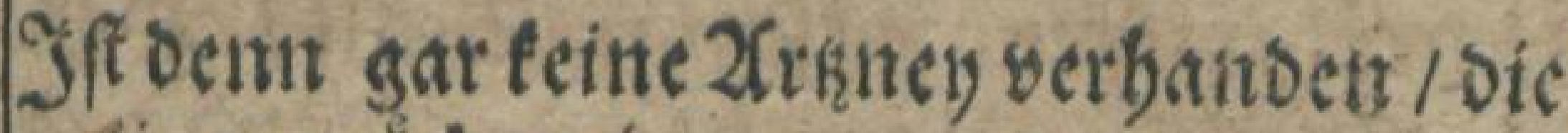
ciner mochte cinnentelt / samit er beat

Stachel bés Yooes nicht su febr febmecten bórffte?

Ya trawen/liebe Geele. Denn fo

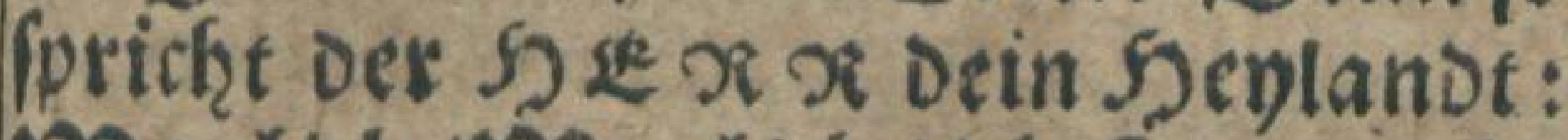
20arlicb/200arlich/icb/age eudb// ruhan.8. fo jemano mein Doort wirs bal: ten/ber woirboen cost nicbt pes ben ewiglicb.

Solve hie / liebe Geele / auff ben Minno dic fes decineo Simlifchen $2 \mathrm{rr}$;

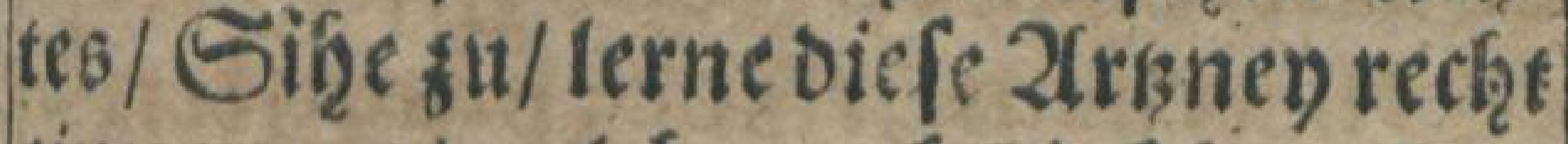
einnemen / welche alsch oie formerizen deßgodes bertreiben fan / Remulich/ Daf 2 Bort beines $\mathfrak{y}$ ex $x$ x $x$ efu Chrift: Richtt DRofes 2hort/weletses if das Befere Cottes: Tiicht Dien" $\Re$ iiii fercen 


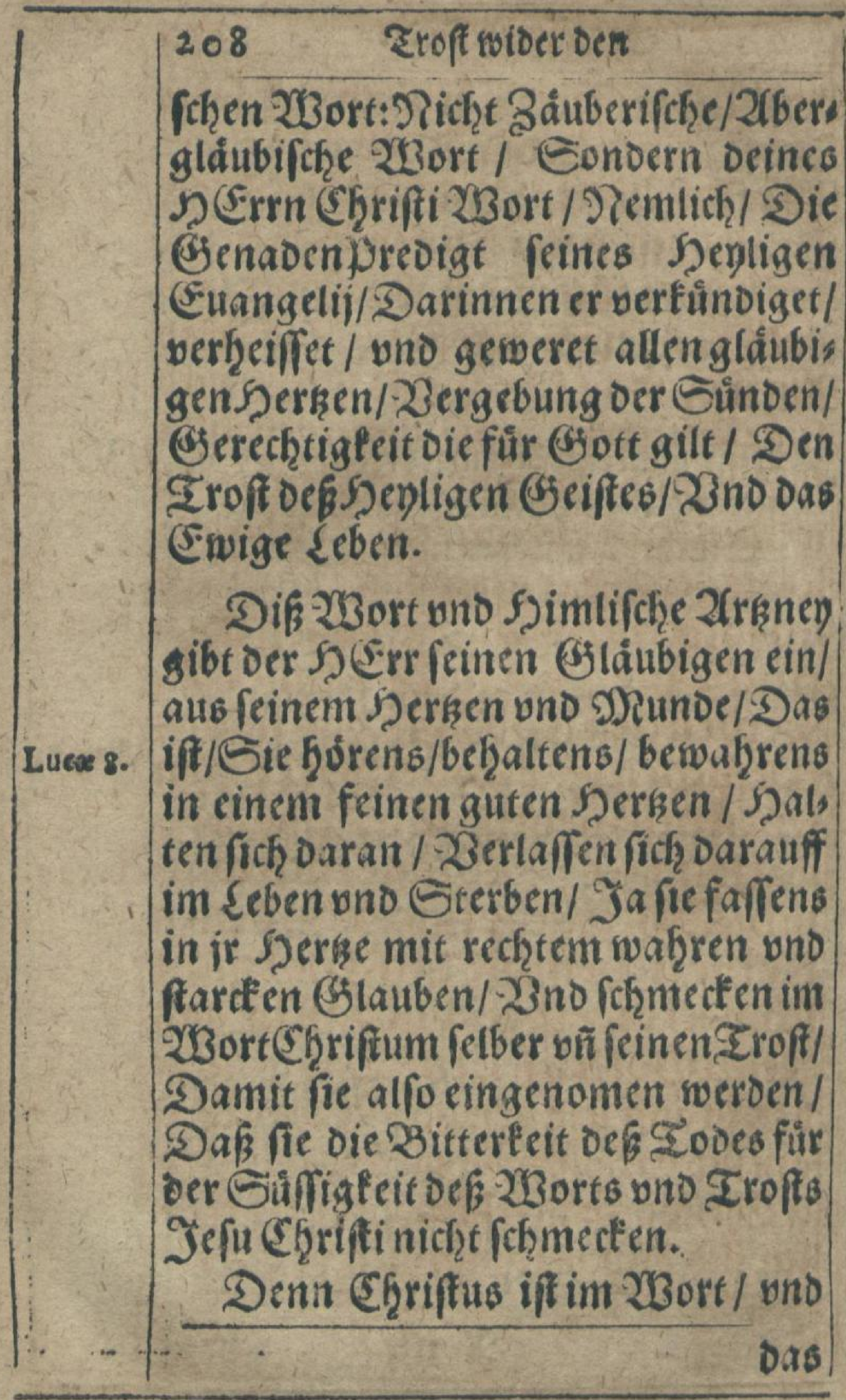




\begin{tabular}{|c|c|}
\hline grawfanten Todt. & \\
\hline 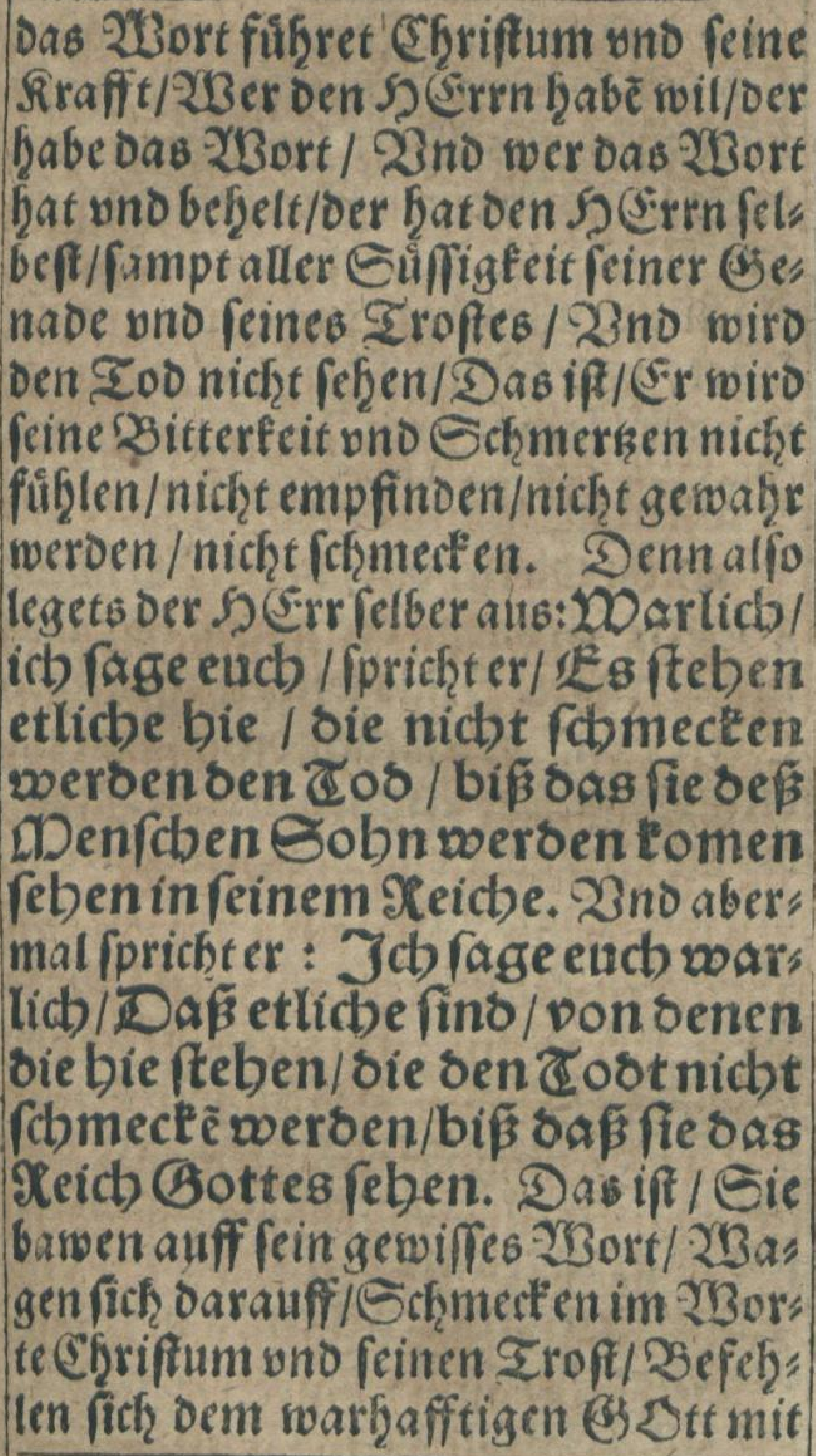 & Lucx 9. \\
\hline $\mathfrak{x}$ & \\
\hline
\end{tabular}




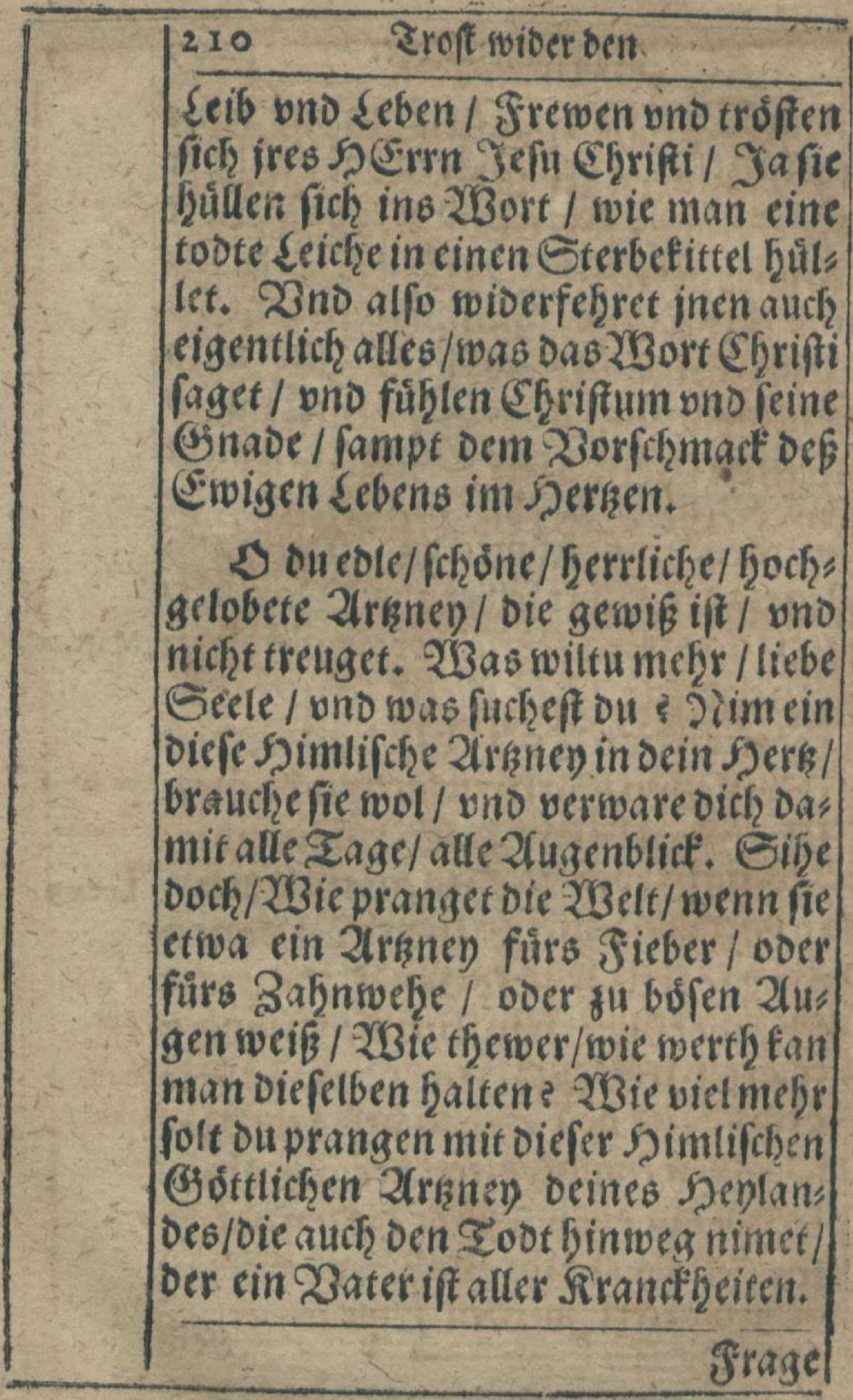


grawfanten zodr. 211

Srage altef nicht/lieke Eeele/ obs Probatum vingewif /en/ Siffe nuran die Exemipal der Sorbilgen / die folche 21rkney gebraucht bab:n. S. Stephanus war voll etlaubens vito I rofes

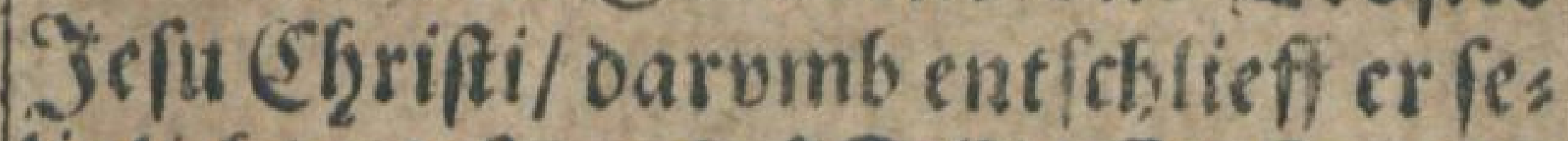
liglich/ond fpract: D DE'TrJefu nim meinen Geift auff. S. Paulus hatte fein serke alich beroaret mit oiefer Soimlifecten 2trsnin/ Darwub funote er tåglich Dem Sode tros bieten / uns fprach: Job babe luft absufdyeys. oen/ono bey Cbrifto 3 a fein.

2lifo thaten auch bie lieben mirs terer : S. Ignatius war erfúflet mit

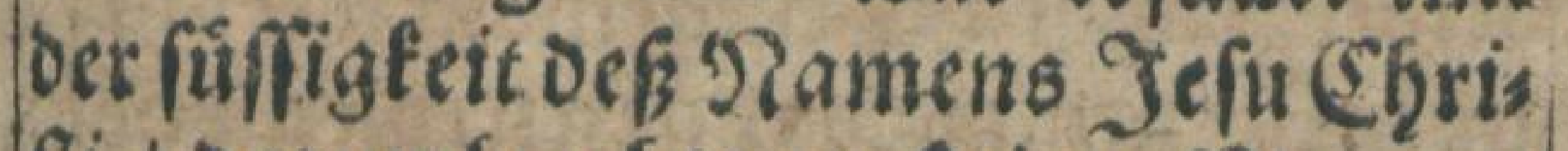
S. Igua. tius.

fii / Darumb achitet er feiner פarter.

S. Polycarpus war entzundet mit Der S. Poly. siebe bã Żrunft Jefu Ehrifti/Darumb carpus. gieng ergetroff zum ferwer. S. Lau. S. Laurentius batte den Gebak deE \{ebens rentius. im Socrien / vnd pottete Deß Invans nen bagu / in allem Dafer lenden mus fie: 2ino da er fabe/ oafiergrewlich folie gemartert werben / fprach er: riad 


\begin{tabular}{|c|c|}
\hline \multirow[b]{2}{*}{$\begin{array}{l}\text { S. Vin- } \\
\text { centius. }\end{array}$} & Sroft iviber bent \\
\hline & 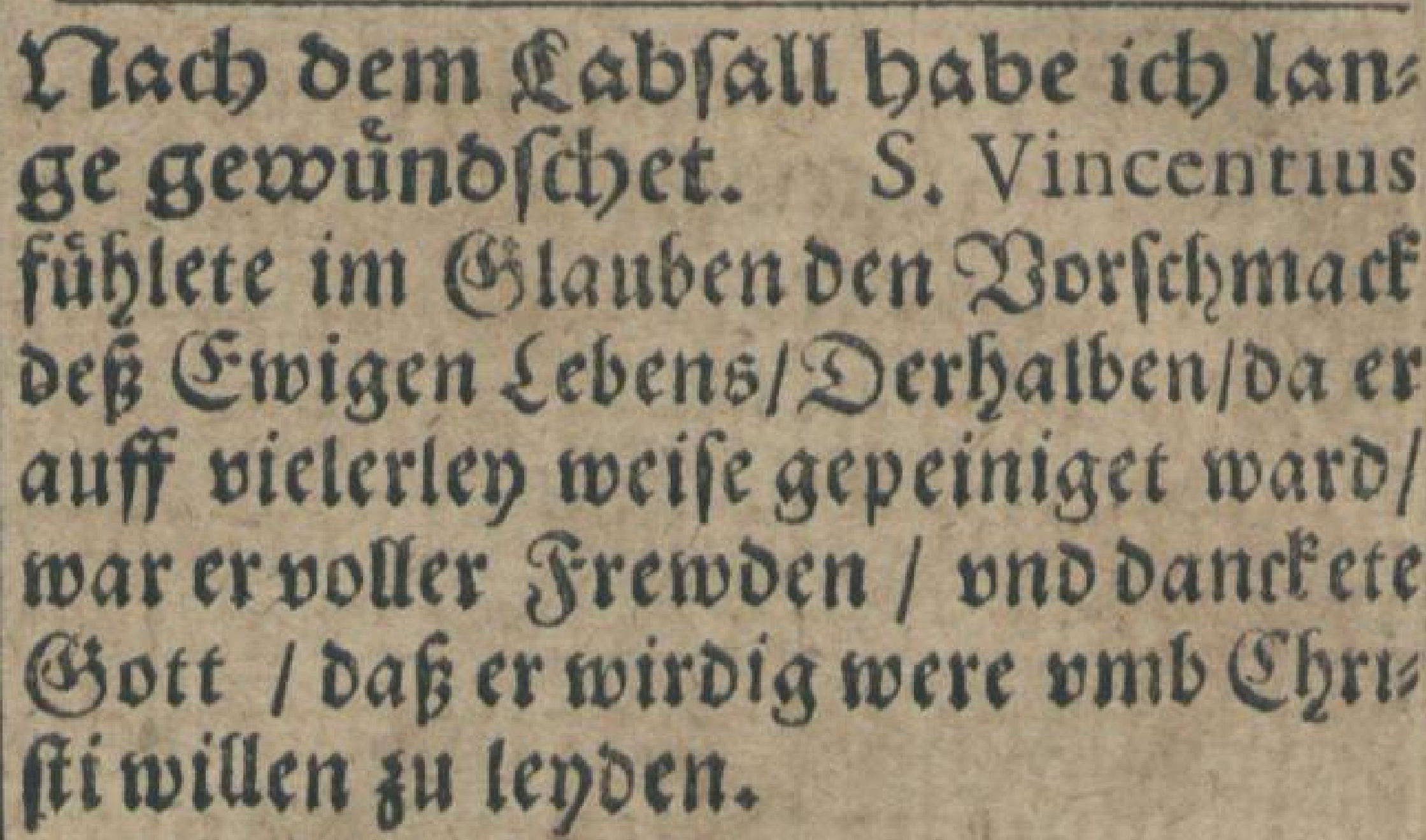 \\
\hline \multirow[b]{2}{*}{$\begin{array}{l}\text { D Mar- } \\
\text { tinus Lu- } \\
\text { therus. }\end{array}$} & 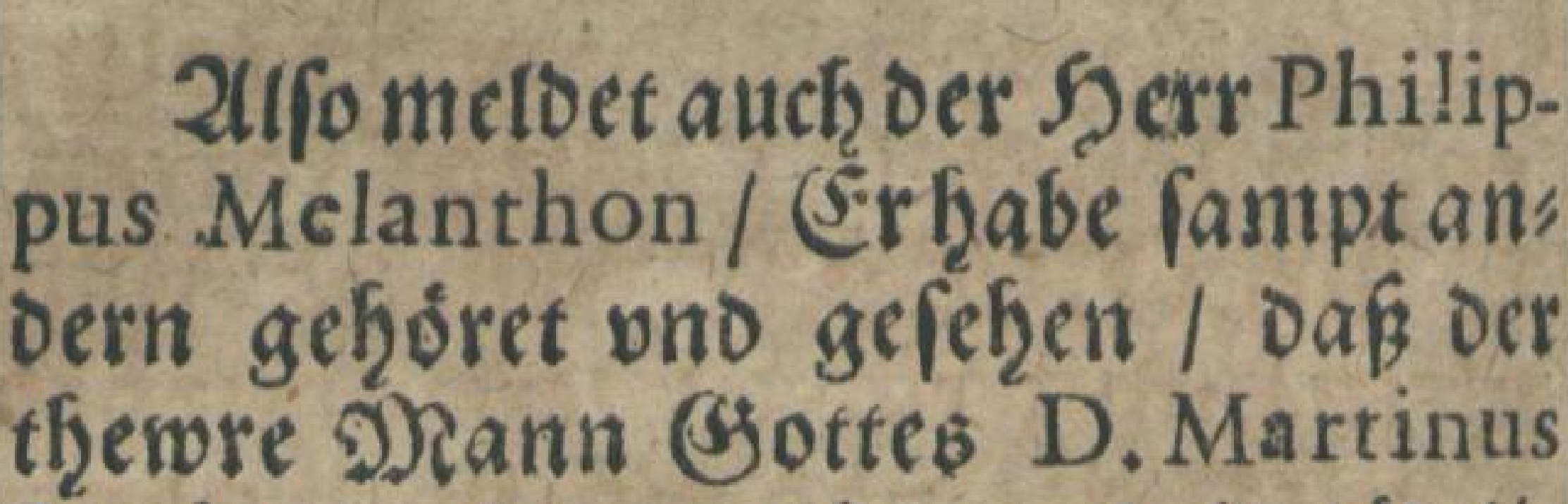 \\
\hline & 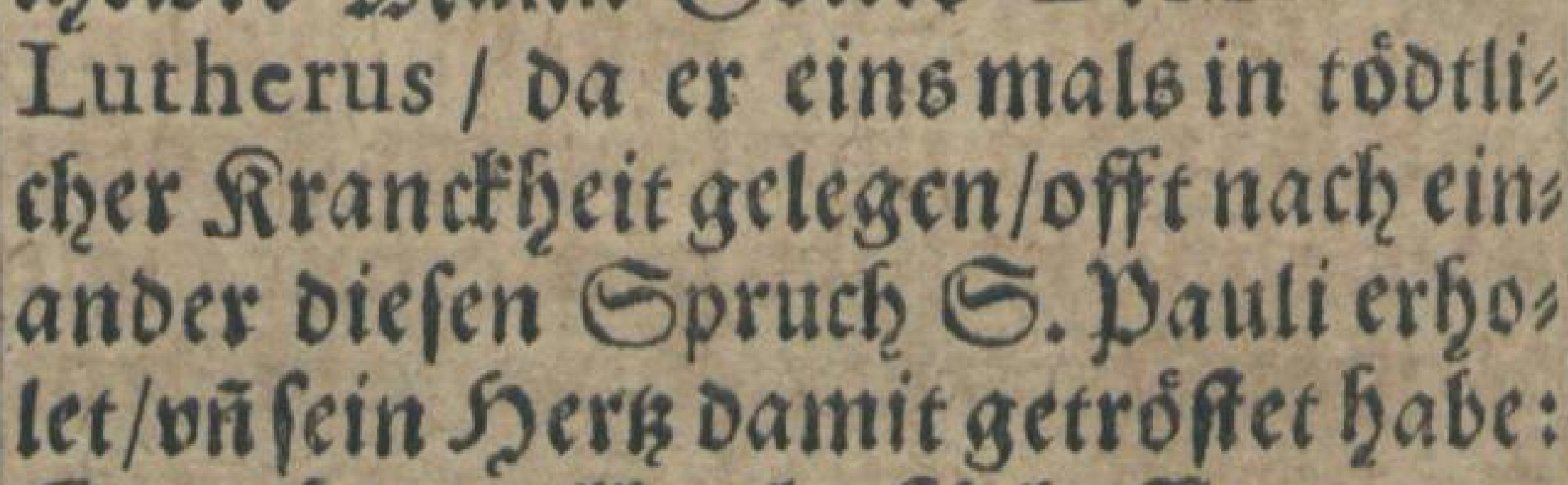 \\
\hline \multirow[t]{2}{*}{ Rom.1x } & 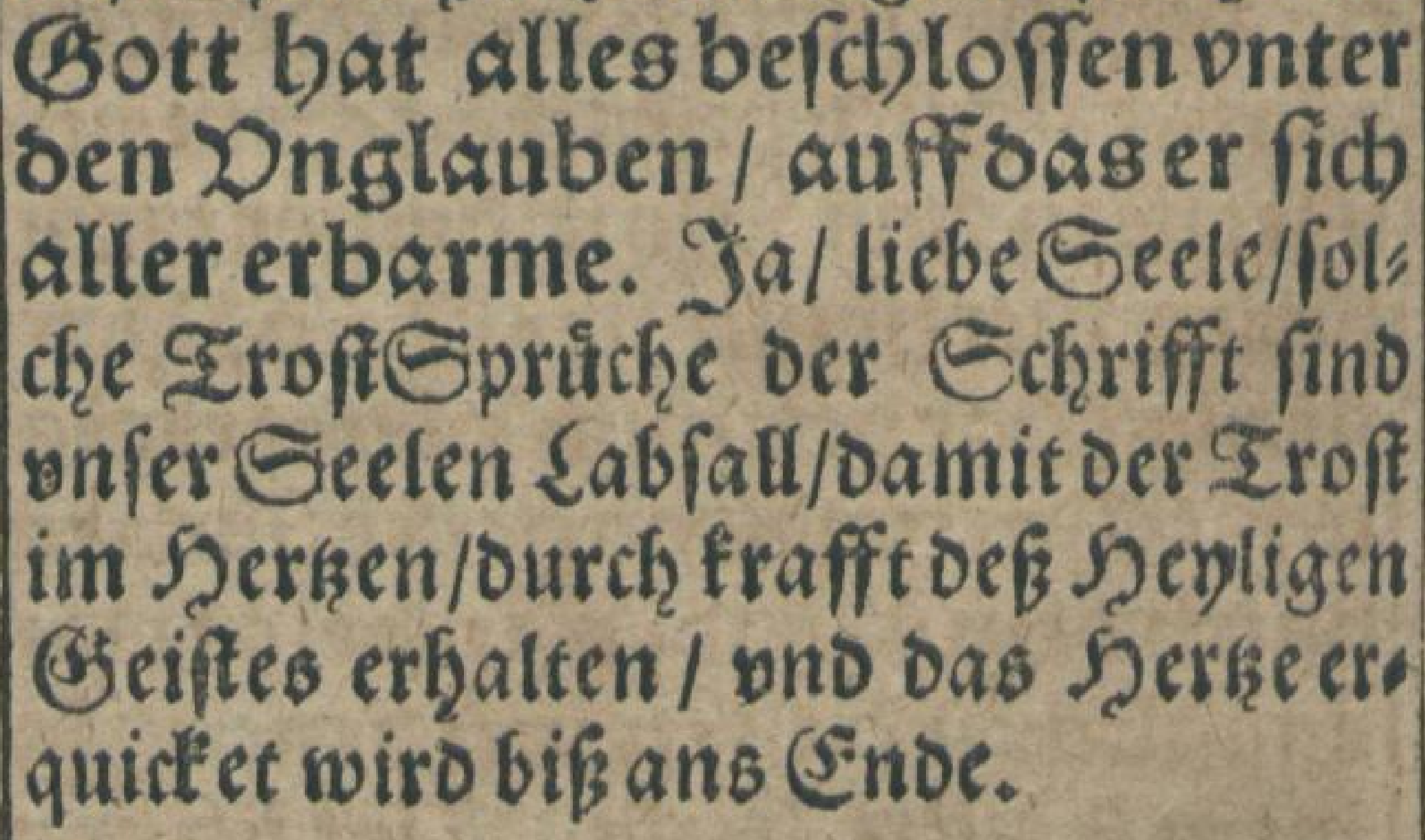 \\
\hline & \\
\hline
\end{tabular}


STE $\Re \Re$ Jefu Shrifte/ Du

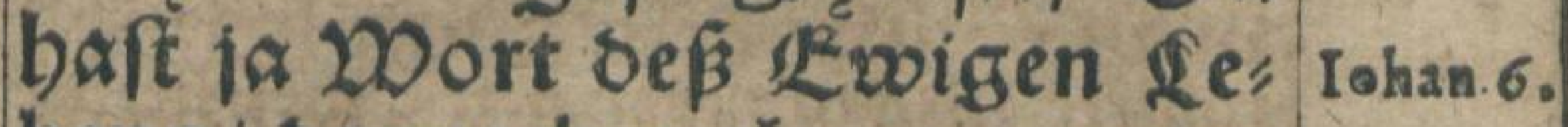
bens/ oarnach Derlanget meine Beele/ ond febnet fich nach beis nem Trofte. Ja/mein Deyland/ woer oein 200 ort bebelt / pns fich oaranff waget/Derbat oie red)" te Dimlifche 2Art3ney / die nicbt treuget / wioer Tost vno notb / pno wiber alle Dellenp forten. $D$ mein D REX Żefia/Rrfullte mein bungeriges Dertse mit beis nem 20 orte/vno nim mich gant; ons gar ein mit beinem đrofte. Gibe/mein(Gott/gleich woie mein Coster feib in ein weiffes Dem: oe gebuillet/ pnósabin getragen wirs / 2tro woiclele ich meine Beele in sas 20 ort beiner trōfts licben Derbeiffung: Dein 20 ort ift die Dorbeit/uns weret ewoig' Ioh. 27. lich / Derbalben voerbe audb ich Ela. $4^{\circ}$. ourch sen (Glauben an bein DDo:t ewiglicb erbalten woerben. Ia/ mein DSRr Jefia / DDer beinem 2000rte 


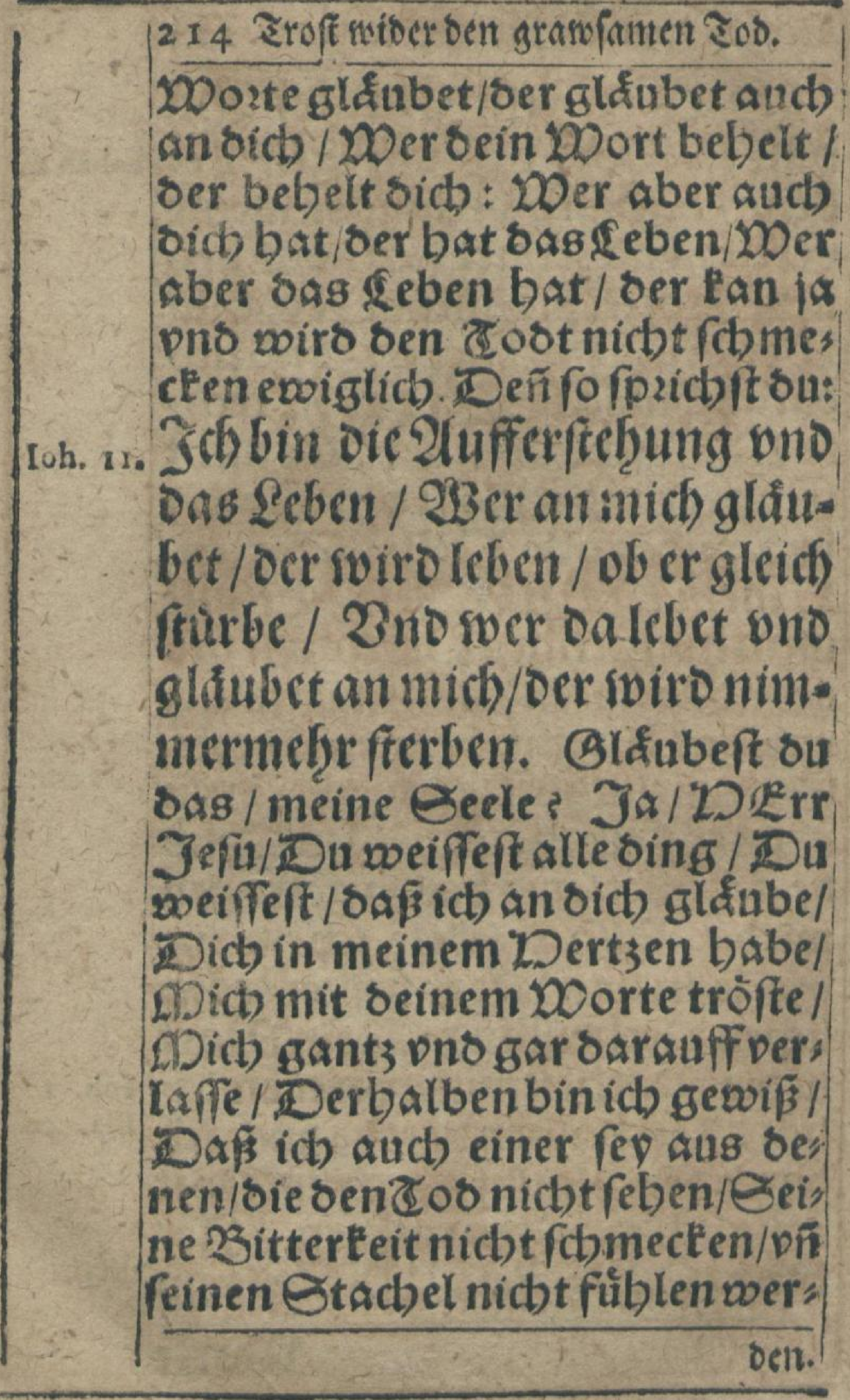


Schoine Spriche fir dic Sterbendè. 2 is oen. Das bilff ono gib mir Derr Tefu Cbrifte/omb ocines beyligen worren 2000rtes willen/ 2 imen.

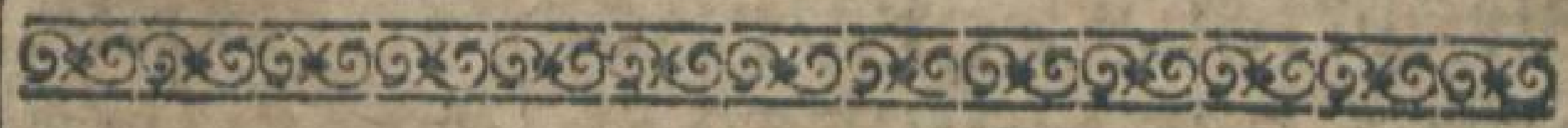

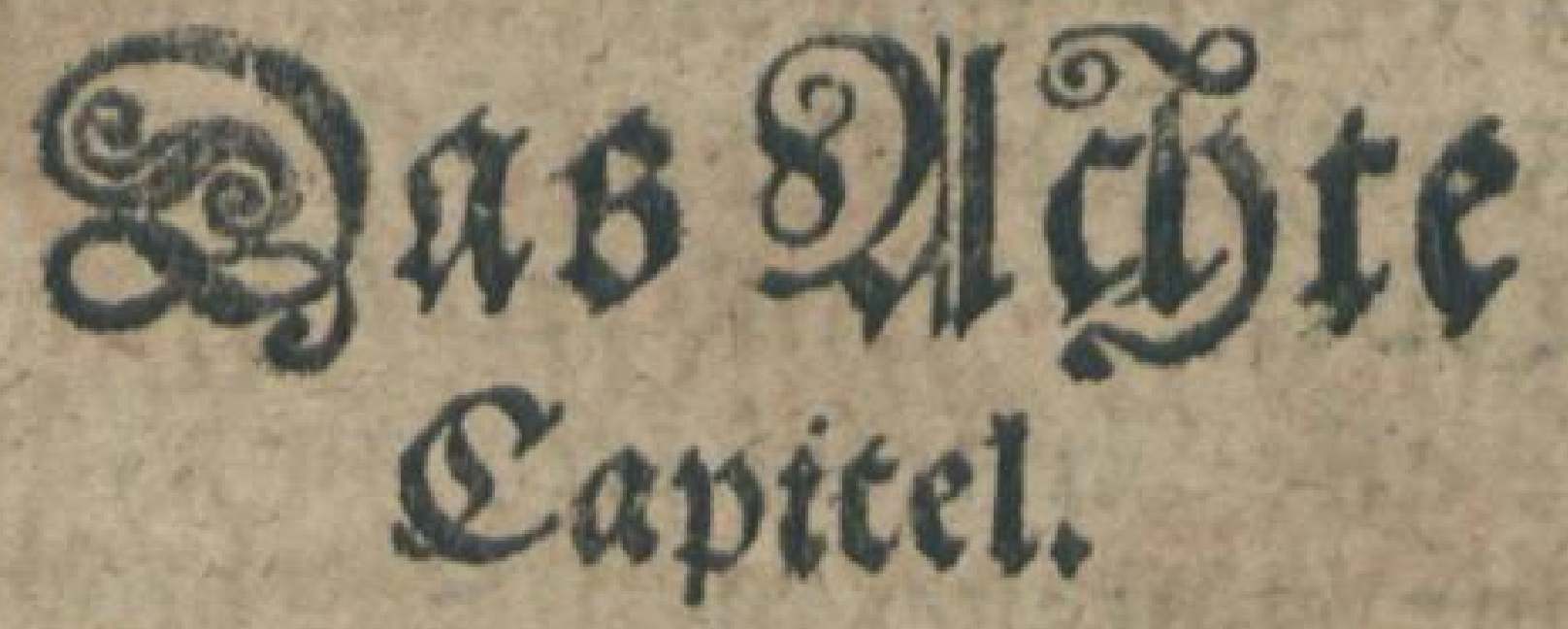

Ergelet biel rofine Sprut che alts Shenliger Schrifit / fo wol edliche andachtige (sebetlein vno Seuffiser/ die man den Gterbenden vor.

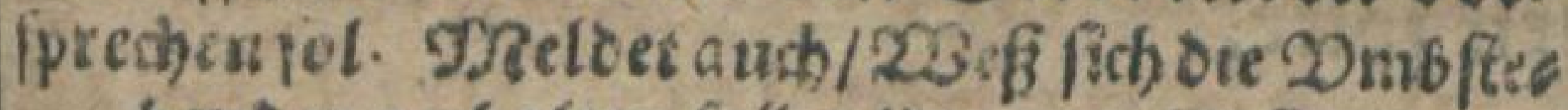
besocn berbalten foller/Die Dem Sefterbes nen ausgerwartet ynd ausges betet baben.

So crseble mir nu eflicte Spriche aus

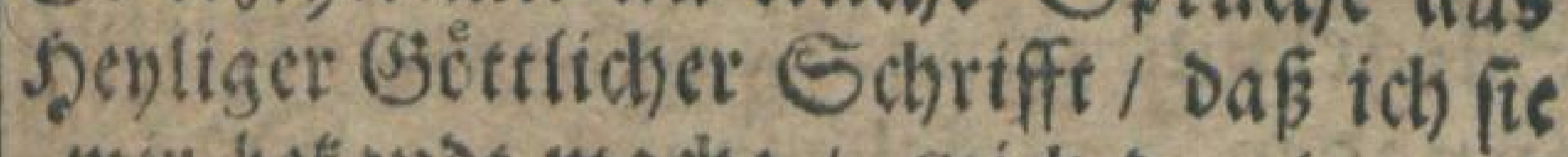
mir betand inache I ntel bamit an

meinem Enoe troften / ono meine Secle laben mogge.

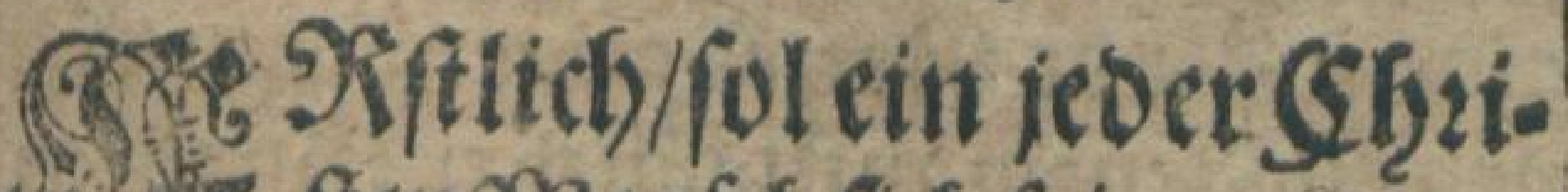

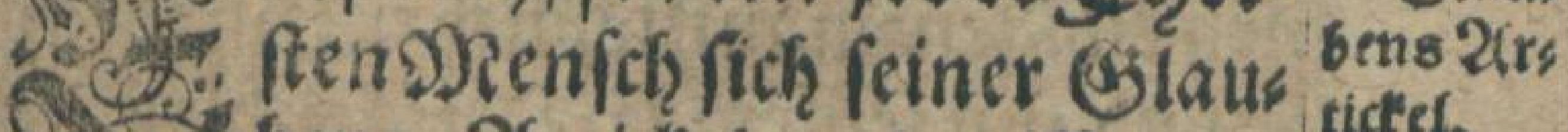
Qus bens 2trtitfel wol wiffen fu ticter. trditen| 


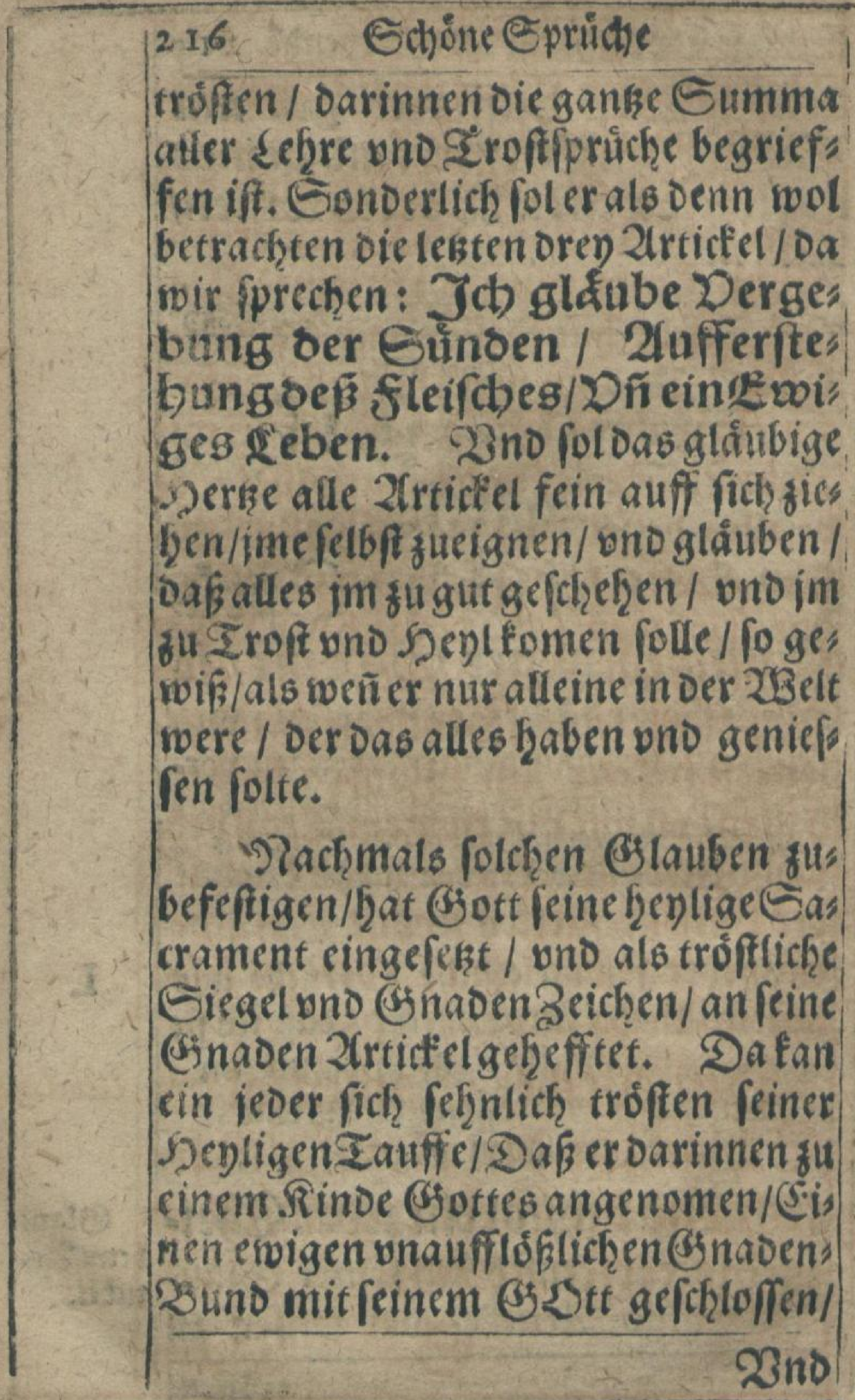


fuir bie Sterbenden. 217

3no mit bem Dolute SGrifti geteinis get vno abgewafehen $\{e y \%$

Darnach troffe er fich auch De ह Seyligen 2fbenomaf̧lo / Daf er fieb als ein Rinot Ejottes bey feines 2 as ters Iifche hat fpeifen bno erquicten laffen / 2 nno nu verfichert ift / Daf er fen cin wahres Slieomaß Shriftimit seib und Eeele / vno cin gewiffer Sir: be alles deffelben / was Çriftus mit Dem Spffer feines scibes und Blutes Gat erworben / Jabas fief Der S Sirt biemit in fein sers fienein gefencfet] Jhen ourch feinen Sepligen Seift als lefeitftárten / Im Btauben fefte er: halten/ vit ime mit ewigem Iroft beys wohnen wolle. Sierauff fan Darnaeh Der Datient allerien fchione Eprichel entweder felber lefen / oder im lefen laffen/alls da find:

Def3 Weibes Santen fol Der Gener. 3. Eiglangen ben Ropff zutretten/ vnd dic Sthlangeswiro in in Die Ferfen ftechen.

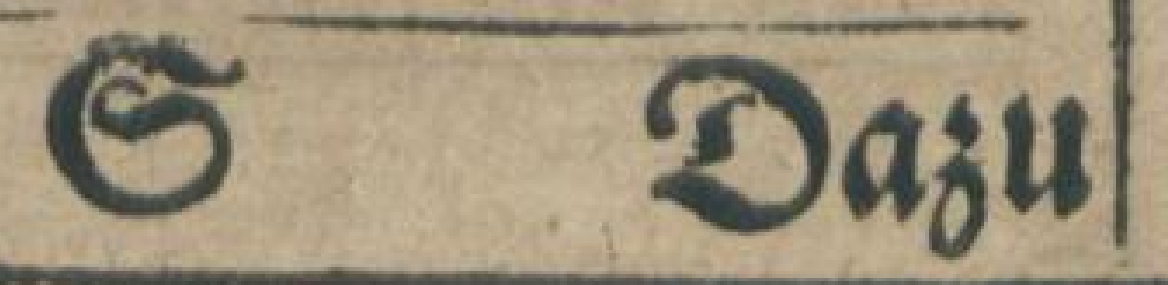




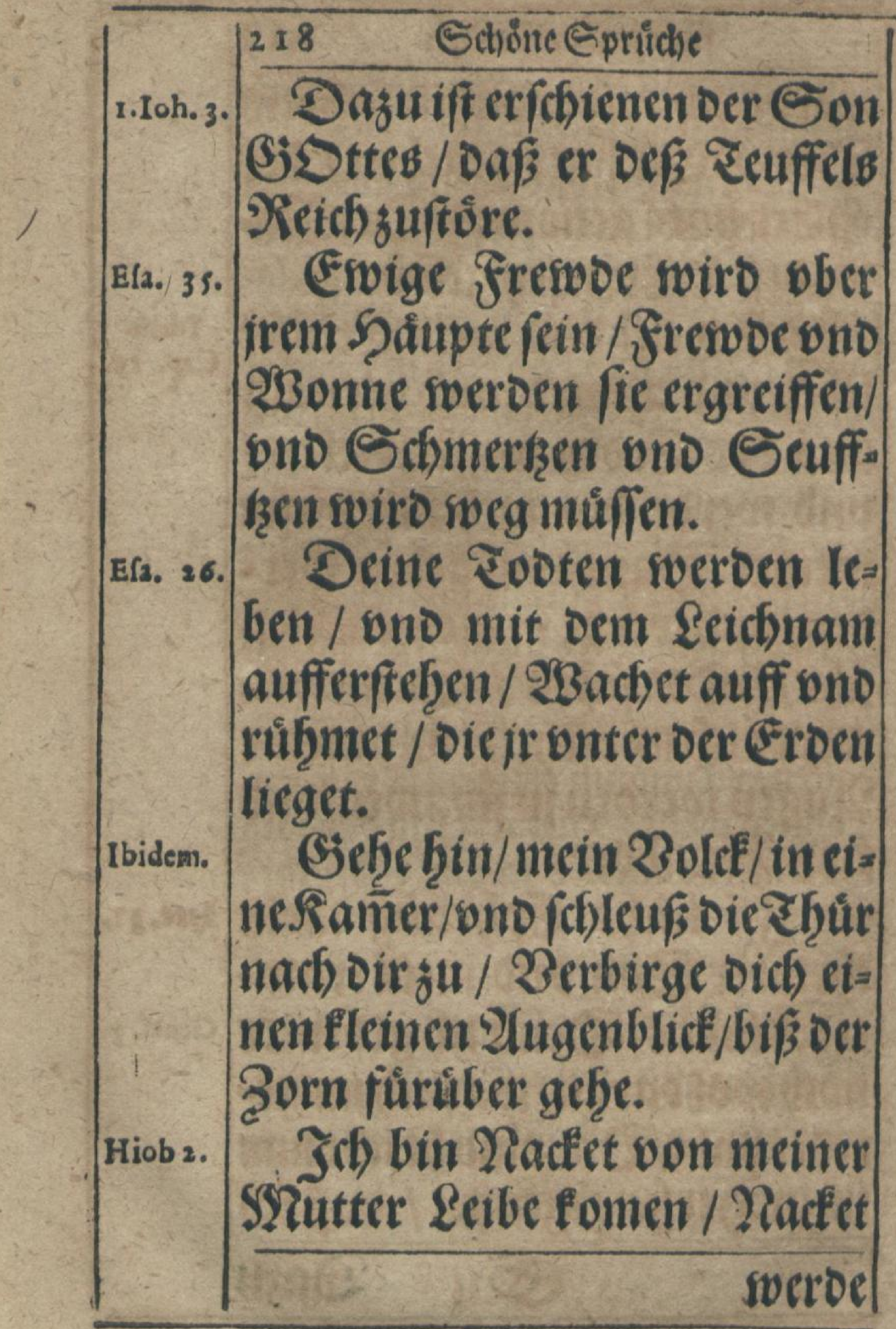


swerde ich swider bahin faberen/ Der SEerr hats gegeben / Der S.Err hat genomen/Der 2ame deß S E errn (ey) gelobet.

Jch weif Das mein Erlojer Idem. lebet/wno er soiro mich hernach aus ber Eroen aufferweden / vno werde barnach mit biefer. meiner Shaut ombgeben soerben/wno soerde in meinem flelfeche Giott fefen / Denfellen foerocid) mir féten / ono meine Qlugen serben in febaswen/ond tein fremboer.

thenorer Son ono mein trawCap. 19. tes Rindt? Denn ich gedende noch) rool baran/fwas ić) im geredet babe/Darbmb bridbe mir

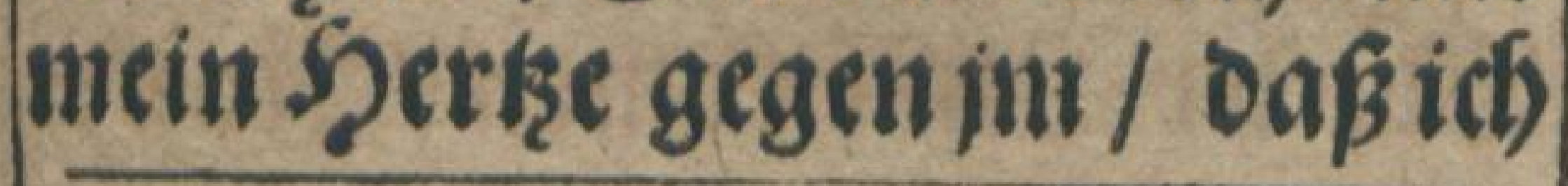

$$
\text { S ij mid }
$$




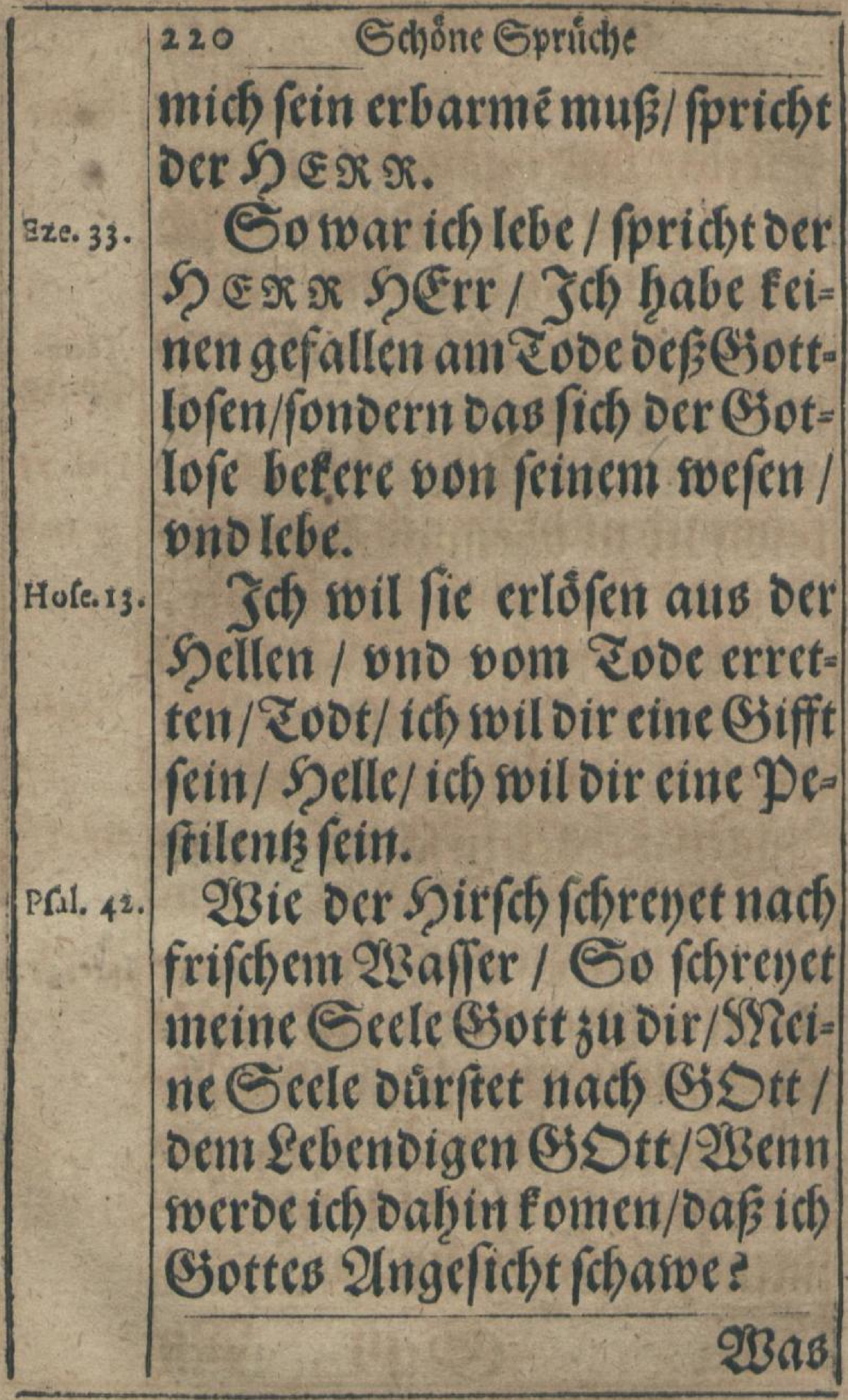




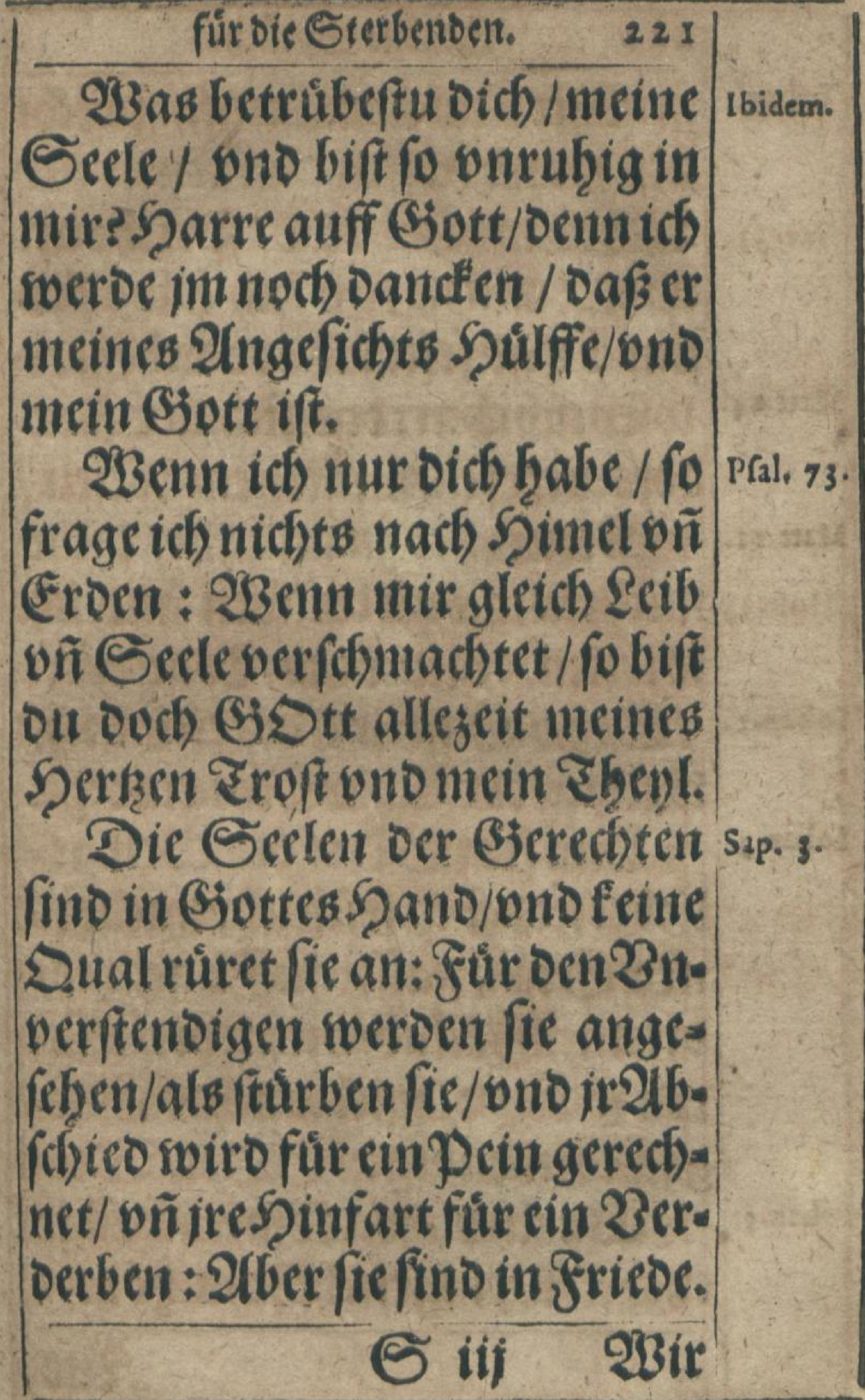


$\frac{\text { fir bie Sterbenben. } 223}{\text { onb glåubet Dent / Der mich) ge- }}$ fano hat/Der hat Das Eswige \&e. ben/bno fontet nicht in oas(5e= richte / Sondern ift bom Tooe zum \&eb he hinourd georungent.

Jch bin Das Broot oef $\mathrm{Ee}=$ Iohan.6. bents / $23 \mathrm{er}$ fu mir fơnet / Den soironickt fbungern / 3 no wer an mich gläubet/Den swiro nimmermefir Durften.

2llles was mir mein 3 ater rbiden. gibt/oaß f dmet fumir/wno iver ;u mir fomet / oen soerde ich. nicht bienauß folfen.

Daf́s ift aber oer $23 i l l e$ oeß rbidem. Baters / Der midg gefano bat / oaß ich nichts verliere von alLem/Daß̂ er mir gegeben b̧at]. fondern oas icbs aufferivecte am Jungften Eage.

$$
\text { E tiij झar: }
$$




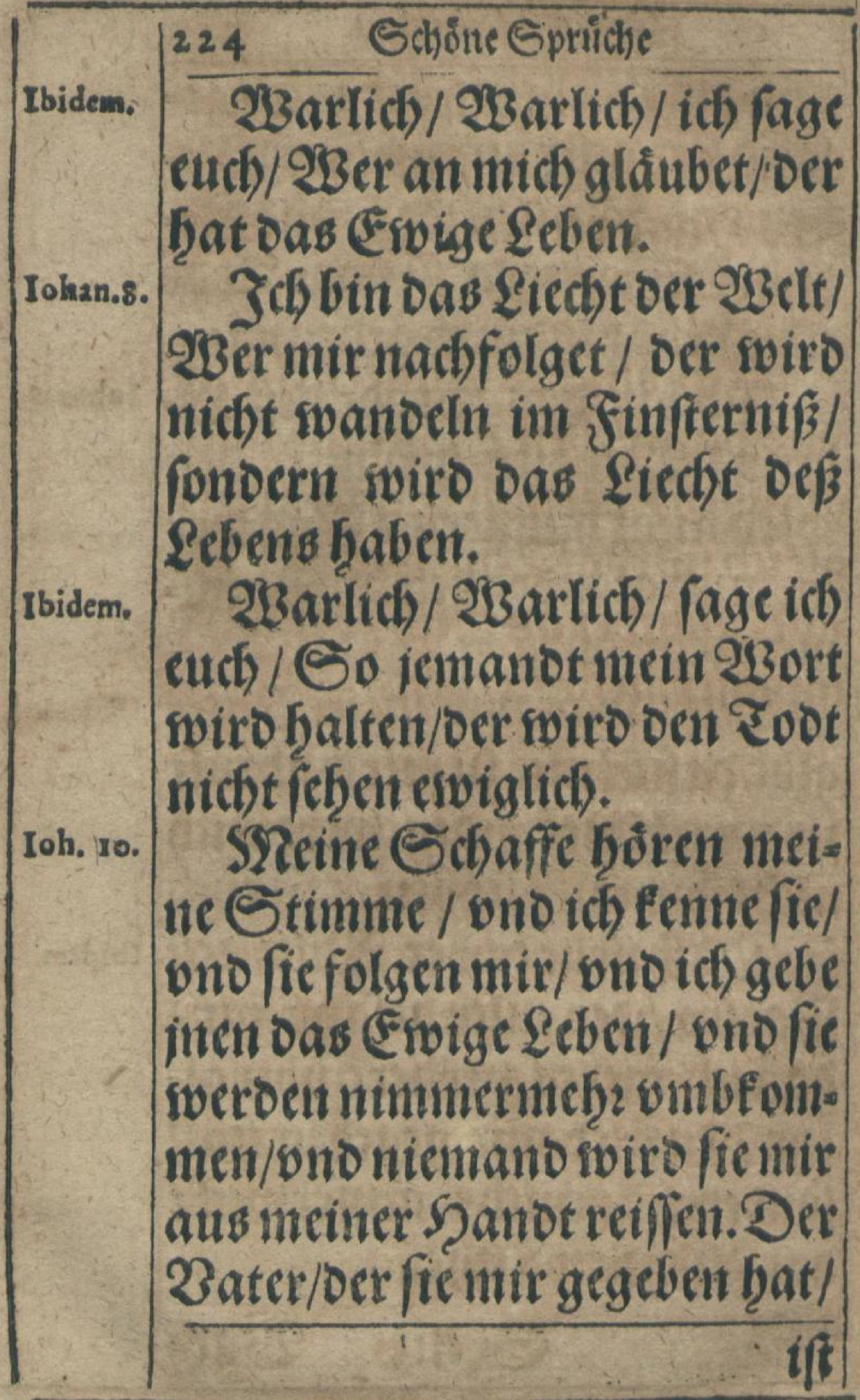


für bie Sterbenden.

225

ift groffer benn alles / ono nie= manot fan fie aus meines $2 \mathfrak{a}=$ ters Shano reiffen/ Joh ond oer Bater fino cins.

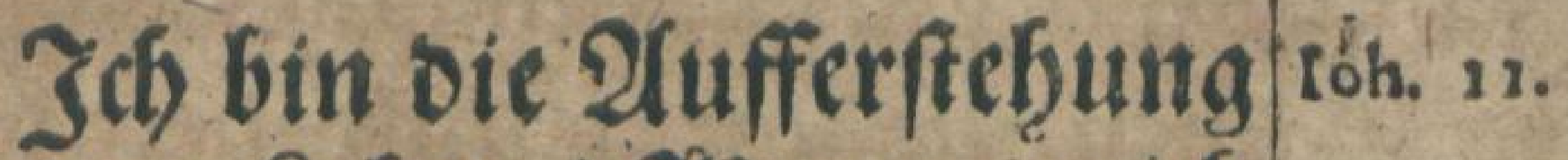
vno das Seben / 2ser an mich gläubet / Der fvird Ieben/ob er glecth fturbe/2no wer oa lebet bno glåubet an mich/ocr woiro nimmermef̧r fterben.

Jumeines 2aters Şaule Ioh. 54. fino viel 23 ofinungen / soenns nicf)t fo were/fo swolt ich) zu euch)

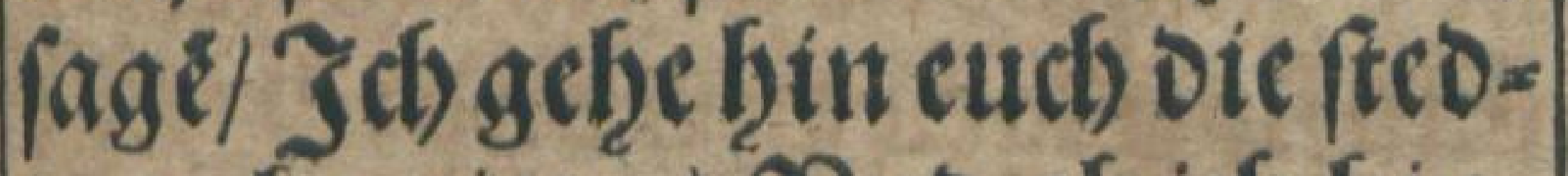
te zubereiten/23no ob ich bin= gienge euch oie ftatote suberen)= ten/2Bil ich Doch) soider fomen/ ono euch su mir nenen / 2luff Das ir (ev) / woo icf bill.

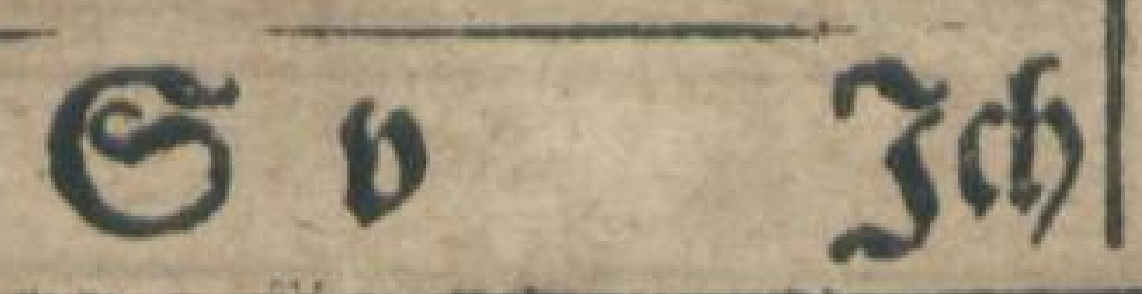




\begin{tabular}{|c|c|}
\hline \multirow{4}{*}{ ibidem. } & Ethone Spríche \\
\hline & Inic ornat. \\
\hline & eity \\
\hline & mict). \\
\hline \multirow{4}{*}{ Ioh, 17} & Dafift aber bas Ewoige $\& e=$ \\
\hline & Iein wabrer Gott bi \\
\hline & 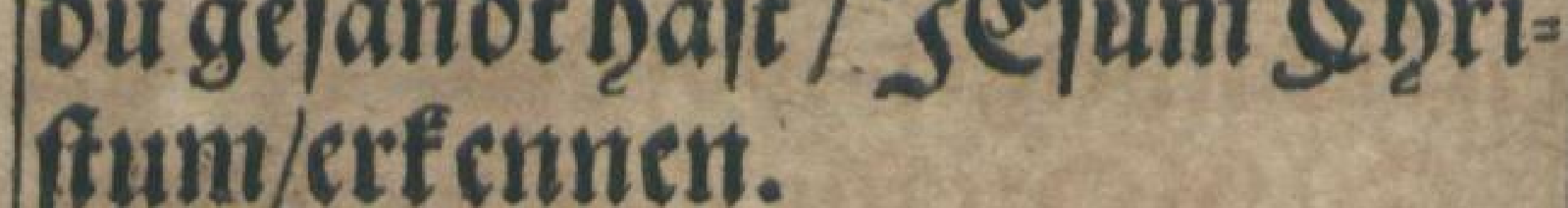 \\
\hline & Bater ich wil/ Daß̧/ woo ich \\
\hline & $\begin{array}{l}\text { bin / audb oie ben mir fein / Die } \\
\text { bu mir acaeben haft / Daf fie }\end{array}$ \\
\hline & meine Spertligf eit febert / oic ou \\
\hline & mich geliebet/effe beun Die2Belt \\
\hline \multirow{5}{*}{ Ator.4. } & gegrundet waro. \\
\hline & if auds fein ander ?ame oen \\
\hline & senffben gegeben / ba \\
\hline & \\
\hline & 3ont \\
\hline
\end{tabular}




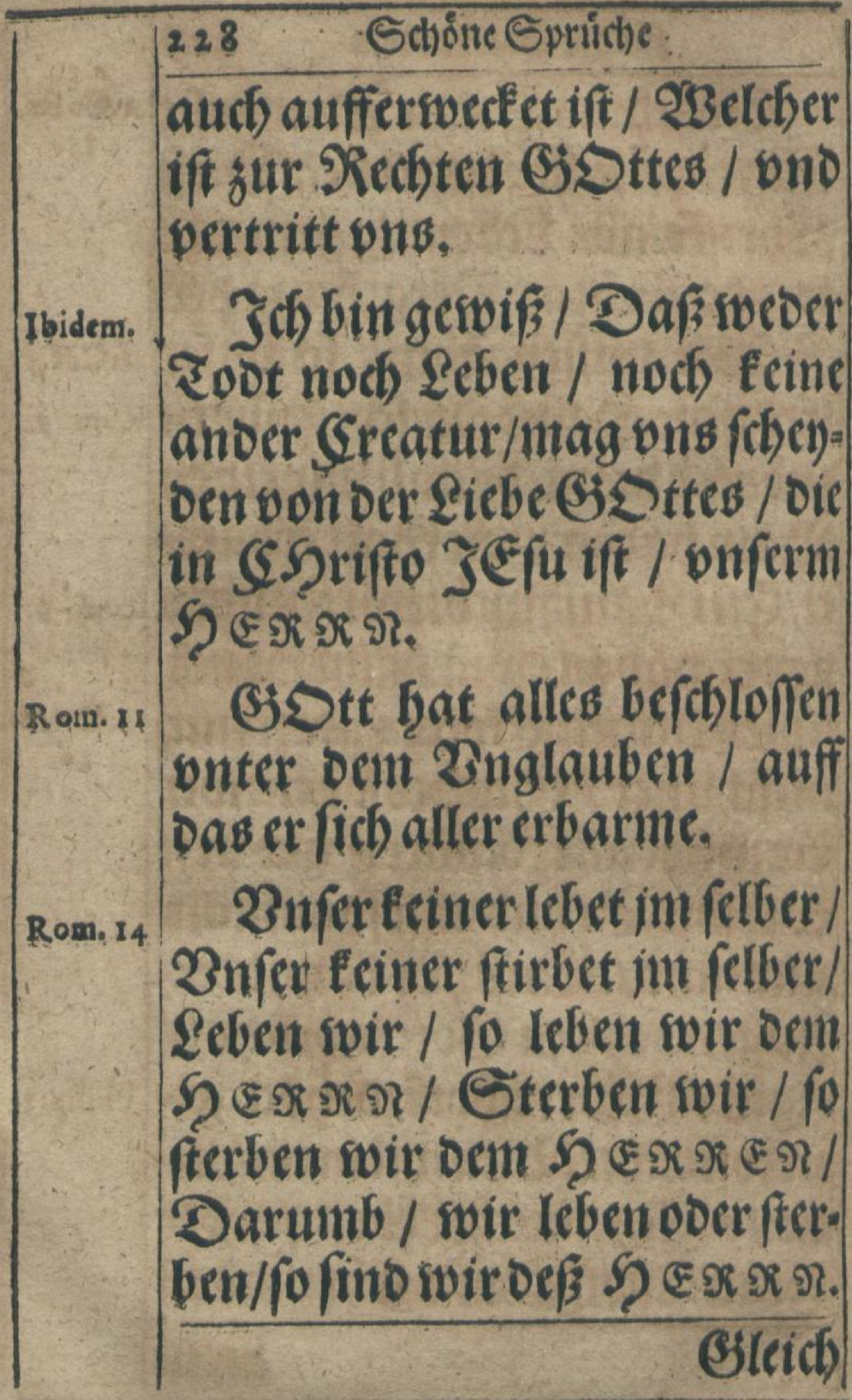


für bie Sterbenden.

229

(Gleich wie fie in 2 doam alle r.Corin. Sterben / 2flip werben fie in Sfrififo alle Eebendig gemad)t soeroent.

PBit wiffen aber / fo vnfer r.Cor.s. irroifth Sauk oiefer Sutten zubrodten wiro/oak svir einen Basv baben/bon Sott erbasw

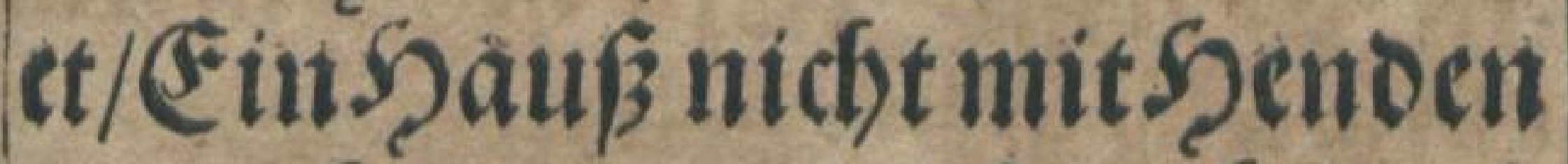
gemacbt/Das eswig ift int Simel.

(S) Dtt ift getreiv / Der eudi) nicht leflet verfuchen bber erver vermogen/ Sondern machet/ Daf oie 2 erfuchung fo cin ende gewinne / Dafs jro fónnet ertrageti.

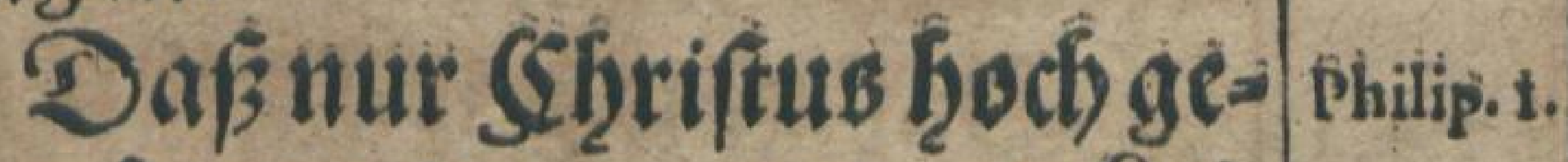
prei) fet weroe an ineinem $\&(i-$ be / eb (ei) Dureds Seben / boer ourcb Evot/Denn SErriftus ift 


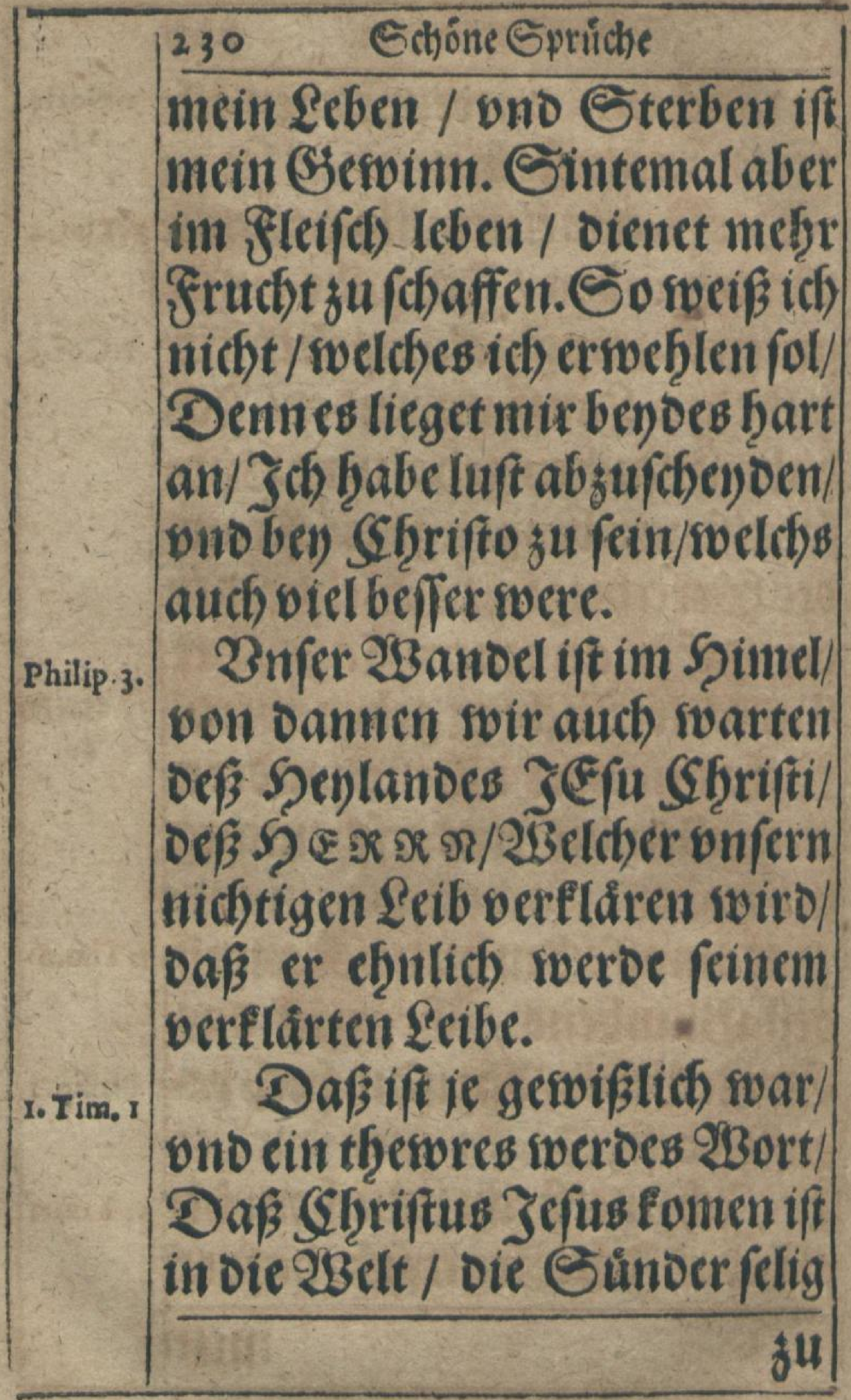


firt bie Sterbenbent. 231

zu madben / onter solchen ich ber vornemefte bin.

Die 3eit meines 216 fobeibens 8. Tim.4 ift berbanden / Jeb frabe cinen. guten Rampfi gef âmpffet/ కch babe den Sauff vollendet / Jch babe Silauben gebalten / Şinfort ift mir bengelegt die Rrone Der Sierechtigfeit / 2 Belebe mir oer ŞErr an ienem Eage / Der (Serecbte Pict)ter geben sviro. Nict)t mir aber alleine/fondern auch allen/o ie feine erfebcinung lieb braben.

Rampfie ben guten Rampff r.Tim.c. ock Sllaubens / Ergreiff Das Ewige Seben/Dagu ou audg beruffen bift.

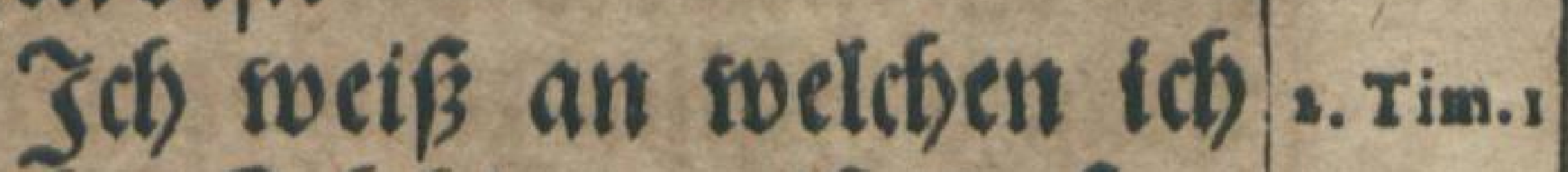

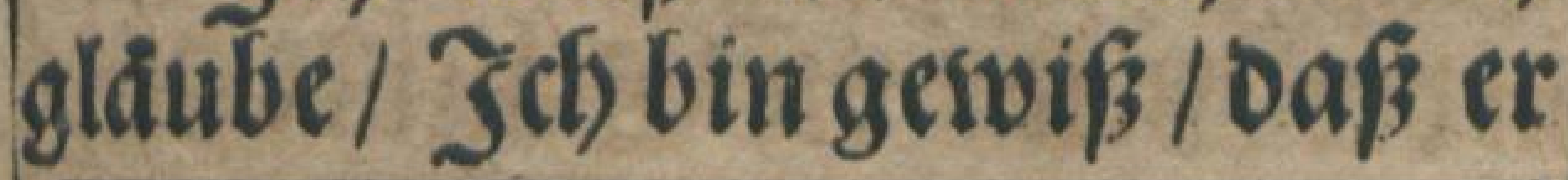
mir 


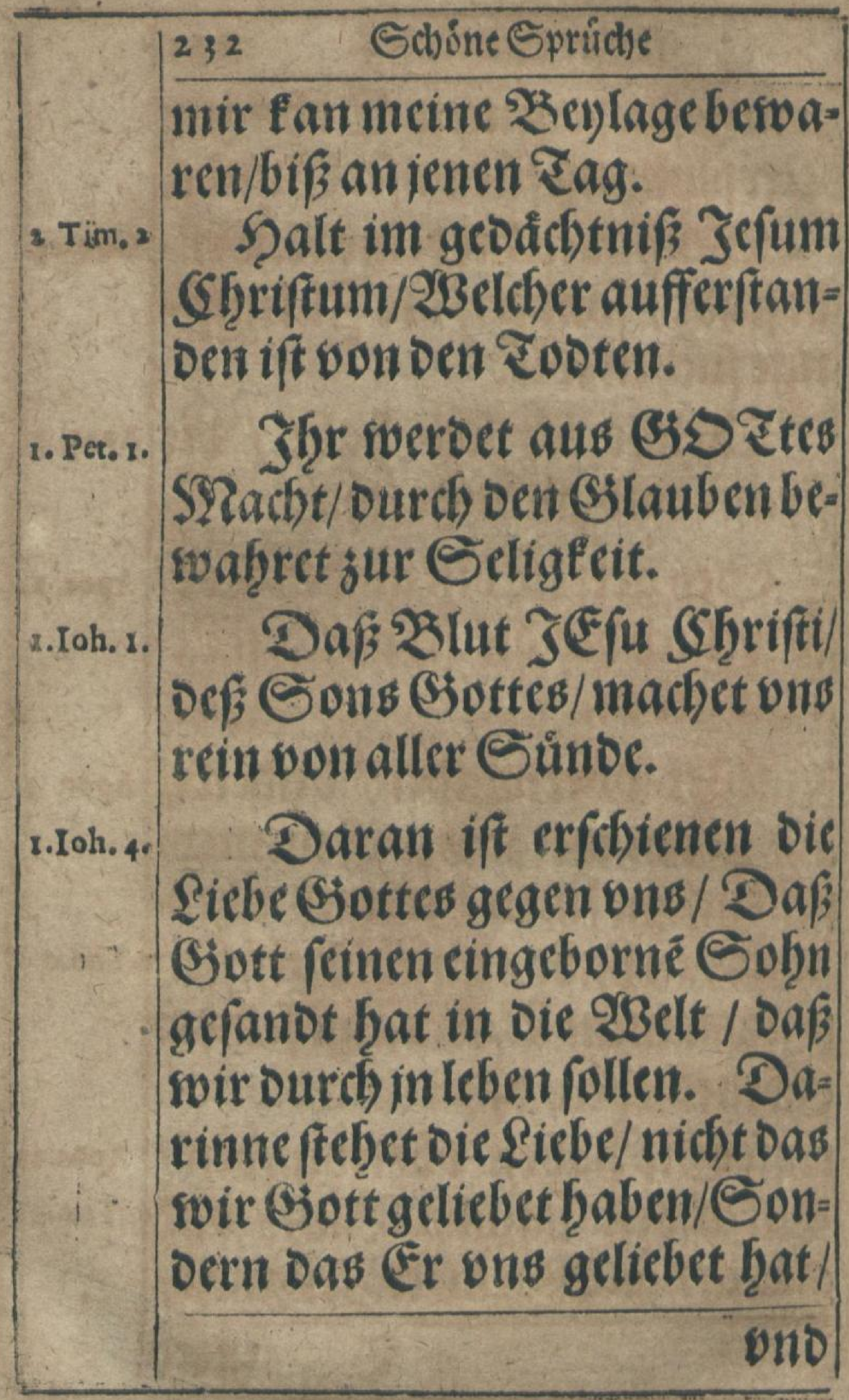




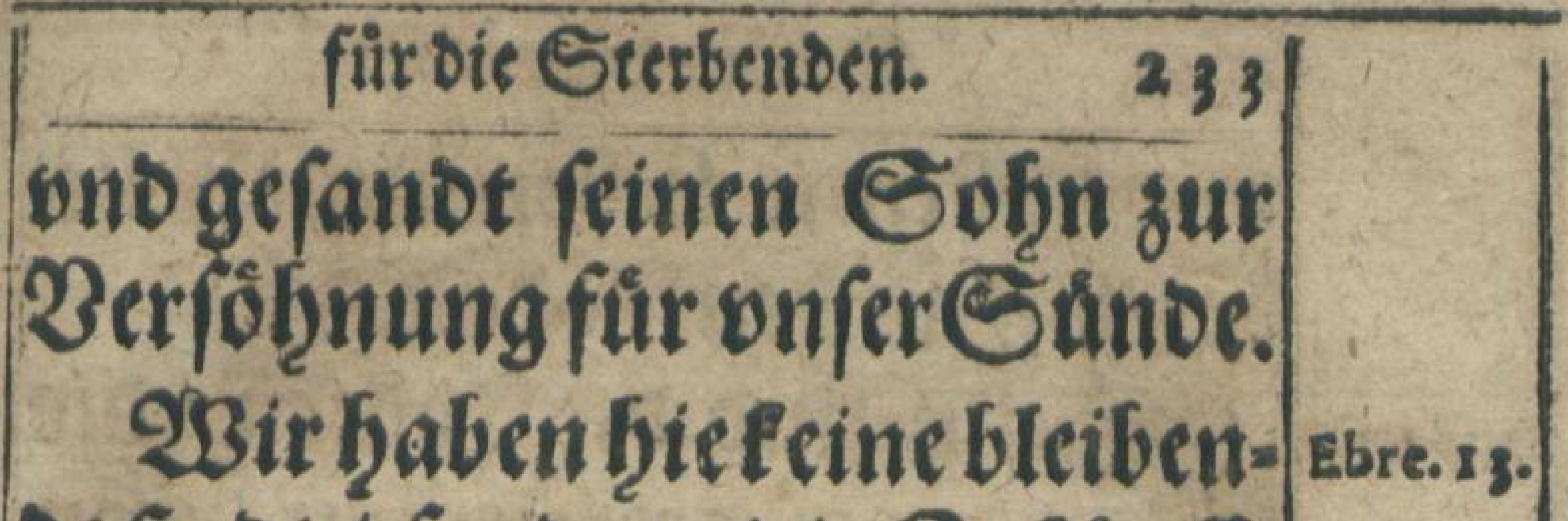
Deftadt / ponoern dic Zutúnftige fuchen swir.

Fibleche ich) lieb babe / Die Apoc. 3. frraffe bno gutchtige ich.

Sen getrew bif an oen Too/ Apoc. 2. fo wil idf oir Die Rrone Def \&e= bens geben.

शुBer oberswinbet / Dem wil ich geben mit mir auff meinem Stuel fu fizen / swie ich vber= wunben frabe / ono bin gefeffen mit meinem Vater auff feinem Stuel.

Sie baben vbersounden / Apocis ourch oef Lambs Blut / ond ourch Das $\mathscr{B}$ ort jer Zetgniß/ ₹ vno Apoc. 3. 


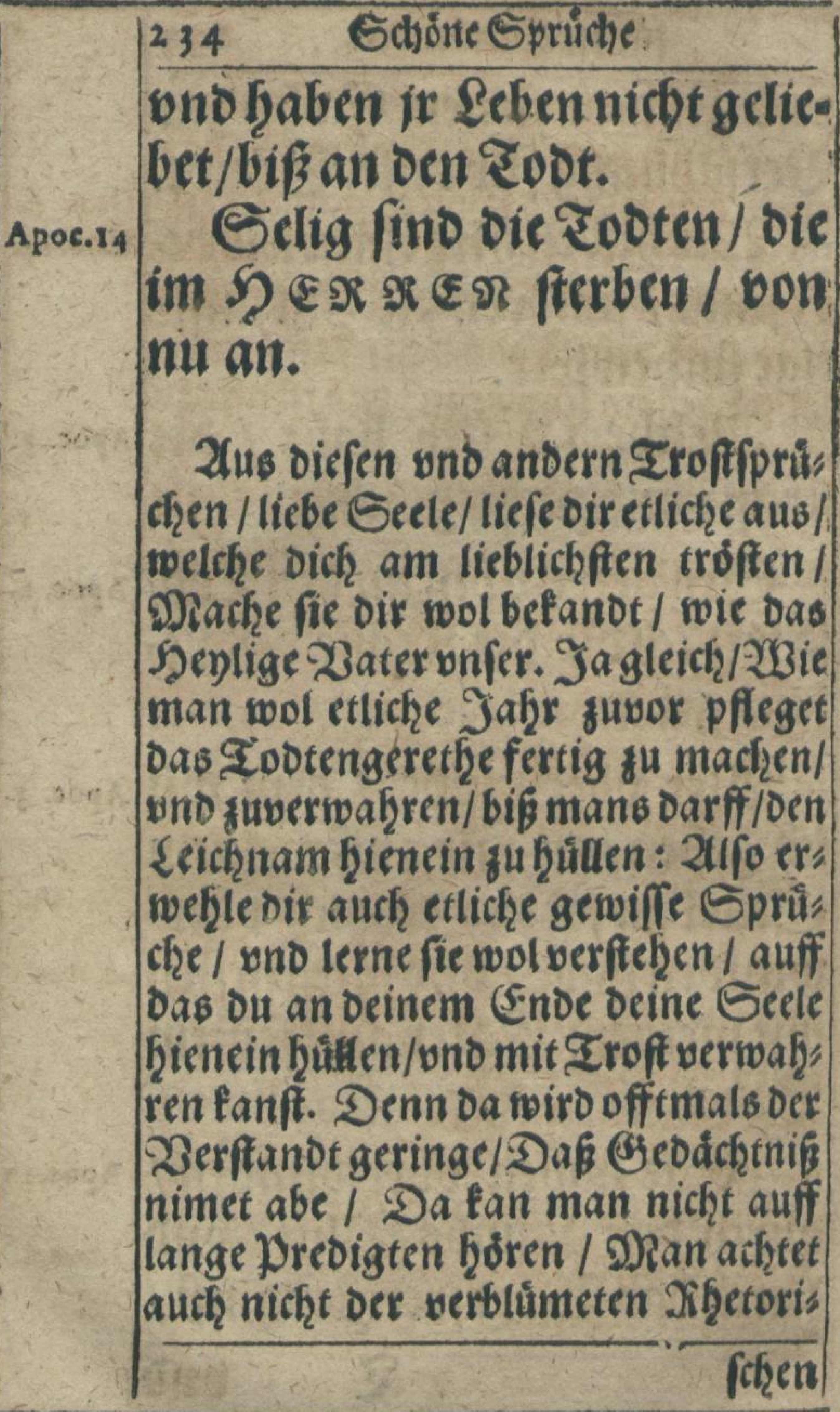


fir ste Seterbenten.

235

fichen gefiedoerten 23 orte / Eondern cin I roff fpruch / ein Iroftwort / Daf ourch Den Seuno Bjottes gangen ift/Deut. 8. Daß erfrewet/ labet/ fpeifet/ erquicfet Matth.4. Sers/ seib ond Gecle. 2Wennmans aber auff bem Todt2Bette erfit lernen fol/Da gefiets fehtwer su/ ono if offit of lange getiarret.

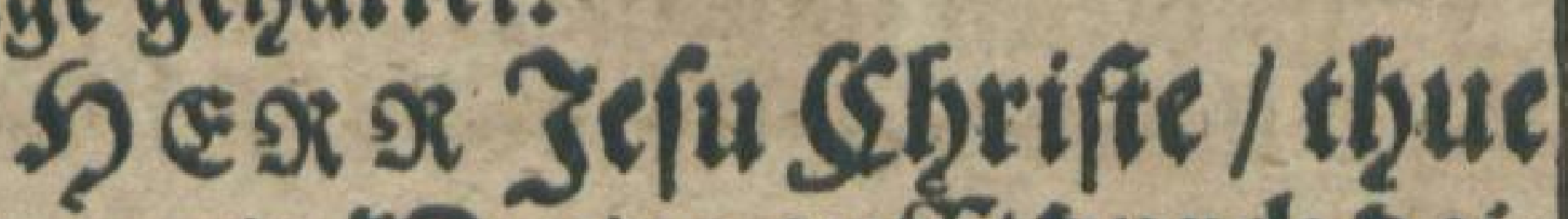
mir mein Dertse auffi/ourdb oei: nen Depligen Geift / oẩ íb oie Eprüctse beines Deyligentwors: tes gerne böre / 50 ol mercke / offt erbole / ons mein Dertse bamit reidb marben möge. Gib das ich bein 20 ort bebalte/ ono nicht $\mathrm{fey}$ wie ein zopff/ber ba auplleuffet/ Syz. \&3. ons teine \&ebre bebelt. Szrōfine mir mein Derftánonißß / Otárcte mir mein (Bebded)tnis/Dno erin: nere midb beines Troftes obn on: terlafí / Dilffoas idb bein Dort bebalte in einem feinen guten Dertzen/ofi frucht bringe in ges/ Luas s. bult/3ur ewigẽ $\Theta$ eliglteit/2Imen.

$$
\text { z if sebre }
$$




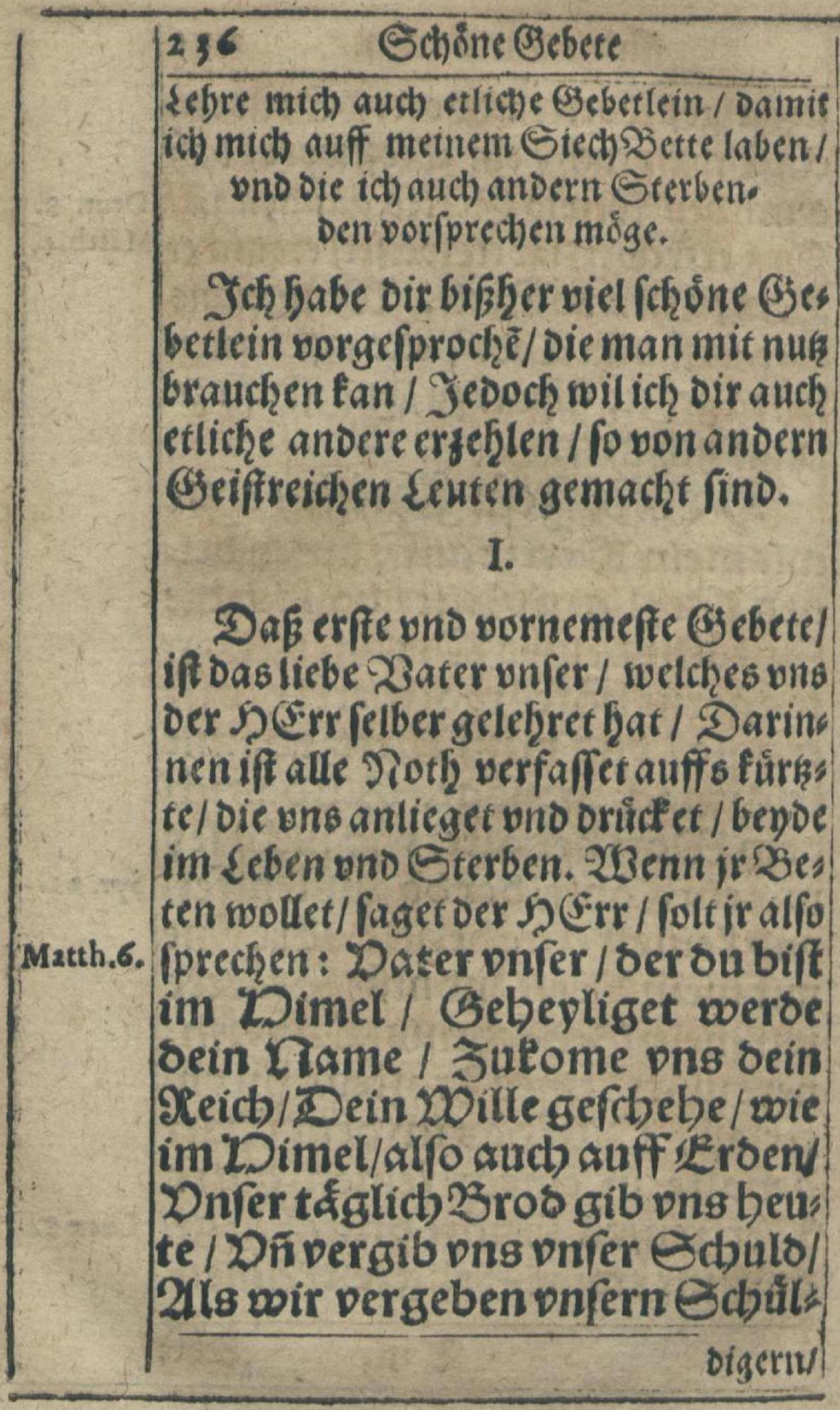


fúr bie Sterbenbent. 237
bigern / Dno fübre Dns nicht in Derfucbung I Gonbern erlöre ons Donbem D bel/Denn bein ift bas Xeich / bie Zurafft / pno bie Derrligteit/in/Rvigteit/2/men.

\section{I.}

D S Erre O5ott/ In meiner Noth/

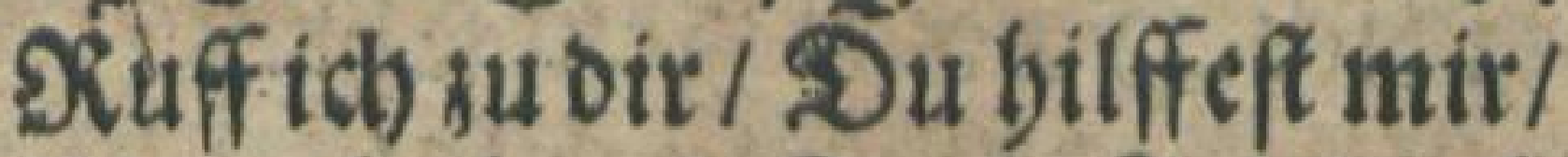
SRein \{eib und Seel $/$ Jch bir befeby Sir beine Jento / Dein Engel feito/ Der mict bewabr/ GSentid binfabr/ 2 fus diefer $23 \mathrm{elt} / 23 \mathrm{enn}$ birs gefelt.

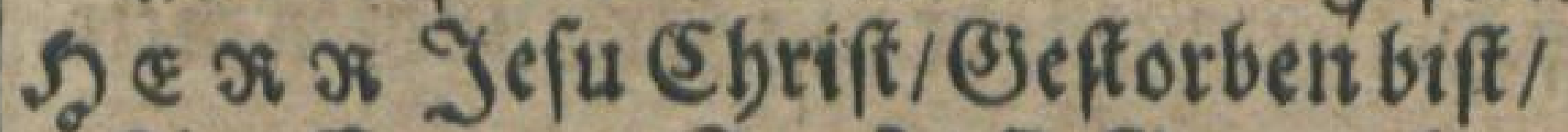
I m Ereuh̆eB Staiñ/D Sottes Samb/ Dein Wuttoen rotb/ In aller Notb/ Dein thesveres. Dlut/ Som mir zu gut/ Dein Eenden und Sterben/:2ach) mich oum Erben/

Sit beinem Dieid)/Dein Engelir gleidy/ Danfict) bey bir leb enviglich.

D Şenliger Eseift/ S) Rcin ?roffter beift/ Un meinem End/Dein Eroft mir fent/

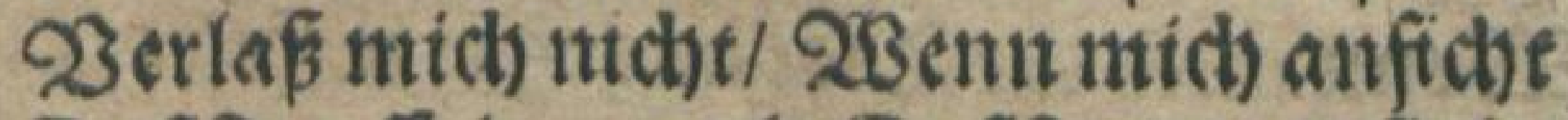
Def Eeuffels gervalt/De

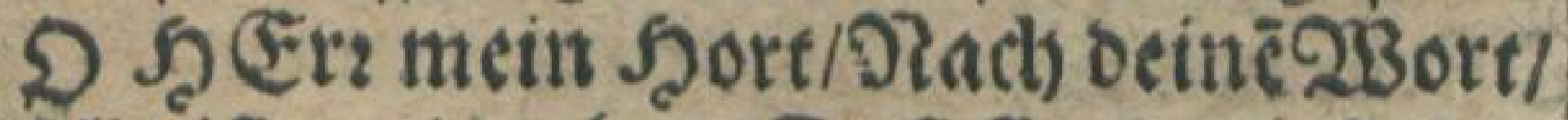
Ojolftu mir geben/ Daf Eneige Seben/ य D E ग.

I iij Silfi




\section{II.}

Silff Selfer f̧ilf in 2lngf uno Rothy Erbarm bid mein ou tretwer (5ott! Jid) bin Doch ja dein liebes Sinde / Trok 20 Belt/ zeuffel yno aller Süno/ Y(b) traw auffoich/ $D$ Bjott mein S Err/ 23enn ich bich bab/was will ich mebr/

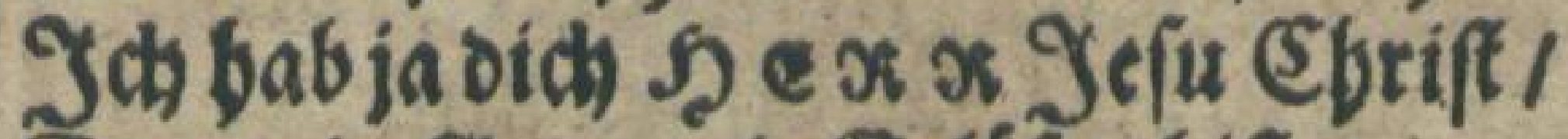
Du mein Bott vind Ertofer bift I Def frewich mids von Serķen feit/ Din gutes SRutbs vino barre bein ! Zerlaßs mich gånklids auff Dein S?amen / Dilff5erffer bilff/ brauff iprech ids 2amen.

\section{IIII.}

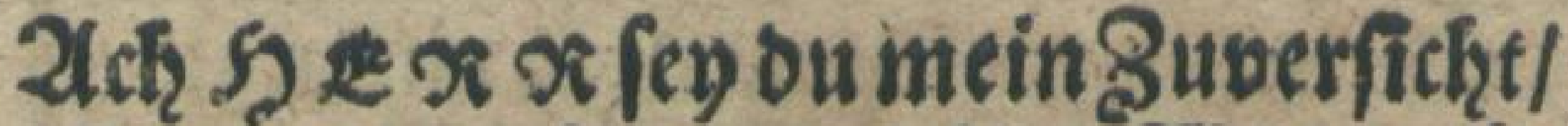
भुenn mein DRumb nu fein 230 ort mebr (pridat)

Ia fo Die Dbren nicht mefor bơren/ Durch Deinen (3eift tbu du mich) lebren.

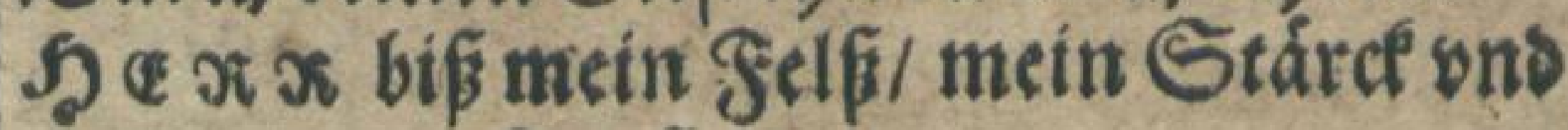
?roft/

QBenn mich ber Eobt alts Serkeftoft। Daburds fich mein 2(ugen verwenden' $D$ ftebe mit bey ond bilff mirs enden. S马 \& $x$ meinen (3) eift befeblich oir I Detingthoigs 2(ngefid)t wend su mir/ 


\section{für bic Sterbenbent.}

Durch bein bitter senden vno Sterben /. sap mich in feiner Sund verberben /

Die mir ber feinto fo grof tbut ntachen/

D S.Evr reißs mich aus feinem Siachèn/ 3noleg Das trofftich WSort in michl Deiner $\mathfrak{B e r}$ fóbnung bit ich) Did). Sas mein Bervifien auch empfindent Daricich rein fey von allen Sunden. $D$ trewer Gott ich bit von Serkent Bib mir (8) edult in meinen Esthmetsen. Durd) Jefum Ebrift erbor metit \$itt/ Saß beinen Diener ferbent int frico. Ertó 3 mich) aus ber s?otf vino ¿uall Belent mein Seet ints Soimels Saal/ Durch Deinen Engel in Dein Setch/

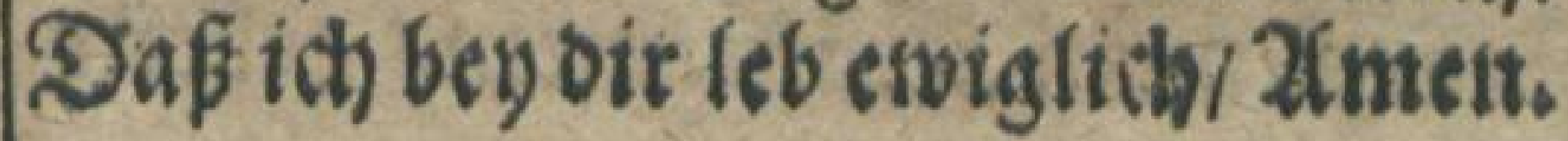

\section{V.}

Ich armer Elinber gar nichto bin/

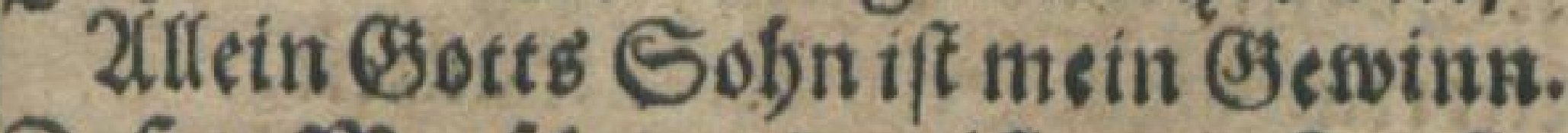
Daß er DRen (t) wardent ift mein zroft I Er bat ntich ourch fein 3 lut erloft.

D) Sott 23 ater regier ou micts DRit Deinem Eseifte fteriglich/ sas Deinen Sobm/mein Zroft unt selen/ 2Uris seit in nteinem Scethen fobweben. Dno wetun bic Stuno verbanden ift

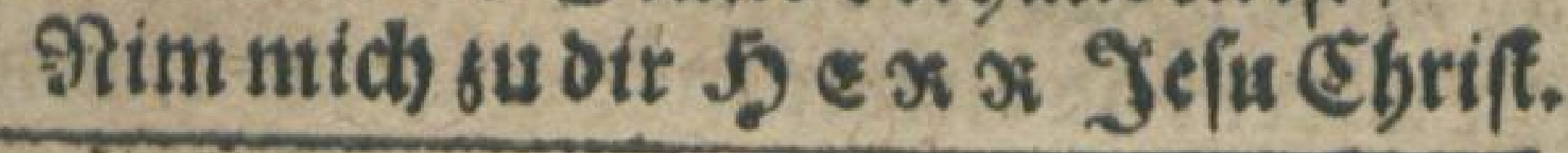
\& iiig Denn 
Denn ich bin bein/vnd ou biff mein/ 23ie gern swolt ich Doch bey dir feitt. S E $x$ Sefu Sbrifte bulff ou mul Dafict) etit Broetglein bleibit birf 23no machmals mit oit sufferftebe I 3u beiner Setrligf eit eingebel Mit Deinent Engeln in Dein Detch/ Dich lob vito prey[e erviglich/24ment.

\section{I.}

DSEr Gefu Chrift/ Du trewer Sport I Du Sobn Botté/vnd erviges 23ort/ DRein Zroft/mein Seyl/ bno ware Freno/ Tit meinet 2 Ang ft vilo groffent \{end/

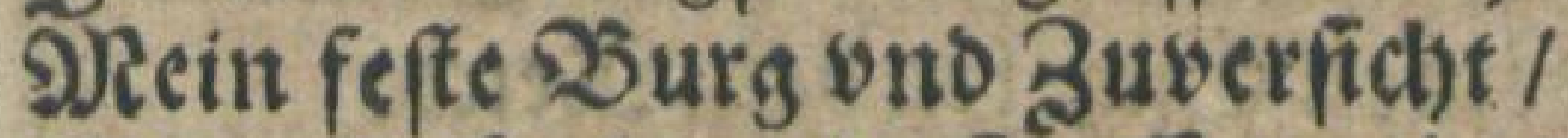
QRein Stårct/mein Sirafft/meint Sebens sied) $t$

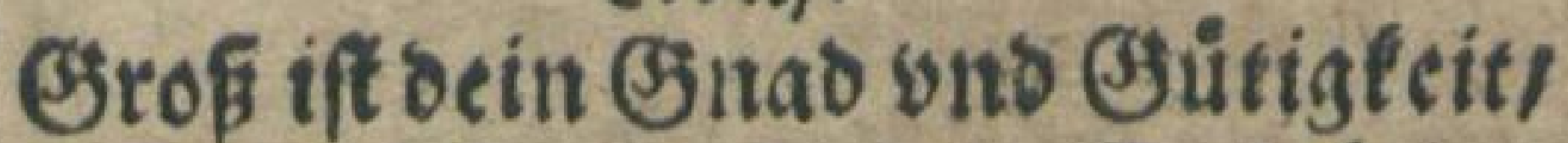
Grundt lop dectin \{teb int Evigteit 1 Durd) ocin grofle $\mathfrak{B}$ armbergigteits

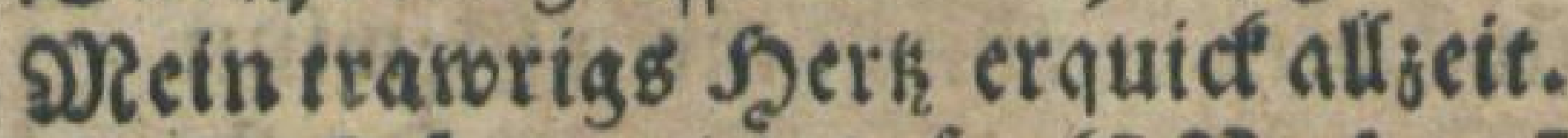

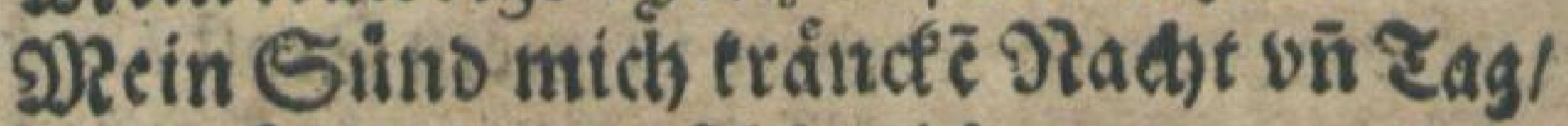
Z(ch trofft mich/ Dafi id) iricht verjag. S3efpreng Hitich ŞE Erz /ont mads micd reis DR it oem Xlofenfarbelt Blute Dein. Erlóp mich) voln oem etoigen Zoo/ Durth beine Seylige Wunten rotb।

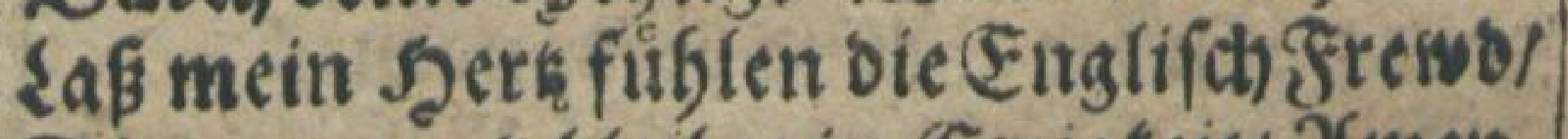
Die weret buro bletbet in Envigfeit/.2fmetr. รูE्Er 


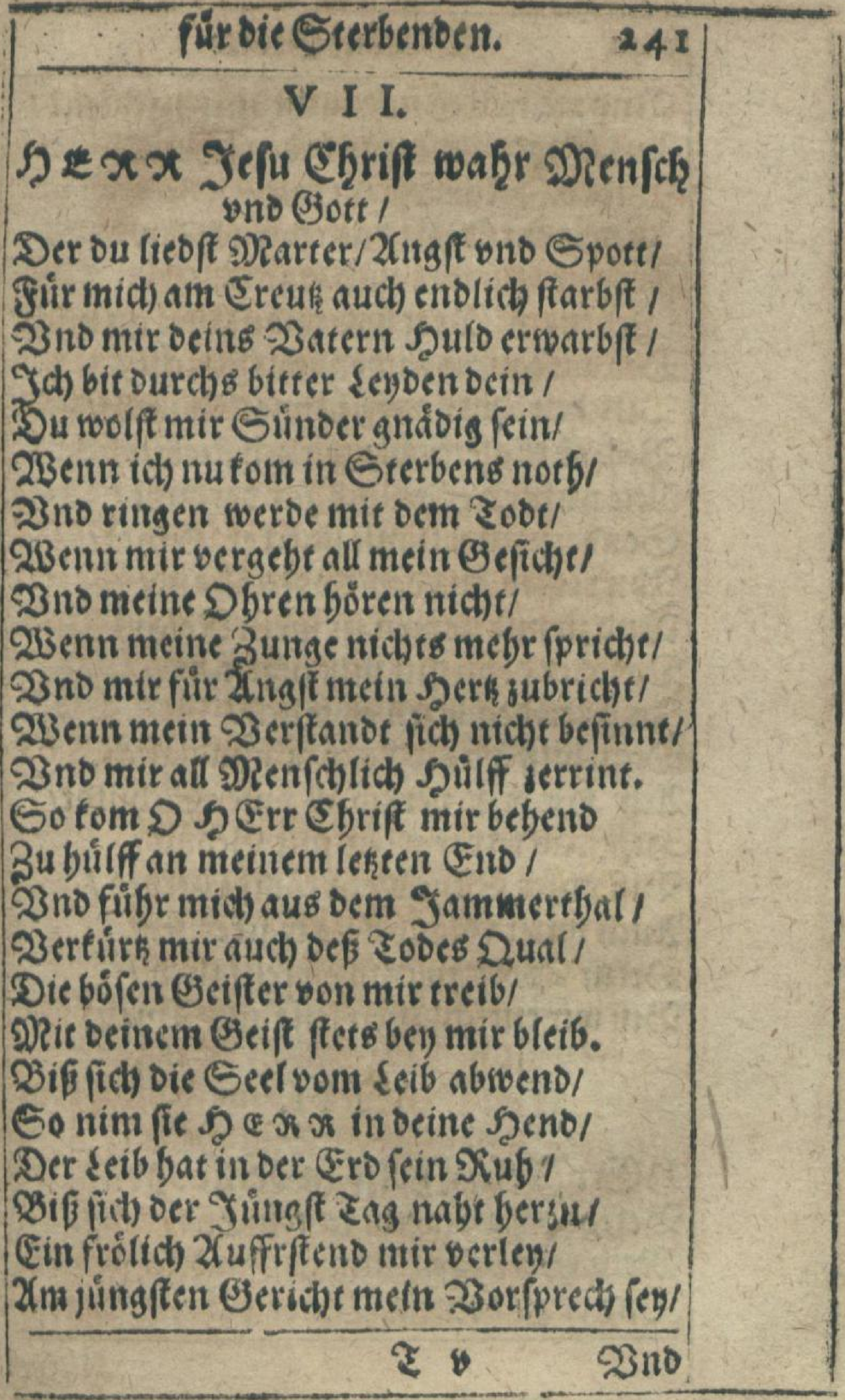




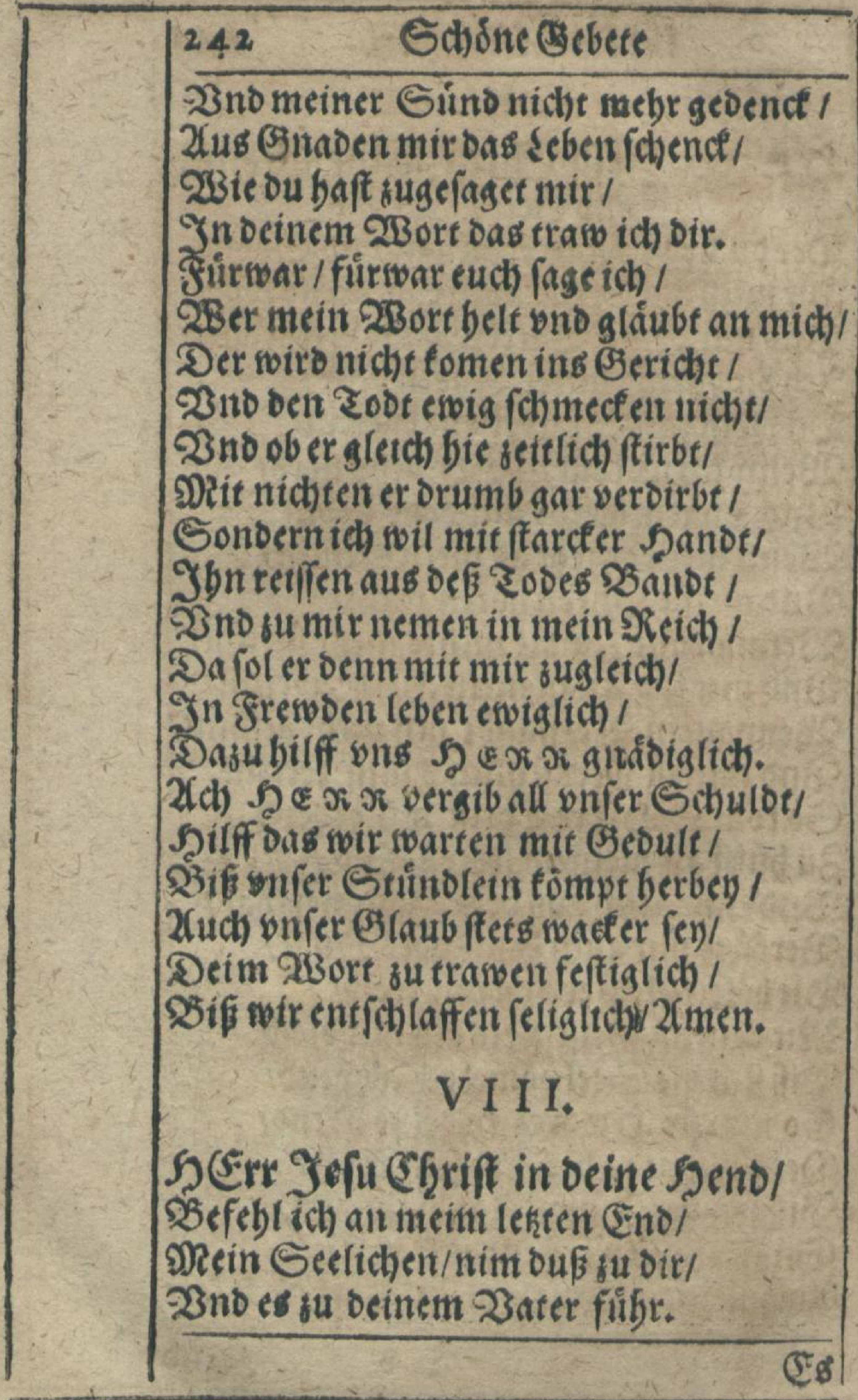


für bic Stetbentoen. 243

Es ift befprengt, mit beinem 23 tut I

Daffelb iff auch mein bocthftes (5)

Ich) ferb ono swil nu beis bie fein/

Dbnalle Rlag / ofn alle Peint.

ORein seben ift bie nichts auff (E)r/

Ẇenn es auct) gleid) viel Jabre weetf.

Sु Errbilff/ baß ich foldhes bedenct/

3no mich in beine Ginabe fenct.

Jc) traso vno glaub ourch Jefum Ebrift I

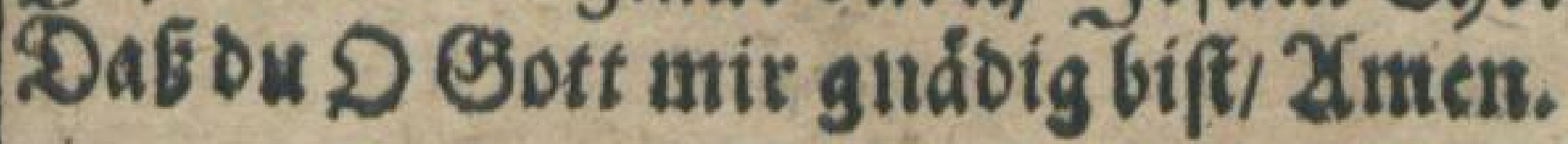

\section{X.}

¿ Sefin Etottes Sammelein/

Sol) leb oder feterb po bin ich oein.

Jch bit la fa mids mit bir jugletchl

Fin Erbe fein in beinem शieich.

Denn nas wer fonft bein Sterbens notb?

So viel Striemen bno 23 unden rotb?

23 enn ich auds nicht ber Seligteit

Geniefien folt in Entigfeit?

Warumb betfu bein \{eib sano seben

Im (3rab verfchlofien uno auffgegeben?

Wुenn niche metn Zoo burch beinen ZoDt/

Werjagt folt werden ou trewer B̈ott?

Darumb D Sefufteb mir bey/

Gerwiflen zroft und Sמúlf verley.

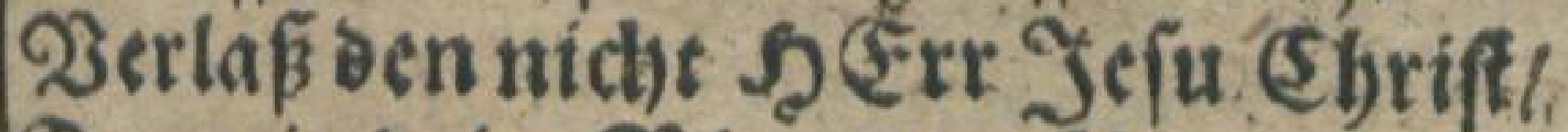

Der mit beim SBlut getwajchen iff. 


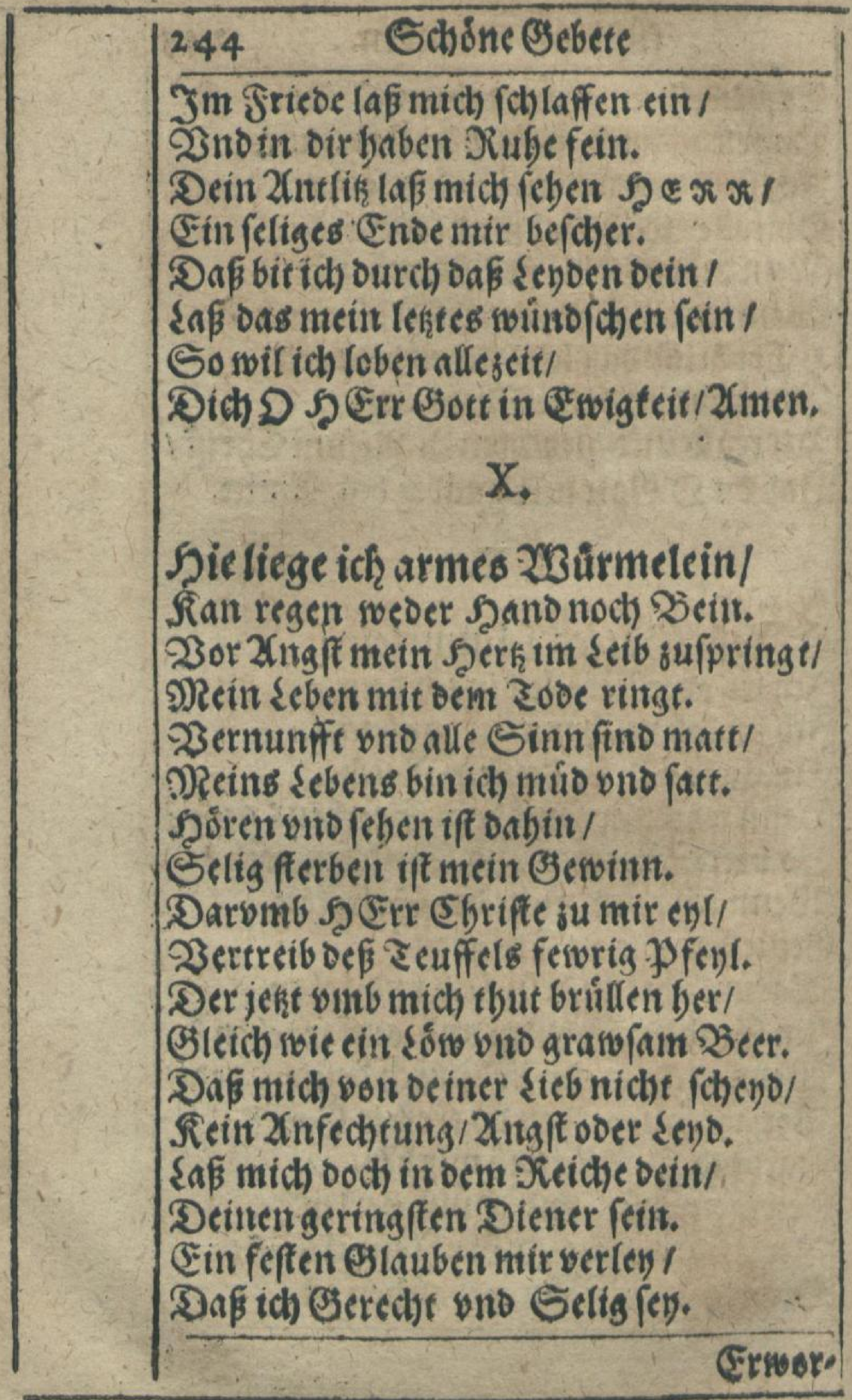




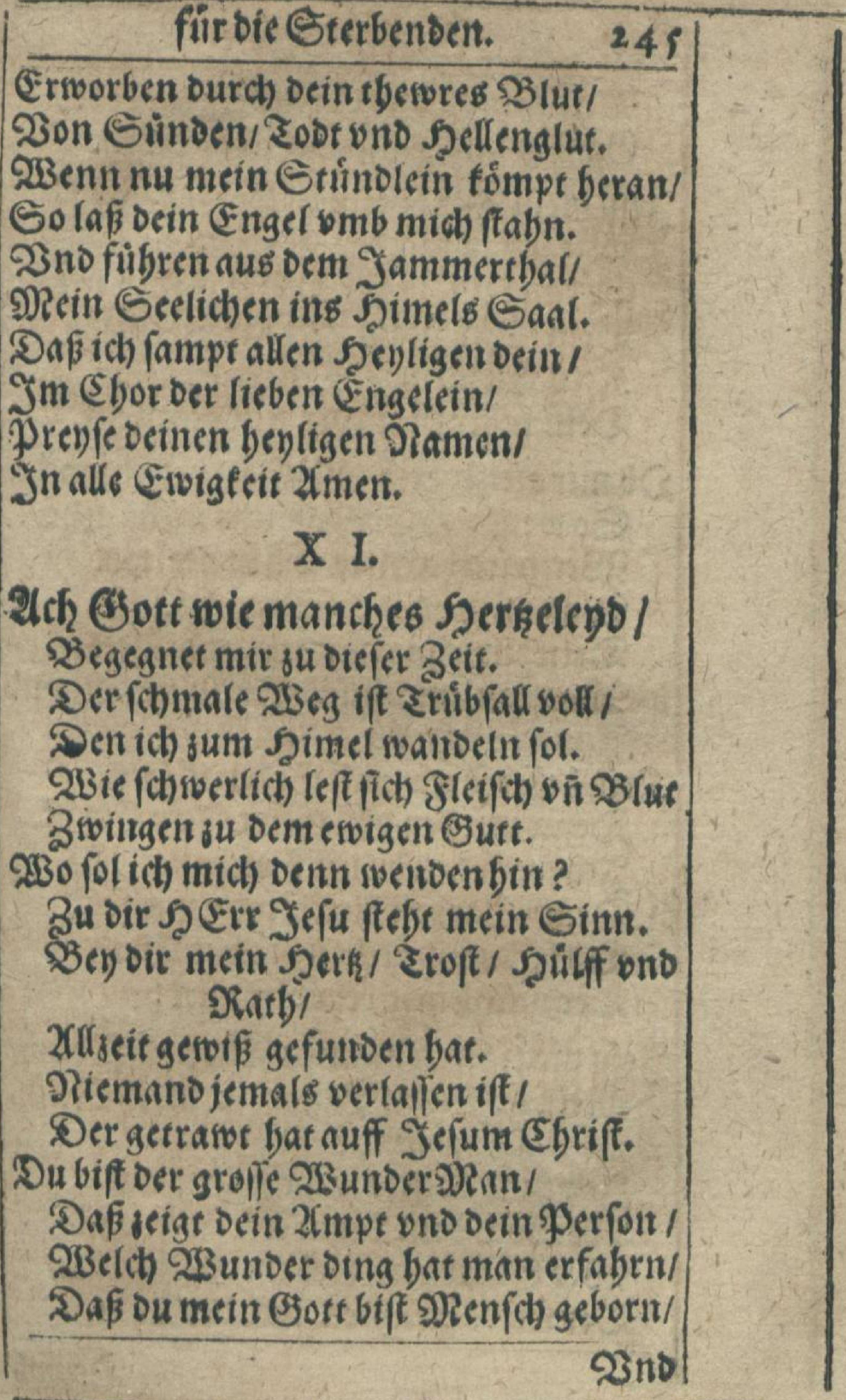


23no fúbreft ons Dutb beinen Zoot I (5)anks sounderlich aus aller Plotb.

Sefu mein Şe $x x$ vno Bott allein/ $23 i e$ fin ift mir Der Pame Decin.

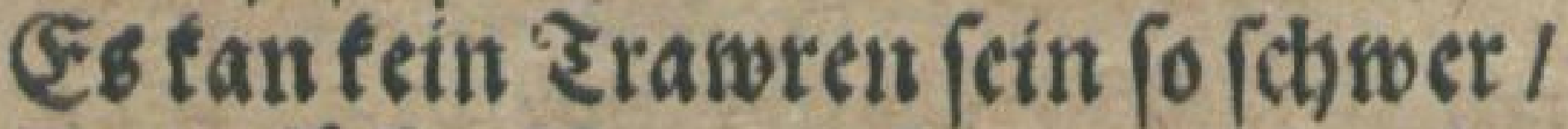
Dein fiffer Dam erfrerbe vief mebr. Sein Eleno mag fobitter fein/ Dein fiij)er glant der linderts feit.

Db mir gleich seib vno Seel verfdsmact) t/ So weiftu Ş Err baf́ ichs nicht acht I (3)ent id) oich $5 a b /$ fo bab ich wol 23 as mits ervig erfrerven fol.

Dein bin ids ja mit \{cib und Scel/ O2Bas tan mir thun / Sinto / Sode und Scill?

Sin beffer Eren auff Erben ift/

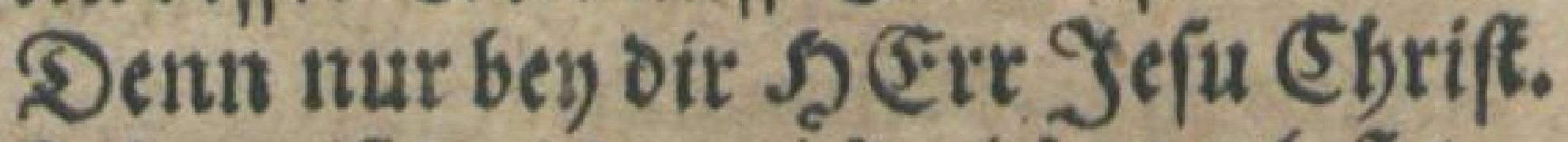
S(b) weif bas ou mich nicht verteft I Dein IB $_{3}$ arbeit bleibt nur erwig feft. Du bift mein rechter trenoer Şirt I Der mich ervig bebuten wirb.

Sefu mein Sretob / mein SEr bno Xibunt

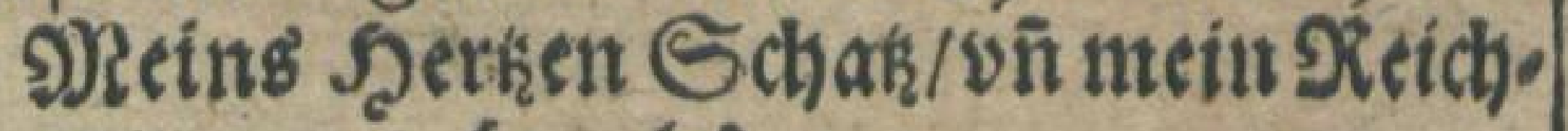
thumb?

Id tans boch ja nicht seigen an/ Wiste boch bein ?Ram erfrefben fant. W3er Staub ono sieb in Şerken bat Der wiros erfabren mit Der Egat. 
firt bic Sterbentoent.

Drumb bab ichs offt und viel gerede I X3enn ich an bir nicht frewoe bet / So wolt ich berl Sodt wind fchen bert Sa das ich ntebr geboren wer. Denth wer bich nicht im Seerthen bat! Der iff genvif lebendig toot.

Sefu bu ebler Breutgam werth, Mein bơchfte Zier auff Diefer (Et). 2(n bir allein ich) mich ergets / शुseit vber alle Búloene Sabda. So offt ich nur gebenct an bich! 2 efr mein semith erfrewet fiot). 2Benn ich mein Şoffnung fell zu bie/ So fublich Srieo ono Eroft in mit. Xjenn ith in Drótgen 2 eetb wno fing So wito mein Seerk rechet guter bing. Dein Seift bejeugt / Dafíf folches frey Deß Ervigen sebens 2 Sorfdimact fey. Drumb soil ich/ reil ich lebenoch/ SRein (Ereul Dir froflich tragen nach I SRein (Sott/ mach mich bajubereit/ E:s dient sum beften alleseit. Shilf mir mein Sach recht greiffen an/ Das ich mein saufft bollentoen fan. Şilff mir auch smingen Sleifch ond 23 lut I Sair Sínd vnd Schanden mich bebuit I Erbalt mein Şerh im Blauben rein/ So leb unto ferb ich oir allein. 


\begin{tabular}{|c|c|}
\hline & 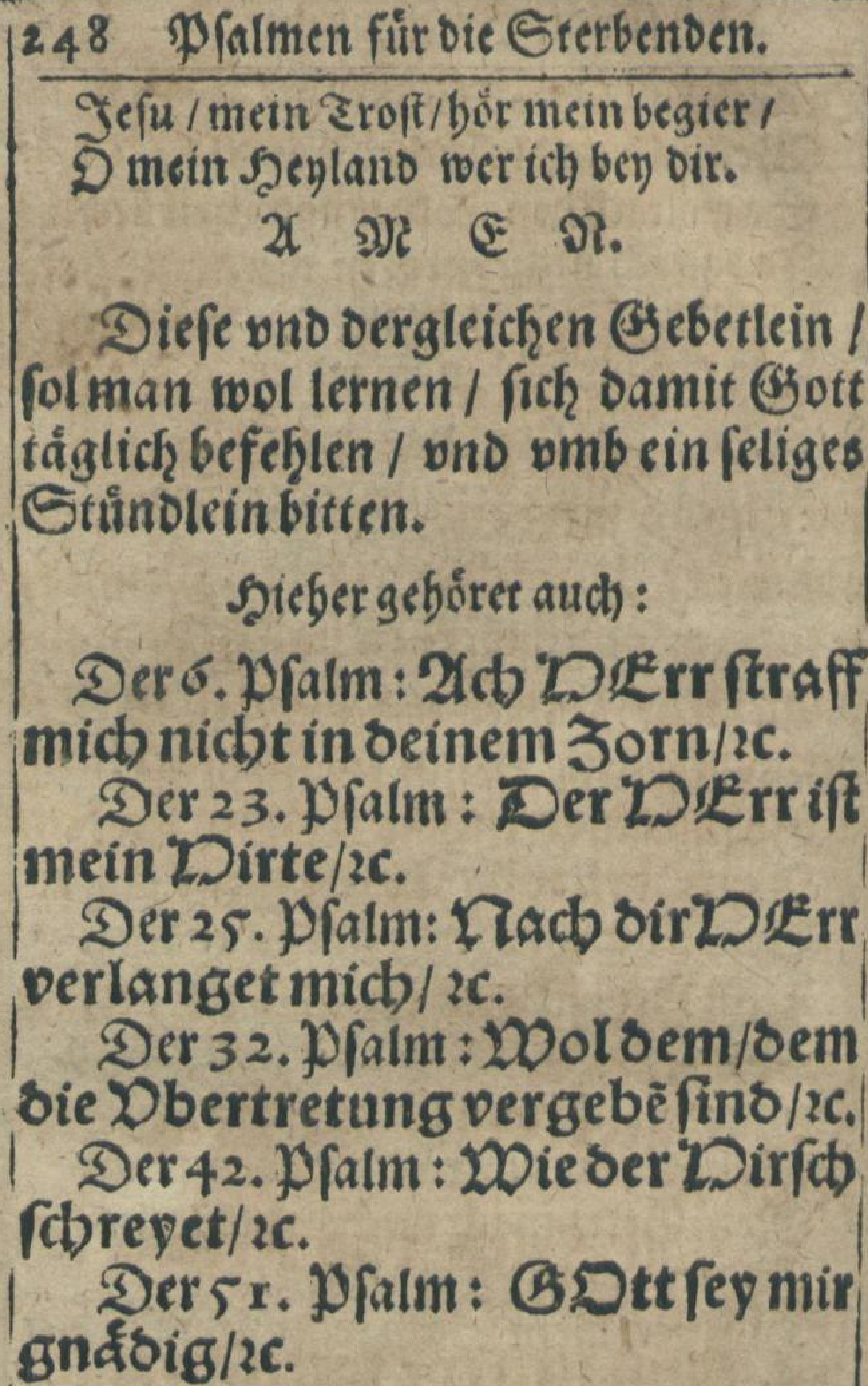 \\
\hline III. & 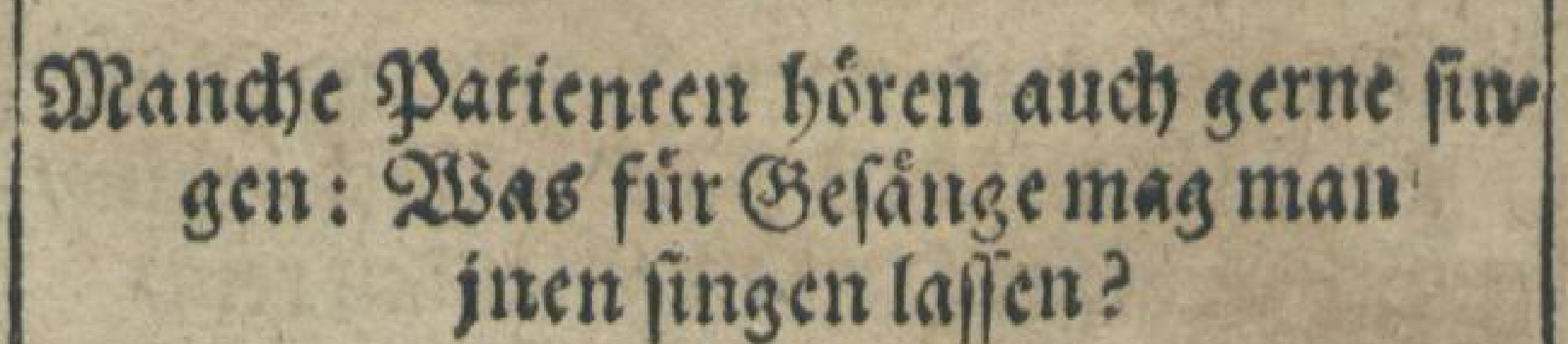 \\
\hline
\end{tabular}


Gefänge für bic Sterbentent. 249

Es gibt bie Erfafirrung/ Daf man ourch fchóne (j)efänge / wenn fie mit 2Inoacht gefiungen weroen/viel traws rigfeit vno fch wermuth als dem Spers ken weg fingen/ Dagegen auch viel Ichơones Grofites f̧ienein fingen fan / daßs die Serken dabey frólich vnd gus tes muthos weroen / Erinnern fich Das curch ber Simlifechen Engel DS uffical welche wir dozt werden halten helffen/ vnd fampt inen (sott preyjen in ewis gen Srewoen. Daan for fich aber nach oem Datienten richten/ond / m / ingen/ was erhaben will / 21 s da find:

r. Woir gléuben alle an einen Gott/2c.

2. Dater vnfer im Dimmels reidh/2c.

3. DDit fries und frewo íd fabroabin/zc.

4. 2uß tieffer Notb/dorey id) $30 \mathrm{oir} / 2 \mathrm{c}$.

5. II bitten wir ben Deyli: gen Geift/2c.

D G.2R




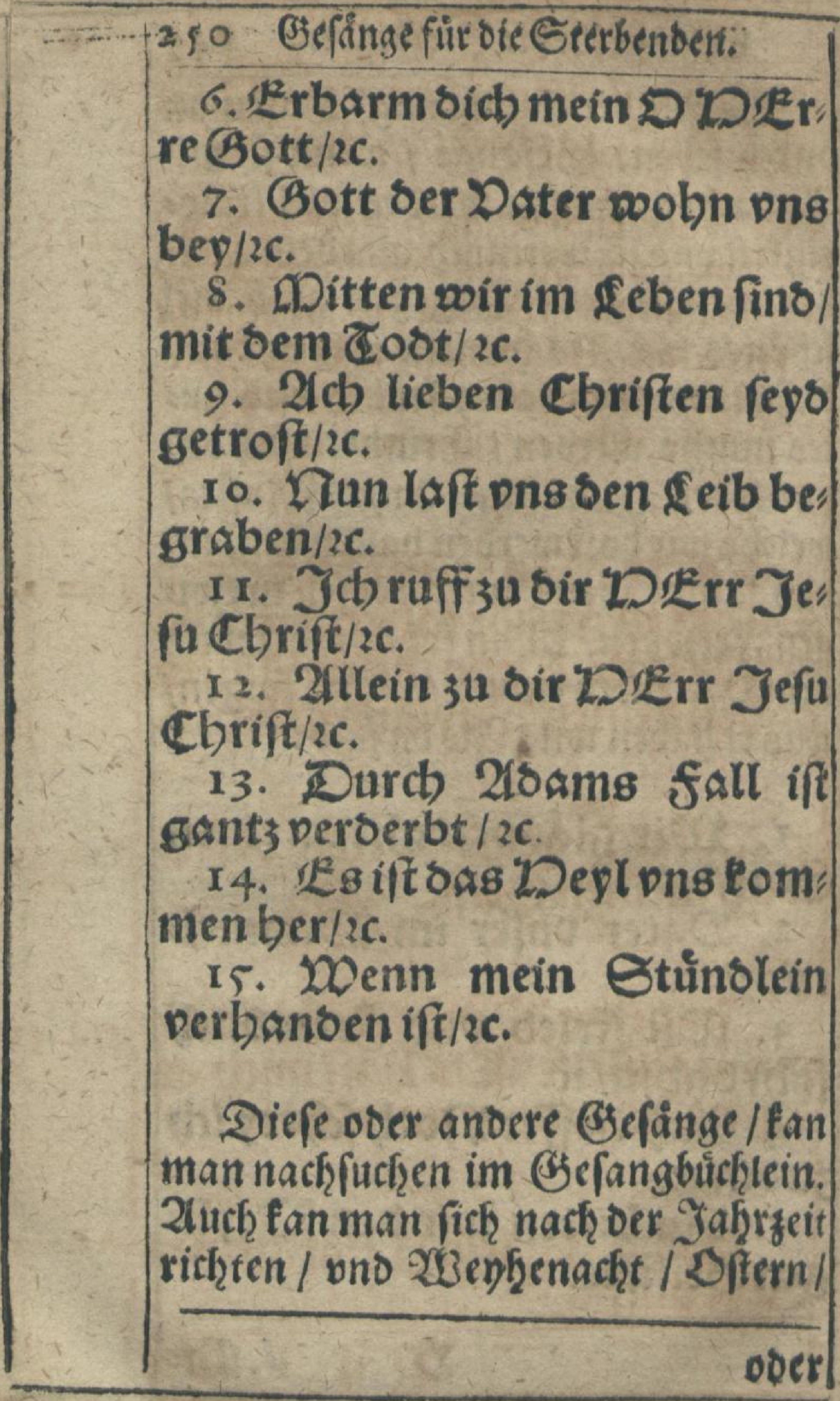


Doer Dfingftgefänge fingen/nach Dem Deß sirande en andacht begeret.

Evrseble mir auth etlich) furke Seuffisen I bie man gebrauch)en mag/ wenn oer Rrait. IIII. ce febr febroads ift/ swenig Doem bat/nicht reden fan / Sonderlich folche Wionts lein/Damit einer feinte Esele Sott befeblen fol.

\section{I.}

DErr / Ru leffeft ou scinen Diener in friese fabren/ wie ou ge [agt baft/Denn meine 2ugen baben beinen Deplano gereben.

$$
\text { II. }
$$

Dater in beine Denbe befeb: (eic) meinen Geift.

\section{III.}

In seine Denoe befeble td) meinen Geift / Du baft mich er: löfet/Dín rr ou trewer Bott.

\section{II.}

DEXX Jefia / flim meinen Acor.y. Geiftanf.

Luce s.

Pfal. 33.

$$
D \text { प EP }
$$




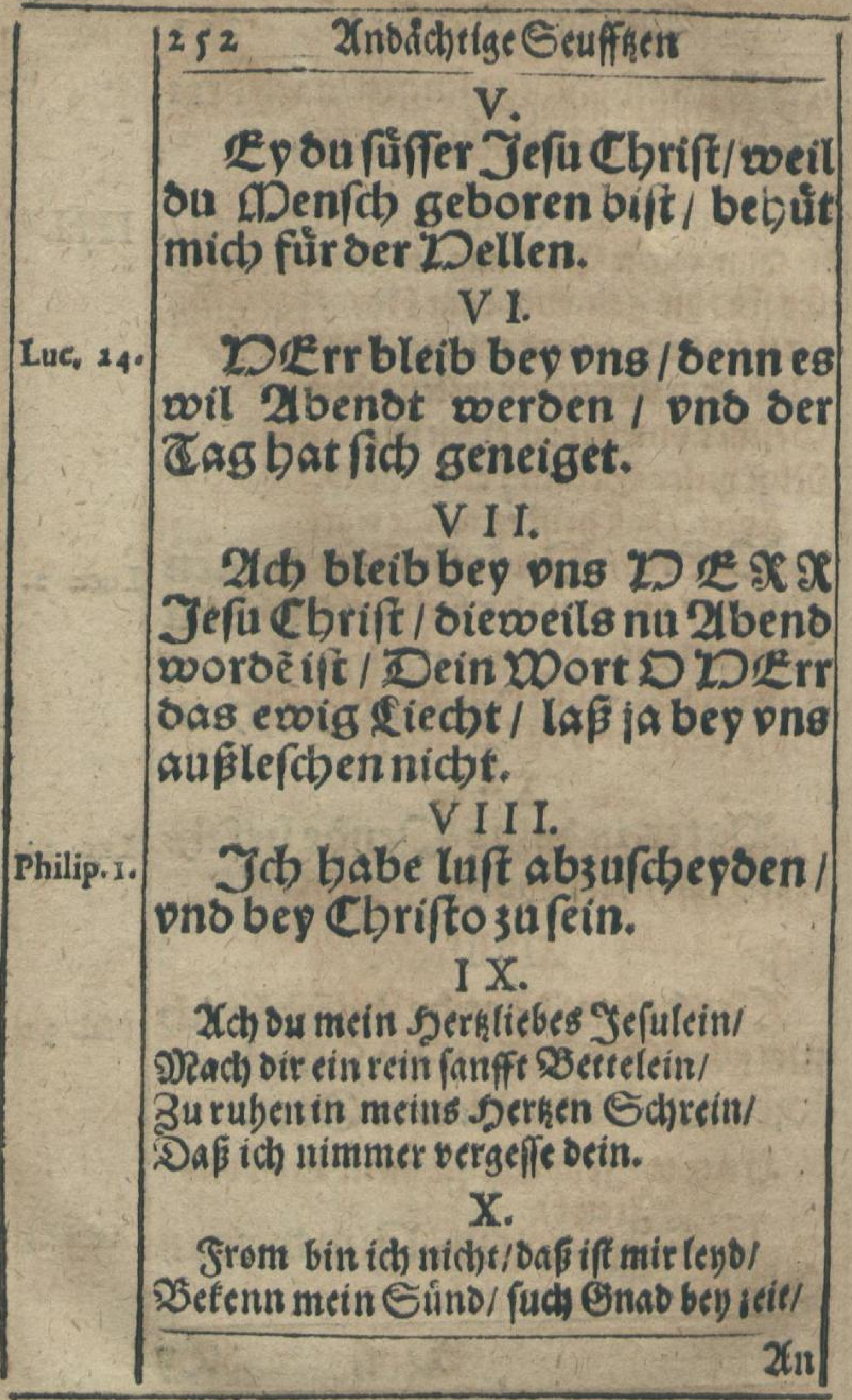




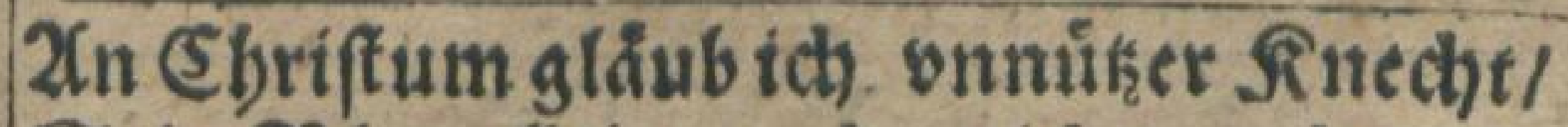
Scin 2 lut allein madht midh gered)t.

\section{I.}

Bon allem $3 b e l$ ons erlé:

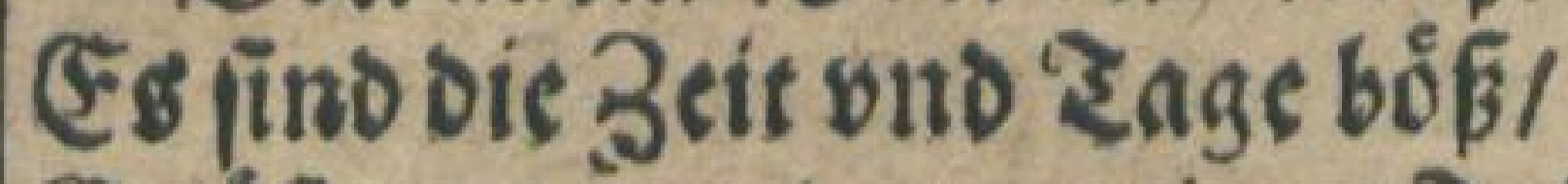
Erlóf ung bon Dem erbigetr Sobt I Wno tróft uns in ber leksten פloth! Befcher un ŞErr ein feligs (Eno/ Pim vnfer Sesl in Deine Secno. 2umen/Daf beift ez nerde war/ Stấref on [ern SIlauben jmmerdar/ Şilff oas wir ja nicht grweiffeln brait/ श्as wit biemit gebeten batt/

2uffocin 230 ort in oem Pamen ocity So fprechen wir bas 2 inten feilt.

$$
\text { XII. }
$$

Sefu ber fiffe Drame beilt !

Im Eodet erquict bis Scele metit.

$$
\text { XIII, }
$$

Die Seele dic bu bafierlofit

Der gib Sุ Err Jefu deinen ?roft.

$$
\text { XIIII. }
$$

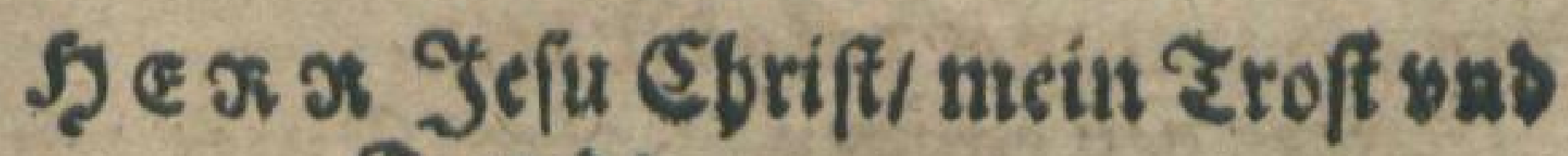
Frenod

Ith ivart auff oich su jeber 3eit! Som wenn bu wilt idh bin bereit. 


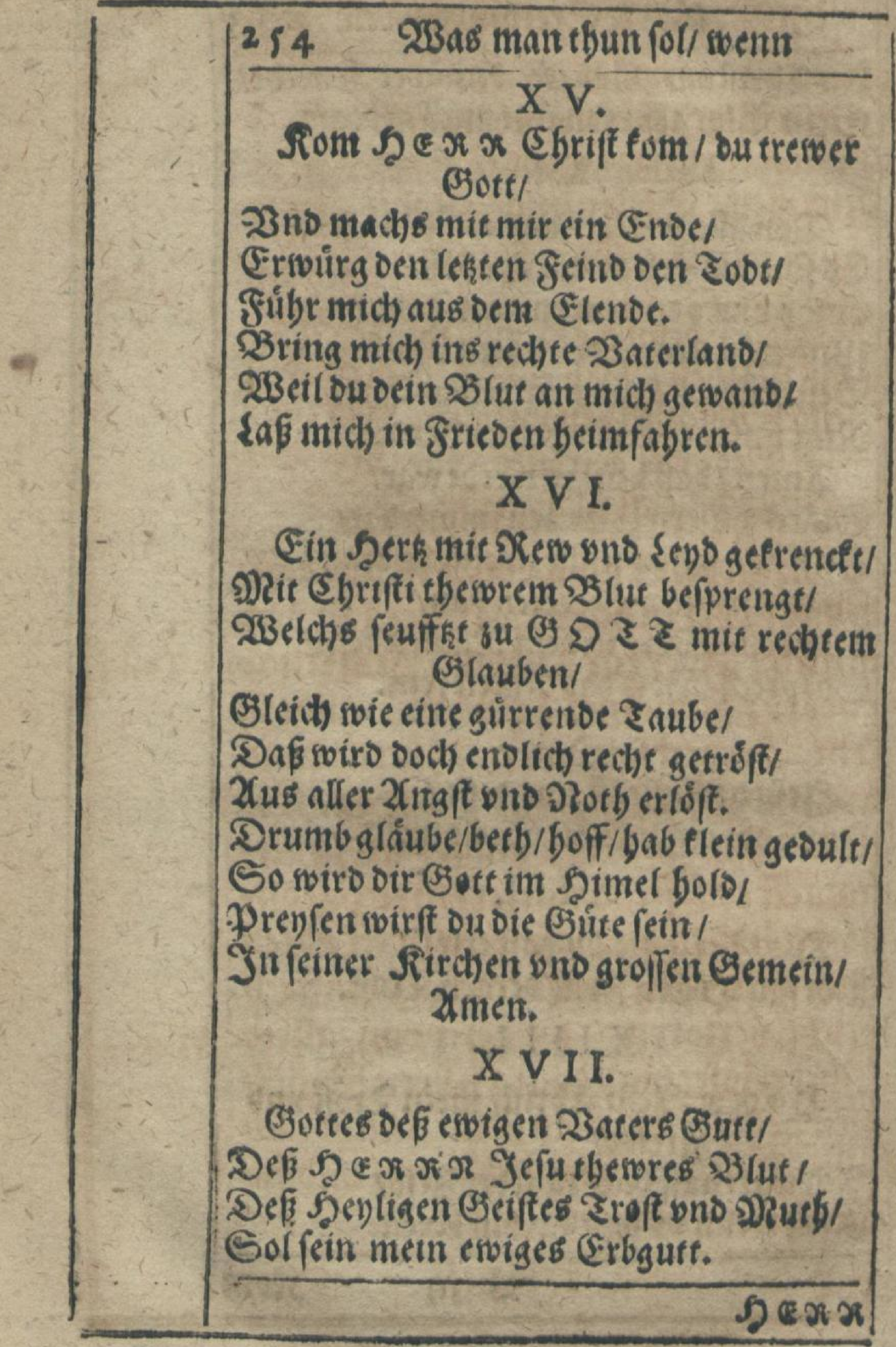




\section{(in ) Menfch (Sbriftlich gefforben. 255 X V II I.}

S马e $x x$ Sefu fon bebendel

Bib mit ein |eliges Entre)

Drim meite Seel in Deine Sachoel

$$
x \text { \& }
$$

23ab follen benn bie 23 mbftebendent thun/ wenn fie feben / Dak ein Sbriften \$Denf ch

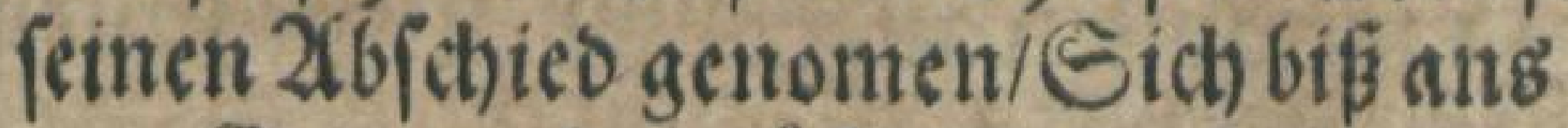

Ende wol getroffet / wird felig entichlaffen ift?

Trawren vno weinen if niemans Den für vbel gu faben / dennes ift $g_{a}$ tuirlich. 2tber fiir Şenonifchem traw" 1. Thef.4 $r$ / für Bettergefectren/ bno andern 2ngeberden fol man fich Guten/Denn wir fino SEffriften / uno follen wns tro: fienlaffen/als die da gláuben/ono oies feffarcte / fefte / vngegiweiffelte 5ooffs nung liaben / Dab vnjere Gooten nicht geftorben / fondern entforlaffen/ Nicht verloren / fondern von allem $23 b e l e r l o f e t / v n o$ fum Ş Errn hinlseis men gebjolet fein/wno nemen nicht taw. feno 2 belt / fo es miglief were/ ono fes men fierwioer in di Eileno.

$$
23 \text { iiij }
$$

Dew

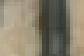




\begin{tabular}{|c|c|}
\hline \multirow{21}{*}{ Ebre. 230} & $233 a s$ man tbun fol/went \\
\hline & DEerhalbenfollen wir viel mefry \\
\hline & (3) Stt fuir folehen peligen 2 bb finied \\
\hline & 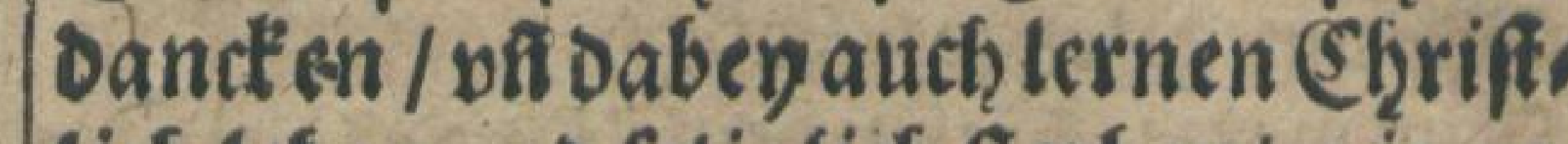 \\
\hline & lich leben und feliglich fterben/ wie ge, \\
\hline & gen ober \&läubigen enve anfchawen \\
\hline & bno irem @lauben nach folgen follen. \\
\hline & $\begin{array}{l}\text { Ya wir follen auff onfer Sinye fallen } \\
\text { ond alfo Deten: }\end{array}$ \\
\hline & Sir Danden oir らุ \\
\hline & Jefu Cbrifte / Du ewiger Der: \\
\hline & $\begin{array}{l}\text { t3og bef febens / Daßj bu biefen } \\
\text { lieben obenfben in wabrer } 2 \text { uf }\end{array}$ \\
\hline & fe ons Glauben beftenoig erbals \\
\hline & ten/vno im ein feligeg oznoe ver; \\
\hline & $\begin{array}{l}\text { lieben baft. D wie lieblich woird } \\
\text { jet3t feine liebe Eeele gen Dimel }\end{array}$ \\
\hline & getragen/ Die wollftir. Denn \\
\hline & $\begin{array}{l}\text { 10 wiro gelaget don oej lieben } \\
\text { Lazari Eeele/ ba er ftarb für be } \beta\end{array}$ \\
\hline & Xeidben DDannes aburre: Sei= \\
\hline & ne Seele soaro getragen oon \\
\hline & Den Eitgelit inn 2lbrabamb \\
\hline & \\
\hline & \\
\hline
\end{tabular}


ein Nenfich (Ebrifflich geftorben. 2 s 7 id) gevoi $/$ Db ich wol mit meis nen fterblicben / leiblicben / tun: ctelen 2lugen nicbt reben tan / oafi viel beylige KEngelein od fino/ oie ombs 23ettbe berumb auff feine Seel gevoartet baben. Iu woirs fie binbeimen gebolet/ III febret fie ein ins eboige $p a s$ rabeif. Hu rubet fie in ibres DEerrn Jefu 21rmen / ons auff reiner Eabố. ITu bat alleibr zrawren ein enbe / Itu wiro ir ber DERr alle jre đbrănen von E[3.26. jren 2lugen wifchen / ons fie mit evoigem friese ons crofte/ mit epoiger frewo ons wonne bes

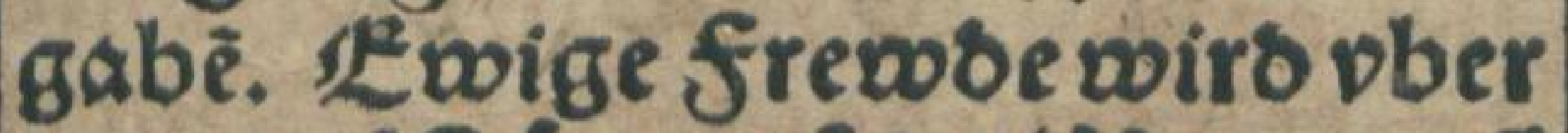
beinem 12 dupte fein/2Donne Dn frewose werben bich ergreiffen/ 2 ber 2ingft ons craworen ift fer: ne pon sir. Doultebe Seele/woie wollift oír nu/ Dubait ja getra: gen Cbrifti Jod) / Sift geftor: ben vnolebeft nodb.

\section{2 $1(6$}




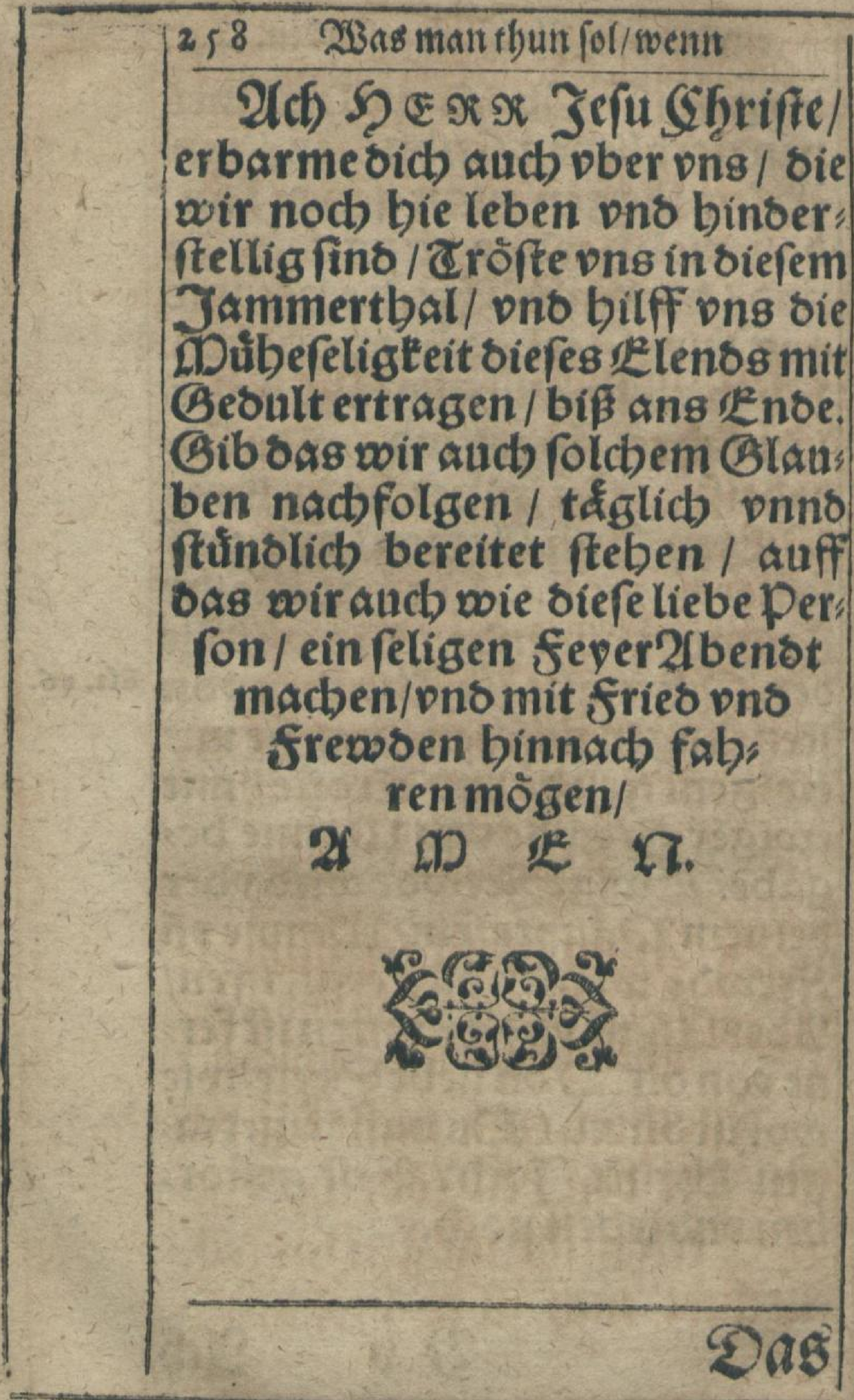




\section{ein Menfch Ebrifftech sefforben. 259

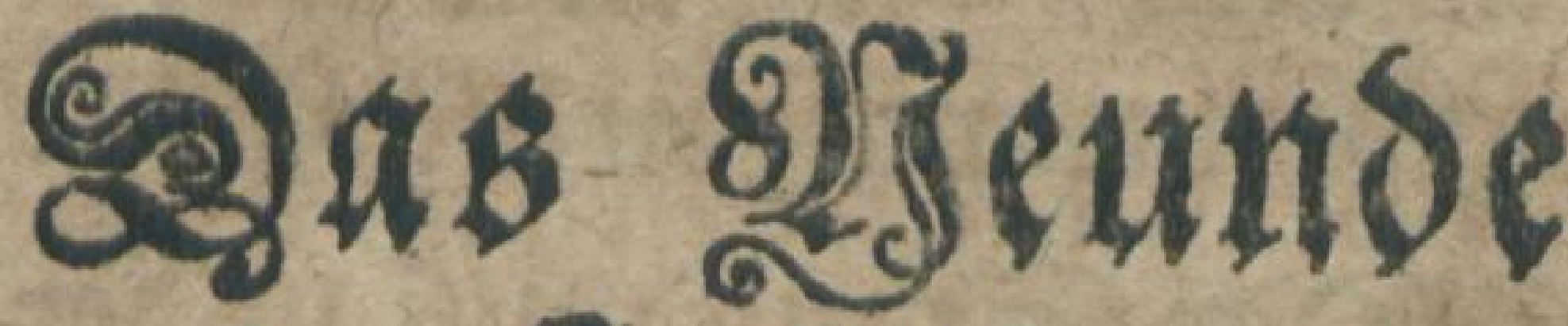 Capitel.}

Thut berictic/20 o bie 23n iterblicten Eeelen nach irem abbcheyde finfomen / vno was it 3w: ftande fer). Betract)tet auch bie 2urffers

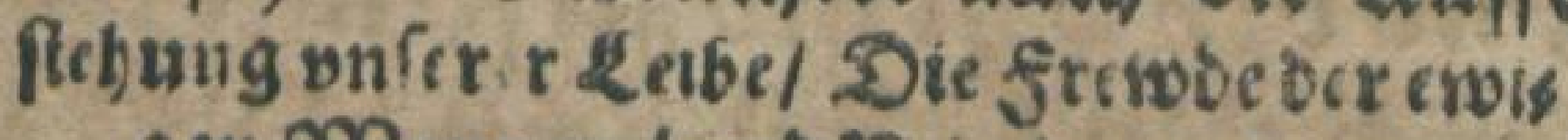
gen 203 onnen / vno pein der ewigen Derbamnis.

23eil nu bic Ecelen snftetblich fino / 250 tomen fie berm bin / vno wie gebet is jnen/wenn fie von Den sciben. abfehenden?

TFe 2 (won berichtet whs bie 6. Echriffit gar fochơn und troffis lich in folgenoen Epríchen : firchtet euch nicf)t fur be $=$ Mat. 30 . nen / Dic ben \&eib tobten / ono die Seele nicht mógen tóoten. furchtet eucb aber vid intąr fưr 


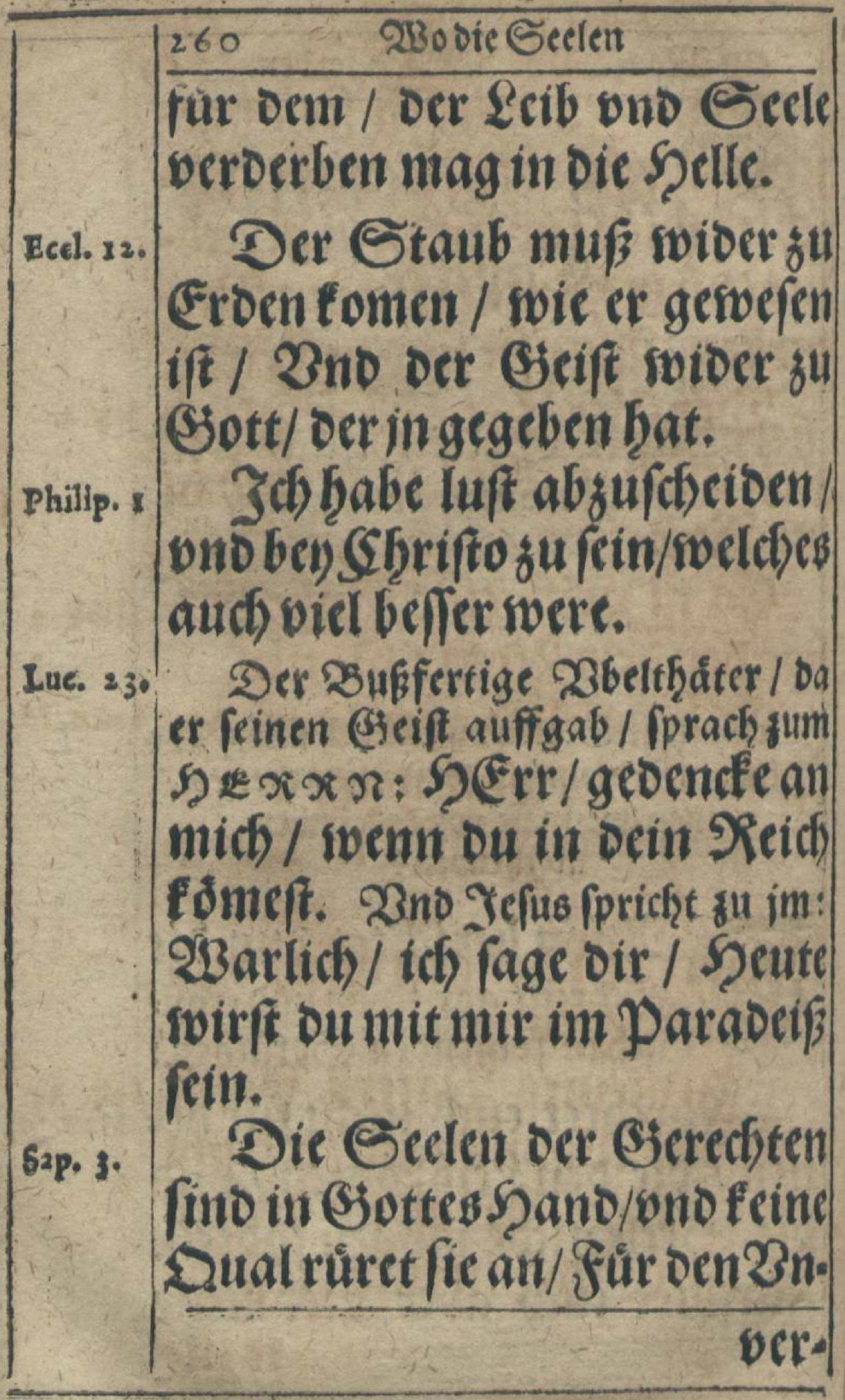




\section{Gin fomen. $\quad 261$}

verffendigen werben fie angem feben/als fturben/ie/vno iralb. (thied wit' fur ein Pein gereshinet / v ñ ir S Sinfart für ein $2 \mathrm{er}$ berben / 2 ber fie find in fricoe:

Der (Berecbte / ob er gleich sap.4. ou zeitlicf) ftirbet / if er boch in Der Puke.

$E_{B}$ ift noch eine Sube ver- Hiob 4. handen oem 20 lefe Gjottes.

Eb begab fiel aber / Daf ber Luez 16 2lrme frarb / ono waro getragen von oen Engeln in 2lbrabams Schof. Dino abermal: ?u aber wiro er getroffet / ono ou wirft gepeiniget.

Jin meines Baters Şaule roh. 14. fino viel 23 ofinungen / wenns inidit fo were/ fo wolt ich) su euch

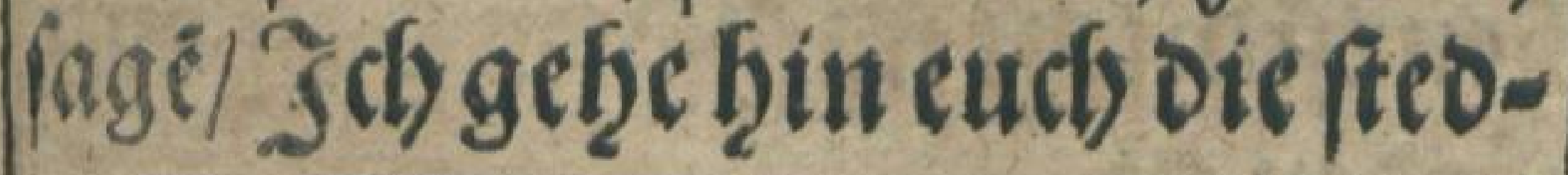

tefut: 


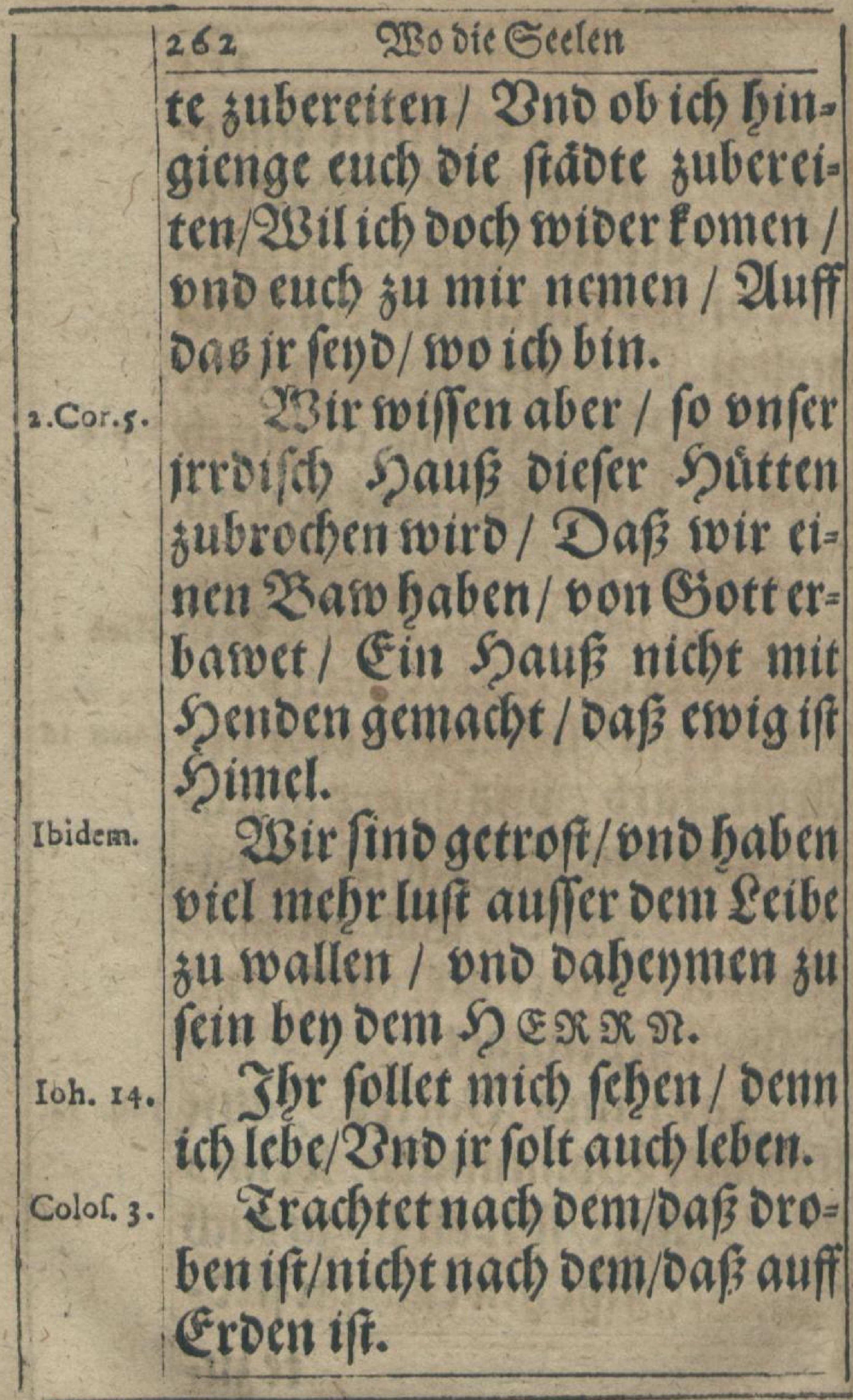




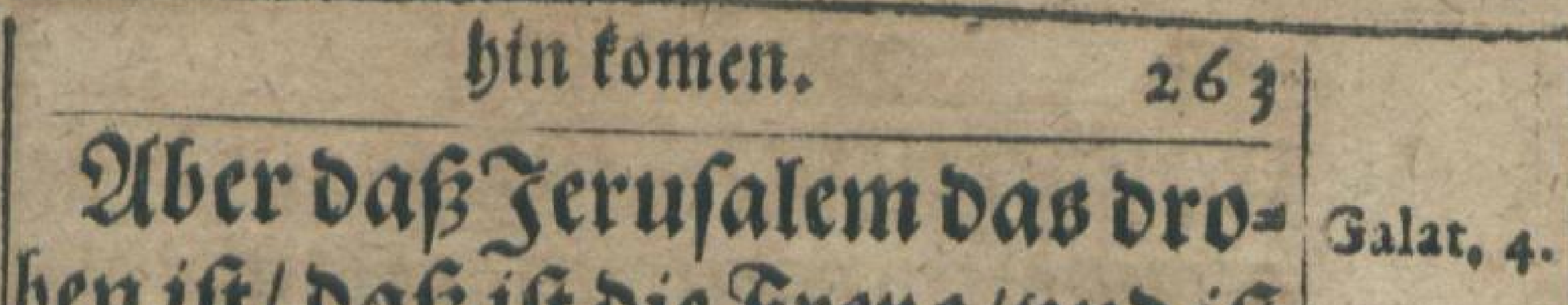
ben ift/ o af ift oic fretse/wond ift onfer aller syutter.

Dut thuft mir funo oen $28 \mathrm{eg}$ sum $\mathrm{Seben}$ / für dir ift frewo oie fülle / vno lieblich $\mathscr{Q B e}$ een $j u$ beciner Siectiten erviglict).

Giffe da / liebe Ecele / diefe wuns

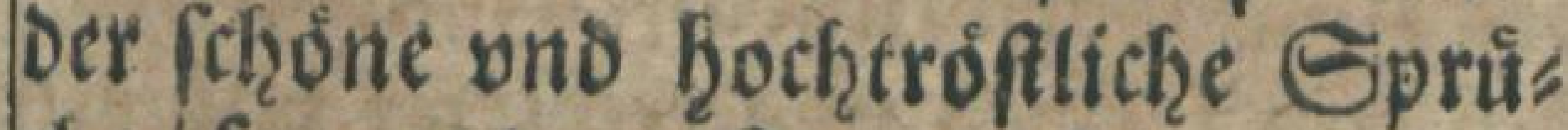
che/ fo att bem Diunde (3)ottes gan: gen find / bezeugen vins / Daf Die Ëes: len Der 9)enfechen gar ein ander bing find/als die \{ribe. Denn fie fino (S) ela

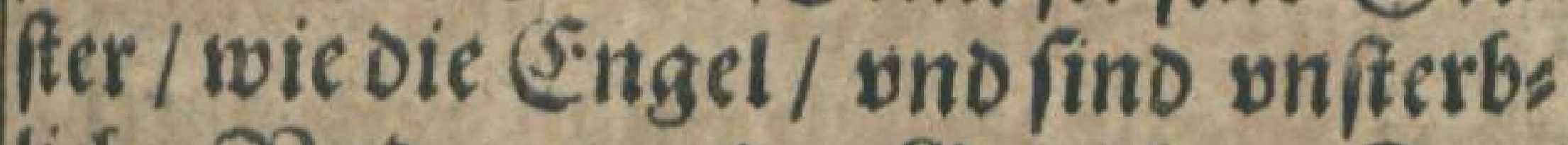
tith. Z3no wenn Der B̈erechtenten Sees lent vonden seiben abfereyoen / wers Den fie von den henligen Eingeln ges frolet vno getragen folt 3 ott / ond find Dafeimen bey oem ste $x x$ / bey Sfgrifo / vnd leben im paradeí / oros ben im Shimel / vno fefrweben in vas auffprechlichen SFren und Jrewben/ 2Werben tieblich getroffet / in Rlars

pifal. 16.

hreit 


\section{0 bic Secle:}

heit ono Sherrligfeit / Sino in Rube I in Sriede/in Sottes Sand / ohn alle Poth / ofne 21ngfi / Irawern vnd )Rangel. Denn wenn dic Ecelen von Den \{eiben ab/chenden/fo fino fie fren/ vno wirten ofine den seib mehre als suborn / Denn Der seib ift in oiefem seben Der Geelen fef̧r befchwerlich vin hinoerlich. W3eil fie aber nu fice auff Evoen in den ferblichen elenden (ei) ben Gott loben / antuffen / jm Dienen/ fich fein frewen und troften / und an alle feinen 23 ercten fich ergessen/2Jiel mehr weroen fie auffer ben Eeiben fols clze oing gank herrlich thun vno aufs richten / Gintemal fie ohne Ginde/ ohne Gefrwach heit fino / ono ourch" aus fein hinoerni liebe Gecle/Dás fan ja fechon vno trópt: lich fein / $2 \mathrm{Bem}$ wolte für Eterben grawen ?

Dagegen aber fomen bie Geelen oer 2 ingláubigen ftrad"s balot in Der Ieuffel gewalt / nno werben getragen an Den Drth oer Sual / 23ie an Deß

Rieichen 
bin fomen. $\quad 265$

Fecichen Diannes Seele zuperten ift I Luce is Zno find oa in 23 nruffe / in 23 fries oe/in 2ungft/in Roth/in 2lch und 2Bes he $/$ ofin allen Froft ono Enabe $/$ in Stanct vnd S infermi $/$ ond in ftetem Geulen vno Seenflapien + bi a an ben Tingften Iag/Dajr Jammer vno Elenot erfe recht grof werden / und nimmermefir auffigoren wiro.

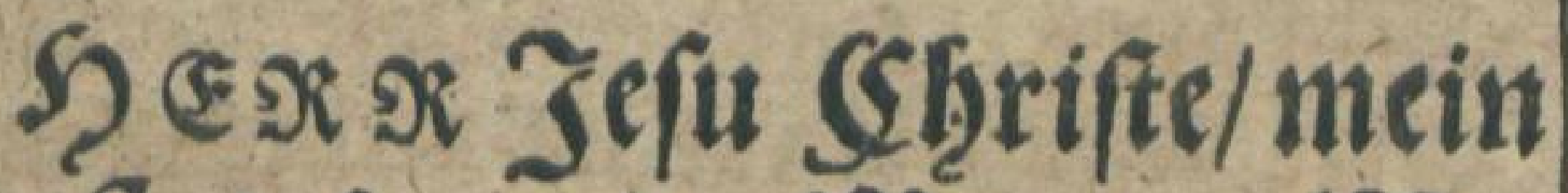
Troft ons meine 200nne/ DDie perlanget meine Eeelenad) bir/ Ja wie der Soirfeh fefreyet nach Pral. 42.

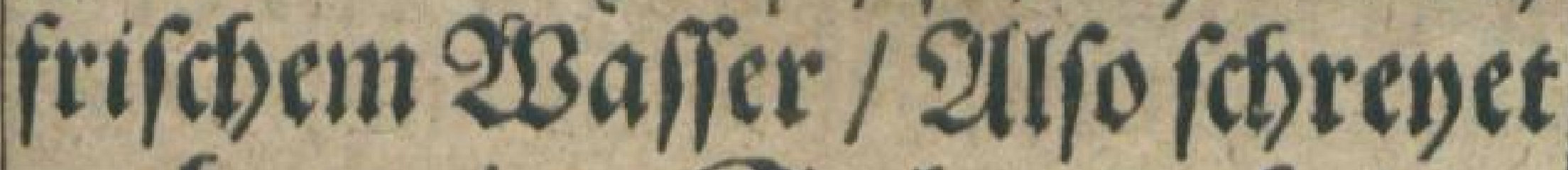
auch meine Seele nach Dir. Sneine Seele Durftet nach bir meinem lebenoigen SD $\mathrm{tt} / \mathrm{D}$ wenn foeroe ich oafinfomen / Dafich Dein 2 (ngeficht f(h)arwe? D wie fatt/wie pberorúffig babe id) Diefes muibefeligen Jammer, tbals / oarinnen ich bir meinem Bott arbeite / 3 um Dienft beiner

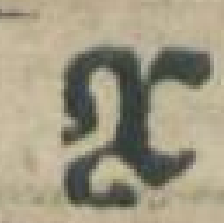

(Be) 


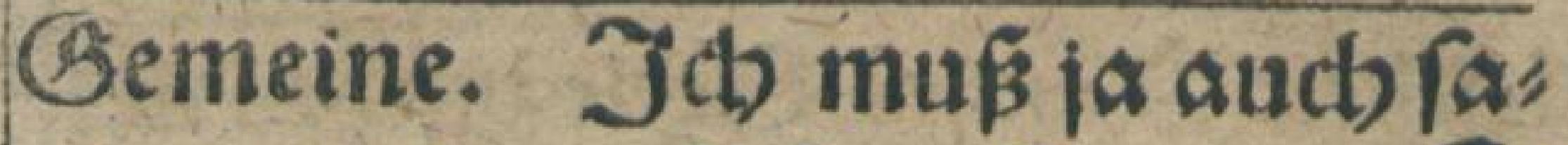
gen mit bem lieben paulo : $\mathfrak{E}_{\mathfrak{B}}$ philip.r. lieget mir beyoes bart an / Jeb begere abzufthenoen / ono bey meinemb ErrnSfrrifro \&u fein/ welebes biel beffer swere / 2lber es ift auth ?oth im ficifs le= ben/ ono Frucht fof affen/ Jed

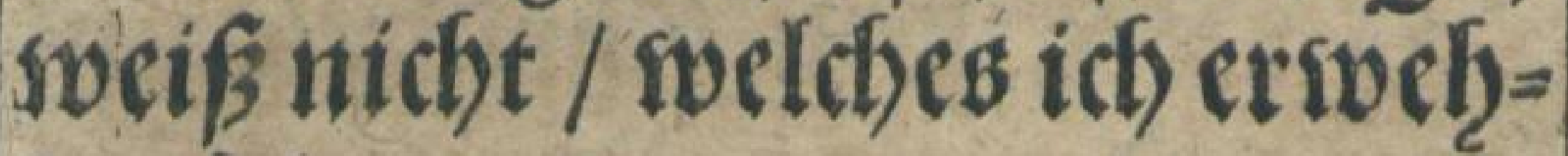
Ien fol. DDErr Jefia/ Du evoige Doeifbeit / Du vociffeft meine Zeit / LDeine argefteben in bei, nen'Densen/Spanne aus/mein DERrr/wenn su wilt/Wein'Ders tze rebnet fich 3ur Stube / Ja zur ewoigen lebenoigen Xube / 21us oiefer finfternip sum fiecht/ 2lus bem Traboren zur frewode/ 2us ber Dnrube sum friebel 2usser DD ib efeligteit 3urDDer: ligkeit / 2 us ber Echwacbeit $3 u$ r Dortéomenbeit. DERr Jefiu / Ioh. 2r. Din weiffeft alle sing/ Du weifs 
Feftaud / bas bich meine Beele bert 3 licb lieb bat / pno tråget ein rebnlidb verlangen / bein 2/nge: fidst 3 u fabaven / Sibe bocb al. lerliebefter Deylanb / Doie flief: fen bie Thránen ber fíebe aus meinen 2ugen / Dercke bed) / Dấ meine 2lugen für frewosen weinen/pno mein "Dert3 fừ fie: be tranct ift / feuffitset / ourfitet / verlanget / vis winofcbet bey oir sabeymen su fein/ pri auffoei ner $\Theta_{\text {dbo }} /$ in beinen 24rmen $3 \mathrm{a}$ ruben. Dein DDÉrr vno mein gott / woie wol wiro mir a a fein / freplich woiro meine $\theta_{e}$ ele aud mit groffen frewoen außffbrey: en/ono /agen : Shic if gut fein / Matt: :79. Sic ift gut /ein. DERr Jefia/ge: bencre an mich in beinem $2(e i d)$ । pns fprich 34 meiner Seelen: Speute folt ou mit mir in Lus. st.

Paraocisfrin/ 2Imen.

$$
\text { X } i j \text { D }
$$




\begin{tabular}{|c|c|}
\hline \multirow{4}{*}{ II. } & 2330 die Geclett \\
\hline & 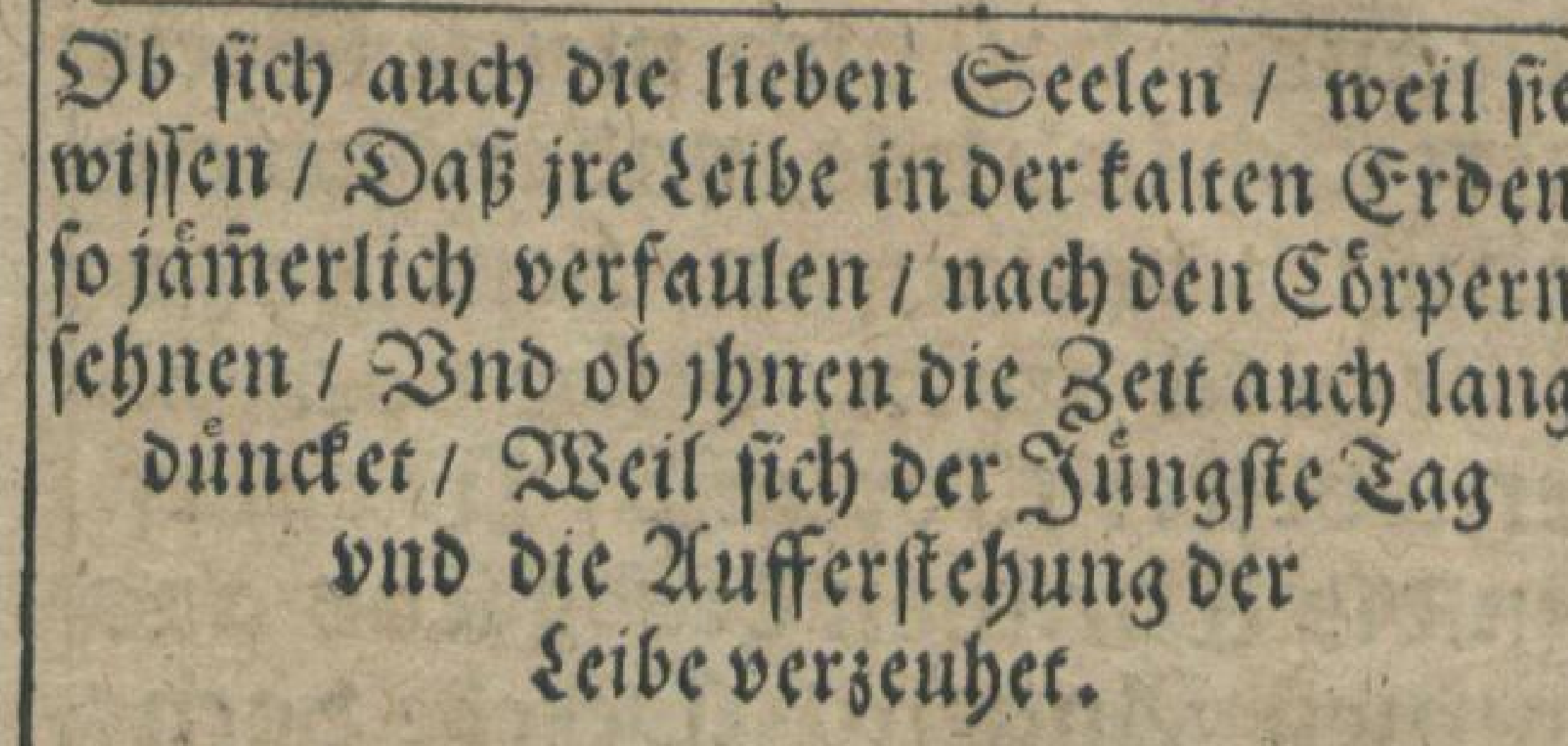 \\
\hline & 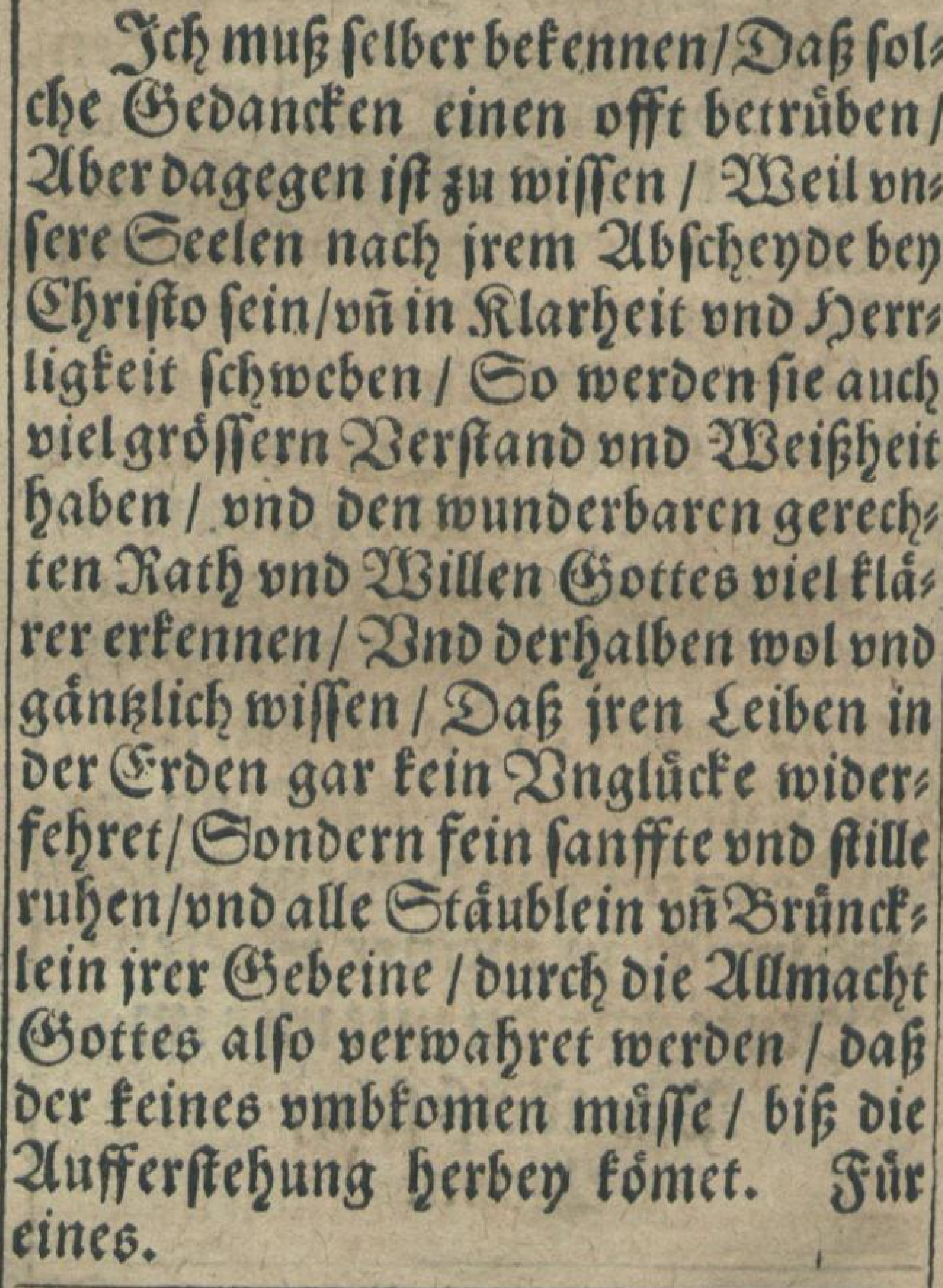 \\
\hline & \\
\hline
\end{tabular}




\section{bin tomen.}

Sum andern / fino die lieben feli: gen Eeelen auch an einem folchen Srthe / in folehen Grewden / Da fein vngeftumes oder trawriges verlangen noch fefgnen feinfan. Denn ob fie wol an ire Seibe of $n$ onterla 3 gedenden! vno vmb oen హ゙erichte anfialten / 23ie in Der Sffenbarung Jofzannis of feften ift / Go ift Doch Cap. 6. folch ir gebentten ond anḩalten inen gar nicht betrublich/Sondern gefioret fu jrer 2 Bonne/Darinnen fie feh weben/ vno fum sobe vno preps Bs Bttes/Dem fie bie Sifre geben/ ono feinen 2rBillen waltenlaffen.

Tfun boch folches die Rinter Bjots tes in diefem elendẽ seben / Da fie nochs in jeren seiben wallen / bno pefinen fieh nach Der enotlichen Errlofunge / vno ferffien nach oer Zufunfft SGriftil Zno wee fich auch Darnach nicht feh" net / oer hat gewiflich feinen Sg Serren Tefum nicht lieb / Golten Denn die lieben Beclen bey EGrifto nicht wins rchen/D af endich alles fündiclie we"

$\mathfrak{X}$ iij pent




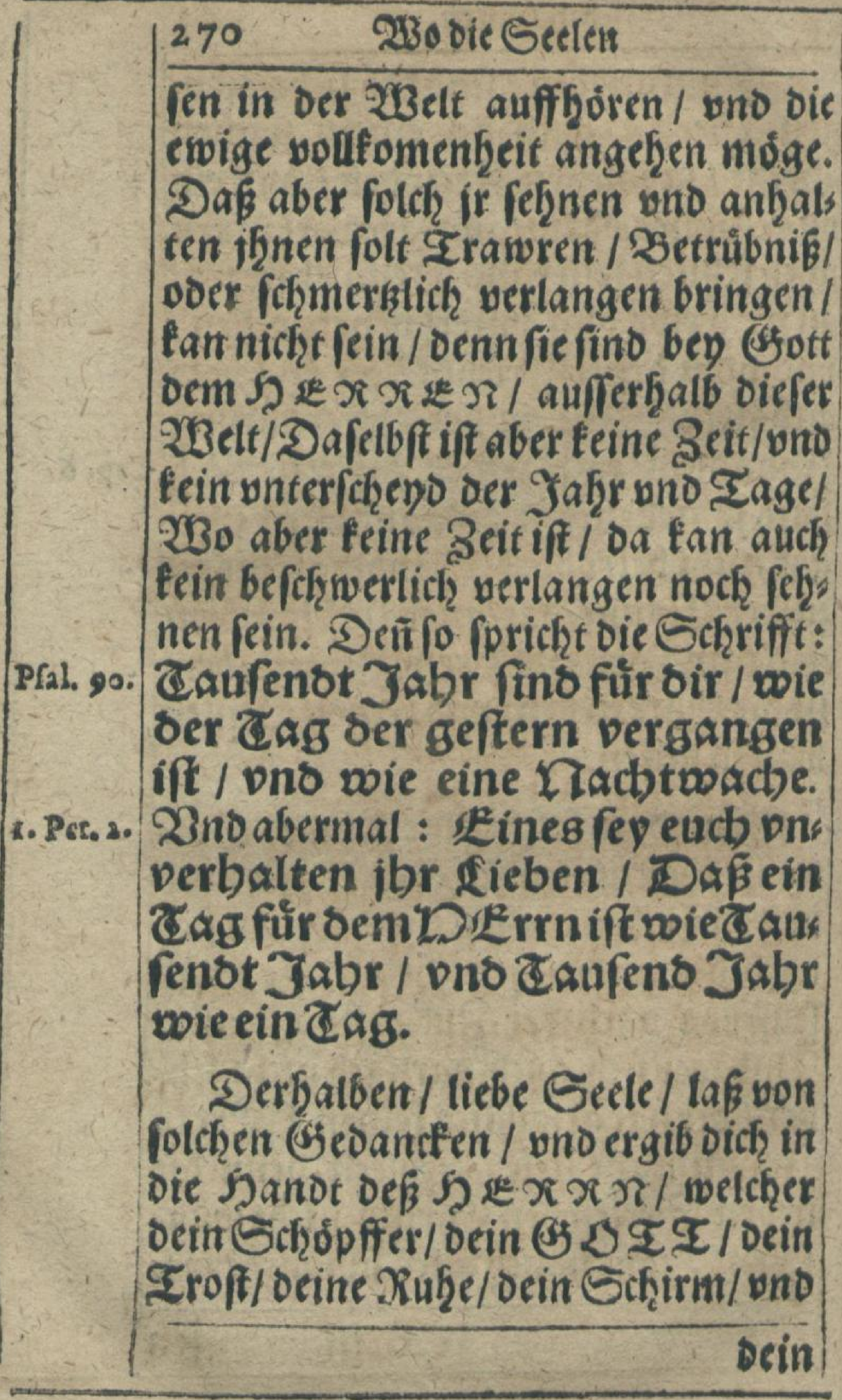




\section{bin fontelt.}

bein volle Bnige ift/bende frie in oies $\mathrm{fem} / \mathrm{v}$ no nach o iefem seben / sno fols gends in alle swigfeit.

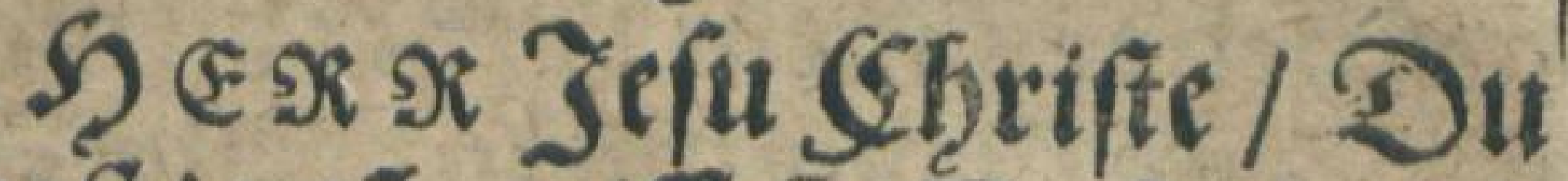
fibeft ia pri weiffeft/ Dafítb beis 2. Tim.4 ne Jerfcbeinung Don Dertzen lieb babe / Dns bitte aus allen Ârafften/Du wolleft burd bei: ne Jutunfft / ठaß̧ Bsottlo/e fund: licbe weltwefen suftòren / 2 in bem reuffel onns allen onfern feinben bein gered) teg (Berichte vben / vno oas evoige frewoens weren laffen angeben / Da ou woirft fein alles in allem. Ja / ${ }^{\text {.Corin. }}$ mein Dokr Jefu / angften ficb oodb alle anbere Creaturen / ons febnenfich/ wie $\Theta$. Paulus fagt/ Rom. s.

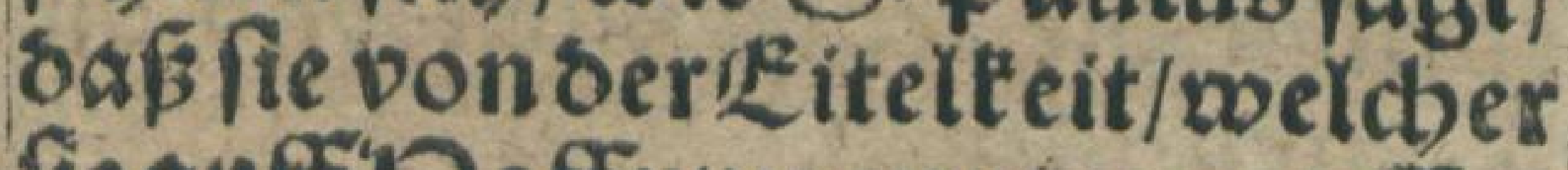
fie auff' Doffinung onterworffen finb / mògen gefreyet werben / Bolten benn wir גinber Gottes pns nicht felsnen nad onfer ens. licben ERlöfung: Ifts bein bey: liger Doille / Dokrr Jefu / ons

$$
\text { ¿ iin bafts }
$$


bafts alfo befdloffen in beinem Xatb/Dafích seine freposenreis d)e Zutunfft erleben fol/ /Ey fo gefbebe es mein 'D Kerr ขñ mein Kerlö/er/D wie woil ich beine po: faune mit fo groffen frewoen anboัzen/pri oir mit pollen fprùn: gen entgegẽ romen inser \&ufft. Wiltu midb aber zudoz ent chlaf: fenlaffen/ons meine Seele 3 u bir ins freworenreidbe Parabeif ne: men / Oibe / bie bin ich / Dno wil gerne mit / J b werbe aber audb borte nicht auffbören seinen framen mit frewosnreicben Geuffizen zudermabnen pnosu Apocal. fagen / Rzple ono tom balbe / ia vlt. Lom mein D Derr Jefu / tom 3 u ricbten bie lebensigen uns bie cooten/2Imen.

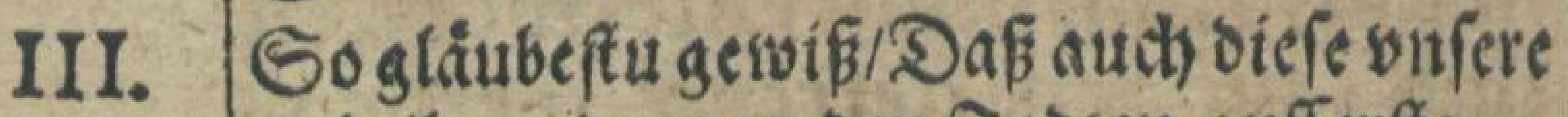
seibe sorber bon ben Eoden aufferfte" ben/but fantpt den Seelen ervig lebent werbent?

Tafrenlich. Derhalben befenne ich in meinen SHlaubens 2drtidteln) 
vno fpreche: Idb gléube eine auff" erftebung oés fleífbes / pno ein 'Evoiges Reben. Denn wenn vnfer

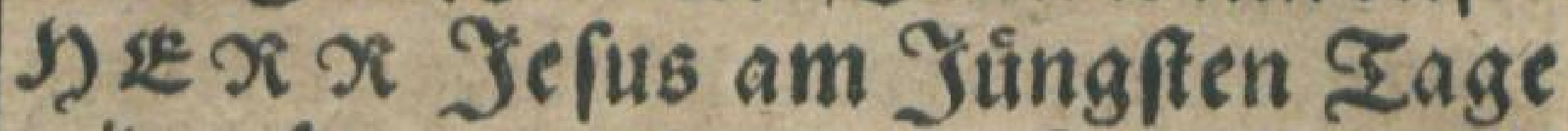
wider fomen wird zum Bserichte/ / fo weroen fuir jm verfamlet werben alle

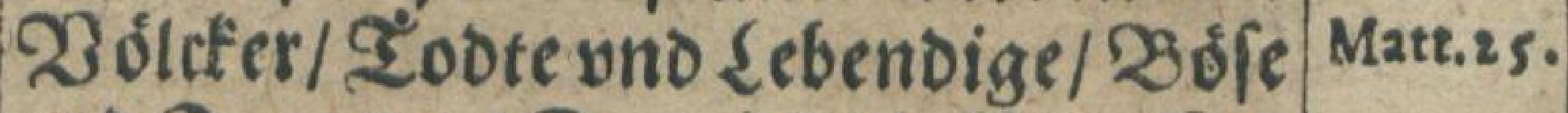
vno fromme / Da wird die Eroe ifre Todten / ond das Dieer feine Tooten herfitr geben / Da werben in einem 2lugenblitf die Seclen wider in jre verfforbene seibe fomen / vno leben:

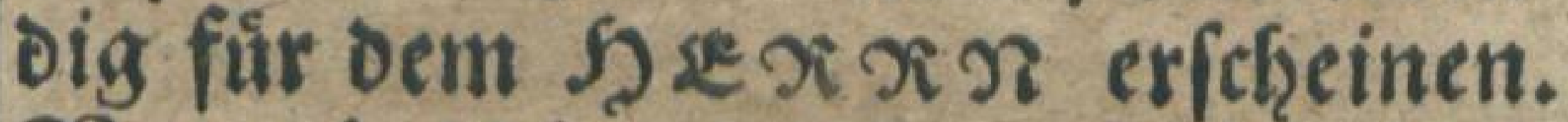
23no wir / bie wirleben yno vberblei, ben / weroen sugleich mit benfelben

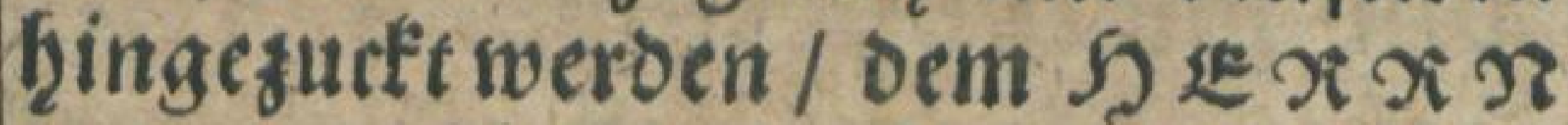
entgegen in ber Sufft : Da wirb fie

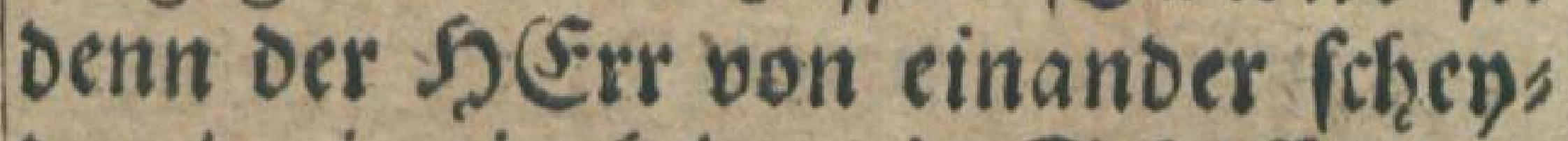
Den / wie ein Sirte die Gichaffe bon Matt.25. ben Borten felendet / 23no wiro bie Bódte zu feiner sintéen / dic Ethaffe aber fu feiner Wecthen fetlen / Dat: auff wiro Er oas 23rtfenl fellen / vnd fagen zu Denen/ glt feiner Rechten: A.omet ber ir Befegneten meis 


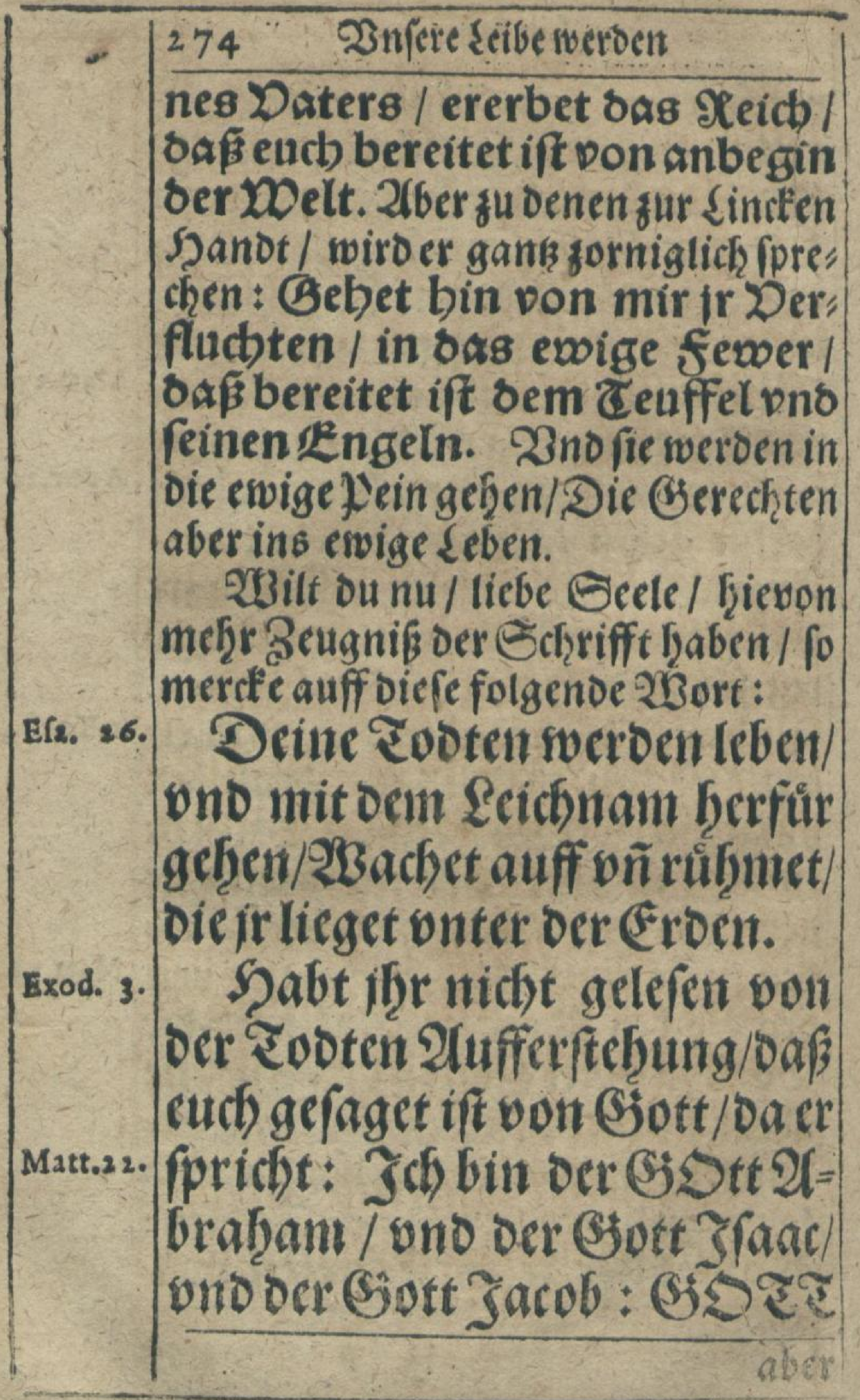




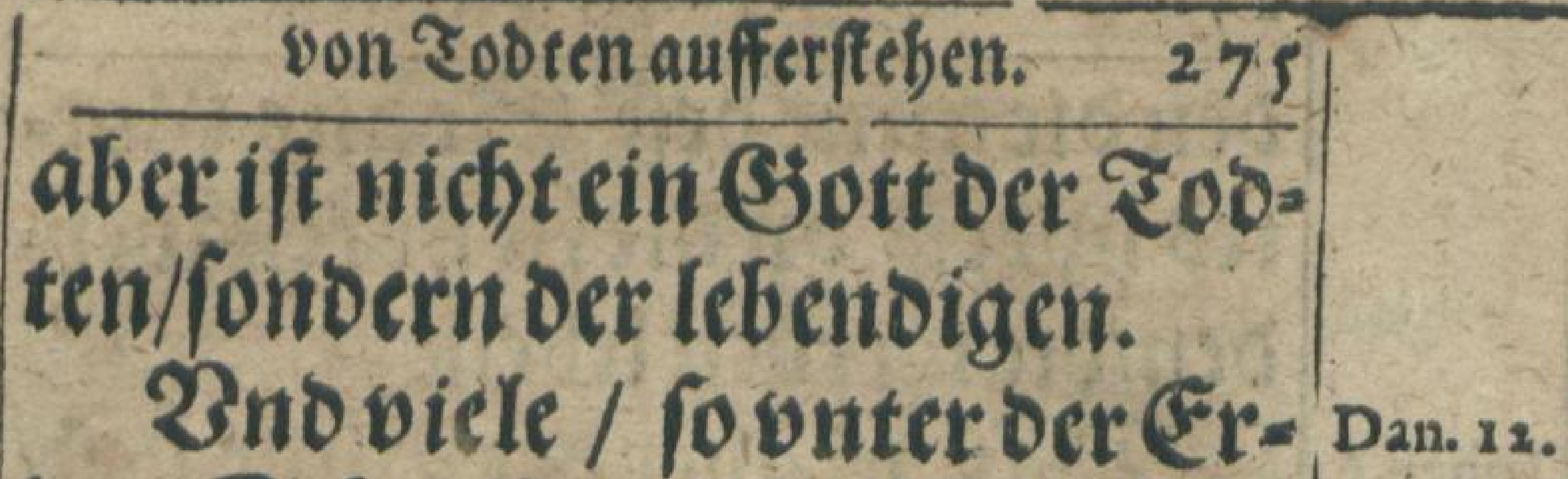
ben Sethlaffen liegen/werden auffitio acben / Etliche sum Eswigen Leben / Etliche zu ewoiger Schmadiond Schanto.

Jich roeif das mein Erlö fer Hiob 19.

lebet/buto er swiro mich) bernad aus ber Erben aufferweden / ono werbe bernach mit oiefer: meiner Spaut bmbgeben werDen/phto werde in meinemgistei. fobe GSOtt feben / Denfelben serbe ich mir fefien / ono meine

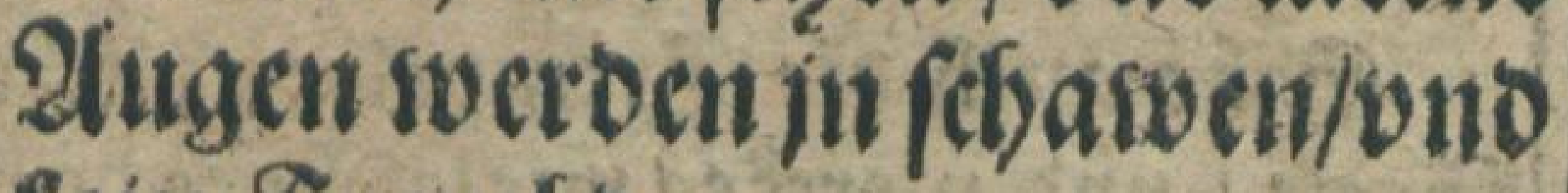
fein fremboer.

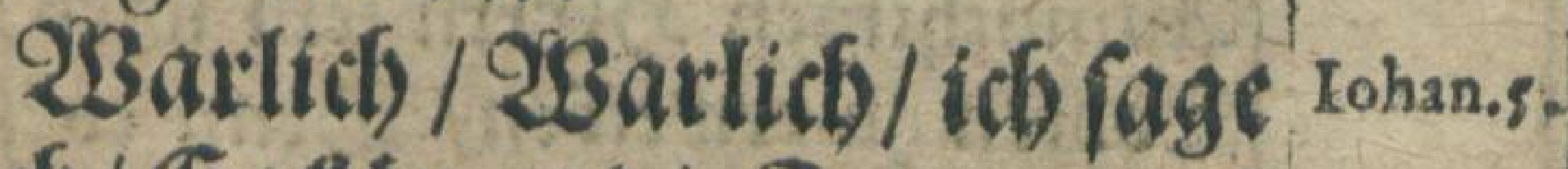
eudb/Es fdinet oie 3eit/ono ift fhoniekt/ Daf oie Toote swet.

oent 


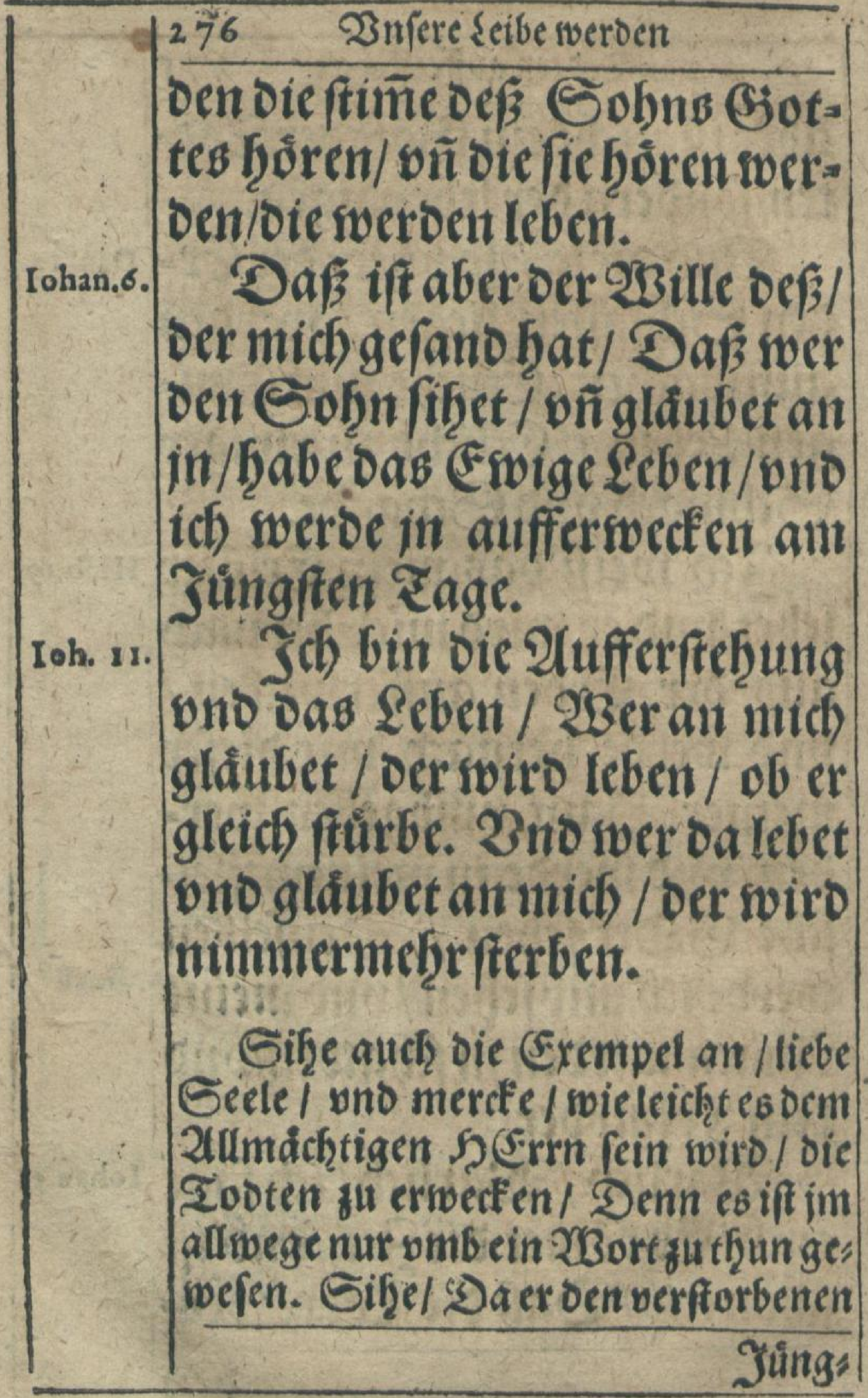




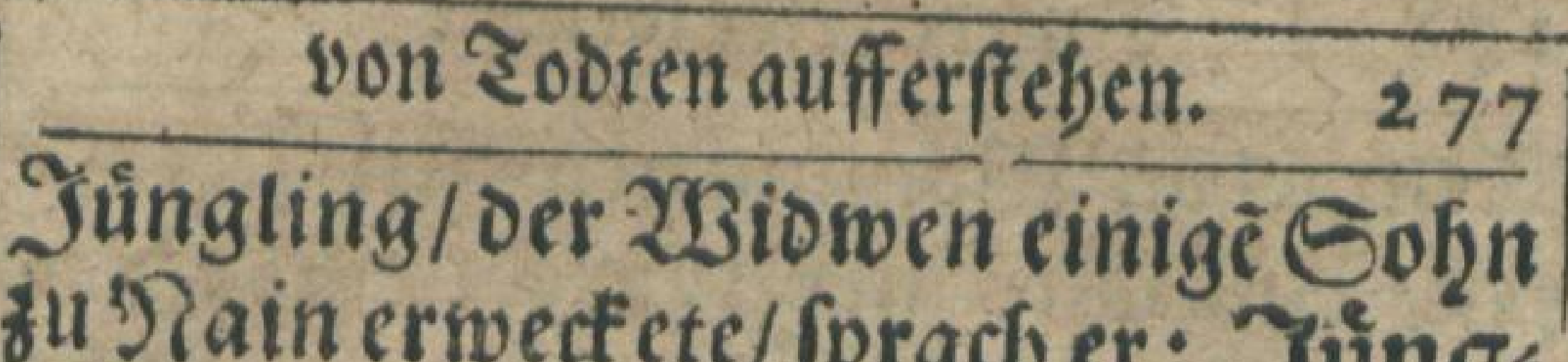
ริ) 'T ain erwedfete/ /prach er: Jüngs Luex 7. ling icb fage bir/ftebe auff. 23 no als balo richtet fich, oer Iodte auff $/$ vin warotebendig. 2llfo erwedtete Er auch mit einem YBorte / Dás, verforbene Ióchterlein/ DefE Eberfiten von Der Echule zu Capernaum / vno fprach: AD \&̊golein ich fage bir/ftebe auff. Marc. s. DeFgleichen gefchach auch mit Dem lieben \{agaro zh Bethania / Der feḩon Ioh. $x$. vier Tage im Esrabe gelegen war: fazare / fom berauk / fprach ber STErr. Zno als bald gieng er leben: Digherfür aus feinem Érabe.

Ja/meine Ecele / für Dienfchen Zugen vno Siraffften fcheinet folches vnmuiglich / 2(ber bey vnferm STerrm vno ES Stt iff fein ding vnmuiglich. Luex r. Stleich wic cine פ) untter wol weis / in welches Rámmerlein oder Betf̨lein fie ir Rindein hat fchlaffen geleget / Da fómet fie dés morgens wider/ wes dfet vno hebet es auff : 2llfo weis auch Der Sgerr / wo unfere Seibe / Die feine Iempel 


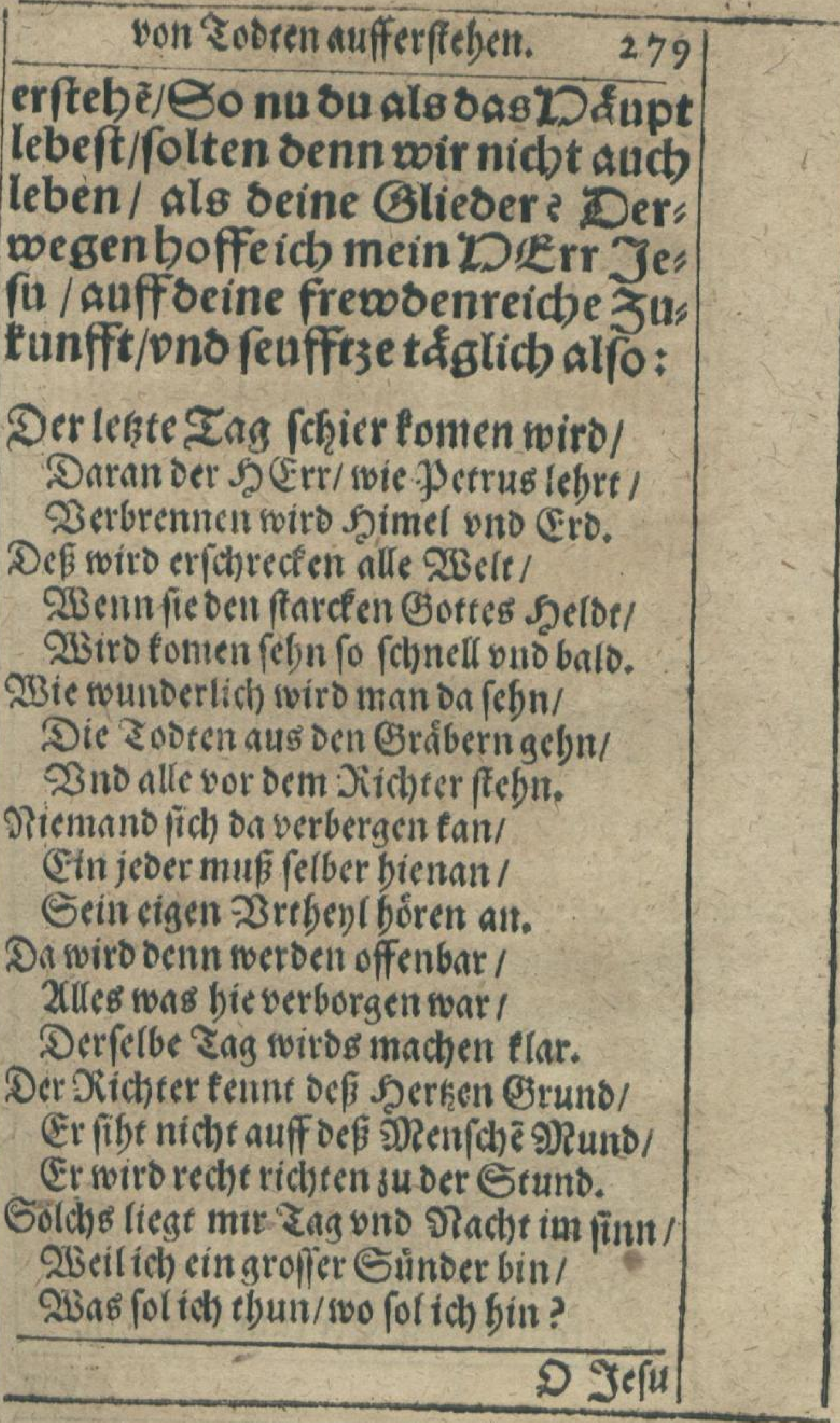


280 Bnfere Ecibe werben

D Sefu trewer Seyland mein /

Du wirft felber ber Dichter fein/

Serweís mir oa die zrewe dein.

Bebenct an beine Gitite grof 3 !

Die mich von Ginden machet lof 1

2(ch) S马 Err von Dir mich uicht verftop.

Dein rodt/Dein 2Angft wno forwere \$sein/ Die bu für mich trugeft allecin!

Saßs an mir nicht verloren feit.

Jch bit und fchrey/D Gott mein Ş (Etr) Shilff Das ich mich) zu dir beter/

Ebe benn ber fechnelle Foot fontpt ber.

Dogleid) mein Sinbe bat fein 3icl/ Soift Doch Deiner Snadent viel/ Darumb ich nicbt verjagen wil.

Da D)Ratia jr Stino beflagt!

Zno der 5 chaicher rieff wnversagt I

Sanfu feillem bein B̈nab ver agt.

Durch folche beine groffe Bitt!

Die allzeit sberfchroenglich thut I

Be efüt mich für Der Setlen E̋tut.

Scifff oas ich ftef but Diechten bein/ Onter bein fieben $S$ chaiffelein/

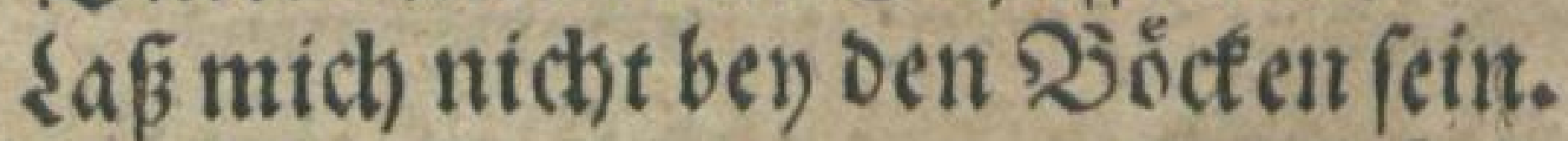

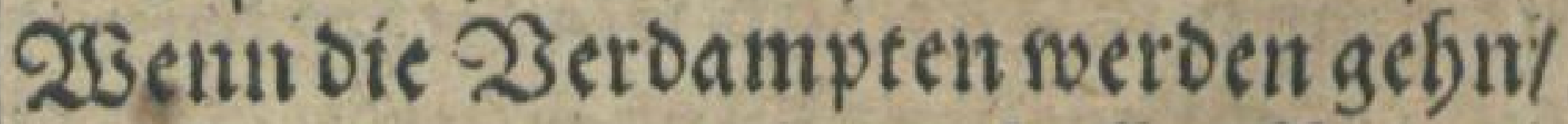

Z3on bir sur erwigen Şellen \$sein/ So fübr mich Ş Err bum \{eben eitr. 


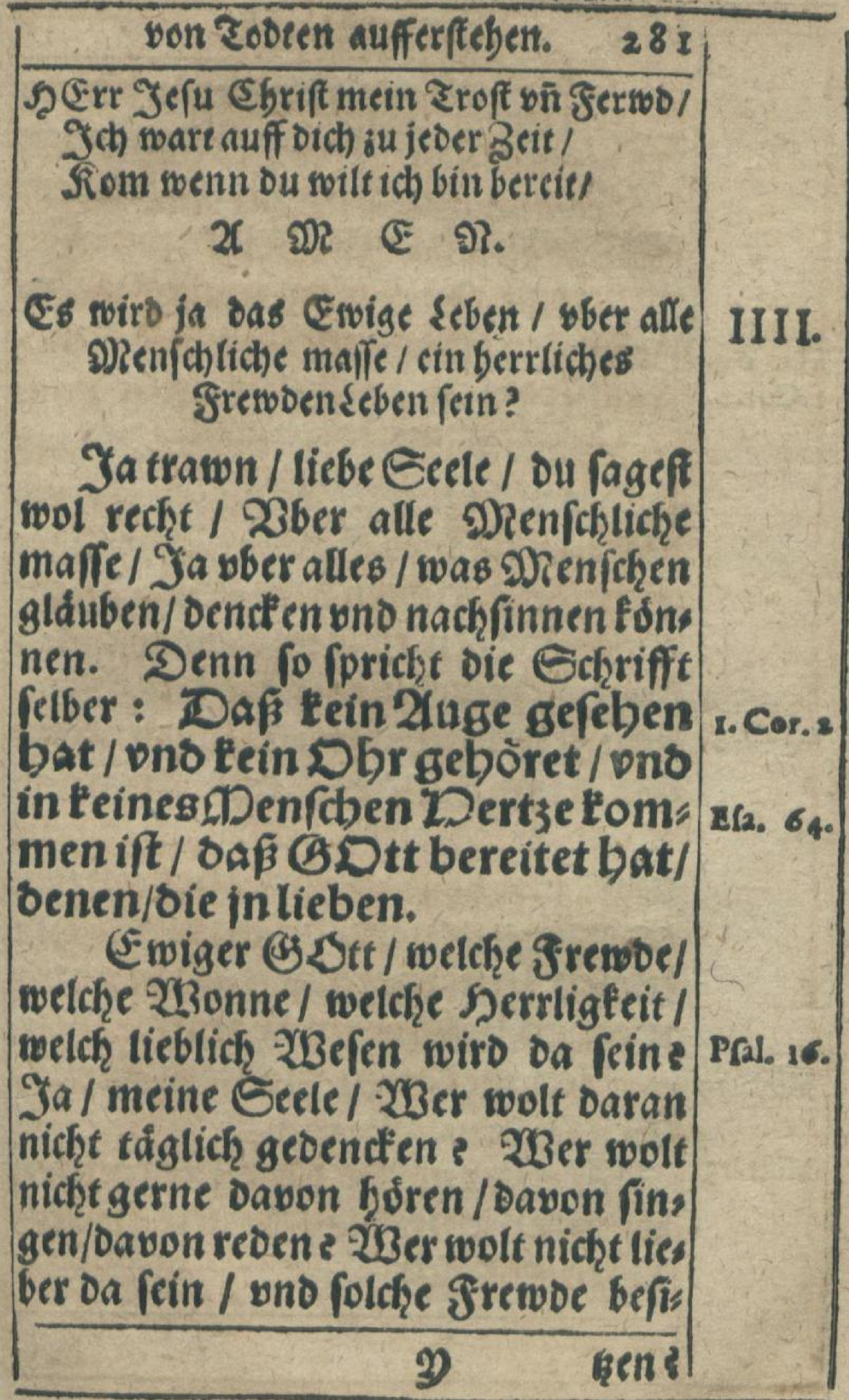




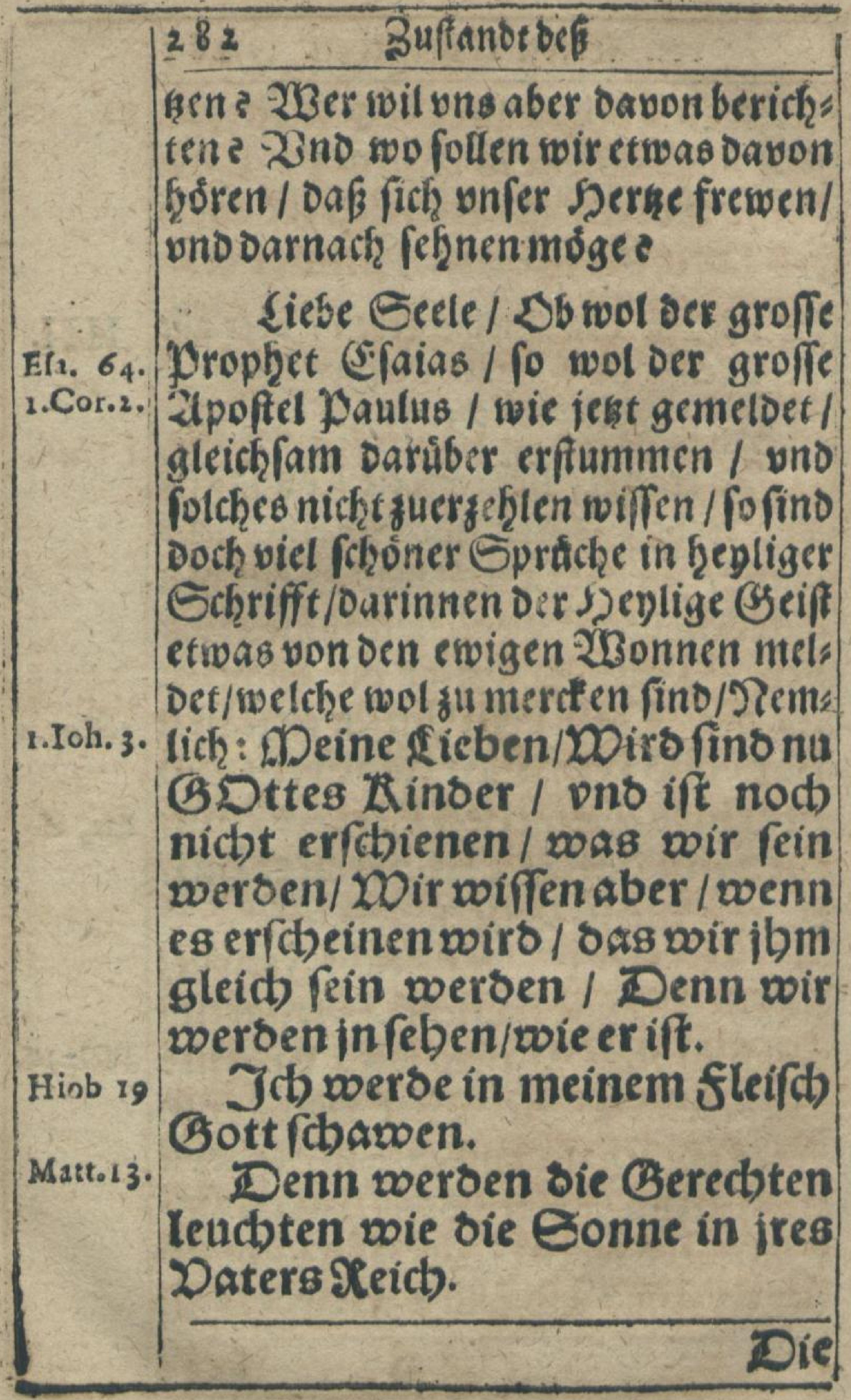




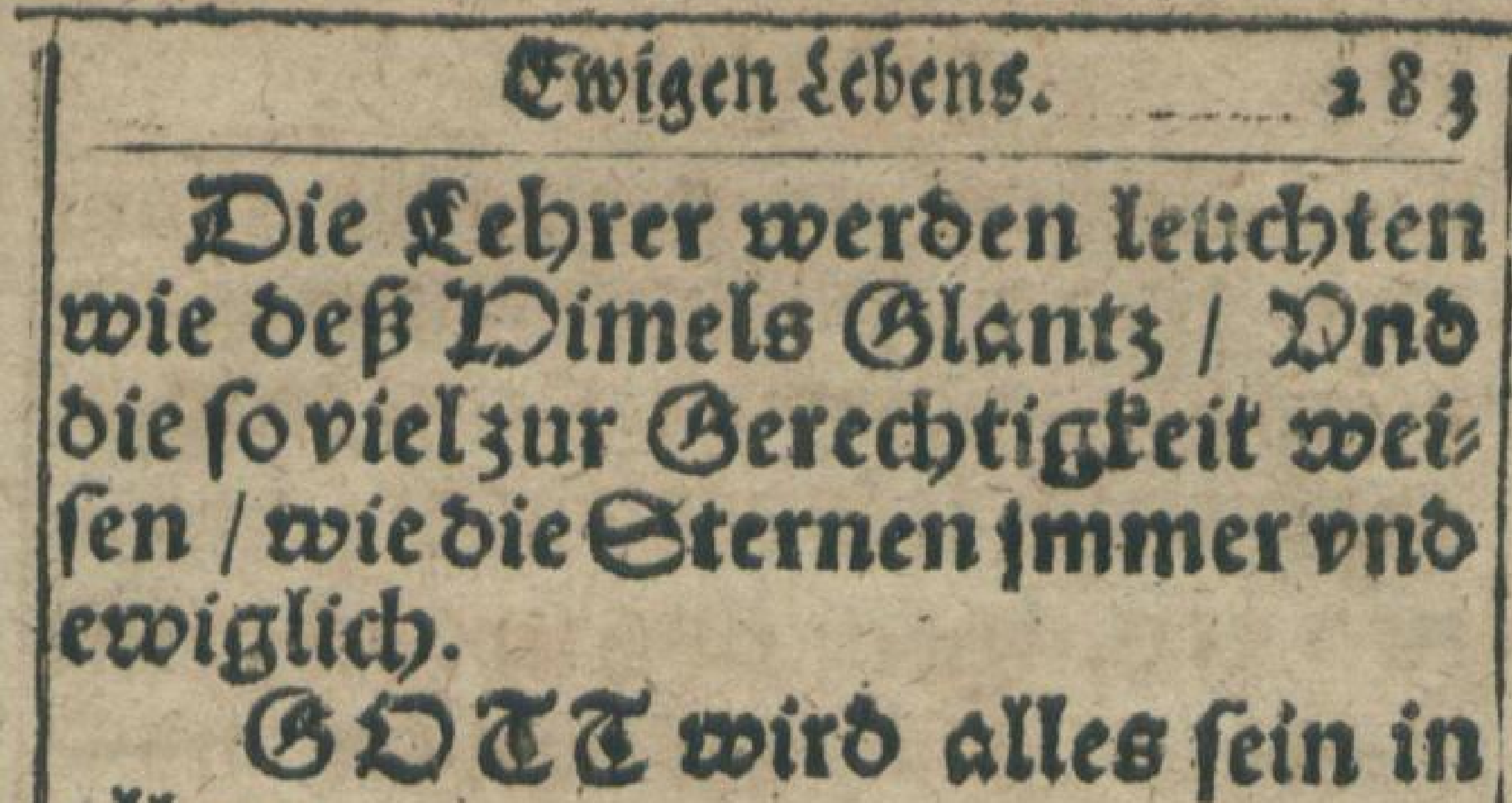
allem. Wir werben bey sem WRE: 1. Ther.4 renfein allezeit.

Der DER wirto Dnfernnichtis Philip.; gen ¿eib verklérē/ baf̧ er cbnlid soerbe feinem verlidirten feibe.

In oer 2 lufferftebung werben fie weeber freven/nod) ficb fireven lafien/fonbern fie fins gleich wote bie Rngel Gottes im 'Dimel.

Dno ich Zobannes-fabe oie beylige Staot / Dapi newe Jeru: falem/ Don Bott aus bem 'Dimel berab fabren / subereit / als eine gefdomuctte Braut irem DDan ne / pns böret eine groffe Etinte Donbem Etuel/sie fpradb: Eibe oa / Rine Dritte Gottes beyoen Den/chen/Dno er wirs bey inen

Matt.82.

.Coris Is. 


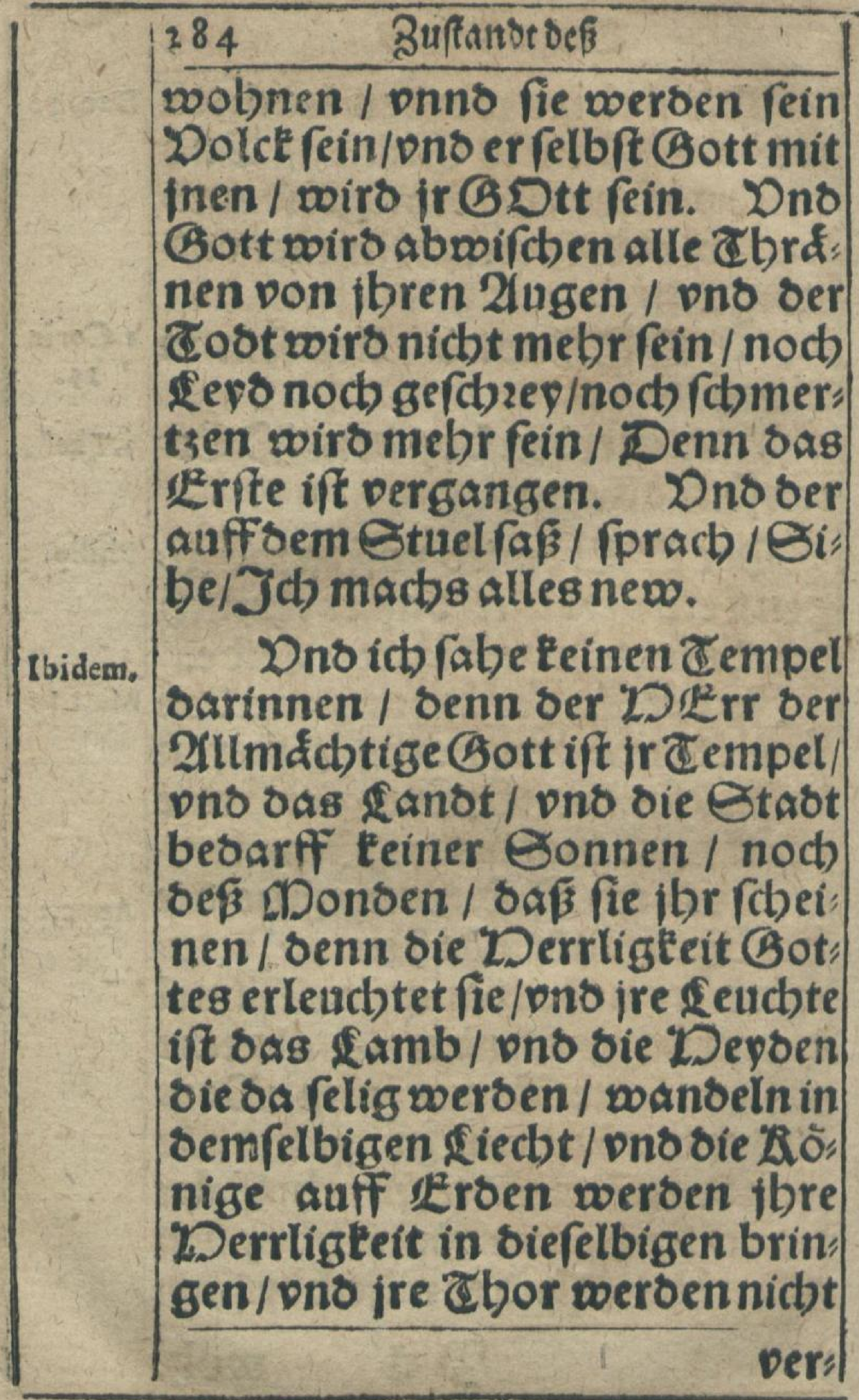


Derfatoffen bef 3 Tages/benn ba wiro teine hacht fein.

Der Stuel GDttes ons befi Apoc.22

Cambs wirs barinnen fein / vns feine Anechte werben ibm bie: nen / pno reben fein 2 ingefidbte/ pno fein name wirs an ibren Stirnen fein / ons wirs Peine Wacbt ba fein / vnno nicbt bes ourffen einer feucbten/ober bef fiechts ber Sonnen/benn Gott oer DERr wirs fie erleucbten/ ons fie werben regieren von $\mathbb{Z}^{\mathbb{2}}$, wigkeit 3 uLwigleit.

Diefes/liebe Seele/find alles furse 2 Bort / aber Doch voller vinaufiprechs licher liebligfeit. Dent wenn gleich die Eclinifft grolfe lange Dreoigten das von thete/ Yft dock unfer elendes Sers B: fufighach/ vno unfer Derfandot gu geringe / ond fans nicht faffen. Ders halben thut ons oer Syenlige (S) eift in polchen fursen Gprüchlein nur ein fleines genfterlein auff/ leflet uns cin wenighienein blicfen / 2uff bas wir

(3) ii wiffen!




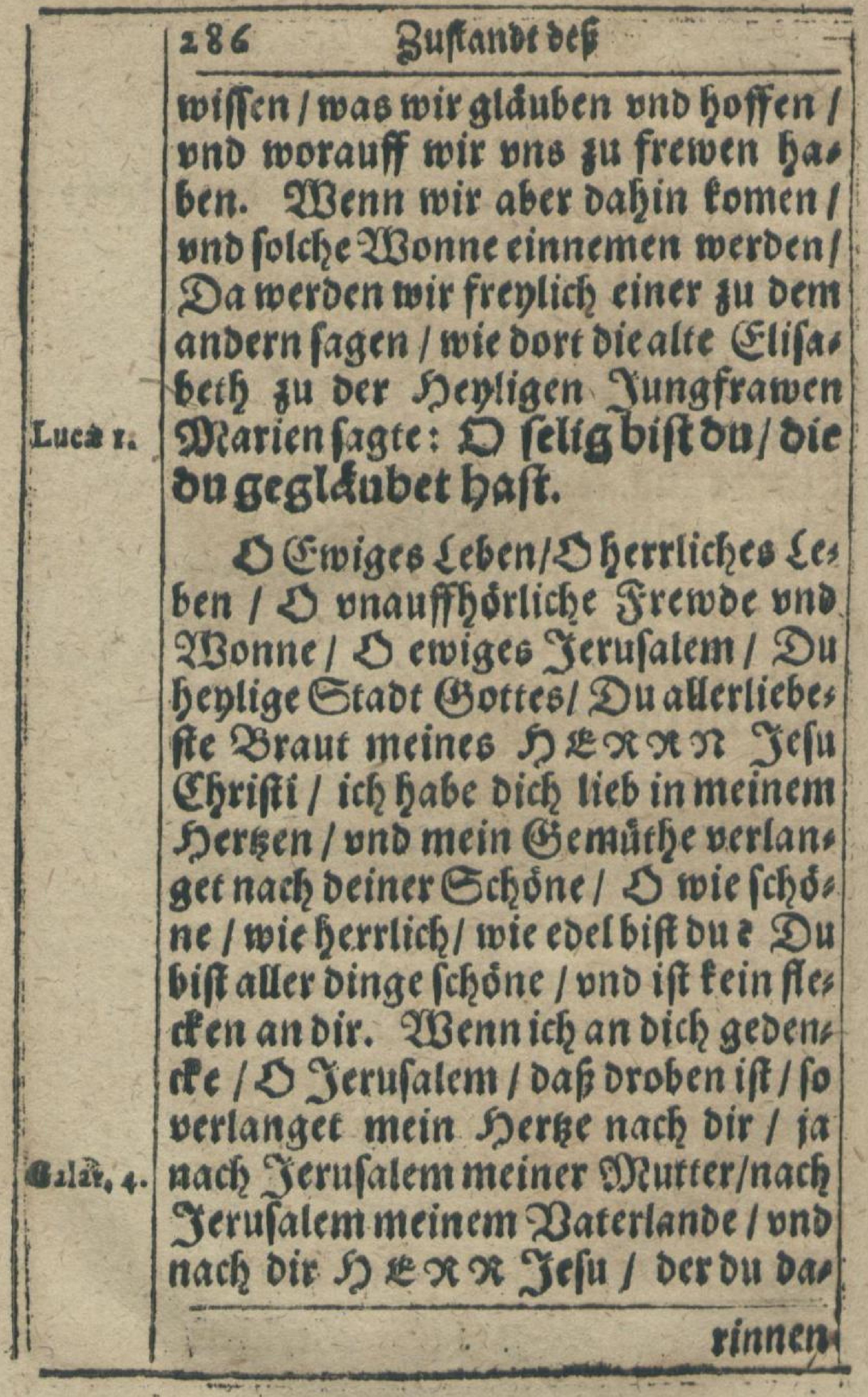


Esoigen \{ebens.

rinnen bift bas Sdupt / Daf siecht / Der S) Serr/Der ewige Sirte.

$\vdots$ heyliges Seben/S feliges \{eben/ DakBott bereitet hat / Denen / Die jn lieben / 5 ou lebendiges seben / Du ftilles Seben / Du friedliches seoen / barinnen fein Ioot / feine Trawrigs feit/feine Etinde / feine Schanersen/ feine Angft / Fcine Giebrechen / fein Echredten / feine Derdnoerung fein wiro. Ein seben voll aller Bier vno Serrligfeit/Da ferin 2 Bioerfacher ift/ Da feine findicke stifte find / Da vollfomene siebe ift/yno feine Furcht ift / Da cin ewiger Iag if / ond cin Beift in allen/Da man Bsott fcharwet won 2Ingeficht fu 2ingeficht / uno cin jedes Sheris mit ber Epeife def sebens vberftiffig gefettiget wirv. Deich ges liiftet zu feçareen beine \&larficit/ vno mein herke bat grof vertangennach Deiner $230 n n e /$ Je melir ich an bich gebenctelie frdinter ich werbe fuir Sies be / die ich gu dir trage.

5 wol dir/meine Seele/ wenn ou 


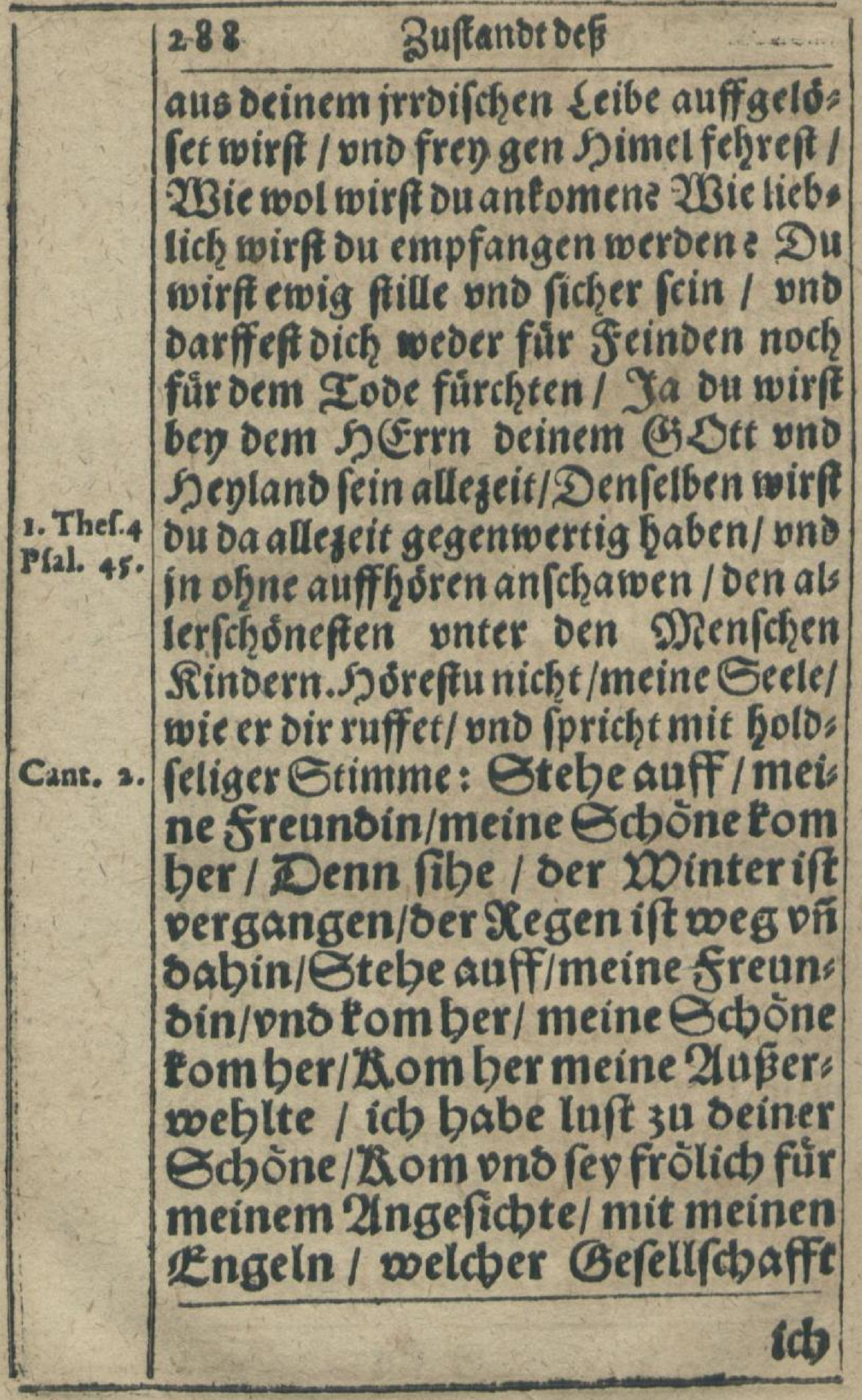




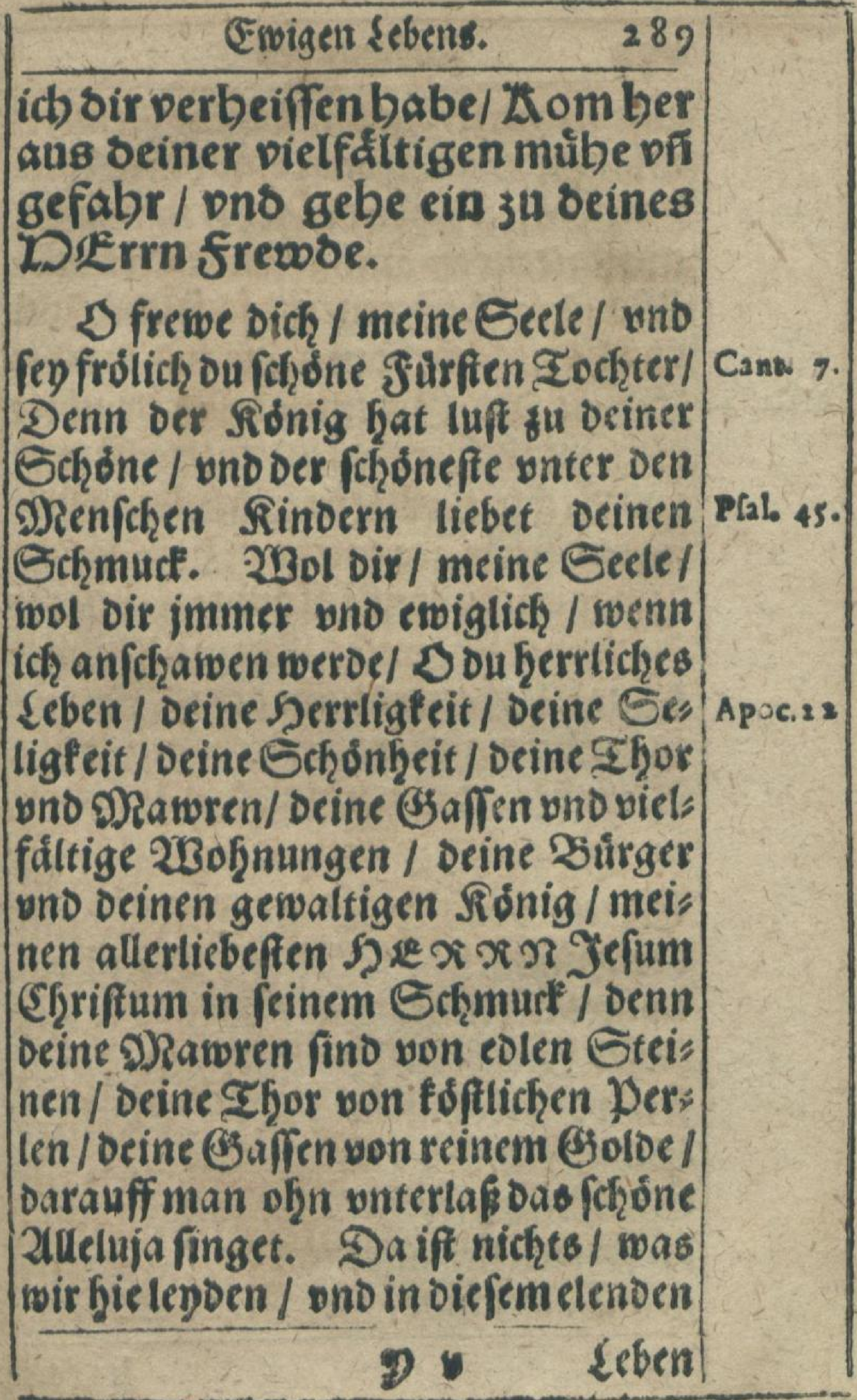




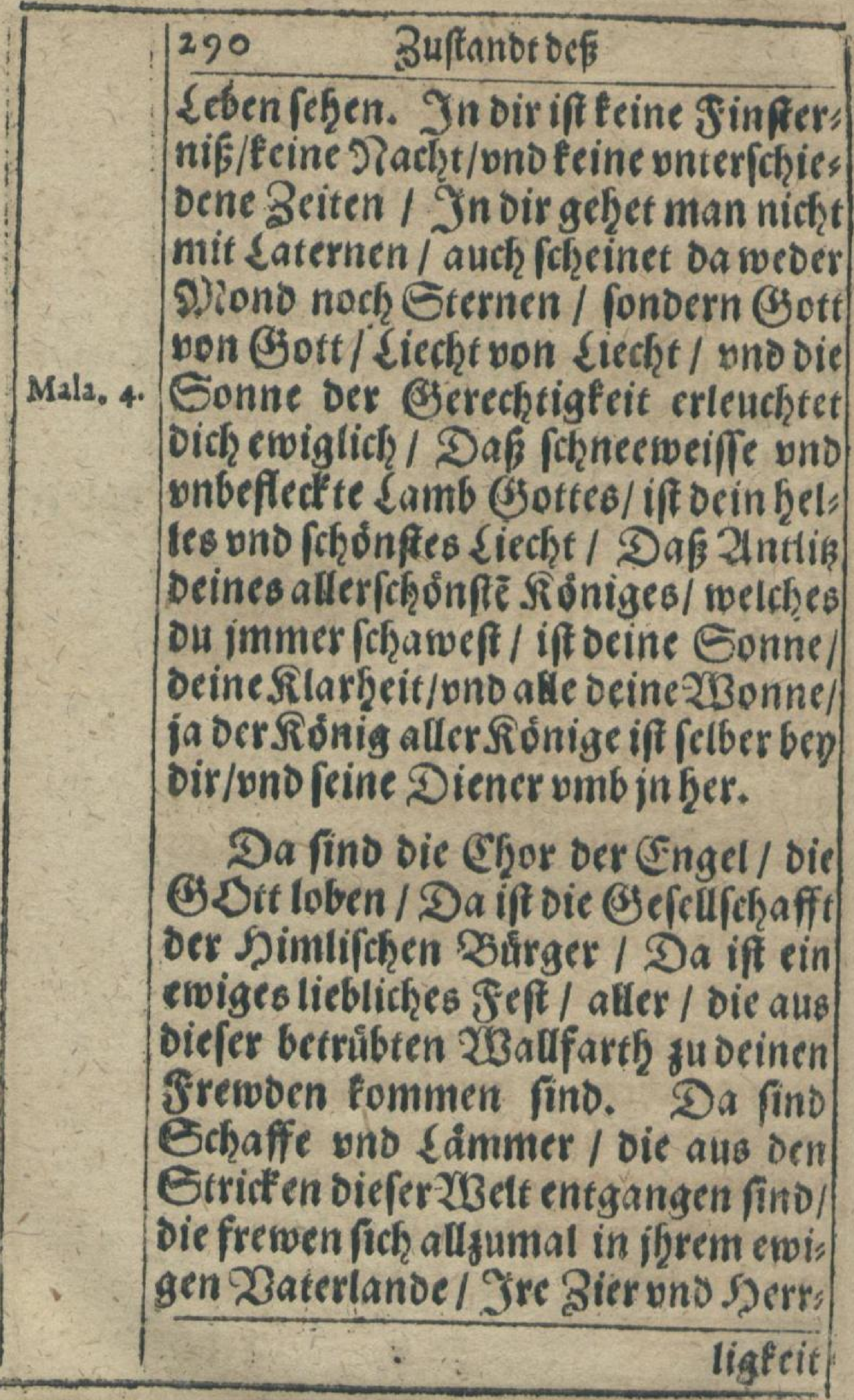


ligfeit if swar vngleich / fie leben aber in gleichen frewoen. Da berrfchet die rechte volfommene siebe / Denn 33ott iff Da alles in allen / welchen fic 1.Corin.

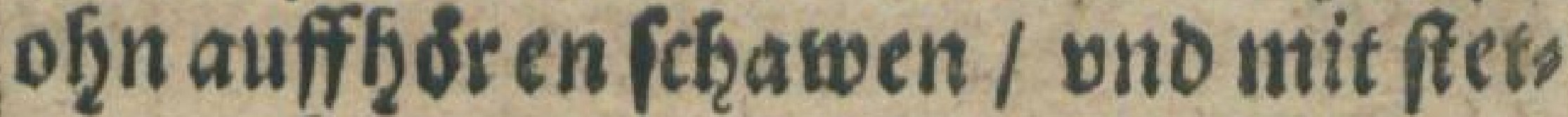
tem anferzawen bons Serken lieben ont loben / Soben vno lieben / vno alle ir thun ift Csott loben ofin ende / ohn ons terlaks/uno ofgn alle mithe.

$\checkmark$ wolmir in Sfroigfeit / wenn ich

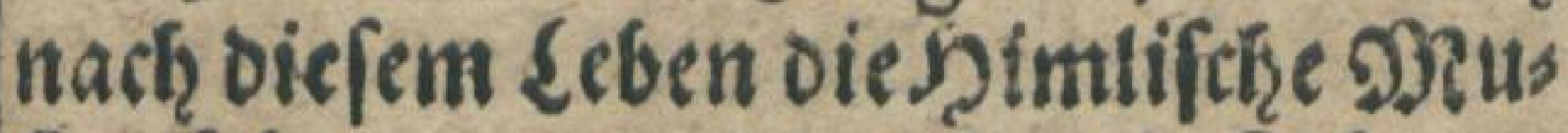
fica hoiren werde / welihe oie Buirger im Simlifchen 23aterland/fampt oen fiegligen Engeln hialten / fu sobe vno

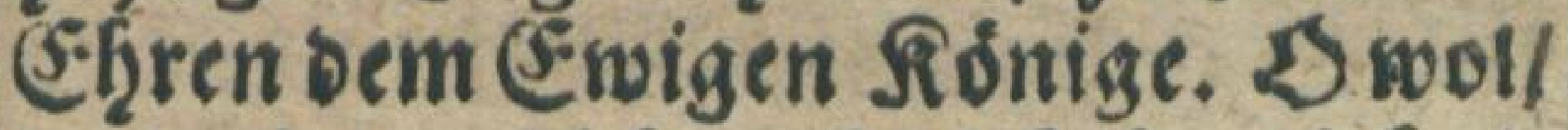
wol mir/ weñ ich auch diefelbe mit bats ten/ono neben meinem Sonige/neben meinem Gott bno SSErrn ftehen/vno in anf cha awen weroe in feiner serrligs feit/2Bie er mir felber verffeiffen ḩat /

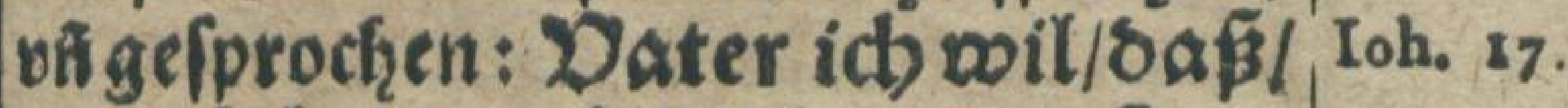
no ich bin/audb bie bey mir fein / die su mir gegeben baft / saffie meine Derrligleit feben $/$ oie id bey bir batte/ebe bie DDelt war. 


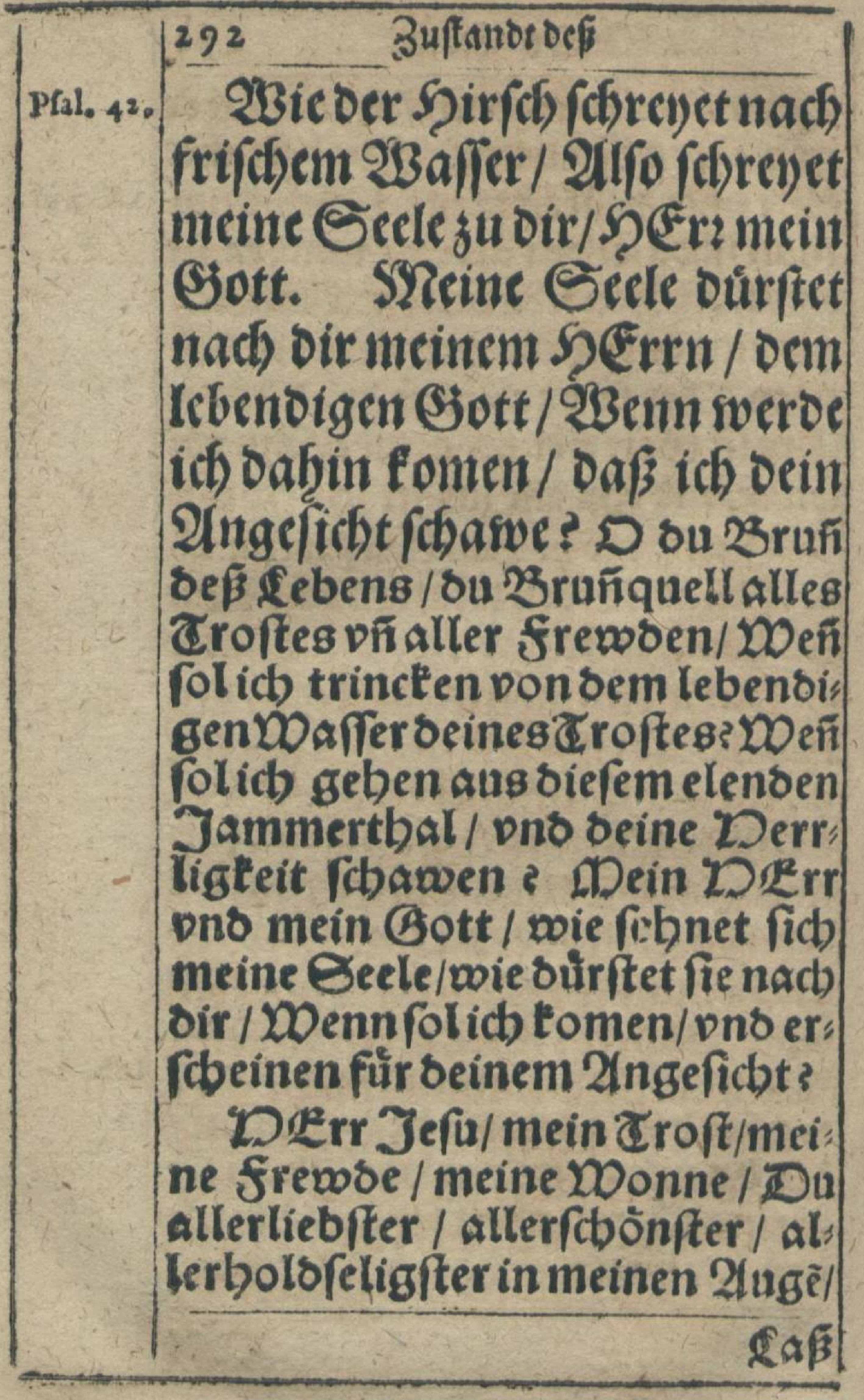




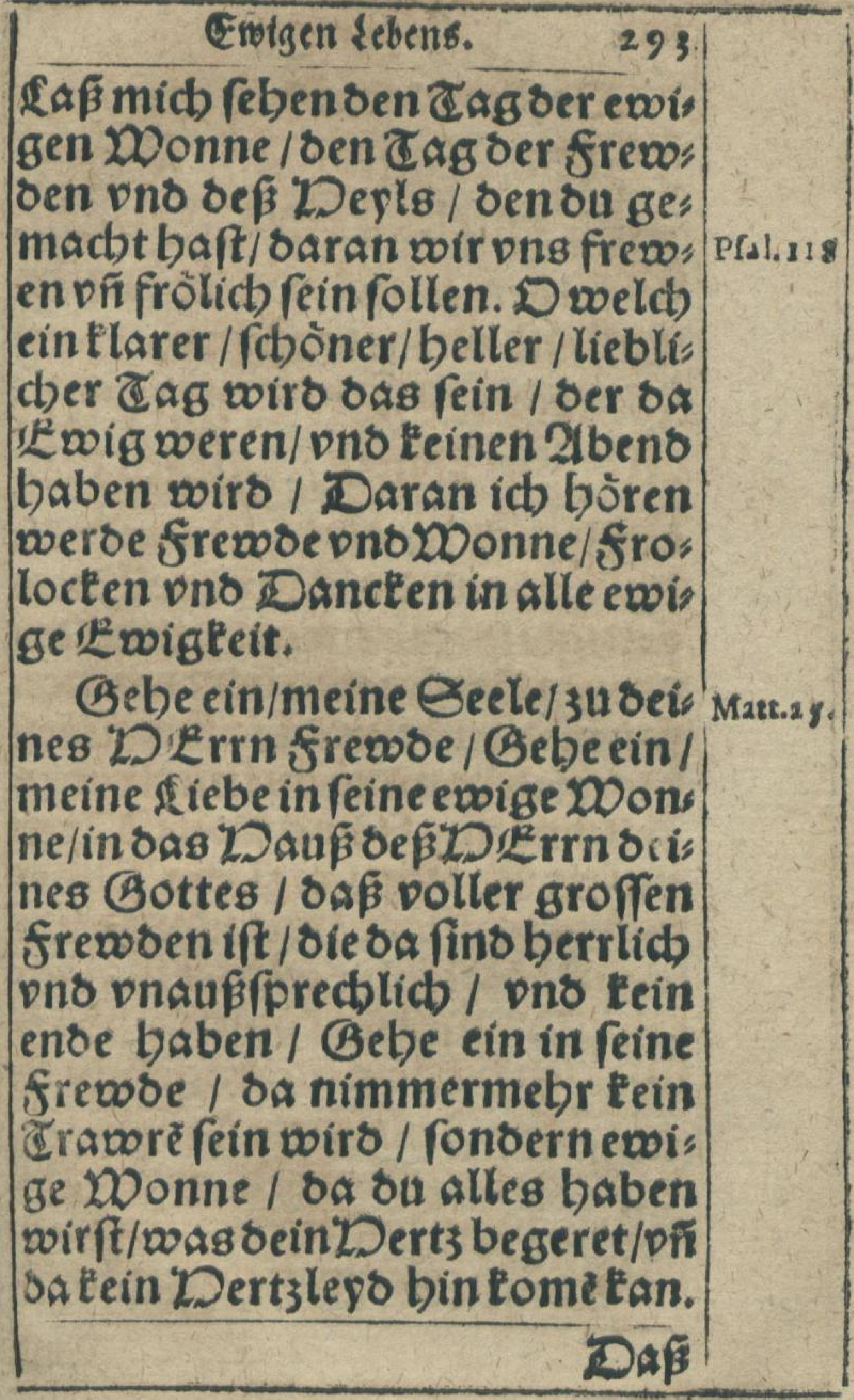




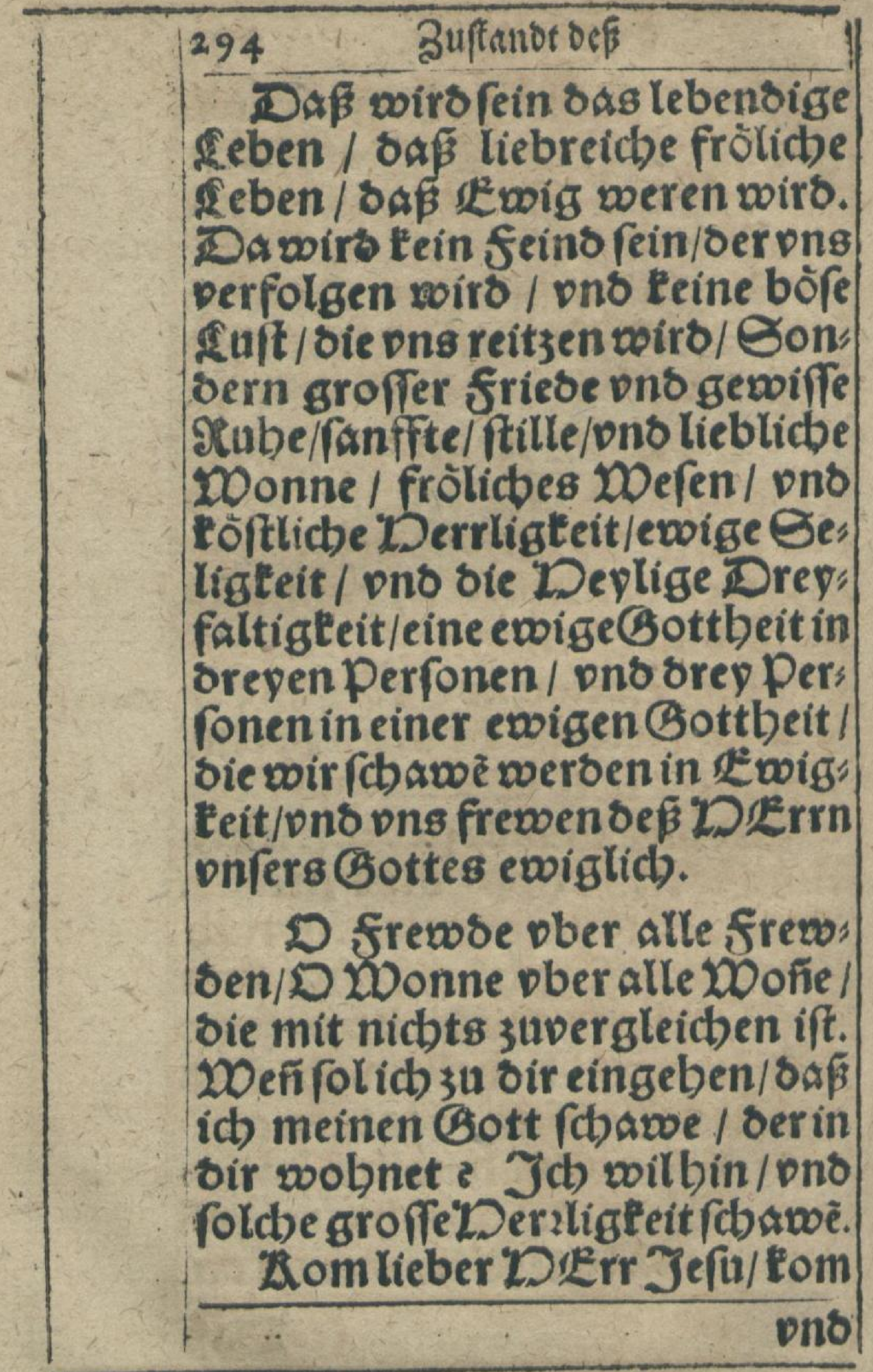


Eroigen 2ebens.

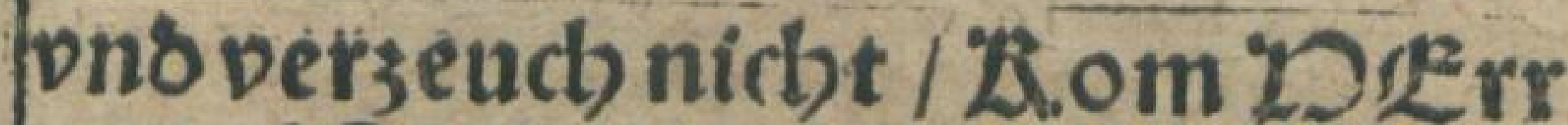
mein Deyland / A om 'DRerr on Troft aller 'Deyoen / A

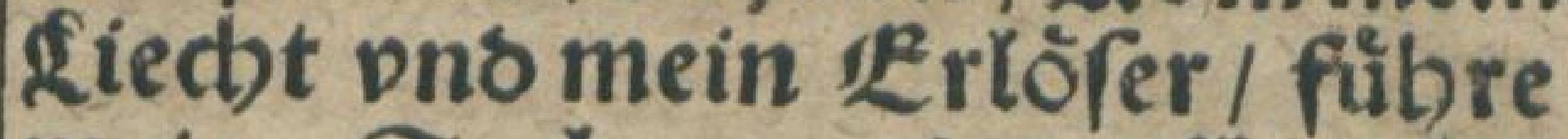
meine Beele aus bem Aercter

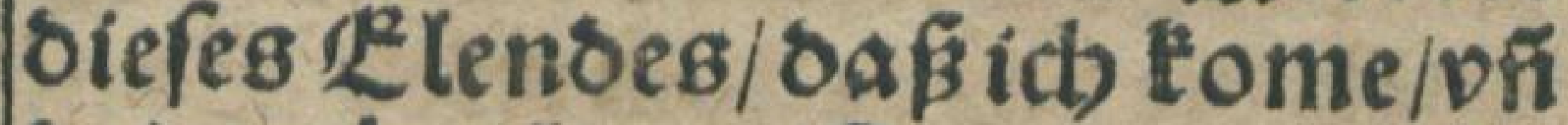
seinen beyligen famen presfe.

$D$ ewiges $X_{e}$ cich/D onver, ganglicbes Dimelreich/in bir ift oas ewige fiecht/ons ber friebe GDttes ber boober ift oennalle Philip.4. Sinn vno Dernunfft/ In sir ru: ben bie Eeelen oer D Deyligen/ Enoige frewobe ift pber ibrem

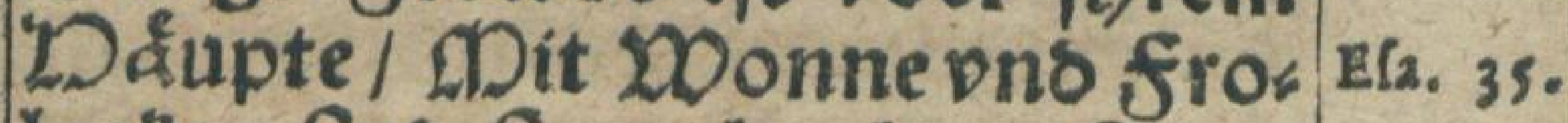
locken fins fie pmbgeben / Zber Angft ons 位lenot ift ferne von inen. DDRr?mein Gott $/$ wie bers licb) ift bein 'Dimelreicb/barifien alle Deyligen mit sir berrfaen/ Dit liecht vns bellem Gtant3 find fie bellepset / Dri tragen gưls bene Âronen auff jren Dakup: 2,Tim, 4 tern. Eie fdawen sicb Don $21 n$ : geficbt ;u 21ngeficht/ onb ou ers

freweft 


\section{6 Suffartot be}

frevoeft fie vmb ons omb mit set, nem friede.

Da ift exoige 200nne/ Dñ tein araboren/Daifteboige frewobl vń fein feyo/Da ift ewige 20ols farth / ons tein a rúbfall / Da ift ein epoiges lieblicbes 20 eren/pns Leine LDubefeligteit / Daiftoas evoige fiecht / ons teine finfter:

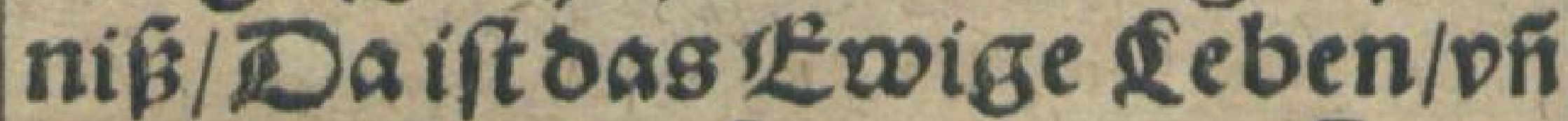
teinสos/Daiftsas ewigeGutt/ ons tein Dbel/ Da ift eitel Iu gens/ons tein 2Alter/Da ift eitel Leben/Dnotein Knse/ Daiftei, tel Sdbmuct Dñ tein (Gebrecben/ Da ift eitel Zier / ons Eeine Dn: geftalt / Da ift eitel fiebe / ono tein DaF/Daift eitel Gefund: beit / Dno leine A ranckbeit / Da if teitel frolocten/ Dno tein auff" börè / Da weís man von teinem Ochmert $3 \tilde{e} /$ Dabjret mantein Debetlagen/Da fibet mantein Zetrubnif / Sie fowewen in ewigen frewosen / ono suirffen fich für Heinem Dbel fürd)ten. 
$\frac{\text { Enigen Sebens. }}{297}$ welcbes nichts anders iff / benn Gott ben 'DERrrn ertennen/ pns in von 2ingeficht 3u 2ingefid)t

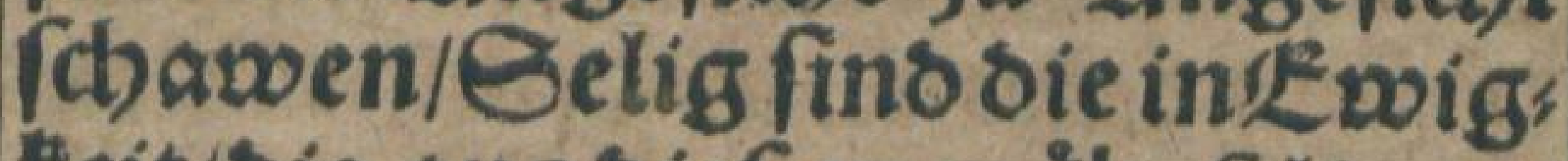
teit/oie aus biefem mulbereligen/ gefebrlicben feben errettet / Dns зu beinen ewigen groffen frew: oen romen fino:

Sroflich pfleg ich) su fingen /

$25 s e n n$ idb folch Frewoe betracht!

Sc) gebe in vollen Springen/

Pein Serts für frerwoen lacht I Mein (Bemit thut fich boch fobroingen / 23on biefer $23 e f($ mit macbt/

Sebne mich ; folchen bingen /

Der $28 \mathrm{elt}$ ich gar nichts acht I

$$
\mathcal{U} \mathfrak{R} \text { E } 2 \text { ? }
$$

23o fretben aber bie ffinctenten 23 icfel Dic Bottlofen bleiben / who wie wirbs jneri geben?

Gienerden mit seib und Eeele in die enige Dein geben. Tal liebe Gee: le / gleich wie die Swige Srewoe und 2 Bonne / Darinnen oie 2ukerweblten fchweben / nicht fan noch mag aufiges 


\begin{tabular}{|c|c|}
\hline & Suftandioce \\
\hline & fprochen werben : 2lifo fan auch nie \\
\hline & manot ermeffen oie vnaufi \\
\hline & 2Angft vno SWual/Darinnen oic @ott \\
\hline & Iofen vno $\mathcal{W}$ erdampen emig gepeini \\
\hline & get und gefiraffet werben. Dis \\
\hline & wennfie \\
\hline & peen reoen / faffens furs \\
\hline & in orey 2 Bort qufamen: Ibi erit, fagen \\
\hline & in multi \\
\hline & tas, ater \\
\hline 1 & Erftlicb/ wirb oie Jecin vno 2 ntaf \\
\hline & Der Berdampten in ber Shellen fo \\
\hline & manclyerley fein / vno fo viel / Dafs fie \\
\hline & fein Dienfel zę̆len fan. De \\
\hline M & Die Echrifft : Eie werben ge \\
\hline & worffen werben in bie enfferite \\
\hline & finfterni $\beta$ bienauß $/$ d woiro fein \\
\hline & Deulen ons Zeentlappen. Gi \\
\hline & fein. Der D $\mathcal{F}^{2} X X X_{\text {woir }}$ \\
\hline & vberfie regne laffenßlit $3 /$ fewer \\
\hline & pns Scbroffel / ons ibnen ein \\
\hline Efa, vltr. & Wetter su fobn geben. Jbr \\
\hline & 20urm wirs nicht fterben / pno \\
\hline & \\
\hline & ont \\
\hline
\end{tabular}


Gotrlofen. 299

ons werben allem fleiftbe ein Grewolfein. Ecbrecten/21ngft pno Edbmertzen wirb fie anto: mẽ / es woiro inen bange fein/ woie einer (Gebadrerin/\&iner wirb ficb Dor bem anbern ent fetzê/ fewor: roth woerben jre 2Angeficbte fein. Dotten werben jre Szette fein/ E/s. I4. Dno so birme ire Decke.

Gife da / liebe Seele / mit biefem yno bergleichen orewoungen feiget die Echrifft den Bottlofen an / Dafs als tes Ereuke / alles sitendt / aller Jam: mer/ aller Irubfall / alle Darter/alle Dein/Sunger/ Rumer/2(ngft/2ot/2/ Durft / Sike / Jrroft / Rrantetheit / Echmerizen / Gterben / Derberben / Derfchmachten/23erzagen/ uno was Da niminermefzr alles fan geneñet noch erzectlet werden/Dak wiro da jre enige Bjeferfechaffit fein/und alle ungeherwie Teuffel werden jre Shender fein.

Zum anbern / fagen fie/ Ibi erit poenarum acerbitas. Das ift + Die mancherien Angft ono Sellen) Dein

3 ij wiro




\begin{tabular}{|c|c|}
\hline & tDet \\
\hline Ạpoc. 9. & 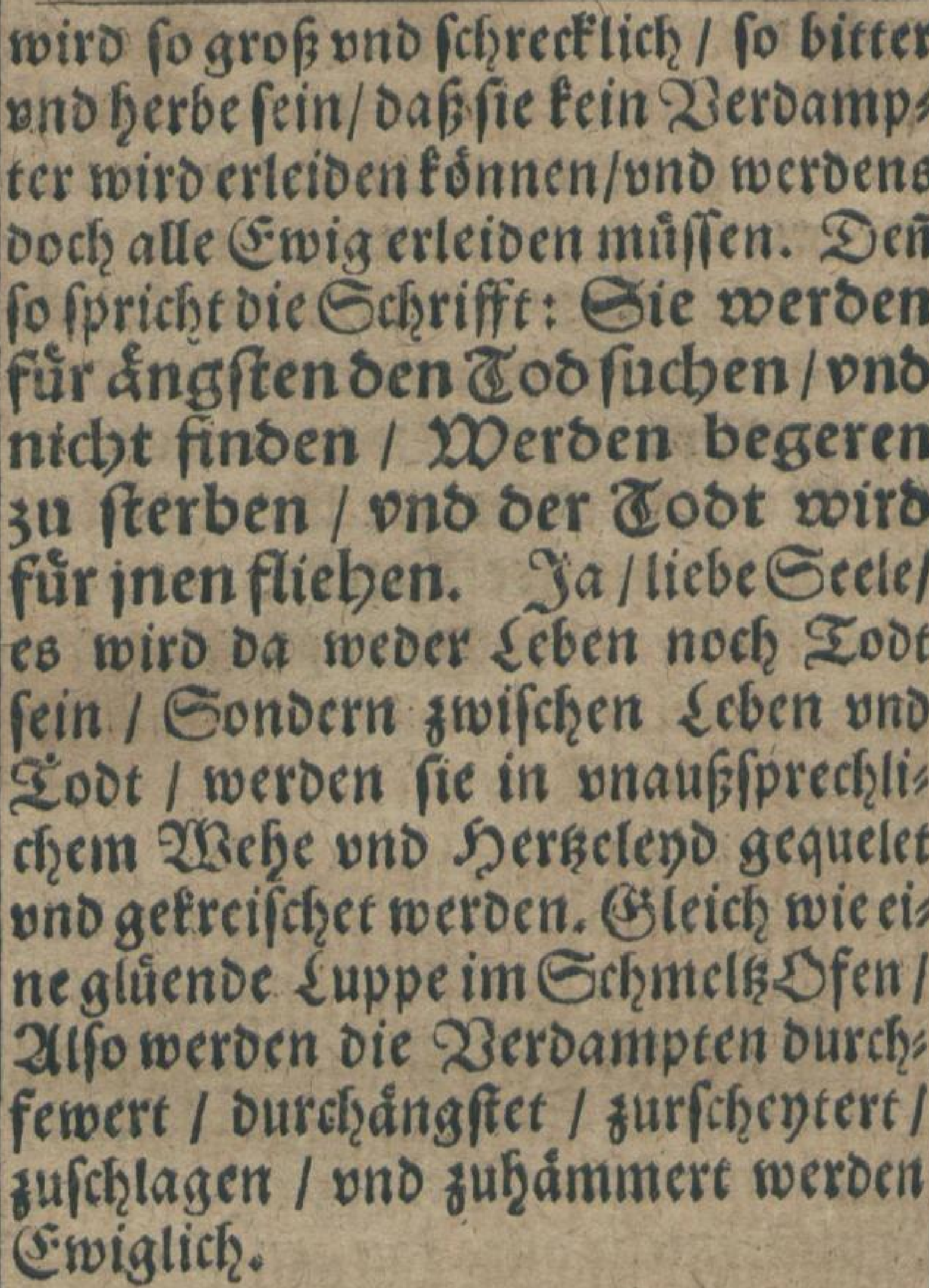 \\
\hline III. & 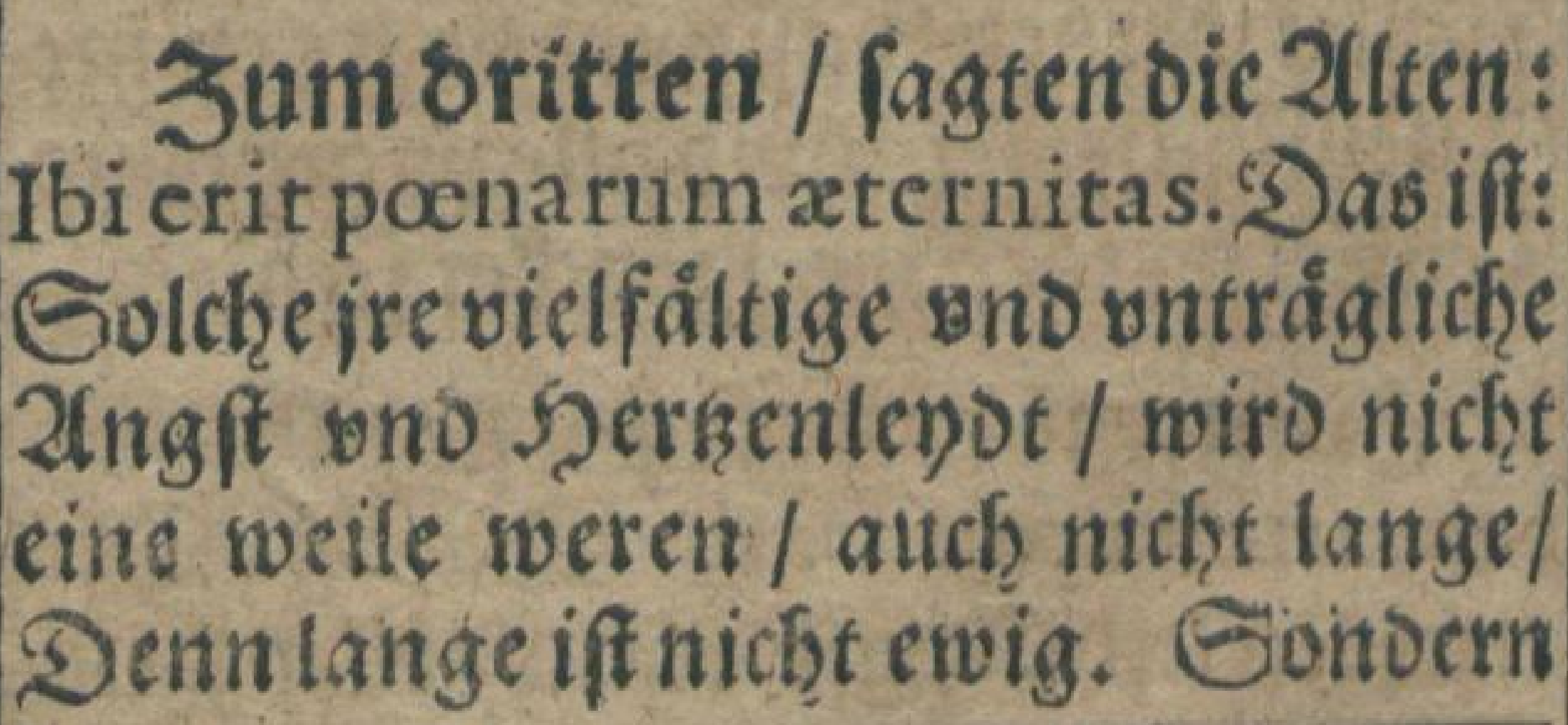 \\
\hline & \\
\hline
\end{tabular}




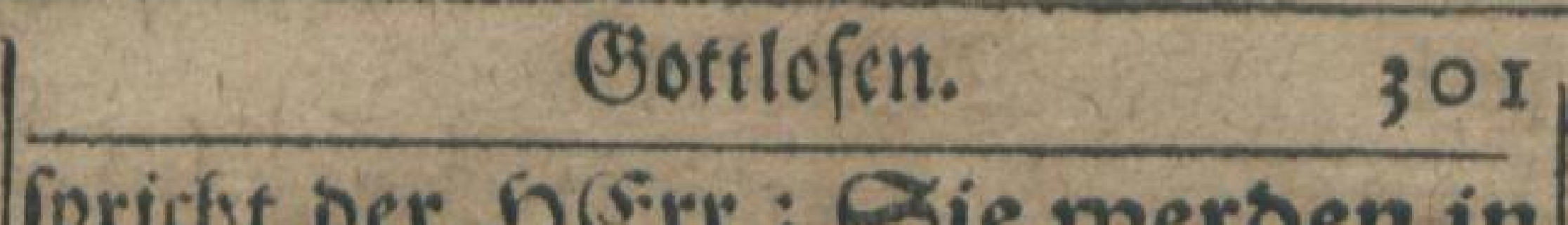

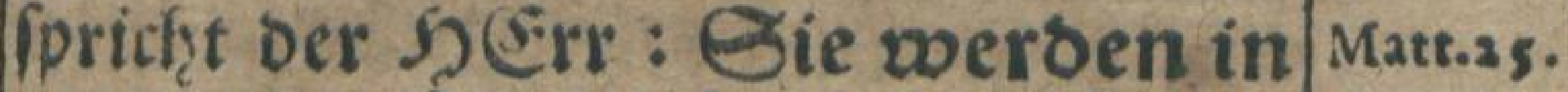
bie ewoige pein geben. Ia/meine Geele / wenns nur lange werete / fo wuirde es endtlich ein mal auffigoren/

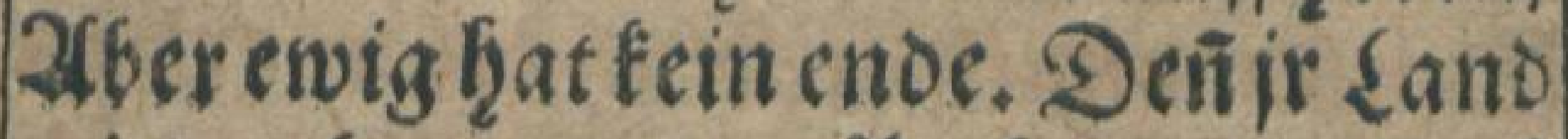
wird su brennendempech weroen/oẩ 5la. 34 weder Lag noch ?acht verlefichen wiro / fondern En wig wiro Nialtch von inen auffigetgen. Ga ber Niaudh jer Apoco is Dual wiro auffeteigen von Swigte gu (Swigfeit / vno werden feine Riufe haben/weder Zag noch ?acḩt.

Bebend"e bodh/liebe Seele/ wenn einer ewiglich folt auff einem weichen Detteliegen / Sitel herrtiche Epeife ond sabfall effen / in cinem ferionen ferrlichen Saal/ wino folte gleichwol auff folchem Dette verbunoen fein /. vno nimermefhr davon fomen / 23 ar: lich ciner witroe ifism auth Das seben nicht wounofehen ? Dort aber follen bie Berbampten nicht in 2130 lhiften/fon:

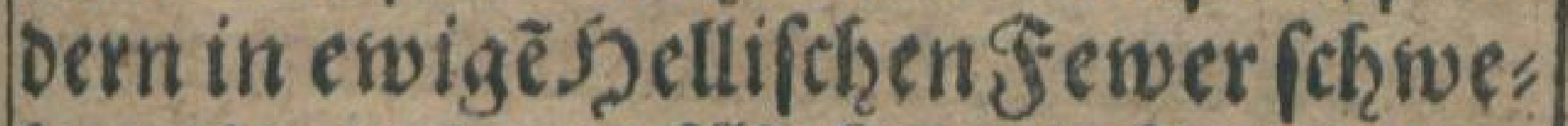
ben / in ewigem 2Belie uno 2lngft / in erfchredtlichem Etantf vno Sinfter:

$$
\text { ¿ iij niß/l }
$$




\begin{tabular}{|c|c|}
\hline & Buftand ber \\
\hline & 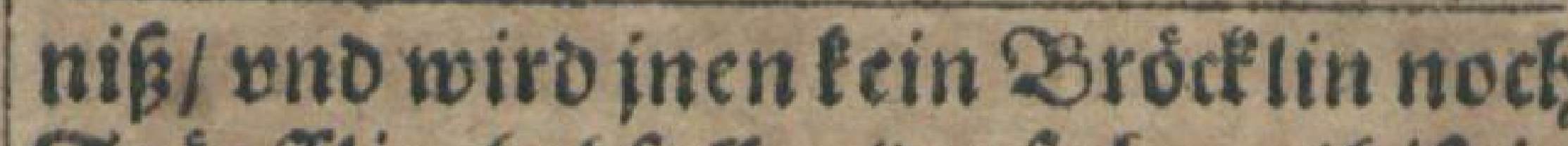 \\
\hline 18 & $\begin{array}{l}\text { Iropfflin sabfall widerfabren/bis in } \\
\text { alle enviae siviafeit. }\end{array}$ \\
\hline & S bore ooch / ou armes / clendet \\
\hline & 2Belt Rinot / Sore ooch / ono nim fu \\
\hline & Serisen/ Du liebeft die 2 Belt / gno jre \\
\hline & gut fein / In oer 2 Balt if gut fein. \\
\hline & Sibe/ es fomet die Beit/vino ift gewif \\
\hline & Reichen verdampten פianne: D icb \\
\hline \multirow[t]{13}{*}{ Luc, $x 6}$. & letbe grofie Pein in biefer flam \\
\hline & men. Ja du wirft flagen vold fagen: \\
\hline & $\begin{array}{l}\text { Die ift nicht gut fein / Die ift } \\
\text { nicht ant fein / und wirft doch (s moia }\end{array}$ \\
\hline & da fein múfen. Du wirft oa ewig ley \\
\hline & Den / vno fanftes nimermef̨r meioen/ \\
\hline & Du wirft da ewig verberben/vno bodh \\
\hline & nimimermefre fterben / in vraltefprech \\
\hline & a lecin entoe wito fein. \\
\hline & ou Sbrenzuboren/ons Dertzen \\
\hline & zuperfteben / oafí idb beine treboe \\
\hline & Anedie ono $230 t b e n$ fleiffig bo: \\
\hline & suffe tbue/ ono mich alleseit \\
\hline & Don \\
\hline
\end{tabular}




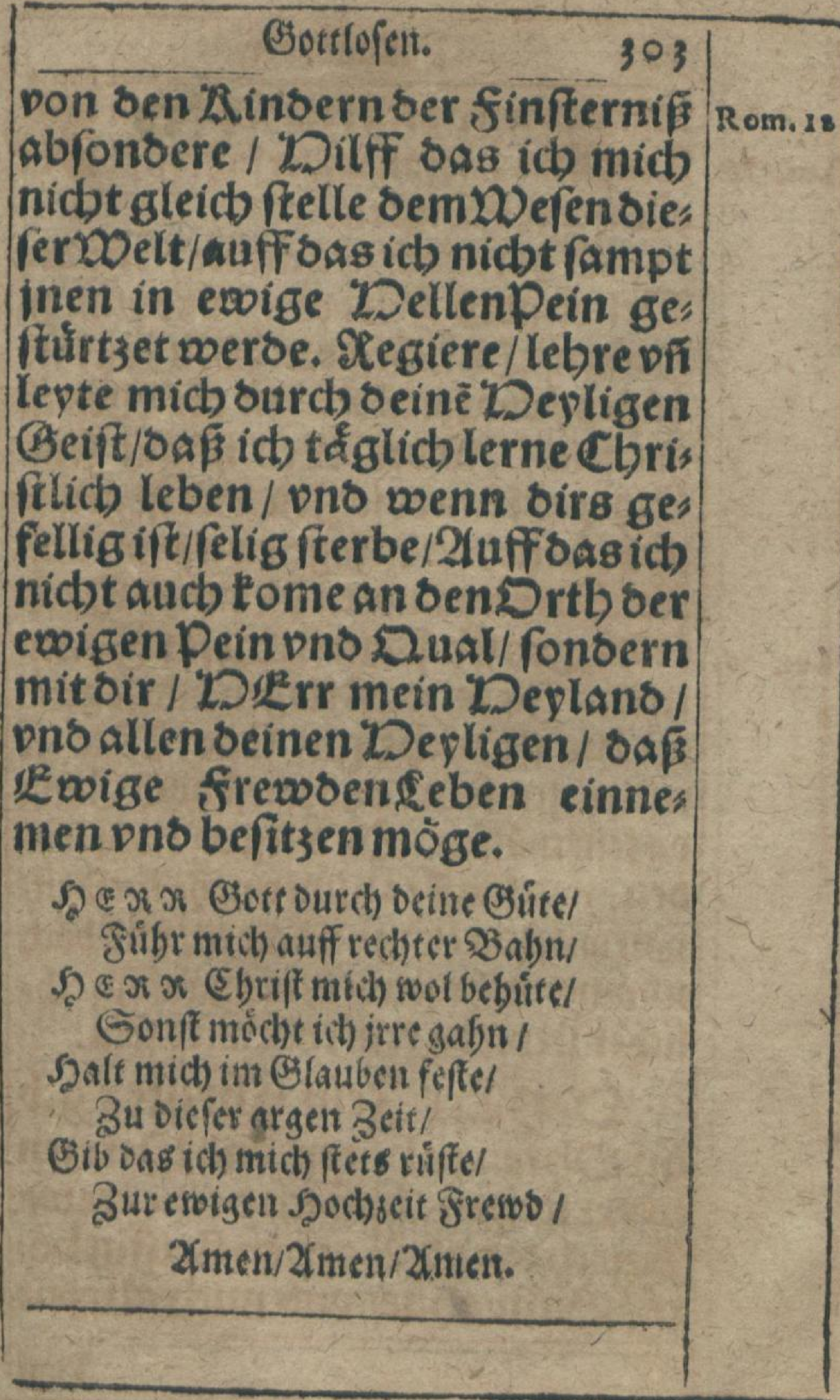




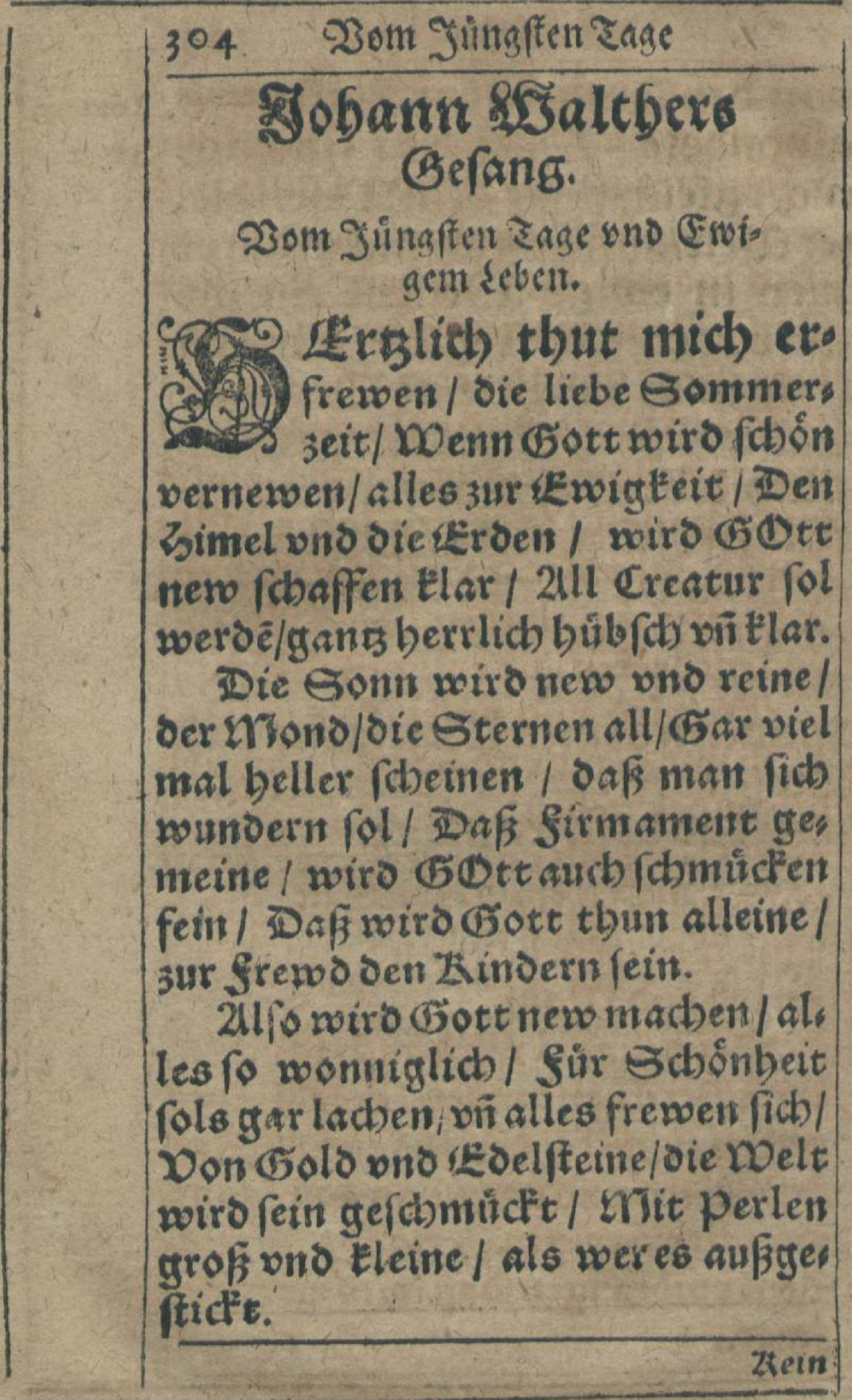


Zin zung Ean nidbe erreichen / Die ewigg Zierbeit gro $\beta$ / Man Lans mit nidbts vergleidbe/oie Doort finto viel su blo 3 / J D rumb mi fien wir fol, cbes fparen / bif an den Junntifen Cay / Denn wollen wirs erfabren / was Gott iff vno vermag.

Denn Gott wiro balo vns allen/ was je ggeboré ift/Durch fein pofaun mit fiballen / in feim Sobn Jos iu Chrift/ 2 in vnferm Slei/ch erwecten/ su groffer berrligketeit / Dno klárlitb vns entoectien/oie ewigg Seligtzeit.

KEr wiro vns vnfer Leben/ dent Leib mit baut vno baar/Gants vóls l'tg wioer geben / Daf ift gewiflich war/Dits Reib vno Geel vertlären/ fibsin bell gleich wie die Soñ/ rhach Luft was wir begeren/ vns geben Srewo vino Donn.

Gein ingel wirb auth fichicten /

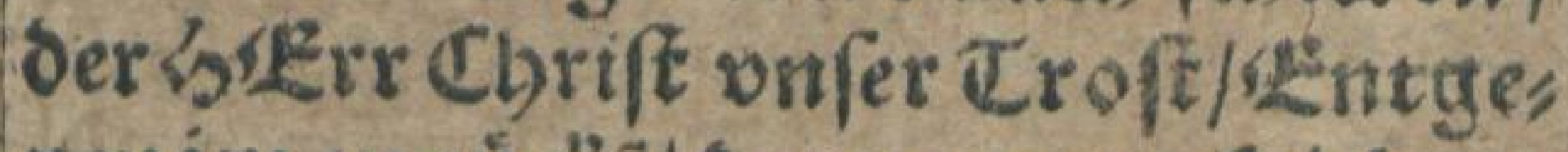
tyen jm 3u zicl'ê/ Der vns aus Lieb er, loft/ Doiro wne gar /chón enipfangê mic aller beylgen Sdbaar I In feine 2 trm omb fangen/oafs wirb er frement 350 ar.

כo 


\section{2om Singfen Eage}

30 a werben wir mit frewden/ben beyland fbawen an/ Der ourdb fein Slut vnd Reyen / Den bimel aufi。 getban/Die lieben patriarcbejpros

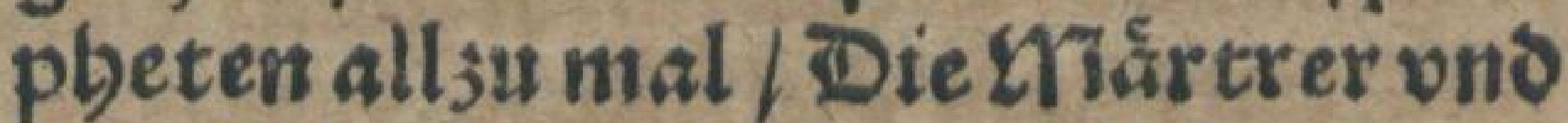
2 lpofteln/bey jum ein groffe כabl.

30 ie werben vns antemen/als jre 25 riberleit / Gidb vnfer gar nidbe (d)enten / vis mengen mittenein / DDir werben alle tretten / 3 ur Rech), ten Jefu Chrift / 2uls vifern GUtt anbeten/ser vnfers Sleifoses ift.

"Zx woiro 3ur Recbten Geyten/vns freunolidh /precbent iu/Kiompt jr ges beneosyten/3u meiner/ebr vnokbu/ Jetst follet jrererben/meins lieb/ten Daters Reich/ Delchs ich euch bab errwozbe / orumb feio jrokrben gleich.

2IIBoenn wiro Gott recht richten/ oie Gottlof bófe Doelt / Dar B bel, lifh ferver fol foblidben/ die Sino mit-barem Gelot 1 Den Ceuffel vno fein Rotte/ die beucbler MTamon Enedht/LDiro Gott 3 us Sdandt pnd Spotte/ all vrtbeyln ongered)t. 
Wird fid gang sornig felien/3u ben 3ur Lincten banot / Kin redbe gleidb Drtbeyl fellen / mit Dorten ro genanot: Gebt bin jr ganb Der, flucbten / zum buellifichen ferwer er, tand / Ins Ceuffels frict geflods, ten/in ewigen Coot wnd 25 anos.

211 fo wiro Gott erlo̊fen/ vns gat vou aller Kotb/ Dom Ceuffel / als

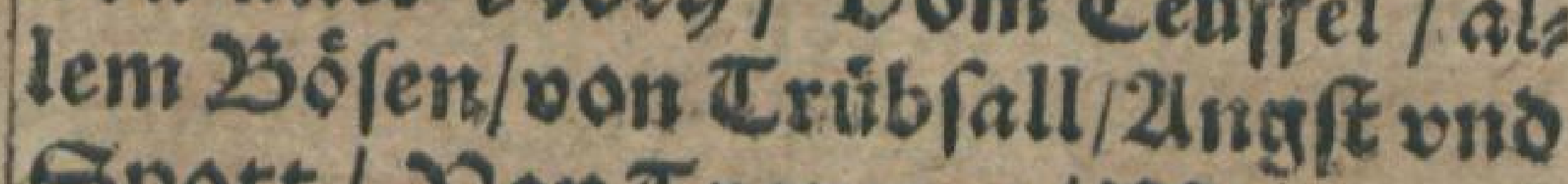
Epott / Don Trawren / Debe ono 23 lagen/ven Zirandibeit/Sdbmern vno Leyo/Don Scbreermutb/Eorg vnd Jaggen/von aller bof cu zeit.

Denn wirb oer bैerr Chrift fübs ren / pns die wir jur vertratve / snit graffen jubilieren/ 3um Dater feine 23 raut / Daf w wird ein beymfartb. merben/ingroffer debr yoso pradat Defigleicben nie aufi serben / Eein Zónig fo gemacbt.

Da wiro die 25 rant tyefiblet / fus Dreutgams berrligteit/Wit frew, Den Triumphiret/oer 25 raut $3 u$ oslor ono Srewo / Da wiro die 25raut ais: fonwen/ Den enigen wabren Gotio/

Den 
308 Dom Sinngfen Enge

Den fie 3 แ⿰氵 im Glauben/imDDort geebret bat.

Der wil vno Ean anseigen/wie Gott ber Dater fich/bolo felig wird erzeigen / vie 2sraut fo gmidoiglich / 42mpfaben wird /ebr freustolid / in frewo vnto fróligketeit / Ono fie eins fithren berrlid/ / in jre Seligteit.

TDie stugel in ben Luffiten/werben mit groffem folll/ Erit allésimels Ziråffen / facon jubiliren all / 2llles was Gote gefchaffen / wiro die zeit

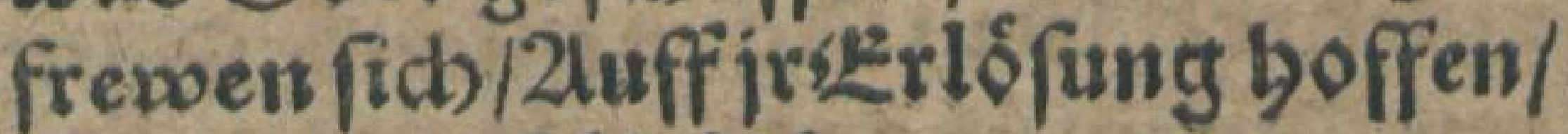
fingen bolofeliglidh.

Eoldbs wird die 2sraut erfrews en/ Jep Daters Sremnotligteit/ Jhr Leib und Geel vernewen/3u wabrer ذ) andibarkete / DDiro Gott 3u Suffe fallen/nacb recber Demutb braucb/ Sit Gortes b́cylgen allen/berblich Sott Dandien au(b).

Dirs beybe zend auffibeben / in Jubt vno Demutb fein/ Gott prey, fen vno bodb loben / fir alle Enade fein / Do iro fur Gott frey betenteri /

จa 
vino Ewigem seben.

309

oaff fie onwirdig fey/fidb eire 25 raut 3u nemen/in Gottes Lieb viี Trew.

G0te wirs die 25raut auffrich: ten/ mit Cbrifto feinem Gobn / Gic bersen vno fein /djlidbten/feit 2 nt wort freunotlich thut / Du folft mit

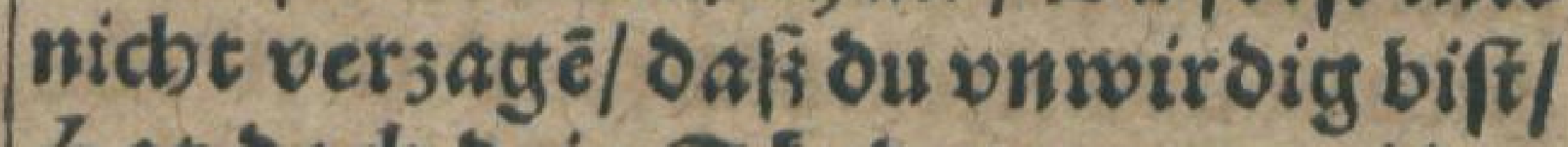
bat doch dein Stind getragen/ Der 25reutgam Je fus Cbrift.

Jdb bab mich sein erbarmet/wie sidh mein Dort gelebrt/Wein ber s if and) erwarmet/meinGnab 3u dir gelebrt / Mein Sobnt für dich geges ben / für bich ans Creus gebend" t / Der bat oir bradbt das Leben / fein Dnfibulot oir tgef benctit.

Deil ou denn foldbs gegláubet / meint Ginà gettomé an/Da âj ich oich bodb ggeliebet / oir foldbs 3u gut ge, than / Darvmb biftu nu reine/ from vñ geredbt fir mir/ De iff alieine/ocin Gabuct vno /abône כెier.

1Es bat mir fo gefallen / baj bu meins Sobns Gemabl/Geinzraut baft werden follen/in meinem Keid) vito 


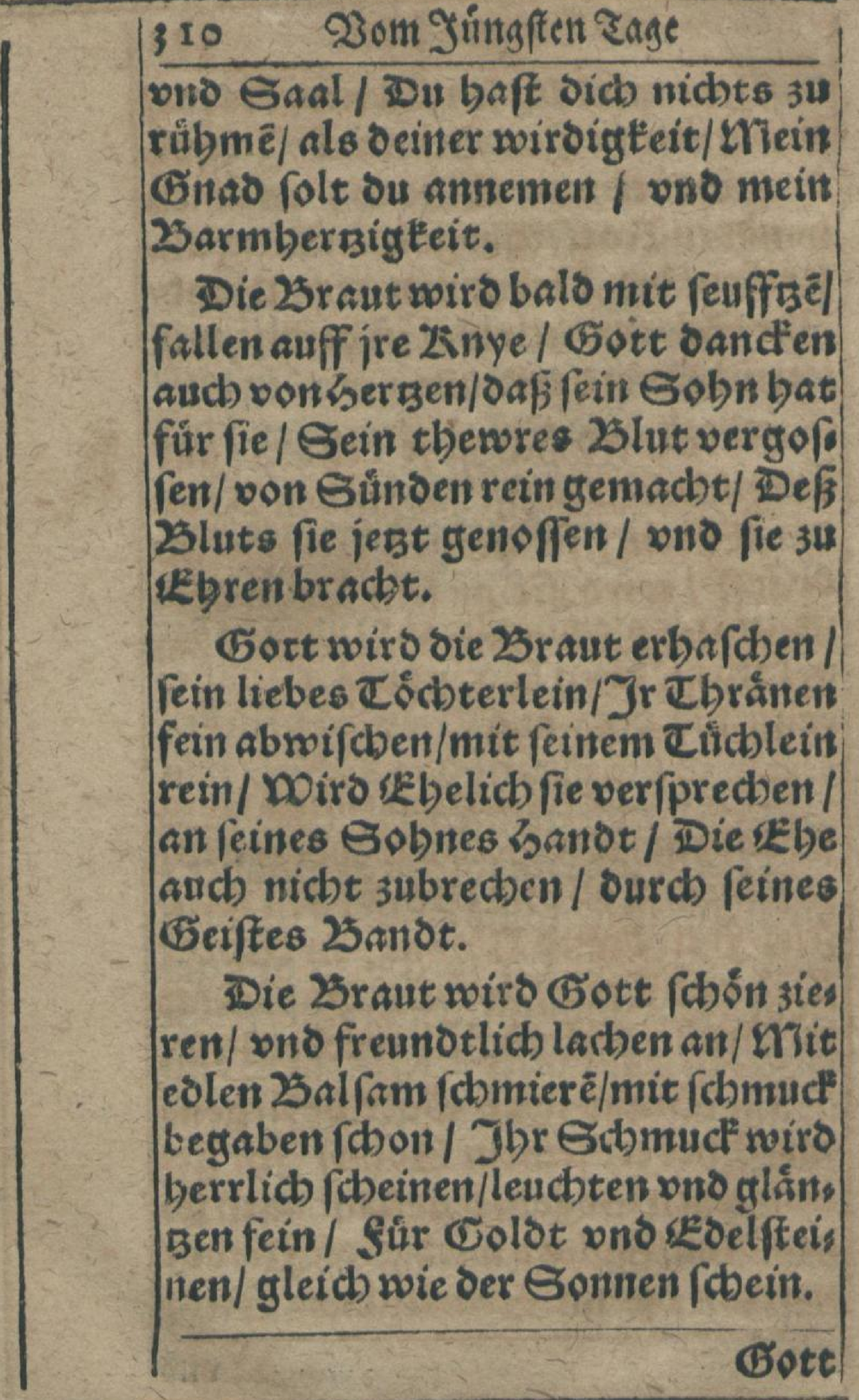


Gott worb die 25 raut new Eleyoê von feinem eigen Scbmad') In guils ben Stúc" vno Seyben / in einen bunoten Rod"/KRein gúlbenking an, fiecten/ Der Lieb zum waren p fand/

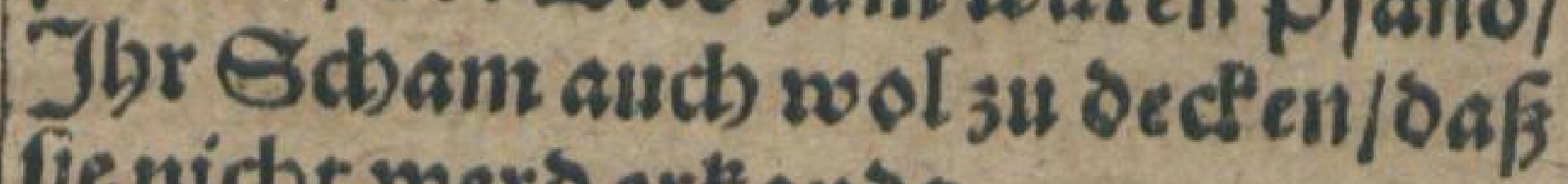
fie nicht werb ertande.

Gott wird fidb 3 ir it tebren / gar berrlicb fezê auff/Kin gúloen Ziron oer ugbren / fie bersen freunotlich orauff / wiro fie an fein 25 ruft orůs dFen/aus Lieb gans Dåterlich / 2 n Leib vno Seel auch / dbmícten / mit Gaben miloiglid).

但r wiro fie frơlicb leiten/ins ewig paradeif / Die boch3eit 3ubereiten/ 31 feinem \& ob vno presf $/$ Da wirs feit frewo vno Donne / in rechter Lieb vno Crew/2lus Gottes Schan vi 23 ruñe/aucb tåglidb werbennew.

Die 25raut wird Gott beylegen / ins (2)bebett feinem Sobn / Jbr ges ben feinen Segen/ fein Luft aud je, bett oran/Golcho noiro grofis Srerobe maben/ gans bimelifbem beer /

Wers 
$\frac{\text { sno Eivigent Seben. }}{\text { Srewo alloa / Denn GOtt wiro se: }}$ noviren/oie ganbe ETinfica.

Ziein triench Ean recbt befareis ben/sic bimlifch ewigg frewo/noiro wol verborgen bleiben / bif ju ber lesten Zeit/24ll Weltlich Gleidnif triegen / fino viel su eng vno formal/ Jft inn Eein bers geftiegen / Die frewo fo werden fol.

Ziein Obr bat nie gebơret / kein men/chlicb 2lug gefebn/Dic Srewd fo den befderet/oie Gottimbat ver: [ebn/Sie werben Gott an/chawen/ von bellem 21ngeficbt / Lieblichmit jren 2lugen/oaf ewig wabre Liecbt.

Gott werben fie ert'ennen/oie beis lig Crinitat/Jn Gottes Liebe brens nen/ fein De e en vn /ein Ratb/Doiro Eott jrn 2lutgen gónnen/ vno was er ift vito bat / 2(ud) feine Zkinber nen" nen / in Gottes Ziraffit ond Cbat.

2lifo wirb GOtterfúllen/alles ourd) feintezizaffe/WDird alles fein in allen/ Durcb feinen Geift vno Saffe/ Diro fich felb/t ganb 3 us cigen / ons

$21 a$

geben 


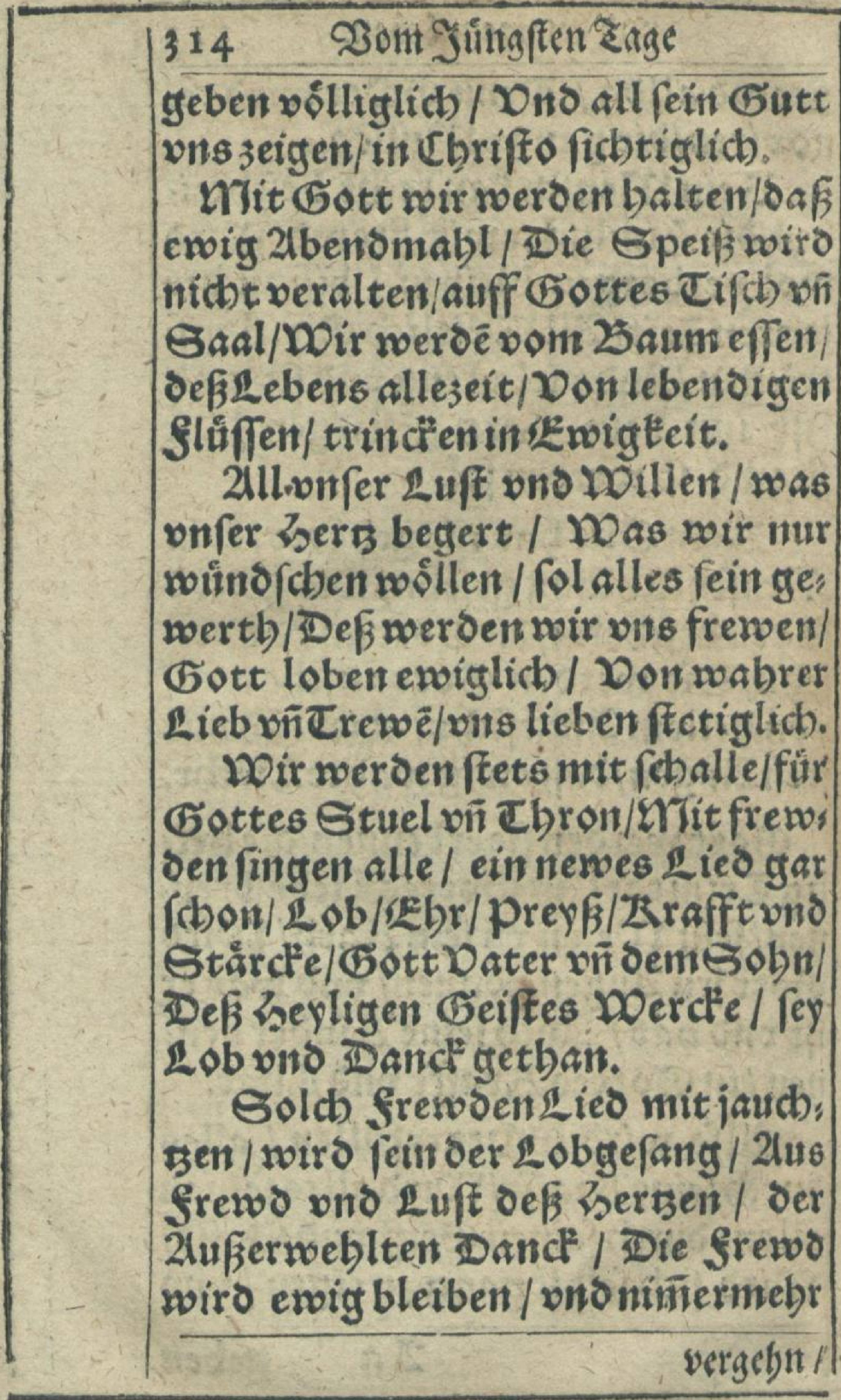




\begin{tabular}{|c|c|}
\hline \multirow{2}{*}{\multicolumn{2}{|c|}{ 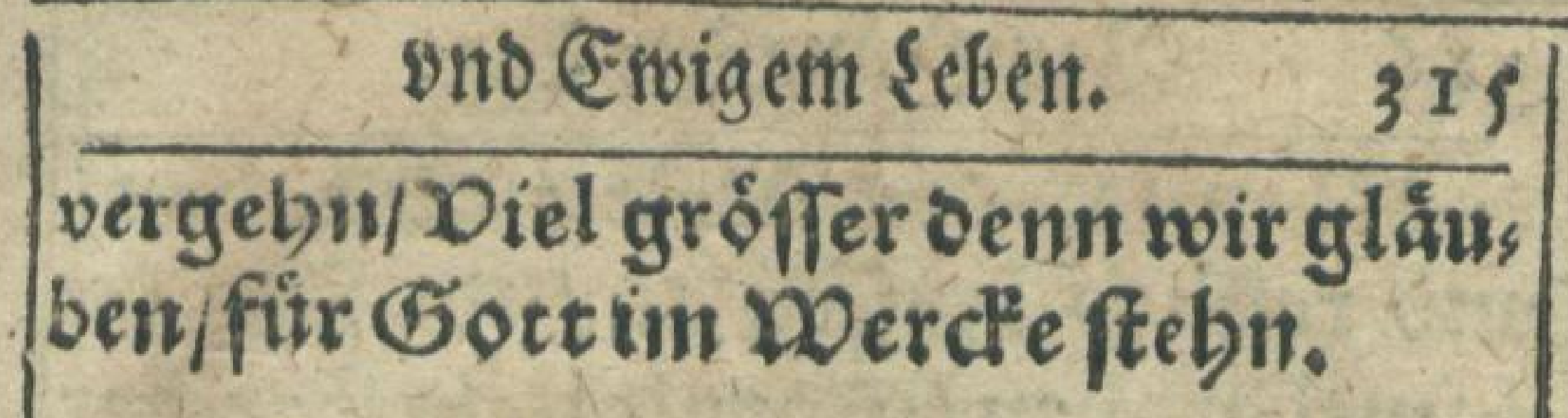 }} \\
\hline & \\
\hline \multicolumn{2}{|l|}{ 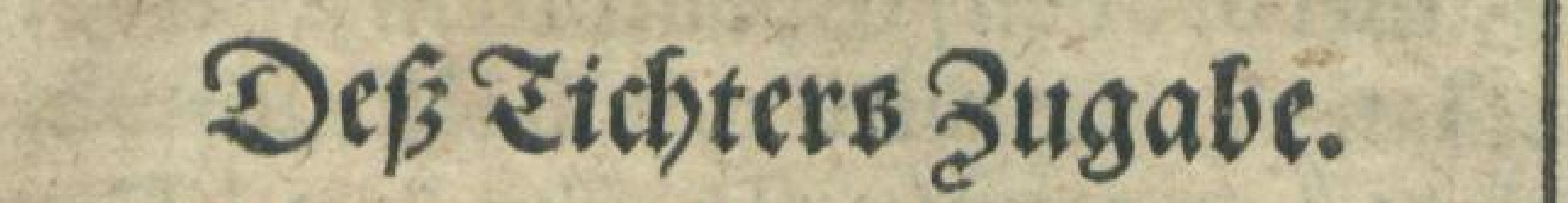 } \\
\hline \multirow{5}{*}{\multicolumn{2}{|c|}{ 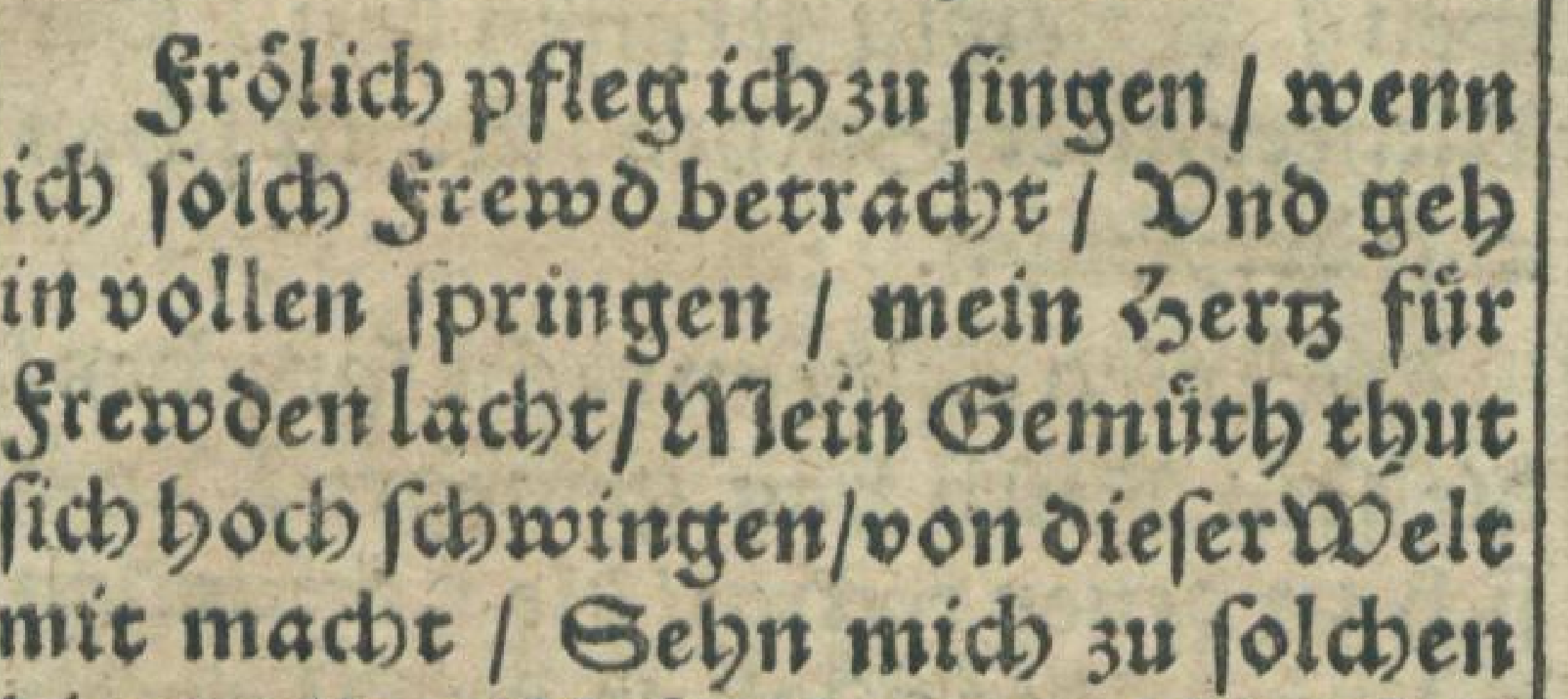 }} \\
\hline & \\
\hline & \\
\hline & \\
\hline & \\
\hline \multirow{2}{*}{\multicolumn{2}{|c|}{$\begin{array}{l}\text { dingen/ber Deltich tyar nidbt adbt. } \\
\text { Dorumb wollen nicht ver3atten/bie }\end{array}$}} \\
\hline & \\
\hline \multicolumn{2}{|l|}{ est in Crub all fint / Dno die die } \\
\hline \multirow{2}{*}{\multicolumn{2}{|c|}{$\begin{array}{l}\text { Welt tbut platgen/vnd ift in pinten } \\
\text { feind/Eic wollen jrCreus tratten/in }\end{array}$}} \\
\hline & \\
\hline friede mit geoult / 2luff GDttes & \\
\hline $\begin{array}{l}\text { Doort ficb wasen / fidb troffen Gots } \\
\text { tes bull. }\end{array}$ & \\
\hline \multirow{5}{*}{\multicolumn{2}{|c|}{ 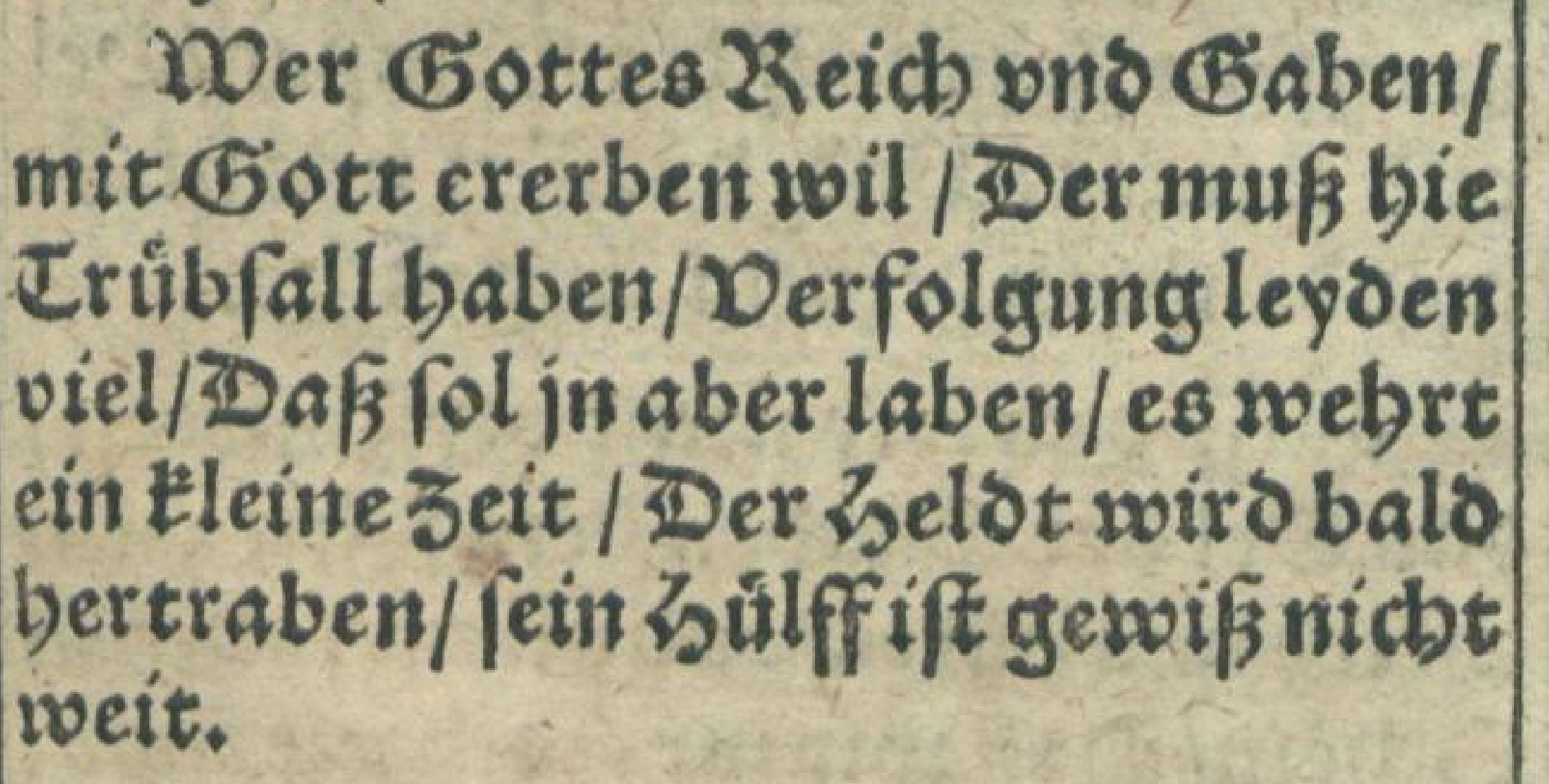 }} \\
\hline & \\
\hline & \\
\hline & \\
\hline & \\
\hline Aa & \\
\hline
\end{tabular}




\section{Som Sungften ?nge}

ITan la j oie Delt audb toben/ vits reolich lauffen an/ KE fint im Zintel oroben / Gott lob ein fard'er LIIanti / $\mathrm{Kr}$ wiro yar bals auffua: chen / ber ewig fraffen Ean / Der Kirbter aller Saben/ 1 Ex iff fon auff oer 25abut.

Der wird alfbentrecht fprecben/ bie fein Reids ban gemebre / Ono fich an bentent rechen/ die Lutgen ban gelebrt/ DDas gilts er wiro fie trefs fen/oie feirte Zirch betrúbt/J br fal,

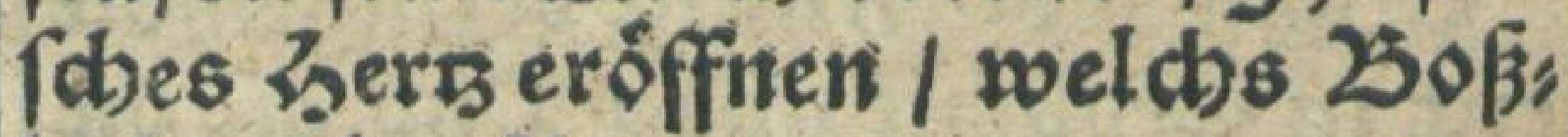
beit viel geutbt.

Der 25reutyam woirs bals ruffen/ Liompt all jo bodbseit Gáft / Zilff GOtt bas wir nicht foblaffen / in Sinden fhlummern fefti 2 alo ban in onfert bántont/ bie Lampen/Del vnt Lieche / Dno oirffent vits nicht wenten/von deinem 2lingefidbt.

Zilff bas wir nidbt verfbutten / Sas Del ber Enaben beit / Sür bew c)eley ons buten/ond allem fal/fben. Gchein/Daf wir 3ur bocbseit frews

Den/ 


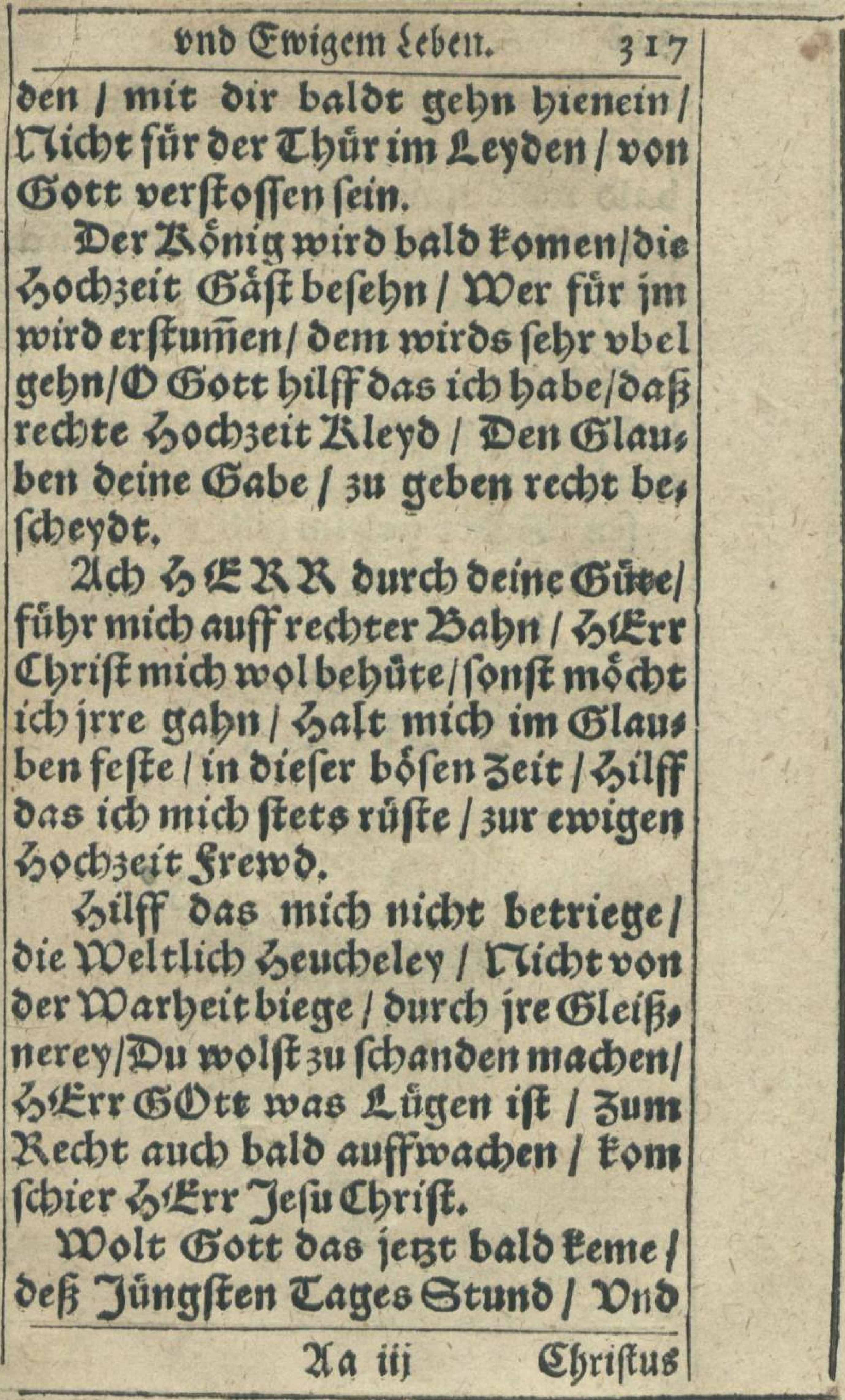


318 Wom Evigem seben.

Chrifus uns binteme / wind (b ich vout berzen grundt / Daf Chrifus bald wolt machen / oie breymfarth feiner 25 raut / Def wolt ith frôlich lacben/für frevoen fingen laut. Ziemit wil ich befablieffen / oaf frólidb Sonmer Lied/佂s wirb gyar balo auffprieffen/ die ewitg Som, merblith / Daf ewig Iabr berfliefa fen / GOtt yeb im felben Jabr/ Daf wir ber fridbt geniefs fen/21mendas woerde. wabrl

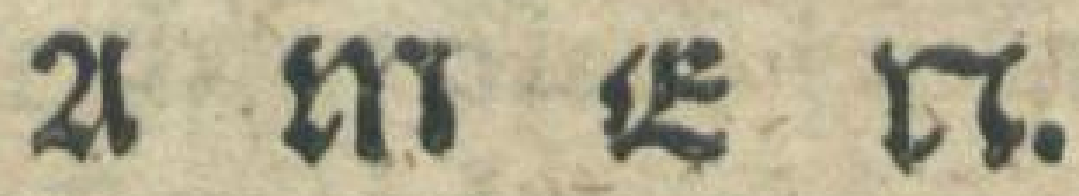

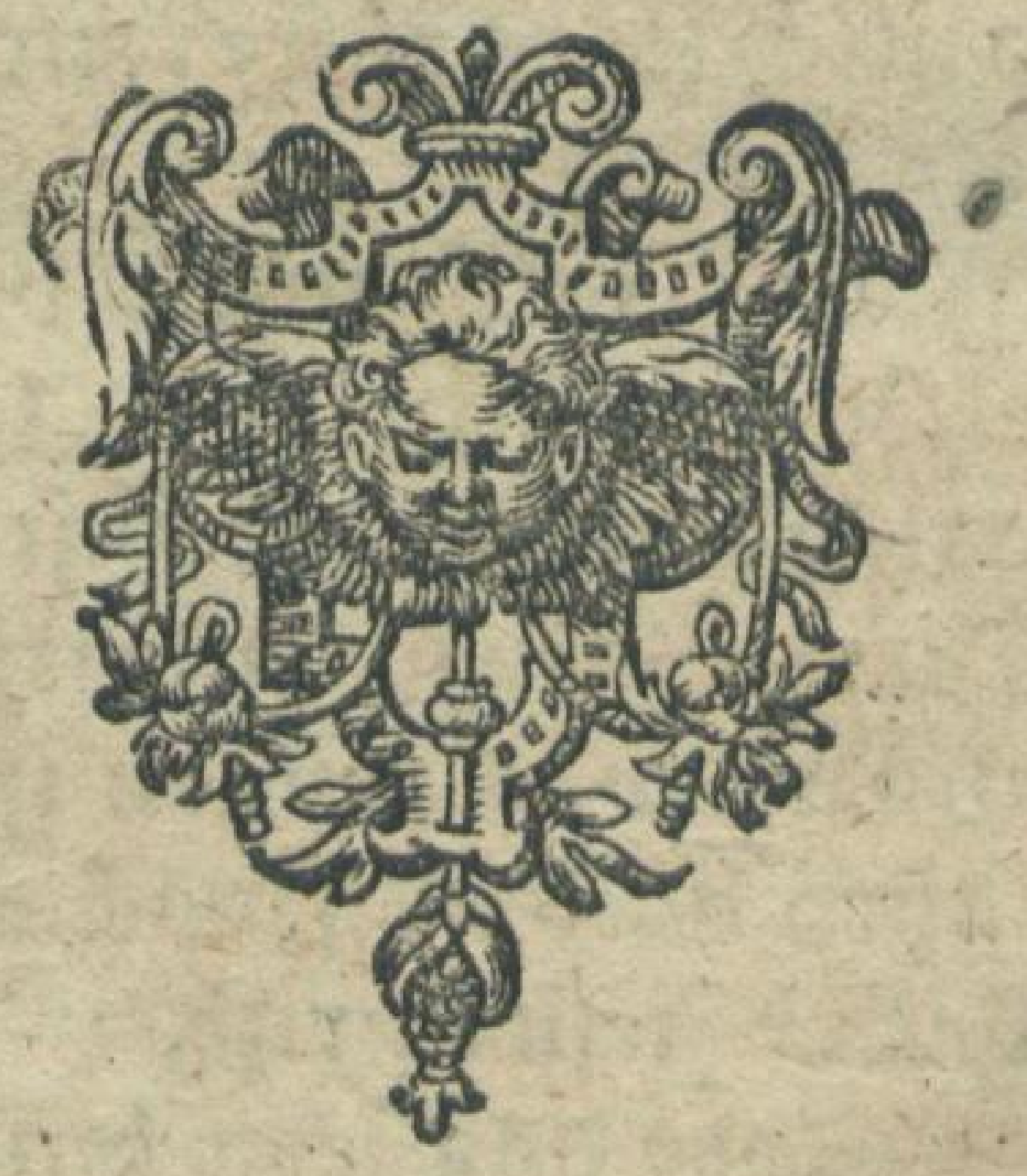




\section{AD MARTINVM MOLLERVM,} ECCIESIASTEN GORLICENSEM, de ipfius Prxparatione ad Mortem.

Mollere potens mortis mollire fagittas,

O Martine novi Martis \& artis honos. Ductrinâ pro tam dulci \& folamine miro

Quid precer aut referam vir venerande tibi? Mollia fint vitx tibi tempora, mollia fata,

Molliaǵ; in tumulo molliter of a cubent. Sint tıbi tot plaufus, tot victâ morte triumphi Hic liber excuffit quot mihi lacrymulas. At mihi tu, libri\& cunctis lectoribus hujus

Vivere da rectè, da bene Chrifte mori.

\section{Nathan Chytraus.}

M. MELIOR IS LAVBA N I SPROTAVI SIIESI.

O DE A M PILOCHIA,

AD MARTINVM MOLLERVM, Virum Clariffinum:

De Tavaro

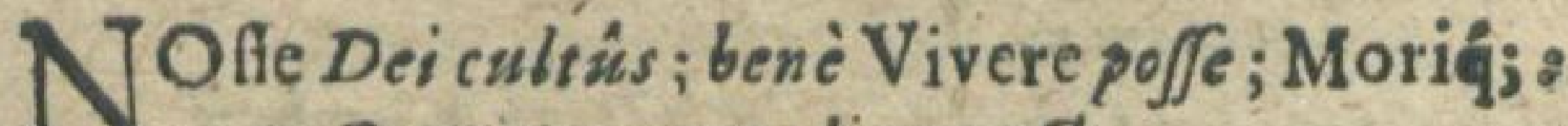

I. Beatitas nortalium est.

Hec Tria quisquis babet Piet ditis Praco; fusumq;

Fideliter docet gregem:

Is non lasidis eget : dignus Ieffeide cingi

Lasere u/que quaque tempora. Queirum adè nomeroes mult is è millibus unus, $M A R T \cdot I N E$, Vates ceelitwm.

2(a iiii Namger 
Namque ea cuxcta tenes; saliq;; mbinde pio rwi

Mulces fonore pectoris.

Nec linguâ docwij]e lat est : jusat if a libellis

Complectser Gigacsbus.

Quo re tota adeo Germania, feraqj; deinceps

Legarit nepotusm facula.

Teftis ab Igne trabens cognomine Prafsul, El alser

Dono vogatus à Dei.

Quostiva docta manus Grajaprius ore logueses

Vertit loquellà T eutonum.

Tefis form A Precum Gariarum è dalcibso hanfa

Patrum ansiquorum ribulis.

Pracipuè Lamenta, twos que, Chrifte, dolores,

In fontis Eo deflent necem.

Ruid memorem, illuftres Regum que dirigit Aulw;

Agapeticam Politicen.

Nuper É ad cunas cantata Genethlia celi

Regis, Jolique, Ef atheris.

Hinc quis certapetat Superos exempla Colendi,

Certamq́; Vitæ regulam.

Sed laudatioperis ne pars tamen una fuperfit:

En Mortis en Scientiam.

Hac sibi, quo doceat Latum expectare pasatu;

Quibus medendum pharmacis

Triftitia E⿱ curis, qua corda agrota fatigant,

Piâ da bit folertiá.

Est aliquid docti tractare Macbaonis arteis;

Seu corpors malagmata

Accelerestrepidante manu;/eu fontica membri

Abigas Genena lang Gidis;

dut GiCtor Canfos /ubigas, Eु quidquid ubique est

Dirarum in orbe Febriums.

Eft aliquid, fateor: me judice nil ismen $\mathrm{wgwam}$ est Hoc omne, qsàm Merum nihil.

VJque adeò MA R T I N E, ommem tua Mufa nodenLonge anteitprodentian.

(

corpos 
Corporeos domat iffa quede mo medicina doloresi

\section{Mentis fed ore perditum}

Topenes est, M A R I I N E, Iuem; pefreing; laterse io Fortinecare dexterá.

Nec Galet in Mortem Medicus : fed re Duce quibis

Nunc Vitior Hujus audiat.

Sternerio ? E mortis se plwrima terret imago?

Heicexcides met us tsbi.

Anne Deximplacer atervis accumbeve menfis?

Visa US fruifciperpere ?

Huc ades; en noft ra hac te Februa rise pjatums

Polibocaburi Gertice.

Quaque beatorum pa Sim Mors effe putaturs

Reappe Vita erit nofia.

O nimium felix, quicumq́; bae jugfa fequestus

Talem Già rit cromine.

Non illum ambitio, nec opes, now quer quera fado

Mens fanciatit crimine.

sed facilesis obitûs omnem expectiàrit ad hor ans:

Solo acquiefcens in Deo.

At tu madte animis, Mar T IN E, Ef munere tanto.

Piöque macte Grasiö.

Perge rudeis pofthatc ment eir jubensmiq; , fenumiq

His 10 Pitare dosibus.

perge precor. Sicfama tuos amplexis labores

Adoreâlasidis beet.

sicribifaderia post fat a suprema Corona

Desus perenne Gondeo.

\section{Sprottavix ad Hebrum,}

clo lo x C III.

\section{Propridie Kalendas Martias,}




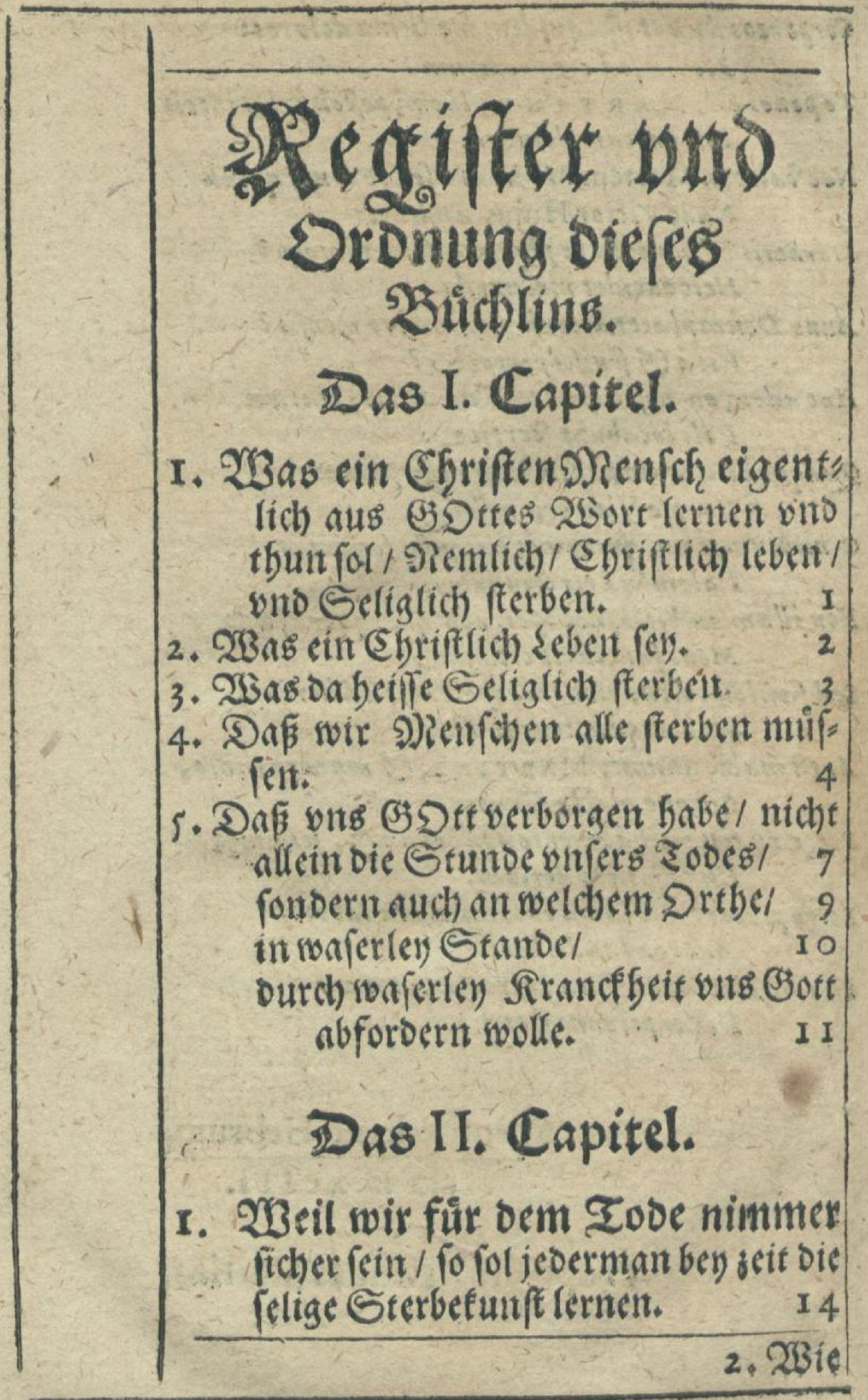




\section{Diegifter.}

2. OBgie man fich ourch wabre 23 ule jum feligen Sterben bereiten fot. 3. W3as rechte wabre (Sbriftliche 5 uffe
fey.

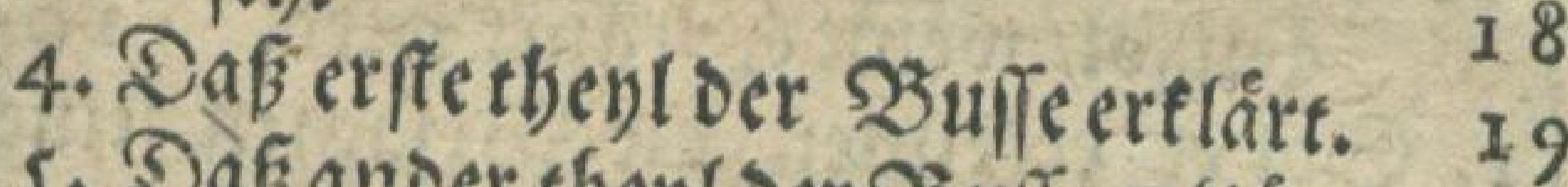

5. Daf ander they) (Der 3 ulfe erflárt. 27

6. Dak oritte thenl Der 3 ulfe erfleirt. 40

7. Dak vinfer gankeg seben fol vno mus sine ftefwerende $\mathfrak{S Z}_{4}$ fe feits.

\section{Das III. Capitel.}

Daffechs Stưfe find / bie sucinem (Sbrifficthen seben sigentlich) gebo: relt.

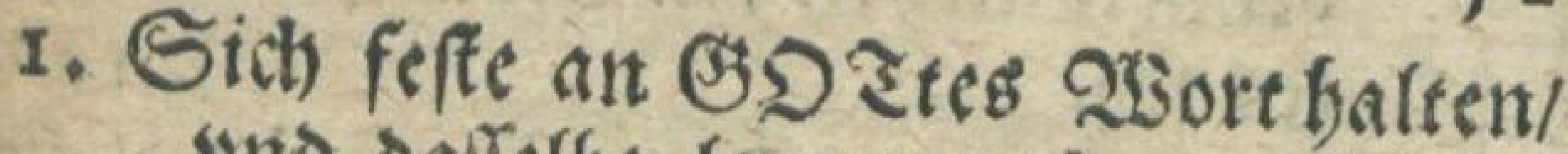
who barlelbe letnen recht verfteben/ rectht thenlen/ wnd recht gebrauchen. 2. Sich ber Şeyligen हnuffe triglich tre:
fent.

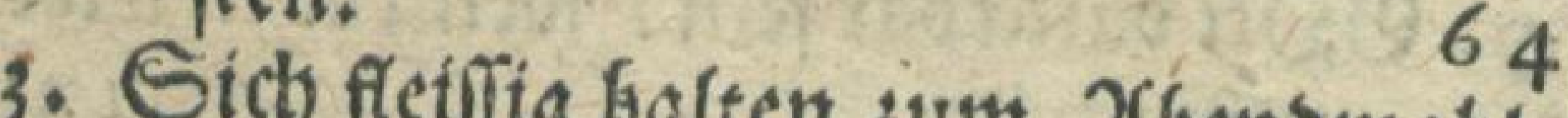
64 4. Zum Ereuke ond seroenfich 68 5. Infecitem eigenen 8 I 6. Sfeillig Bott antufente 95 . Sinlg bott amruffer alle ₹age. 102 


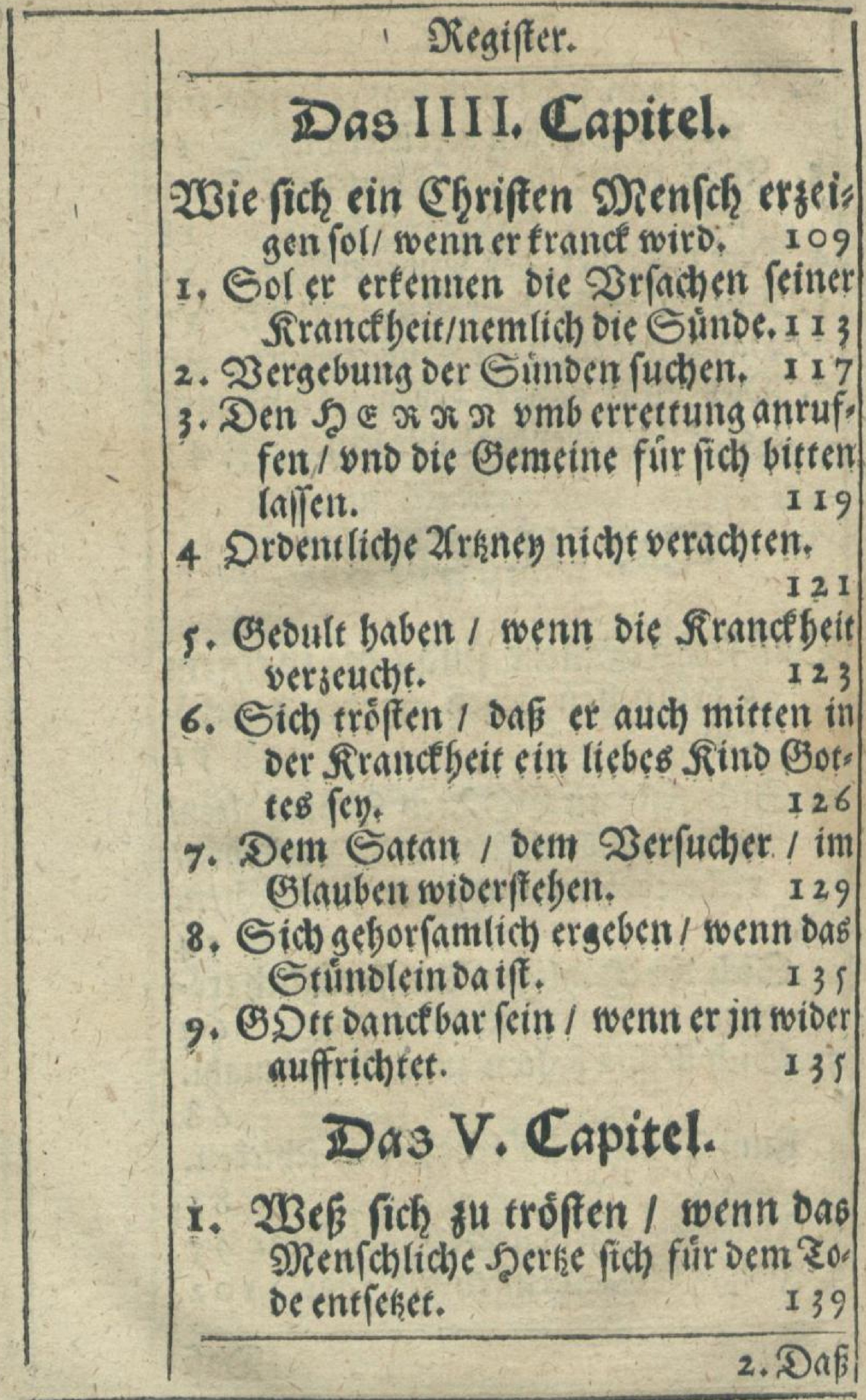


Niegifter.

2. Daß viel seute ein febnlich verlangen nach bem Sode tragen.

3. $\$ 6 \mathrm{ein}$ ग) vmb ein lenger seben bitten moge.

4. Orang eineti geen 145 Daf er fich swillig sum Gterben er. sebe.

5. WSie mtat das Gerke verwafren 148 vider oen fchonen saufe bert wiber ben fechonen sauffit ber 23 elt.

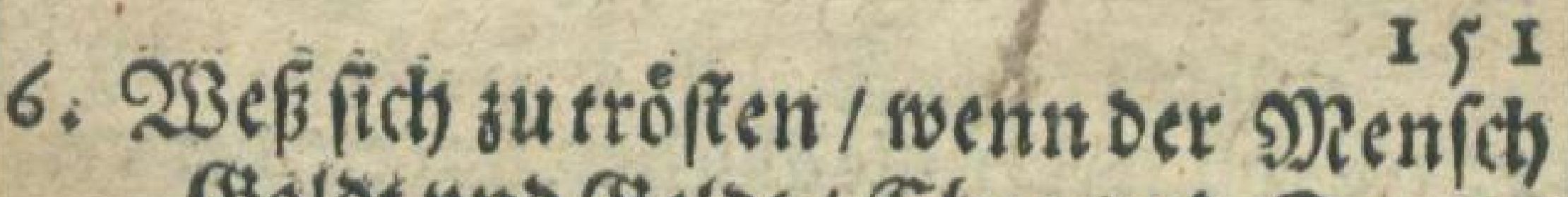
Goldt und Seldot / Ebte und Frende

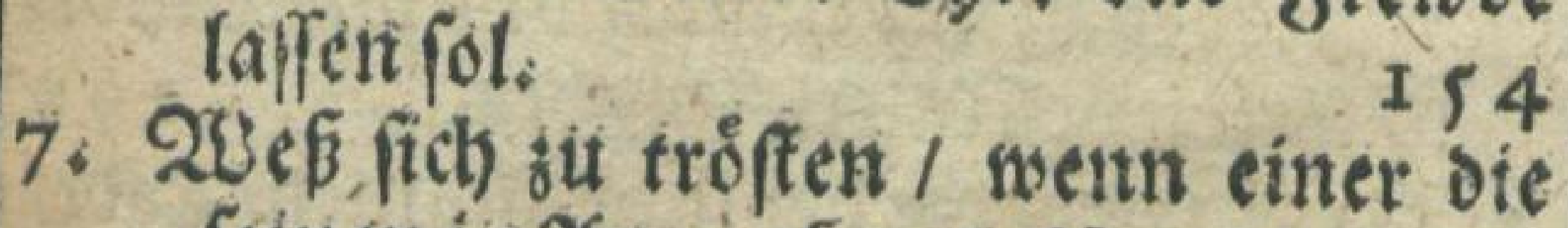
fetnen in 2 (rmutth wnd 2 nrichtigfeit bindertalfen for.

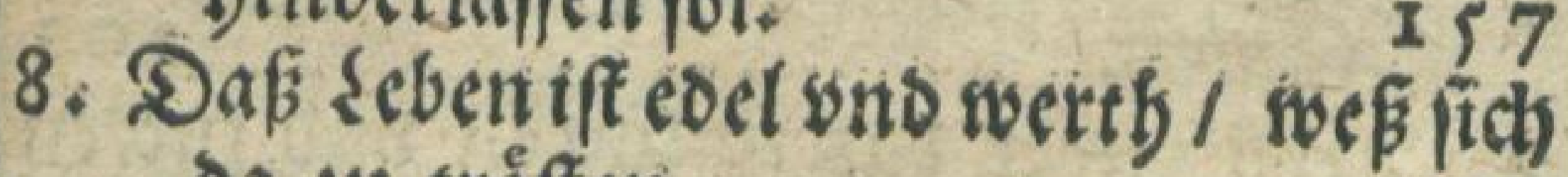
ba zu troftent. 164

\section{Das VI. Capitel.}

1. 2Bomit bas glàubige Serke bie furcht bes ?oDeE ftillen/ wñ fich frew. Dig vito fróstich ergeben fơnne. $\quad$ 71

2. OZGie Das gláubige Szerke fict) vbent vito troffen fol / beftendig bié ants Ende suverbarrent:

i 78

3. 25ent 


\section{SRegifter.}

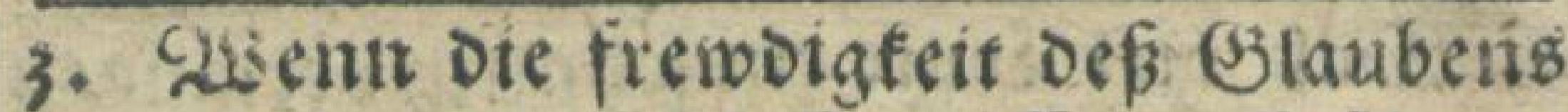
bisweile nachleflet 1 offt grofie foch wa cheit fiiblet/auch bey forge tregt/ob es bejêtenoig verfarren/ ja ob er auch er= weblet fey / oder nicht / we f fich on zu troffen.

\section{Das V II. Capitel.}

1. Iroft wiber die abfehendigfeit Def soder.

2. Eroft wiber bie absichervligfeit ber vers forbenen seichnam/ uno onfreullot:

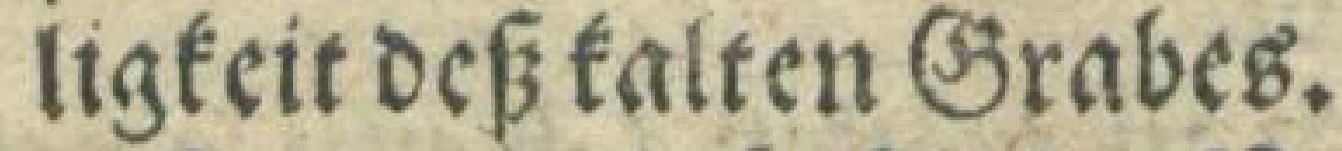

197

3. Iroft noider bie befitiche Diamen/ Sobt/ Sterben / Grab/3u/charren/2 $3 \mathrm{cr}$. faulen.

201

4. Sroft fwider bas fchlucten snd zucten der Sterbenden.

5. Göttlicte bewerte 2(rsiney roiber den Etachel vno 3 itterteit Deß Fodes.

$$
.207
$$

\section{Das V III, Capitel.}

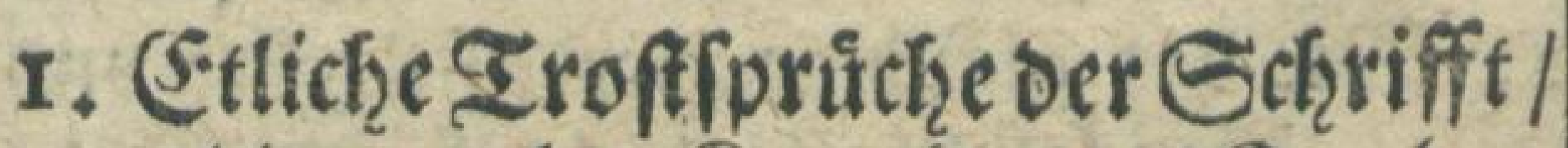
Die man bin Rrancten vid Eterben oen gebrauchen for.

2. Etli- 


\section{Diegiffer.}

2. Etliche febone (3̈ebetin/Die man ben

Eterbenden vorfprechen for. 236

3. Etliche (jefänge angemeloet / Die man bei) Den Siranefen und Sterbenden fingen mige.

4. Etticke furke Eeufe Den Sarbendenter Damit man 5. 23as die 23 mbfebensen fol. 25 I Dic Dem 2 etforber vid berforbenen aupgenartet vno aufigebetet baben.

\section{Das IX. Capitel.}

255

1. Wo die vnfterblichen Seelent fints tonren / vito wic eb jnen ergebet nach Demi 2lofdsted vonden seiben. 259

2. $\$ 6$ pith Die feligen Seelen auch febnen nach jeen Sorpern/weil bie 2lufferfte. bung fo verzeucht. $\quad 268$

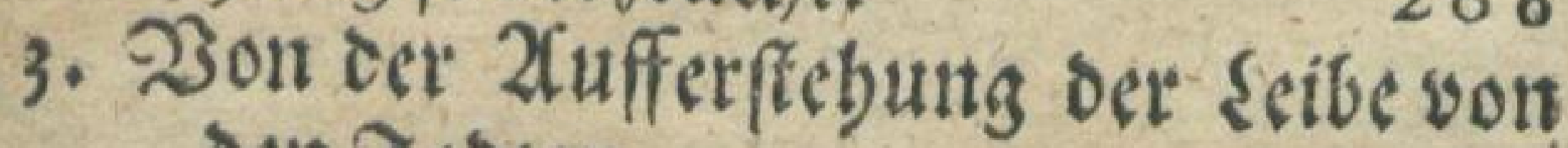
Den Sobten.

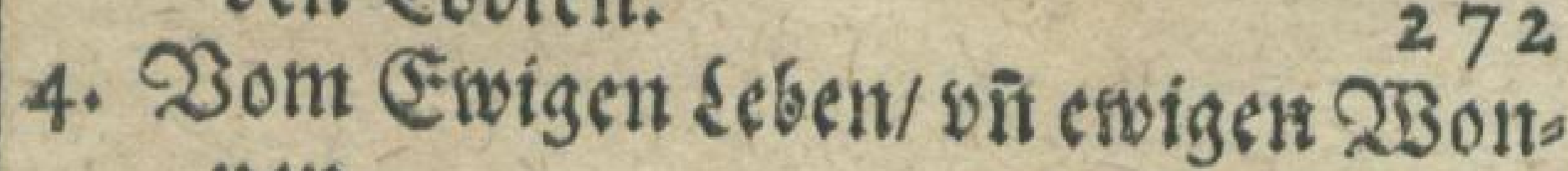
nent.

$5.230 n$ Der eswigent 28 I Damnís ber Bottlolen.
(E) $\mathfrak{R}$
(2)
E.

3uGörlit: Srucḱts vno vorlegts Jobann Rbanibaw.

$$
\text { CIJ I } \mathrm{C} \text { V. }
$$




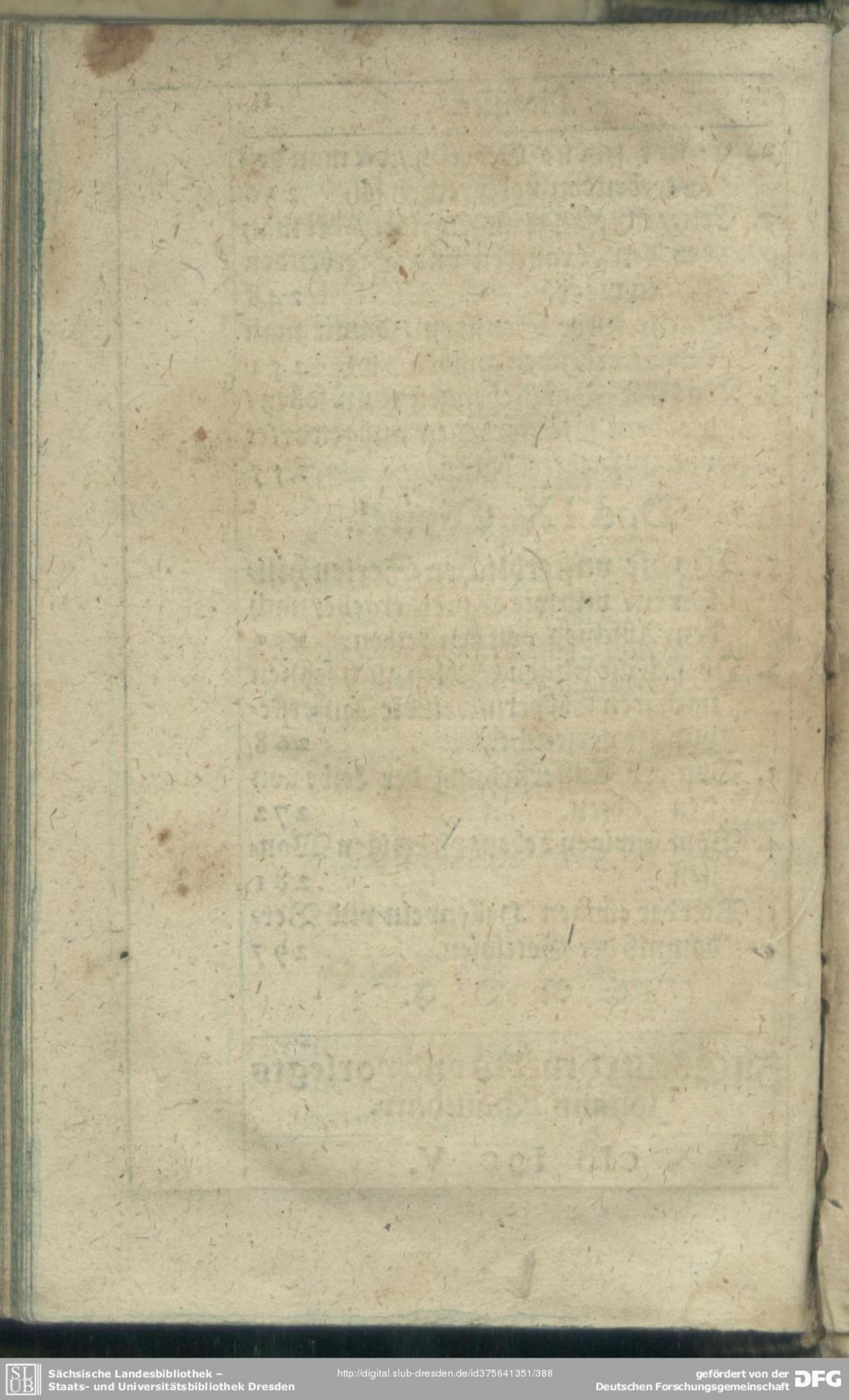




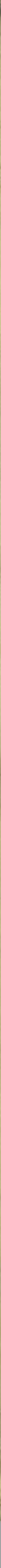

\begin{tabular}{l||l} 
SEächsische Landesbibliothek - \\
UB \\
Staats- und Universitätsbibliothek Dresden
\end{tabular} 
Datum der Entleihung bitte hier einstempe

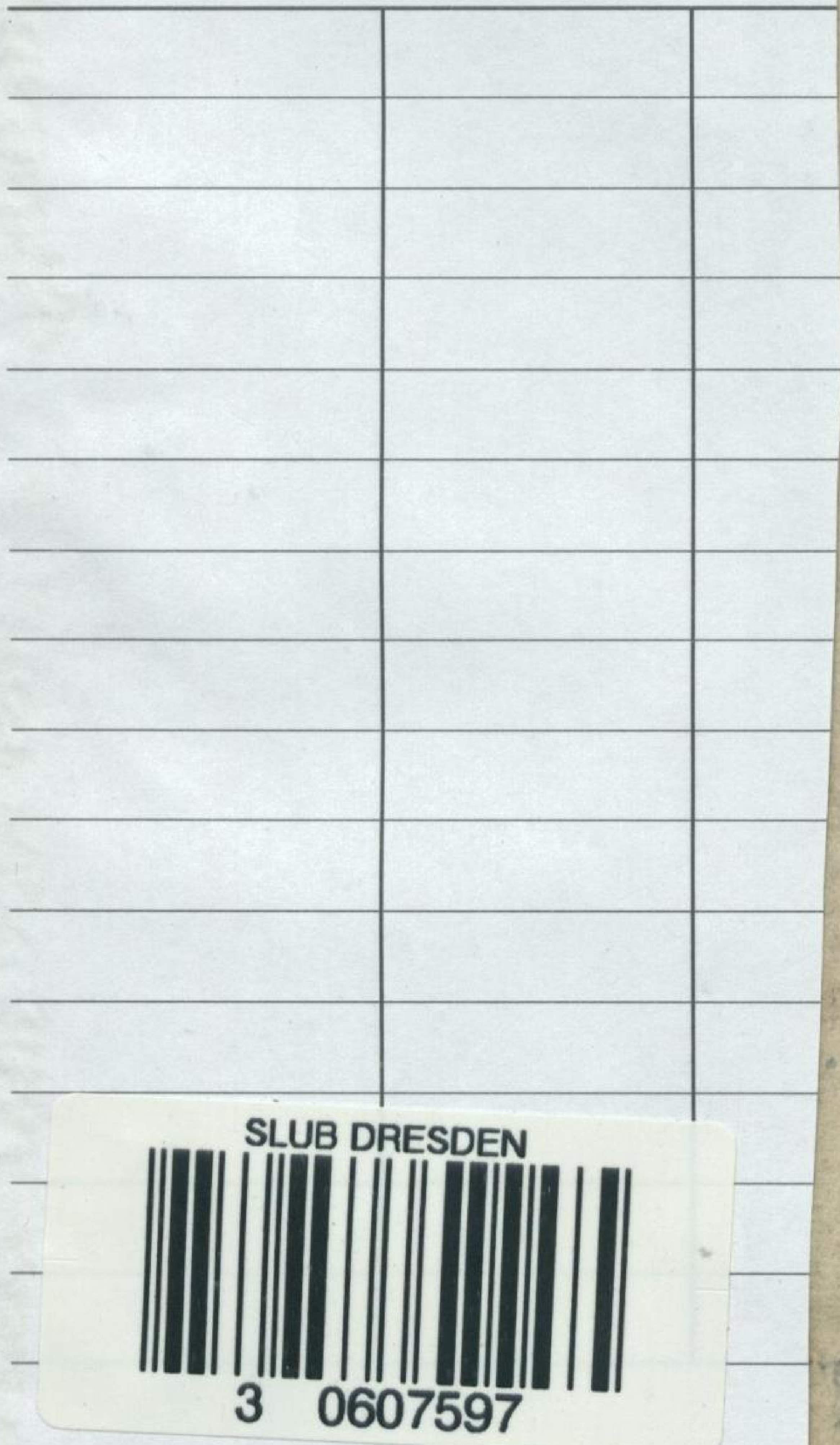

SL Sachsische Landesbibliothek - 
